\title{
Dynamic Kinetic Asymmetric Amination of Alcohols Assisted by Microwave: Stereo-convergent Access to Tetralin- and Indane-Derived Chiral Amines
}

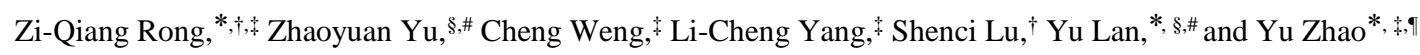 \\ ${ }^{\dagger}$ Frontiers Science Center for Flexible Electronics (FSCFE), Shaanxi Institute of Flexible Electronics (SIFE) \& Shaanxi Institute \\ of Biomedical Materials and Engineering (SIBME), Northwestern Polytechnical University (NPU), 127 West Youyi Road, Xi'an \\ 710072, China \\ Department of Chemistry, National University of Singapore, 3 Science Drive 3, Republic of Singapore, 117543 \\ ${ }^{\S}$ School of Chemistry and Chemical Engineering, Chongqing University, Chongqing, 400030, China \\ ${ }^{\mathbb{I} C h i n a}$ Joint School of National University of Singapore and Tianjin University, International Campus of Tianjin University, \\ Binhai New City, Fuzhou 350207, China \\ ${ }^{\#}$ College of Chemistry and Molecular Engineering, Zhengzhou University, Zhengzhou 450001, China \\ *Corresponding Author: iamzqrong@nwpu.edu.cn; zhaoyu@nus.edu.sg; lanyu@cqu.edu.cn
}

\section{Table of Contents}

1. General information

2. Representative Procedure for Dynamic Kinetic Asymmetric Amination of Alcohols

3. Optimization of DYKAT

4. Analytical data of the substrates

5. Analytical data of the products

6. Analytical data of acyclic products under microwave conditions

7. General procedure for the deprotection of $\mathbf{6 h}$

8. X-ray crystallographic analysis and determination of configuration of $\mathbf{6} \mathbf{f}$

9. NMR spectra of the substrates

10. NMR spectra of the products

11. References 


\section{General Information}

Thin layer chromatography (TLC) was performed on Merck pre-coated TLC plates (Merck 60 F254), and compounds were visualized with a UV light at 254nm. Further visualization was achieved by staining with iodine, or potassium permanganate solution followed by heating using a heat gun. Flash chromatography separations were performed on Merck 60 (0.040-0.063 mm) mesh silica gel. ${ }^{\mathbf{1}} \mathbf{H}$ and ${ }^{{ }^{13}} \mathbf{C}$ NMR spectra were recorded on a Bruker ACF300 (300 MHz) or AMX500 (500 MHz) spectrometer. Chemical shifts were reported in parts per million (ppm), and the residual solvent peak was used as an internal reference: proton (chloroform $\delta 7.26$ ), carbon (chloroform $\delta 77.0$ ) or tetramethylsilane (TMS $\delta 0.00$ ) was used as a reference. Data are reported as follows: chemical shift, multiplicity ( $\mathrm{s}=$ singlet, $\mathrm{d}=$ doublet, $\mathrm{t}=$ triplet, $\mathrm{q}=$ quartet, $\mathrm{m}=$ multiplet, $\mathrm{br}=$ broad $)$, coupling constants $(\mathrm{Hz})$ and integration. High resolution mass spectra (HRMS) were obtained on a Finnigan/MAT 95XL$\mathrm{T}$ spectrometer. Optical rotations were recorded on an mrc AP81 automatic polarimeter. Enantiomeric excesses (ee) were determined by HPLC analysis on Shimadzu HPLC units, including the following instruments: pump, LC-20AD; detector, SPD-20A; column, Chiralcel OD-H, OJ-H, Chiralpak AS-H, IC.

A Discover microwave reactor (Discover SP - Microwave Synthesizer, CEM, North Carolina, USA) was used to heat the reactions.

All reactions were carried out under nitrogen atmosphere. All solvents were purified and dried according to standard methods prior to use. The Iridium complex ${ }^{1}$, chiral phosphoric acid $^{1,2}$, all substrates ${ }^{3}$ were prepared based on the general procedure. 


\section{Representative Procedure for Dynamic Kinetic Asymmetric Amination of Alcohols.}

Scheme S1. Representative Procedure for Dynamic Kinetic Asymmetric Amination of Alcohols

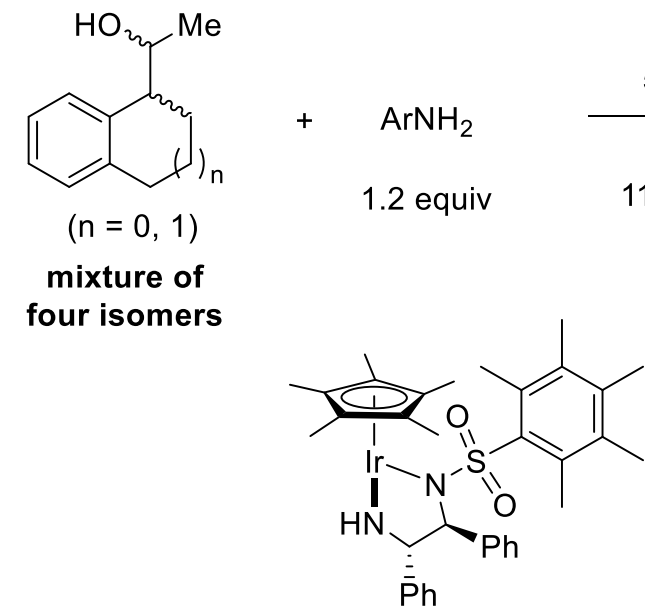

$2(S, S)$-Ir complex
$5 \mathrm{~mol} \% 2,10 \mathrm{~mol} \% 3$

$4 \AA$ MS, toluene $110{ }^{\circ} \mathrm{C}$, time, Microwave

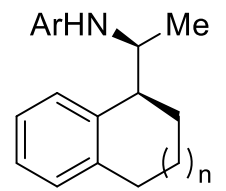

To a $10 \mathrm{~mL}$ microwave tube equipped with a stir bar was added alcohol $(0.20 \mathrm{mmol}$, 1.0 equiv.), aniline (0.24 mmol, 1.2 equiv.) and chiral phosphoric acid 3 (0.0200 mmol, $14.4 \mathrm{mg}$ ). The mixture was taken into the glovebox, where iridium complex 2 $(0.0100 \mathrm{mmol}, 7.5 \mathrm{mg}), 100 \mathrm{mg}$ molecular sieves and anhydrous toluene $(0.5 \mathrm{~mL})$ were added. The reaction mixture was sealed with a rubber septum and taken outside the glovebox. The tube was then put into the microwave reactor and allowed to stir at $110{ }^{\circ} \mathrm{C}$. After completion of the reaction (monitored by TLC), the reaction was directly purified by silica gel chromatography $($ Hexane/EA $=50 / 1)$. 


\section{Optimization of DYKAT.}

Table S1. Optimization of DYKAT<smiles>CC(O)C1CCCc2ccccc21</smiles>

$( \pm)-1 \mathrm{a}$

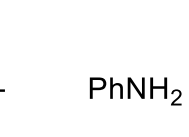

(1.2 equiv.)
$5 \mathrm{~mol} \%$ [Ir]

$10 \mathrm{~mol} \%$ acid

A MS, solven

$110^{\circ} \mathrm{C}, 120 \mathrm{~h}$

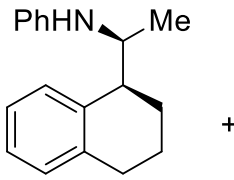

$4 a$<smiles>C[C@H](Nc1ccccc1)[C@H]1CCCc2ccccc21</smiles>

epi-4a

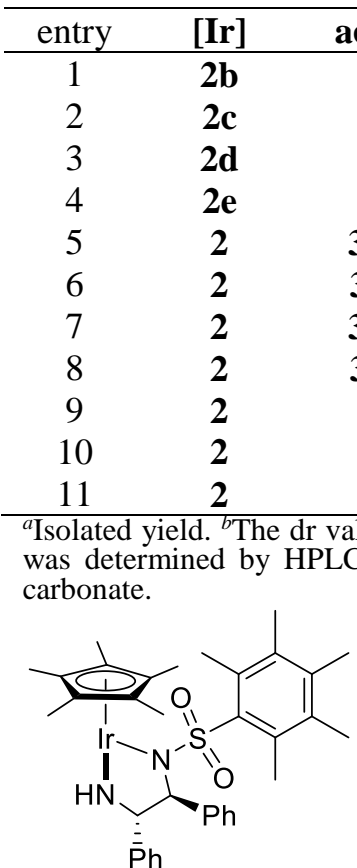

2

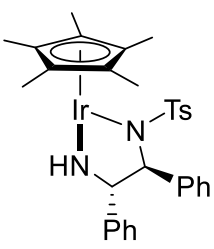

2b

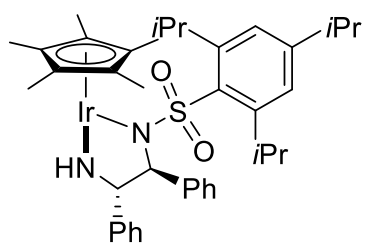

2c<smiles>CC1=C(C)C2(C)C(C)=C(C)C(C)(C1)N2S(C)(=O)=O</smiles>

2d<smiles>CC1=C(C)C(C)(C)N(I2N[C@@H]3CCCC[C@@H]3N2S(=O)(=O)c2c(C)c(C)c(C)c(C)c2C)C1</smiles>

$2 e$

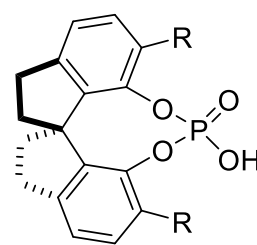

$3\left(\mathrm{R}=2,4,6-i \mathrm{Pr}_{3} \mathrm{C}_{6} \mathrm{H}_{2}\right)$<smiles>[R]c1cc2ccccc2c(-c2c(OP(=O)(O)O)c([R])cc3ccccc23)c1OP(=O)(O)O</smiles>

3b, $\mathrm{R}=2,4,6-i \mathrm{Pr}_{3} \mathrm{C}_{6} \mathrm{H}_{2}$ 3c, $\mathrm{R}=9$-anthracyl 3d, $\mathrm{R}=3,5-\left(\mathrm{CF}_{3}\right)_{2} \mathrm{C}_{6} \mathrm{H}_{3}$<smiles></smiles>

$3 e$ 


\section{Analytical data of substrates.}

\section{1-(5-methoxy-1,2,3,4-tetrahydronaphthalen-1-yl)ethanol (1b).}

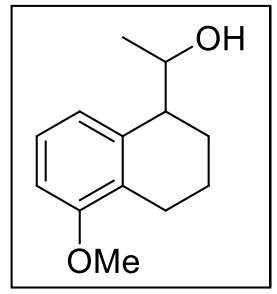

Colorless oil, 1:1 dr. ${ }^{1} \mathbf{H}$ NMR $\left(500 \mathrm{MHz}, \mathrm{CD}_{2} \mathrm{Cl}_{2}\right) \delta 7.19-7.08(\mathrm{~m}, 2 \mathrm{H})$, $6.93(\mathrm{~d}, J=7.5 \mathrm{~Hz}, 1 \mathrm{H}), 6.83(\mathrm{~d}, J=8.0 \mathrm{~Hz}, 1 \mathrm{H}), 6.73-6.69(\mathrm{~m}, 2 \mathrm{H})$, $4.30-4.25(\mathrm{~m}, 1 \mathrm{H}), 4.02-3.97(\mathrm{~m}, 1 \mathrm{H}), 3.81(\mathrm{~s}, 6 \mathrm{H}), 2.80-2.70(\mathrm{~m}$, $3 \mathrm{H}), 2.67-2.64(\mathrm{~m}, 2 \mathrm{H}), 2.55-2.48(\mathrm{~m}, 1 \mathrm{H}), 2.03-1.96(\mathrm{~m}, 1 \mathrm{H}), 1.91$ $-1.76(\mathrm{~m}, 6 \mathrm{H}), 1.74-1.68(\mathrm{~m}, 1 \mathrm{H}), 1.24(\mathrm{~d}, J=6.4 \mathrm{~Hz}, 3 \mathrm{H}), 1.16(\mathrm{~d}, J=$ $6.3 \mathrm{~Hz}, 3 \mathrm{H}) .{ }^{13} \mathbf{C}$ NMR $\left(126 \mathrm{MHz}, \mathrm{CD}_{2} \mathrm{Cl}_{2}\right) \delta 157.9,157.7,139.8,139.3,128.3,126.9,126.7$, 125.9, 122.0, 120.8, 107.9, 107.6, 70.9, 70.6, 55.7, 55.6, 45.6, 44.9, 39.7, 31.0, 24.3, 23.4, 20.9, 20.7, 20.1, 19.6. HRMS-EI (m/z): [M] calcd for $\mathrm{C}_{13} \mathrm{H}_{18} \mathrm{O}_{2}: 206.1307$; Found: 206.1309.

\section{1-(6-methoxy-1,2,3,4-tetrahydronaphthalen-1-yl)ethanol (1c).}

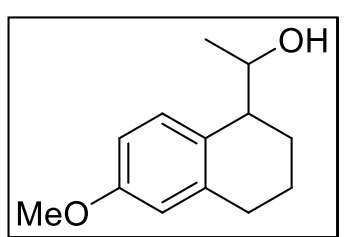

Colorless oil, 5:1 dr. ${ }^{1} \mathbf{H}$ NMR (500 MHz, $\mathrm{CD}_{2} \mathrm{Cl}_{2}$ ): $\delta 7.33$ (d, $J=8.1$ $\mathrm{Hz}, 0.19 \mathrm{H}), 7.12(\mathrm{~d}, J=8.3 \mathrm{~Hz}, 1 \mathrm{H}), 6.78-6.57(\mathrm{~m}, 2 \mathrm{H}), 6.03(\mathrm{td}, J$ $=4.7,1.3 \mathrm{~Hz}, 0.18 \mathrm{H}), 4.83(\mathrm{qd}, J=6.5,1.3 \mathrm{~Hz}, 0.18 \mathrm{H}), 4.02-3.93$ (m, 1H), $3.79(\mathrm{~s}, 1 \mathrm{H}), 3.76(\mathrm{~s}, 3 \mathrm{H}), 2.80-2.62(\mathrm{~m}, 3 \mathrm{H}), 2.36-2.11$ (m, $0.45 \mathrm{H}), 1.91-1.73(\mathrm{~m}, 3 \mathrm{H}), 1.73-1.61(\mathrm{~m}, 1 \mathrm{H}), 1.57(\mathrm{~s}, 1 \mathrm{H}), 1.41(\mathrm{~d}, J=6.4 \mathrm{~Hz}, 1 \mathrm{H})$, $1.15(\mathrm{~d}, J=6.3 \mathrm{~Hz}, 3 \mathrm{H}) .{ }^{13} \mathrm{C} \mathbf{~ N M R}\left(125 \mathrm{MHz}, \mathrm{CD}_{2} \mathrm{Cl}_{2}\right): \delta 158.9,158.3,139.8,139.4,130.8$, 130.1, 124.6, 121.5, 114.3, 114.3, 111.8, 111.2, 71.2, 67.8, 55.6, 55.5, 44.8, 30.2, 29.2, 25.0, 23.2, 22.9, 20.6, 20.2. HRMS-EI (m/z): [M] calcd for $\mathrm{C}_{13} \mathrm{H}_{18} \mathrm{O}_{2}$ : 206.1307; Found: 206.1312 .

\section{1-(6-chloro-1,2,3,4-tetrahydronaphthalen-1-yl)ethanol (1d).}

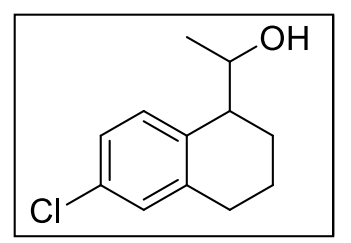

Colorless oil, 1:1 dr. ${ }^{1} \mathbf{H}$ NMR (500 MHz, $\left.\mathrm{CDCl}_{3}\right): \delta 7.24(\mathrm{~d}, J=8.2$ $\mathrm{Hz}, 1 \mathrm{H}), 7.18-7.05(\mathrm{~m}, 4 \mathrm{H}), 4.27$ (qd, $J=6.4,4.0 \mathrm{~Hz}, 1 \mathrm{H}), 4.00$ (p, $J=6.4 \mathrm{~Hz}, 1 \mathrm{H}), 2.86-2.61(\mathrm{~m}, 6 \mathrm{H}), 2.04-1.93(\mathrm{~m}, 1 \mathrm{H}), 1.90-$

$1.76(\mathrm{~m}, 5 \mathrm{H}), 1.73-1.58(\mathrm{~m}, 2 \mathrm{H}), 1.25(\mathrm{~d}, J=6.4 \mathrm{~Hz}, 3 \mathrm{H}), 1.20(\mathrm{~d}, J$

$=6.2 \mathrm{~Hz}, 3 \mathrm{H}) .{ }^{13} \mathbf{C} \mathbf{N M R}\left(126 \mathrm{MHz}, \mathrm{CDCl}_{3}\right): \delta 141.1,139.9,135.9,135.8,131.7,131.6$, 130.8, 129.7, 129.1, 129.0, 126.0, 125.5, 70.8, 70.5, 44.4, 44.0, 29.8, 29.3, 24.2, 22.6, 21.0, 20.2, 20.0, 19.8. HRMS-EI (m/z): [M] $]^{+}$calcd for $\mathrm{C}_{12} \mathrm{H}_{15} \mathrm{ClO}$ : 210.0811; Found: 210.0807.

\section{1-(7-methoxy-1,2,3,4-tetrahydronaphthalen-1-yl)ethanol (1e).}

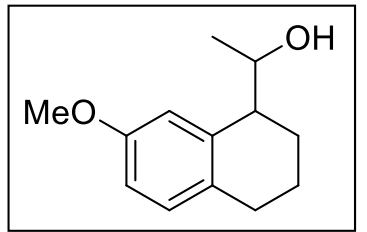

Colorless oil, 5:1 dr. ${ }^{1} \mathbf{H}$ NMR (500 MHz, $\left.\mathrm{CDCl}_{3}\right): \delta 7.02(\mathrm{dd}, J=$ $8.4,4.8 \mathrm{~Hz}, 2 \mathrm{H}), 6.86(\mathrm{~d}, J=2.7 \mathrm{~Hz}, 1 \mathrm{H}), 6.78(\mathrm{~d}, J=2.7 \mathrm{~Hz}, 1 \mathrm{H})$, $6.72(\mathrm{ddd}, J=8.5,5.9,2.7 \mathrm{~Hz}, 2 \mathrm{H}), 4.34(\mathrm{qd}, J=6.4,3.8 \mathrm{~Hz}, 1 \mathrm{H})$, $4.03(\mathrm{p}, J=6.5 \mathrm{~Hz}, 1 \mathrm{H}), 3.78(\mathrm{~s}, 3 \mathrm{H}), 3.78(\mathrm{~s}, 3 \mathrm{H}), 2.85-2.75(\mathrm{~m}$, $2 \mathrm{H}), 2.73-2.62(\mathrm{~m}, 4 \mathrm{H}), 2.06-1.91(\mathrm{~m}, 1 \mathrm{H}), 1.90-1.75(\mathrm{~m}, 5 \mathrm{H}), 1.73-1.60(\mathrm{~m}, 2 \mathrm{H}), 1.28$ $(\mathrm{d}, J=6.4 \mathrm{~Hz}, 3 \mathrm{H}), 1.21(\mathrm{~d}, J=6.3 \mathrm{~Hz}, 3 \mathrm{H}) .{ }^{13} \mathbf{C} \mathbf{~ N M R}\left(126 \mathrm{MHz}, \mathrm{CDCl}_{3}\right): \delta 157.8,157.3$, 
$138.4,131.5,130.2,130.1,130.0,114.4,113.2,112.2,112.0,70.8,70.5,55.2,45.2,44.6,29.1$, 28.6, 24.2, 22.5, 21.6, 20.3, 20.1, 19.8. HRMS-EI (m/z): [M] ${ }^{+}$calcd for $\mathrm{C}_{13} \mathrm{H}_{18} \mathrm{O}_{2}:$ 206.1307; Found: 206.1306.

\section{1-(7-fluoro-1,2,3,4-tetrahydronaphthalen-1-yl)ethanol (1f).}

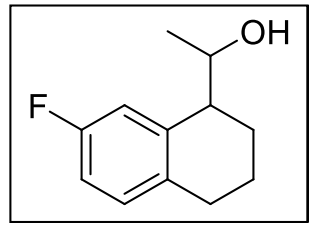

Colorless oil, 1:1 dr. ${ }^{1} \mathbf{H}$ NMR $\left(500 \mathrm{MHz}, \mathrm{CDCl}_{3}\right): \delta 7.10-6.99(\mathrm{~m}$, $3 \mathrm{H}), 6.96(\mathrm{dd}, J=10.2,2.7 \mathrm{~Hz}, 1 \mathrm{H}), 6.84(\mathrm{td}, J=8.3,3.5 \mathrm{~Hz}, 2 \mathrm{H})$, $4.29(\mathrm{qd}, J=6.3,3.9 \mathrm{~Hz}, 1 \mathrm{H}), 4.05(\mathrm{p}, J=6.4 \mathrm{~Hz}, 1 \mathrm{H}), 2.82(\mathrm{dd}, J=$ 7.4, $4.2 \mathrm{~Hz}, 2 \mathrm{H}), 2.76-2.65(\mathrm{~m}, 4 \mathrm{H}), 2.04-1.94(\mathrm{~m}, 1 \mathrm{H}), 1.92-$

$1.73(\mathrm{~m}, 5 \mathrm{H}), 1.72-1.59(\mathrm{~m}, 2 \mathrm{H}), 1.27(\mathrm{~d}, J=6.4 \mathrm{~Hz}, 3 \mathrm{H}), 1.19(\mathrm{~d}, J=6.2 \mathrm{~Hz}, 3 \mathrm{H}) .{ }^{13} \mathrm{C}$ NMR $\left(126 \mathrm{MHz}, \mathrm{CDCl}_{3}\right): \delta 162.1,161.6,160.2,159.7,139.4,139.3,139.3,134.7,134.7$, $133.4,133.4,130.5,130.5,130.5,130.4,115.7,115.5,114.5,114.3,113.2,113.1,113.0$, $112.9,70.8,70.5,44.9,44.5,29.3,28.8,23.8,22.4,21.5,20.4,20.2,19.8$. HRMS-EI (m/z): $[\mathrm{M}]^{+}$calcd for $\mathrm{C}_{12} \mathrm{H}_{15} \mathrm{FO}$ : 194.1107; Found: 194.1098.

\section{1-(7-chloro-1,2,3,4-tetrahydronaphthalen-1-yl)ethanol (1g)}

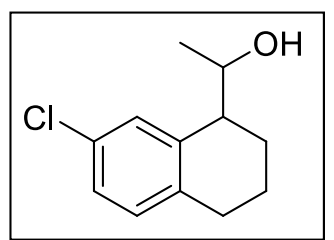

Colorless oil, 1:1 dr. ${ }^{1} \mathbf{H}$ NMR $\left(500 \mathrm{MHz}, \mathrm{CDCl}_{3}\right): \delta 7.31(\mathrm{~d}, J=2.1$ $\mathrm{Hz}, 1 \mathrm{H}), 7.23(\mathrm{~d}, J=2.2 \mathrm{~Hz}, 1 \mathrm{H}), 7.09$ (ddd, $J=7.8,5.3,2.2 \mathrm{~Hz}, 2 \mathrm{H})$, $7.02(\mathrm{dd}, J=8.3,3.6 \mathrm{~Hz}, 2 \mathrm{H}), 4.29(\mathrm{qd}, J=6.4,3.7 \mathrm{~Hz}, 1 \mathrm{H}), 4.04(\mathrm{p}$, $J=6.4 \mathrm{~Hz}, 1 \mathrm{H}), 2.82-2.78(\mathrm{~m}, 2 \mathrm{H}), 2.73-2.67(\mathrm{~m}, 4 \mathrm{H}), 2.05-1.94$ (m, 1H), $1.92-1.74(\mathrm{~m}, 5 \mathrm{H}), 1.72-1.57(\mathrm{~m}, 2 \mathrm{H}), 1.27$ (d, $J=6.3 \mathrm{~Hz}, 3 \mathrm{H}), 1.19$ (d, $J=6.2$ $\mathrm{Hz}, 3 \mathrm{H}) .{ }^{13} \mathbf{C}$ NMR $\left(126 \mathrm{MHz}, \mathrm{CDCl}_{3}\right): \delta 139.4,139.3,137.6,136.4,131.3,130.8,130.5$, $130.2,129.2,128.1,126.2,126.1,70.7,70.5,44.8,44.3,29.4,28.9,23.9,22.4,21.2,20.2$, 20.1, 19.9. HRMS-EI (m/z): [M] ${ }^{+}$calcd for $\mathrm{C}_{12} \mathrm{H}_{15} \mathrm{ClO}: 210.0811$; Found: 210.0802.

\section{1-(6,7-dimethoxy-1,2,3,4-tetrahydronaphthalen-1-yl)ethanol (1h).}

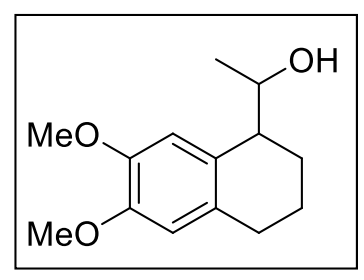

Colorless oil, 1:1 dr. ${ }^{1} \mathbf{H}$ NMR $\left(500 \mathrm{MHz}, \mathrm{CDCl}_{3}\right): \delta 6.81(\mathrm{~s}, 1 \mathrm{H})$, $6.74(\mathrm{~s}, 1 \mathrm{H}), 6.59(\mathrm{~s}, 1 \mathrm{H}), 6.58(\mathrm{~s}, 1 \mathrm{H}), 4.29(\mathrm{qd}, J=6.4,3.7 \mathrm{~Hz}$, $1 \mathrm{H}), 4.02-3.91(\mathrm{~m}, 1 \mathrm{H}), 3.84-3.83(\mathrm{~m}, 12 \mathrm{H}), 2.77(\mathrm{td}, J=7.1$, $3.7 \mathrm{~Hz}, 1 \mathrm{H}), 2.72-2.61(\mathrm{~m}, 5 \mathrm{H}), 2.05-1.91(\mathrm{~m}, 1 \mathrm{H}), 1.87-1.77$ $(\mathrm{m}, 5 \mathrm{H}), 1.74-1.65(\mathrm{~m}, 2 \mathrm{H}), 1.26(\mathrm{~d}, J=6.4 \mathrm{~Hz}, 3 \mathrm{H}), 1.21(\mathrm{~d}, J=$ $6.2 \mathrm{~Hz}, 3 \mathrm{H}) .{ }^{13} \mathbf{C} \mathbf{N M R}\left(126 \mathrm{MHz}, \mathrm{CDCl}_{3}\right): \delta 147.5,147.3,147.2,146.7,131.5,130.0,129.0$, 128.8, 112.7, 112.2, 112.0, 111.2, 71.0, 70.5, 56.0, 55.8, 55.8, 44.6, 43.9, 29.5, 29.0, 24.5, 22.7, 21.5, 20.1, 20.0, 19.9. HRMS-EI (m/z): $[\mathrm{M}]^{+}$calcd for $\mathrm{C}_{14} \mathrm{H}_{20} \mathrm{O}_{3}: 236.1412$; Found: 236.1405 .

\section{1-(5,7-dimethyl-1,2,3,4-tetrahydronaphthalen-1-yl)ethanol (1i).}




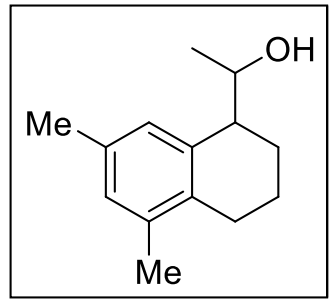

Colorless oil, 6:1 dr. ${ }^{1} \mathbf{H}$ NMR $\left(500 \mathrm{MHz}, \mathrm{CD}_{2} \mathrm{Cl}_{2}\right): \delta 6.98(\mathrm{~s}, 0.18 \mathrm{H})$, $6.88(\mathrm{~s}, 1 \mathrm{H}), 6.87(\mathrm{~s}, 1 \mathrm{H}), 6.85(\mathrm{~s}, 0.18 \mathrm{H}), 4.28(\mathrm{qd}, J=6.4,4.2 \mathrm{~Hz}$, $0.18 \mathrm{H}), 3.97(\mathrm{dq}, J=7.3,6.3 \mathrm{~Hz}, 1 \mathrm{H}), 2.79-2.73(\mathrm{~m}, 0.20 \mathrm{H}), 2.73-$ $2.67(\mathrm{~m}, 1 \mathrm{H}), 2.63-2.58(\mathrm{~m}, 2 \mathrm{H}), 2.30(\mathrm{~s}, 0.46 \mathrm{H}), 2.28(\mathrm{~s}, 3 \mathrm{H}), 2.20$ (s, 4H), $1.97-1.72(\mathrm{~m}, 5 \mathrm{H}), 1.68(\mathrm{~s}, 1 \mathrm{H}), 1.25(\mathrm{~d}, J=6.3 \mathrm{~Hz}, 1 \mathrm{H})$, $1.19(\mathrm{~d}, J=6.3 \mathrm{~Hz}, 3 \mathrm{H}) .{ }^{13} \mathrm{C}$ NMR $\left(125 \mathrm{MHz}, \mathrm{CD}_{2} \mathrm{Cl}_{2}\right): \delta 137.7$, $137.0,134.5,133.4,129.1,128.3,70.9,46.0,26.5,24.7,21.1,20.3,20.1,19.8$. HRMS-EI (m/z): $[\mathrm{M}]^{+}$calcd for $\mathrm{C}_{14} \mathrm{H}_{20} \mathrm{O}: 204.1514$; Found: 204.1513 .

\section{1-(2,3-dihydro-1H-inden-1-yl)ethanol (5a).}

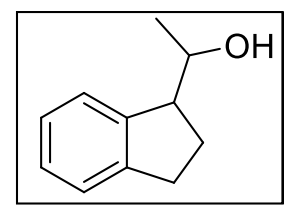

Colorless oil, 1:1 dr. ${ }^{1} \mathbf{H}$ NMR $\left(500 \mathrm{MHz}, \mathrm{CDCl}_{3}\right): \delta 7.43(\mathrm{~d}, J=6.7 \mathrm{~Hz}$, $1 \mathrm{H}), 7.33-7.25(\mathrm{~m}, 3 \mathrm{H}), 7.25-7.17(\mathrm{~m}, 4 \mathrm{H}), 4.29(\mathrm{qd}, J=6.3,3.5 \mathrm{~Hz}$, $1 \mathrm{H}), 3.95(\mathrm{p}, J=6.4 \mathrm{~Hz}, 1 \mathrm{H}), 3.27(\mathrm{td}, J=7.5,3.6 \mathrm{~Hz}, 1 \mathrm{H}), 3.25-3.20$ $(\mathrm{m}, 1 \mathrm{H}), 3.00(\mathrm{dt}, J=14.5,7.2 \mathrm{~Hz}, 2 \mathrm{H}), 2.95-2.83(\mathrm{~m}, 2 \mathrm{H}), 2.30-$ $2.09(\mathrm{~m}, 3 \mathrm{H}), 2.00-1.90(\mathrm{~m}, 1 \mathrm{H}), 1.30(\mathrm{~d}, J=6.3 \mathrm{~Hz}, 3 \mathrm{H}), 1.26(\mathrm{~d}, J=6.3 \mathrm{~Hz}, 3 \mathrm{H}) .{ }^{13} \mathrm{C}$ NMR $\left(126 \mathrm{MHz}, \mathrm{CDCl}_{3}\right): \delta 145.4,144.7,144.1,143.5,127.0,126.9,126.2,126.1,125.2$, 124.7, 124.6, 124.0, 71.0, 68.9, 52.4, 52.1, 31.6, 31.5, 28.0, 25.0, 20.8, 20.4. HRMS-EI (m/z): $[\mathrm{M}]^{+}$calcd for $\mathrm{C}_{11} \mathrm{H}_{14} \mathrm{O}: 162.1045$; Found: 162.1042 .

\section{1-(5-methoxy-2,3-dihydro-1H-inden-1-yl)ethanol (5b).}

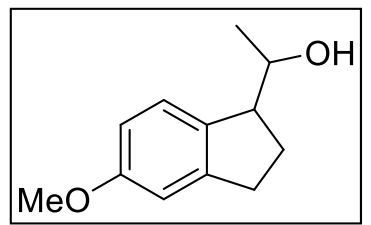

Colorless oil, $1: 1 \mathrm{dr} .{ }^{1} \mathbf{H}$ NMR $\left(500 \mathrm{MHz}, \mathrm{CDCl}_{3}\right): \delta 7.29(\mathrm{~d}, J=$ $8.3 \mathrm{~Hz}, 1 \mathrm{H}), 7.16(\mathrm{~d}, J=8.2 \mathrm{~Hz}, 1 \mathrm{H}), 6.79$ (s, 2H), 6.73 (ddd, $J=$ $11.0,8.2,2.5 \mathrm{~Hz}, 2 \mathrm{H}), 4.19$ (qd, $J=6.4,3.7 \mathrm{~Hz}, 1 \mathrm{H}), 3.86$ (p, $J=$ $6.5 \mathrm{~Hz}, 1 \mathrm{H}), 3.79(\mathrm{~s}, 6 \mathrm{H}), 3.17(\mathrm{td}, J=7.3,3.6 \mathrm{~Hz}, 1 \mathrm{H}), 3.11(\mathrm{td}, J$ $=7.6,4.8 \mathrm{~Hz}, 1 \mathrm{H}), 2.99-2.88(\mathrm{~m}, 2 \mathrm{H}), 2.88-2.75(\mathrm{~m}, 2 \mathrm{H}), 2.27-2.04(\mathrm{~m}, 3 \mathrm{H}), 1.95-1.85$ $(\mathrm{m}, 1 \mathrm{H}), 1.24(\mathrm{~d}, J=6.4 \mathrm{~Hz}, 3 \mathrm{H}), 1.22(\mathrm{~d}, J=6.3 \mathrm{~Hz}, 3 \mathrm{H}) .{ }^{13} \mathbf{C} \mathbf{N M R}\left(125 \mathrm{MHz}, \mathrm{CDCl}_{3}\right): \delta$ 159.2, 159.2, 146.9, 146.3, 136.1, 135.5, 125.8, 124.5, 112.3, 112.0, 110.1, 71.2, 69.0, 55.4, 55.3, 51.7, 51.3, 31.8, 31.7, 28.5, 25.4, 20.9, 20.3. HRMS-EI (m/z): $[\mathrm{M}]^{+}$calcd for $\mathrm{C}_{12} \mathrm{H}_{16} \mathrm{O}_{2}$ : 192.1150; Found: 192.1153.

\section{1-(5-chloro-2,3-dihydro-1H-inden-1-yl)ethanol (5c).}

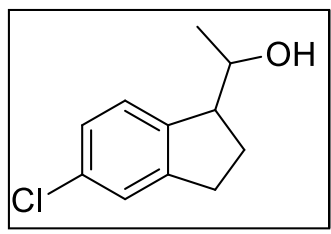

Colorless oil, 1:1 dr. ${ }^{1} \mathbf{H}$ NMR $\left(500 \mathrm{MHz}, \mathrm{CDCl}_{3}\right): \delta 7.33(\mathrm{~d}, J=8.0$ $\mathrm{Hz}, 1 \mathrm{H}), 7.22-7.10(\mathrm{~m}, 5 \mathrm{H}), 4.20$ (qd, $J=6.3,3.7 \mathrm{~Hz}, 1 \mathrm{H}), 3.88$ (p, $J=6.4 \mathrm{~Hz}, 1 \mathrm{H}), 3.19(\mathrm{td}, J=7.7,3.7 \mathrm{~Hz}, 1 \mathrm{H}), 3.13(\mathrm{td}, J=7.6,5.3$ $\mathrm{Hz}, 1 \mathrm{H}), 3.00-2.88(\mathrm{~m}, 2 \mathrm{H}), 2.88-2.78(\mathrm{~m}, 2 \mathrm{H}), 2.27-2.04(\mathrm{~m}$, $3 \mathrm{H}), 1.93-1.87(\mathrm{~m}, 1 \mathrm{H}), 1.23(\mathrm{~d}, J=6.3 \mathrm{~Hz}, 3 \mathrm{H}), 1.21(\mathrm{~d}, J=6.2 \mathrm{~Hz}, 3 \mathrm{H}) .{ }^{13} \mathbf{C}$ NMR $(125$ $\left.\mathrm{MHz}, \mathrm{CDCl}_{3}\right): \delta 147.3,146.7,142.7,142.1,132.6,132.6,126.4,126.3,126.2,125.1,124.9$, 
124.7, 71.0, 68.9, 51.8, 51.5, 31.6, 31.4, 28.2, 25.3, 21.0, 20.4. HRMS-EI (m/z): $[\mathrm{M}]^{+}$calcd for $\mathrm{C}_{11} \mathrm{H}_{13} \mathrm{ClO}$ : 196.0655; Found: 196.0662 .

\section{1-(6-methyl-2,3-dihydro-1H-inden-1-yl)ethanol (5d).}

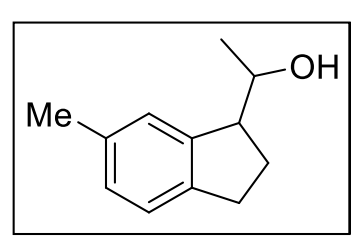

Colorless oil, 8:1 dr. ${ }^{1} \mathbf{H}$ NMR (500 MHz, Acetone-d6): $\delta 7.28$ (s, $1 \mathrm{H}), 7.13(\mathrm{~s}, 0.24 \mathrm{H}), 7.05(\mathrm{~d}, J=7.6 \mathrm{~Hz}, 1 \mathrm{H}), 6.99(\mathrm{~d}, J=7.7 \mathrm{~Hz}$, $0.16 \mathrm{H}), 6.93(\mathrm{~d}, J=7.6 \mathrm{~Hz}, 1 \mathrm{H}), 4.07(\mathrm{~h}, J=6.0 \mathrm{~Hz}, 0.11 \mathrm{H}), 3.90(\mathrm{~h}$, $J=6.0 \mathrm{~Hz}, 1 \mathrm{H}), 3.82(\mathrm{dt}, J=3.8,1.6 \mathrm{~Hz}, 0.13 \mathrm{H}), 3.58(\mathrm{ddt}, J=5.0$, $3.3,1.6 \mathrm{~Hz}, 1 \mathrm{H}), 3.33(\mathrm{dd}, J=3.3,1.9 \mathrm{~Hz}, 0.10 \mathrm{H}), 3.14(\mathrm{q}, J=7.0 \mathrm{~Hz}, 1 \mathrm{H}), 2.92-2.68(\mathrm{~m}$, $3 \mathrm{H}), 2.30$ (s, 0.49H), 2.27 (s, 3H), $2.15-2.06(\mathrm{~m}, 1 \mathrm{H}), 1.93-1.80$ (m, 1H), 1.16 (dd, $J=6.4$, $1.0 \mathrm{~Hz}, 0.36 \mathrm{H}), 1.09$ (dd, $J=6.2,1.0 \mathrm{~Hz}, 3 \mathrm{H}) .{ }^{13} \mathrm{C}$ NMR (126 MHz, Acetone-d6): $\delta 146.3$, 142.2, 135.8, 129.1, 128.0, 126.8, 126.3, 125.1, 124.7, 71.4, 69.1, 53.3, 43.3, 31.9, 31.5, 28.5, 28.3, 21.4, 20.9. HRMS-EI (m/z): [M] $]^{+}$calcd for $\mathrm{C}_{12} \mathrm{H}_{16} \mathrm{O}$ : 176.1201; Found: 176.1198.

\section{1-(6-methoxy-2,3-dihydro-1H-inden-1-yl)ethanol (5e).}

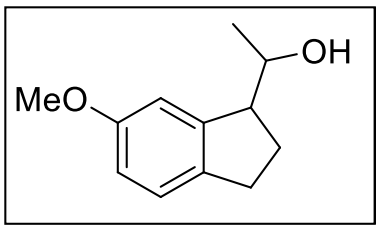

Colorless oil, 1:1 dr. ${ }^{1} \mathbf{H}$ NMR $\left(500 \mathrm{MHz}, \mathrm{CDCl}_{3}\right): \delta 7.13$ (dd, $J$ $=8.3,3.6 \mathrm{~Hz}, 2 \mathrm{H}), 6.98(\mathrm{~d}, J=2.3 \mathrm{~Hz}, 1 \mathrm{H}), 6.83(\mathrm{~d}, J=2.3 \mathrm{~Hz}$, $1 \mathrm{H}), 6.75(\mathrm{dd}, J=8.2,2.5 \mathrm{~Hz}, 2 \mathrm{H}), 4.25(\mathrm{qd}, J=6.4,3.6 \mathrm{~Hz}, 1 \mathrm{H})$, $3.90(\mathrm{p}, J=6.4 \mathrm{~Hz}, 1 \mathrm{H}), 3.80-3.79(\mathrm{~m}, 6 \mathrm{H}), 3.29-3.08(\mathrm{~m}$, 2H), $2.95-2.74(\mathrm{~m}, 4 \mathrm{H}), 2.26-2.06(\mathrm{~m}, 3 \mathrm{H}), 1.97-1.83(\mathrm{~m}, 1 \mathrm{H}), 1.26(\mathrm{~d}, J=6.3 \mathrm{~Hz}, 3 \mathrm{H})$, $1.23(\mathrm{~d}, J=6.2 \mathrm{~Hz}, 3 \mathrm{H}) .{ }^{13} \mathrm{C}$ NMR $\left(126 \mathrm{MHz}, \mathrm{CDCl}_{3}\right): \delta 158.8,158.5,145.7,145.0,137.3$, 136.6, 125.2, 125.0, 112.8, 112.8, 110.9, 109.6, 71.0, 68.9, 55.5, 52.7, 52.3, 30.7, 30.6, 28.6, 25.5, 20.9, 20.4. HRMS-EI (m/z): [M] $]^{+}$calcd for $\mathrm{C}_{12} \mathrm{H}_{16} \mathrm{O}_{2}:$ 192.1150; Found: 192.1152.

\section{1-(5-chloro-6-methoxy-2,3-dihydro-1H-inden-1-yl)ethanol (5f).}

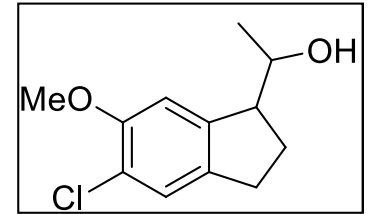

Colorless oil, 1.6:1 dr. ${ }^{1} \mathbf{H}$ NMR $\left(500 \mathrm{MHz}, \mathrm{CDCl}_{3}\right): \delta 7.22(\mathrm{~s}, 1 \mathrm{H})$, $7.20(\mathrm{~s}, 1 \mathrm{H}), 7.07(\mathrm{~s}, 1 \mathrm{H}), 6.87(\mathrm{~s}, 1 \mathrm{H}), 4.23(\mathrm{qd}, J=6.3,3.5 \mathrm{~Hz}$, $1 \mathrm{H}), 3.91-3.85(\mathrm{~m}, 7 \mathrm{H}), 3.22(\mathrm{td}, J=9.0,7.9,3.5 \mathrm{~Hz}, 1 \mathrm{H}), 3.16-$

$3.07(\mathrm{~m}, 1 \mathrm{H}), 2.93-2.68(\mathrm{~m}, 4 \mathrm{H}), 2.27-2.04(\mathrm{~m}, 3 \mathrm{H}), 1.94-1.82$ $(\mathrm{m}, 1 \mathrm{H}), 1.24(\mathrm{~d}, J=6.1 \mathrm{~Hz}, 6 \mathrm{H}) .{ }^{13} \mathrm{C}$ NMR $\left(126 \mathrm{MHz}, \mathrm{CDCl}_{3}\right): \delta 153.8,153.6,144.2,143.3$, 137.9, 137.2, 126.1, 125.8, 121.3, 121.1, 109.6, 108.2, 71.2, 69.0, 56.3, 56.3, 52.6, 52.1, 30.8, 30.7, 28.6, 25.6, 21.2, 20.3. HRMS-EI (m/z): $[\mathrm{M}]^{+}$calcd for $\mathrm{C}_{12} \mathrm{H}_{15} \mathrm{ClO}_{2}: 226.0761$; Found: 226.0756 . 
1-(4-bromo-7-methoxy-2,3-dihydro-1H-inden-1-yl)ethanol (5g).

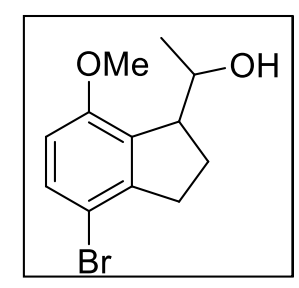

Colorless oil, 5:1 dr. ${ }^{1} \mathbf{H}$ NMR $\left(500 \mathrm{MHz}, \mathrm{CDCl}_{3}\right): \delta 7.30(\mathrm{~d}, J=8.8 \mathrm{~Hz}$, $1 \mathrm{H}), 6.61(\mathrm{~d}, J=8.6 \mathrm{~Hz}, 1 \mathrm{H}), 4.21(\mathrm{qd}, J=6.4,2.8 \mathrm{~Hz}, 1 \mathrm{H}), 4.06-3.97$ (m, 0.19H), $3.83-3.82(\mathrm{~m}, 4 \mathrm{H}), 3.65-3.54(\mathrm{~m}, 1 \mathrm{H}), 3.46(\mathrm{td}, J=8.0$, $3.7 \mathrm{~Hz}, 0.18 \mathrm{H}), 3.07-2.90(\mathrm{~m}, 1 \mathrm{H}), 2.89-2.77(\mathrm{~m}, 1 \mathrm{H}), 2.39(\mathrm{~s}, 1 \mathrm{H})$, $2.23-2.10(\mathrm{~m}, 1 \mathrm{H}), 1.99(\mathrm{ddt}, J=13.6,9.3,4.8 \mathrm{~Hz}, 1 \mathrm{H}), 1.16(\mathrm{~d}, J=6.3$ $\mathrm{Hz}, 1 \mathrm{H}), 1.09(\mathrm{~d}, J=6.4 \mathrm{~Hz}, 3 \mathrm{H}) .{ }^{13} \mathbf{C}$ NMR $\left(125 \mathrm{MHz}, \mathrm{CDCl}_{3}\right): \delta 155.3,147.2,146.6,133.7$, 132.5, 131.0, 111.3, 111.3, 110.5, 110.4, 70.2, 69.5, 55.6, 55.5, 52.7, 52.2, 33.8, 33.7, 26.9, 25.5, 20.6, 19.7. HRMS-EI (m/z): [M] calcd for $\mathrm{C}_{12} \mathrm{H}_{15} \mathrm{BrO}_{2}: 270.0255$; Found: 270.0252 . 


\section{Analytical data of products.}

\section{$N$-((S)-1-((S)-1,2,3,4-tetrahydronaphthalen-1-yl)ethyl)aniline (4a).}

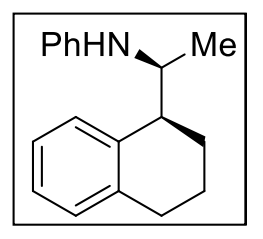

Colorless oil, $60 \%$ yield, 83:17 dr. ${ }^{1} \mathbf{H}$ NMR $\left(500 \mathrm{MHz}, \mathrm{CDCl}_{3}\right): \delta 7.25$ (d, $J=7.5 \mathrm{~Hz}, 0.18 \mathrm{H}), 7.18-7.12(\mathrm{~m}, 1 \mathrm{H}), 7.12-7.05$ (m, 2H), $7.05-6.99$ $(\mathrm{m}, 3 \mathrm{H}), 6.64-6.55(\mathrm{~m}, 1 \mathrm{H}), 6.50(\mathrm{~d}, J=7.4 \mathrm{~Hz}, 2 \mathrm{H}), 4.14-4.03(\mathrm{~m}$, $0.16 \mathrm{H}), 3.81-3.67(\mathrm{~m}, 1 \mathrm{H}), 3.50(\mathrm{~s}, 1 \mathrm{H}), 3.22(\mathrm{q}, J=7.7,7.0 \mathrm{~Hz}, 0.20 \mathrm{H})$, $2.98(\mathrm{q}, J=6.5,6.1 \mathrm{~Hz}, 1 \mathrm{H}), 2.78-2.61(\mathrm{~m}, 2 \mathrm{H}), 1.99-1.82(\mathrm{~m}, 2 \mathrm{H}), 1.79-1.65(\mathrm{~m}, 1 \mathrm{H})$, $1.65-1.47(\mathrm{~m}, 1 \mathrm{H}), 1.03(\mathrm{~d}, J=6.5 \mathrm{~Hz}, 3 \mathrm{H}), 0.93(\mathrm{~d}, J=6.6 \mathrm{~Hz}, 0.50 \mathrm{H}) .{ }^{13} \mathbf{C}$ NMR $(126$ $\left.\mathrm{MHz}, \mathrm{CDCl}_{3}\right): \delta 147.5,147.1,138.6,138.5,138.1,137.5,129.3,129.3,129.2,129.0,128.8$, $127.4,125.8,125.5,125.4,117.2,117.0,113.3,113.2,52.3,52.2,42.1,39.5,30.3,29.7,25.5$, 22.8, 21.9, 21.3, 18.4, 16.1. HRMS (ESI) m/z Calcd for $\left[\mathrm{C}_{18} \mathrm{H}_{22} \mathrm{~N}, \mathrm{M}+\mathrm{H}\right]^{+}:$252.1747; Found: 252.1748 .

93\% ee (major isomer), $89 \%$ ee (minor isomer). (HPLC condition: Chiralpak IC following by another Chiralcel OD-H column, $n$-hexane $/ i$-PrOH $=99: 1$, flow rate $=1.0$ $\mathrm{ml} / \mathrm{min}$, wavelength $=254 \mathrm{~nm}, \mathrm{t}_{\mathrm{R} 1}=11.51 \mathrm{~min}$ for minor isomer, $\mathrm{t}_{\mathrm{R} 2}=13.59 \mathrm{~min}$ for minor isomer, $\mathrm{t}_{\mathrm{R} 3}=14.26 \mathrm{~min}$ for major isomer, $\mathrm{t}_{\mathrm{R} 4}=14.71 \mathrm{~min}$ for major isomer $)$.

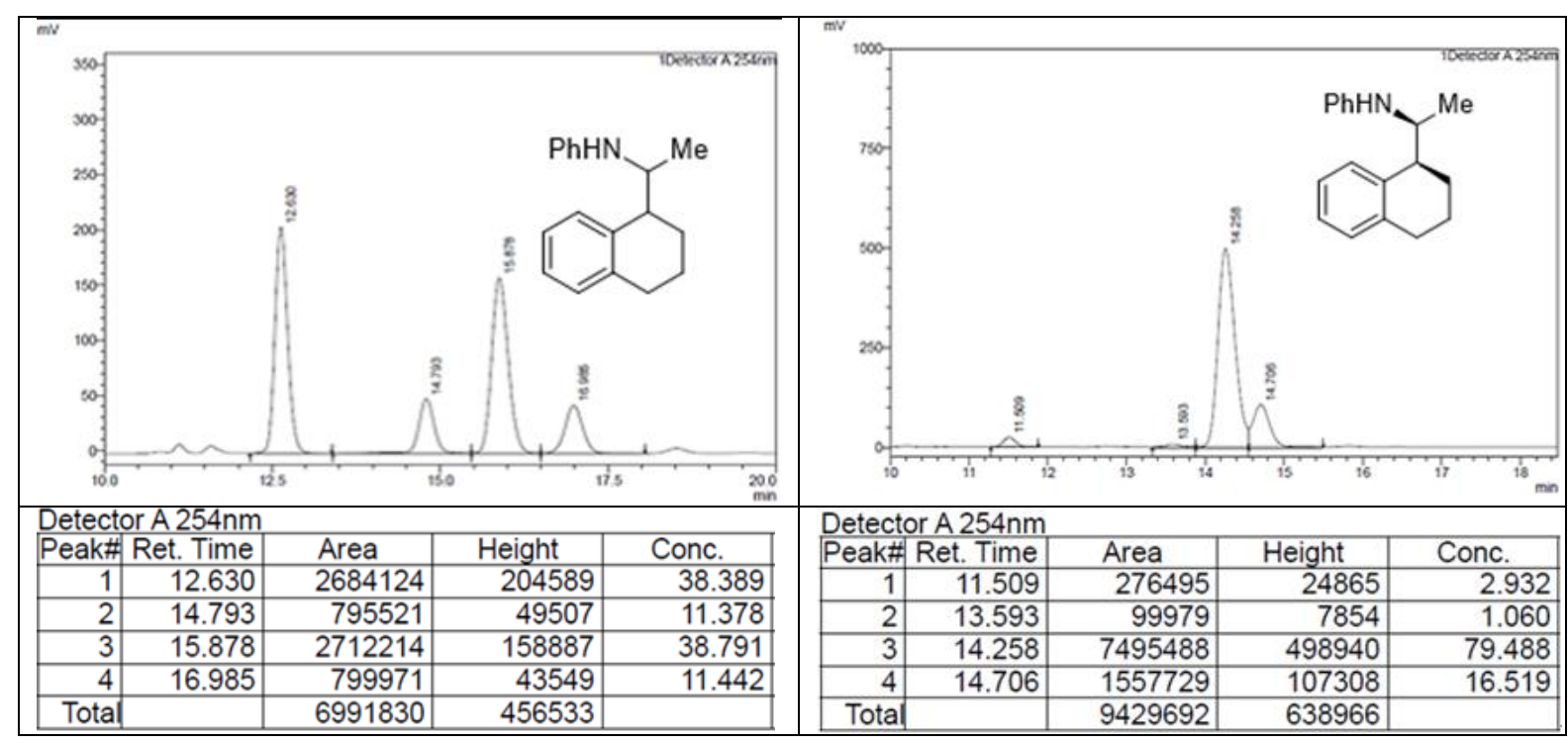

\section{$\underline{N-((S)-1-((S)-5-m e t h o x y-1,2,3,4-t e t r a h y d r o n a p h t h a l e n-1-y l) e t h y l) a n i l i n e ~(4 b)}$}

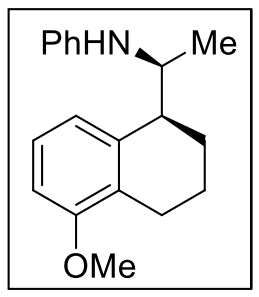

Colorless oil, 50\% yield, 87:13 dr. ${ }^{1} \mathbf{H}$ NMR $\left(500 \mathrm{MHz}, \mathrm{CDCl}_{3}\right): \delta 7.24-$ $7.15(\mathrm{~m}, 2 \mathrm{H}), 7.10(\mathrm{t}, J=8.0 \mathrm{~Hz}, 1 \mathrm{H}), 7.00(\mathrm{~d}, J=7.9 \mathrm{~Hz}, 0.16 \mathrm{H}), 6.89(\mathrm{~d}$, $J=7.7 \mathrm{~Hz}, 1 \mathrm{H}), 6.76-6.66(\mathrm{~m}, 2 \mathrm{H}), 6.61(\mathrm{~d}, J=7.8 \mathrm{~Hz}, 2 \mathrm{H}), 3.86-3.80$ (m, 1H), $3.83(\mathrm{~s}, 3 \mathrm{H}), 3.04(\mathrm{q}, J=5.9 \mathrm{~Hz}, 1 \mathrm{H}), 2.83-2.77(\mathrm{~m}, 1 \mathrm{H}), 2.65-$ $2.54(\mathrm{~m}, 1 \mathrm{H}), 2.03-1.90(\mathrm{~m}, 2 \mathrm{H}), 1.90-1.81(\mathrm{~m}, 1 \mathrm{H}), 1.73-1.62(\mathrm{~m}$,

$1 \mathrm{H}), 1.15(\mathrm{~d}, J=6.5 \mathrm{~Hz}, 3 \mathrm{H}), 1.03(\mathrm{~d}, J=6.6 \mathrm{~Hz}, 1 \mathrm{H}) .{ }^{13} \mathbf{C ~ N M R}\left(126 \mathrm{MHz}, \mathrm{CDCl}_{3}\right): \delta 157.1$, 
147.5, 138.8, 129.3, 129.3, 127.1, 126.0, 125.5, 121.2, 119.6, 117.0, 113.2, 107.2, 55.2, 52.0, 42.4, 25.0, 23.3, 22.8, 22.3, 21.1, 20.3, 18.6. HRMS (ESI) m/z Calcd for $\left[\mathrm{C}_{19} \mathrm{H}_{24} \mathrm{NO}, \mathrm{M}+\mathrm{H}\right]^{+}$: 282.1852; Found: 282.1854.

95\% ee (major isomer), 91\% ee (minor isomer). (HPLC condition: Chiralcel OD-H column, $n$-hexane $/ i-\mathrm{PrOH}=99: 1$, flow rate $=0.5 \mathrm{ml} / \mathrm{min}$, wavelength $=254 \mathrm{~nm}, \mathrm{t}_{\mathrm{R} 1}=17.22$ $\min$ for minor isomer, $\mathrm{t}_{\mathrm{R} 2}=19.94 \mathrm{~min}$ for major isomer, $\mathrm{t}_{\mathrm{R} 3}=22.06 \mathrm{~min}$ for minor isomer, $\mathrm{t}_{\mathrm{R} 4=}$ $25.75 \mathrm{~min}$ for major isomer).

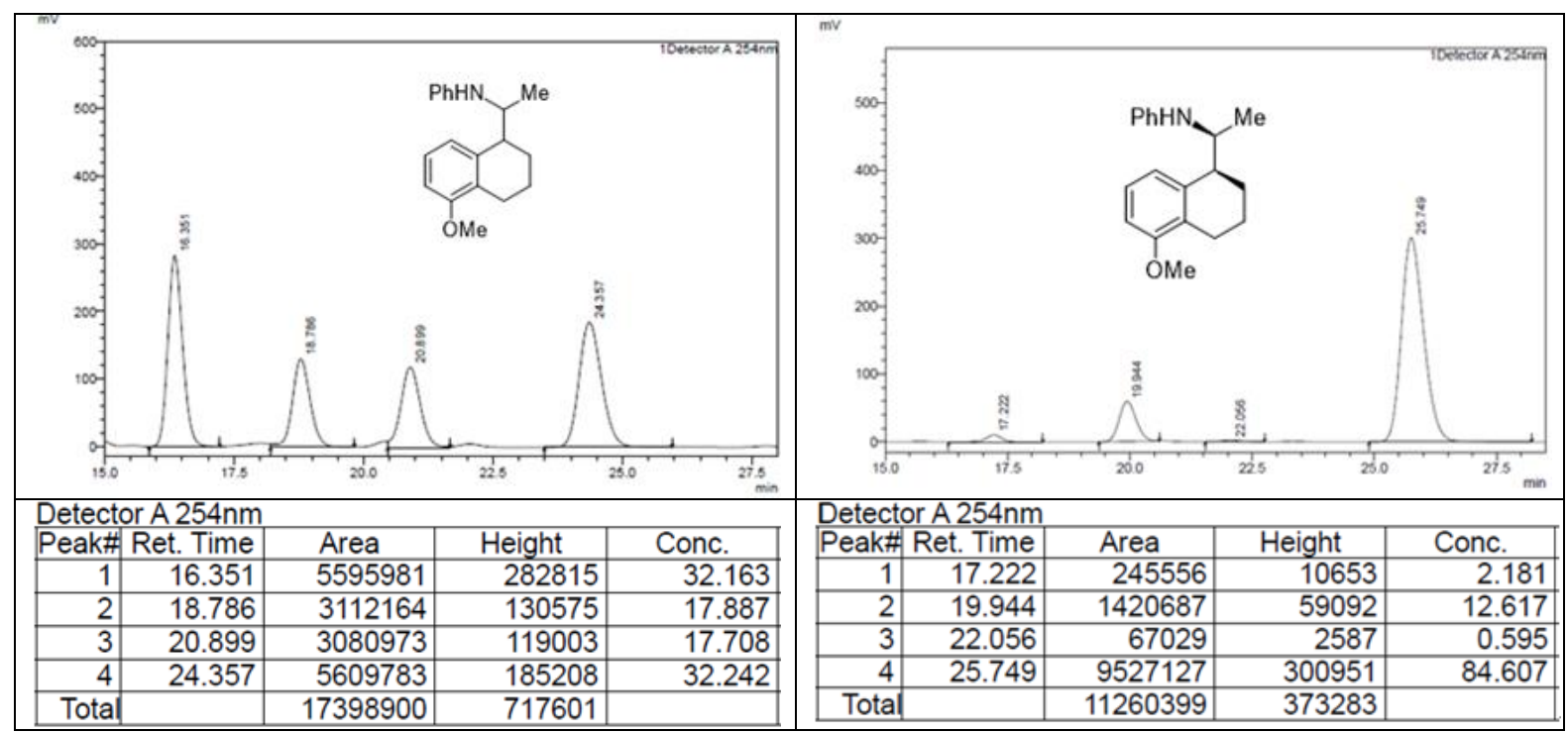

\section{$\underline{N-((S)-1-((S)-6 \text {-methoxy-1,2,3,4-tetrahydronaphthalen-1-yl)ethyl)aniline (4c). }}$}

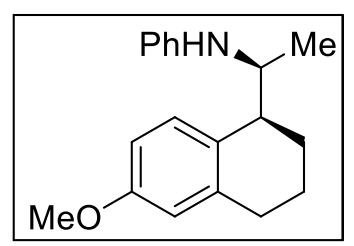

White solid, 53\% yield, 87:13 dr. ${ }^{1} \mathbf{H}$ NMR (500 MHz, $\left.\mathrm{CDCl}_{3}\right): \delta$ $7.24-7.07(\mathrm{~m}, 3 \mathrm{H}), 6.78-6.54(\mathrm{~m}, 5 \mathrm{H}), 3.78(\mathrm{~s}, 3 \mathrm{H}), 3.63(\mathrm{~s}, 1 \mathrm{H})$, $3.02(\mathrm{q}, J=6.0 \mathrm{~Hz}, 1 \mathrm{H}), 2.82-2.63(\mathrm{~m}, 2 \mathrm{H}), 2.05-1.91(\mathrm{~m}, 2 \mathrm{H})$, $1.86-1.74(\mathrm{~m}, 1 \mathrm{H}), 1.74-1.50(\mathrm{~m}, 2 \mathrm{H}), 1.13(\mathrm{~d}, J=6.4 \mathrm{~Hz}, 3 \mathrm{H})$. ${ }^{13} \mathrm{C}$ NMR (126 MHz, $\left.\mathrm{CDCl}_{3}\right): \delta 157.6,147.4,139.7,129.8,129.5,129.3,129.3,128.4,117.0$, 113.8, 113.6, 113.2, 112.0, 111.6, 55.1, 55.1, 52.2, 41.4, 38.8, 30.6, 30.1, 25.5, 23.0, 21.9, 21.2, 18.3, 16.0. HRMS (ESI) $\mathrm{m} / \mathrm{z}$ Calcd for $\left[\mathrm{C}_{19} \mathrm{H}_{24} \mathrm{NO}, \mathrm{M}+\mathrm{H}\right]^{+}:$282.1852; Found: 282.1856.

96\% ee (major isomer), 96\% ee (minor isomer). (HPLC condition: Chiralcel OD-H column, $n$-hexane $/ i-\mathrm{PrOH}=99: 1$, flow rate $=0.5 \mathrm{ml} / \mathrm{min}$, wavelength $=254 \mathrm{~nm}, \mathrm{t}_{\mathrm{R} 1}=18.17$ $\min$ for minor isomer, $\mathrm{t}_{\mathrm{R} 2}=22.41 \mathrm{~min}$ for minor isomer, $\mathrm{t}_{\mathrm{R} 3}=22.97 \mathrm{~min}$ for major isomer, $\mathrm{t}_{\mathrm{R} 4}=$ $24.54 \mathrm{~min}$ for major isomer). 


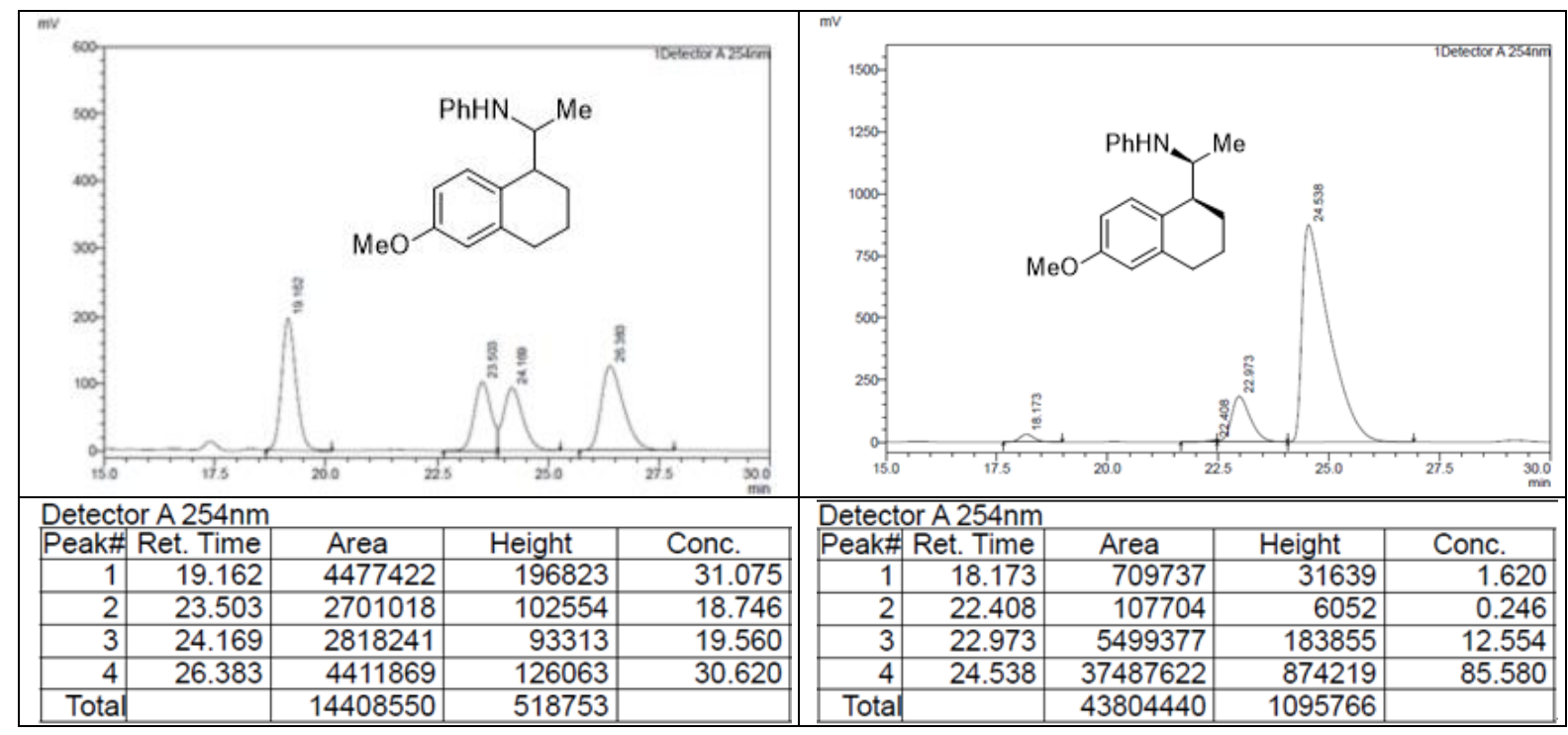

$\underline{N-((S)-1-((S)-6-c h l o r o-1,2,3,4-t e t r a h y d r o n a p h t h a l e n-1-y l) e t h y l) a n i l i n e ~(4 d)}$

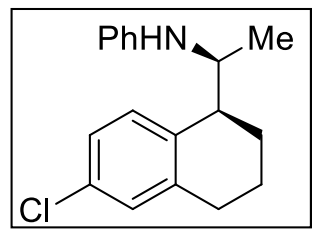

Colorless oil, 50\% yield, 79:21 dr. ${ }^{1} \mathbf{H}$ NMR (500 $\left.\mathrm{MHz}, \mathrm{CDCl}_{3}\right): \delta$ $7.21-7.17(\mathrm{~m}, 3 \mathrm{H}), 7.08-7.06(\mathrm{~m}, 2 \mathrm{H}), 6.70(\mathrm{t}, J=7.3 \mathrm{~Hz}, 1 \mathrm{H}), 6.60$ $(\mathrm{d}, J=8.0 \mathrm{~Hz}, 2 \mathrm{H}), 4.14-4.04(\mathrm{~m}, 0.28 \mathrm{H}), 3.77(\mathrm{dt}, J=11.5,6.2 \mathrm{~Hz}$, $1 \mathrm{H}), 3.66(\mathrm{~s}, 1 \mathrm{H}), 3.28-3.21(\mathrm{~m}, 0.25 \mathrm{H}), 3.06(\mathrm{q}, J=6.5 \mathrm{~Hz}, 1 \mathrm{H})$, $2.75-2.69(\mathrm{~m}, 2 \mathrm{H}), 2.08-1.85(\mathrm{~m}, 2 \mathrm{H}), 1.85-1.72(\mathrm{~m}, 1 \mathrm{H}), 1.72-1.50(\mathrm{~m}, 2 \mathrm{H}), 1.09(\mathrm{~d}, J$ $=6.5 \mathrm{~Hz}, 3 \mathrm{H}), 1.02(\mathrm{~d}, J=6.5 \mathrm{~Hz}, 1 \mathrm{H}) .{ }^{13} \mathbf{C ~ N M R}\left(126 \mathrm{MHz}, \mathrm{CDCl}_{3}\right): \delta 140.5,140.4,135.9$, $131.4,131.1,130.2,129.4,129.4,128.9,128.9,128.7,126.0,125.5,117.3,113.3,52.4,41.3$, 39.0, 30.1 , 29.7 , 25.4, 22.6, 21.6, 21.1, 18.2. HRMS (ESI) m/z Calcd for $\left[\mathrm{C}_{18} \mathrm{H}_{21} \mathrm{ClN}, \mathrm{M}+\mathrm{H}\right]^{+}$: 286.1357; Found: 286.1360.

93\% ee (major isomer), 88\% ee (minor isomer). (HPLC condition: Chiralpark IB column followed by Chiralcel OD-H column, $n$-hexane $/ i$-PrOH $=99: 1$, flow rate $=0.5 \mathrm{ml} / \mathrm{min}$, wavelength $=230 \mathrm{~nm}, \mathrm{t}_{\mathrm{R} 1}=29.84 \mathrm{~min}$ for minor isomer, $\mathrm{t}_{\mathrm{R} 2}=44.20 \mathrm{~min}$ for major isomer, $\mathrm{t}_{\mathrm{R} 3}=$ $45.60 \mathrm{~min}$ for minor isomer, $\mathrm{t}_{\mathrm{R} 4}=47.08 \mathrm{~min}$ for major isomer).

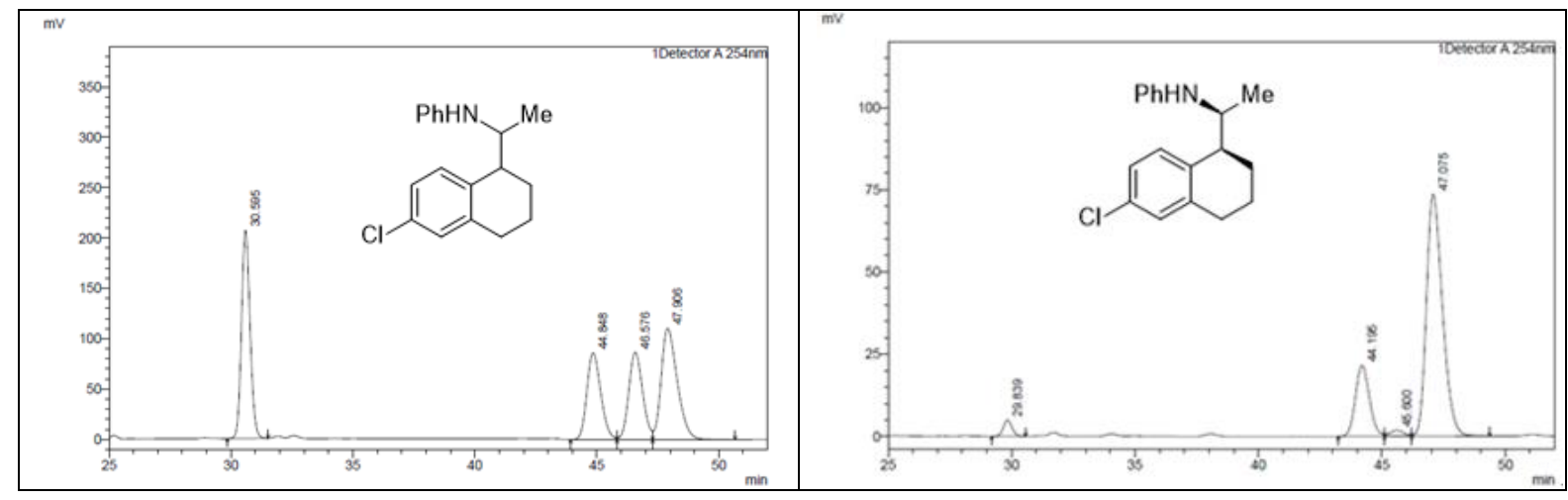




\begin{tabular}{|c|c|c|c|c|}
\hline \multicolumn{5}{|c|}{ Detector A 254nm } \\
\hline Peak\# & et. Time & Area & Height & Conc. \\
\hline 1 & 30.595 & 5043791 & 206623 & 29.779 \\
\hline 2 & 44.848 & 3410211 & 86053 & 20.134 \\
\hline 3 & 46.576 & 3419958 & 86728 & 20.192 \\
\hline 4 & 47.906 & 5063225 & 110454 & 29.894 \\
\hline Total & & 16937186 & 489859 & \\
\hline
\end{tabular}

\begin{tabular}{|r|r|r|r|r|}
\multicolumn{5}{|c|}{ Detector A 254nm } \\
\hline Peak\# Ret. Time & \multicolumn{1}{c|}{ Area } & Height & \multicolumn{1}{c|}{ Conc. } \\
\hline 1 & 29.839 & 122029 & 4934 & 2.803 \\
\hline 2 & 44.195 & 849580 & 21592 & 19.518 \\
\hline 3 & 45.600 & 70329 & 1791 & 1.616 \\
\hline 4 & 47.075 & 3310856 & 73596 & 76.063 \\
\hline Total & & 4352794 & 101913 & \\
\hline
\end{tabular}

\section{$\underline{N-((S)-1-((S)-7-m e t h o x y-1,2,3,4-t e t r a h y d r o n a p h t h a l e n-1-y l) e t h y l) a n i l i n e ~(4 e) ~}$}

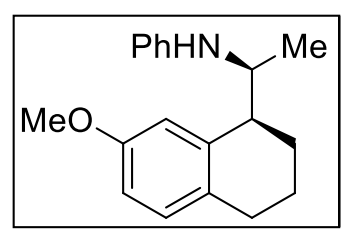

Colorless oil, 49\% yield, 90:10 dr. ${ }^{1} \mathbf{H}$ NMR (500 MHz, $\left.\mathrm{CDCl}_{3}\right): \delta$ $7.19(\mathrm{t}, J=7.9 \mathrm{~Hz}, 2 \mathrm{H}), 7.01(\mathrm{~d}, J=8.4 \mathrm{~Hz}, 1 \mathrm{H}), 6.81(\mathrm{~d}, J=2.2 \mathrm{~Hz}$, $1 \mathrm{H}), 6.76-6.67(\mathrm{~m}, 2 \mathrm{H}), 6.62(\mathrm{~d}, J=7.9 \mathrm{~Hz}, 2 \mathrm{H}), 3.82(\mathrm{~s}, 0.47 \mathrm{H})$, $3.81-3.78(\mathrm{~m}, 1 \mathrm{H}), 3.74(\mathrm{~s}, 3 \mathrm{H}), 3.15-3.02(\mathrm{~m}, 1 \mathrm{H}), 2.70(\mathrm{t}, J=6.3$ $\mathrm{Hz}, 2 \mathrm{H}), 2.06-1.93(\mathrm{~m}, 2 \mathrm{H}), 1.83-1.72(\mathrm{~m}, 1 \mathrm{H}), 1.69-1.59(\mathrm{~m}, 1 \mathrm{H}), 1.13(\mathrm{~d}, J=6.5 \mathrm{~Hz}$, $3 \mathrm{H}), 1.05(\mathrm{~d}, J=6.6 \mathrm{~Hz}, 0.34 \mathrm{H}) .{ }^{13} \mathbf{C} \mathbf{N M R}\left(125 \mathrm{MHz}, \mathrm{CDCl}_{3}\right): \delta 157.3,138.4,130.8,129.7$, 129.3 117.1, 113.9, 113.3, 111.8, 55.2, 52.5, 41.8, 29.0, 25.6, 21.6, 18.2. HRMS (ESI) m/z Calcd for $\left[\mathrm{C}_{19} \mathrm{H}_{24} \mathrm{NO}, \mathrm{M}+\mathrm{H}\right]^{+}$: 282.1852; Found: 282.1851.

93\% ee (major isomer), 88\% ee (minor isomer). (HPLC condition: Chiralpak IA column, $n$-hexane $/ i-\mathrm{PrOH}=99: 1$, flow rate $=1.0 \mathrm{ml} / \mathrm{min}$, wavelength $=254 \mathrm{~nm}, \mathrm{t}_{\mathrm{R} 1}=6.46 \mathrm{~min}$ for minor isomer, $\mathrm{t}_{\mathrm{R} 2}=7.62 \mathrm{~min}$ for major isomer, $\mathrm{t}_{\mathrm{R} 3}=9.08 \mathrm{~min}$ for major isomer, $\mathrm{t}_{\mathrm{R} 4}=10.39 \mathrm{~min}$ for minor isomer).

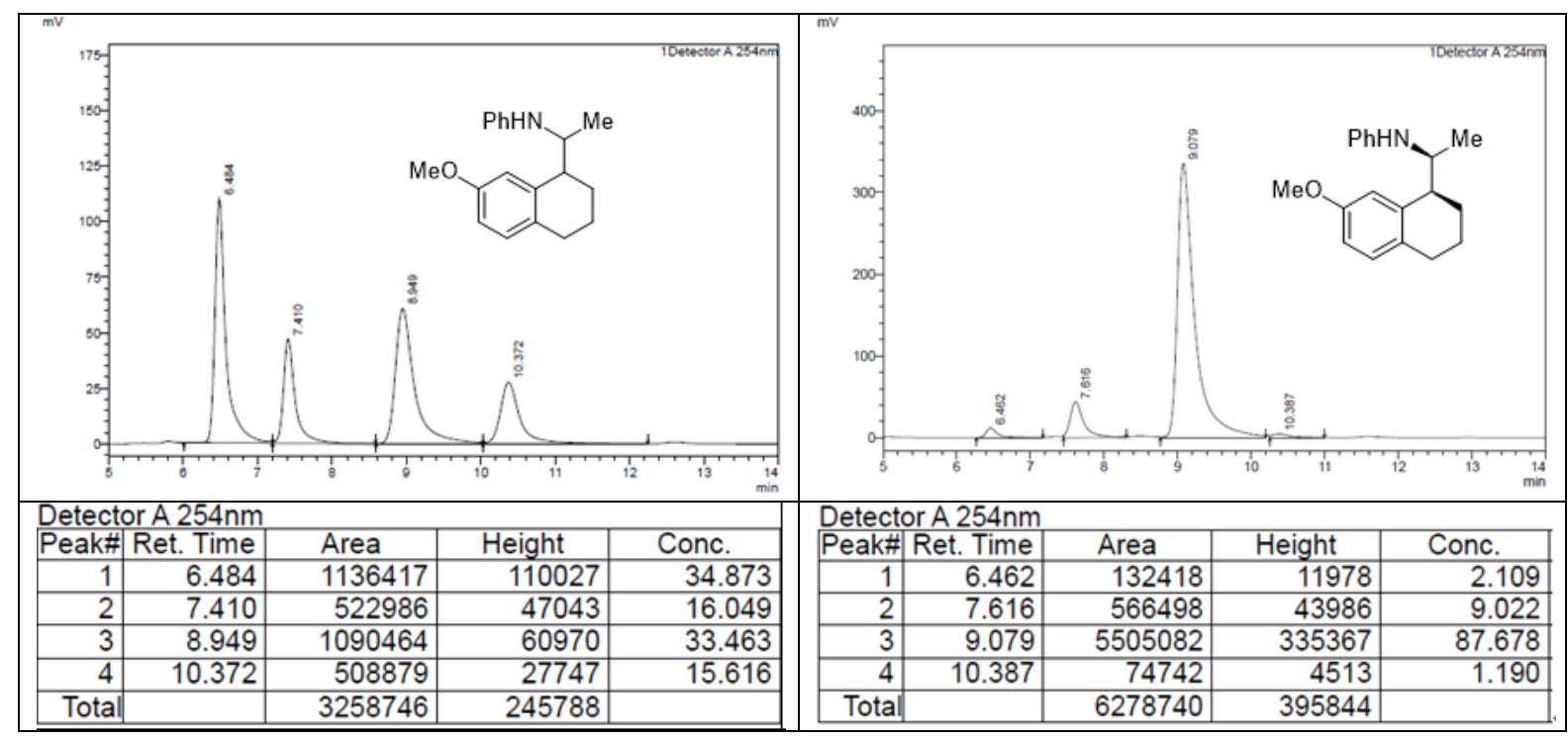

$\underline{N-((S)-1-((S)-7-f l u o r 0-1,2,3,4-t e t r a h y d r o n a p h t h a l e n-1-y l) e t h y l) a n i l i n e ~(4 f) ~}$

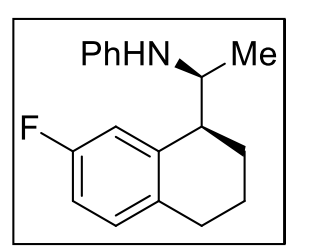

Colorless oil, $54 \%$ yield, 73:27 dr. ${ }^{1} \mathbf{H}$ NMR $\left(500 \mathrm{MHz}, \mathrm{CDCl}_{3}\right): \delta 7.25$ $-7.17(\mathrm{~m}, 2 \mathrm{H}), 7.09-6.97(\mathrm{~m}, 2 \mathrm{H}), 6.88-6.78(\mathrm{~m}, 1 \mathrm{H}), 6.75(\mathrm{~d}, J=$ $7.3 \mathrm{~Hz}, 0.27 \mathrm{H}), 6.71(\mathrm{t}, J=7.2 \mathrm{~Hz}, 1 \mathrm{H}), 6.62(\mathrm{~d}, J=7.9 \mathrm{~Hz}, 2 \mathrm{H}), 4.12$ (qd, $J=6.5,4.2 \mathrm{~Hz}, 0.31 \mathrm{H}), 3.78(\mathrm{tt}, J=6.6,3.2 \mathrm{~Hz}, 1 \mathrm{H}), 3.65(\mathrm{~s}, 1 \mathrm{H})$, $3.29(\mathrm{q}, J=6.9 \mathrm{~Hz}, 0.31 \mathrm{H}), 3.11(\mathrm{q}, J=7.2,6.8 \mathrm{~Hz}, 1 \mathrm{H}), 2.72(\mathrm{t}, J=6.2 \mathrm{~Hz}, 2 \mathrm{H}), 2.09-1.91$ 
(m, 2H), $1.91-1.84(\mathrm{~m}, 0.38 \mathrm{H}), 1.81-1.69(\mathrm{~m}, 1 \mathrm{H}), 1.69-1.50(\mathrm{~m}, 2 \mathrm{H}), 1.11(\mathrm{~d}, J=6.5 \mathrm{~Hz}$, 3H), $1.04(\mathrm{~d}, J=6.6 \mathrm{~Hz}, 1 \mathrm{H}) .{ }^{13} \mathbf{C}$ NMR $\left(126 \mathrm{MHz}, \mathrm{CDCl}_{3}\right): \delta 162.2,161.7,160.2,159.8$, $147.1,140.1,140.0,139.4,139.3,134.1,134.1,130.4,130.3,130.1,130.0,129.4,129.3$, $117.5,117.2,115.1,114.9,113.8,113.6,113.5,113.2,112.8,112.6,112.5,52.5,41.6,39.5$, 29.6, 29.2, 25.5, 22.3, 21.9, 21.5, 18.0, 15.9. HRMS (ESI) $\mathrm{m} / \mathrm{z}$ Calcd for $\left[\mathrm{C}_{18} \mathrm{H}_{21} \mathrm{FN}, \mathrm{M}+\mathrm{H}\right]^{+}$: 270.1653; Found: 270.1646.

91\% ee (major isomer), $85 \%$ ee (minor isomer). (HPLC condition: Chiralpak IB column, $n$-hexane $/ i-\mathrm{PrOH}=99: 1$, flow rate $=1.0 \mathrm{ml} / \mathrm{min}$, wavelength $=254 \mathrm{~nm}, \mathrm{t}_{\mathrm{R} 1}=6.11$ $\min$ for minor isomer, $t_{\mathrm{R} 2}=7.10 \mathrm{~min}$ for minor isomer, $\mathrm{t}_{\mathrm{R} 3}=7.33 \mathrm{~min}$ for major isomer, $\mathrm{t}_{\mathrm{R} 4}=$ $8.83 \mathrm{~min}$ for major isomer).

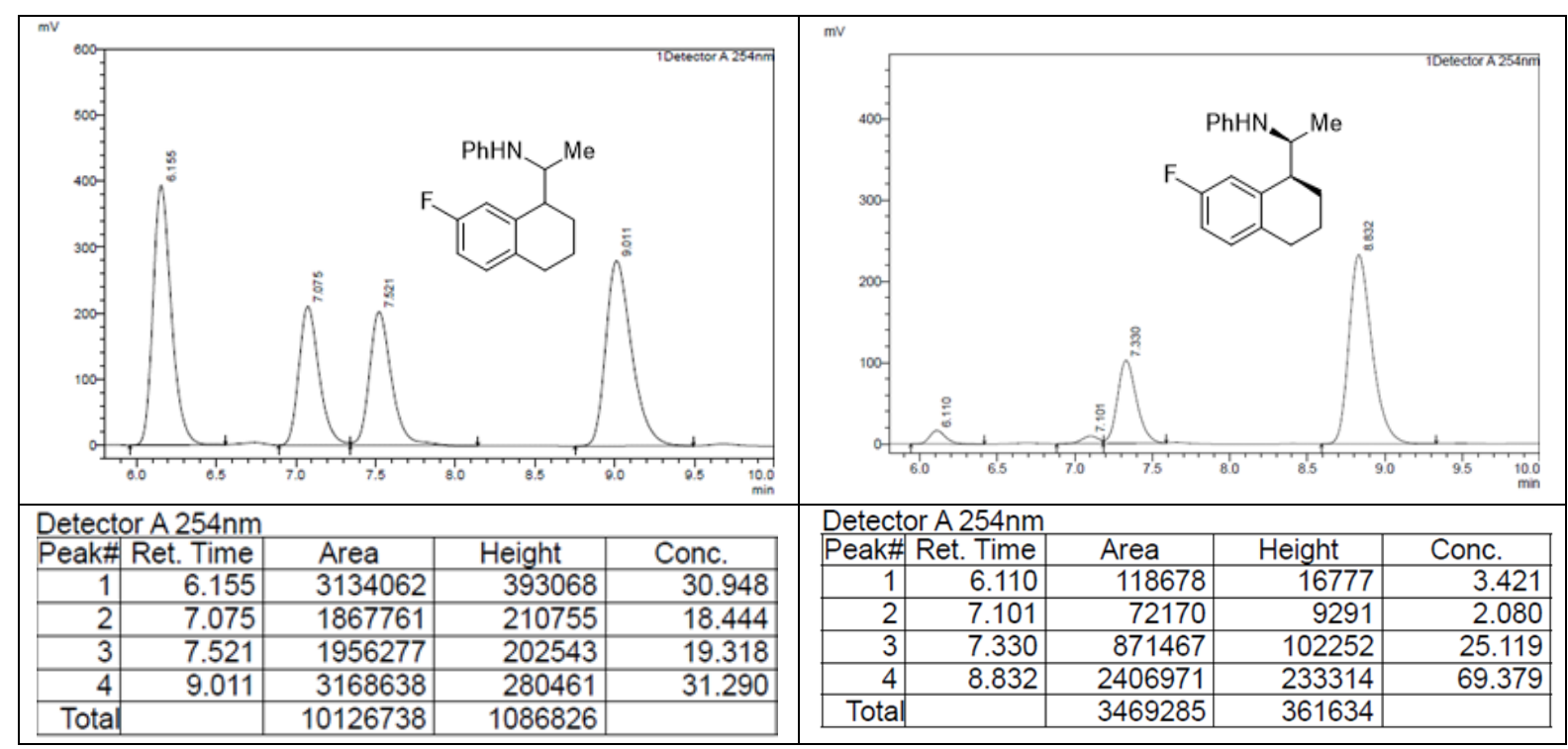

\section{$\underline{N-((S)-1-((S)-7-c h l o r o-1,2,3,4-t e t r a h y d r o n a p h t h a l e n-1-y l) e t h y l) a n i l i n e ~(4 g) ~}$}

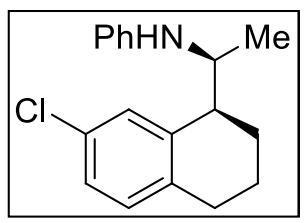

Colorless oil, $42 \%$ yield, $79: 21 \mathrm{dr} .{ }^{1} \mathbf{H}$ NMR $\left(500 \mathrm{MHz}, \mathrm{CDCl}_{3}\right): \delta 7.31$ $-7.30(\mathrm{~m}, 0.18 \mathrm{H}), 7.26(\mathrm{~d}, J=2.2 \mathrm{~Hz}, 1 \mathrm{H}), 7.24-7.17(\mathrm{~m}, 2 \mathrm{H}), 7.08$ $(\mathrm{dd}, J=8.1,1.9 \mathrm{~Hz}, 1 \mathrm{H}), 7.04-7.00(\mathrm{~m}, 1 \mathrm{H}), 6.76-6.70(\mathrm{~m}, 1 \mathrm{H})$, $6.62(\mathrm{~d}, J=7.9 \mathrm{~Hz}, 2 \mathrm{H}), 4.18-4.03(\mathrm{~m}, 0.24 \mathrm{H}), 3.82-3.71(\mathrm{~m}, 1 \mathrm{H})$, $3.68(\mathrm{~s}, 1 \mathrm{H}), 3.35-3.21(\mathrm{~m}, 0.23 \mathrm{H}), 3.08(\mathrm{q}, J=6.7 \mathrm{~Hz}, 1 \mathrm{H}), 2.72-2.70(\mathrm{~m}, 2 \mathrm{H}), 2.06-$ $1.90(\mathrm{~m}, 2 \mathrm{H}), 1.83-1.71(\mathrm{~m}, 1 \mathrm{H}), 1.69-1.59(\mathrm{~m}, 1 \mathrm{H}), 1.09(\mathrm{~d}, J=6.6 \mathrm{~Hz}, 3 \mathrm{H}), 1.03(\mathrm{~d}, J=$ $6.7 \mathrm{~Hz}, 1 \mathrm{H}) .{ }^{13} \mathbf{C} \mathbf{N M R}\left(126 \mathrm{MHz}, \mathrm{CDCl}_{3}\right): \delta 147.0,139.3,137.1,137.0,131.4,130.8,130.5$, $130.2,129.4,129.4,128.7,127.3,125.9,125.7,117.4,113.4,52.6,41.5,39.2,29.8,29.3,25.5$, 22.3, 21.7, 21.2, 18.1, 15.9. HRMS (ESI) $\mathrm{m} / \mathrm{z}$ Calcd for $\left[\mathrm{C}_{18} \mathrm{H}_{21} \mathrm{ClN}, \mathrm{M}+\mathrm{H}\right]^{+}:$: 286.1357; Found: 286.1359.

92\% ee (major isomer), $75 \%$ ee (minor isomer). (HPLC condition: Chiralcel OD-H column, $n$-hexane $/ i-\mathrm{PrOH}=99: 1$, flow rate $=1.0 \mathrm{ml} / \mathrm{min}$, wavelength $=254 \mathrm{~nm}, \mathrm{t}_{\mathrm{R} 1}=6.85 \mathrm{~min}$ 
for minor isomer, $\mathrm{t}_{\mathrm{R} 2}=8.33 \mathrm{~min}$ for minor isomer, $\mathrm{t}_{\mathrm{R} 3}=9.41 \mathrm{~min}$ for major isomer, $\mathrm{t}_{\mathrm{R} 4}=11.75$ min for major isomer).

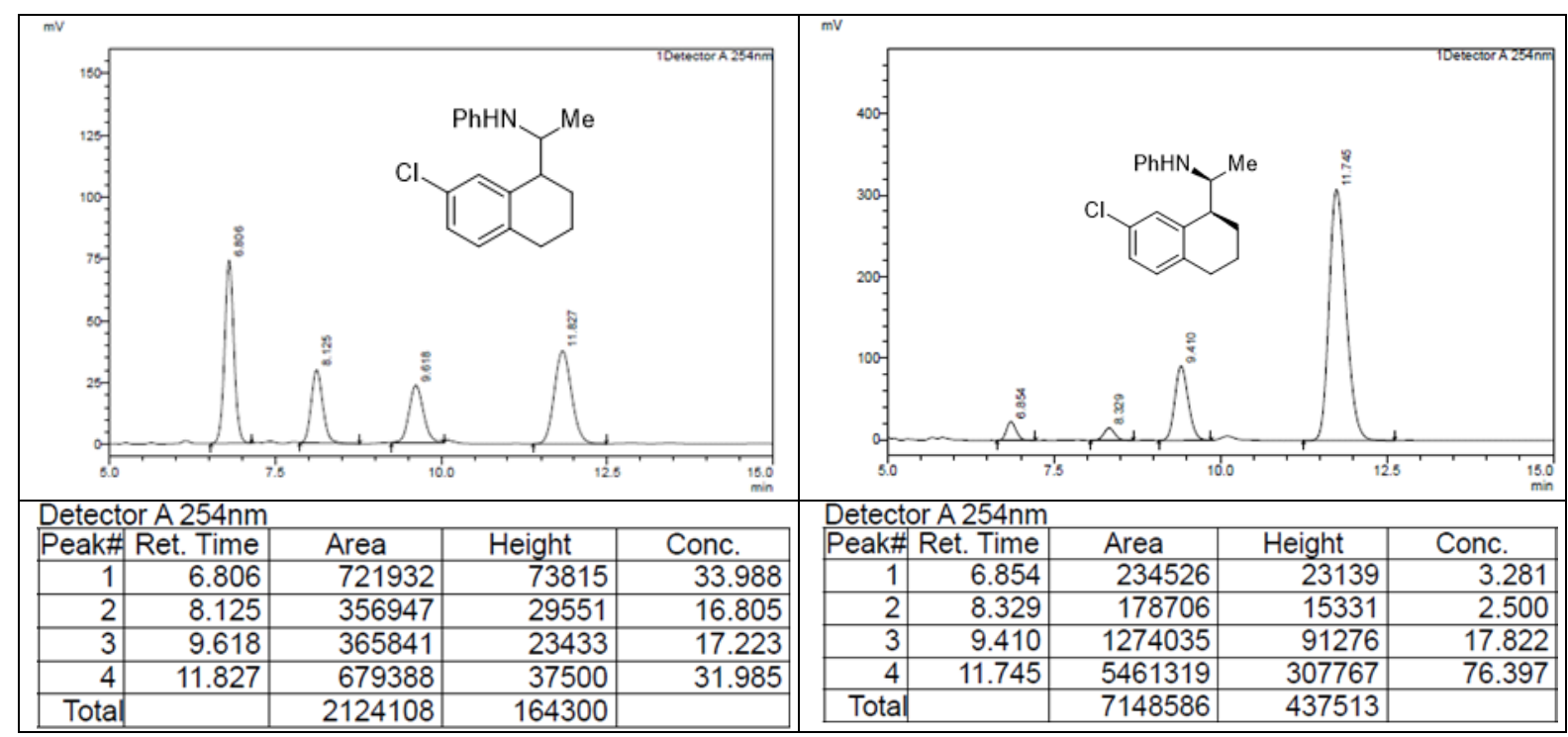

$\underline{N-((S)-1-((S)-6,7-d i m e t h o x y-1,2,3,4-t e t r a h y d r o n a p h t h a l e n-1-y l) e t h y l) a n i l i n e ~(4 h) ~}$

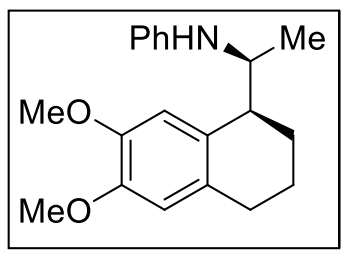

Colorless oil, 53\% yield, 94:6 dr. ${ }^{1} \mathbf{H}$ NMR $\left(500 \mathrm{MHz}, \mathrm{CDCl}_{3}\right): \delta$ $7.20-7.14(\mathrm{~m}, 2 \mathrm{H}), 6.74(\mathrm{~s}, 1 \mathrm{H}), 6.69(\mathrm{t}, J=7.2 \mathrm{~Hz}, 1 \mathrm{H}), 6.64-$ $6.56(\mathrm{~m}, 3 \mathrm{H}), 3.85(\mathrm{~s}, 3 \mathrm{H}), 3.79(\mathrm{dd}, J=6.6,4.7 \mathrm{~Hz}, 1 \mathrm{H}), 3.75(\mathrm{~s}$, $3 \mathrm{H}), 3.04(\mathrm{q}, J=6.6,6.1 \mathrm{~Hz}, 1 \mathrm{H}), 2.76-2.64(\mathrm{~m}, 2 \mathrm{H}), 2.05-1.93$ $(\mathrm{m}, 2 \mathrm{H}), 1.79-1.71(\mathrm{~m}, 1 \mathrm{H}), 1.70-1.60(\mathrm{~m}, 1 \mathrm{H}), 1.14(\mathrm{~d}, J=6.4$ $\mathrm{Hz}, 3 \mathrm{H}) .{ }^{13} \mathrm{C}$ NMR $\left(126 \mathrm{MHz}, \mathrm{CDCl}_{3}\right): \delta 147.4,147.1,146.6,130.6,129.3,129.0,117.1$, 113.1, 111.9, 111.6, 55.8, 55.7, 52.4, 41.2, 29.3, 25.3, 21.5, 18.3. HRMS (ESI) m/z Calcd for $\left[\mathrm{C}_{20} \mathrm{H}_{25} \mathrm{NaNO}_{2}, \mathrm{M}+\mathrm{Na}\right]^{+}: 334.1778$; Found: 334.1780 .

96\% ee (major isomer). (HPLC condition: Chiralcel OD-H column, $n$-hexane/ $i-\mathrm{PrOH}=$ 90:10, flow rate $=1.0 \mathrm{ml} / \mathrm{min}$, wavelength $=254 \mathrm{~nm}, \mathrm{t}_{\mathrm{R} 1}=8.62 \mathrm{~min}$ for minor isomer, $\mathrm{t}_{\mathrm{R} 2}=$ $10.39 \mathrm{~min}$ for minor isomer, $\mathrm{t}_{\mathrm{R} 3}=12.5 \mathrm{~min}$ for major isomer, $\mathrm{t}_{\mathrm{R} 4}=13.89 \mathrm{~min}$ for major isomer).

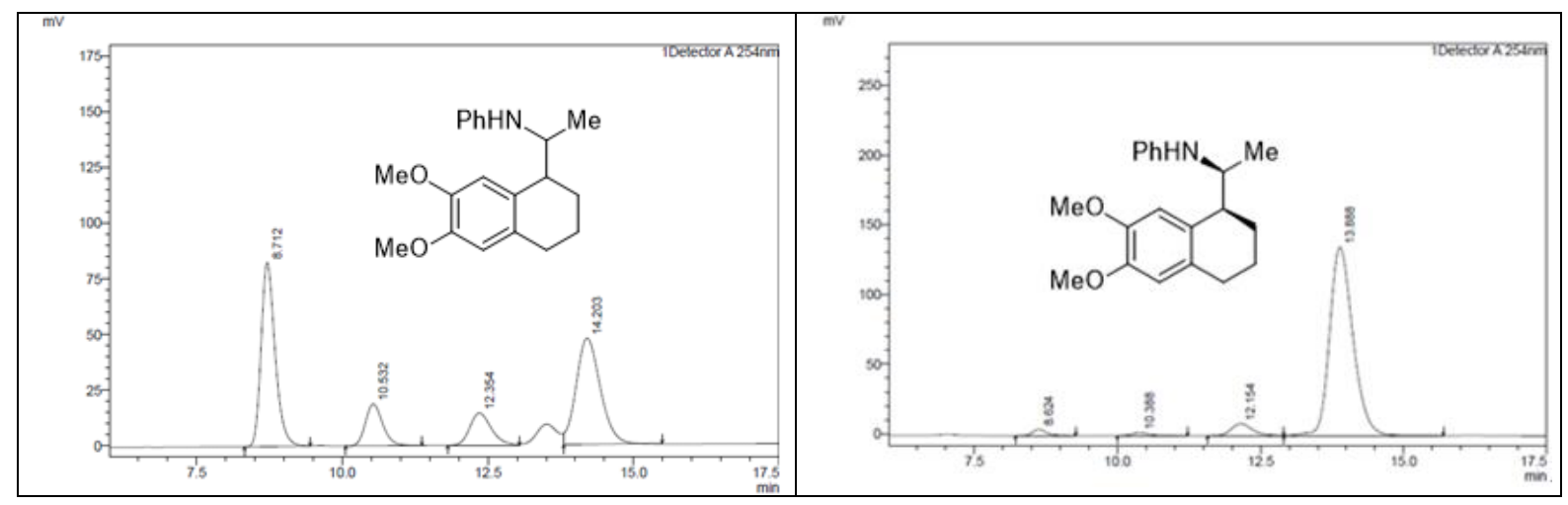




\begin{tabular}{|c|c|c|c|c|c|c|c|c|c|}
\hline \multicolumn{5}{|c|}{ Detector A 254nm } & \multicolumn{5}{|c|}{ Detector A 254nm } \\
\hline Peak\# & et. Time & Area & Height & Conc. & Peak\# & et. Time & Area & Height & Conc. \\
\hline \begin{tabular}{|l|}
1 \\
\end{tabular} & 8.712 & 1383265 & 82549 & 39.352 & $\begin{array}{l}1 \\
\end{array}$ & 8.624 & 76841 & 4522 & 1.838 \\
\hline 2 & 10.532 & 387486 & 18786 & 11.023 & 2 & 10.388 & 44314 & 2181 & 1.060 \\
\hline 3 & 12.354 & 374493 & 14488 & 10.654 & 3 & 12.154 & 218157 & 8594 & 5.219 \\
\hline 4 & 14.203 & 1369879 & 47672 & 38.971 & 4 & 13.888 & 3840866 & 135299 & 91.883 \\
\hline Total & & 3515123 & 163495 & & Tota & & 4180178 & 150596 & \\
\hline
\end{tabular}

\section{$\underline{N-((S)-1-((S)-5,7-d i m e t h y l-1,2,3,4-t e t r a h y d r o n a p h t h a l e n-1-y l) e t h y l) a n i l i n e ~(4 i)}$.}

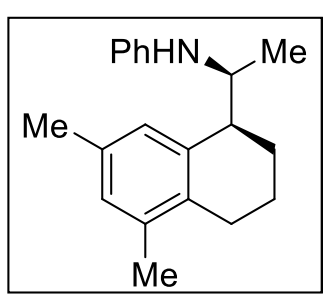

Colorless oil, 50\% yield, 95:5 dr. ${ }^{1} \mathbf{H}$ NMR (500 $\left.\mathrm{MHz} \mathrm{CDCl}_{3}\right): \delta$ $7.20(\mathrm{t}, J=7.2 \mathrm{~Hz}, 2 \mathrm{H}), 6.93(\mathrm{~s}, 1 \mathrm{H}), 6.89(\mathrm{~s}, 1 \mathrm{H}), 6.71(\mathrm{t}, J=7.3 \mathrm{~Hz}$, $1 \mathrm{H}), 6.63(\mathrm{~d}, J=8.0 \mathrm{~Hz}, 2 \mathrm{H}), 3.81(\mathrm{t}, J=6.5 \mathrm{~Hz}, 1 \mathrm{H}), 3.59(\mathrm{~s}, 1 \mathrm{H})$, $2.97(\mathrm{q}, J=6.0 \mathrm{~Hz}, 1 \mathrm{H}), 2.75-2.55(\mathrm{~m}, 2 \mathrm{H}), 2.29$ (s, 3H), 2.23 (s, $3 \mathrm{H}), 2.06-1.96(\mathrm{~m}, 1 \mathrm{H}), 1.96-1.88(\mathrm{~m}, 2 \mathrm{H}), 1.77-1.72(\mathrm{~m}, 1 \mathrm{H})$,

$1.17(\mathrm{~d}, J=6.5 \mathrm{~Hz}, 3 \mathrm{H}) .{ }^{13} \mathrm{C}$ NMR $\left(126 \mathrm{MHz}, \mathrm{CDCl}_{3}\right): \delta 147.6,137.3,136.1,133.9,133.4$, 129.3, 129.2, 128.6, 127.6, 116.9, 113.3, 52.0, 43.1, 26.1, 25.0, 21.0, 20.5, 19.7, 18.9. HRMS (ESI) $\mathrm{m} / \mathrm{z}$ Calcd for $\left[\mathrm{C}_{20} \mathrm{H}_{26} \mathrm{~N}, \mathrm{M}+\mathrm{H}\right]^{+}: 280.2060$; Found: 280.2064.

97\% ee (major isomer). (HPLC condition: Chiralcel OD-H column, $n$-hexane $/ i$-PrOH $=$ $99: 1$, flow rate $=0.5 \mathrm{ml} / \mathrm{min}$, wavelength $=254 \mathrm{~nm}, t_{\mathrm{R} 1}=10.29 \min$ for minor isomer, $t_{\mathrm{R} 2}=$ $11.88 \mathrm{~min}$ for minor isomer, $\mathrm{t}_{\mathrm{R} 3}=12.33 \mathrm{~min}$ for major isomer, $\mathrm{t}_{\mathrm{R} 4}=12.67 \mathrm{~min}$ for major isomer).

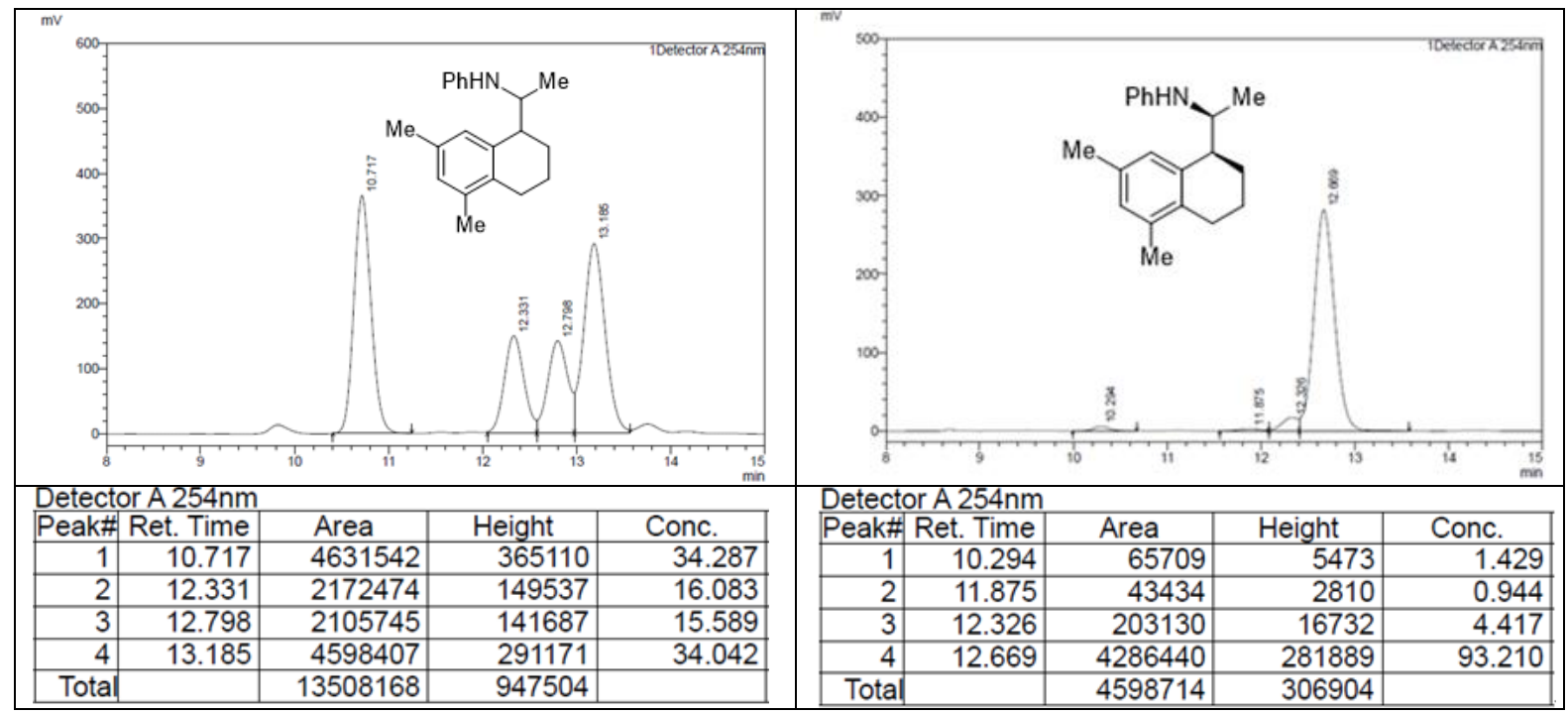

\section{4-methoxy- $N-((S)-1-((S)-1,2,3,4-t e t r a h y d r o n a p h t h a l e n-1-y l) e t h y l)$ aniline $(4 \mathbf{j})$}

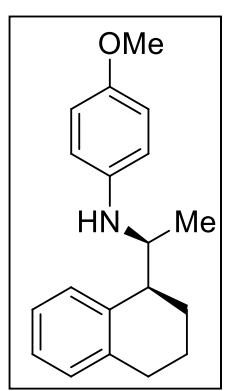

Colorless oil, $58 \%$ yield, 86:14 dr. ${ }^{1} \mathbf{H}$ NMR (500 MHz, $\left.\mathrm{CD}_{3} \mathrm{OD}\right): \delta 7.24-$ $7.16(\mathrm{~m}, 1 \mathrm{H}), 7.09-6.98(\mathrm{~m}, 3 \mathrm{H}), 6.71(\mathrm{~d}, J=8.9 \mathrm{~Hz}, 2 \mathrm{H}), 6.56(\mathrm{~d}, J=8.9$ $\mathrm{Hz}, 2 \mathrm{H}), 4.81(\mathrm{~s}, 3 \mathrm{H}), 3.68-3.64(\mathrm{~m}, 4 \mathrm{H}), 3.00(\mathrm{q}, J=6.4 \mathrm{~Hz}, 1 \mathrm{H}), 2.79-$ $2.63(\mathrm{~m}, 2 \mathrm{H}), 2.00-1.82(\mathrm{~m}, 3 \mathrm{H}), 1.60(\mathrm{ddd}, J=17.6,8.9,4.5 \mathrm{~Hz}, 1 \mathrm{H})$, $1.04(\mathrm{~d}, J=6.5 \mathrm{~Hz}, 3 \mathrm{H}) .{ }^{13} \mathrm{C}$ NMR (126 MHz, CD $\left.\mathrm{CD}_{3} \mathrm{O}\right): \delta 153.2,143.7$, 
139.6, 139.2, 130.1, 129.9, 126.7, 126.3, 116.1, 115.9, 56.3, 54.9, 43.4, 30.7, 26.6, 22.4, 18.7 .

HRMS (ESI) $\mathrm{m} / \mathrm{z}$ Calcd for $\left[\mathrm{C}_{19} \mathrm{H}_{24} \mathrm{NO}, \mathrm{M}+\mathrm{H}\right]^{+}:$282.1852; Found: 282.1856.

88\% ee (major isomer). (HPLC condition: Chiralcel OD-H column, $n$-hexane/ $i-\mathrm{PrOH}=$ 95:5, flow rate $=1.0 \mathrm{ml} / \mathrm{min}$, wavelength $=254 \mathrm{~nm}, \mathrm{t}_{\mathrm{R}}=6.13 \mathrm{~min}$ for minor isomer, $\mathrm{t}_{\mathrm{R}}=7.60$ min for major isomer).

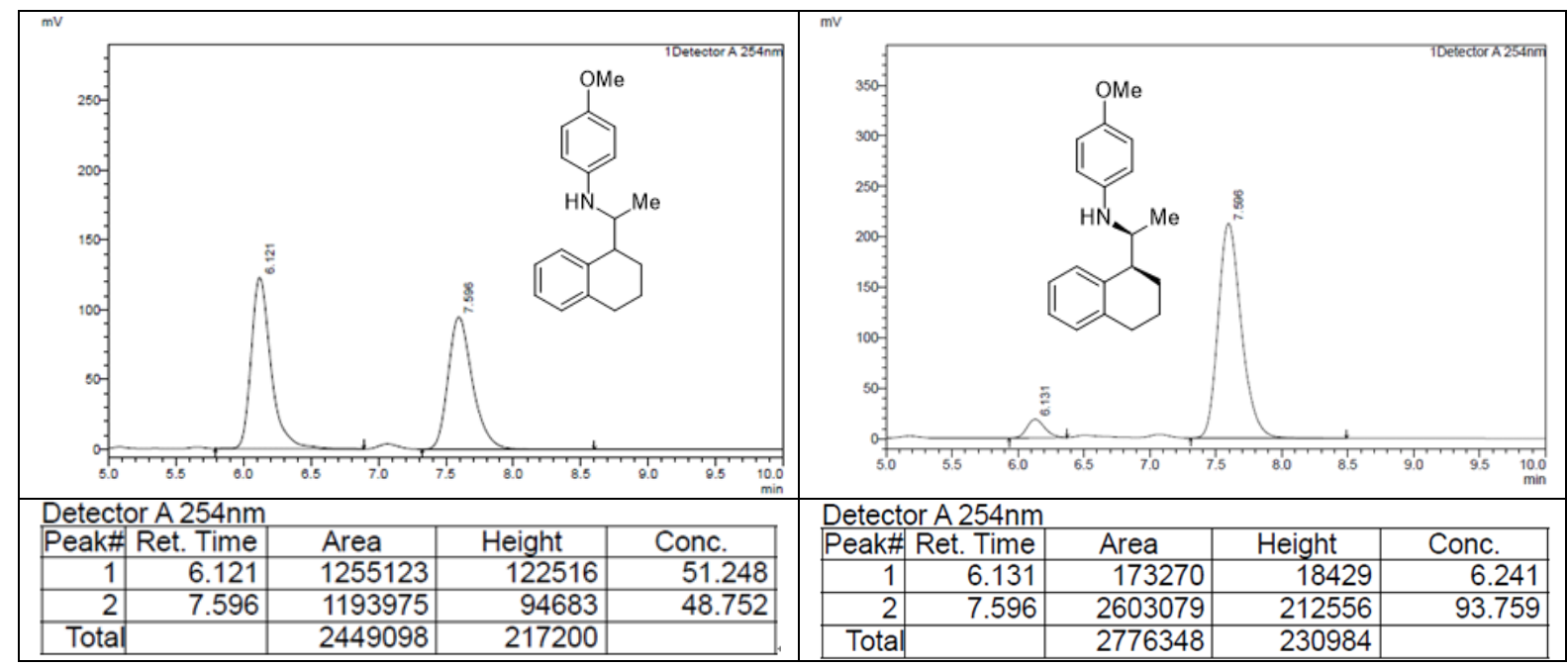

\section{$\underline{N-((S)-1-((S)-5,7-d i m e t h y l-1,2,3,4-t e t r a h y d r o n a p h t h a l e n-1-y l) e t h y l)-4-m e t h o x y a n i l i n e ~(4 k) ~}$}

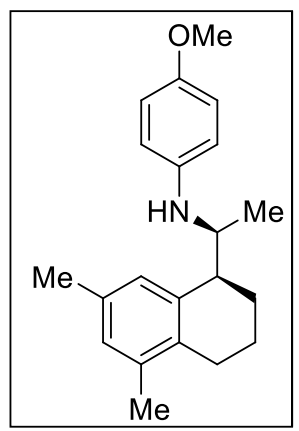

Colorless oil, $52 \%$ yield, $92: 8 \mathrm{dr} .{ }^{1} \mathbf{H}$ NMR $\left(500 \mathrm{MHz}, \mathrm{CDCl}_{3}\right): \delta 6.91$ (s, 1H), $6.85(\mathrm{~s}, 1 \mathrm{H}), 6.78(\mathrm{~d}, J=8.1 \mathrm{~Hz}, 2 \mathrm{H}), 6.72-6.35(\mathrm{~m}, 2 \mathrm{H}), 3.76$ (s, 3H), $2.94(\mathrm{~s}, 1 \mathrm{H}), 2.61$ (tq, $J=17.0,10.3,8.2 \mathrm{~Hz}, 2 \mathrm{H}), 2.26(\mathrm{~s}, 3 \mathrm{H})$, $2.21(\mathrm{~s}, 3 \mathrm{H}), 2.06-1.95(\mathrm{~m}, 1 \mathrm{H}), 1.95-1.85(\mathrm{~m}, 2 \mathrm{H}), 1.76-1.69(\mathrm{~m}$, $1 \mathrm{H}) .{ }^{13} \mathbf{C} \mathbf{N M R}\left(126 \mathrm{MHz}, \mathrm{CDCl}_{3}\right): \delta 137.4,136.1,134.0,133.5,128.6$, 127.4, 115.0, 55.8, 26.1, 24.9, 21.0, 20.7, 19.7. HRMS (ESI) m/z Calcd for $\left[\mathrm{C}_{21} \mathrm{H}_{28} \mathrm{NO}, \mathrm{M}+\mathrm{H}\right]^{+}: 310.2165$; Found: 310.2167.

92\% ee. (HPLC condition: Chiralcel OD-H column, $n$-hexane $/ i-\mathrm{PrOH}=99: 1$, flow rate $=$ $1.0 \mathrm{ml} / \mathrm{min}$, wavelength $=254 \mathrm{~nm}, \mathrm{t}_{\mathrm{R}}=6.49 \mathrm{~min}$ for minor isomer, $\mathrm{t}_{\mathrm{R}}=7.92 \mathrm{~min}$ for major isomer).

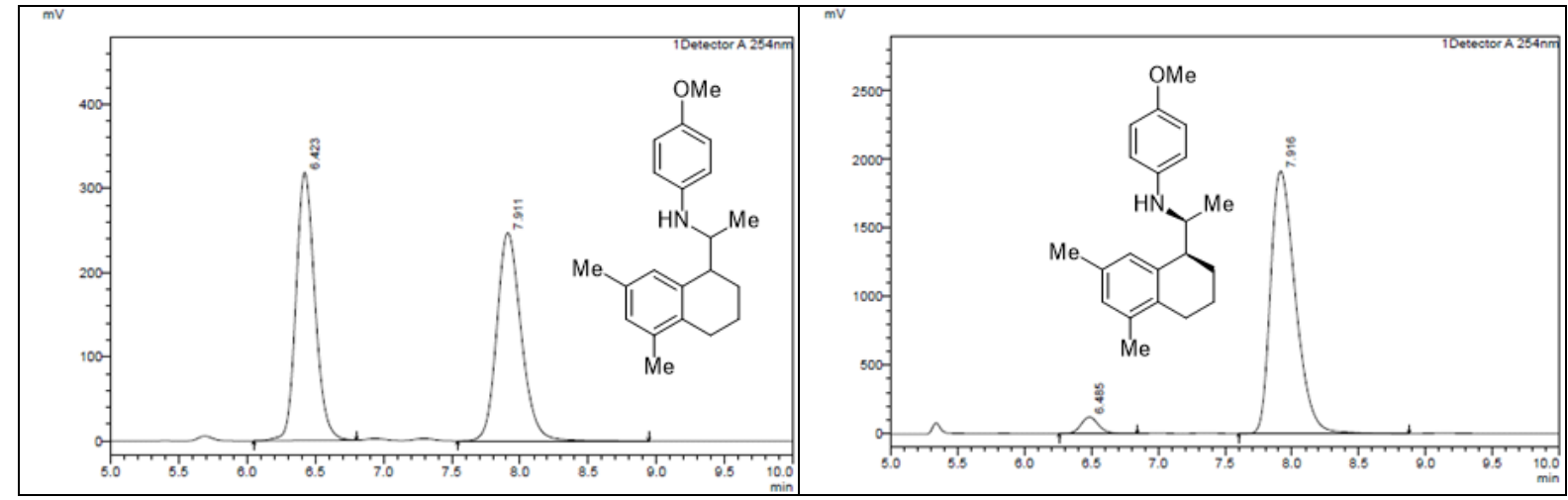




\begin{tabular}{|c|c|c|c|c|}
\hline \multicolumn{5}{|c|}{ Detector A 254nm } \\
\hline Peak\# & Ret. Time & Area & Height & Conc. \\
\hline 1 & 6.423 & 3063938 & 318397 & 49.856 \\
\hline 2 & 7.911 & 3081586 & 247222 & 50.144 \\
\hline Total & & 6145524 & 565619 & \\
\hline
\end{tabular}

\begin{tabular}{|c|c|c|c|c|}
\hline \multirow{2}{*}{\multicolumn{5}{|c|}{ Detector A 254nm }} \\
\hline & t. Time & Area & Height & Conc. \\
\hline 1 & 6.485 & 1090432 & 120476 & 4.233 \\
\hline 2 & 7.916 & 24670554 & 1914656 & 95.767 \\
\hline Total & & 25760986 & 2035133 & \\
\hline
\end{tabular}

\section{$\underline{N-((S)-1-((S)-c h r o m a n-3-y l) e t h y l) a n i l i n e ~(4 I)}$}

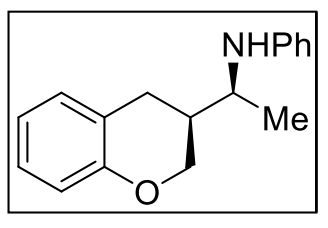

Colorless oil, 53\% yield, 68:32 dr. ${ }^{1} \mathbf{H}$ NMR (500 MHz, $\left.\mathrm{CDCl}_{3}\right): \delta$ $7.21-7.16(\mathrm{~m}, 3 \mathrm{H}), 7.12(\mathrm{t}, J=7.7 \mathrm{~Hz}, 1 \mathrm{H}), 7.07(\mathrm{t}, J=8.2 \mathrm{~Hz}, 1 \mathrm{H})$, $6.91-6.81(\mathrm{~m}, 3 \mathrm{H}), 6.73(\mathrm{t}, J=6.8 \mathrm{~Hz}, 1 \mathrm{H}), 6.63(\mathrm{~d}, J=8.0 \mathrm{~Hz}, 2 \mathrm{H})$, $6.60(\mathrm{~d}, J=8.0 \mathrm{~Hz}, 1 \mathrm{H}), 4.48(\mathrm{~d}, J=10.7 \mathrm{~Hz}, 1 \mathrm{H}), 4.33(\mathrm{~d}, J=10.7$ $\mathrm{Hz}, 1 \mathrm{H}), 4.08-3.96(\mathrm{~m}, 1 \mathrm{H}), 3.91(\mathrm{t}, J=10.1 \mathrm{~Hz}, 1 \mathrm{H}), 3.58(\mathrm{p}, J=6.6 \mathrm{~Hz}, 1 \mathrm{H}), 3.51(\mathrm{p}, J=$ $6.5 \mathrm{~Hz}, 1 \mathrm{H}), 2.97(\mathrm{dd}, J=16.3,5.1 \mathrm{~Hz}, 1 \mathrm{H}), 2.89(\mathrm{dd}, J=16.0,4.3 \mathrm{~Hz}, 1 \mathrm{H}), 2.80(\mathrm{dd}, J=$ $16.3,9.2 \mathrm{~Hz}, 1 \mathrm{H}), 2.70(\mathrm{dd}, J=16.1,10.2 \mathrm{~Hz}, 1 \mathrm{H}), 2.24-2.07$ (m, 2H), $1.39-1.20$ (m, 6H). ${ }^{13} \mathrm{C}$ NMR (126 MHz, $\left.\mathrm{CDCl}_{3}\right): \delta 154.7,154.6,147.2,130.0,129.9,129.4,127.3,127.2,121.5$, 121.3, 120.4, 120.4, 117.6, 117.5, 116.5, 116.4, 113.5, 113.4, 68.5, 68.1, 50.0, 49.9, 38.7, 38.4, 28.3, 28.1, 18.4, 18.1. HRMS (ESI) $\mathrm{m} / \mathrm{z}$ Calcd for $\left[\mathrm{C}_{17} \mathrm{H}_{20} \mathrm{NO}, \mathrm{M}+\mathrm{H}\right]^{+}:$254.1539; Found: 254.1535 .

94\% ee (major isomer), 93\% ee (minor isomer). (HPLC condition: Chiralpark IB column, $n$-hexane $/ i-\mathrm{PrOH}=95: 5$, flow rate $=1.0 \mathrm{ml} / \mathrm{min}$, wavelength $=254 \mathrm{~nm}, \mathrm{t}_{\mathrm{R} 1}=13.36 \mathrm{~min}$ for minor isomer, $\mathrm{t}_{\mathrm{R} 2}=14.57 \mathrm{~min}$ for minor isomer, $\mathrm{t}_{\mathrm{R} 3}=21.56 \mathrm{~min}$ for major isomer, $\mathrm{t}_{\mathrm{R} 4}=22.72$ min for major isomer).

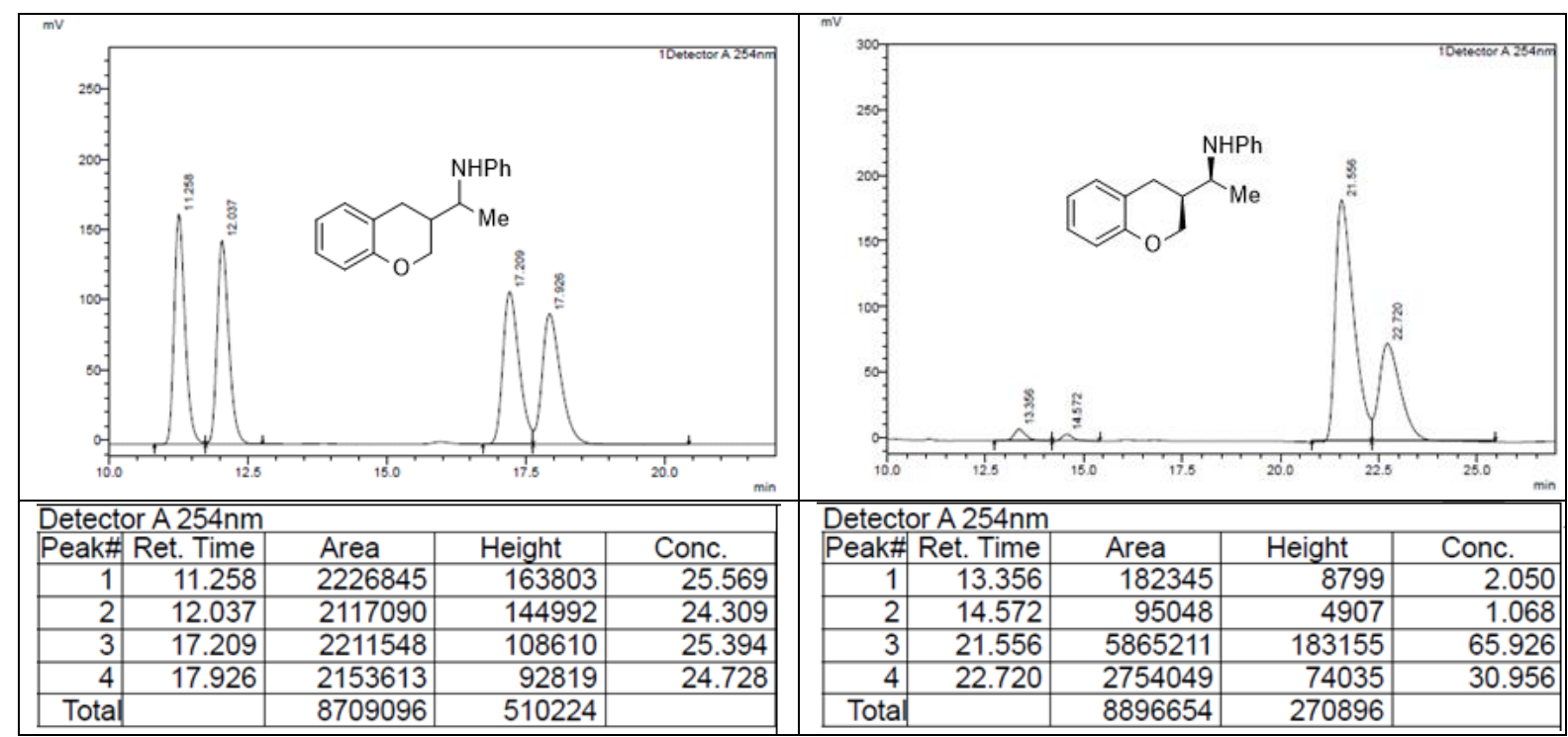

$\underline{N-((S)-1-((S)-2,3-d i h y d r o-1 H-i n d e n-1-y l) e t h y l) a n i l i n e ~(6 a)}$

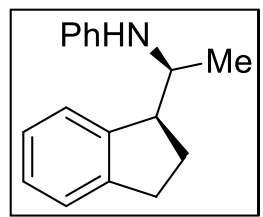

Colorless oil, 83\% yield, 81:19 dr. ${ }^{1} \mathbf{H}$ NMR (500 MHz, $\mathrm{CDCl}_{3}$ ): $\delta 7.51-$

$7.38(\mathrm{~m}, 0.25 \mathrm{H}), 7.35-7.17(\mathrm{~m}, 6 \mathrm{H}), 6.81-6.66(\mathrm{~m}, 3 \mathrm{H}), 3.98(\mathrm{dt}, J=$ 
$12.1,6.3 \mathrm{~Hz}, 0.23 \mathrm{H}), 3.86(\mathrm{p}, J=6.2 \mathrm{~Hz}, 1 \mathrm{H}), 3.71(\mathrm{~s}, 1 \mathrm{H}), 3.57(\mathrm{dt}, J=9.3,5.0 \mathrm{~Hz}, 1 \mathrm{H})$, $3.08(\mathrm{dt}, J=16.2,8.1 \mathrm{~Hz}, 1 \mathrm{H}), 3.01(\mathrm{dd}, J=9.1,5.9 \mathrm{~Hz}, 0.18 \mathrm{H}), 3.00-2.86(\mathrm{~m}, 1 \mathrm{H}), 2.42-$ $2.30(\mathrm{~m}, 1 \mathrm{H}), 2.29-2.20(\mathrm{~m}, 0.26 \mathrm{H}), 2.06$ (ddt, $J=13.1,8.7,4.6 \mathrm{~Hz}, 1 \mathrm{H}), 2.03-1.97$ (m, $0.18 \mathrm{H}), 1.18(\mathrm{~d}, J=6.5 \mathrm{~Hz}, 3 \mathrm{H}) .{ }^{13} \mathbf{C}$ NMR $\left(126 \mathrm{MHz}, \mathrm{CDCl}_{3}\right): \delta 147.3,144.9,144.8,143.4$, 129.3, 126.8, 126.7, 126.2, 126.0, 125.0, 124.6, 124.5, 124.4, 117.0, 113.2, 51.2, 49.0, 31.8, 31.5, 28.1, 27.1, 17.5, 16.8. HRMS (ESI) $\mathrm{m} / \mathrm{z}$ Calcd for $\left[\mathrm{C}_{17} \mathrm{H}_{20} \mathrm{~N}, \mathrm{M}+\mathrm{H}\right]^{+}: 238.1590$; Found: 238.1587 .

96\% ee (major isomer), 99\% ee (minor isomer). (HPLC condition: Chiralcel OD-H column, $n$-hexane $/ i-\mathrm{PrOH}=99: 1$, flow rate $=0.5 \mathrm{ml} / \mathrm{min}$, wavelength $=254 \mathrm{~nm}, \mathrm{t}_{\mathrm{R} 1}=7.31 \mathrm{~min}$ for minor isomer, $t_{\mathrm{R} 2}=8.24 \mathrm{~min}$ for minor isomer, $\mathrm{t}_{\mathrm{R} 3}=8.88 \mathrm{~min}$ for major isomer, $\mathrm{t}_{\mathrm{R} 4}=9.44$ min for major isomer).

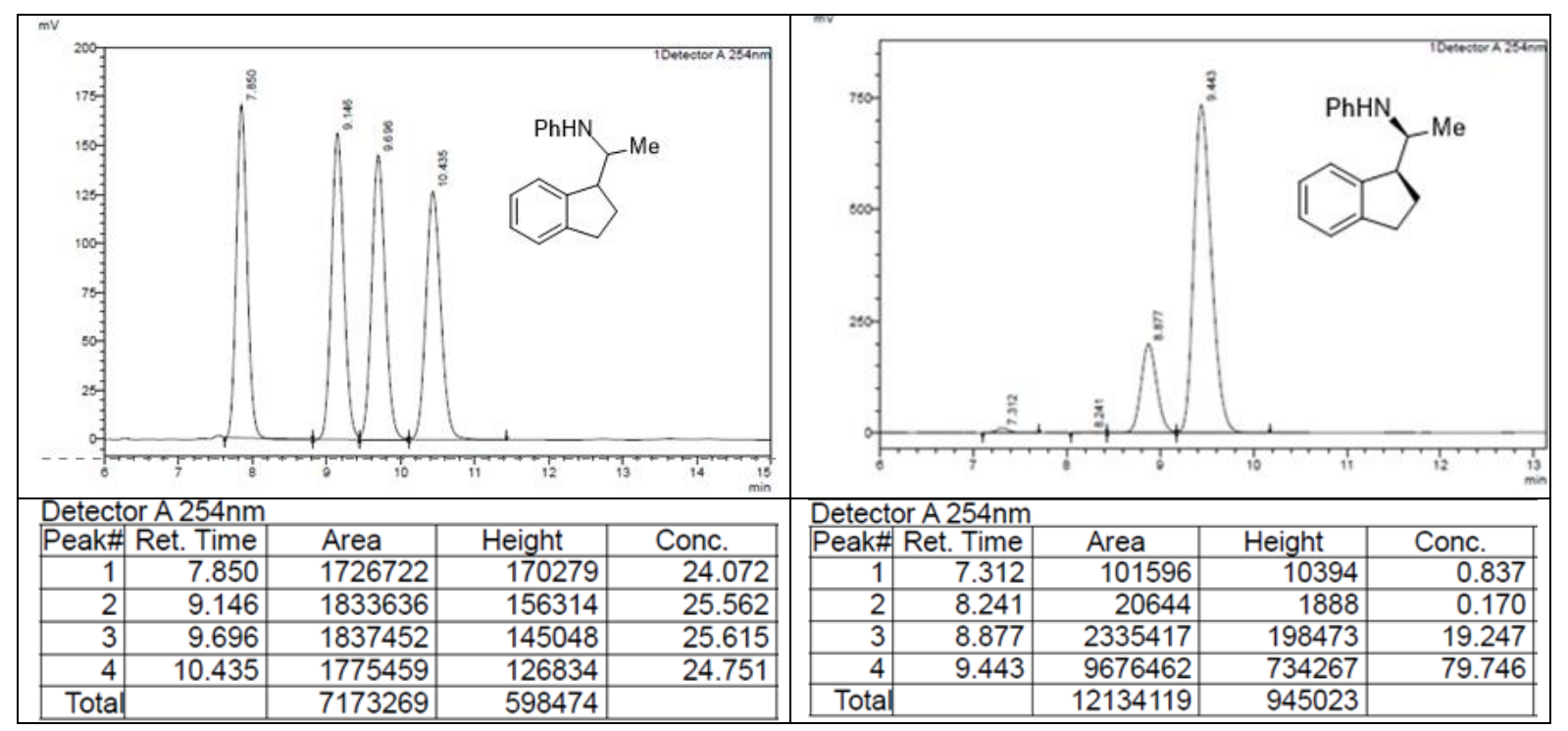

$\underline{N-((S)-1-((S)-5-m e t h o x y-2,3-d i h y d r o-1 H-i n d e n-1-y l) e t h y l) a n i l i n e ~(6 b)}$

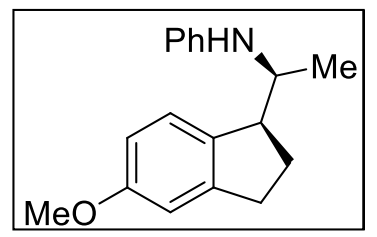

Colorless oil, $86 \%$ yield, $81: 19$ dr. ${ }^{1} \mathbf{H}$ NMR (500 MHz, $\left.\mathrm{CDCl}_{3}\right): \delta$ $7.29(\mathrm{~d}, J=8.4 \mathrm{~Hz}, 0.21 \mathrm{H}), 7.21(\mathrm{t}, J=8.0 \mathrm{~Hz}, 2 \mathrm{H}), 7.13(\mathrm{~d}, J=$ $8.3 \mathrm{~Hz}, 1 \mathrm{H}), 6.82(\mathrm{~s}, 1 \mathrm{H}), 6.78-6.70(\mathrm{~m}, 2 \mathrm{H}), 6.69(\mathrm{~d}, J=7.5 \mathrm{~Hz}$, $0.46 \mathrm{H}), 6.66(\mathrm{~d}, J=7.8 \mathrm{~Hz}, 2 \mathrm{H}), 3.81(\mathrm{~s}, 3 \mathrm{H}), 3.80-3.73(\mathrm{~m}, 1 \mathrm{H})$, $3.45(\mathrm{qd}, J=5.0,3.5,2.6 \mathrm{~Hz}, 1 \mathrm{H}), 3.01(\mathrm{dt}, J=16.2,8.1 \mathrm{~Hz}, 1 \mathrm{H}), 2.96-2.91(\mathrm{~m}, 0.19 \mathrm{H})$, $2.87(\mathrm{ddd}, J=14.0,8.9,4.9 \mathrm{~Hz}, 1 \mathrm{H}), 2.32(\mathrm{dq}, J=13.8,9.1 \mathrm{~Hz}, 1 \mathrm{H}), 2.26-2.18(\mathrm{~m}, 0.25 \mathrm{H})$, 2.02 (ddt, $J=13.1,8.8,4.5 \mathrm{~Hz}, 1 \mathrm{H}), 1.97(\mathrm{~d}, J=6.4 \mathrm{~Hz}, 0.16 \mathrm{H}), 1.13(\mathrm{~d}, J=6.4 \mathrm{~Hz}, 3 \mathrm{H}) .{ }^{13} \mathrm{C}$ NMR $\left(126 \mathrm{MHz}, \mathrm{CDCl}_{3}\right): \delta 159.0,147.4,146.5,146.4,136.2,135.5,129.3,125.4,125.0$, 117.0, 113.4, 113.2, 112.1, 112.0, 109.9, 109.8, 55.3, 51.4, 48.4, 48.3, 32.0, 31.7, 28.5, 27.8, 17.4. HRMS (ESI) $\mathrm{m} / \mathrm{z}$ Calcd for $\left[\mathrm{C}_{18} \mathrm{H}_{22} \mathrm{NO}, \mathrm{M}+\mathrm{H}\right]^{+}:$268.1696; Found: 268.1694. 
97\% ee (major isomer), 97\% ee (minor isomer). (HPLC condition: Chiralcel OD-H column, $n$-hexane $/ i-\mathrm{PrOH}=99: 1$, flow rate $=0.5 \mathrm{ml} / \mathrm{min}$, wavelength $=254 \mathrm{~nm}, \mathrm{t}_{\mathrm{R} 1}=9.77 \mathrm{~min}$ for minor isomer, $\mathrm{t}_{\mathrm{R} 2}=10.35 \mathrm{~min}$ for minor isomer, $\mathrm{t}_{\mathrm{R} 3}=12.40 \mathrm{~min}$ for major isomer, $\mathrm{t}_{\mathrm{R} 4}=14.12$ min for major isomer).

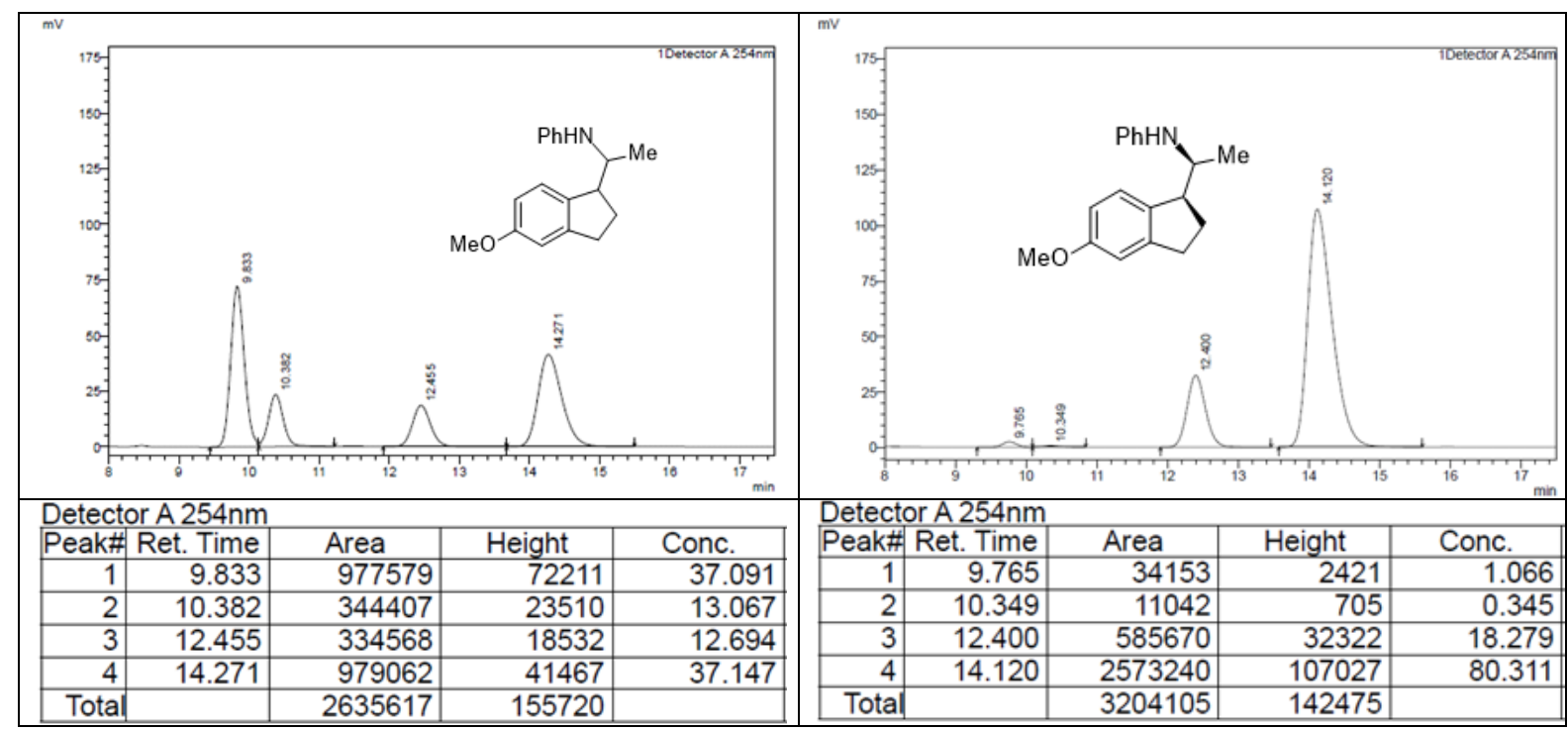

$\underline{N-((S)-1-((S)-5-c h l o r o-2,3-d i h y d r o-1 H-i n d e n-1-y l) e t h y l) a n i l i n e ~(6 c)}$

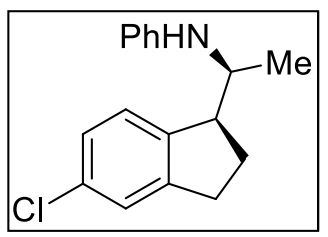

Colorless oil, $78 \%$ yield, 75:25 dr. ${ }^{1} \mathbf{H}$ NMR (500 MHz, $\left.\mathrm{CDCl}_{3}\right): \delta$ $7.30(\mathrm{~d}, J=8.1 \mathrm{~Hz}, 0.30 \mathrm{H}), 7.24-7.17(\mathrm{~m}, 3 \mathrm{H}), 7.17-7.09(\mathrm{~m}, 2 \mathrm{H})$, $6.75-6.70(\mathrm{~m}, 1 \mathrm{H}), 6.67(\mathrm{~d}, J=8.6 \mathrm{~Hz}, 0.51 \mathrm{H}), 6.64(\mathrm{~d}, J=8.4 \mathrm{~Hz}$, 2H), $3.87(\mathrm{p}, J=6.4 \mathrm{~Hz}, 0.33 \mathrm{H}), 3.78(\mathrm{p}, J=6.3 \mathrm{~Hz}, 1 \mathrm{H}), 3.63$ (s, $1 \mathrm{H}), 3.55-3.41(\mathrm{~m}, 1 \mathrm{H}), 3.00(\mathrm{dt}, J=16.5,8.1 \mathrm{~Hz}, 1 \mathrm{H}), 2.95-2.92(\mathrm{~m}, 0.30 \mathrm{H}), 2.90-2.83$ (m, 1H), $2.40-2.26(\mathrm{~m}, 1 \mathrm{H}), 2.24-2.16(\mathrm{~m}, 0.36 \mathrm{H}), 2.08-1.91(\mathrm{~m}, 1 \mathrm{H}), 1.12(\mathrm{~d}, J=6.6$ $\mathrm{Hz}, 1 \mathrm{H}), 1.10(\mathrm{~d}, J=6.5 \mathrm{~Hz}, 3 \mathrm{H}) .{ }^{13} \mathbf{C}$ NMR $\left(125 \mathrm{MHz}, \mathrm{CDCl}_{3}\right): \delta 147.1,147.0,146.8,142.9$, $142.0,132.5,132.4,129.4,126.4,126.2,125.9,125.4,124.8,124.7,117.5,117.2,113.5$, 113.2, 52.2, 51.2, 48.6, 48.3, 31.7, 31.5, 28.2, 27.4, 17.3, 16.9. HRMS (ESI) m/z Calcd for $\left[\mathrm{C}_{17} \mathrm{H}_{19} \mathrm{ClN}, \mathrm{M}+\mathrm{H}\right]^{+}:$272.1201; Found: 272.1207.

98\% ee (major isomer), 97\% ee (minor isomer). (HPLC condition: Chiralcel OD-H column, $n$-hexane $/ i-\mathrm{PrOH}=99: 1$, flow rate $=1.0 \mathrm{ml} / \mathrm{min}$, wavelength $=254 \mathrm{~nm}, \mathrm{t}_{\mathrm{R} 1}=9.57 \mathrm{~min}$ for minor isomer, $\mathrm{t}_{\mathrm{R} 2}=11.02 \mathrm{~min}$ for minor isomer, $\mathrm{t}_{\mathrm{R} 3}=12.28 \mathrm{~min}$ for major isomer, $\mathrm{t}_{\mathrm{R} 4}=14.90$ min for major isomer). 


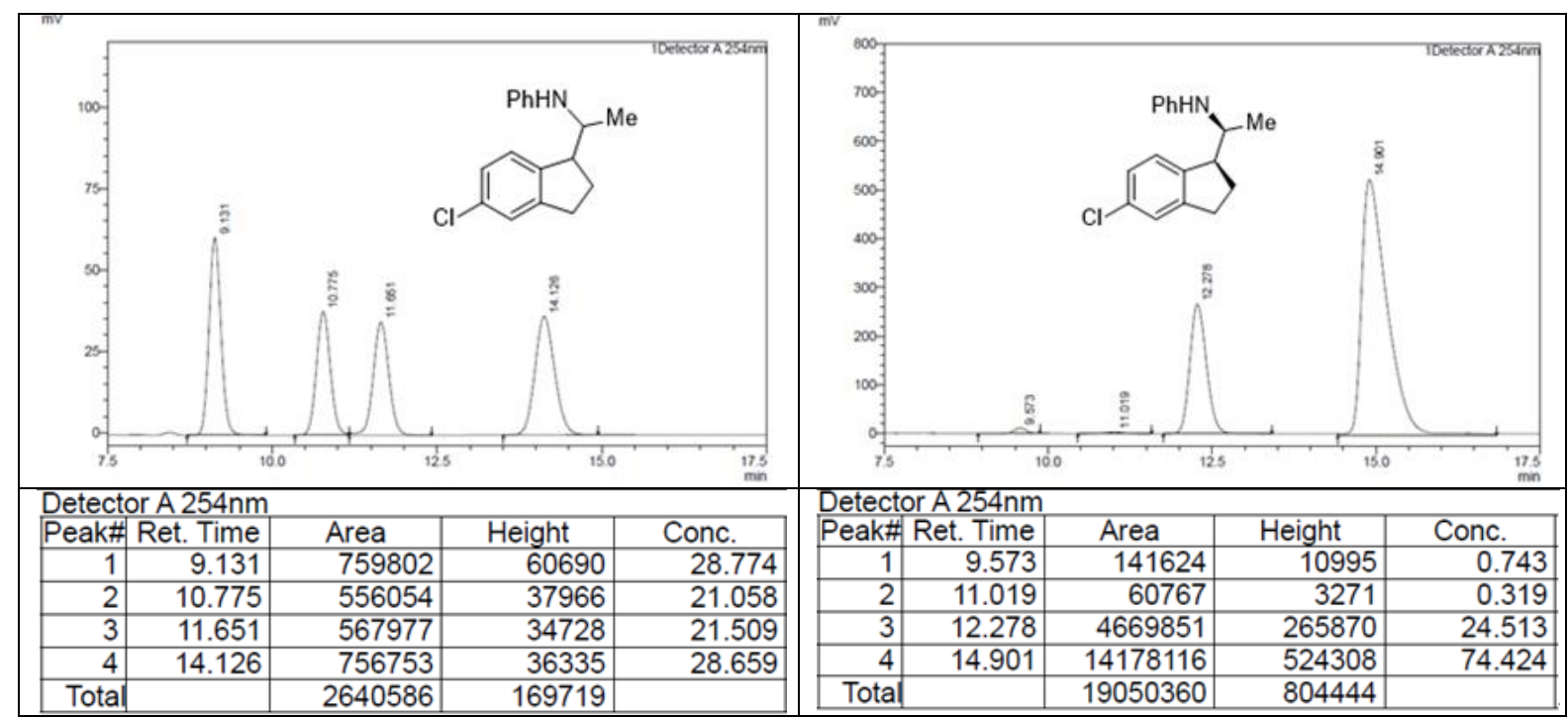

$\underline{N-((S)-1-((S)-6-m e t h y l-2,3-d i h y d r o-1 H-i n d e n-1-y l) e t h y l) a n i l i n e ~(6 d) ~}$

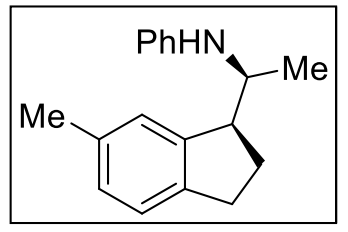

Colorless oil, 83\% yield, 81:19 dr. ${ }^{1} \mathbf{H}$ NMR (500 $\mathrm{MHz}, \mathrm{CDCl}_{3}$ ): $\delta$ $7.31-7.25(\mathrm{~m}, 2 \mathrm{H}), 7.20(\mathrm{~d}, J=7.6 \mathrm{~Hz}, 1 \mathrm{H}), 7.12(\mathrm{~s}, 1 \mathrm{H}), 7.08$ (d, $J$ $=7.4 \mathrm{~Hz}, 1 \mathrm{H}), 6.78(\mathrm{t}, J=7.7 \mathrm{~Hz}, 1 \mathrm{H}), 6.72(\mathrm{~d}, J=7.8 \mathrm{~Hz}, 2 \mathrm{H})$, $4.00(\mathrm{tt}, J=6.6,3.3 \mathrm{~Hz}, 0.21 \mathrm{H}), 3.85(\mathrm{p}, J=6.3 \mathrm{~Hz}, 1 \mathrm{H}), 3.73(\mathrm{~s}$, 1H), 3.59 (dd, $J=9.0,4.9 \mathrm{~Hz}, 0.31 \mathrm{H}), 3.53(\mathrm{dt}, J=9.2,4.8 \mathrm{~Hz}, 1 \mathrm{H}), 3.05(\mathrm{dt}, J=16.1,8.1$ $\mathrm{Hz}, 1 \mathrm{H}), 2.98(\mathrm{dd}, J=9.0,6.1 \mathrm{~Hz}, 0.20 \mathrm{H}), 2.96-2.86(\mathrm{~m}, 1 \mathrm{H}), 2.43(\mathrm{~s}, 1 \mathrm{H}), 2.40(\mathrm{~s}, 3 \mathrm{H})$, $2.39-2.32(\mathrm{~m}, 1 \mathrm{H}), 2.29-2.20(\mathrm{~m}, 0.28 \mathrm{H}), 2.08(\mathrm{ddt}, J=13.0,8.7,4.5 \mathrm{~Hz}, 1 \mathrm{H}), 2.04-1.96$ $(\mathrm{m}, 0.19 \mathrm{H}), 1.20(\mathrm{~d}, J=6.5 \mathrm{~Hz}, 3 \mathrm{H}) .{ }^{13} \mathrm{C}$ NMR $\left(126 \mathrm{MHz}, \mathrm{CDCl}_{3}\right): \delta 147.4,144.4,143.6$, $141.9,141.8,135.7,135.4,129.3,127.6,127.6,125.6,125.0,124.3,124.2,117.1,116.9$, 113.4, 113.2, 52.1, 51.2, 49.1, 48.8, 31.4, 31.1, 28.3, 27.1, 21.3, 17.5, 16.6. HRMS (ESI) m/z Calcd for $\left[\mathrm{C}_{18} \mathrm{H}_{22} \mathrm{~N}, \mathrm{M}+\mathrm{H}\right]^{+}:$252.1747; Found: 252.1746 .

98\% ee (major isomer) and 98\% ee (minor isomer). (HPLC condition: Chiralcel OJ-H column, $n$-hexane $/ i-\mathrm{PrOH}=99: 1$, flow rate $=1.0 \mathrm{ml} / \mathrm{min}$, wavelength $=254 \mathrm{~nm}, \mathrm{t}_{\mathrm{R} 1}=13.46$ $\min$ for minor isomer, $\mathrm{t}_{\mathrm{R} 2}=16.86 \mathrm{~min}$ for major isomer, $\mathrm{t}_{\mathrm{R} 3}=26.01 \mathrm{~min}$ for minor isomer, $\mathrm{t}_{\mathrm{R} 4}=$ $27.25 \mathrm{~min}$ for major isomer).

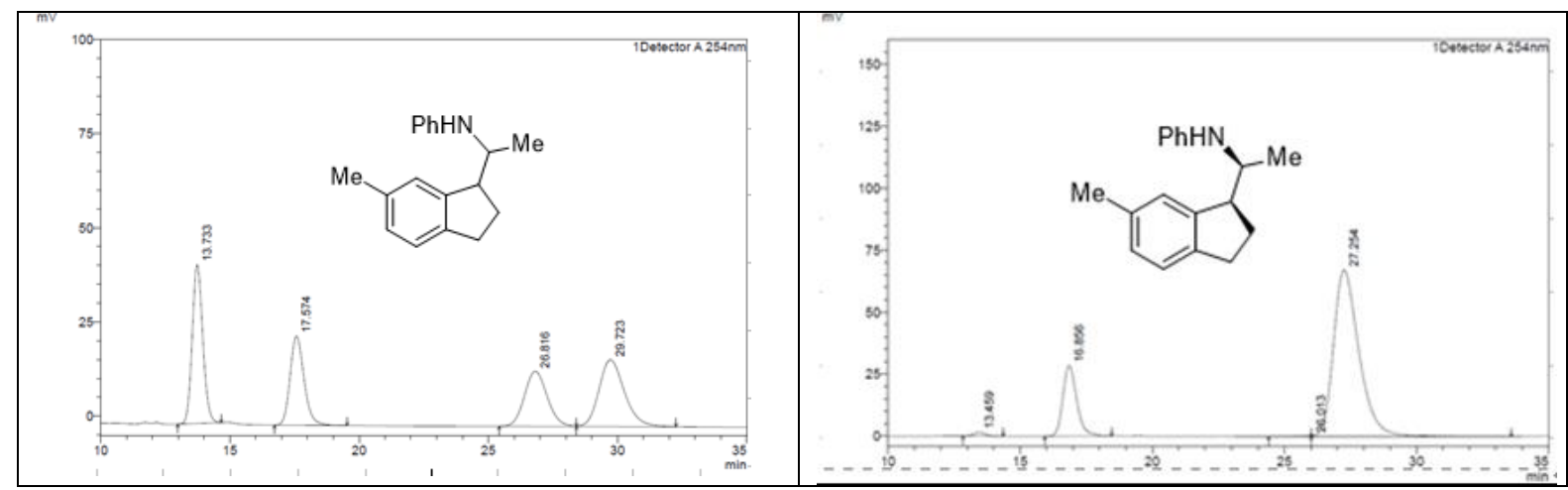




\begin{tabular}{|c|c|c|c|c|c|c|c|c|c|}
\hline \multicolumn{5}{|c|}{ Detector A 254nm } & \multicolumn{5}{|c|}{ Detector A $254 \mathrm{~nm}$} \\
\hline Peak\# & et. Time & Area & Height & Conc. & Peak\# & Ret. Time & Area & Height & Conc. \\
\hline 1 & 13.733 & 1202015 & 42137 & 28.626 & 1 & 13.459 & 43435 & 1479 & 0.774 \\
\hline 2 & 17.574 & 890826 & 23685 & 21.215 & 2 & 16.856 & 1043015 & 28471 & 18.589 \\
\hline 3 & 26.816 & 894822 & 14626 & 21.310 & 3 & 26.013 & 9648 & 240 & 0.172 \\
\hline 4 & 29.723 & 1211398 & 17716 & 28.849 & 4 & 27.254 & 4514952 & 67216 & 80.465 \\
\hline Total & & 4199061 & 98163 & & Total & & 5611049 & 97406 & \\
\hline
\end{tabular}

$\underline{N-((S)-1-((S)-6-m e t h o x y-2,3-d i h y d r o-1 H-i n d e n-1-y l) e t h y l) a n i l i n e ~(6 e) ~}$

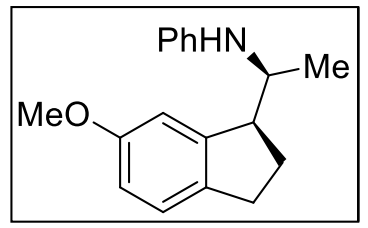

Colorless oil, 81\% yield, 83:17 dr. ${ }^{1} \mathbf{H}$ NMR (500 $\left.\mathrm{MHz}, \mathrm{CDCl}_{3}\right): \delta$

$7.22(\mathrm{t}, J=7.9 \mathrm{~Hz}, 2 \mathrm{H}), 7.16(\mathrm{~d}, J=8.1 \mathrm{~Hz}, 1 \mathrm{H}), 6.96(\mathrm{~s}, 0.17 \mathrm{H})$,

$6.81(\mathrm{~s}, 1 \mathrm{H}), 6.78(\mathrm{dd}, J=8.1,2.2 \mathrm{~Hz}, 1 \mathrm{H}), 6.73(\mathrm{t}, J=6.9 \mathrm{~Hz}, 1 \mathrm{H})$,

$6.67(\mathrm{~d}, J=7.7 \mathrm{~Hz}, 2 \mathrm{H}), 3.85-3.82(\mathrm{~m}, 1 \mathrm{H}), 3.81(\mathrm{~s}, 1 \mathrm{H}), 3.79$ (s,

$3 \mathrm{H}), 3.52(\mathrm{dt}, J=9.3,4.7 \mathrm{~Hz}, 1 \mathrm{H}), 2.98(\mathrm{dt}, J=16.0,8.1 \mathrm{~Hz}, 1 \mathrm{H}), 2.91(\mathrm{dd}, J=8.9,6.0 \mathrm{~Hz}$, $0.16 \mathrm{H}$ ), $2.89-2.77(\mathrm{~m}, 1 \mathrm{H}), 2.42-2.27(\mathrm{~m}, 1 \mathrm{H}), 2.26-2.17$ (m, 0.22H), 2.03 (ddt, $J=13.2$, 8.8, $4.5 \mathrm{~Hz}, 1 \mathrm{H}), 1.14(\mathrm{~d}, J=6.4 \mathrm{~Hz}, 3 \mathrm{H}) .{ }^{13} \mathrm{C}$ NMR $\left(126 \mathrm{MHz}, \mathrm{CDCl}_{3}\right): \delta 158.4,147.3$, $144.9,129.3,124.9,117.1,113.3,112.5,110.7$, $55.4,51.4,49.0$, 30.7 , 28.6 , 17.4. HRMS (ESI) m/z Calcd for $\left[\mathrm{C}_{18} \mathrm{H}_{22} \mathrm{NO}, \mathrm{M}+\mathrm{H}\right]^{+}:$268.1696; Found: 268.1702.

98\% ee (major isomer), 98\% ee (minor isomer). (HPLC condition: Chiralcel OD-H column, $n$-hexane $/ i-\mathrm{PrOH}=99: 1$, flow rate $=0.5 \mathrm{ml} / \mathrm{min}$, wavelength $=254 \mathrm{~nm}, \mathrm{t}_{\mathrm{R} 1}=16.81$ min for minor isomer, $t_{\mathrm{R} 2}=19.15 \mathrm{~min}$ for major isomer, $\mathrm{t}_{\mathrm{R} 3}=20.98 \mathrm{~min}$ for minor isomer, $\mathrm{t}_{\mathrm{R} 4}=$ $26.14 \mathrm{~min}$ for major isomer).

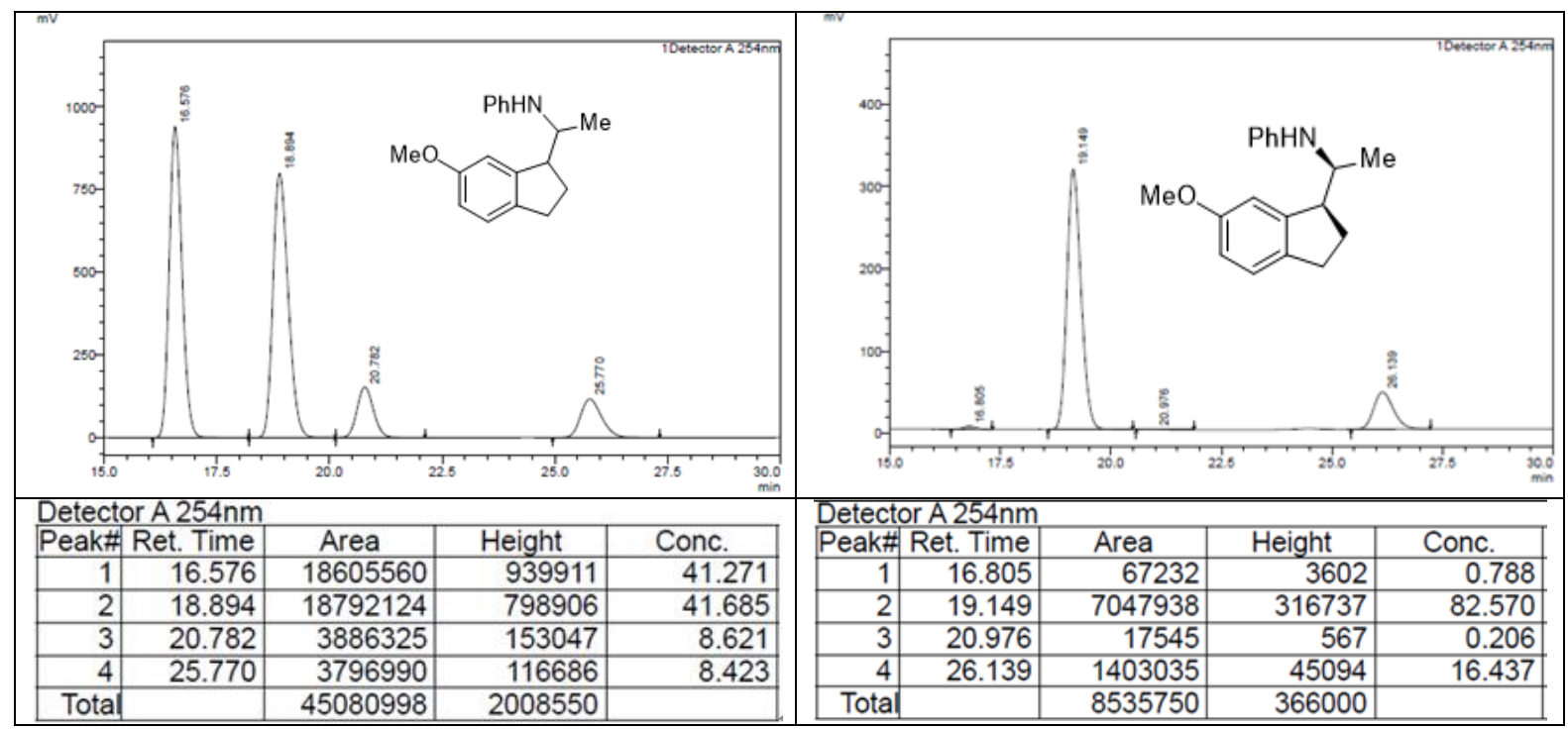

$\underline{N-((S)-1-((S)-5-c h l o r o-6-m e t h o x y-2,3-d i h y d r o-1 H-i n d e n-1-y l) e t h y l) a n i l i n e ~(6 f) ~}$<smiles>COc1cc2c(cc1Cl)CCC2[C@H](C)N</smiles>

Colorless oil, $76 \%$ yield, $95: 5$ dr. ${ }^{1} \mathbf{H}$ NMR $\left(500 \mathrm{MHz}, \mathrm{CDCl}_{3}\right): \delta$

$7.25-7.15(\mathrm{~m}, 3 \mathrm{H}), 6.78(\mathrm{~s}, 1 \mathrm{H}), 6.72(\mathrm{t}, J=7.3 \mathrm{~Hz}, 1 \mathrm{H}), 6.65$ (d, $J=8.0 \mathrm{~Hz}, 2 \mathrm{H}), 3.86-3.77(\mathrm{~m}, 4 \mathrm{H}), 3.67(\mathrm{~s}, 1 \mathrm{H}), 3.52(\mathrm{dt}, J=9.1$, 
$4.7 \mathrm{~Hz}, 1 \mathrm{H}), 2.95(\mathrm{dt}, J=16.0,8.0 \mathrm{~Hz}, 1 \mathrm{H}), 2.88-2.73(\mathrm{~m}, 1 \mathrm{H}), 2.33(\mathrm{dq}, J=13.1,8.8 \mathrm{~Hz}$, 1H), 2.00 (ddt, $J=13.0,8.7,4.4 \mathrm{~Hz}, 1 \mathrm{H}), 1.11(\mathrm{~d}, J=6.5 \mathrm{~Hz}, 3 \mathrm{H}) .{ }^{13} \mathbf{C}$ NMR $(125 \mathrm{MHz}$, $\left.\mathrm{CDCl}_{3}\right): \delta 153.5,147.2,143.2,137.6,129.4,125.8,121.0,117.3,113.2,109.1,56.3,51.4$, 48.6, 30.8, 28.4, 17.3. HRMS (ESI) m/z Calcd for $\left[\mathrm{C}_{18} \mathrm{H}_{21} \mathrm{ClNO}, \mathrm{M}+\mathrm{H}\right]^{+}:$302.1306; Found: 302.1314 .

Optical Rotation: $[\alpha]^{25} \mathrm{D}=17.2\left(0.6, \mathrm{CHCl}_{3}\right) .99 \%$ ee. (HPLC condition: Chiralcel OD-H column, $n$-hexane $/ i-\mathrm{PrOH}=99: 1$, flow rate $=0.5 \mathrm{ml} / \mathrm{min}$, wavelength $=254 \mathrm{~nm}, \mathrm{t}_{\mathrm{R}}=30.21$ $\min$ for major isomer, $t_{\mathrm{R}}=37.73 \mathrm{~min}$ for minor isomer).

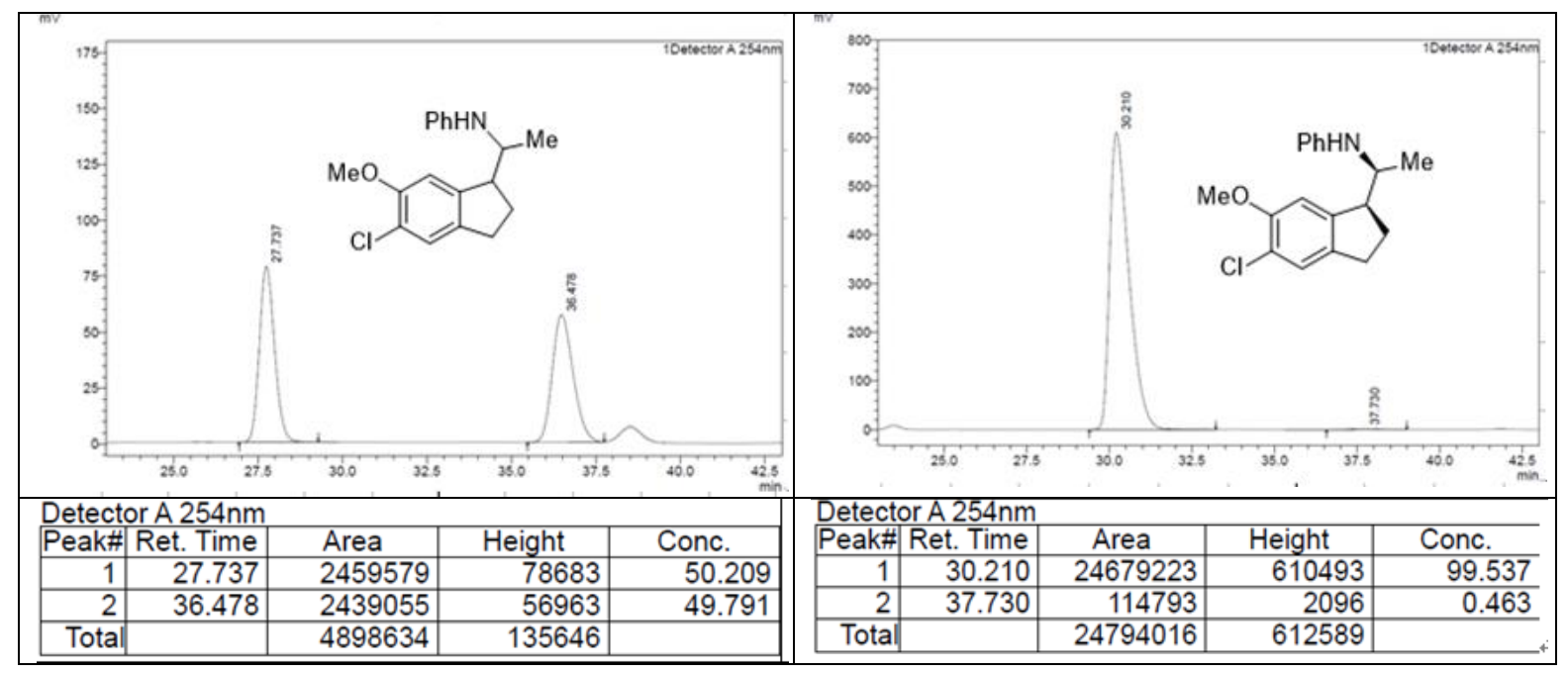

\section{$\underline{N-((S)-1-((S)-4-b r o m 0-7-m e t h o x y-2,3-d i h y d r o-1 H-i n d e n-1-y l) e t h y l) a n i l i n e ~(6 g) ~}$}

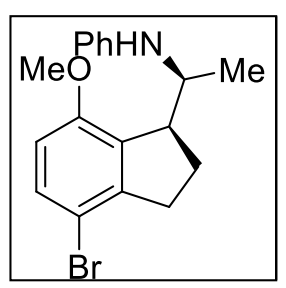

Colorless oil, $40 \%$ yield, $>95: 5$ dr. ${ }^{1} \mathbf{H}$ NMR $\left(500 \mathrm{MHz}, \mathrm{CDCl}_{3}\right): \delta 7.28$ $(\mathrm{d}, J=8.6 \mathrm{~Hz}, 1 \mathrm{H}), 7.14(\mathrm{t}, J=7.7 \mathrm{~Hz}, 2 \mathrm{H}), 6.66(\mathrm{t}, J=7.4 \mathrm{~Hz}, 1 \mathrm{H})$, $6.59(\mathrm{dd}, J=13.3,8.2 \mathrm{~Hz}, 3 \mathrm{H}), 3.97-3.86(\mathrm{~m}, 1 \mathrm{H}), 3.84(\mathrm{~s}, 3 \mathrm{H}), 3.71$ $(\mathrm{dt}, J=8.5,3.7 \mathrm{~Hz}, 1 \mathrm{H}), 3.02(\mathrm{dt}, J=16.7,8.4 \mathrm{~Hz}, 1 \mathrm{H}), 2.87$ (ddd, $J=$ $16.7,9.7,4.0 \mathrm{~Hz}, 1 \mathrm{H}), 2.38-2.26(\mathrm{~m}, 1 \mathrm{H}), 1.99$ (ddt, $J=13.3,8.9,4.1$ $\mathrm{Hz}, 1 \mathrm{H}), 1.06(\mathrm{~d}, J=6.5 \mathrm{~Hz}, 3 \mathrm{H}) .{ }^{13} \mathbf{C}$ NMR $\left(126 \mathrm{MHz}, \mathrm{CDCl}_{3}\right): \delta 155.6,147.5,146.9,132.9$, 130.9, 129.2, 117.0, 113.5, 111.1, 110.5, 55.5, 52.6, 49.9, 33.8, 28.1, 17.2. HRMS (ESI) m/z Calcd for $\left[\mathrm{C}_{18} \mathrm{H}_{21} \mathrm{BrNO}, \mathrm{M}+\mathrm{H}\right]^{+}: 346.0801$; Found: 346.0805 .

Optical Rotation: $[\alpha]^{24} \mathrm{D}=-88.0\left(0.4, \mathrm{CHCl}_{3}\right) .98 \%$ ee. (HPLC condition: Chiralcel OD$\mathrm{H}$ column, $n$-hexane $/ i-\mathrm{PrOH}=99: 1$, flow rate $=1.0 \mathrm{ml} / \mathrm{min}$, wavelength $=254 \mathrm{~nm}, \mathrm{t}_{\mathrm{R}}=10.36$ $\min$ for minor isomer, $\mathrm{t}_{\mathrm{R}}=11.41 \mathrm{~min}$ for major isomer). 


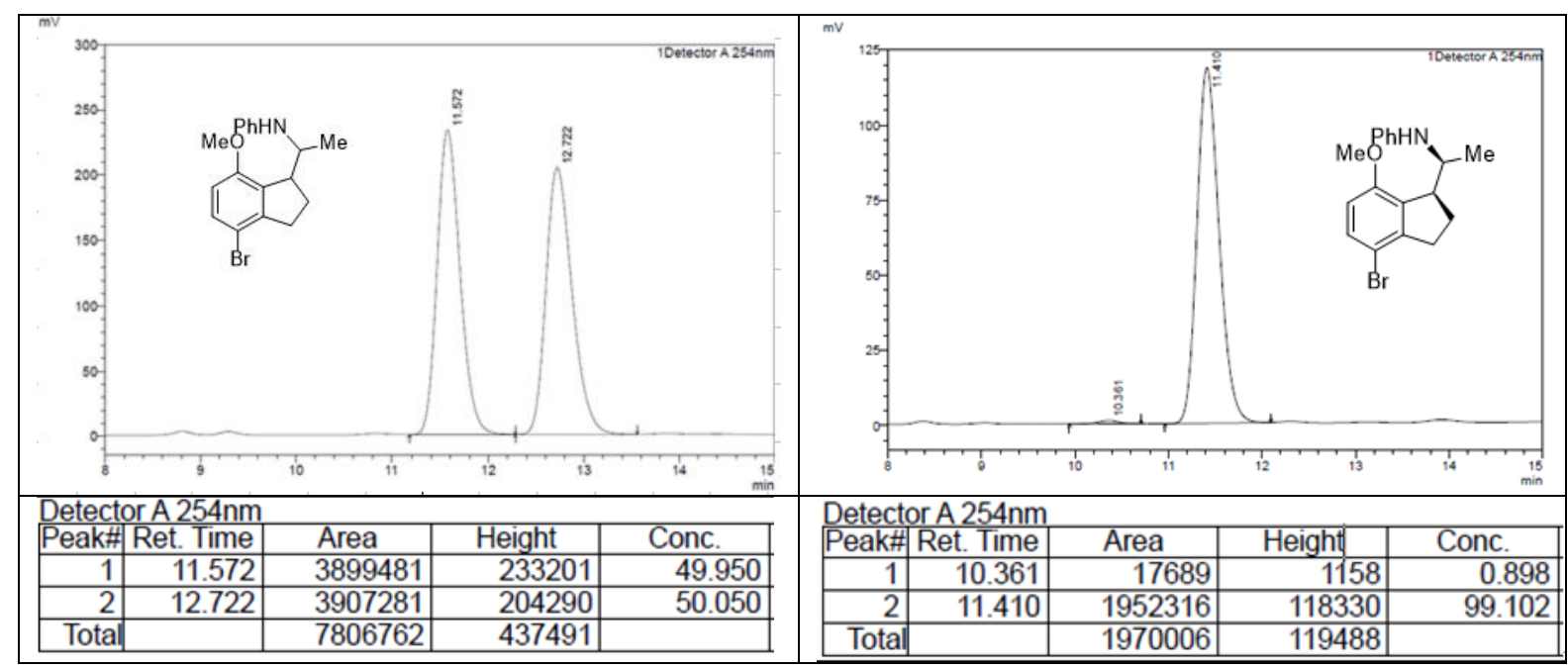

N-((S)-1-((S)-5-chloro-6-methoxy-2,3-dihydro-1H-inden-1-yl)ethyl)-4-methoxyaniline (6h)

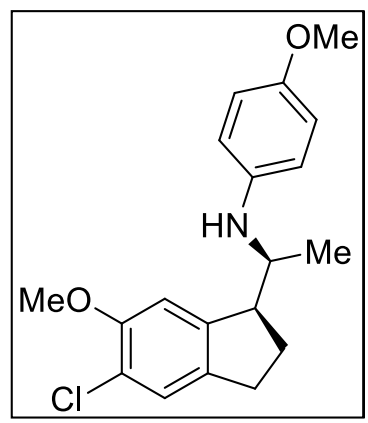

Colorless oil, $73 \%$ yield, 94:6 dr. ${ }^{1} \mathbf{H}$ NMR (500 $\left.\mathrm{MHz}, \mathrm{CDCl}_{3}\right): \delta$

$7.20(\mathrm{~s}, 1 \mathrm{H}), 6.89-6.73(\mathrm{~m}, 3 \mathrm{H}), 6.45-6.43(\mathrm{~m}, 1 \mathrm{H}), 3.82(\mathrm{~s}, 3 \mathrm{H})$,

$3.76(\mathrm{~s}, 3 \mathrm{H}), 3.55-3.37(\mathrm{~m}, 1 \mathrm{H}), 2.93(\mathrm{dt}, J=15.7,7.9 \mathrm{~Hz}, 1 \mathrm{H})$,

$2.86-2.70(\mathrm{~m}, 1 \mathrm{H}), 2.38-2.20(\mathrm{~m}, 1 \mathrm{H}), 1.99(\mathrm{ddt}, J=13.2,8.8$,

$4.5 \mathrm{~Hz}, 1 \mathrm{H}), 1.08(\mathrm{~d}, J=6.4 \mathrm{~Hz}, 3 \mathrm{H}) .{ }^{13} \mathrm{C}$ NMR $(126 \mathrm{MHz}$,

$\left.\mathrm{CDCl}_{3}\right): \delta 153.5,143.3,137.7,125.8,121.0,115.0,109.1,56.3$,

55.8, 30.8, 28.3. HRMS (ESI) $\mathrm{m} / \mathrm{z}$ Calcd for $\left[\mathrm{C}_{19} \mathrm{H}_{23} \mathrm{ClNO}_{2}\right.$,

$\mathrm{M}+\mathrm{H}]^{+}:$332.1412; Found: 332.1414.

Optical Rotation: $[\alpha]_{\mathrm{D}}^{25}=-88.0\left(0.7, \mathrm{CHCl}_{3}\right) .96 \%$ ee. (HPLC condition: Chiralcel OD-

H column, $n$-hexane $/ i-\mathrm{PrOH}=90: 10$, flow rate $=1.0 \mathrm{ml} / \mathrm{min}$, wavelength $=254 \mathrm{~nm}, \mathrm{t}_{\mathrm{R}}=7.43$ min for minor isomer, $\mathrm{t}_{\mathrm{R}}=10.54 \mathrm{~min}$ for major isomer).
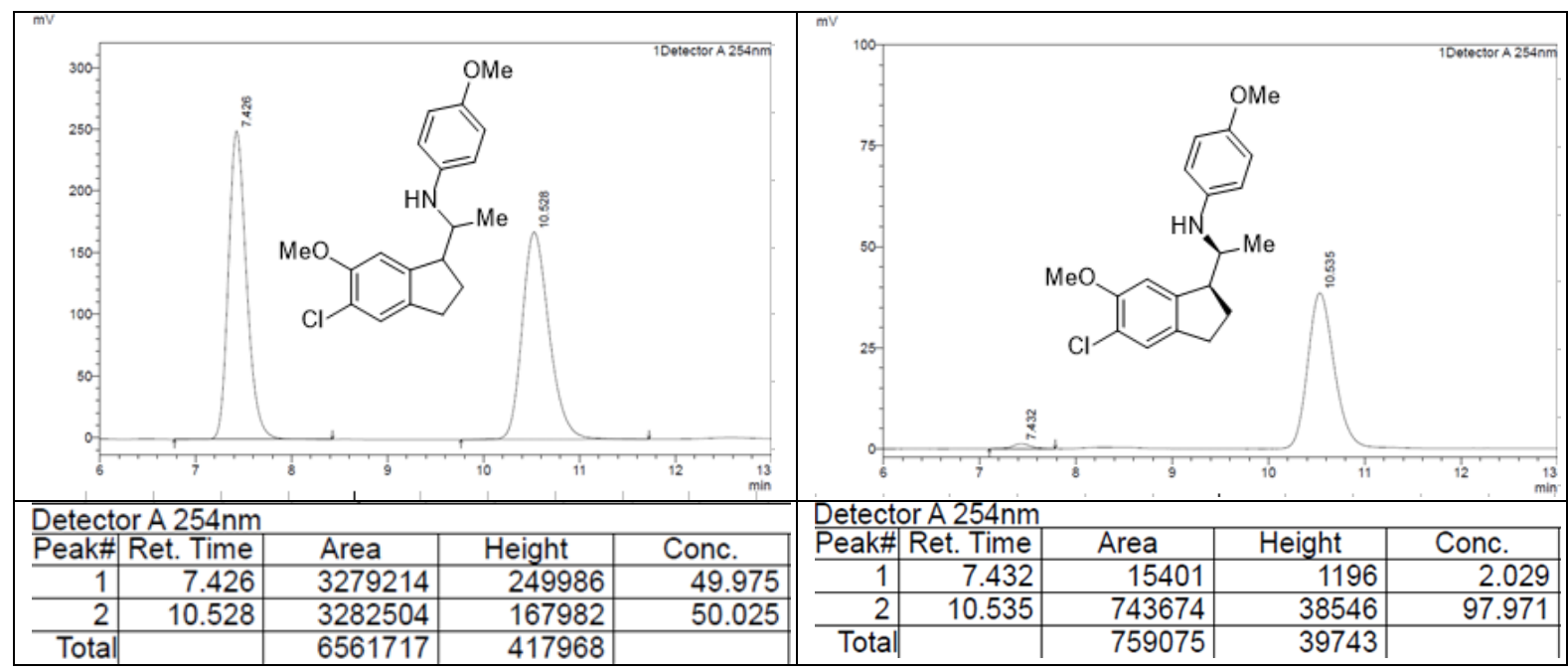


\section{$\mathrm{N}$-((S)-1-((S)-5-chloro-6-methoxy-2,3-dihydro-1H-inden-1-yl)ethyl)-4-methylaniline (6i)}

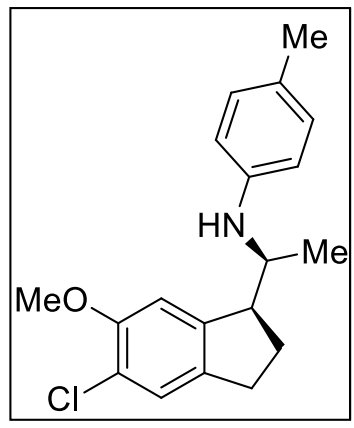

Colorless oil, $81 \%$ yield, 94:6 dr. ${ }^{1} \mathbf{H}$ NMR $\left(500 \mathrm{MHz}, \mathrm{CDCl}_{3}\right): \delta$ $7.22(\mathrm{~s}, 1 \mathrm{H}), 7.02(\mathrm{~d}, J=8.0 \mathrm{~Hz}, 2 \mathrm{H}), 6.79(\mathrm{~s}, 1 \mathrm{H}), 6.58(\mathrm{~d}, J=8.3$ $\mathrm{Hz}, 2 \mathrm{H}), 3.83(\mathrm{~s}, 3 \mathrm{H}), 3.81-3.70(\mathrm{~m}, 1 \mathrm{H}), 3.52(\mathrm{dt}, J=9.1,4.5 \mathrm{~Hz}$, $1 \mathrm{H}), 3.37(\mathrm{~s}, 1 \mathrm{H}), 2.94(\mathrm{dt}, J=16.0,8.1 \mathrm{~Hz}, 1 \mathrm{H}), 2.87-2.75(\mathrm{~m}$, $1 \mathrm{H}), 2.38-2.29(\mathrm{~m}, 1 \mathrm{H}), 2.27$ (s, 3H), 1.99 (ddt, $J=13.1,8.7,4.4$ $\mathrm{Hz}, 1 \mathrm{H}), 1.09$ (d, $J=6.6 \mathrm{~Hz}, 3 \mathrm{H}) .{ }^{13} \mathbf{C} \mathbf{N M R}\left(126 \mathrm{MHz}, \mathrm{CDCl}_{3}\right): \delta$ $153.5,144.8,143.3,137.7,129.9,126.6,125.8,121.0,113.6,109.1$,

56.3, 51.8, 48.6, 48.6, 30.8, 28.4, 20.3, 17.3. HRMS (ESI) m/z Calcd for $\left[\mathrm{C}_{19} \mathrm{H}_{23} \mathrm{ClNO}\right.$, $\mathrm{M}+\mathrm{H}]^{+}:$316.1463; Found: 316.1466.

Optical Rotation: $[\alpha]^{25} \mathrm{D}=-86.3\left(0.7, \mathrm{CHCl}_{3}\right) .96 \%$ ee. (HPLC condition: Chiralcel OD$\mathrm{H}$ column, $n$-hexane $/ i-\mathrm{PrOH}=99: 1$, flow rate $=1.0 \mathrm{ml} / \mathrm{min}$, wavelength $=254 \mathrm{~nm}, \mathrm{t}_{\mathrm{R}}=10.57$ $\min$ for minor isomer, $t_{\mathrm{R}}=17.34 \mathrm{~min}$ for major isomer).

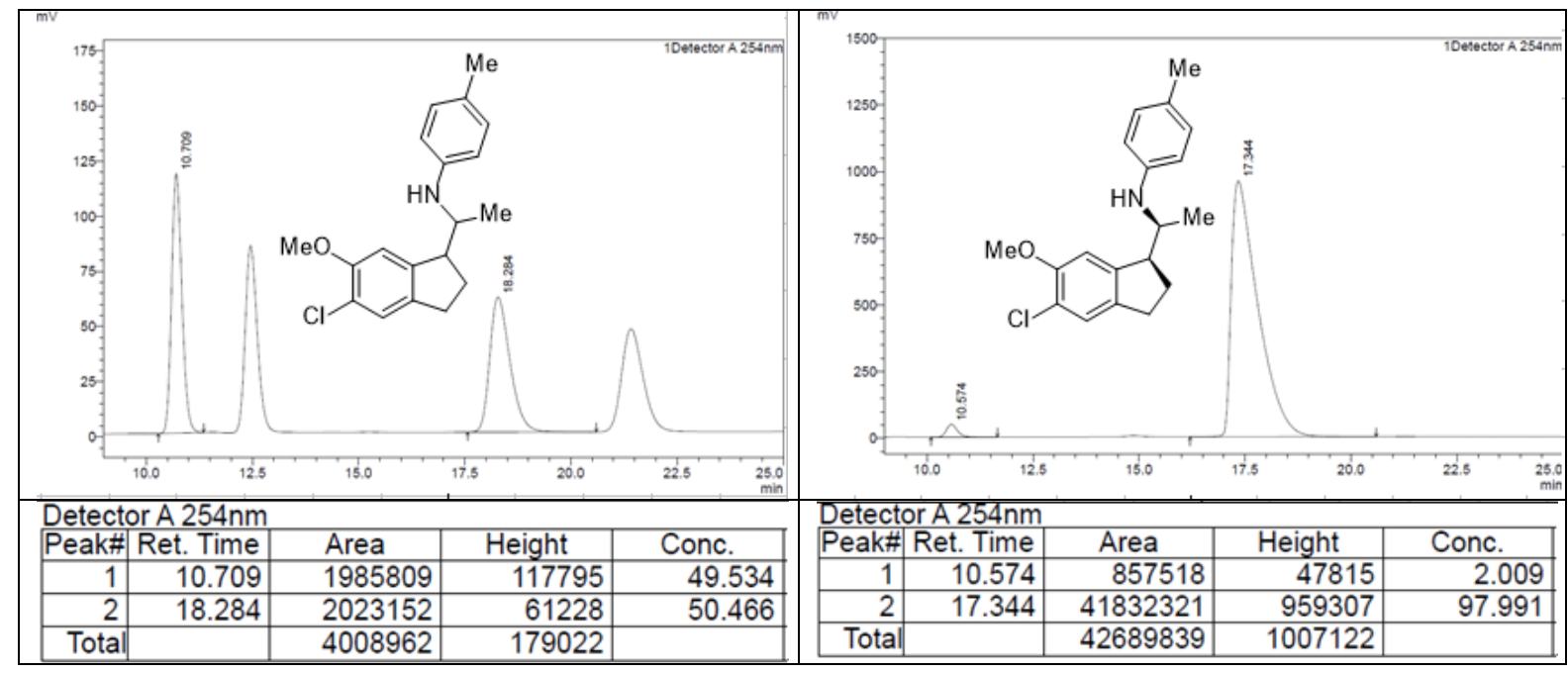

4-chloro-N-((S)-1-((S)-5-chloro-6-methoxy-2,3-dihydro-1H-inden-1-yl)ethyl)aniline (6j)

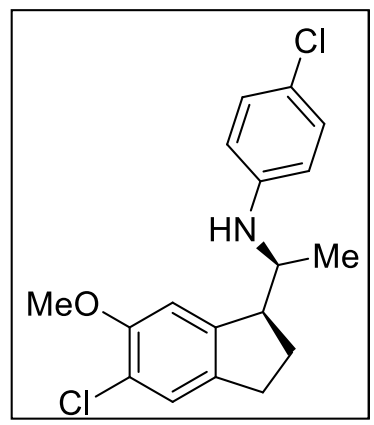

Colorless oil, $83 \%$ yield, $94: 6 \mathrm{dr} .{ }^{1} \mathbf{H}$ NMR $\left(500 \mathrm{MHz}, \mathrm{CDCl}_{3}\right): \delta$ $7.20(\mathrm{~s}, 1 \mathrm{H}), 7.12(\mathrm{~d}, J=8.7 \mathrm{~Hz}, 2 \mathrm{H}), 6.72(\mathrm{~s}, 1 \mathrm{H}), 6.55$ (d, $J=8.6$ $\mathrm{Hz}, 2 \mathrm{H}), 3.80(\mathrm{~s}, 3 \mathrm{H}), 3.76-3.71(\mathrm{~m}, 1 \mathrm{H}), 3.47$ (dt, $J=9.2,4.7$ $\mathrm{Hz}, 1 \mathrm{H}), 2.92$ (dt, $J=15.9,8.0 \mathrm{~Hz}, 1 \mathrm{H}), 2.80$ (ddd, $J=15.9,9.3$, $4.6 \mathrm{~Hz}, 1 \mathrm{H}), 2.31(\mathrm{dtd}, J=13.2,9.0,7.3 \mathrm{~Hz}, 1 \mathrm{H}), 1.96$ (ddt, $J=$ 13.2, 8.8, 4.6 Hz, 1H), 1.09 (d, $J=6.5 \mathrm{~Hz}, 3 \mathrm{H}) .{ }^{13} \mathbf{C}$ NMR $(126$ $\left.\mathrm{MHz}, \mathrm{CDCl}_{3}\right): \delta 153.6,145.7,143.0,137.7,129.2,125.9,122.0$, 121.3, 114.4, 109.0, 56.3, 51.7, 48.7, 30.8, 17.4. HRMS (ESI) m/z Calcd for $\left[\mathrm{C}_{18} \mathrm{H}_{20} \mathrm{Cl}_{2} \mathrm{NO}\right.$, $\mathrm{M}+\mathrm{H}]^{+}:$336.0916; Found: 336.0918. 
Optical Rotation: $[\alpha]^{23} \mathrm{D}=-96.9\left(0.4, \mathrm{CHCl}_{3}\right) .96 \%$ ee. (HPLC condition: Chiralcel AS-H column, $n$-hexane $/ i-\mathrm{PrOH}=99: 1$, flow rate $=1.0 \mathrm{ml} / \mathrm{min}$, wavelength $=254 \mathrm{~nm}, \mathrm{t}_{\mathrm{R}}=13.07$ $\min$ for minor isomer, $\mathrm{t}_{\mathrm{R}}=21.45$ min for major isomer).

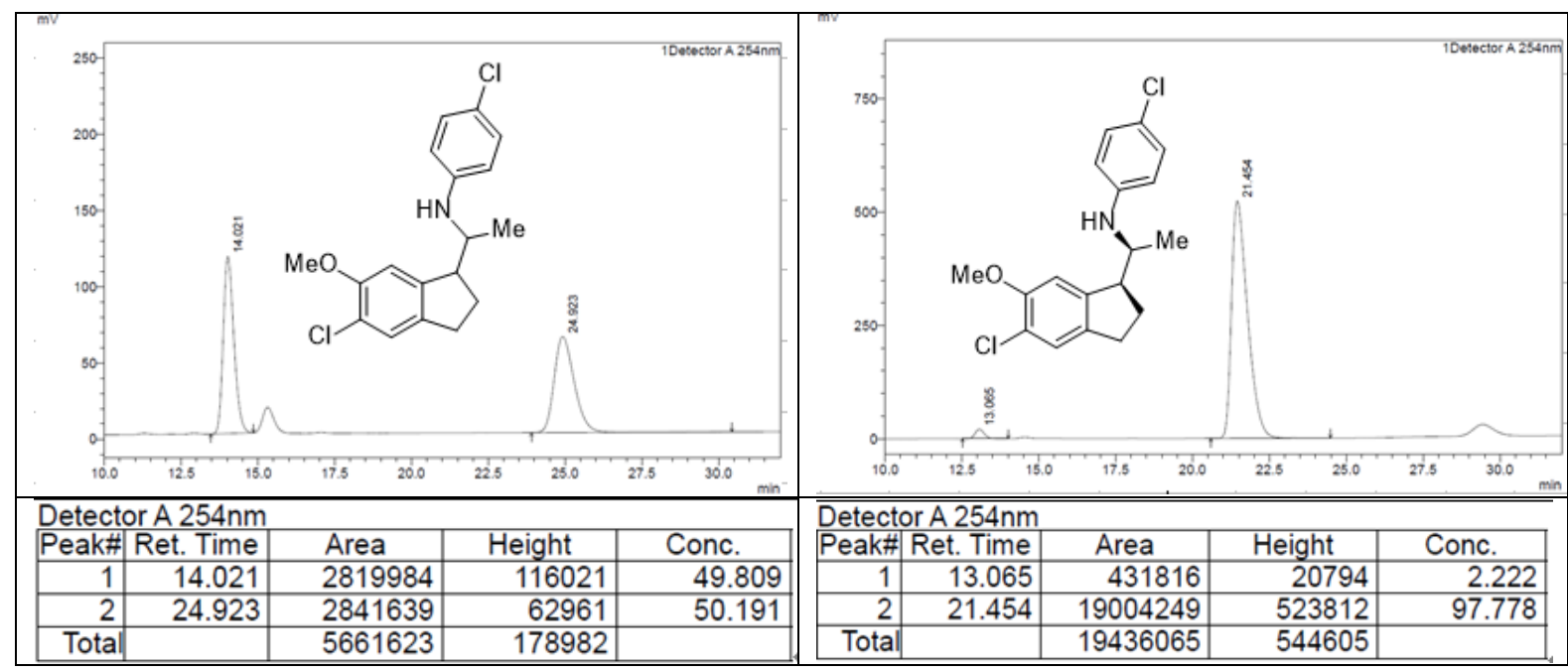

\section{$\underline{N-((S)-1-((S)-5-c h l o r 0-6-m e t h o x y-2,3-d i h y d r o-1 H-i n d e n-1-y l) e t h y l) b i p h e n y l-4-a m i n e ~(6 k) ~}$}

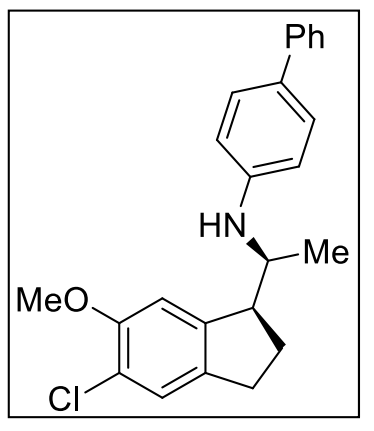

Colorless oil, $82 \%$ yield, $94: 6$ dr. ${ }^{1} \mathbf{H}$ NMR $\left(500 \mathrm{MHz}, \mathrm{CDCl}_{3}\right): \delta$

$7.57(\mathrm{~d}, J=7.6 \mathrm{~Hz}, 2 \mathrm{H}), 7.48(\mathrm{~d}, J=8.4 \mathrm{~Hz}, 2 \mathrm{H}), 7.41(\mathrm{t}, J=7.6$

$\mathrm{Hz}, 2 \mathrm{H}), 7.28(\mathrm{t}, J=7.3 \mathrm{~Hz}, 1 \mathrm{H}), 7.24(\mathrm{~s}, 1 \mathrm{H}), 6.81(\mathrm{~s}, 1 \mathrm{H}), 6.73(\mathrm{~d}$, $J=7.3 \mathrm{~Hz}, 2 \mathrm{H}), 3.94-3.78(\mathrm{~m}, 4 \mathrm{H}), 3.55(\mathrm{dt}, J=8.9,4.5 \mathrm{~Hz}, 1 \mathrm{H})$, $2.96(\mathrm{dt}, J=16.0,8.0 \mathrm{~Hz}, 1 \mathrm{H}), 2.87-2.77(\mathrm{~m}, 1 \mathrm{H}), 2.35$ (ddd, $J=$ $16.7,13.3,9.0 \mathrm{~Hz}, 1 \mathrm{H}), 2.02$ (ddt, $J=13.1,8.7,4.4 \mathrm{~Hz}, 1 \mathrm{H}$ ), 1.14 $(\mathrm{d}, J=6.5 \mathrm{~Hz}, 3 \mathrm{H}),{ }^{13} \mathbf{C}$ NMR $\left(126 \mathrm{MHz}, \mathrm{CDCl}_{3}\right): \delta 153.57$, $143.14,137.67,128.61,128.06,126.18,126.07,125.87,121.15,113.59,109.15,56.31,51.57$, 48.74, 30.83, 28.38, 17.40. HRMS (ESI) $\mathrm{m} / \mathrm{z}$ Calcd for $\left[\mathrm{C}_{24} \mathrm{H}_{25} \mathrm{ClNO}, \mathrm{M}+\mathrm{H}\right]^{+}$: 378.1619 ; Found: 378.1626.

Optical Rotation: $[\alpha]^{23}=+63.7\left(0.9, \mathrm{CHCl}_{3}\right) .97 \%$ ee. (HPLC condition: Chiralcel OD$\mathrm{H}$ column, $n$-hexane $/ i-\mathrm{PrOH}=99: 1$, flow rate $=1.0 \mathrm{ml} / \mathrm{min}$, wavelength $=254 \mathrm{~nm}, \mathrm{t}_{\mathrm{R}}=24.69$ $\min$ for minor isomer, $t_{R}=59.56$ min for major isomer). 


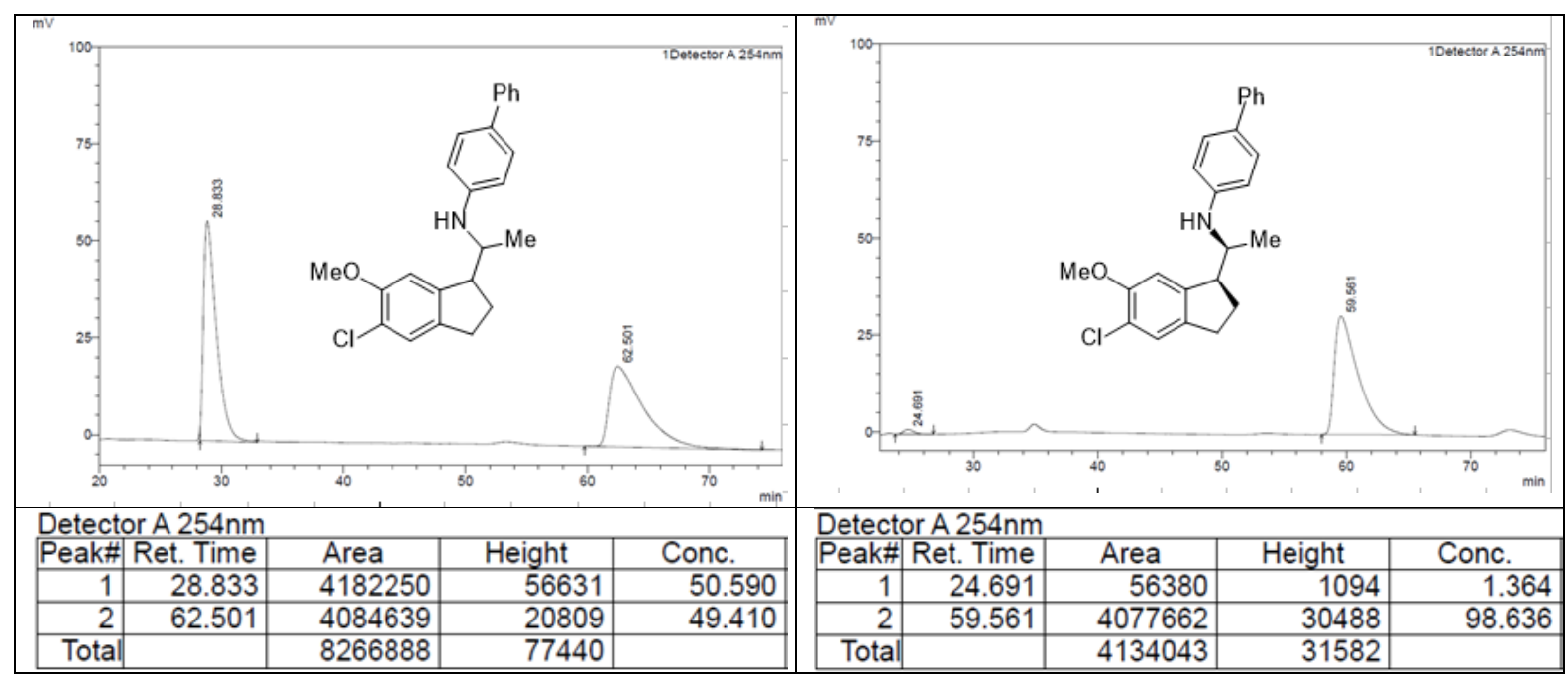

$\underline{N-((S)-1-((S)-5-c h l o r o-6-m e t h o x y-2,3-d i h y d r o-1 H-i n d e n-1-y l) e t h y l)-3-m e t h o x y a n i l i n e ~(61) ~}$

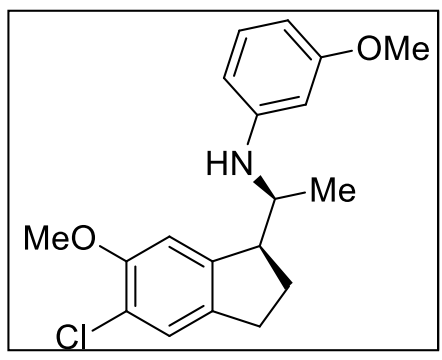

Colorless oil, $72 \%$ yield, 94:6 dr. ${ }^{1} \mathbf{H}$ NMR (500 MHz, $\left.\mathrm{CDCl}_{3}\right): \delta 7.20(\mathrm{~s}, 1 \mathrm{H}), 7.09(\mathrm{t}, J=8.1 \mathrm{~Hz}, 1 \mathrm{H}), 6.77(\mathrm{~s}, 1 \mathrm{H})$, $6.26(\mathrm{ddd}, J=16.0,8.1,2.3 \mathrm{~Hz}, 2 \mathrm{H}), 6.20(\mathrm{t}, J=2.3 \mathrm{~Hz}, 1 \mathrm{H})$, $3.82(\mathrm{~s}, 3 \mathrm{H}), 3.78(\mathrm{~s}, 3 \mathrm{H}), 3.77-3.73(\mathrm{~m}, 1 \mathrm{H}), 3.51(\mathrm{dt}, J=$ 9.0, 4.6 Hz, 1H), $2.93(\mathrm{dt}, J=16.0,8.1 \mathrm{~Hz}, 1 \mathrm{H}), 2.85-2.71$ $(\mathrm{m}, 1 \mathrm{H}), 2.31(\mathrm{dtd}, J=13.2,9.1,7.5 \mathrm{~Hz}, 1 \mathrm{H}), 1.97(\mathrm{ddt}, J=$ 13.1, 8.7, 4.4 Hz, 1H), 1.09 (d, $J=6.5 \mathrm{~Hz}, 3 \mathrm{H}) .{ }^{13} \mathbf{C ~ N M R}\left(126 \mathrm{MHz}, \mathrm{CDCl}_{3}\right): \delta 161.0,153.5$, $148.5,143.2$, 137.7, 130.1, 125.8, 121.1, 109.2, 106.6, 102.3, 99.5, 56.3, 55.1, 51.6, 48.7, 30.9, 28.4, 17.3 HRMS (ESI) $\mathrm{m} / \mathrm{z}$ Calcd for $\left[\mathrm{C}_{19} \mathrm{H}_{23} \mathrm{ClNO}_{2}, \mathrm{M}+\mathrm{H}\right]^{+}$: 332.1412 ; Found: 332.1413.

Optical Rotation: $[\alpha]_{\mathrm{D}}^{24}=+72.5\left(0.3, \mathrm{CHCl}_{3}\right) .98 \%$ ee. (HPLC condition: Chiralcel OD$\mathrm{H}$ column, $n$-hexane $/ i$-PrOH $=91: 10$, flow rate $=1.0 \mathrm{ml} / \mathrm{min}$, wavelength $=254 \mathrm{~nm}, \mathrm{t}_{\mathrm{R}}=14.14$ min for minor isomer, $\mathrm{t}_{\mathrm{R}}=18.16 \mathrm{~min}$ for major isomer).

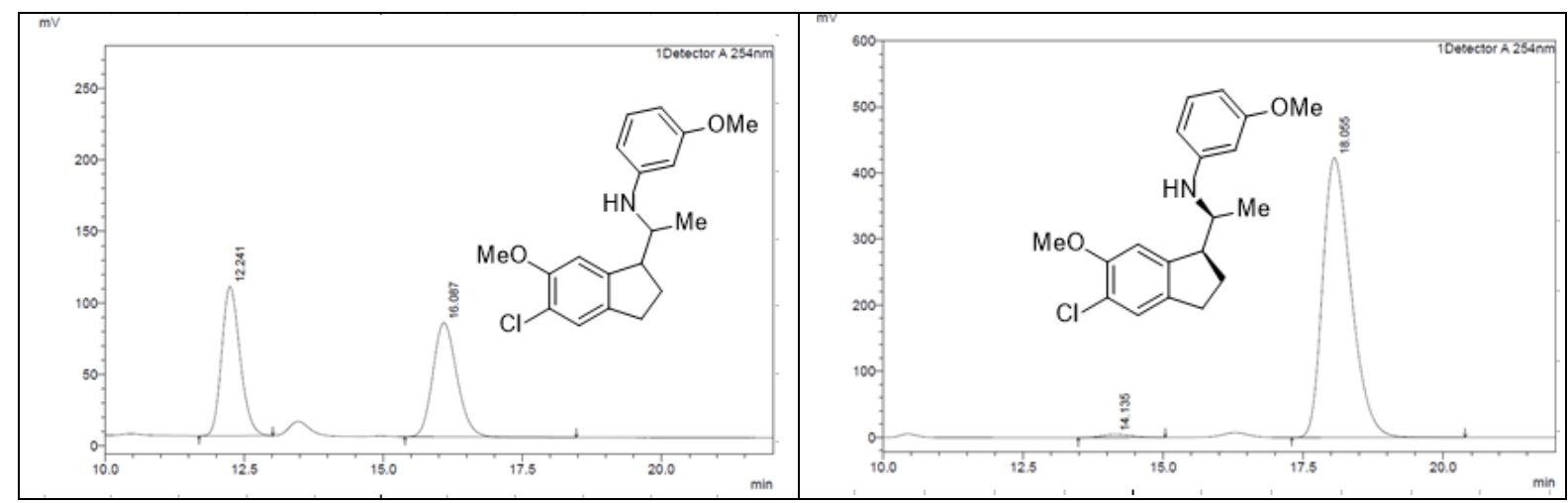




\begin{tabular}{|r|r|r|r|r||}
\hline \multicolumn{5}{|c|}{ Detector A 254nm } \\
\hline Peak\# & Ret. Time & \multicolumn{1}{l|}{ Area } & \multicolumn{1}{c|}{ Height } & \multicolumn{1}{c|}{ Conc. } \\
\hline 1 & 12.241 & 2408236 & 104528 & 50.026 \\
\hline 2 & 16.087 & 2405693 & 79868 & 49.974 \\
\hline Total & & 4813929 & 184396 & \\
\hline
\end{tabular}

\begin{tabular}{|c|c|c|c|c|}
\hline \multirow{2}{*}{\multicolumn{5}{|c|}{ Detector A 254nm }} \\
\hline & Ret. Time & Area & Height & Conc. \\
\hline 1 & 14.135 & 169381 & 4888 & 1.122 \\
\hline 2 & 18.055 & 14922901 & 423095 & 98.878 \\
\hline Total & & 15092282 & 427983 & \\
\hline
\end{tabular}




\section{General procedure for the deprotection of $6 h^{4}$}

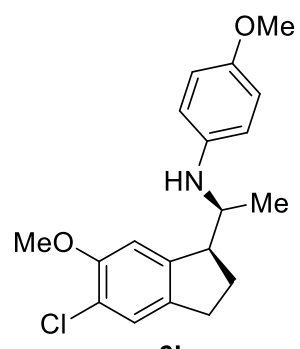

$6 \mathrm{~h}$
$\underset{\mathrm{MeCN} / \mathrm{H}_{2} \mathrm{O}(1: 1, \mathrm{v} / \mathrm{v})}{\stackrel{\mathrm{H}_{5} \mathrm{IO}_{6}(1.5 \text { equiv. })}{\longrightarrow}}$

$61 \%$<smiles>COc1cc2c(cc1Cl)CCC2[C@H](C)N</smiles>

7

To a solution of $N$-((S)-1-((S)-5-chloro-6-methoxy-2,3-dihydro-1H-inden-1-yl)ethyl)4-methoxyaniline 6h (116 mg, 96\% ee, $0.35 \mathrm{mmol})$ in $\mathrm{MeCN} / \mathrm{H}_{2} \mathrm{O}(10 \mathrm{~mL}, 1: 1$, v/v) was added $\mathrm{H}_{5} \mathrm{IO}_{6}(120 \mathrm{mg}, 1.5 \mathrm{eq})$ and $1 \mathrm{M}$ aqueous solution of $\mathrm{H}_{2} \mathrm{SO}_{4}(0.5 \mathrm{~mL})$. The mixture was stirred for $14 \mathrm{~h}$ at room temperature, and then washed with $\mathrm{CH}_{2} \mathrm{Cl}_{2}(4 \times$ $50 \mathrm{~mL}$ ). The organic phase was discarded and $5 \mathrm{M}$ aqueous $\mathrm{KOH}$ solution was added dropwise to the resulting colorless aqueous phase until pH 11 was reached. The basic aqueous solution was extracted with EtOAc $(4 \times 60 \mathrm{~mL})$. The combined organic layer was dried over $\mathrm{Na}_{2} \mathrm{SO}_{4}$, and concentrated to afford the 7 (48 $\mathrm{mg}, 61 \%$ yield) as slightly brown oil.

\section{$\underline{(S)-1-((S)-5-c h l o r o-6-m e t h o x y-2,3-d i h y d r o-1 H-i n d e n-1-y l) e t h a n a m i n e ~(7) ~}$}

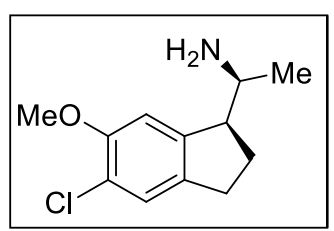

Colorless oil, $61 \%$ yield, ${ }^{1} \mathbf{H}$ NMR $\left(500 \mathrm{MHz}, \mathrm{CDCl}_{3}\right): \delta 7.18(\mathrm{~s}$, $1 \mathrm{H}), 6.81(\mathrm{~s}, 1 \mathrm{H}), 3.87(\mathrm{~s}, 3 \mathrm{H}), 3.41(\mathrm{qd}, J=6.5,3.8 \mathrm{~Hz}, 1 \mathrm{H})$, $3.15(\mathrm{td}, J=7.7,3.7 \mathrm{~Hz}, 1 \mathrm{H}), 2.89-2.74(\mathrm{~m}, 2 \mathrm{H}), 2.10(\mathrm{dtd}, J$ $=12.8,8.6,5.4 \mathrm{~Hz}, 1 \mathrm{H}), 1.99(\mathrm{ddd}, J=13.0,9.0,6.6 \mathrm{~Hz}, 1 \mathrm{H}), 1.13(\mathrm{~d}, J=6.6 \mathrm{~Hz}$, 3H). ${ }^{13} \mathbf{C}$ NMR (126 MHz, $\left.\mathrm{CDCl}_{3}\right): \delta 153.7,144.3,137.6,125.9,120.8,107.9,56.3$, 52.1, 48.6, 30.8, 25.2, 21.1. HRMS (ESI) $\mathrm{m} / \mathrm{z}$ Calcd for $\left[\mathrm{C}_{12} \mathrm{H}_{16} \mathrm{ClNO}, \mathrm{M}+\mathrm{H}\right]^{+}$: 226.0993; Found: 226.0992. Optical Rotation: $[\alpha]^{23} \mathrm{D}=+36.3\left(0.4, \mathrm{CHCl}_{3}\right)$. 


\section{Analytical data of acyclic products under microwave conditions.}

\section{$\underline{N-((2 S, 3 S)-3-p h e n y l b u t a n-2-y l) a n i l i n e ~(9 a)}$.}

Colorless oil, $82 \%$ yield, 97:3 dr. ${ }^{1} \mathbf{H}$ NMR (500 MHz, Chloroform-d) $\delta 7.33$ (t,<smiles>CC(Nc1ccccc1)C(C)c1ccccc1</smiles>
$J=7.4 \mathrm{~Hz}, 2 \mathrm{H}), 7.28-7.16(\mathrm{~m}, 5 \mathrm{H}), 6.71(\mathrm{tt}, J=7.2,1.0 \mathrm{~Hz}, 1 \mathrm{H}), 6.66-6.56$ (m, 2H), 3.69 (p, $J=6.4 \mathrm{~Hz}, 1 \mathrm{H}), 3.43(\mathrm{~s}, 1 \mathrm{H}), 3.14-2.93(\mathrm{~m}, 1 \mathrm{H}), 1.40(\mathrm{~d}, J$ $=7.2 \mathrm{~Hz}, 3 \mathrm{H}), 1.00(\mathrm{~d}, J=6.5 \mathrm{~Hz}, 3 \mathrm{H}) .{ }^{13} \mathbf{C} \mathbf{~ N M R}\left(126 \mathrm{MHz}, \mathrm{CDCl}_{3}\right) \delta 147.44$, $142.96,129.34,128.32,128.09,126.36,116.94,113.29,53.25,43.77,18.09$,

17.24 .

Optical Rotation: $[\alpha]^{23}{ }_{\mathrm{D}}=-69.8\left(0.4, \mathrm{CHCl}_{3}\right) .98 \%$ ee. (HPLC condition: Chiralcel OD-H column, $n$-hexane $/ i-\mathrm{PrOH}=99: 1$, flow rate $=0.5 \mathrm{ml} / \mathrm{min}$, wavelength $=254 \mathrm{~nm}, \mathrm{t}_{\mathrm{R}}=$ $12.94 \mathrm{~min}$ for minor isomer, $\mathrm{t}_{\mathrm{R}}=13.92 \mathrm{~min}$ for major isomer).

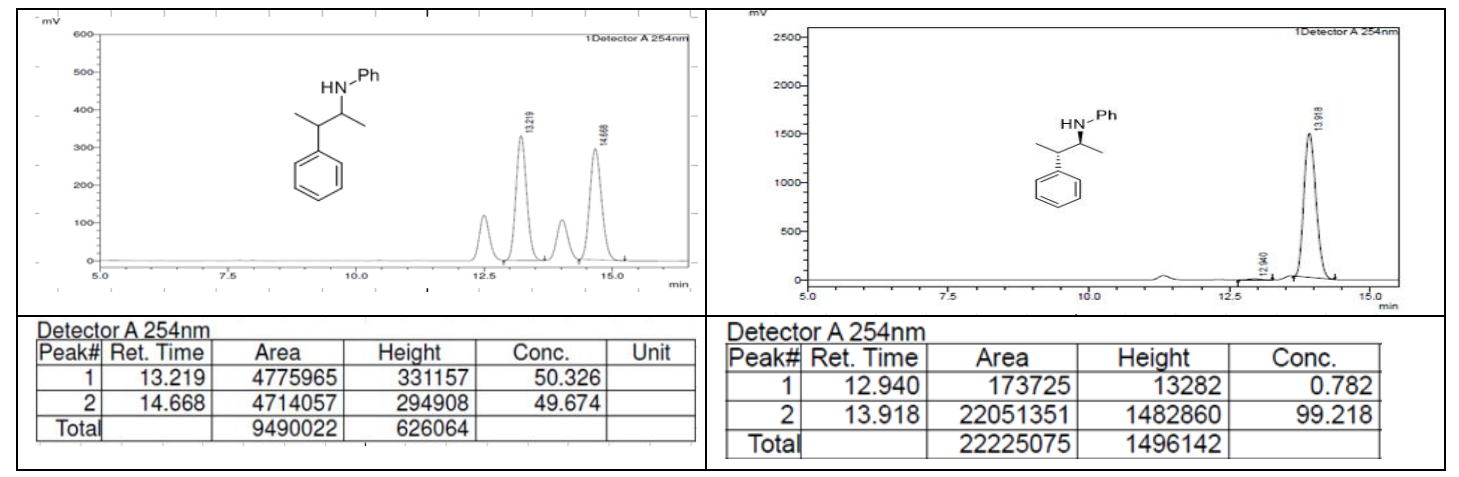

\section{$\underline{N-((2 S, 3 S)-3-(4-c h l o r o p h e n y l) b u t a n-2-y l) a n i l i n e ~(9 b)}$}

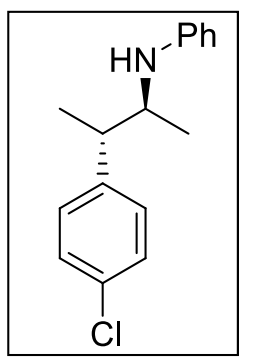

Colorless oil, $83 \%$ yield, 96:4 dr. ${ }^{1} \mathbf{H}$ NMR (500 MHz, Chloroform-d) $\delta$ $7.28(\mathrm{dd}, J=8.5,2.1 \mathrm{~Hz}, 2 \mathrm{H}), 7.20(\mathrm{ddd}, J=8.7,7.3,2.2 \mathrm{~Hz}, 2 \mathrm{H}), 7.11(\mathrm{dd}$, $J=8.5,2.1 \mathrm{~Hz}, 2 \mathrm{H}), 6.70(\mathrm{t}, J=7.3 \mathrm{~Hz}, 1 \mathrm{H}), 6.59$ (d, $J=7.8 \mathrm{~Hz}, 2 \mathrm{H}), 3.66$ (p, $J=6.5 \mathrm{~Hz}, 1 \mathrm{H}), 3.36$ (s, 1H), 3.03 (p, $J=7.2 \mathrm{~Hz}, 1 \mathrm{H}), 1.36$ (d, $J=7.2$ $\mathrm{Hz}, 3 \mathrm{H}), 0.98(\mathrm{~d}, J=6.5 \mathrm{~Hz}, 3 \mathrm{H}) \cdot{ }^{13} \mathbf{C}$ NMR $\left(126 \mathrm{MHz}, \mathrm{CDCl}_{3}\right) \delta 147.21$, 141.37 , 132.10, 129.64, 129.40, 128.18, 117.15, 113.29, 53.12, 42.93,

$17.83,17.11$.

Optical Rotation: $[\alpha]_{\mathrm{D}}^{23}=-97.3\left(0.4, \mathrm{CHCl}_{3}\right) .98 \%$ ee. (HPLC condition: Chiralcel OJ-H column, $n$-hexane $/ i-\mathrm{PrOH}=95: 5$, flow rate $=1.0 \mathrm{ml} / \mathrm{min}$, wavelength $=254 \mathrm{~nm}, \mathrm{t}_{\mathrm{R}}=13.47$ $\min$ for minor isomer, $t_{R}=16.69 \min$ for major isomer). 


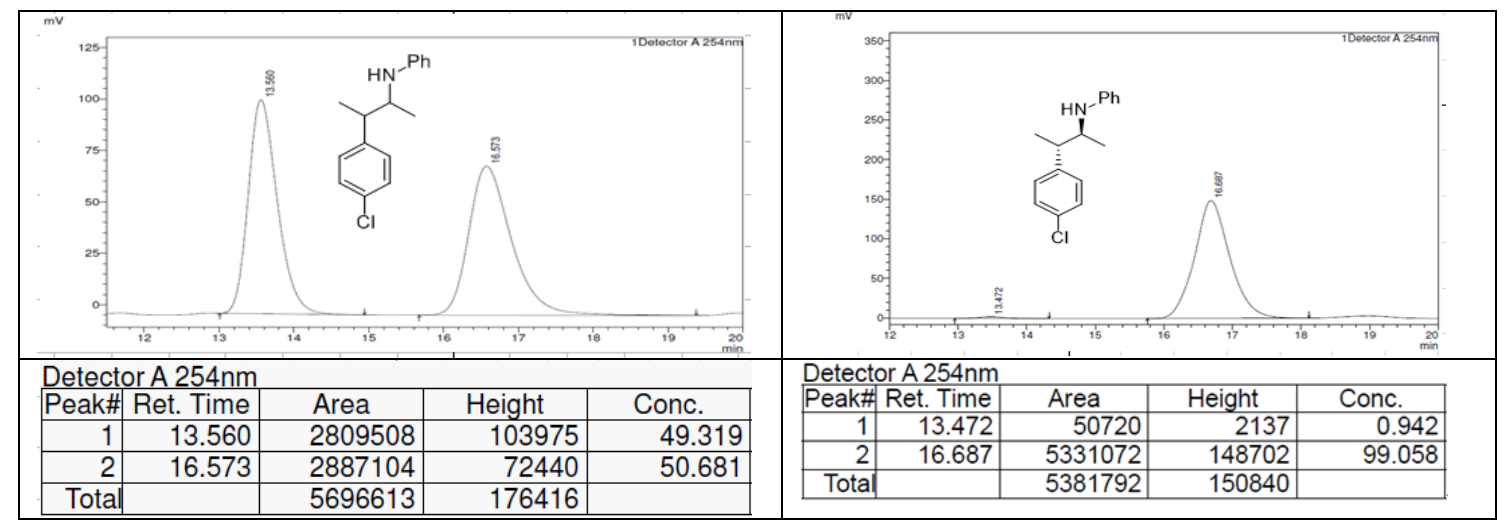

$\underline{N-((2 S, 3 S)-3-p h e n y l h e x a n-2-y l) \text { aniline }(9 \mathrm{c})}$

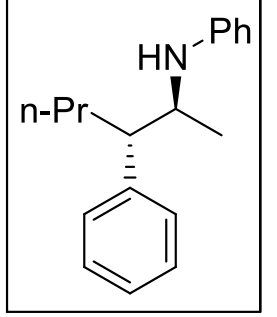

Colorless oil, 60\% yield, 97:3 dr. ${ }^{1} \mathbf{H}$ NMR (500 MHz, Chloroform-d) $\delta$ $7.35(\mathrm{t}, J=7.5 \mathrm{~Hz}, 1 \mathrm{H}), 7.28(\mathrm{~d}, J=6.1 \mathrm{~Hz}, 0 \mathrm{H}), 7.26-7.21(\mathrm{~m}, 1 \mathrm{H})$, $7.20-7.15(\mathrm{~m}, 1 \mathrm{H}), 6.73(\mathrm{td}, J=7.3,1.2 \mathrm{~Hz}, 1 \mathrm{H}), 6.68-6.58(\mathrm{~m}, 1 \mathrm{H})$, $3.77(\mathrm{p}, J=6.3 \mathrm{~Hz}, 1 \mathrm{H}), 3.41(\mathrm{~s}, 1 \mathrm{H}), 2.90(\mathrm{dt}, J=10.5,5.3 \mathrm{~Hz}, 1 \mathrm{H})$, $1.97-1.68(\mathrm{~m}, 1 \mathrm{H}), 1.32-1.23(\mathrm{~m}, 2 \mathrm{H}), 1.02(\mathrm{~d}, J=6.5 \mathrm{~Hz}, 2 \mathrm{H}), 0.92(\mathrm{t}$, $J=7.3 \mathrm{~Hz}, 2 \mathrm{H}) .{ }^{13} \mathbf{C}$ NMR $(126 \mathrm{MHz}, \mathrm{CDCl} 3) \delta 147.41,141.26,129.36$, 129.05, 128.04, 126.36, 116.94, 113.34, 52.20, 49.65, 34.54, 20.98, 17.21, 14.19.

Optical Rotation: $[\alpha]^{24}=-76.8\left(0.3, \mathrm{CHCl}_{3}\right) .94 \%$ ee. (HPLC condition: Chiralcel OD$\mathrm{H}$ column, $n$-hexane $/ i-\mathrm{PrOH}=99: 1$, flow rate $=0.5 \mathrm{ml} / \mathrm{min}$, wavelength $=254 \mathrm{~nm}, \mathrm{t}_{\mathrm{R}}=10.74$ $\min$ for minor isomer, $t_{R}=11.21$ min for major isomer).

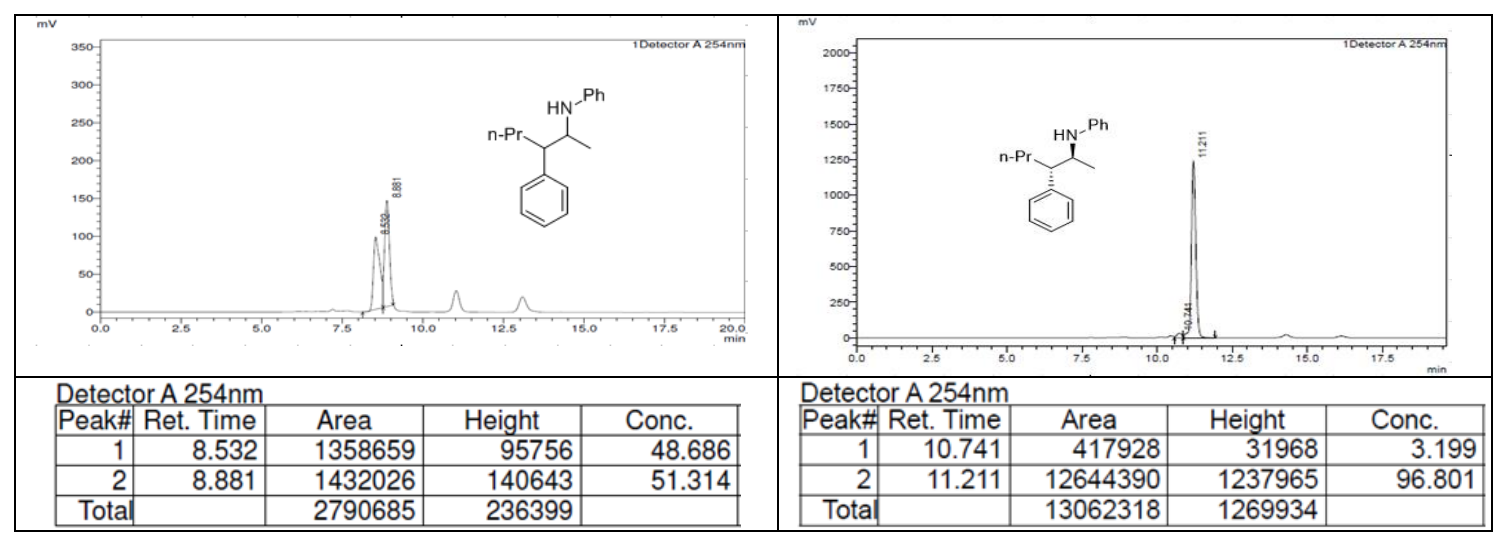

\section{$\underline{N-((2 S, 3 R)-3-(t e r t-b u t y l d i m e t h y l s i l y l o x y) b u t a n-2-y l) a n i l i n e ~(9 d)}$}

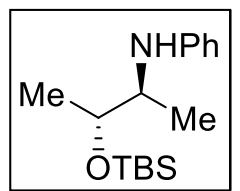

Colorless oil, $61 \%$ yield, >98:2 dr. ${ }^{1} \mathbf{H}$ NMR (500 MHz, Chloroform-d) $\delta$ $7.22-7.13(\mathrm{~m}, 2 \mathrm{H}), 6.68(\mathrm{td}, J=7.3,1.1 \mathrm{~Hz}, 1 \mathrm{H}), 6.64-6.52(\mathrm{~m}, 2 \mathrm{H})$, $4.06-3.95(\mathrm{~m}, 1 \mathrm{H}), 3.80(\mathrm{~s}, 1 \mathrm{H}), 3.39(\mathrm{qd}, J=6.4,3.0 \mathrm{~Hz}, 1 \mathrm{H}), 1.18(\mathrm{~d}, J$ $=6.3 \mathrm{~Hz}, 3 \mathrm{H}), 1.14(\mathrm{~d}, J=6.4 \mathrm{~Hz}, 3 \mathrm{H}), 0.94(\mathrm{~s}, 9 \mathrm{H}), 0.05(\mathrm{~d}, J=9.5 \mathrm{~Hz}$, 6H). ${ }^{13} \mathrm{C}$ NMR $\left(126 \mathrm{MHz}, \mathrm{CDCl}_{3}\right) \delta 147.29,129.23,116.94,113.43,77.25,77.00,76.75$, $69.90,53.50,20.80,18.05,13.55,-4.24,-4.86$. 
Optical Rotation: $[\alpha]^{24}=5.5\left(0.6, \mathrm{CHCl}_{3}\right) .94 \%$ ee. (HPLC condition: Chiralcel OD-H column, $n$-hexane $/ i-\mathrm{PrOH}=99: 1$, flow rate $=0.5 \mathrm{ml} / \mathrm{min}$, wavelength $=254 \mathrm{~nm}, \mathrm{t}_{\mathrm{R}}=7.77$ or $8.14 \mathrm{~min}$ for minor isomer, $\mathrm{t}_{\mathrm{R}}=8.29$ or $8.73 \mathrm{~min}$ for major isomer).

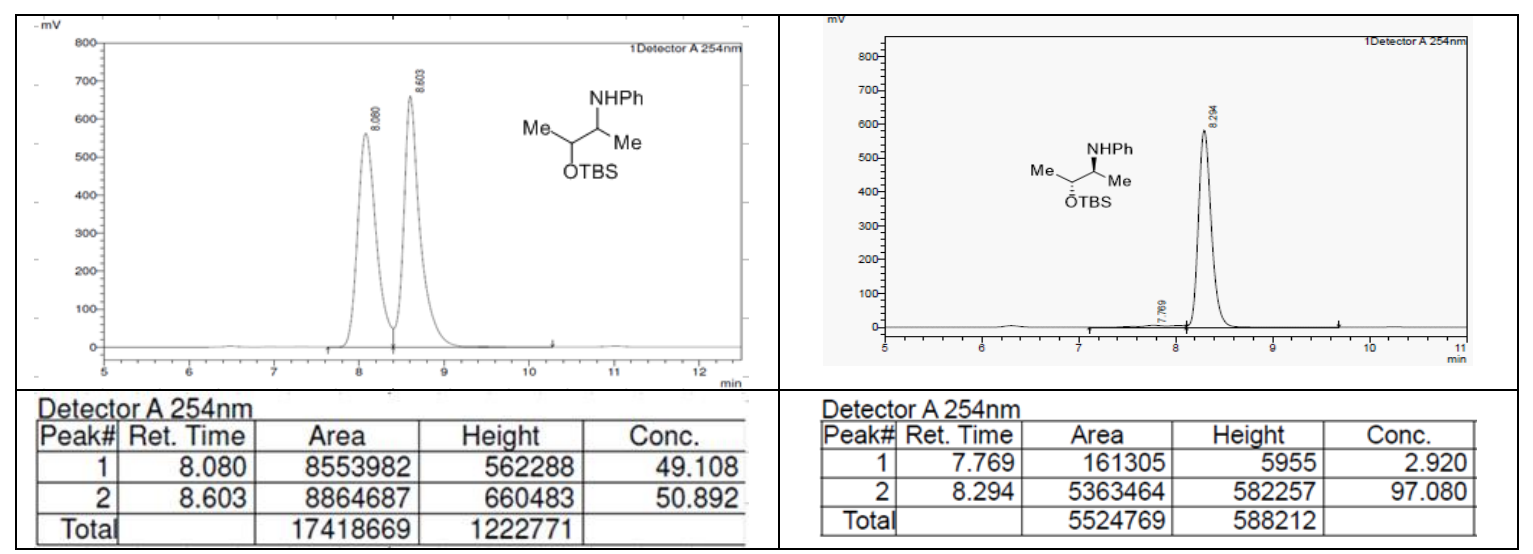




\section{X-ray crystallographic analysis and determination of configuration of $6 \mathrm{f}(\mathrm{HCl}$ salt $)$.}

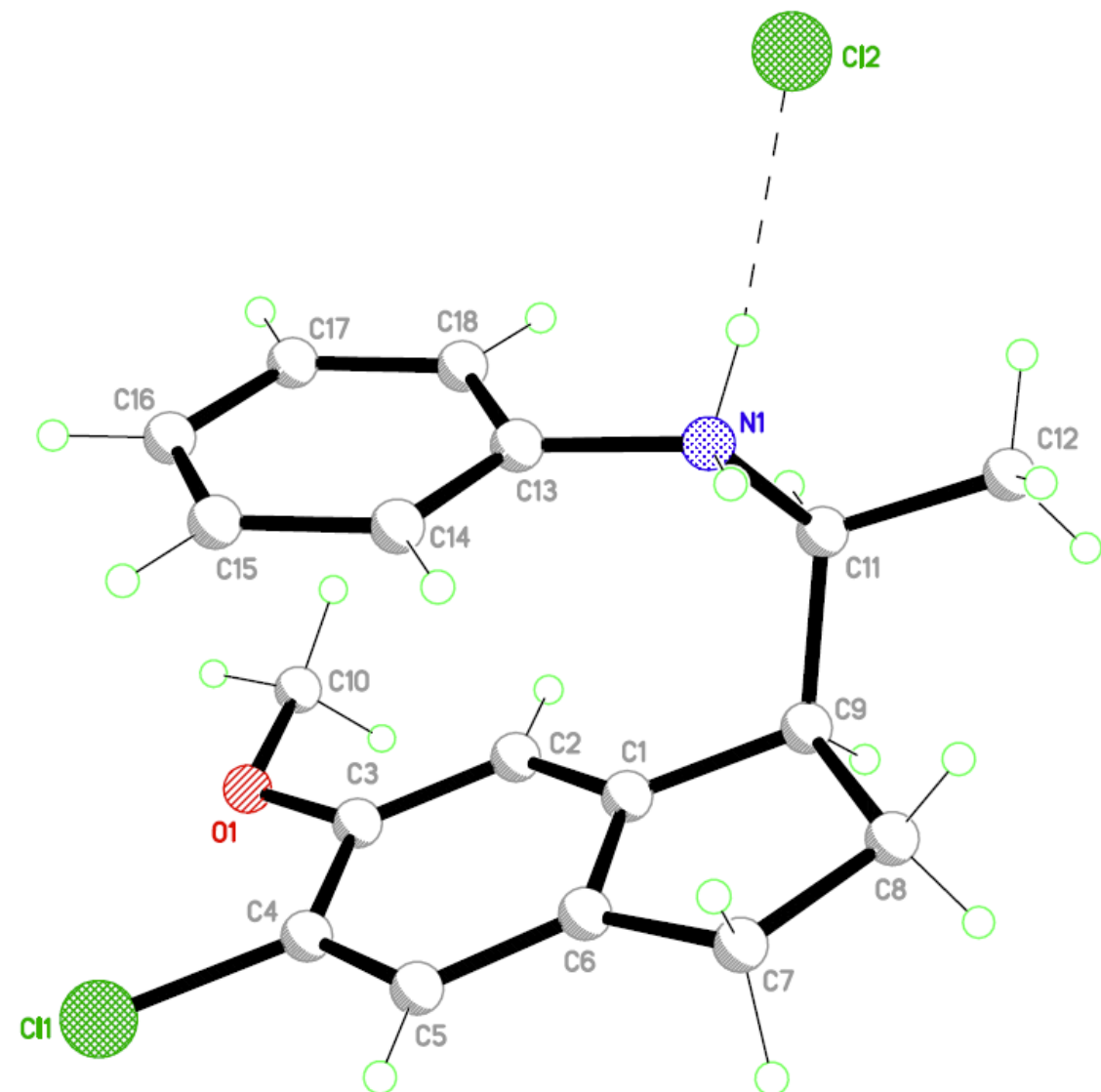

Table S2. Crystal data and structure refinement for f532.

Identification code

Empirical formula

Formula weight

Temperature

Wavelength

Crystal system

Space group

Unit cell dimensions

Volume

$\mathrm{Z}$

Density (calculated)

Absorption coefficient f532

$\mathrm{C} 18 \mathrm{H} 21 \mathrm{Cl} 2 \mathrm{~N} \mathrm{O}$

338.26

100(2) K

$0.71073 \AA$

Monoclinic

P21

$\mathrm{a}=7.6233(4) \AA \quad \alpha=90^{\circ}$.

$\mathrm{b}=7.8881(4) \AA \quad \beta=91.9018(16)^{\circ}$.

$\mathrm{c}=13.5115(7) \AA \quad \gamma=90^{\circ}$.

812.04(7) $\AA^{3}$

2

$1.383 \mathrm{Mg} / \mathrm{m}^{3}$

$0.401 \mathrm{~mm}^{-1}$ 
$\mathrm{F}(000)$

Crystal size

Theta range for data collection

Index ranges

Reflections collected

Independent reflections

Completeness to theta $=25.242^{\circ}$

Absorption correction

Max. and min. transmission

Refinement method

Data / restraints / parameters

Goodness-of-fit on $\mathrm{F}^{2}$

Final $\mathrm{R}$ indices [I>2sigma(I)]

$\mathrm{R}$ indices (all data)

Absolute structure parameter

Extinction coefficient

Largest diff. peak and hole
356

$0.539 \times 0.466 \times 0.162 \mathrm{~mm}^{3}$

2.673 to $28.277^{\circ}$.

$-9<=\mathrm{h}<=10,-10<=\mathrm{k}<=10,-17<=\mathrm{l}<=18$

20317

$4039[\mathrm{R}($ int $)=0.0497]$

$99.9 \%$

Semi-empirical from equivalents

0.7459 and 0.6439

Full-matrix least-squares on $\mathrm{F}^{2}$

4039 / 1 / 209

1.065

$\mathrm{R} 1=0.0247, \mathrm{wR} 2=0.0644$

$\mathrm{R} 1=0.0259, \mathrm{wR} 2=0.0652$

$-0.013(18)$

$\mathrm{n} / \mathrm{a}$

0.257 and -0.234 e. $\AA^{-3}$ 
9. NMR Spectra of Substrates.

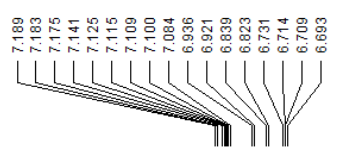

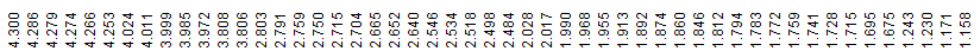

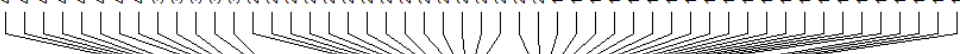

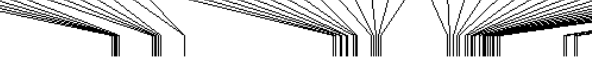

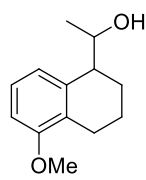

$1 \mathrm{~b}$
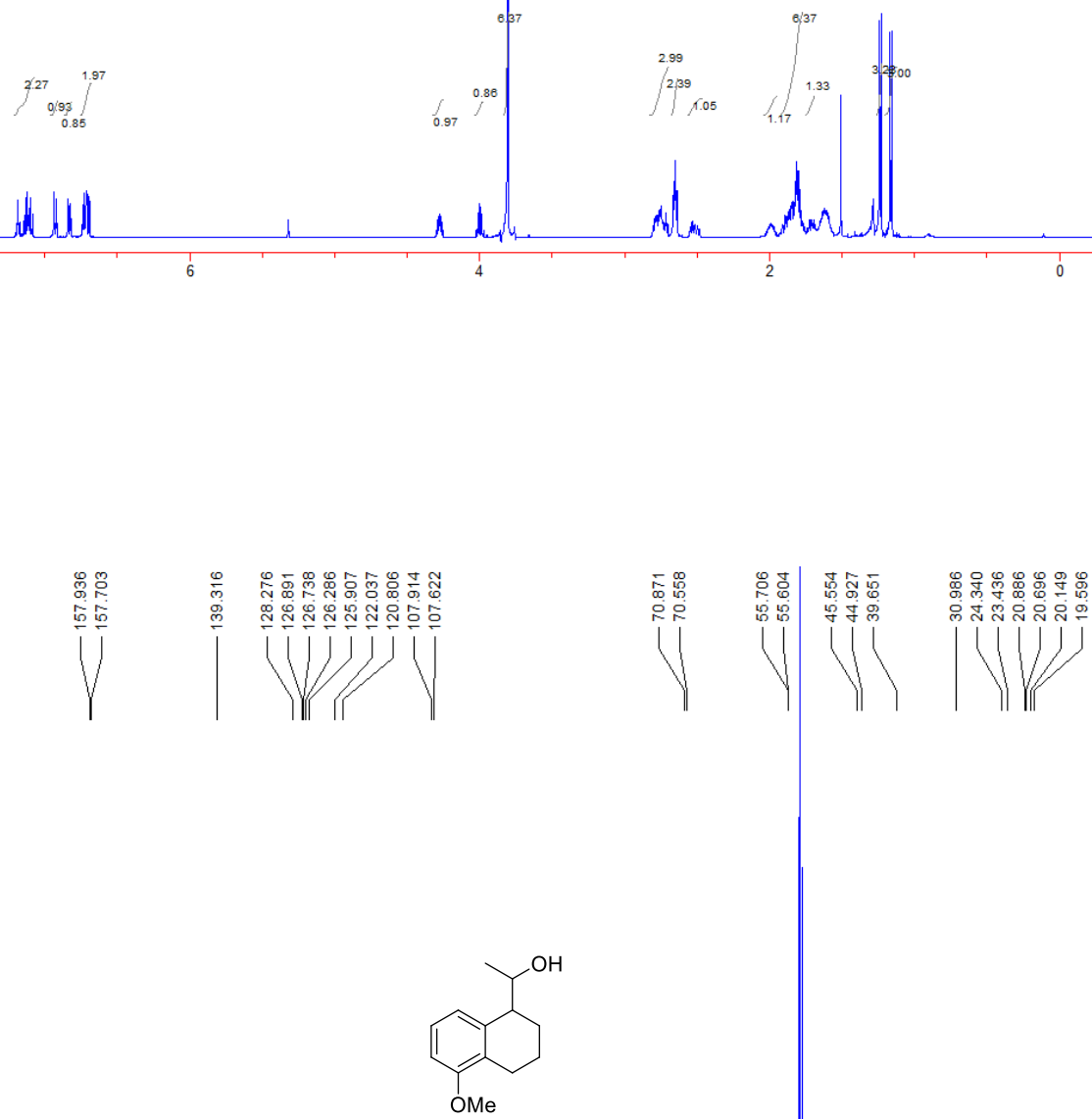

$1 \mathbf{b}$ 


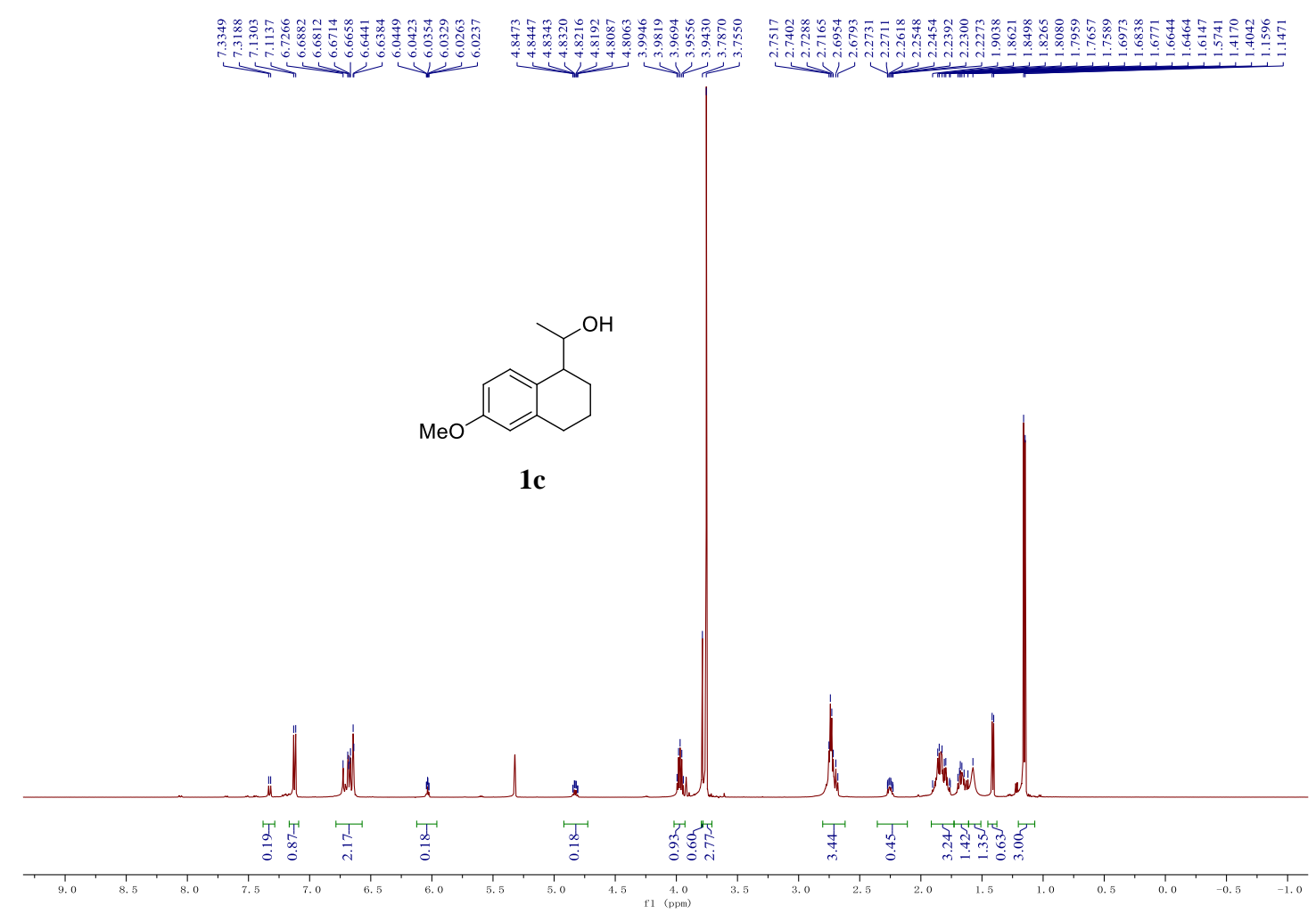

6-OMe-CD2C12/4

$13 \mathrm{C}$ AMX500
rzq-6 $-5-500 \mathrm{C}$

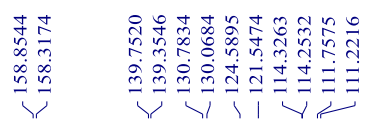

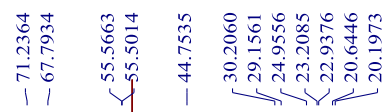

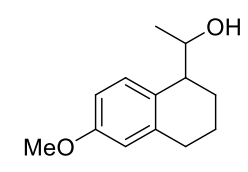

1c

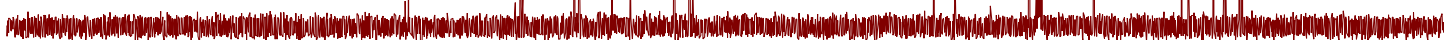




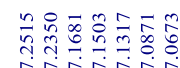

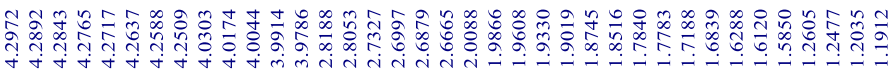 \\ inising

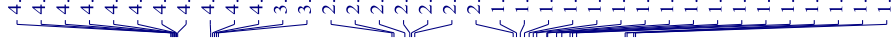

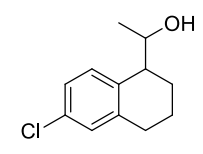

1d
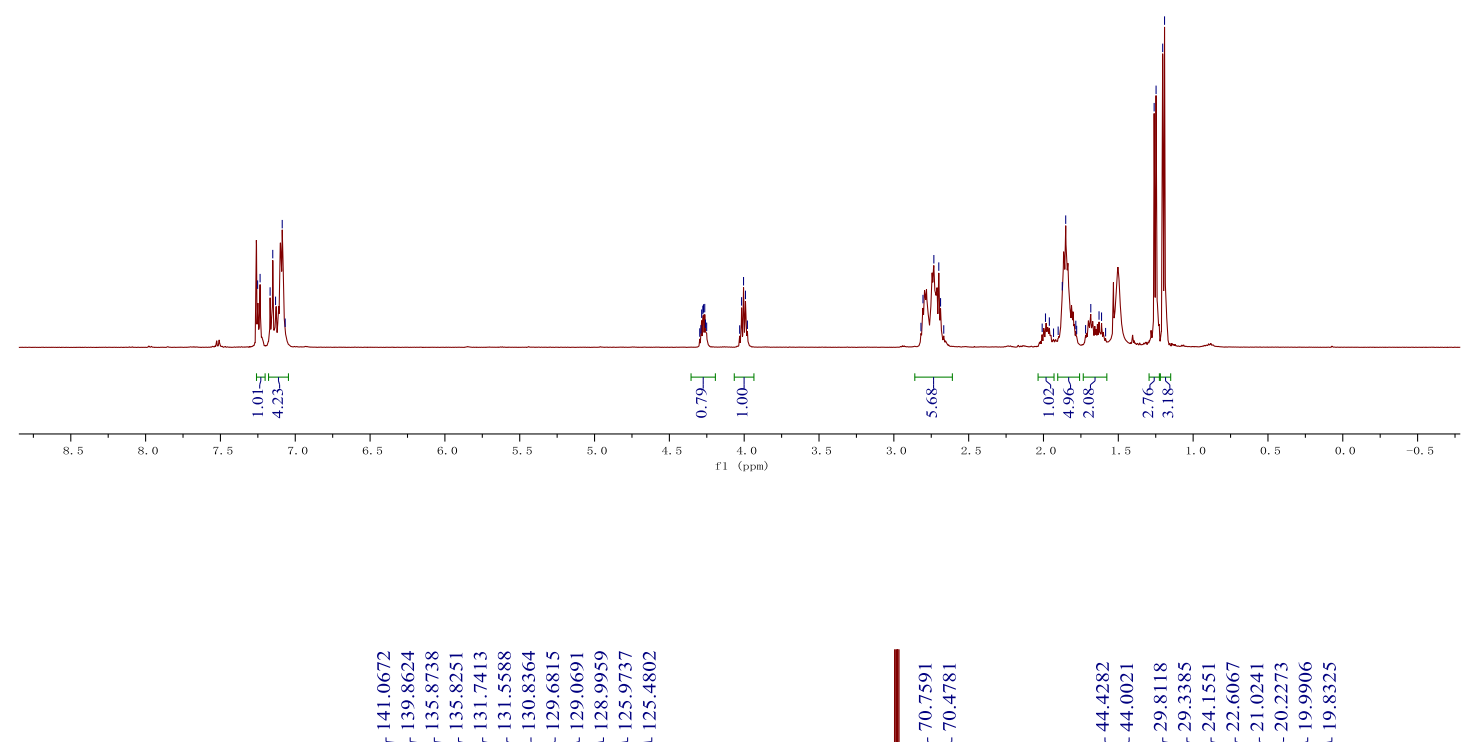

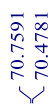

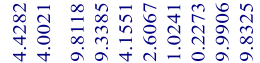

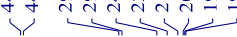
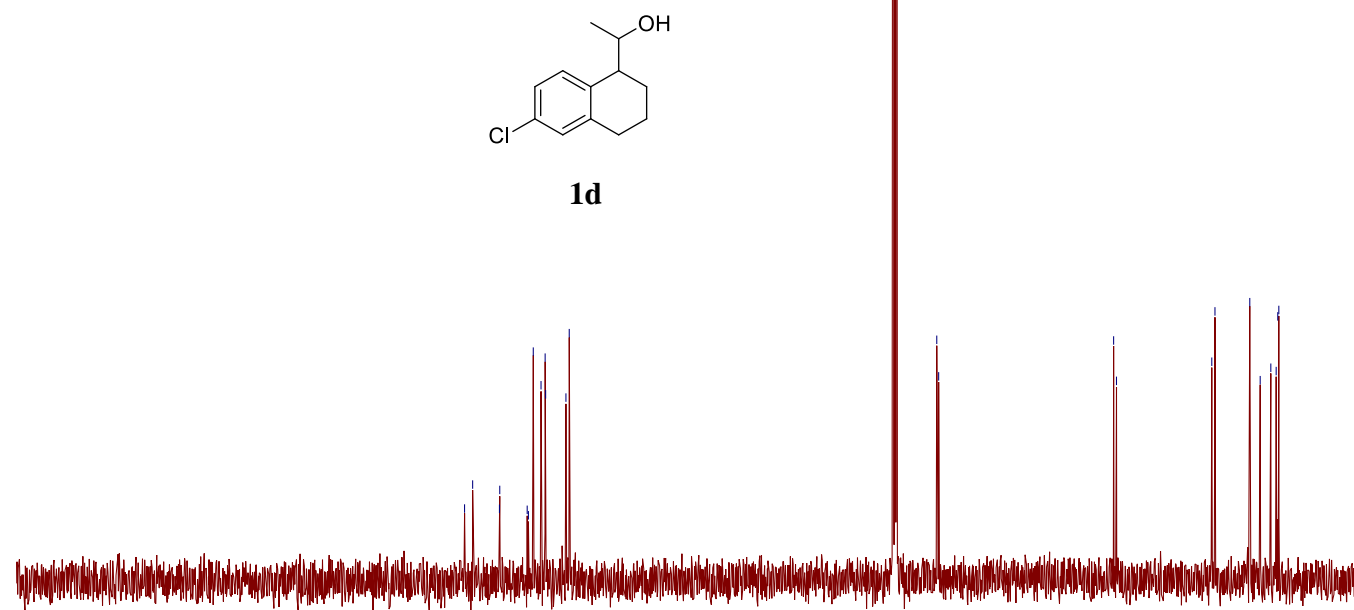

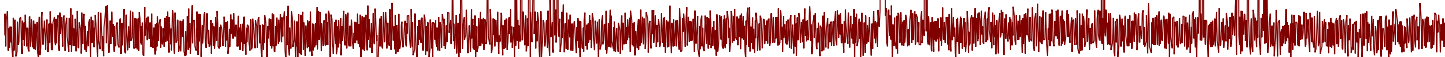

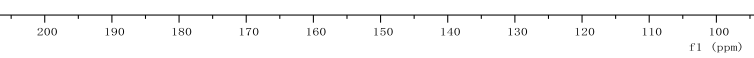




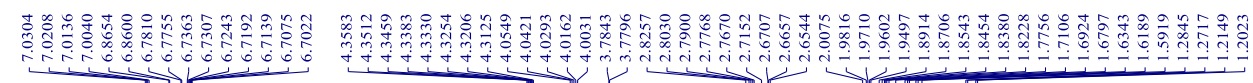
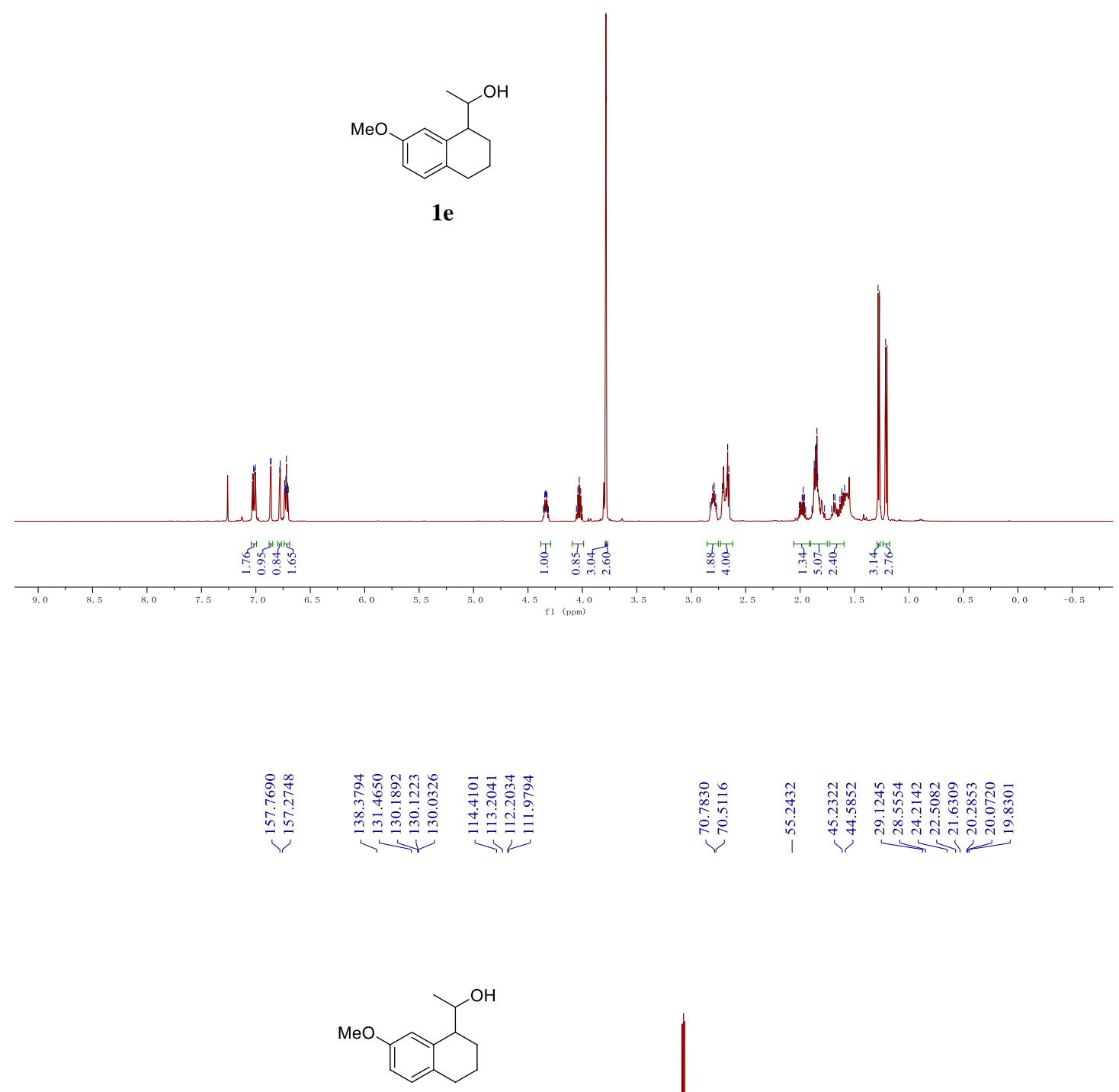

$1 \mathrm{e}$

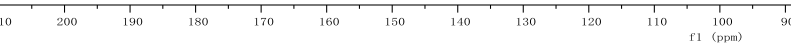



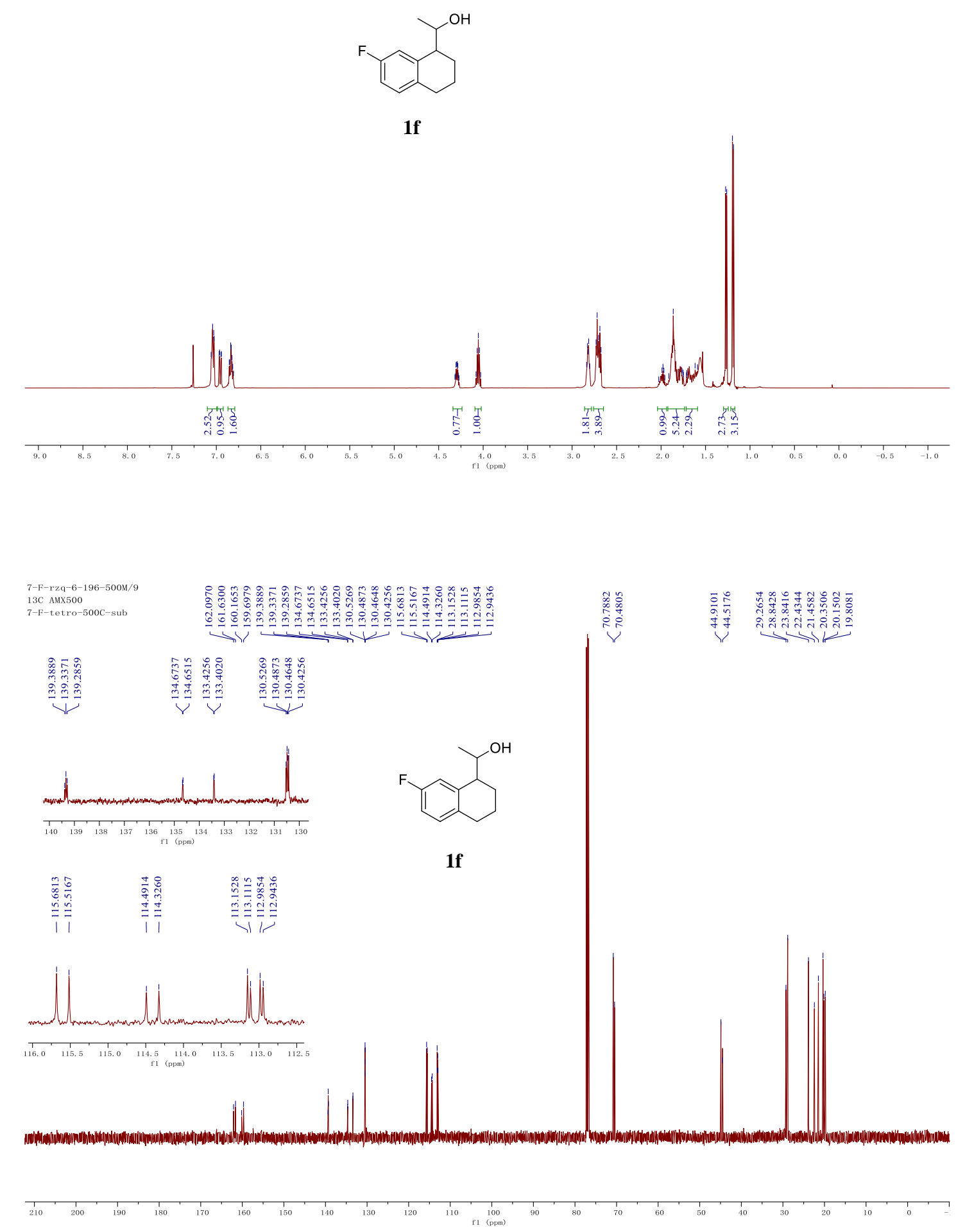


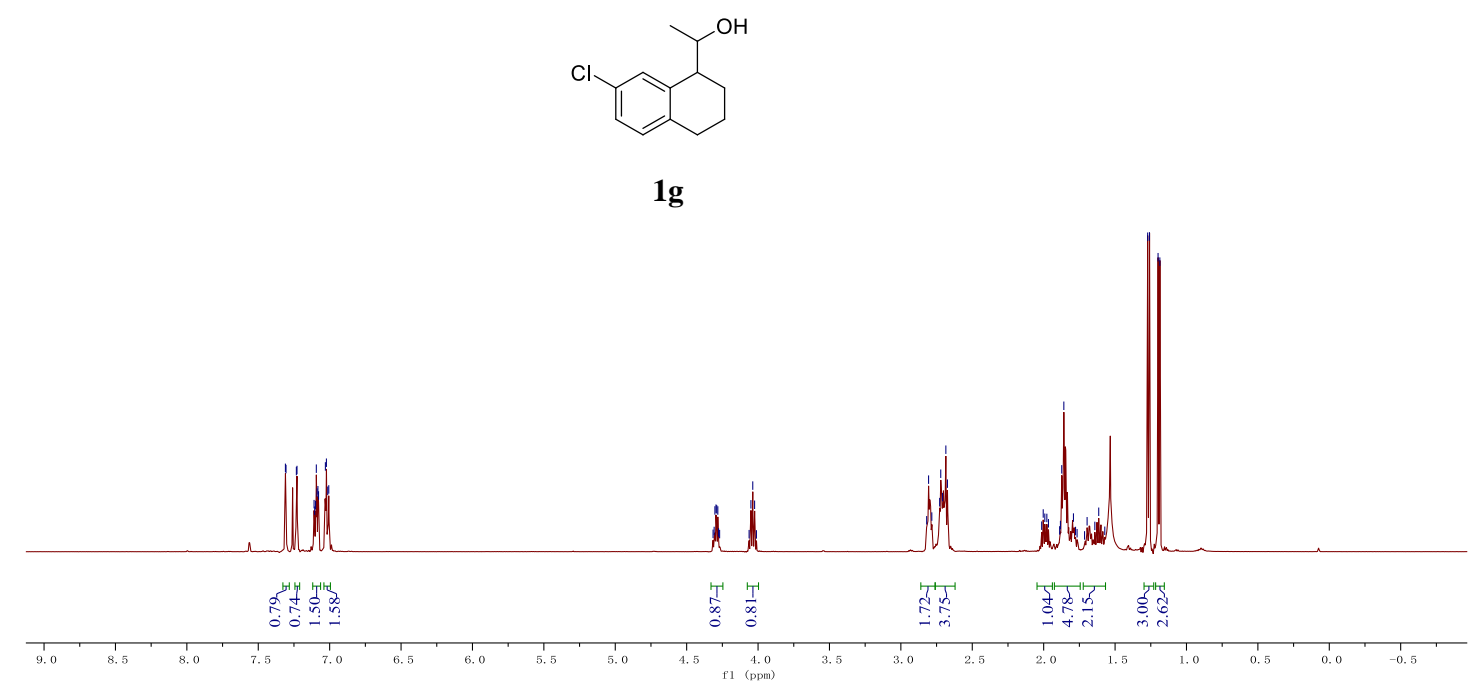

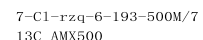

13C AMX500
$7-$ Cl-tetro-sub-50oC

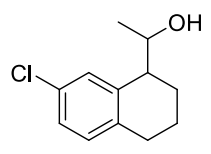

$1 \mathrm{~g}$

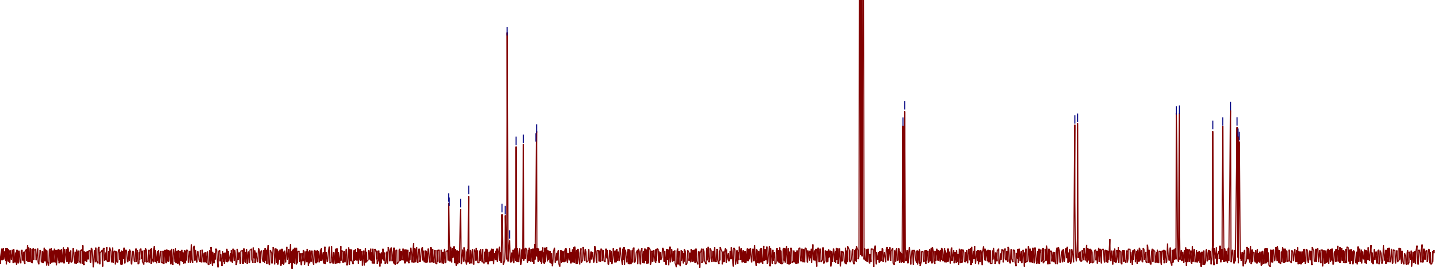




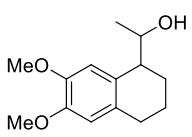

$1 \mathbf{h}$

6-OMe-7-OMe/8

$13 \mathrm{C}$ AMX500
rzq- $-6-3-500 \mathrm{C}$

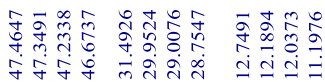

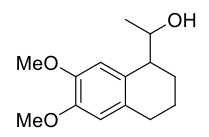

$1 \mathbf{h}$
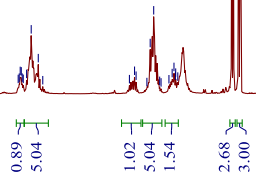

$\stackrel{\infty}{+}$

要,


5, 7-dimethy1-CD2C12/13

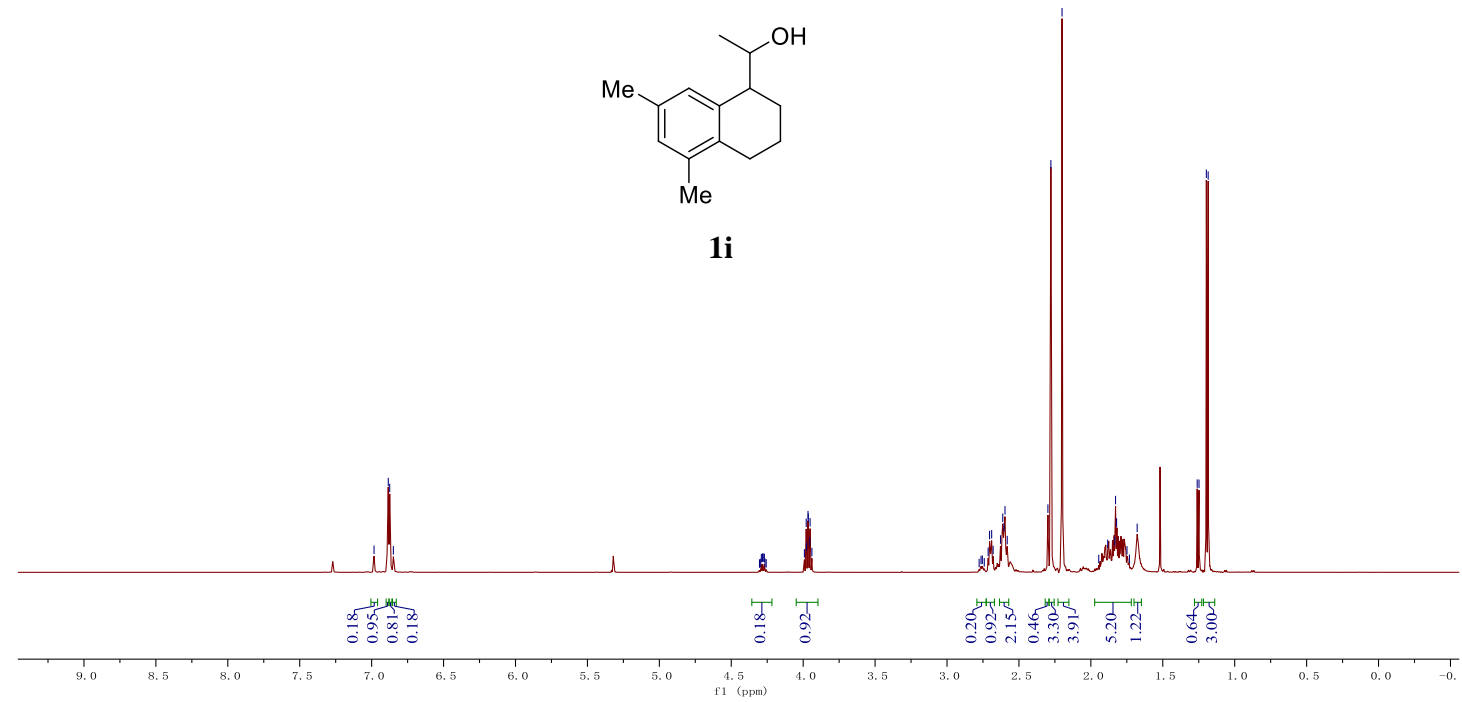

$13 \mathrm{C}$ AMX500
rzq-6-4-500C

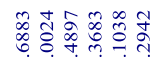

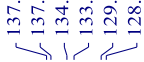

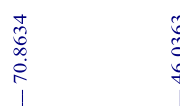

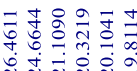

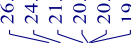

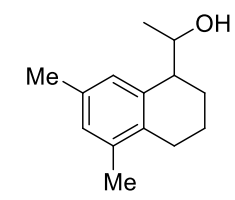

1i

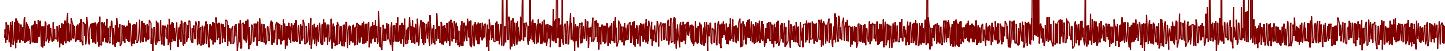

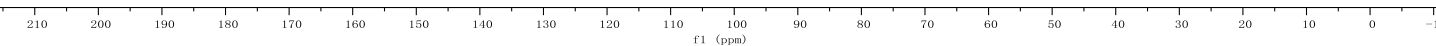




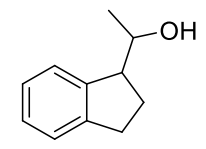

$5 \mathbf{a}$

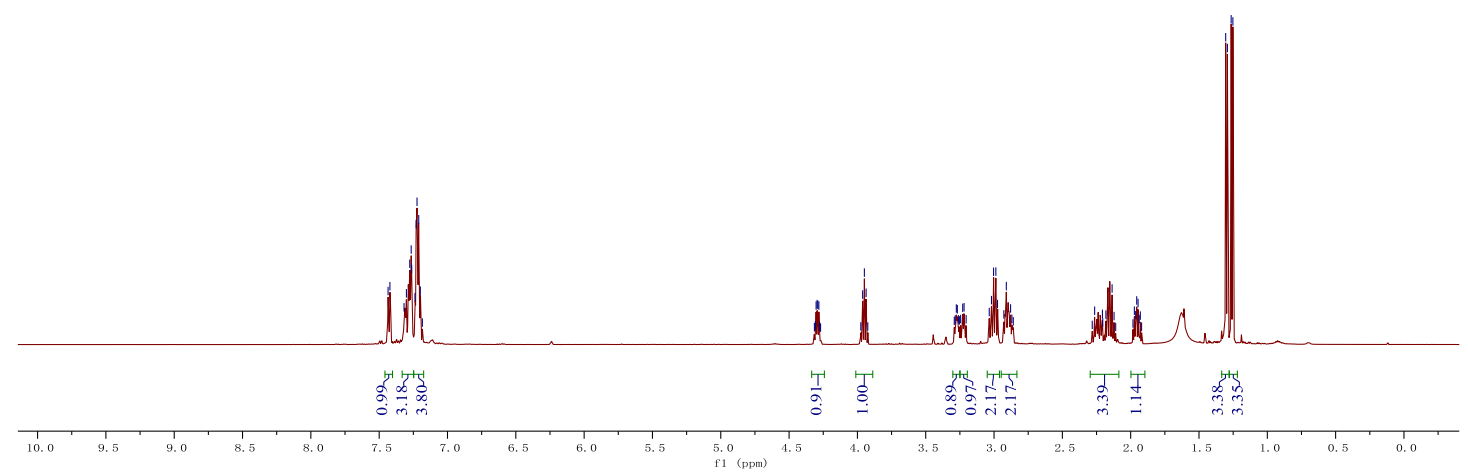

$\mathrm{Ph} / 2$
$13 \mathrm{C}$ AMX500

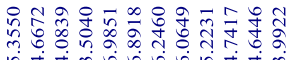

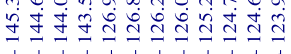

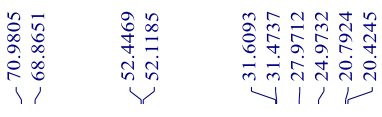

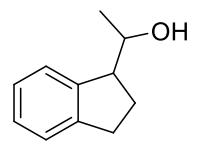

$5 \mathbf{a}$

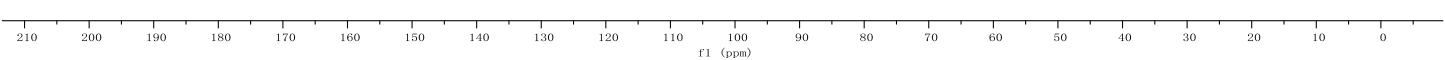


$5-\mathrm{OMe} / 3$
$1 \mathrm{H}$ MMX500

rzq-5-2-500H

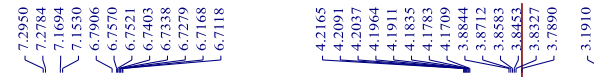

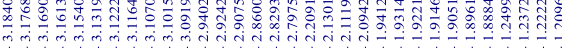<smiles>COc1ccc2c(c1)CCC2C(C)O</smiles>

5b

5-OMe $/ 4$

13C AMX500

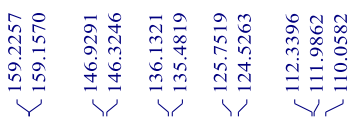

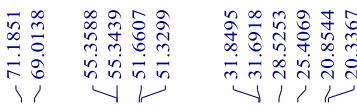

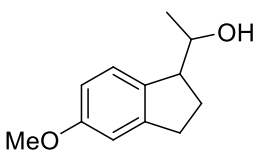

$5 \mathbf{b}$

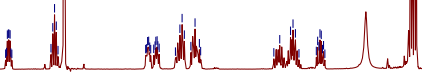

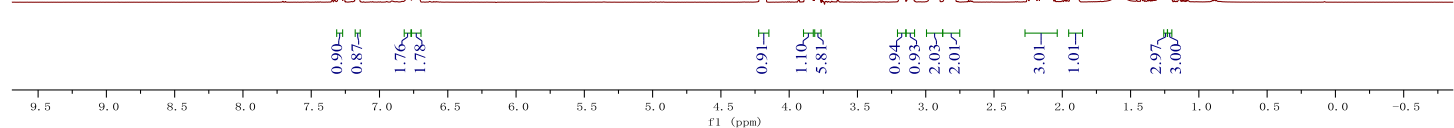

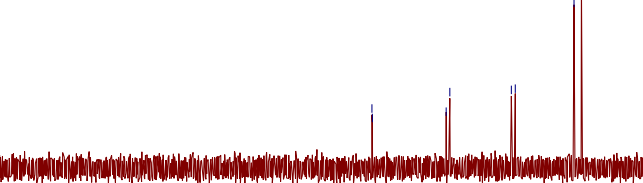

$\underset{220}{1}+\frac{1}{210} \quad 1$ 


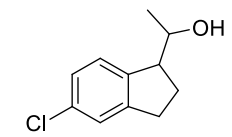

5 c

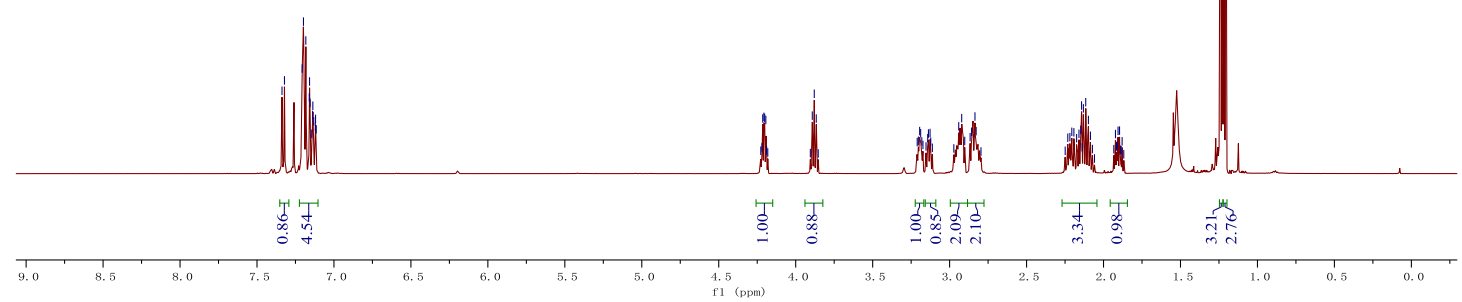

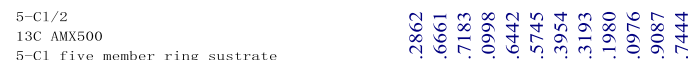

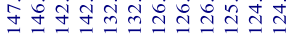

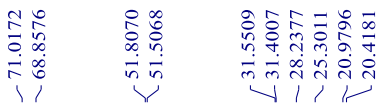

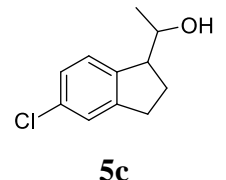

$5 c$

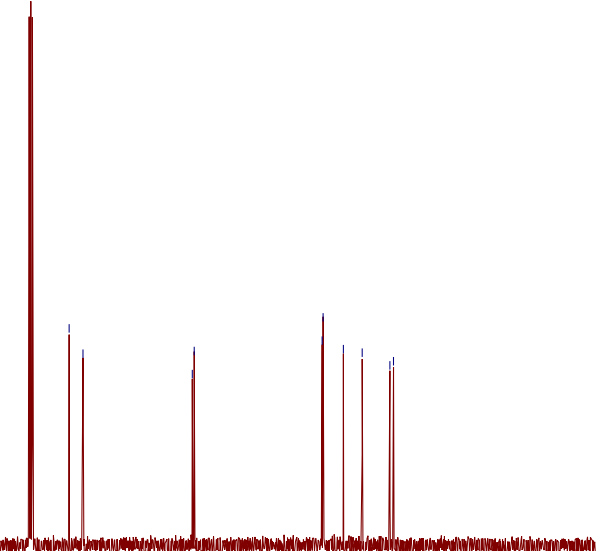

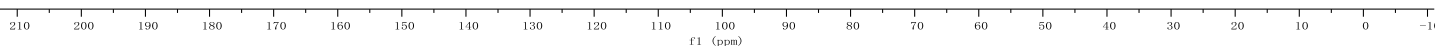



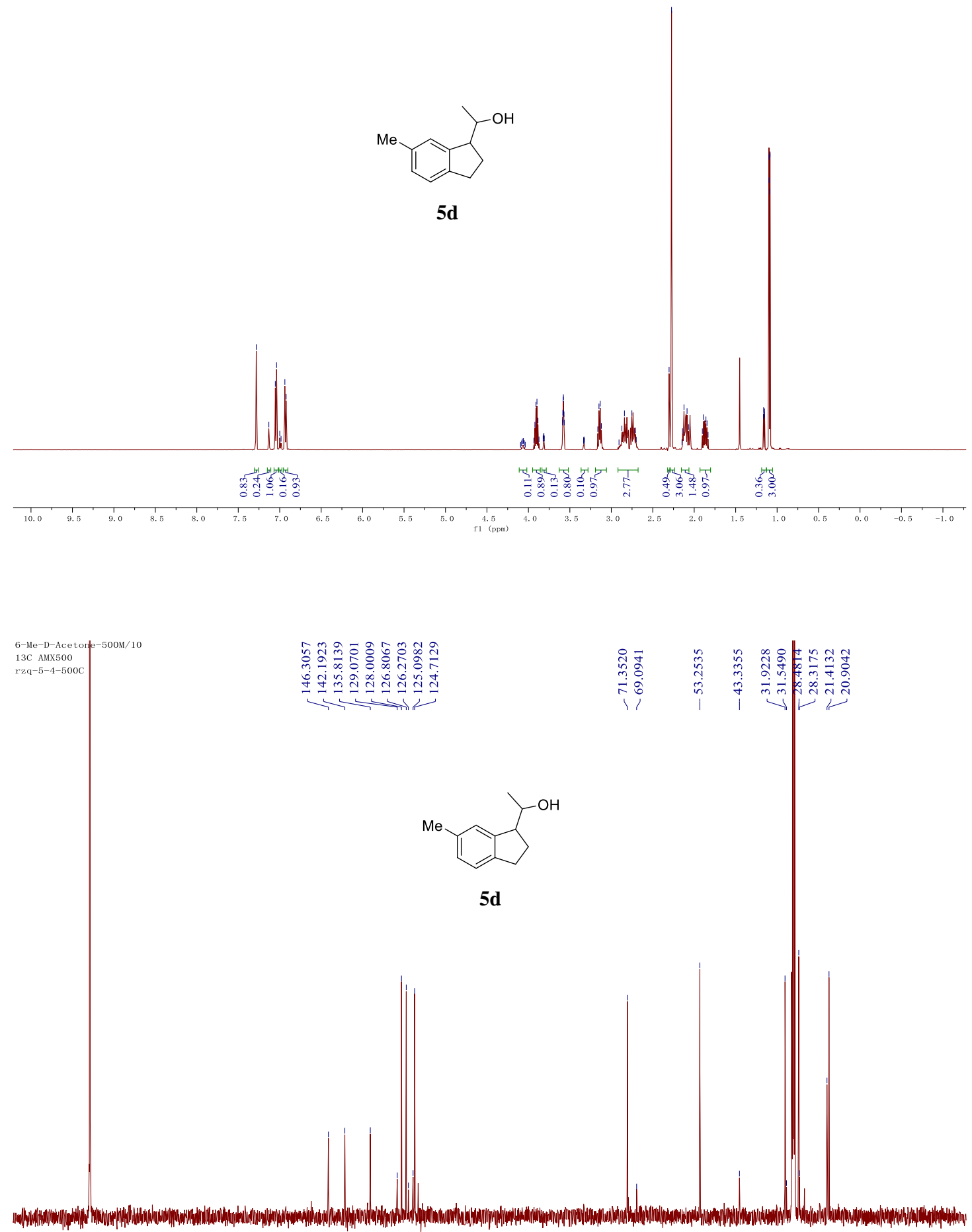

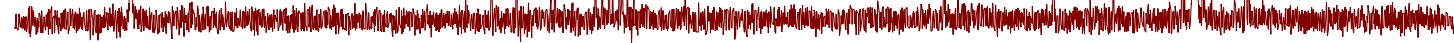

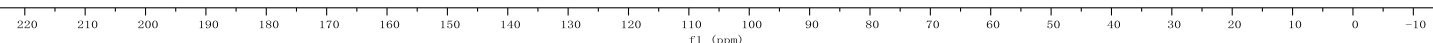




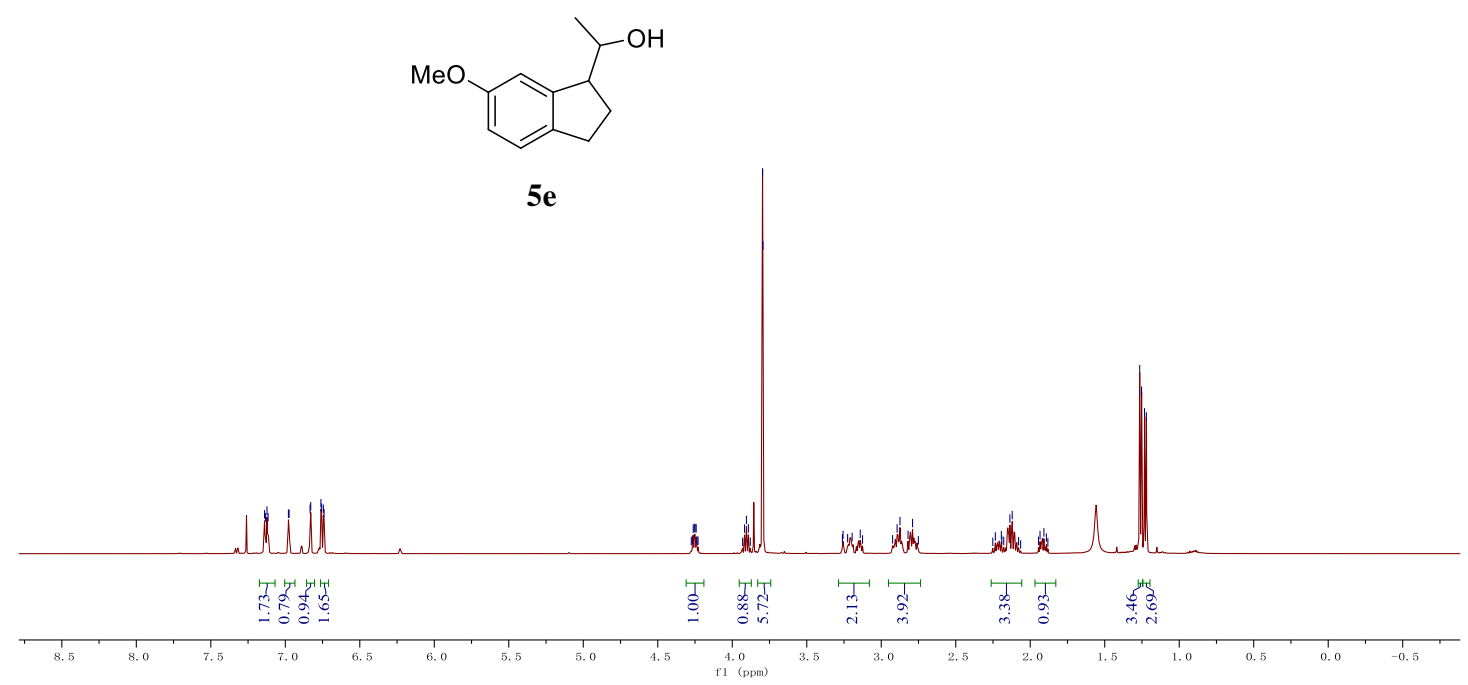

6-OMe-500M-re/13

$13 \mathrm{C}$ AMX500
rzq-5-6-500C

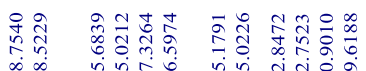

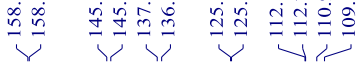

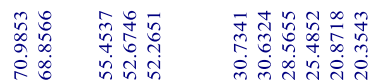

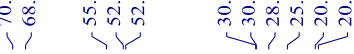

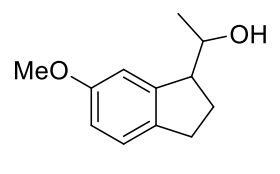

$5 \mathbf{e}$

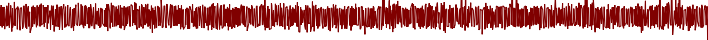

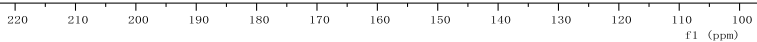




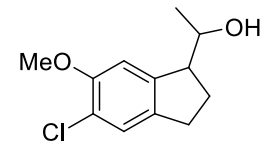

$5 f$

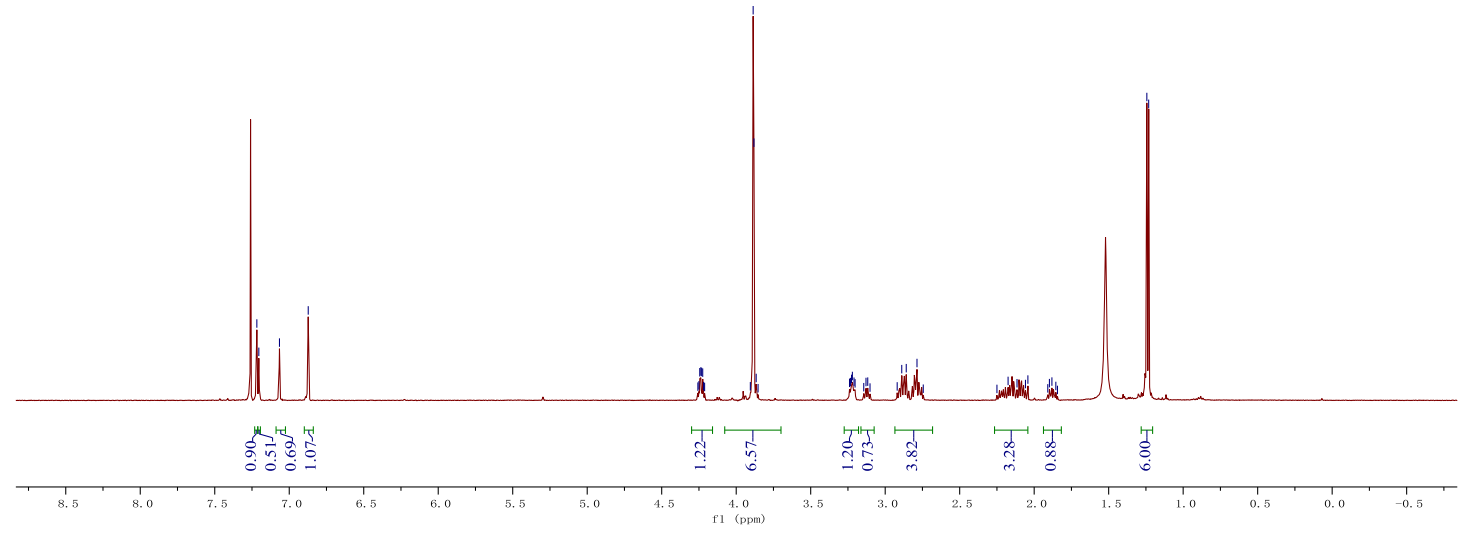

5-Cl-6-OMe- $500 \mathrm{M} / 2$

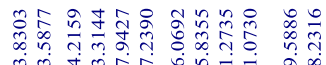

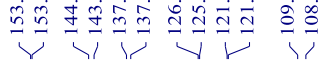

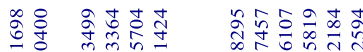

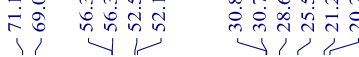

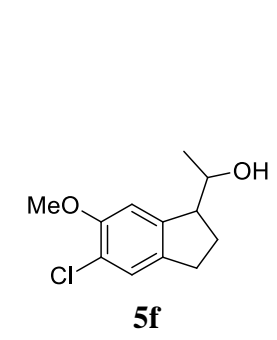

5f

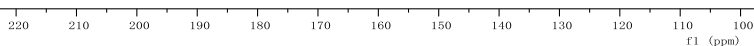




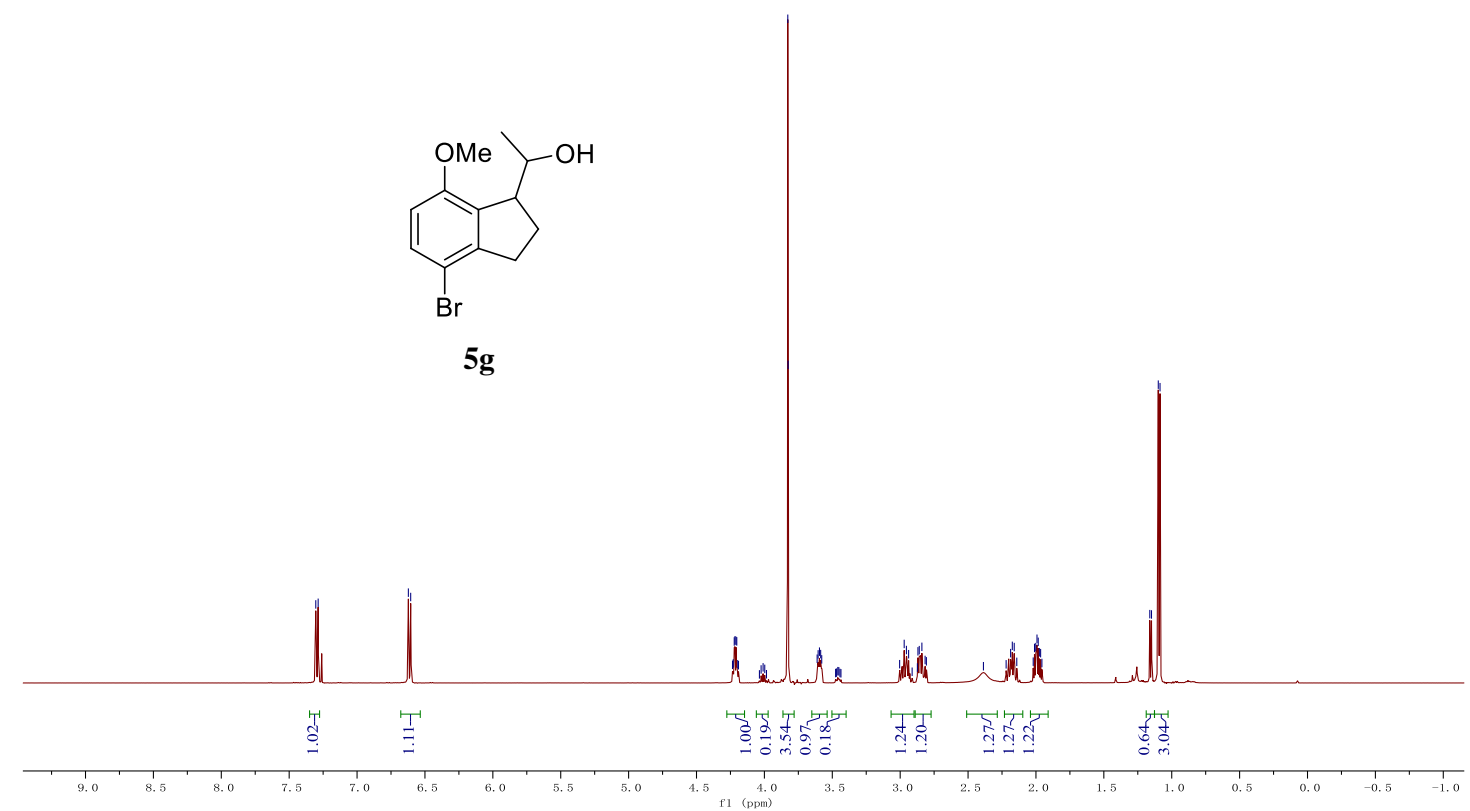

$4-\mathrm{Br}-7-\mathrm{OMe} / 2$

$13 \mathrm{C}$ AMX500
rzq $-5-7-500 \mathrm{C}$

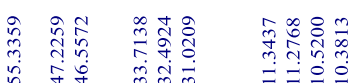

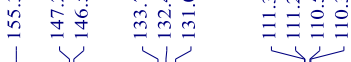

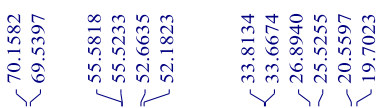

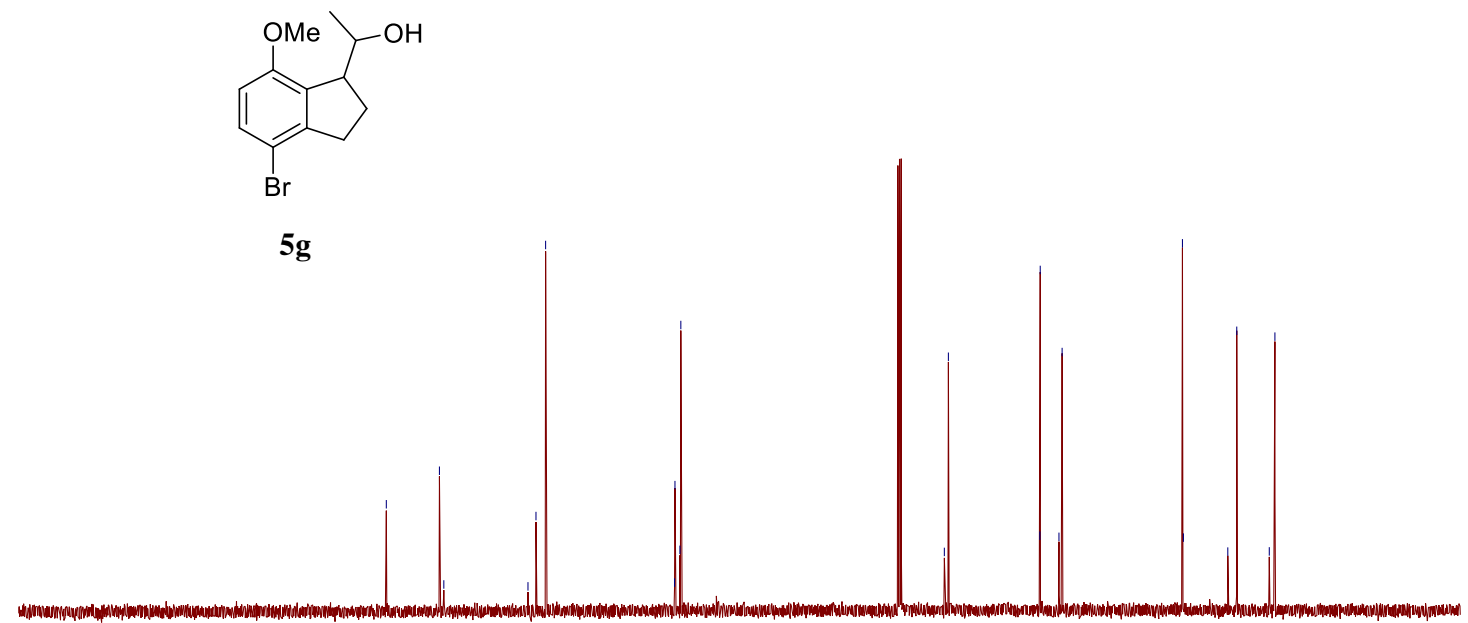


10. NMR Spectra of Products.

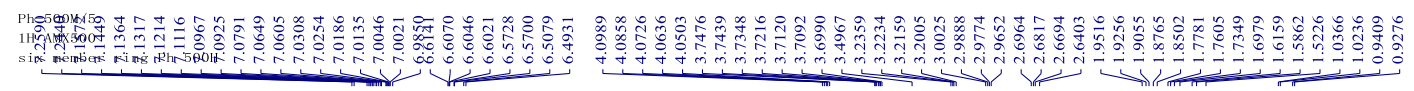

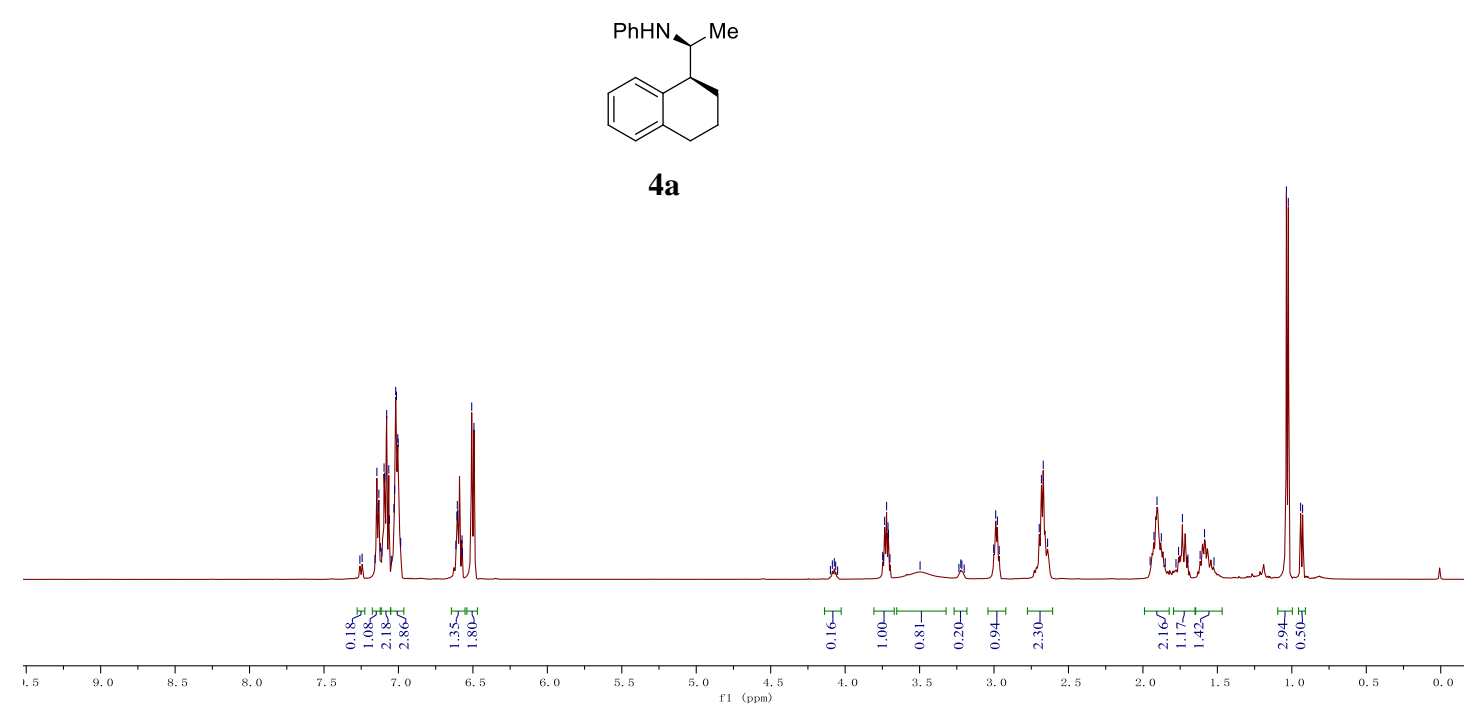

$\mathrm{Ph}-500 \mathrm{M} / 6$
$13 \mathrm{C}$ AMX500

sil menber ring ph 5000

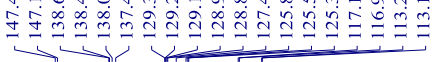

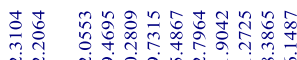

กู

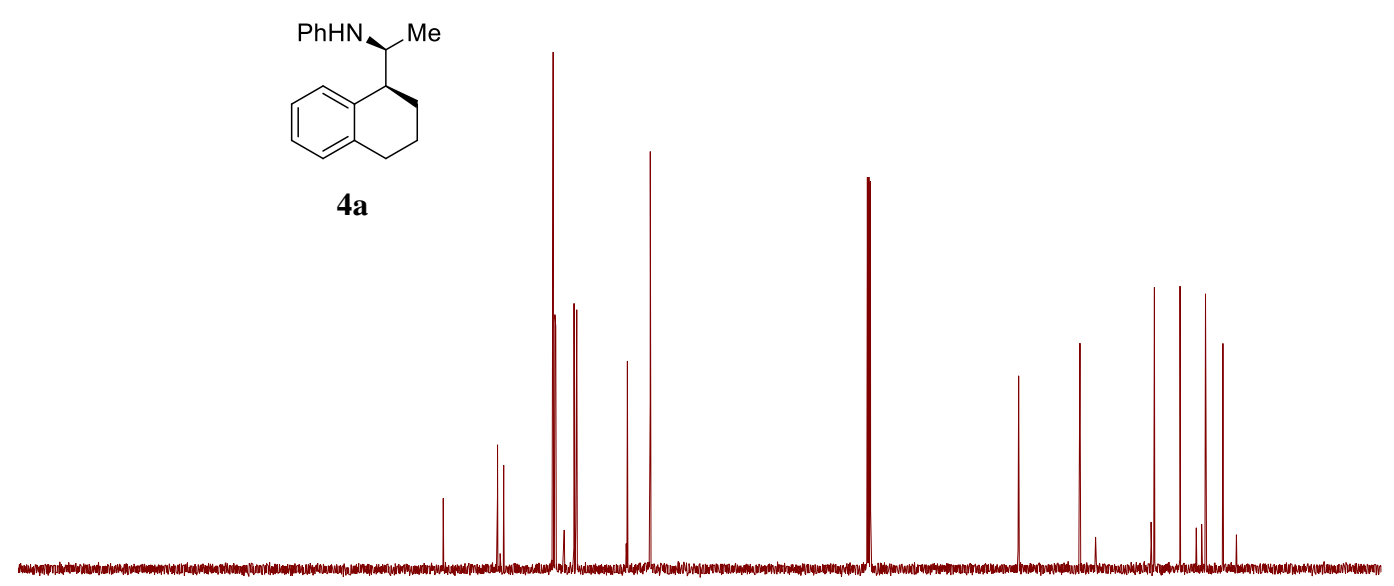




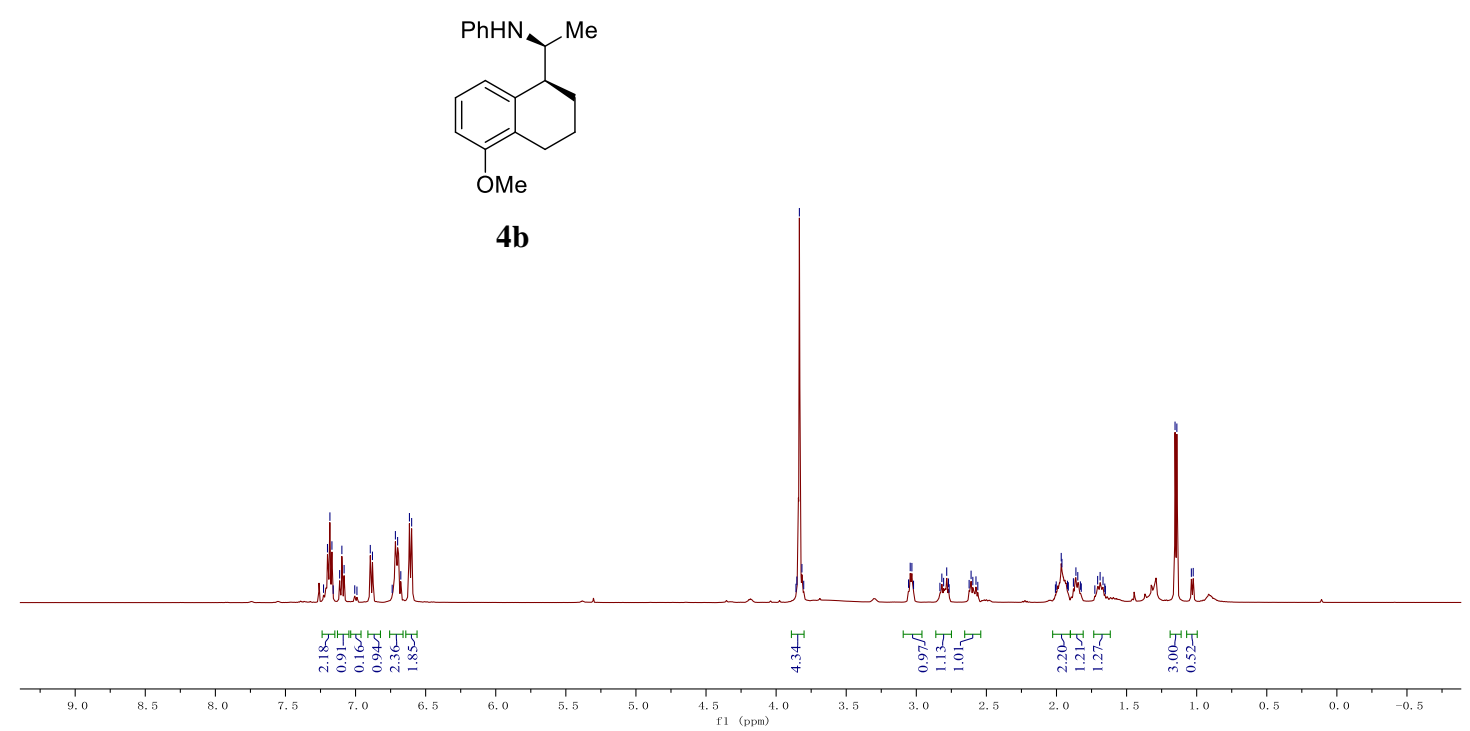

5-OMe-rzq-6-182-500M/3

$13 \mathrm{C}$ AMX500

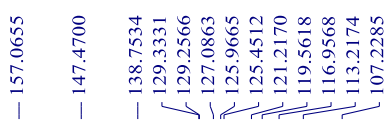

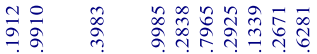

i)

in के तु

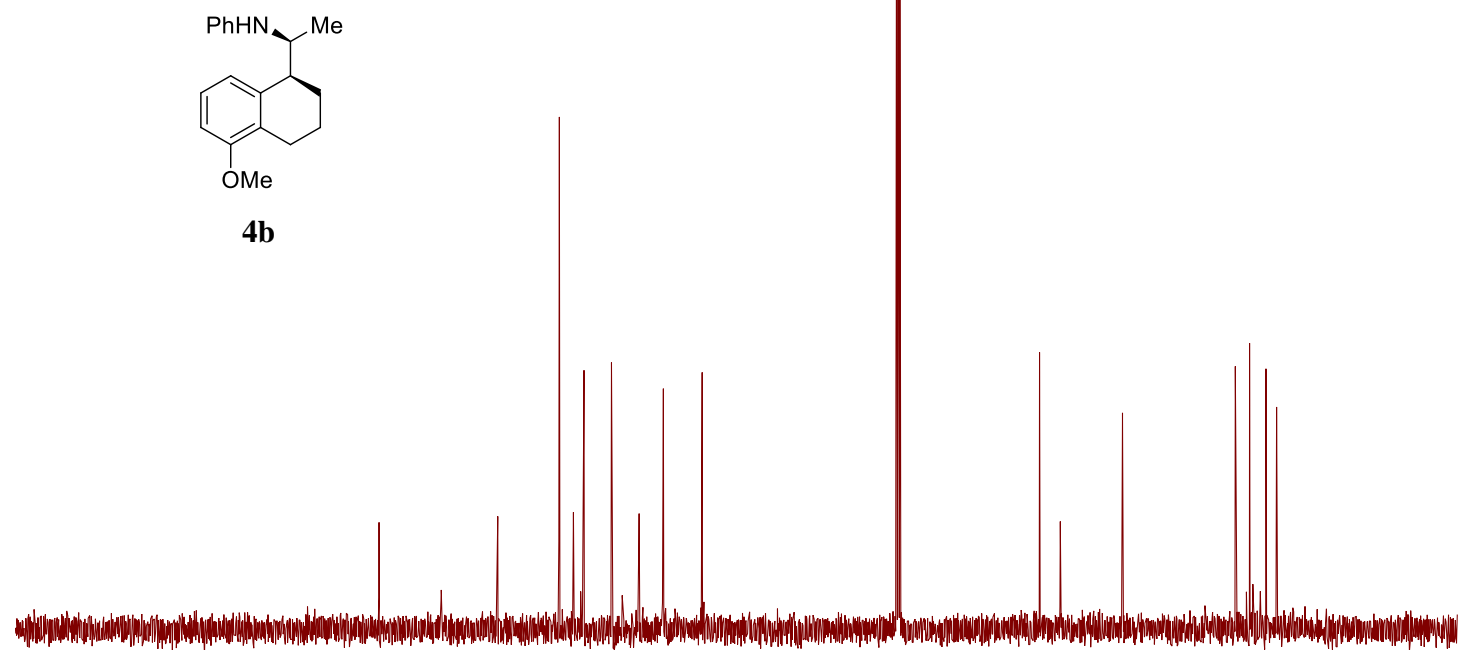

$4 b$

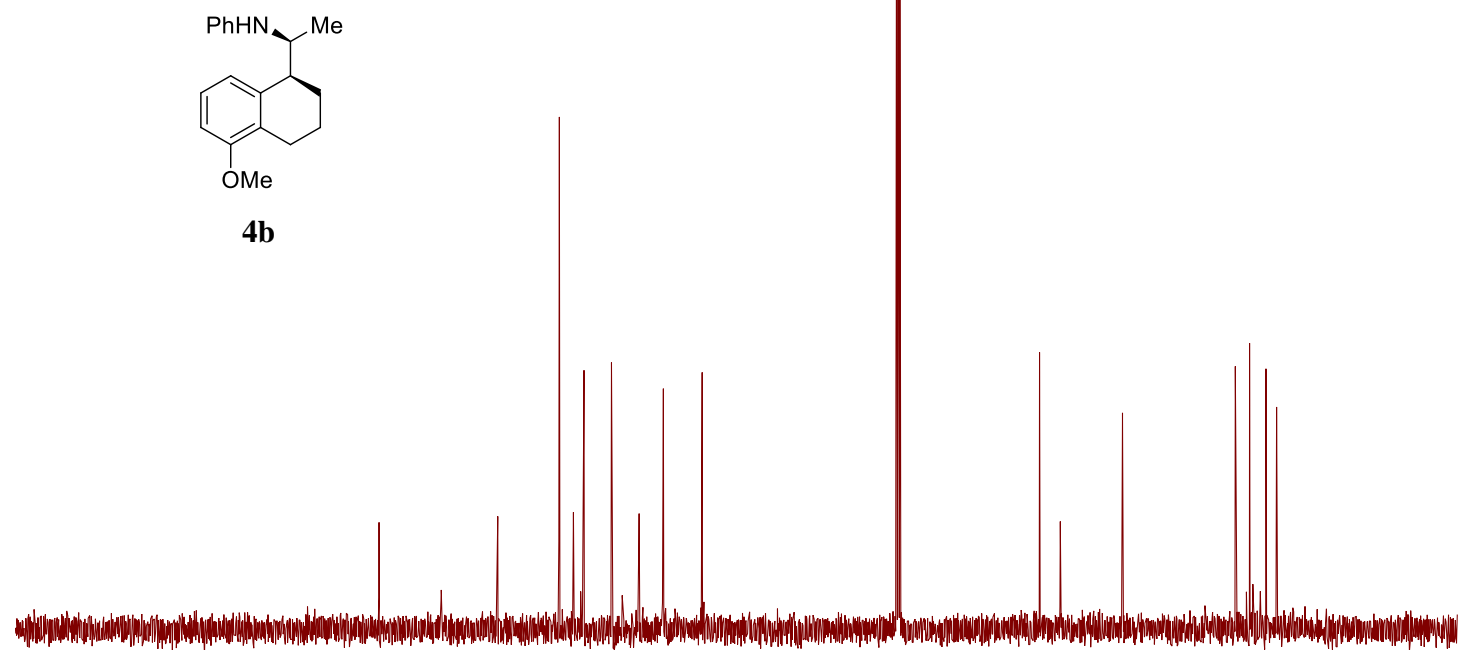




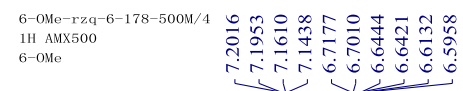

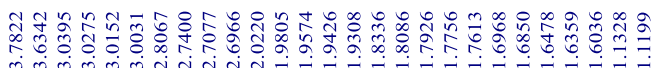

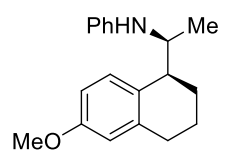

$4 c$

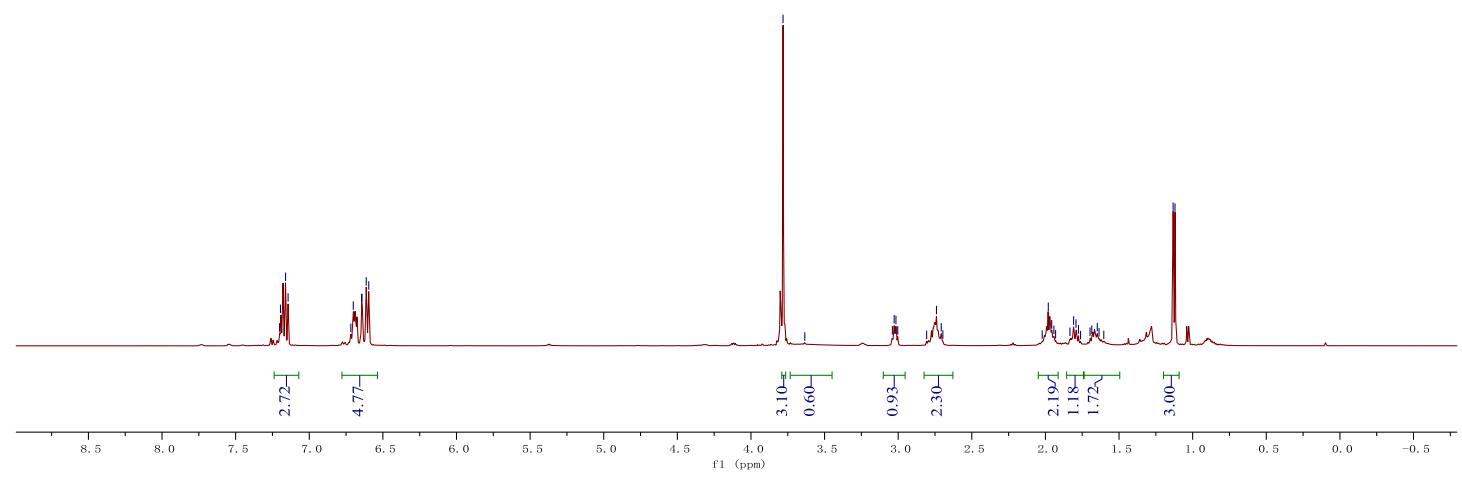

6-OMe-rzq-6-178-500M/6

13C AMx50
$6-\mathrm{MMe}$

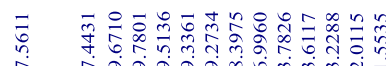

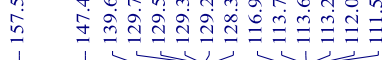

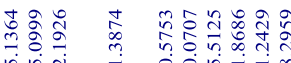

nin च
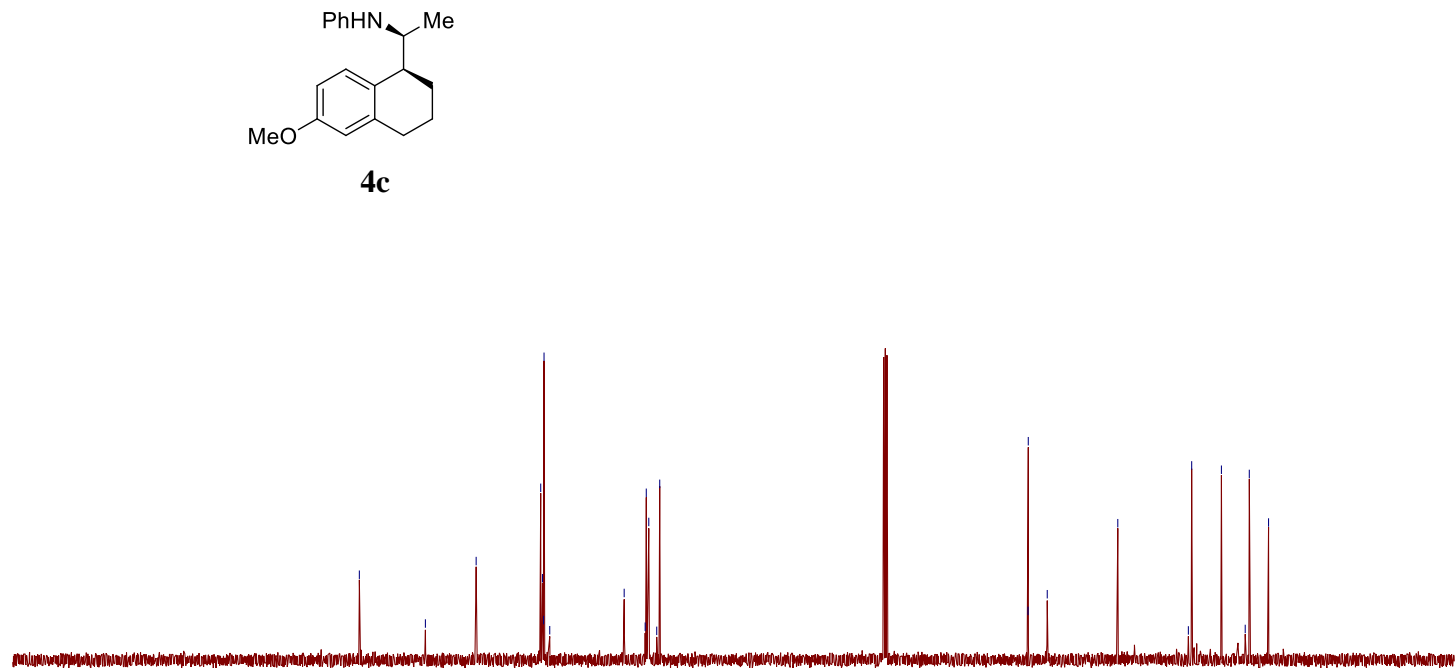


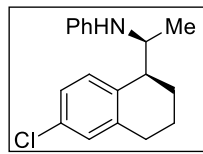

4d
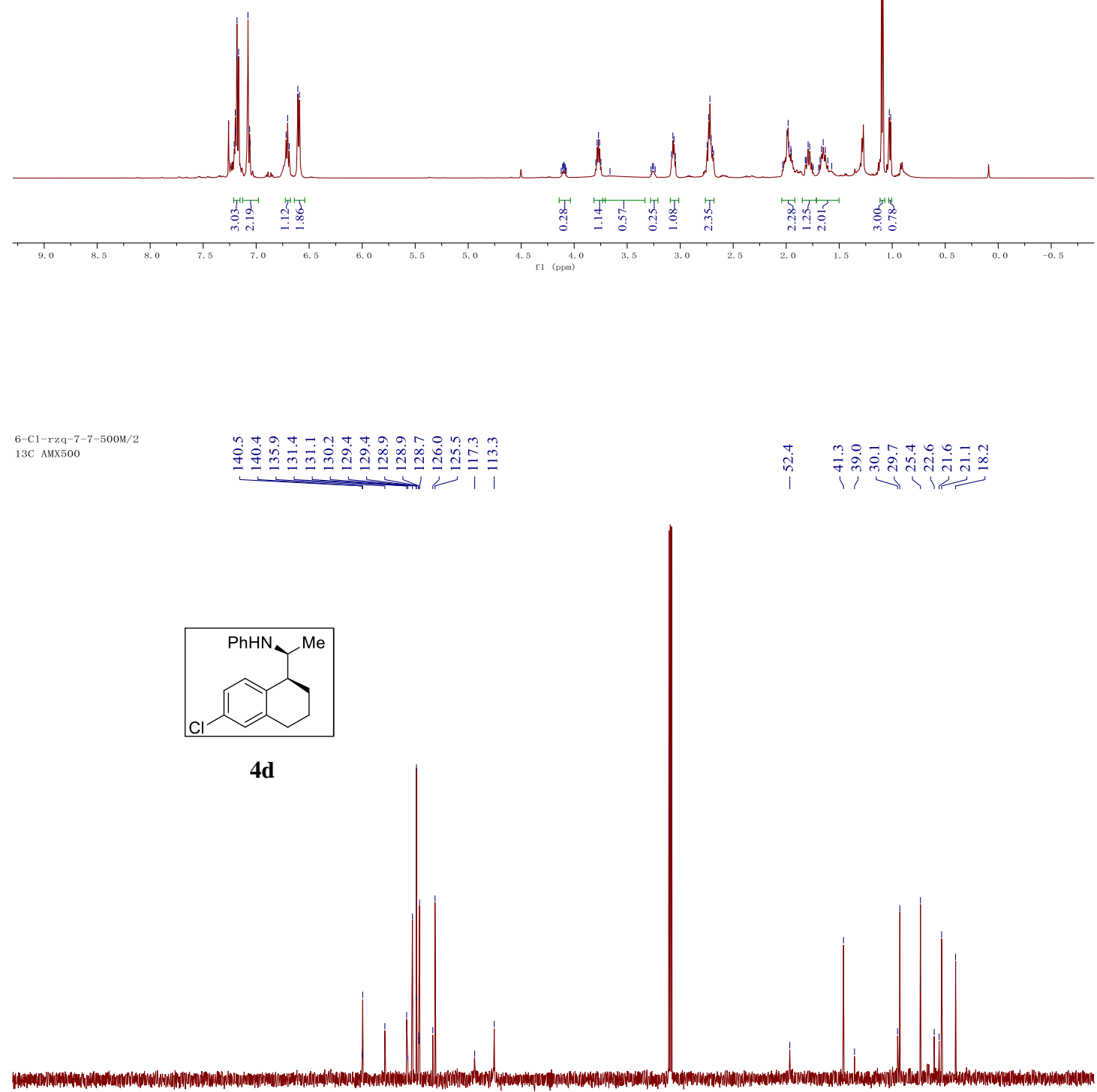


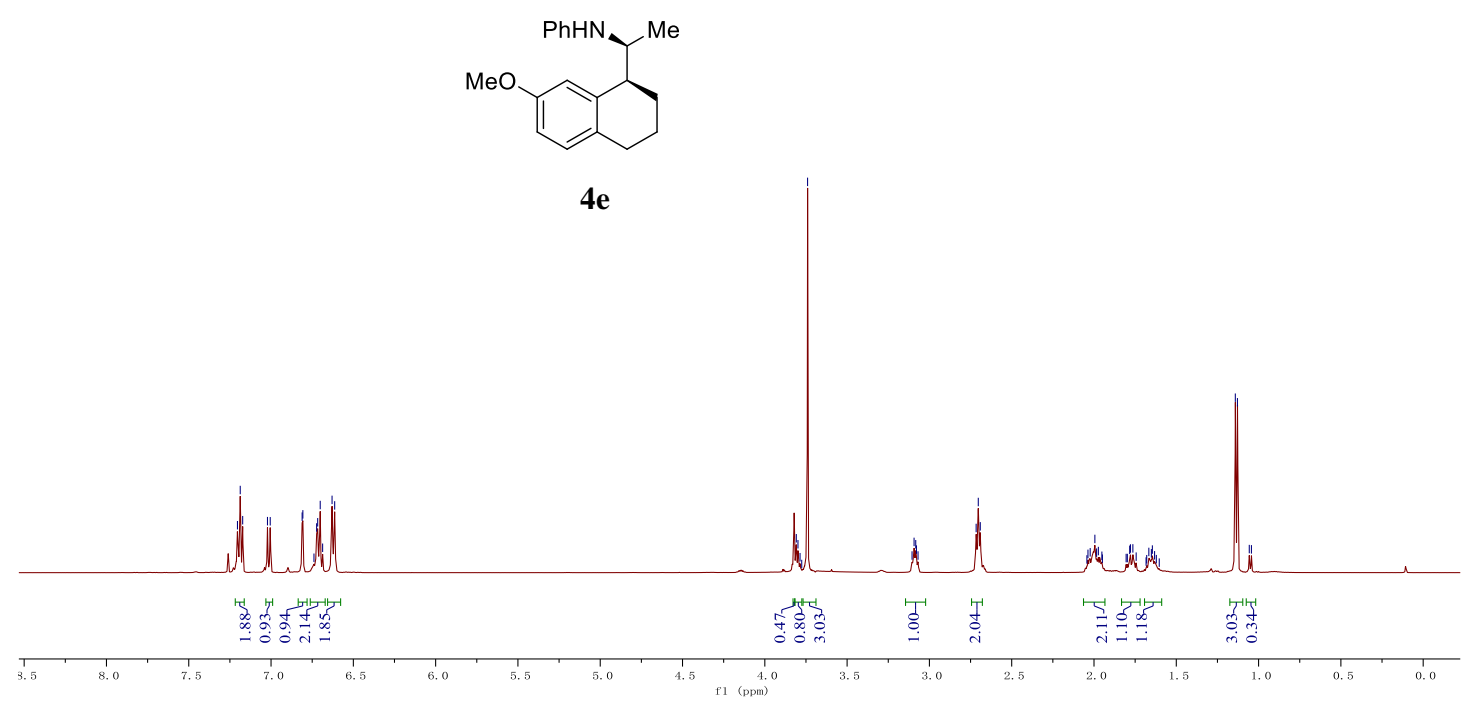

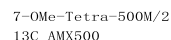

7-OMe-Tetra-product-500C

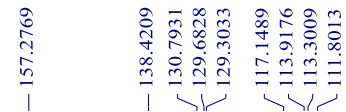

$\begin{array}{llll} & \\ 1 & & \\ 1 & 1 & 1 & 1\end{array}$

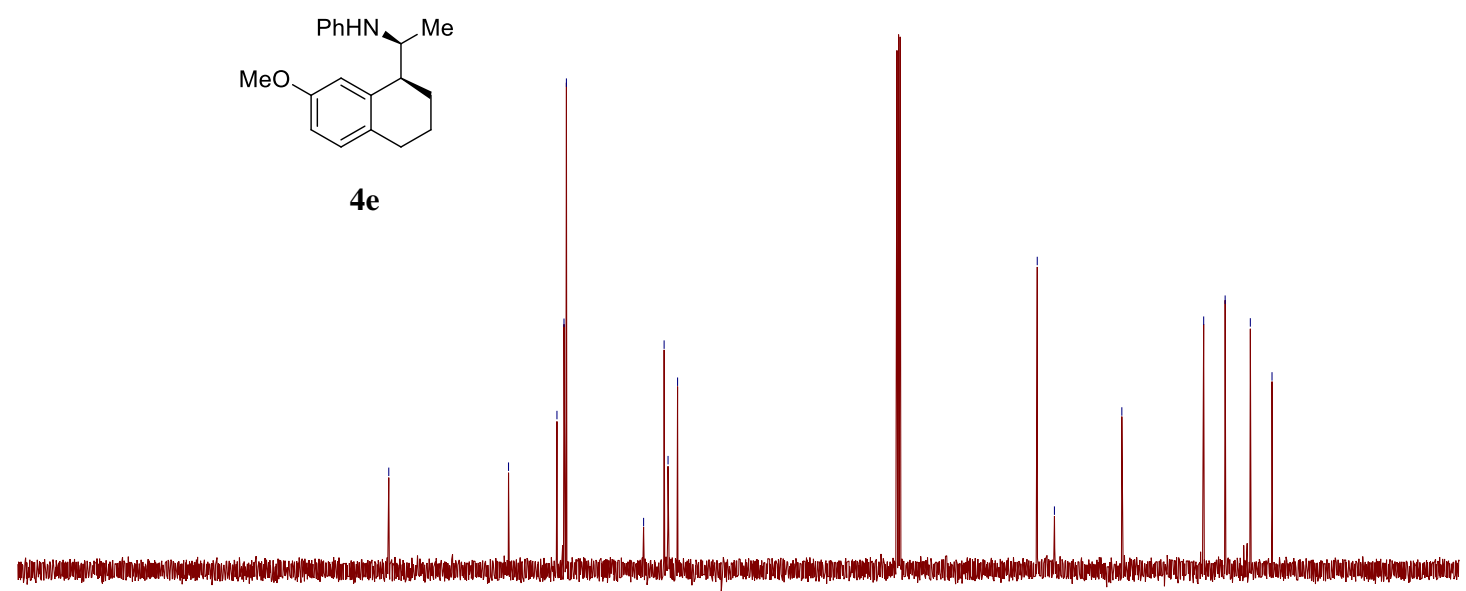



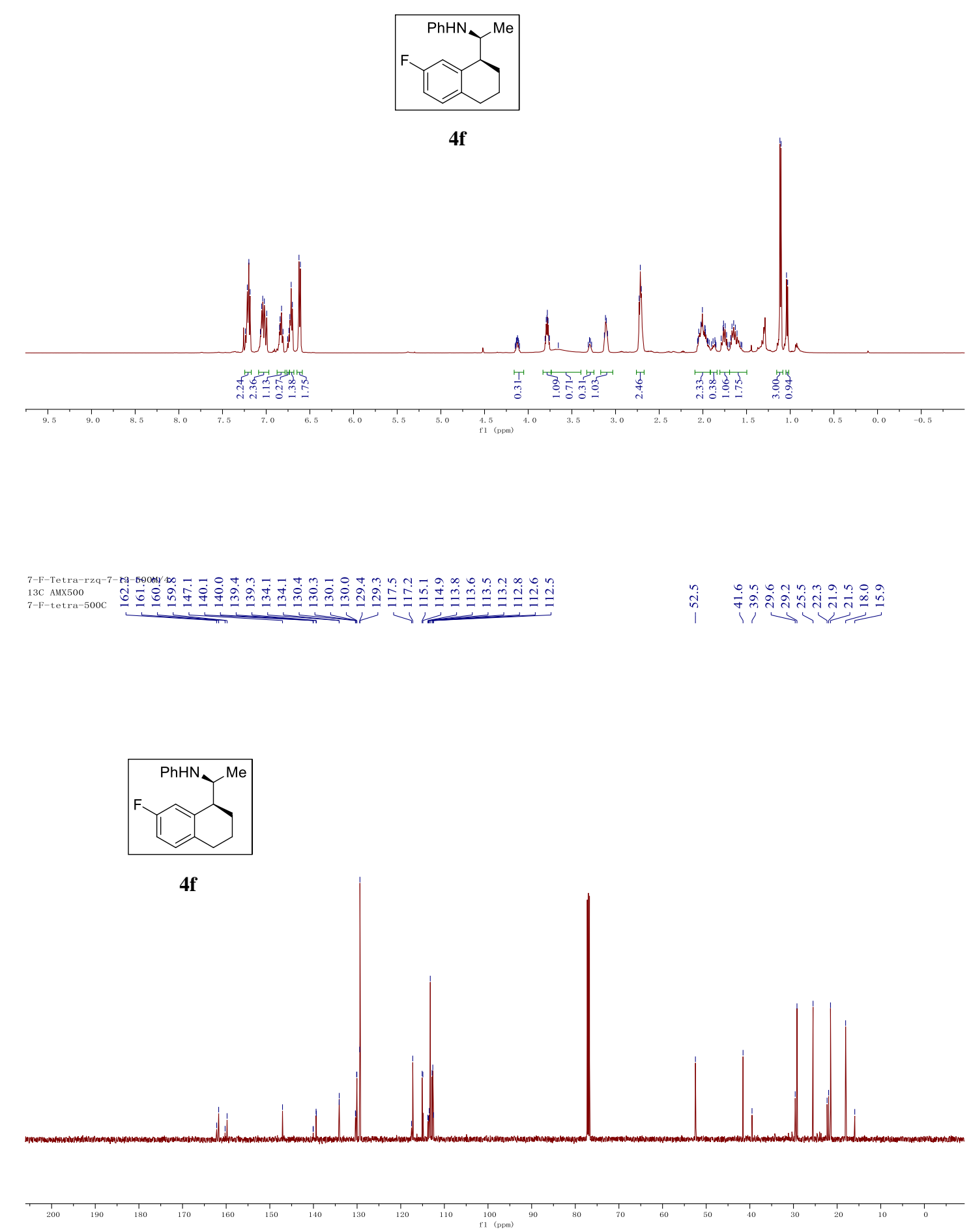


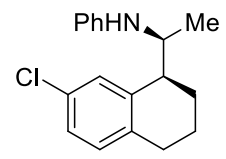

$4 \mathrm{~g}$

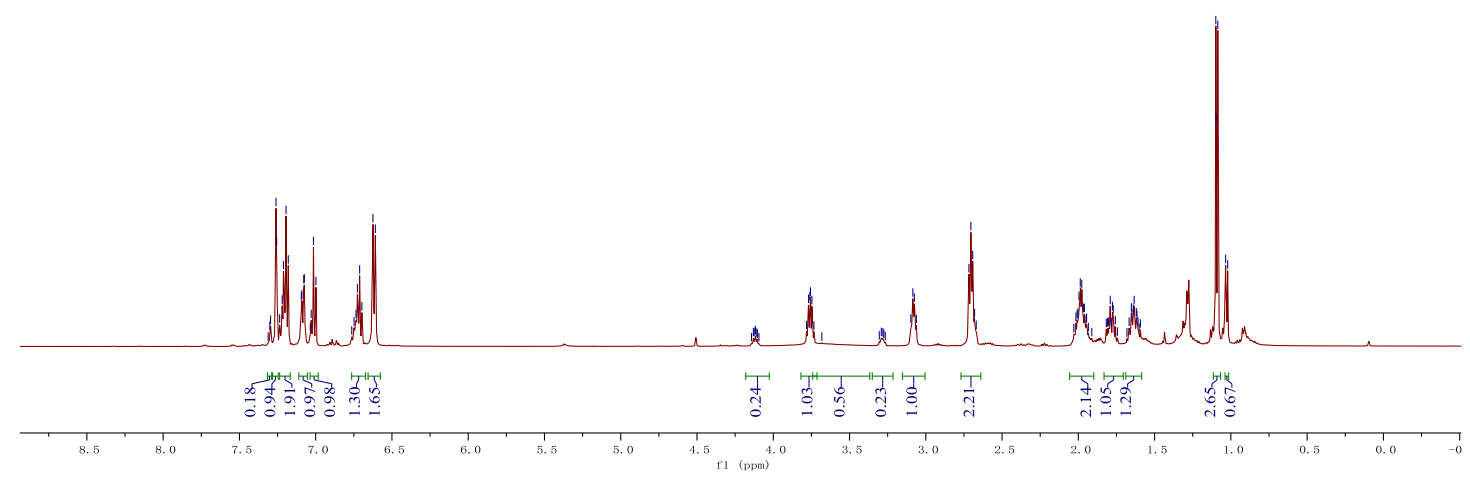

7-C1-rzq-8-500M/12

13C AMX500
7-Cl-tetro-

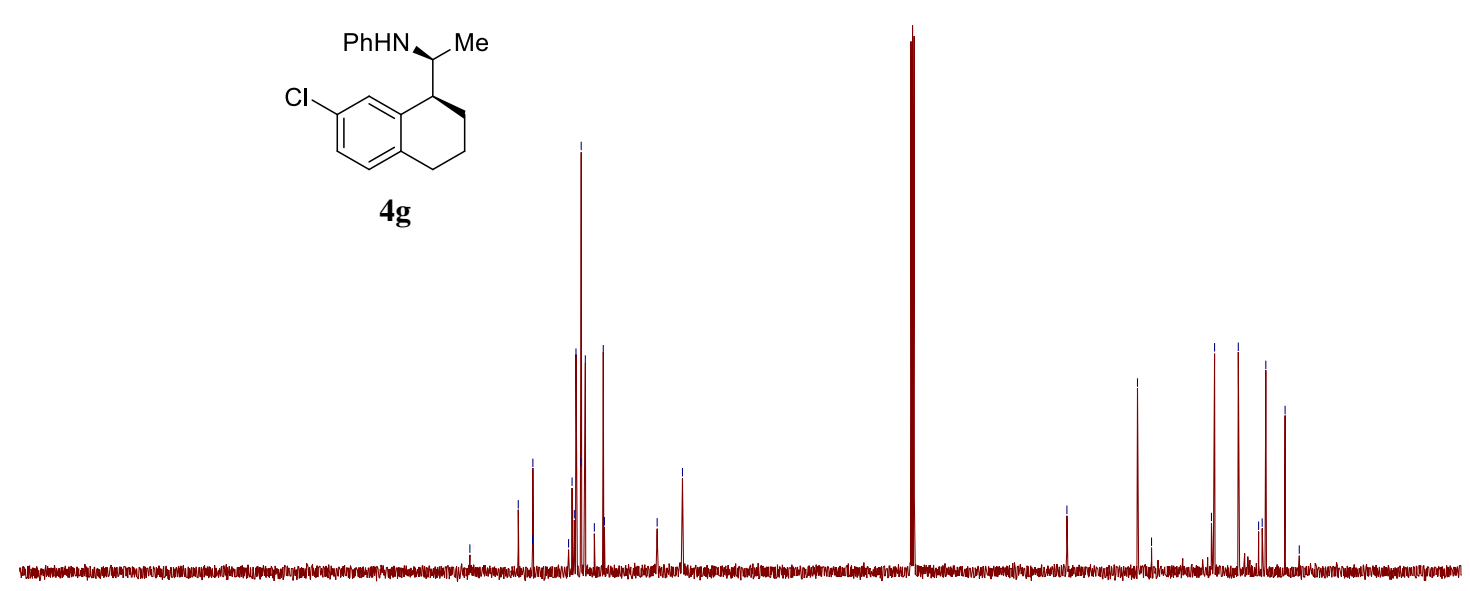




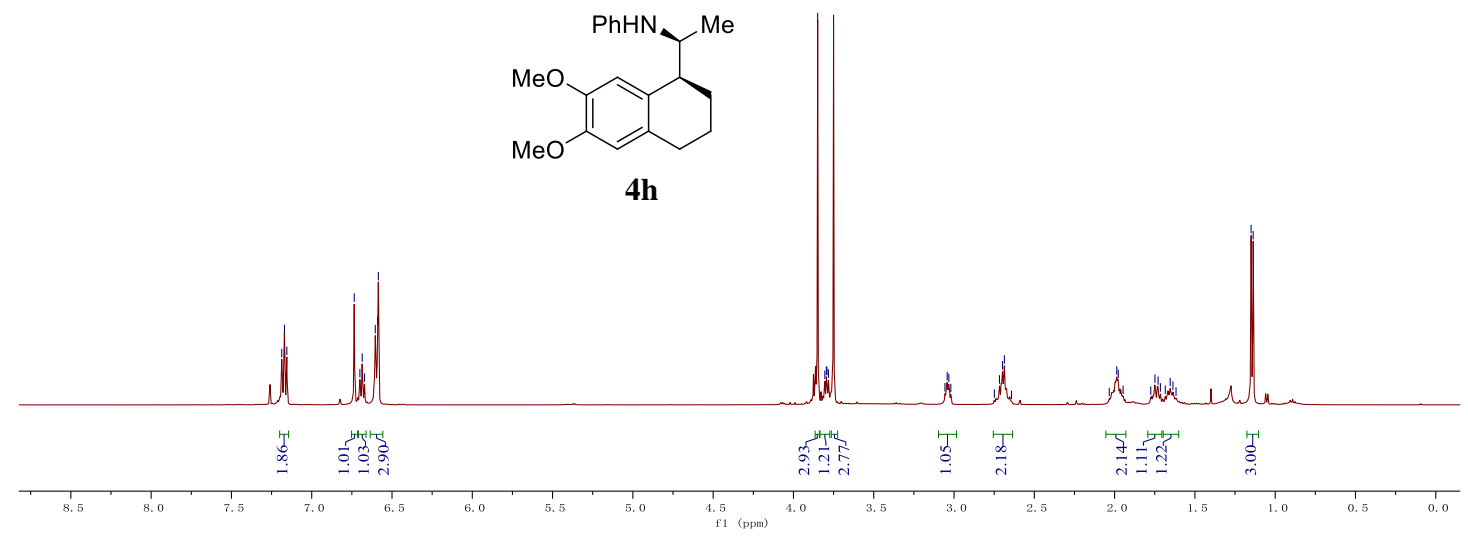

6. 7-dimethoxy1-rzq-6-186-500M/2

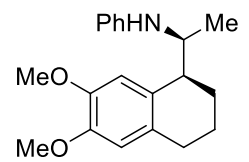

4h

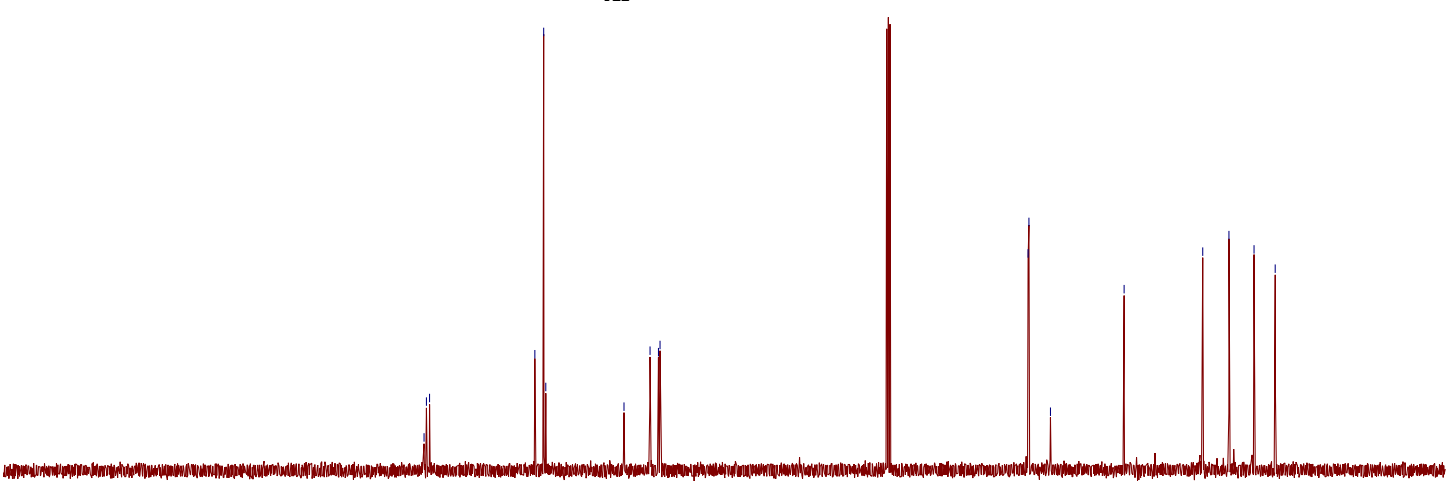

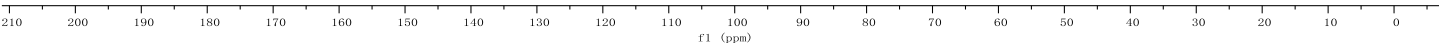




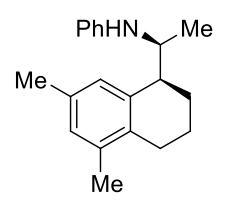

$4 i$

5, 7-dimethy 1-rzq-6-183-500m/3

5, 7-dimethy

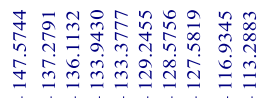

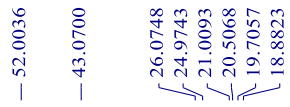

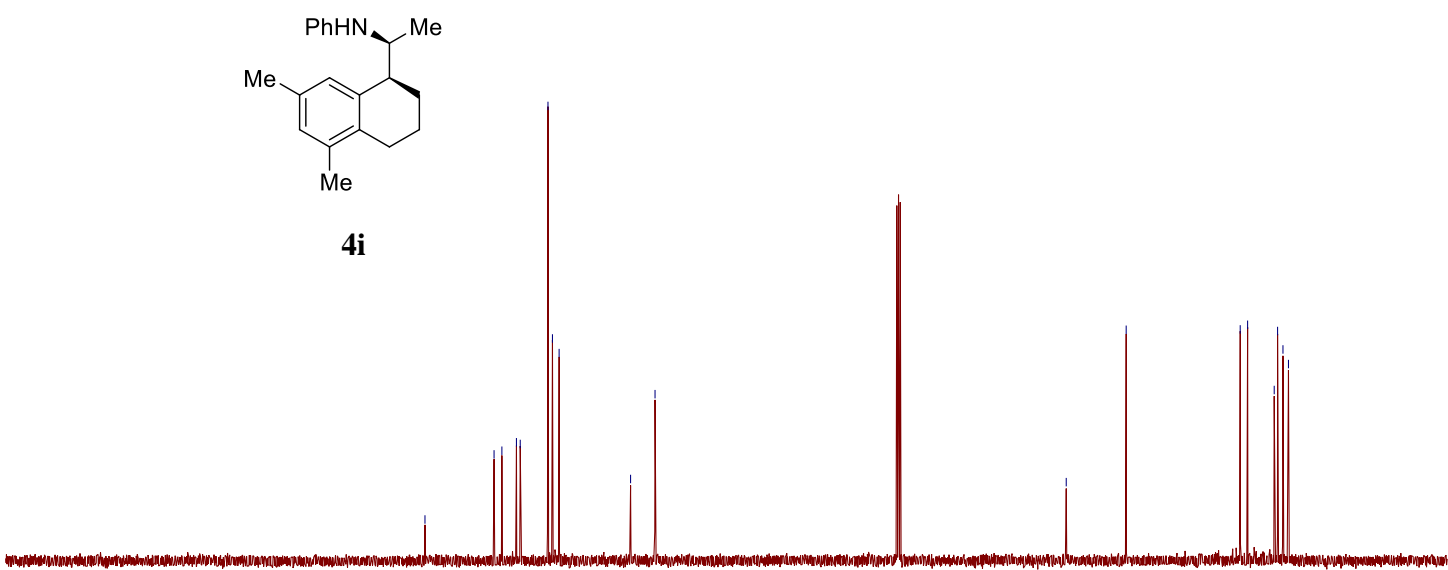

$4 \mathbf{i}$

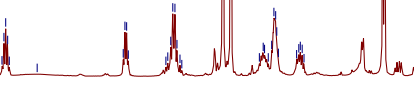

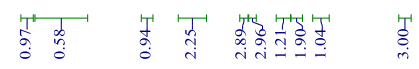




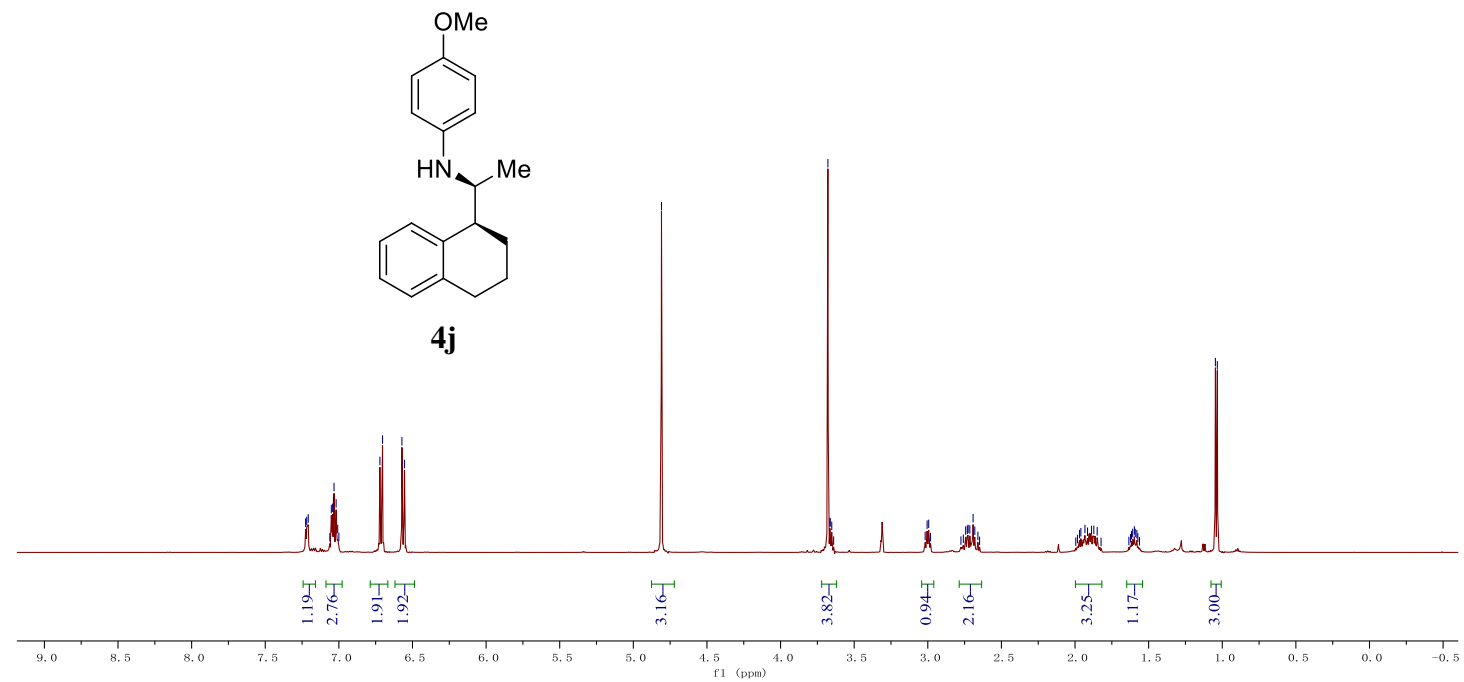

PMP-CD30D-500M-Major/8

ix member ring PMP 500

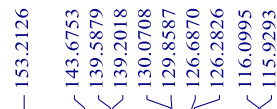

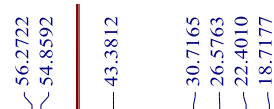

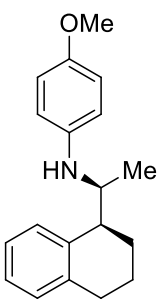

$4 \mathbf{j}$ 


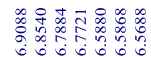

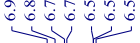

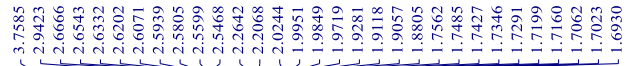

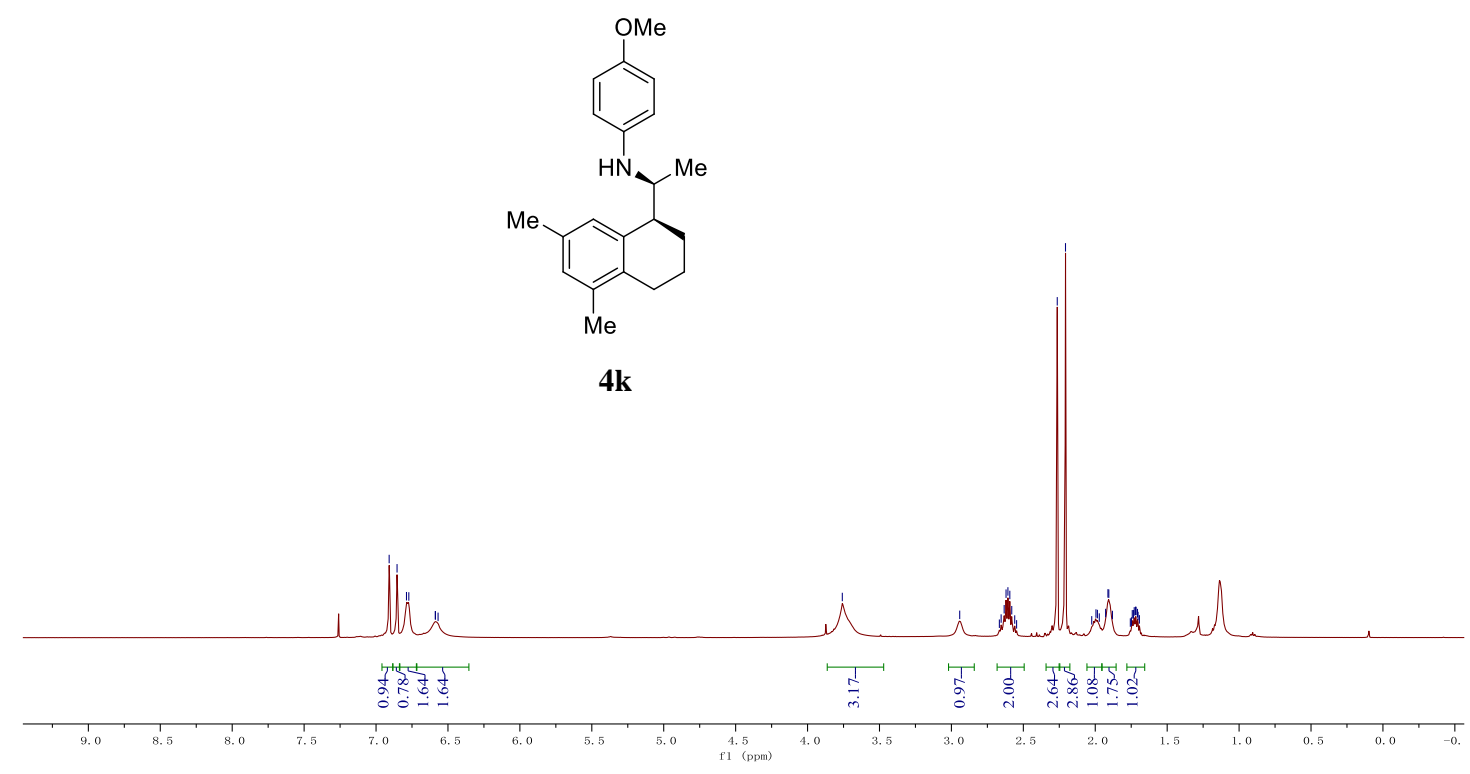

5, 7-dimethy1-PMP-rzq-7--500M/

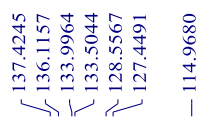

$n$
$\infty$
$\infty$
$i$
$i$
1

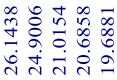

$\sqrt{9 \sqrt{4}}$

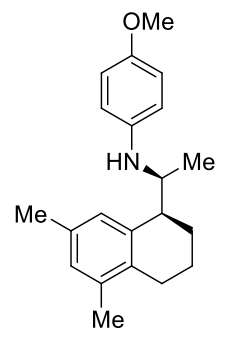

$4 k$ 


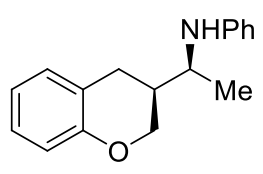

41

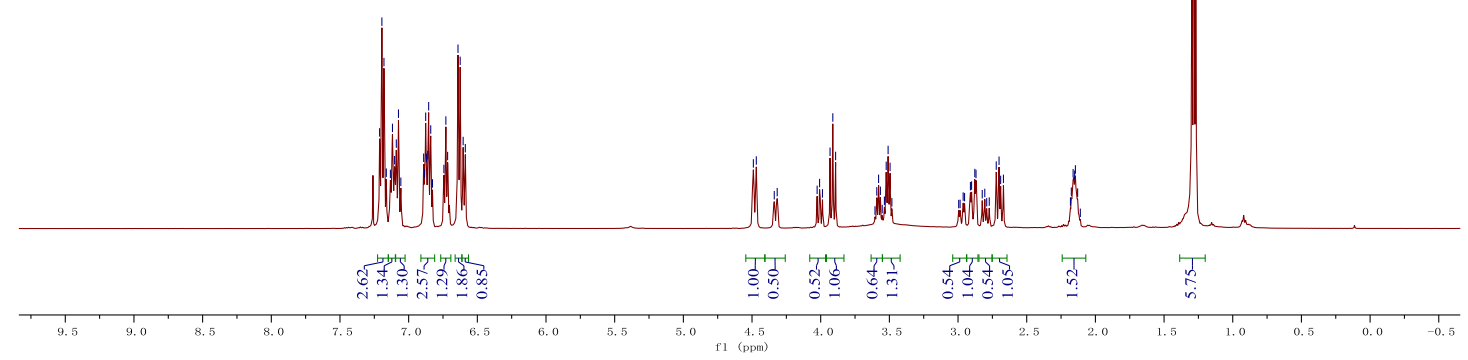

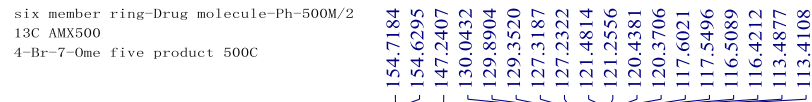

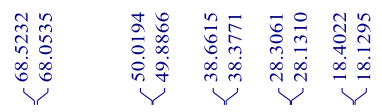<smiles>C[C@H](Nc1ccccc1)[C@H]1COc2ccccc2C1</smiles>

41 


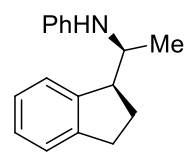

6a

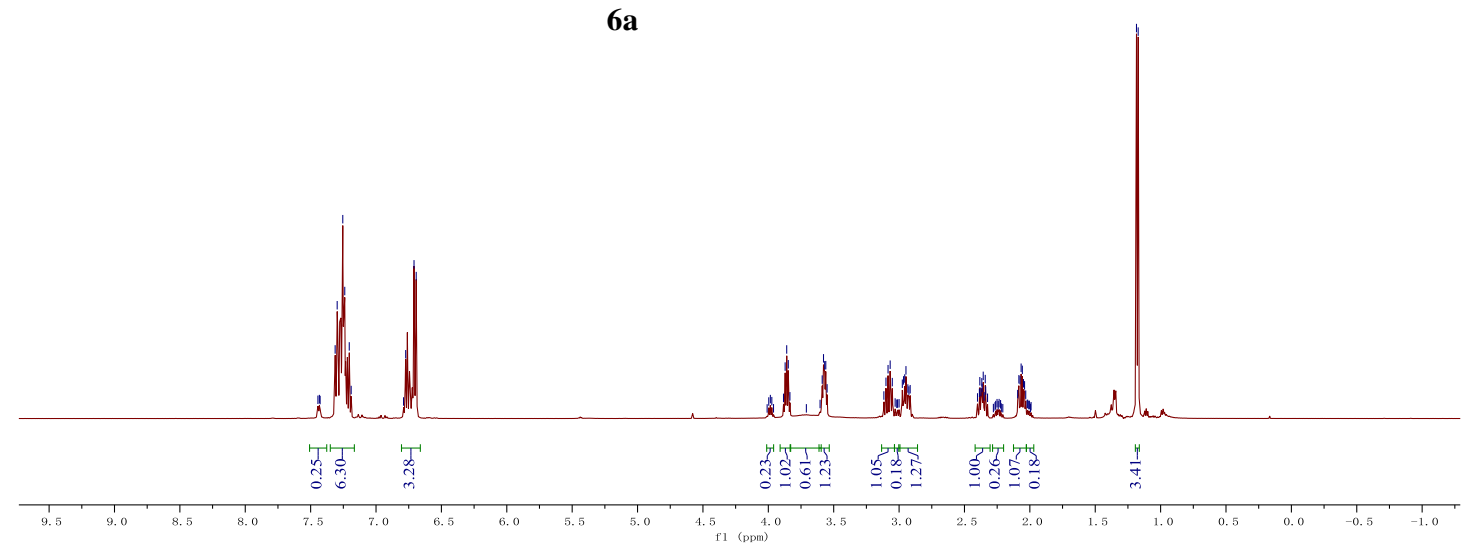

Ph-rzq-6-177-500M/3
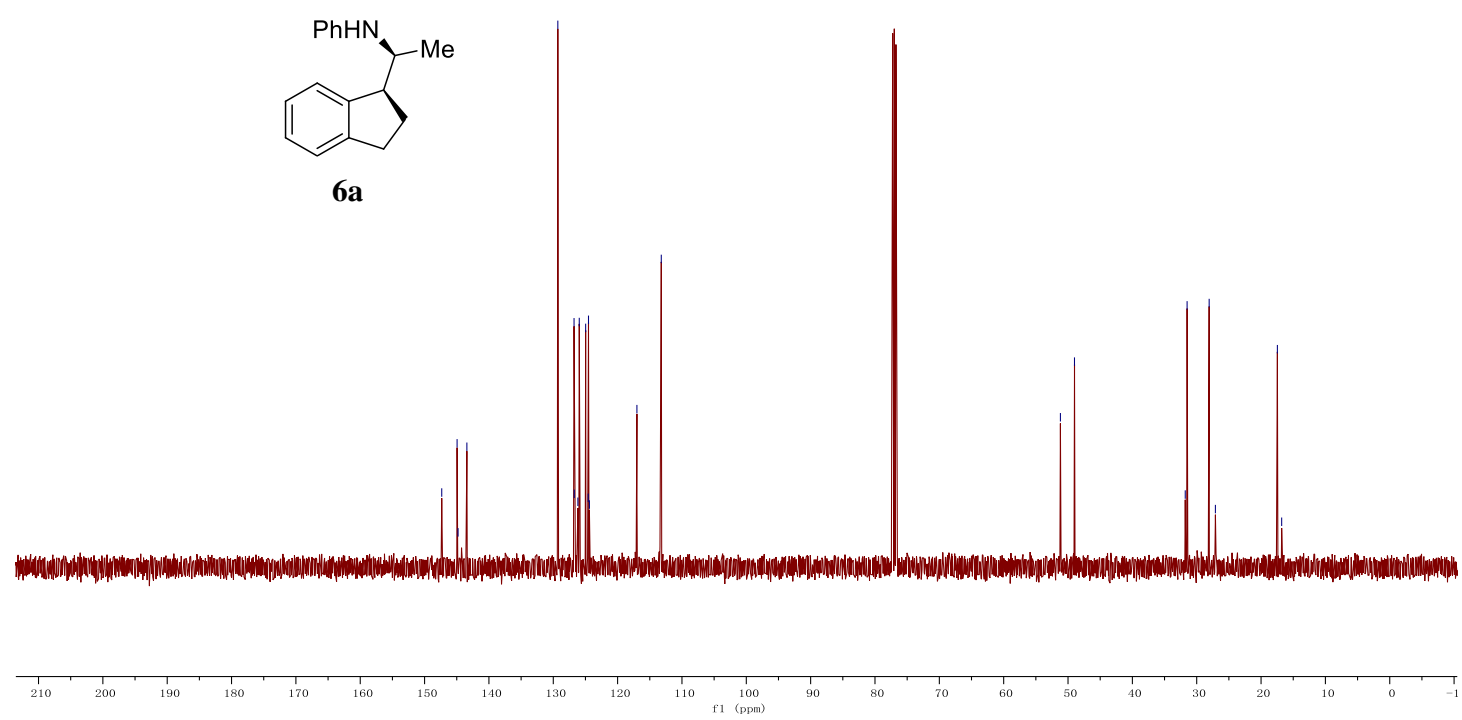


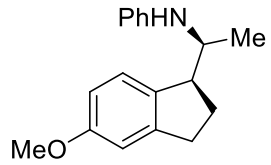

6b

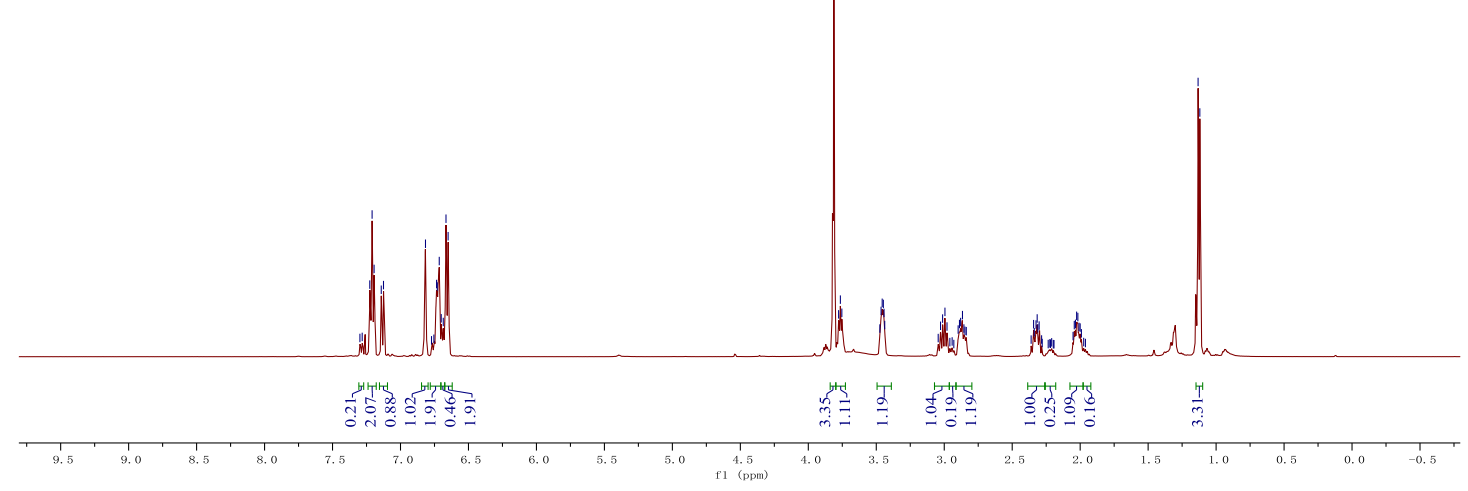

$5-\mathrm{OMe}-\mathrm{rzq}-6-184 / 3$

$13 \mathrm{C}$ AMX500

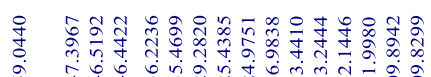

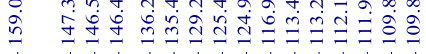

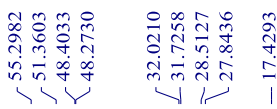

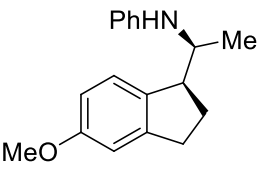

6b 


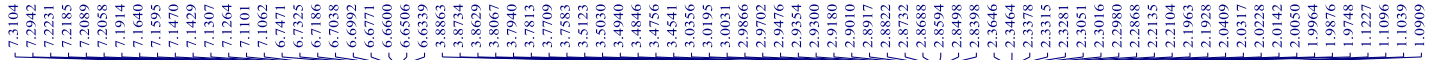

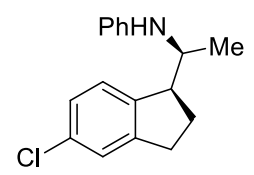

6c

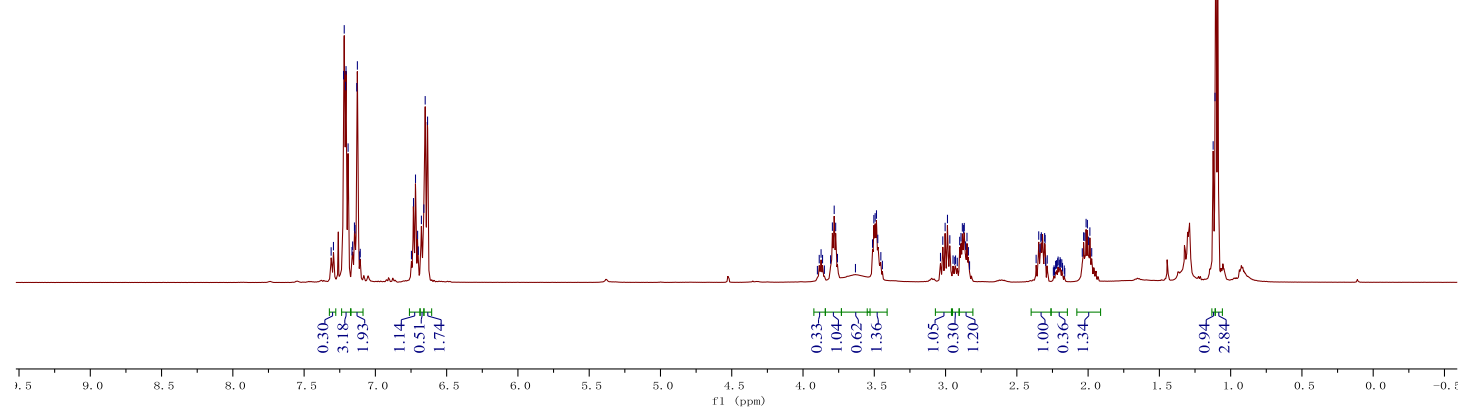

5-C1-rzq-6-187-500M/6

13C AMX500
5-Cl five

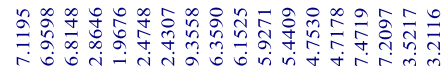

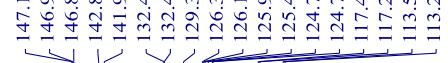

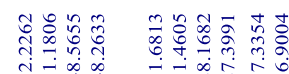

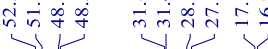

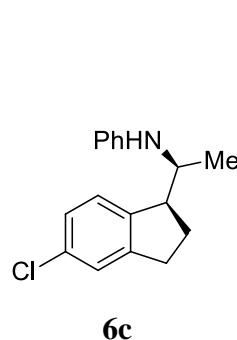

6c 


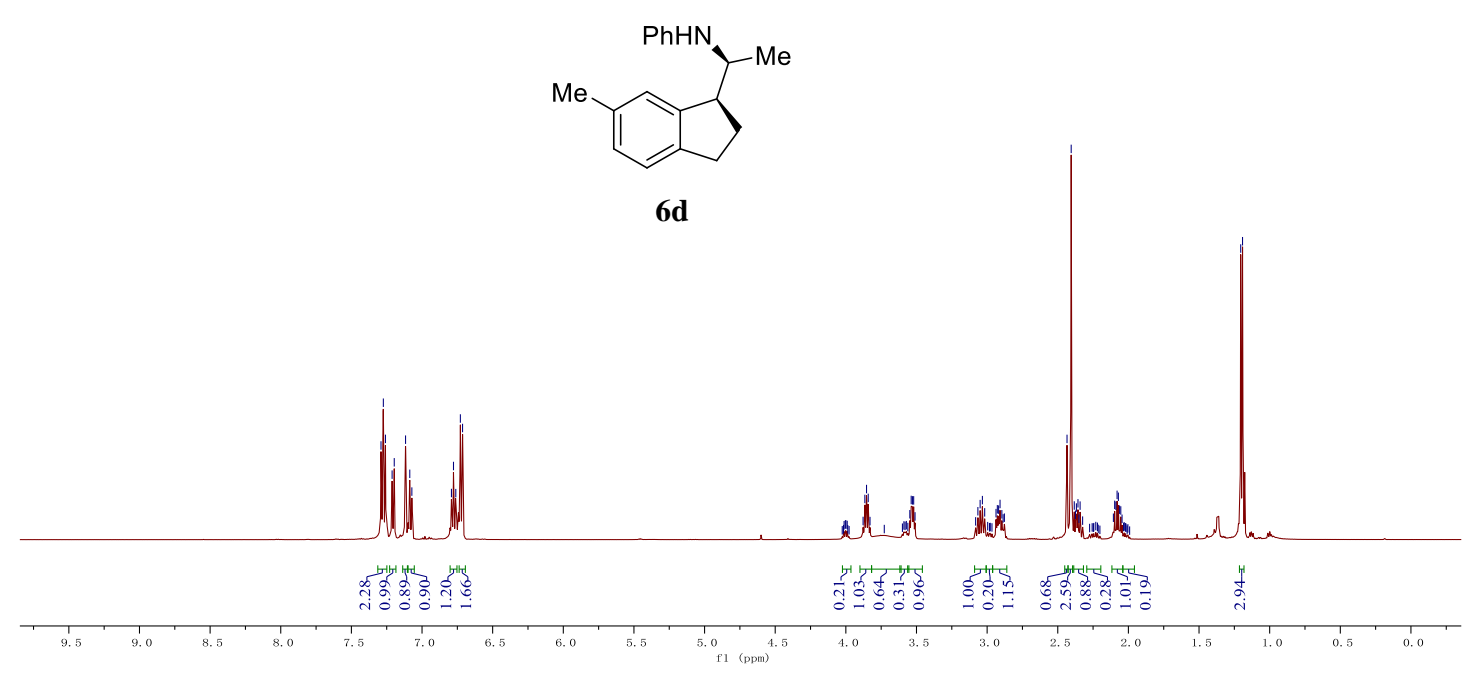

$6-\mathrm{Me}-\mathrm{rzq}-6-185 / 6$

13C AMX500

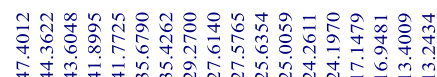

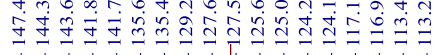

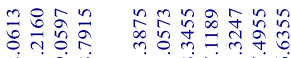

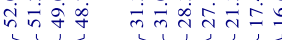

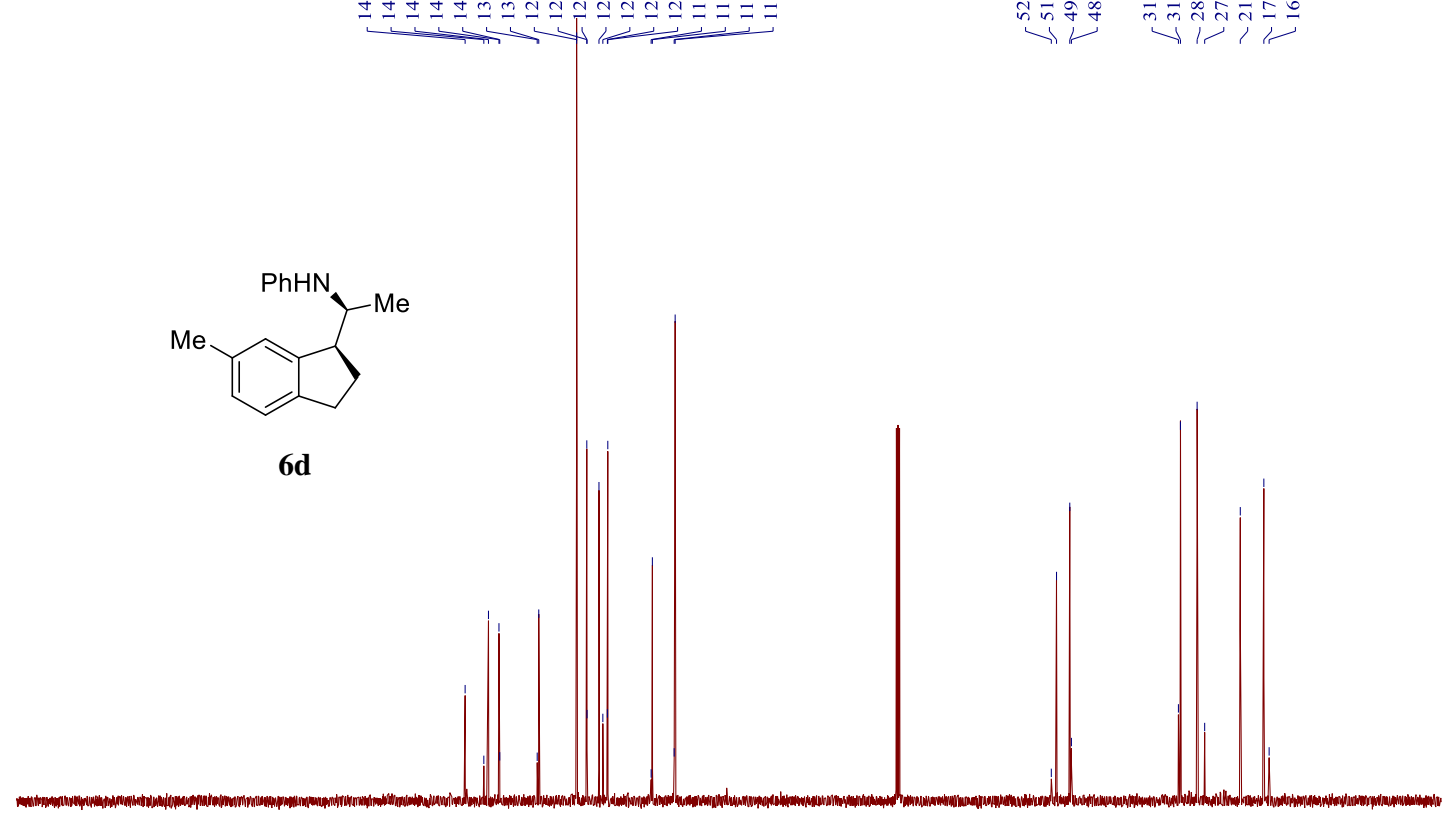

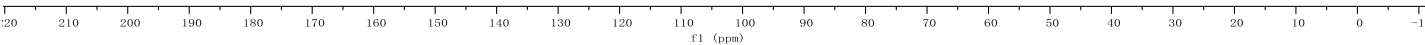



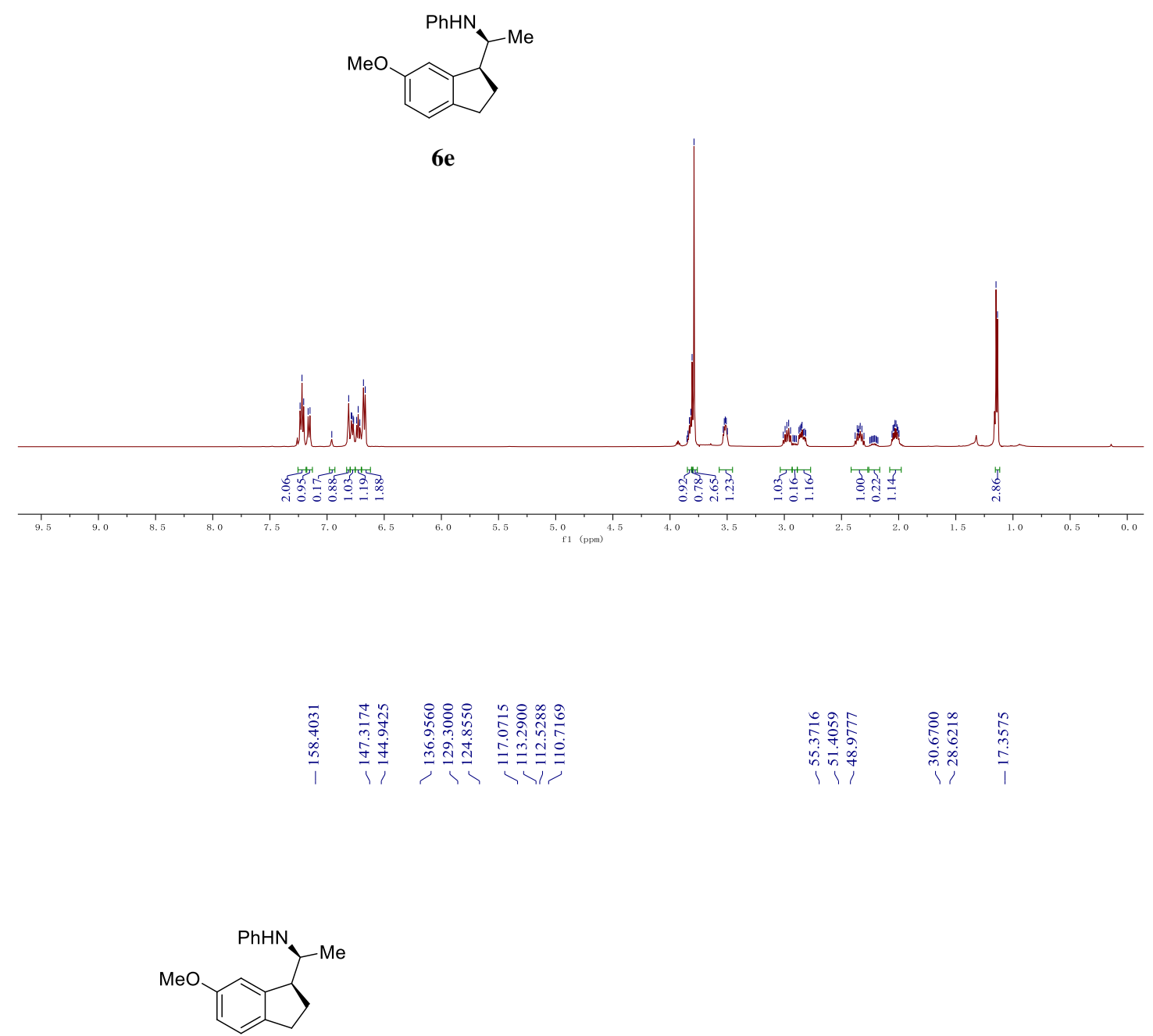

$6 e$ 

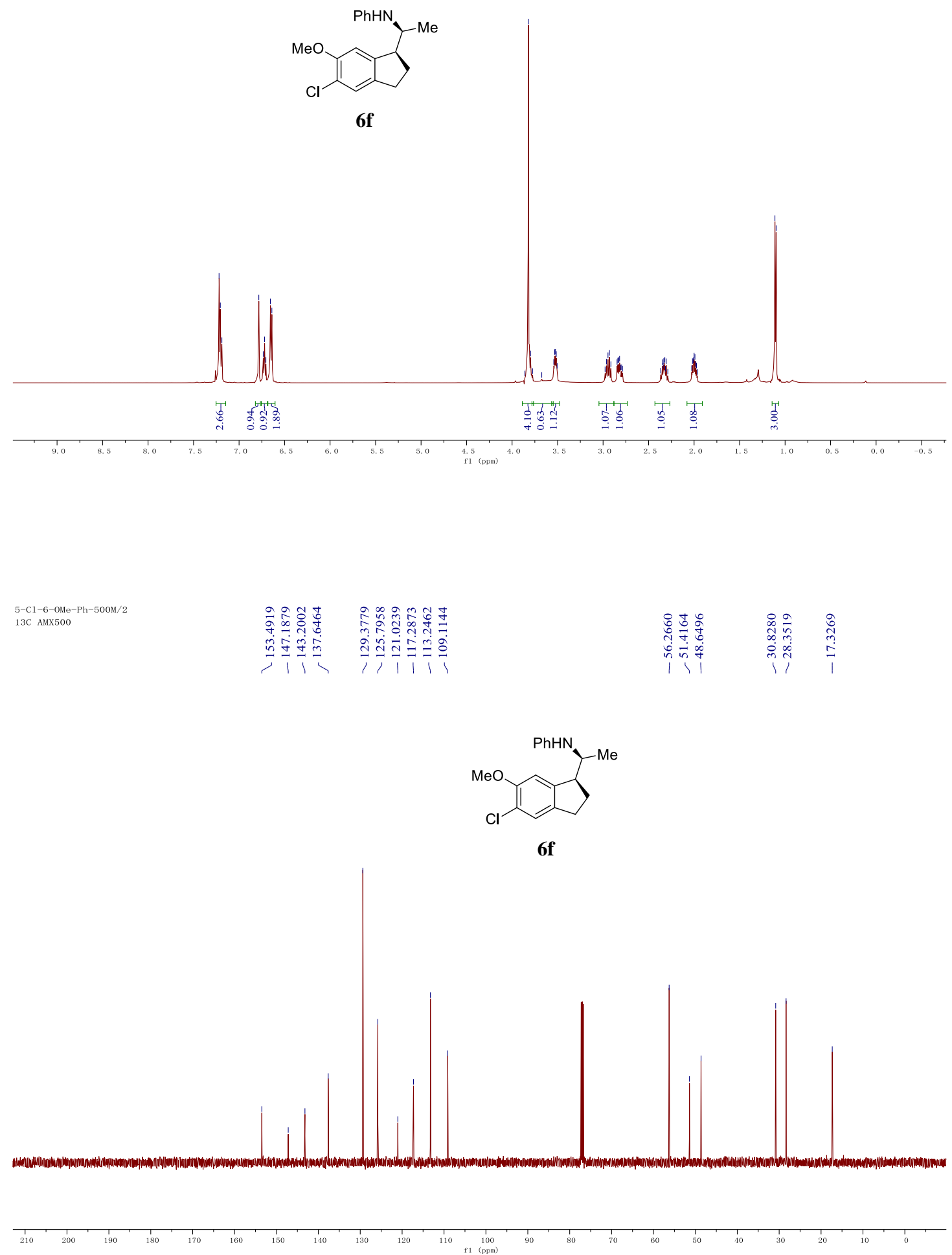


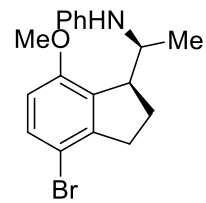

$6 \mathrm{~g}$

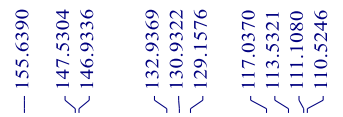

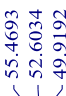

$\begin{array}{ccc}0 & 0 \\ 0 & 0 \\ 0 & 0 \\ 0 & 0 \\ 0 & 0 & 0 \\ 1 & 1 & 1 \\ 1 & 1 & 1\end{array}$<smiles>COc1ccc(Br)c2c1C([C@H](C)NP)CC2</smiles>

$6 \mathrm{~g}$

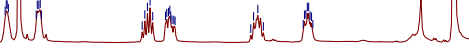

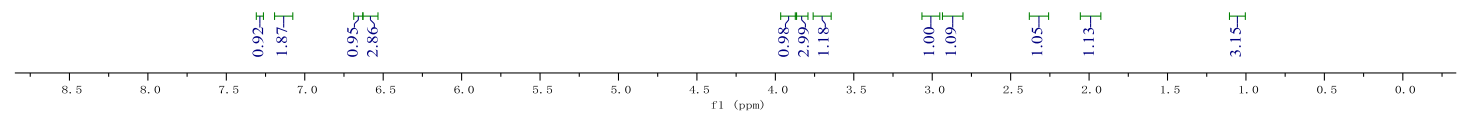

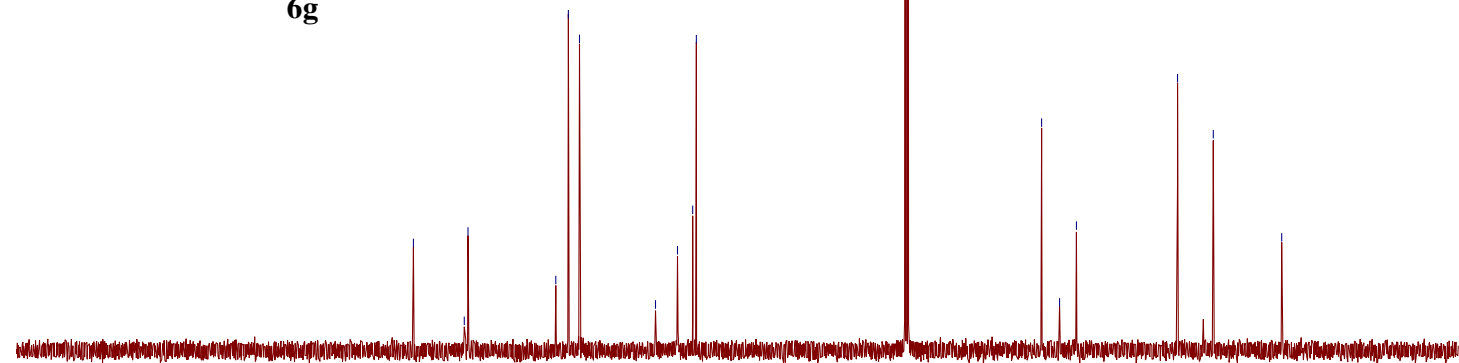




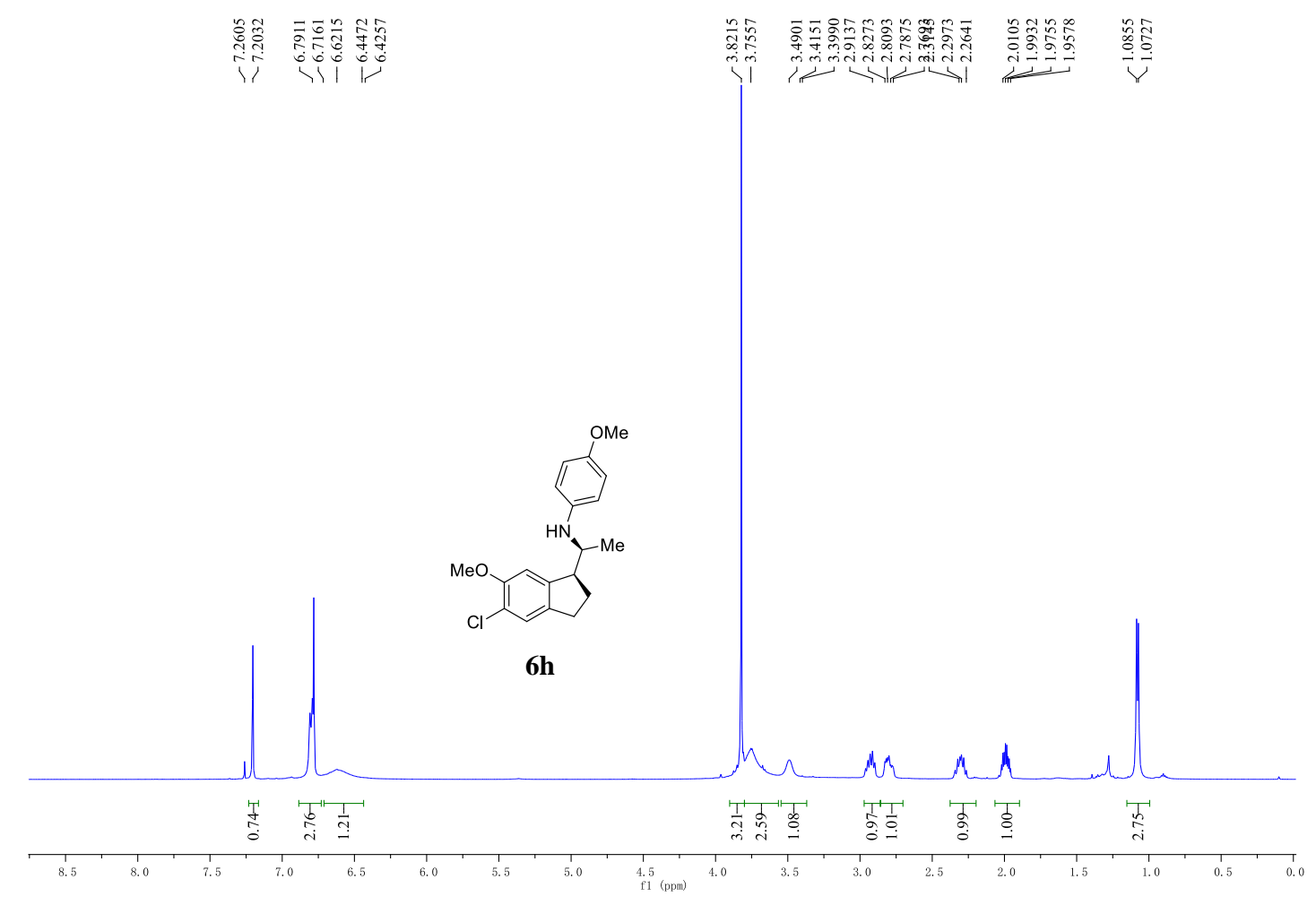

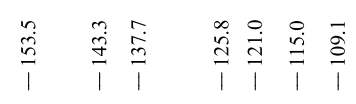

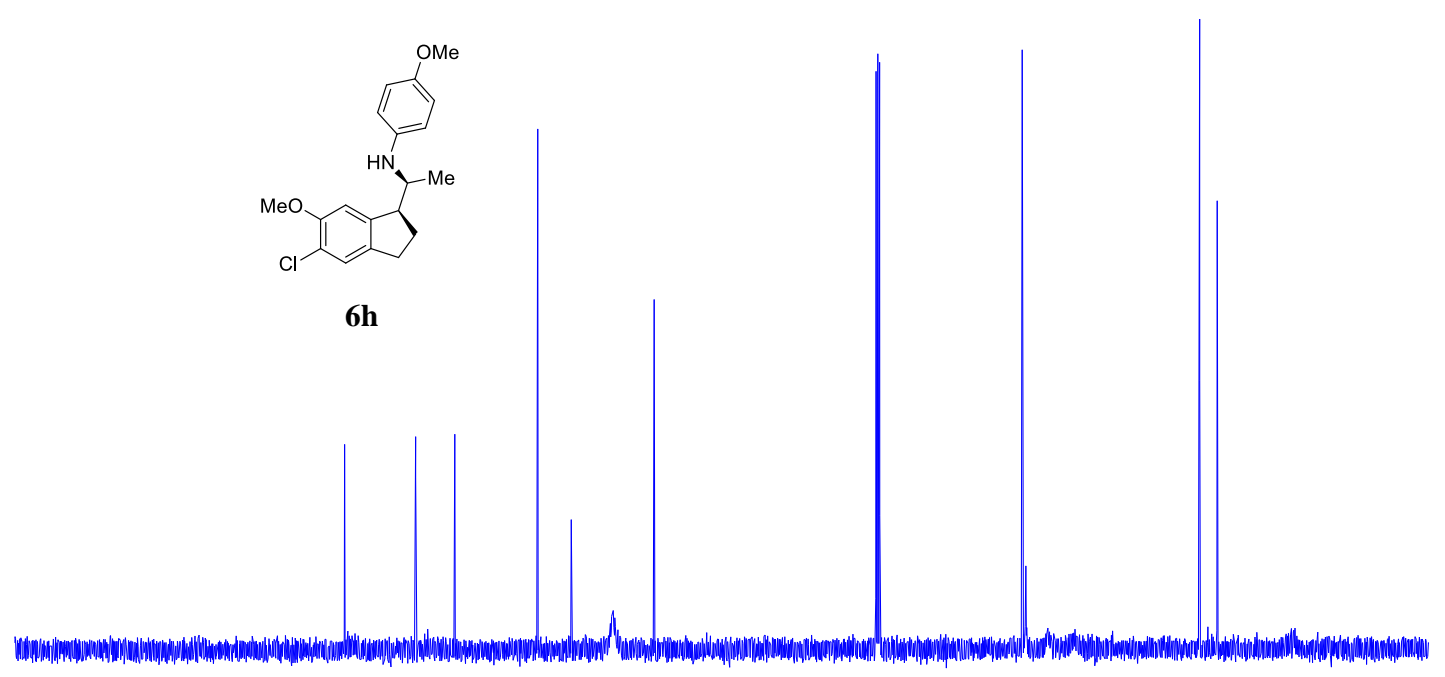




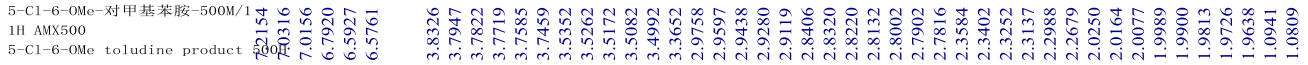

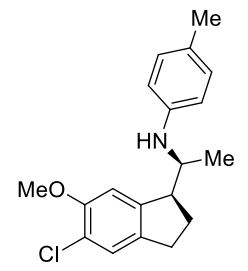

6i

5 - Cl- 6 - OMe-对甲基苯胺 $500 \mathrm{M} / 2$

$13 \mathrm{C}$ AMX500

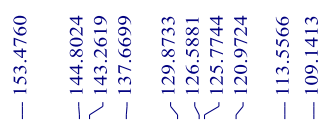

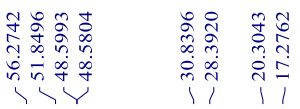

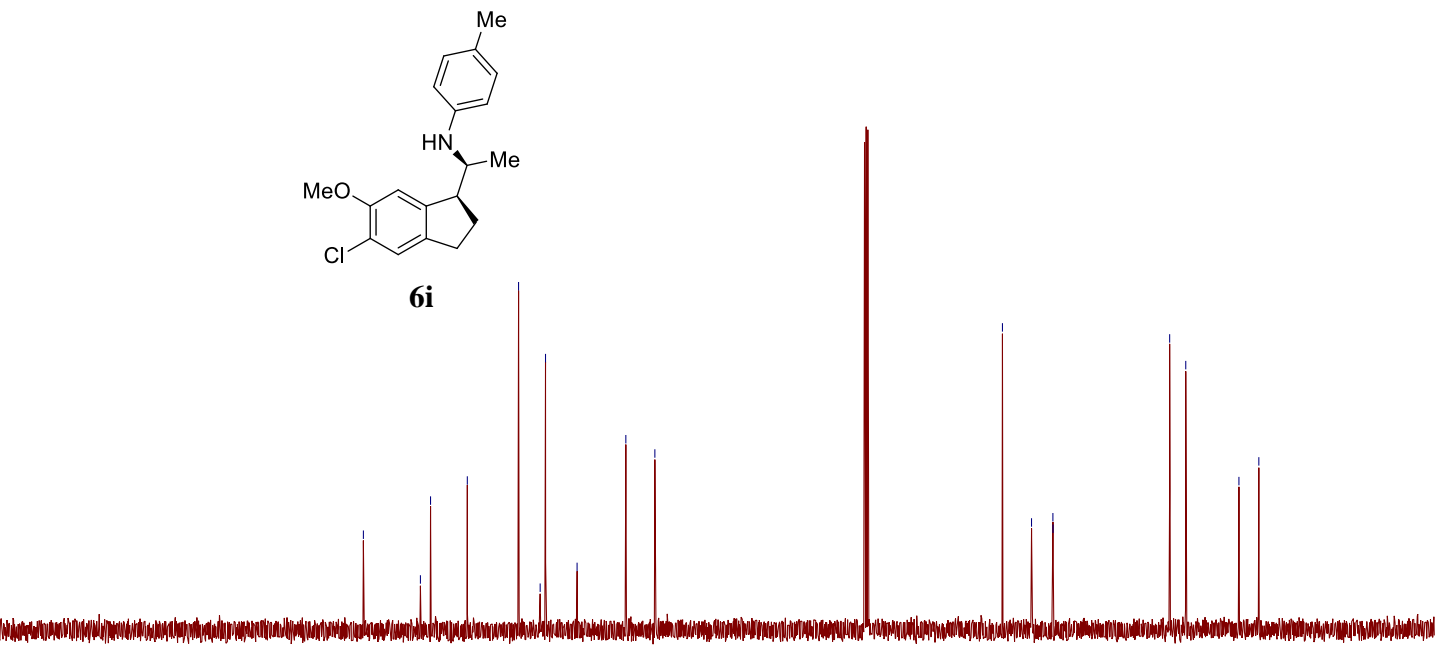

6i
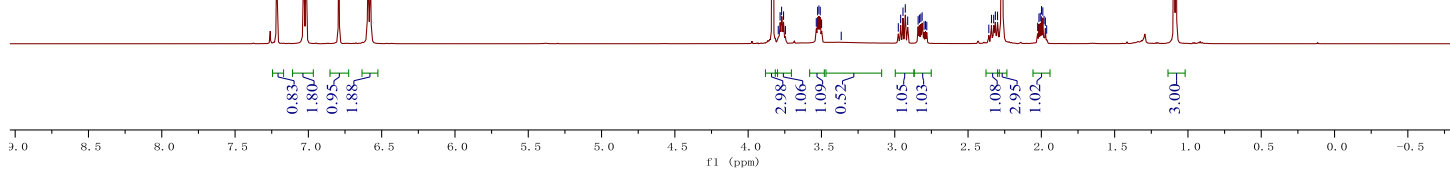


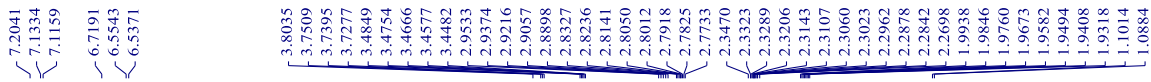

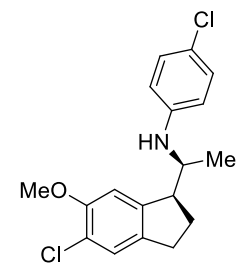

6j

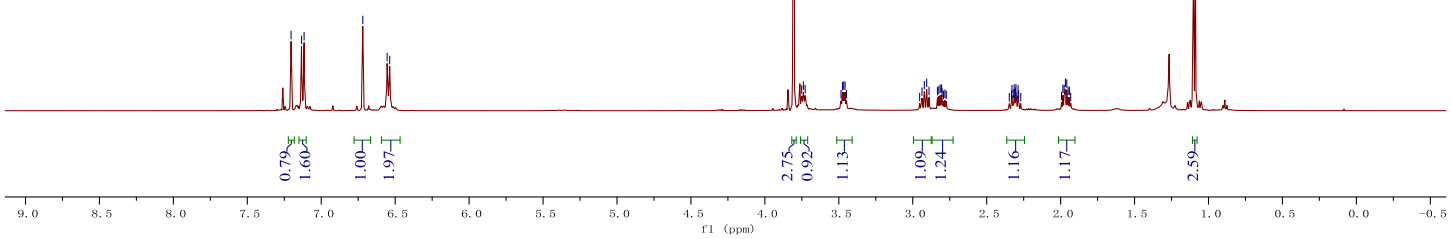

5-Cl-6-OMe-4-Cl-aniline-500M/4

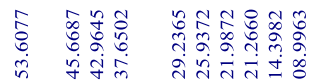

5-C1-6-OMe 4-C1 product $500 \mathrm{C}$

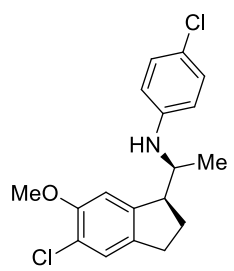

6j

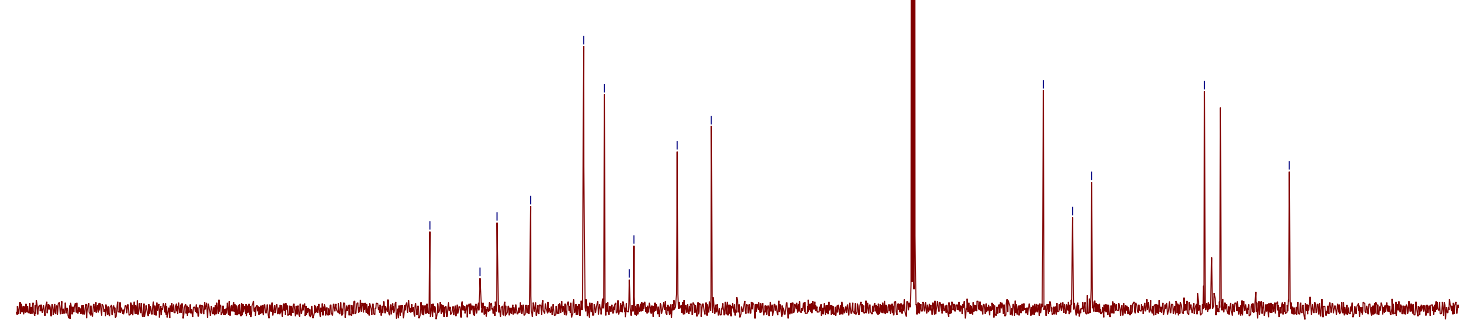



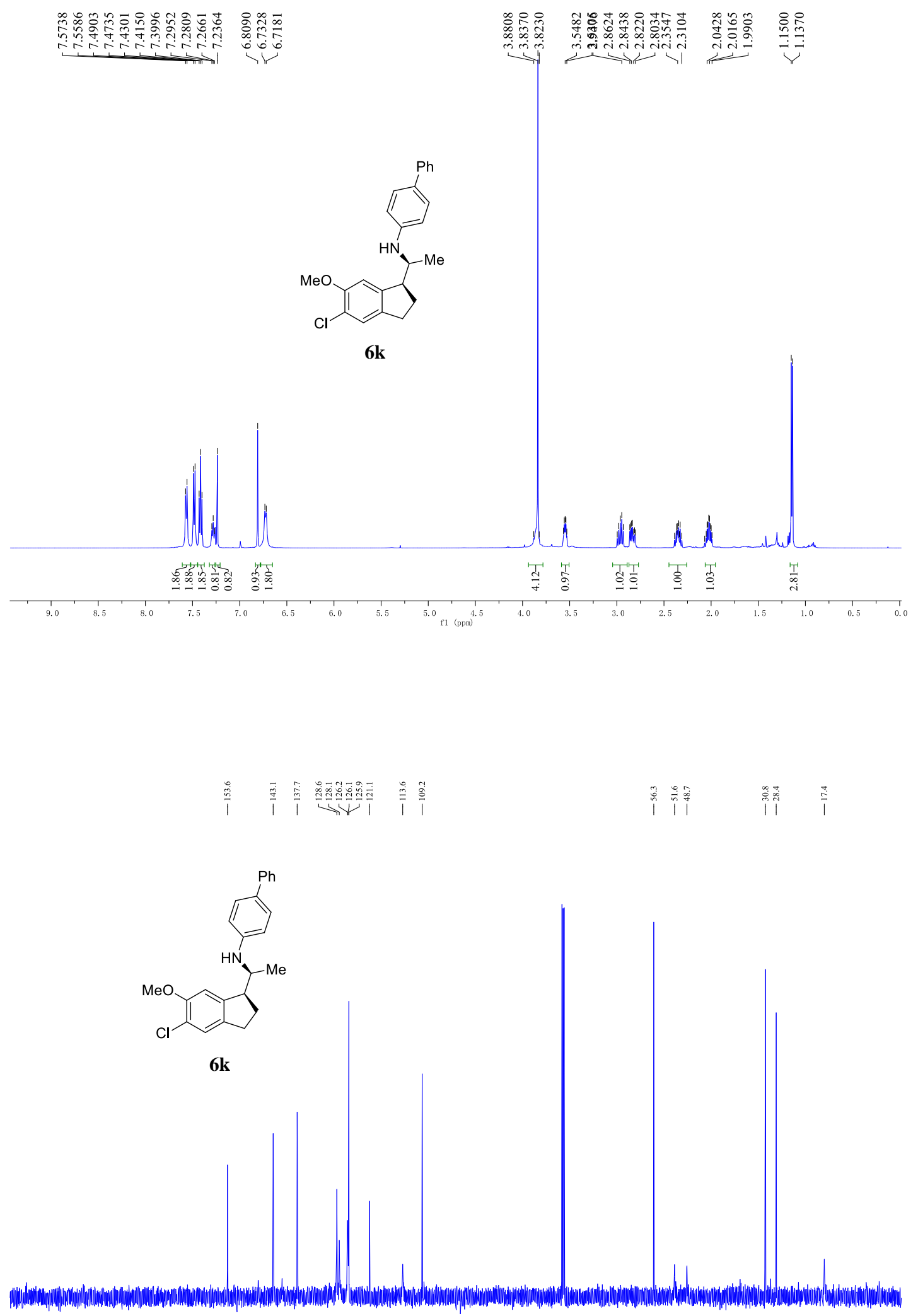


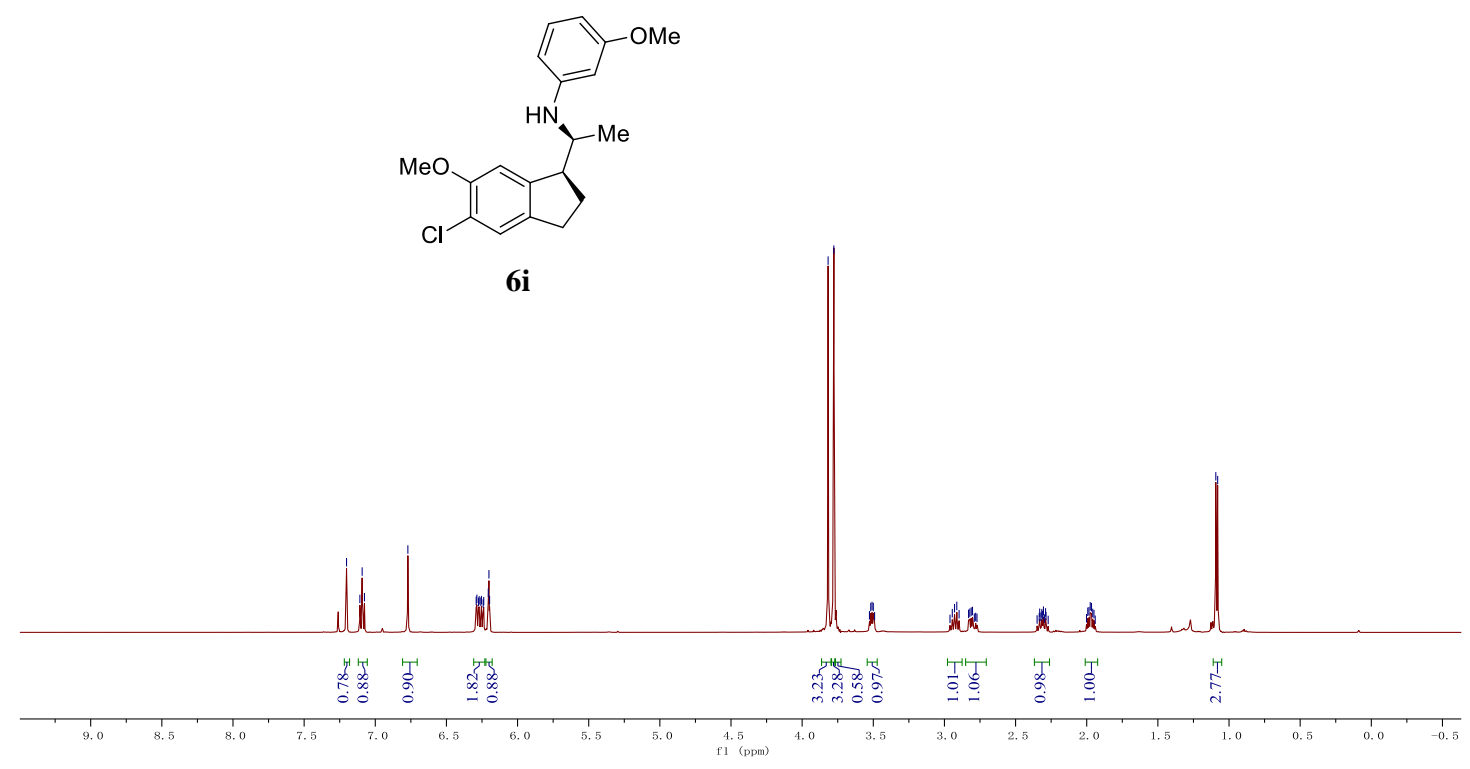

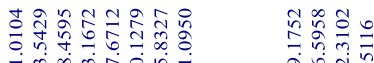

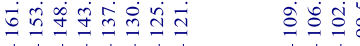

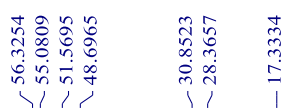

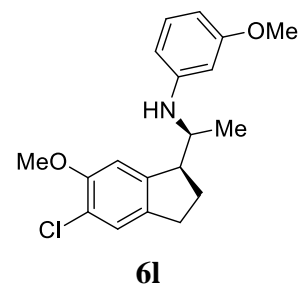

$6 \mathbf{1}$

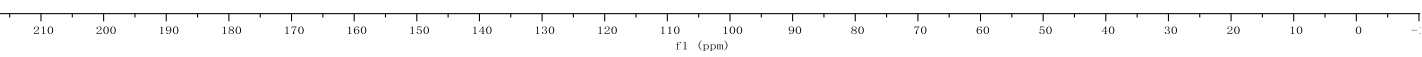



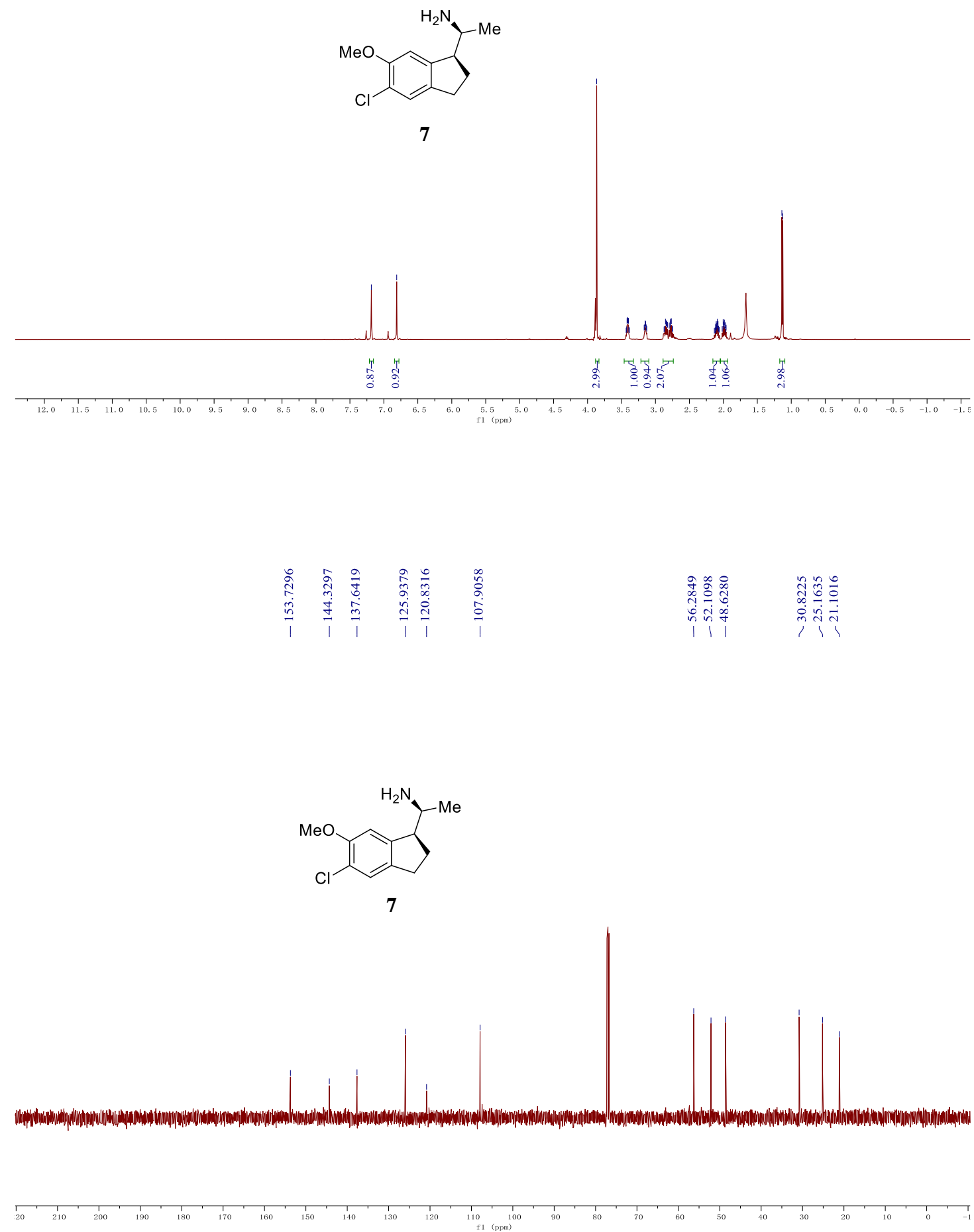


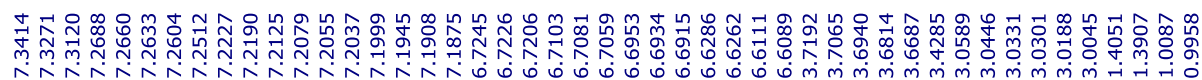

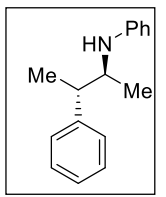

9a
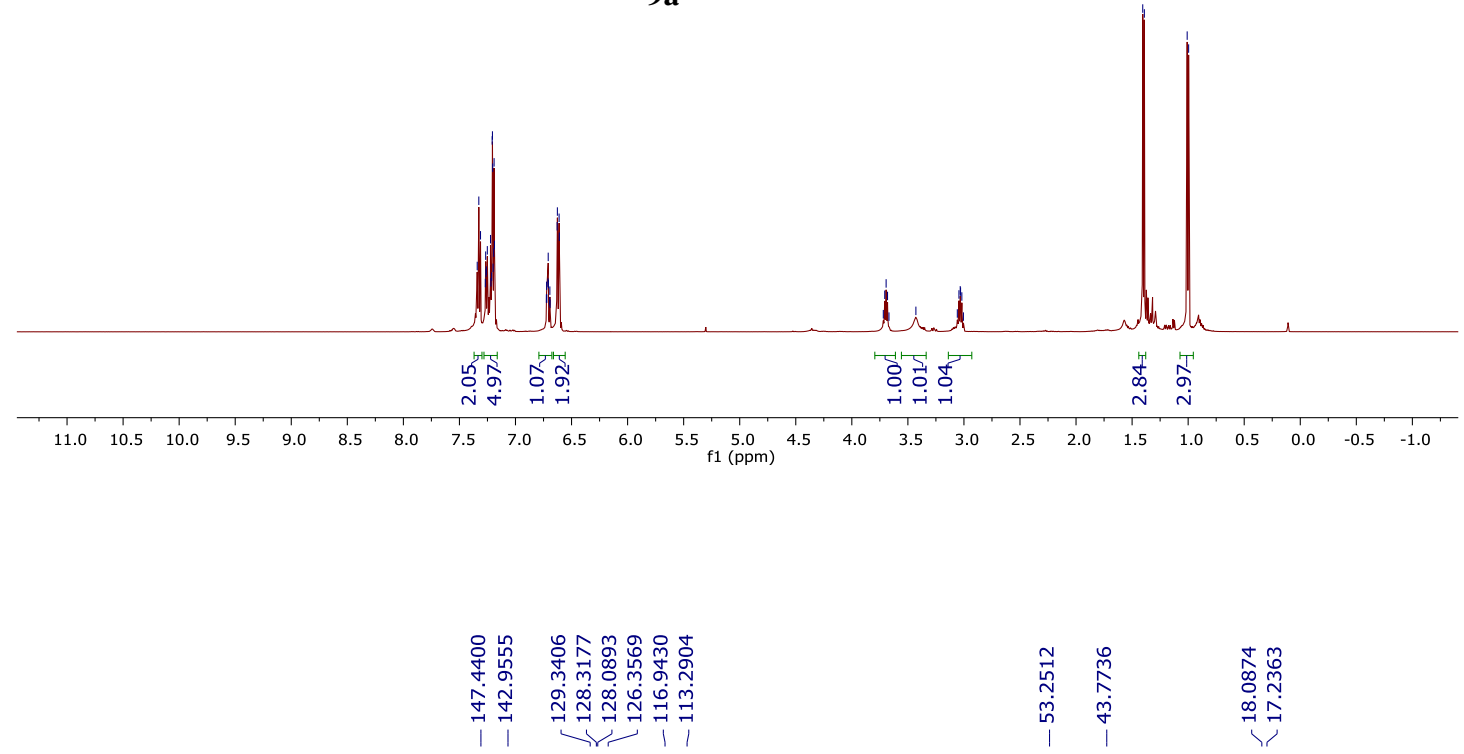

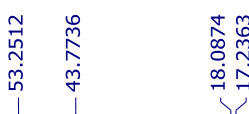
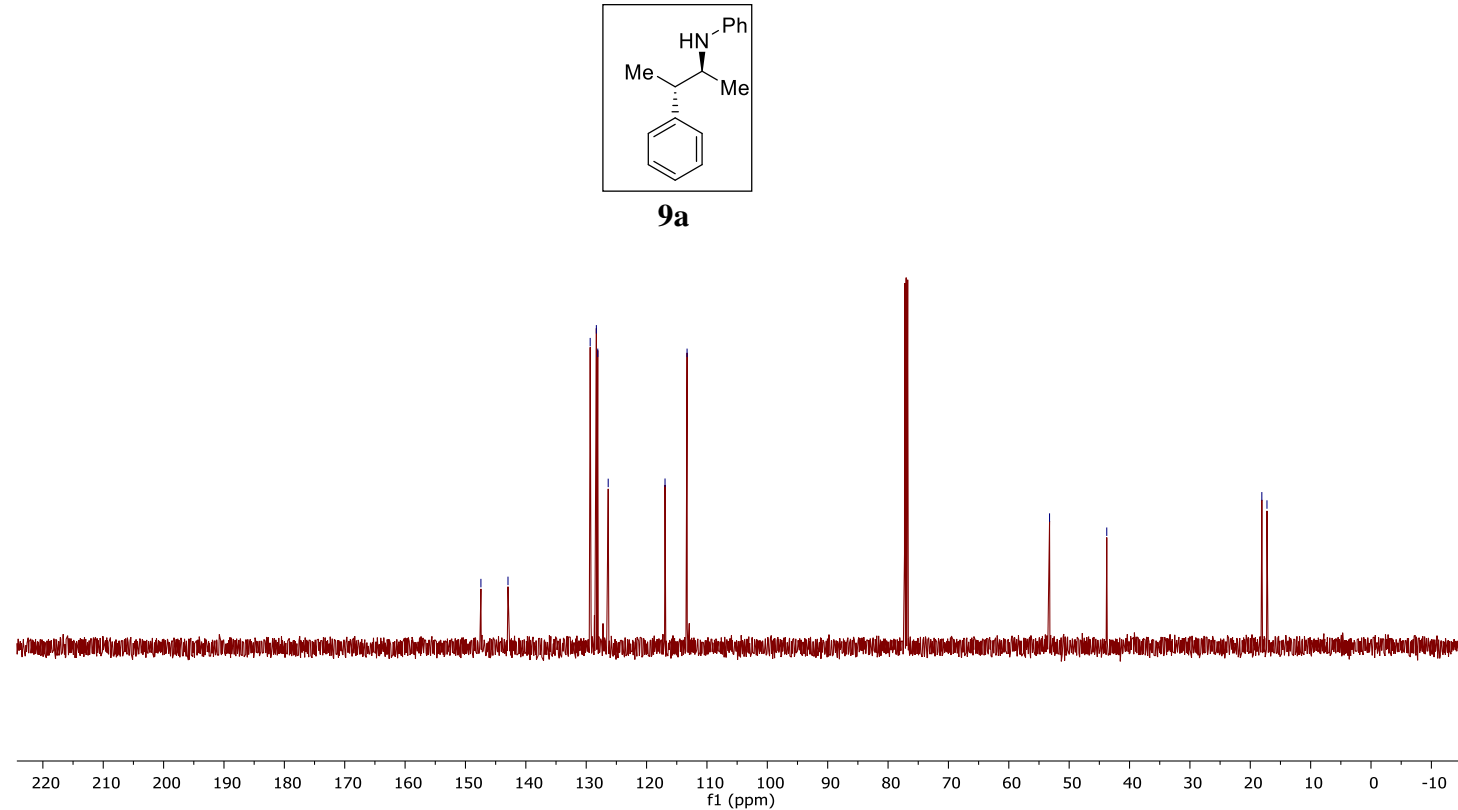


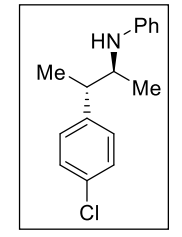

9b

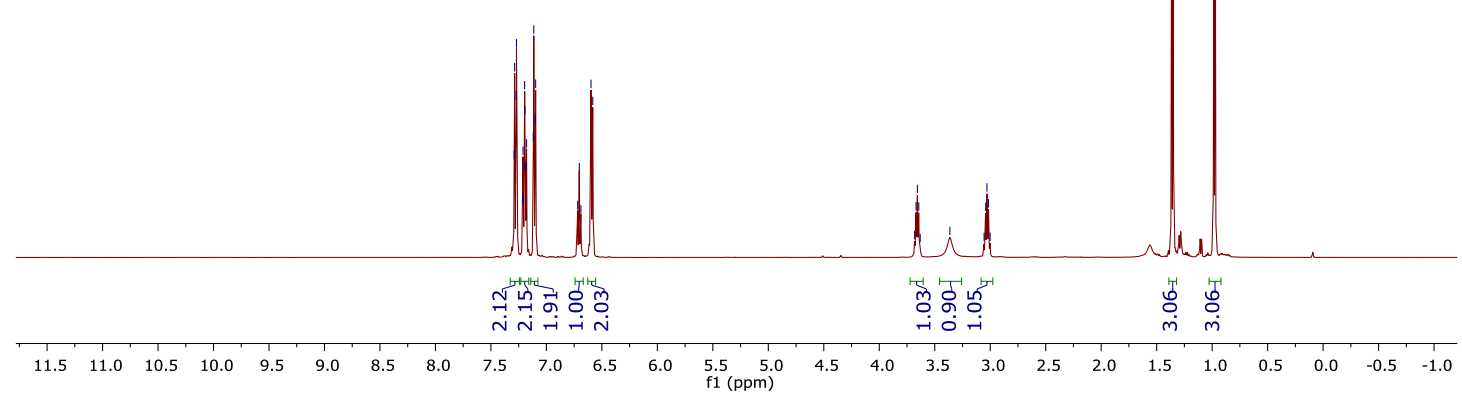

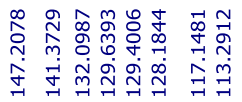

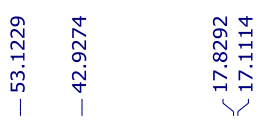
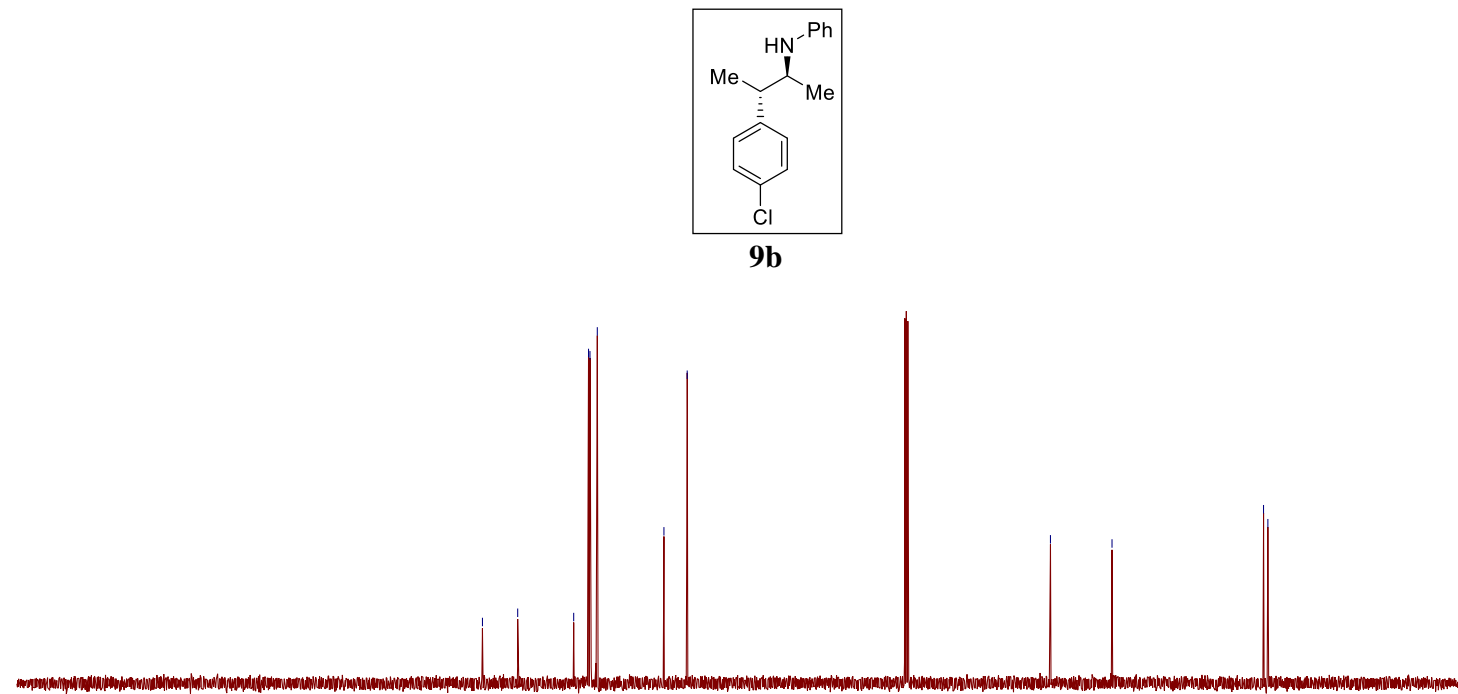

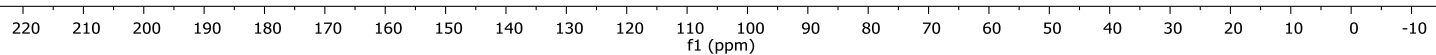




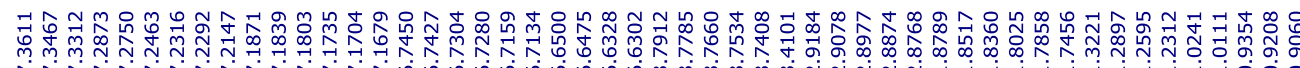
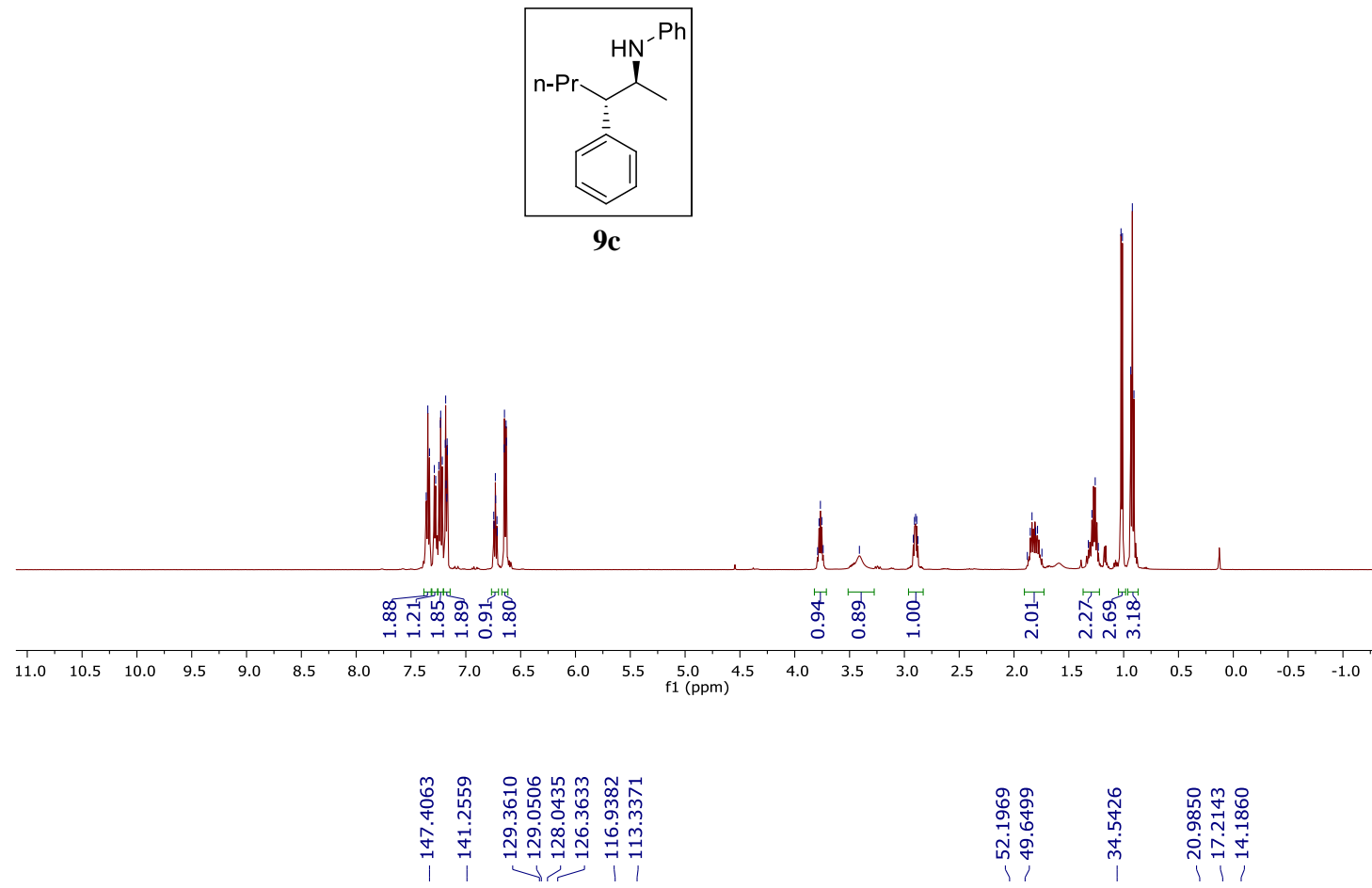

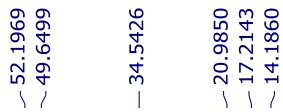
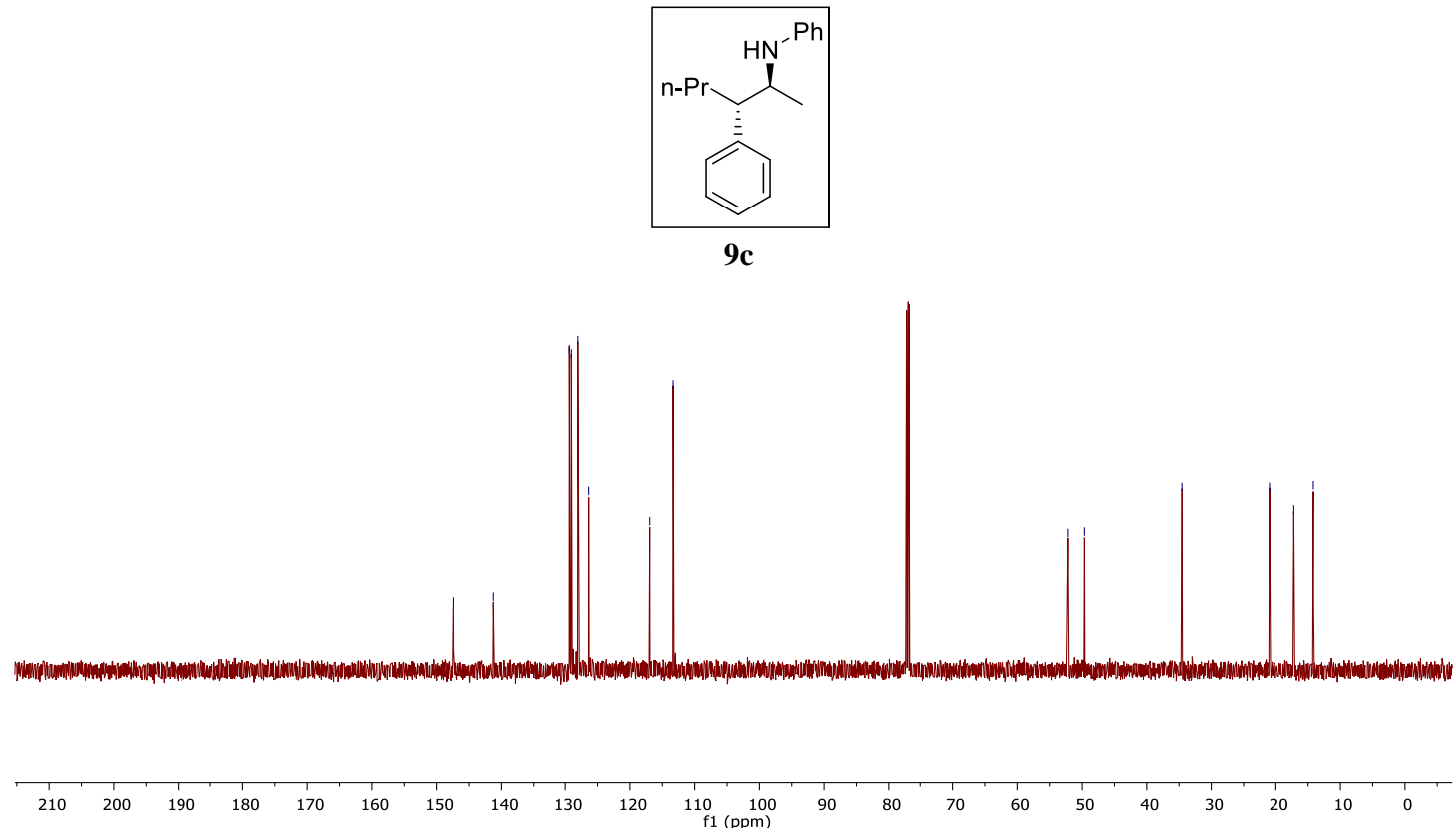


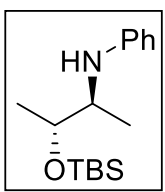

9d
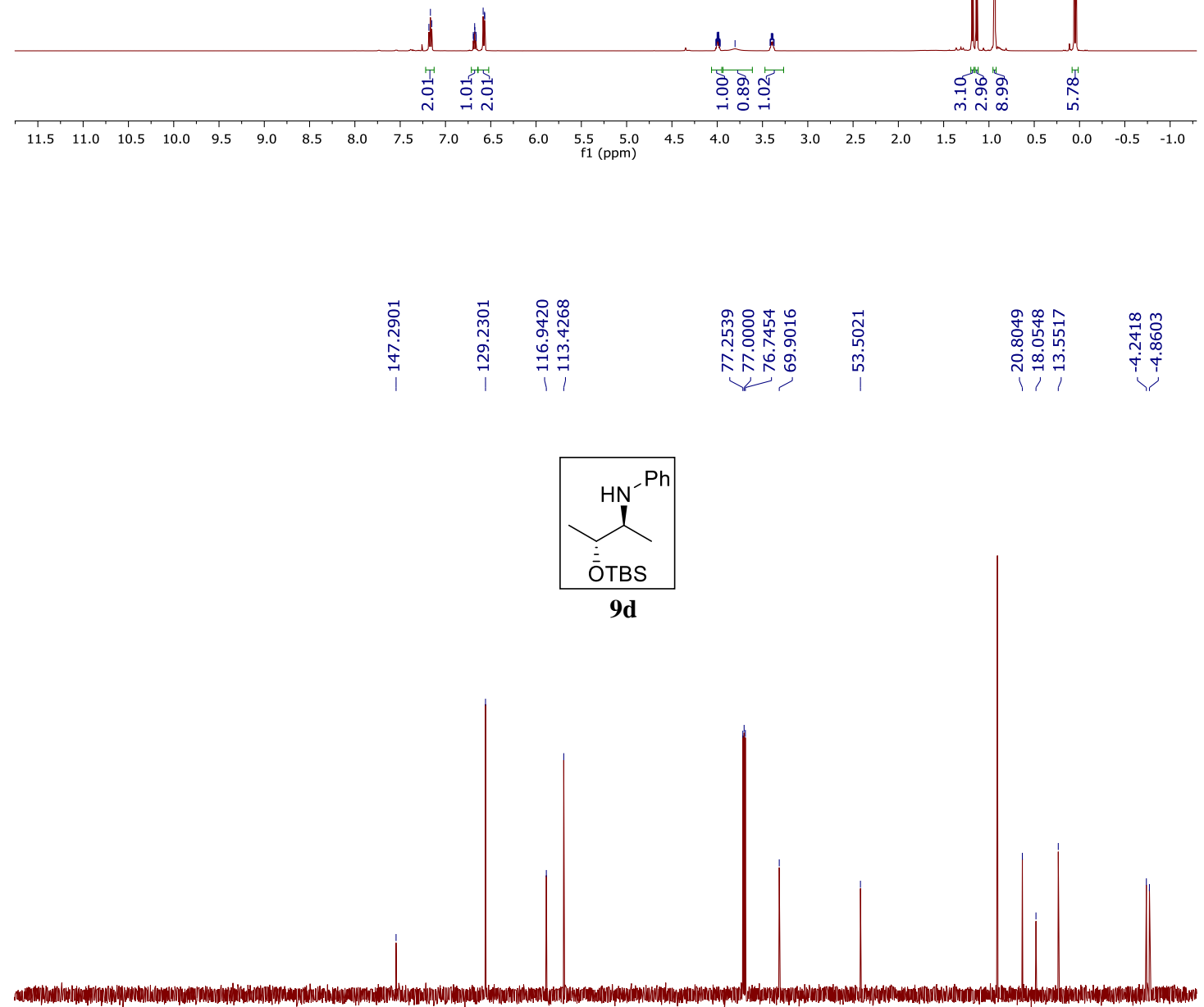

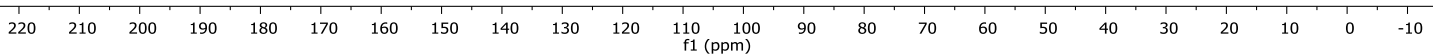




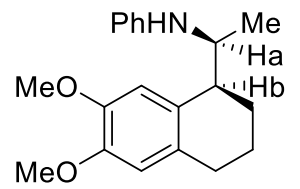

The cis relative configuration of $\mathbf{4 h}$ was determined by the NOESY correlation between the Ha and $\mathrm{Hb}$ (Scheme $\mathrm{S} 2)$.

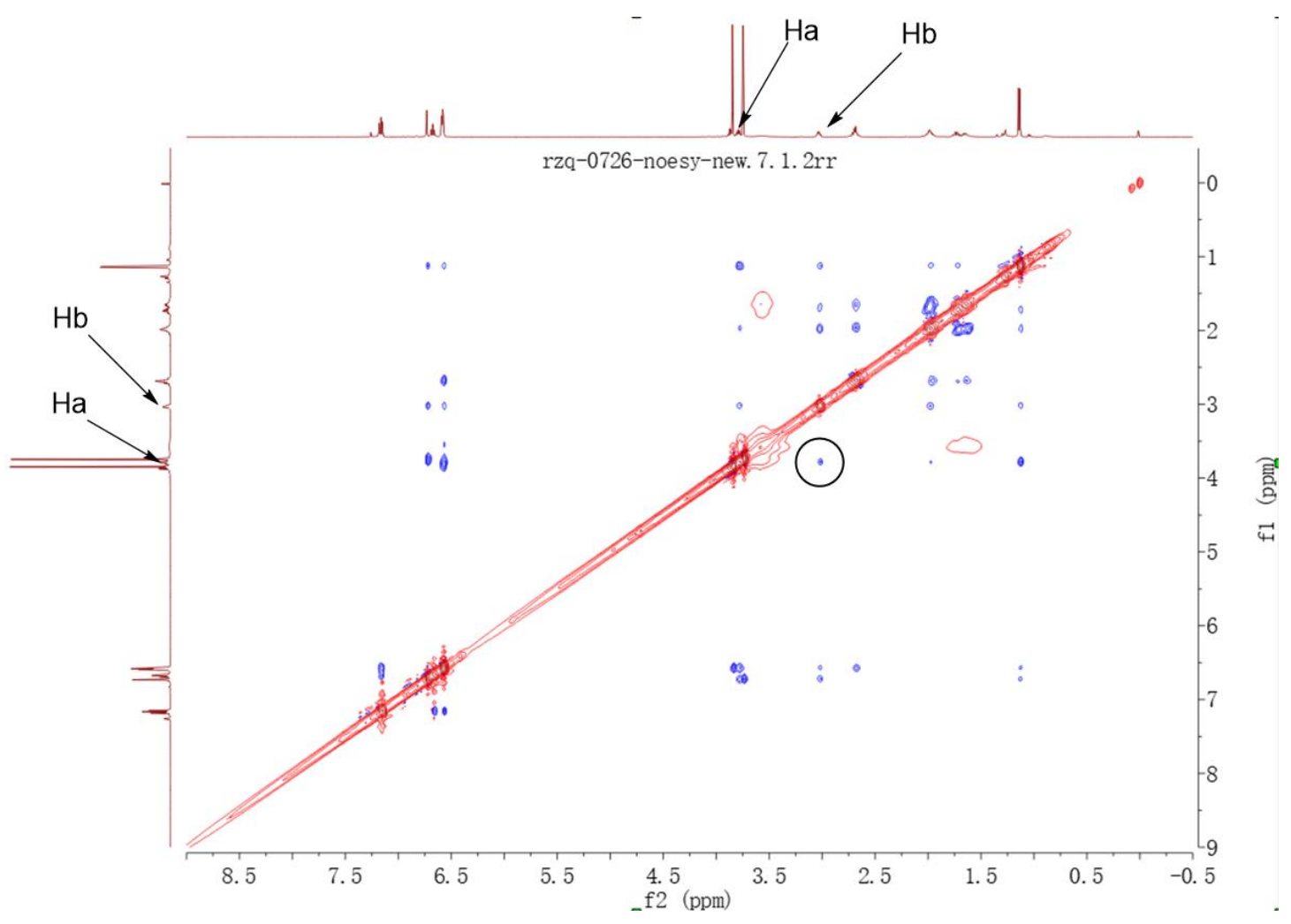

Scheme S2. The NOESY spectrum of $\mathbf{4 h}$ 


\section{References}

1. Zhang, Y.; Lim, C.-S.; Sim, D. S. B.; Pan, H.-J.; Zhao, Y. Angew. Chem. Int. Ed. 2014, 53, 1399-1403.

2. (a) Čorić, I.; Müller, S.; List, B. J. Am. Chem. Soc. 2010, 132, 17370. (b) Xu, B.; Zhu, S.F.; Xie, X.-L.; Shen, J.-J.; Zhou, Q.-L. Angew. Chem. Int. Ed. 2011, 50, 11483.

3. (a) Rong, Z.-Q.; Zhang, Y.; Chua, H. B. R.; Pan, H.-J.; Zhao, Y. J. Am. Chem. Soc. 2015, 137, 4944. (b) Hoffmann, S.; Nicoletti, M.; List, B. J. Am. Chem. Soc. 2006, 128, 13074. (c) Xie, J.-H.; Zhou, Z.-T.; Kong, W.-L.; Zhou, Q.-L. J. Am. Chem. Soc. 2007, 129, 1868.

4. Wakchaure, V. N.; Zhou, J.; Hoffmann, S.; List, B. Angew. Chem. Int. Ed. 2010, 49, 4612. 


\section{Computation}

\section{Table of contents}

1. Completes reference for Gaussian 09

Page S82

2. Computational Methods

Page S82

3. Free energy profiles of the first hydrogen transfer step with reactant 1a (Figure S1), and free energy profilels of Ir-catalyzed dynamic kinetic asymmetric amination reaction of reactant $\mathbf{8 a}$ (Figure S2, S3 and S4)

Page S83

4. B3LYP and M0b-2X absolute calculation energies, enthalpies, and free energies

Page S87

5. B3LYP geometries for all the optimized compounds and transition states

Page S90

6. References

Page S104 


\section{Complete reference for Gaussian 09}

Frisch, M. J.; Trucks, G. W.; Schlegel, H. B.; Scuseria, G. E.; Robb, M. A.; Cheeseman, J. R.; Montgomery, J. A., Jr.; Vreven, T.; Kudin, K. N.; Burant, J. C.; Millam, J. M.; Iyengar, S. S.; Tomasi, J.; Barone, V.; Mennucci, B.; Cossi, M.; Scalmani, G.; Rega, N.; Petersson, G. A.; Nakatsuji, H.; Hada, M.; Ehara, M.; Toyota, K.; Fukuda, R.; Hasegawa, J.; Ishida, M.; Nakajima, T.; Honda, Y.; Kitao, O.; Nakai, H.; Klene, M.; Li, X.; Knox, J. E.; Hratchian, H. P.; Cross, J. B.; Adamo, C.; Jaramillo, J.; Gomperts, R.; Stratmann, R. E.; Yazyev, O.; Austin, A. J.; Cammi, R.; Pomelli, C.; Ochterski, J. W.; Ayala, P. Y.; Morokuma, K.; Voth, G. A.; Salvador, P.; Dannenberg, J. J.; Zakrzewski, V. G.; Dapprich, S.; Daniels, A. D.; Strain, M. C.; Farkas, O.; Malick, D. K.; Rabuck, A. D.; Raghavachari, K.; Foresman, J. B.; Ortiz, J. V.; Cui, Q.; Baboul, A. G.; Clifford, S.; Cioslowski, J.; Stefanov, B. B.; Liu, G.; Liashenko, A.; Piskorz, P.; Komaromi, I.; Martin, R. L.; Fox, D. J.; Keith, T.; Al-Laham, M. A.; Peng, C. Y.; Nanayakkara, A.; Challacombe, M.; Gill, P. M. W.; Johnson, B.; Chen, W.; Wong, M. W.; Gonzalez, C.; Pople, J. A. Gaussian 09, revision D.01; Gaussian, Inc.: Wallingford, CT, 2013.

\section{Computational methods.}

All of the DFT calculations conducted in this study were carried out using the GAUSSIAN 09 series of programs. DFT method B3LYP ${ }^{1}$ with a standard 6-31G(d) ${ }^{2}$ basis set (SDD basis set ${ }^{3}$ for Ir) was used for the geometry optimizations. The M06-2X, proposed by Truhlar et al., ${ }^{4}$ was used with a 6-311G(d,p) ${ }^{5}$ basis set (LANL08 (f) ${ }^{6}$ basis set for Ir) to calculate the single point energies in toluene solvent. Solvent effects were taken into consideration using single point calculations based on the gas-phase stationary points with the C-PCM ${ }^{7}$ solvation model. The energies presented in this paper are the M06-2X calculated Gibbs free energies in toluene solvent with B3LYP calculated thermodynamic corrections. 


\section{Free energy profiles of the first hydrogen transfer step with reactant 1a (Figure S1), and free energy profilels}

of Ir-catalyzed dynamic kinetic asymmetric amination reaction of reactant 1a (Figure S2, S3 and S4).

a)

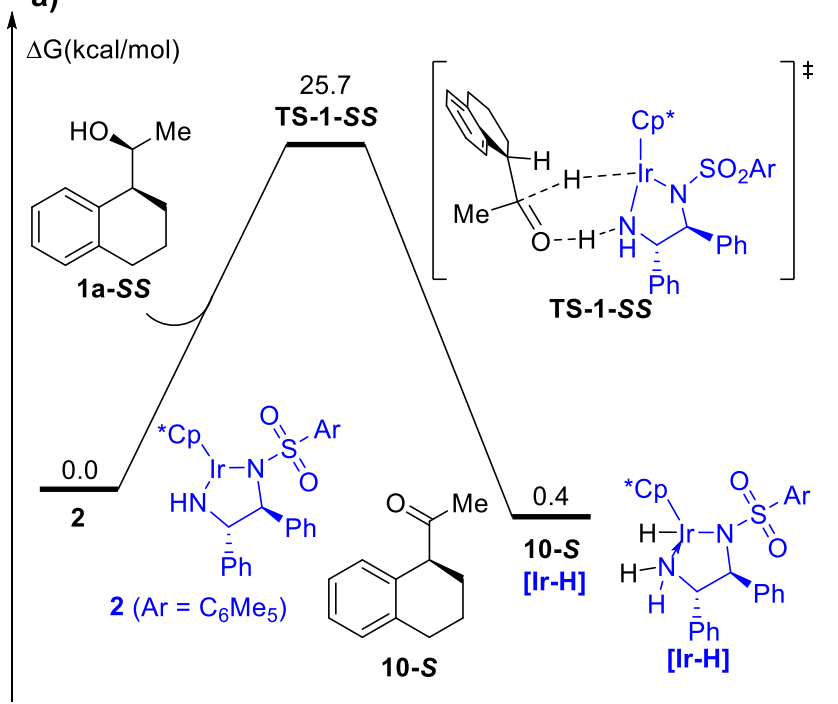

b)

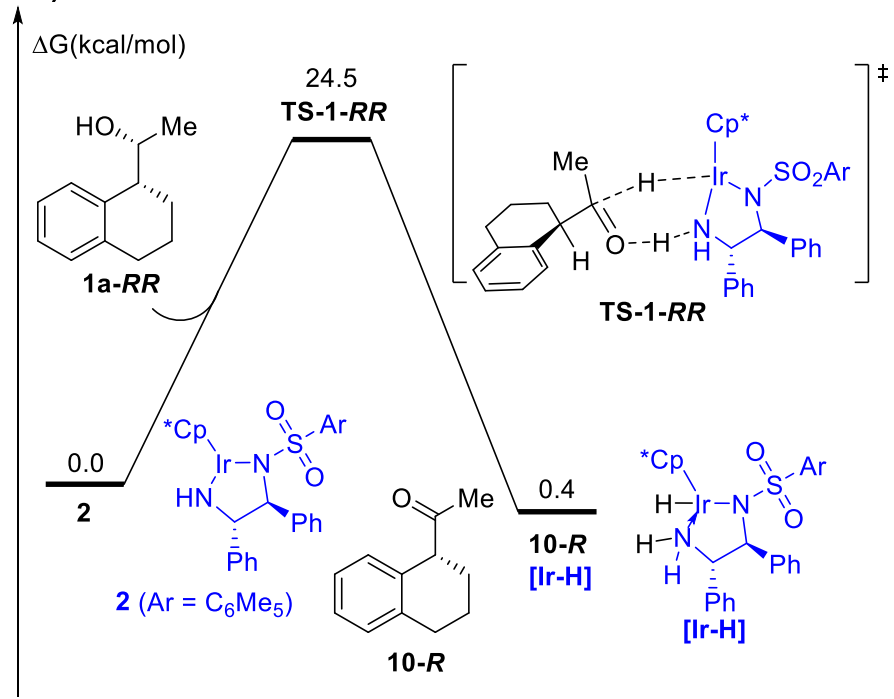

c)

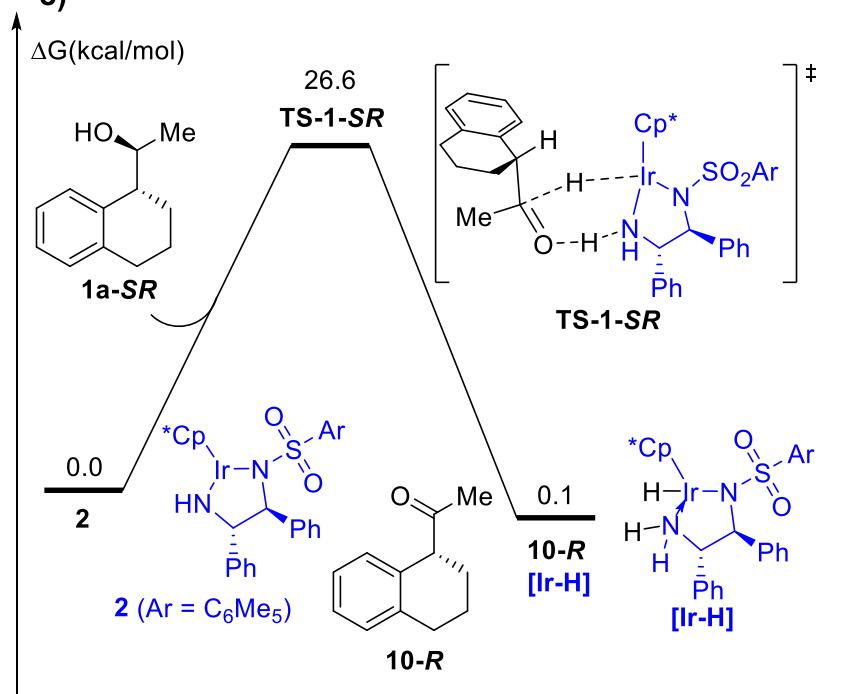

d)

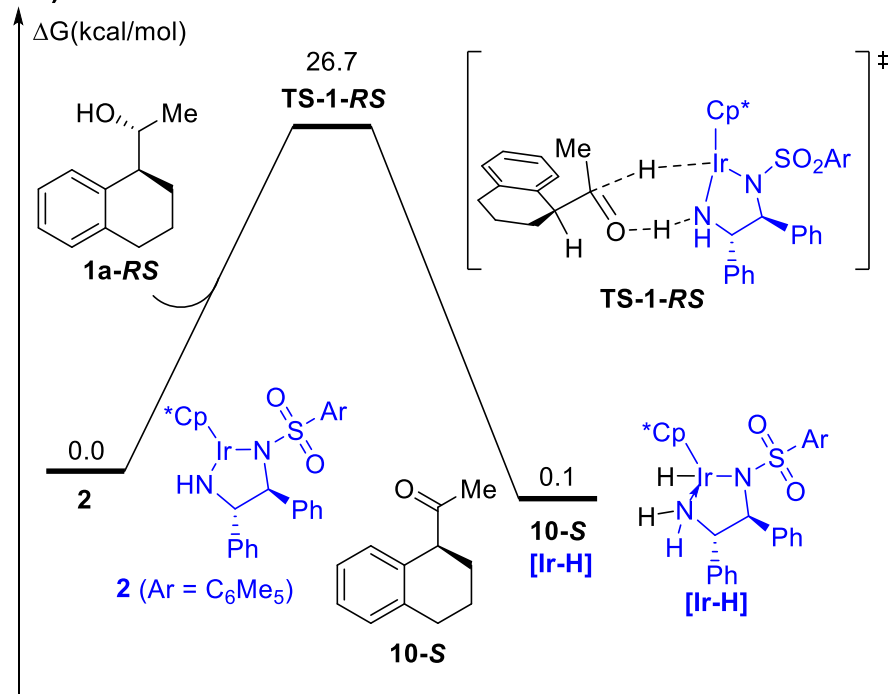

Figure S1. Free energy profiles of the first hydrogen transfer step of Ir-catalyzed dynamic kinetic asymmetric amination reaction of reactant 1a. The energy values are given in $\mathrm{kcal} / \mathrm{mol}$ and represent the relative free energies calculated by the M06-2X in toluene solvent. 
a)

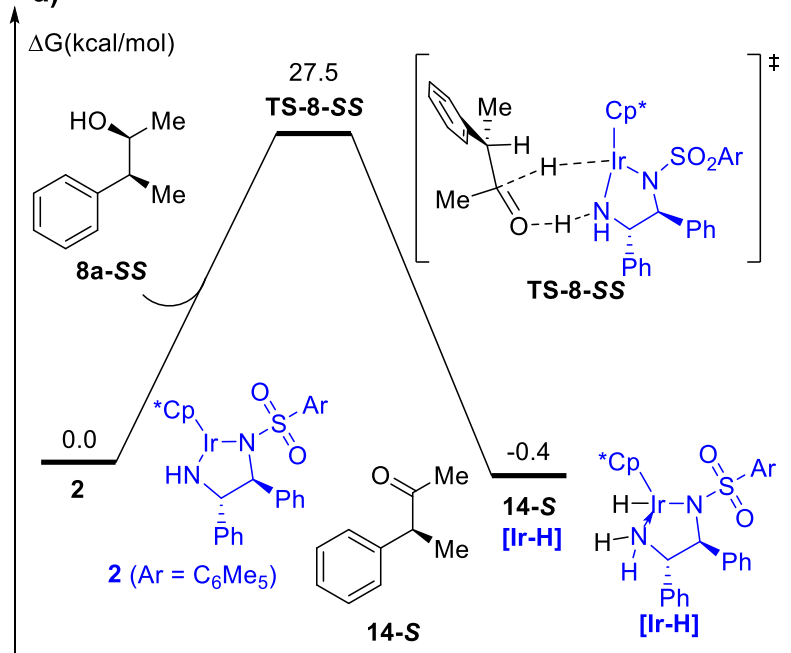

c)

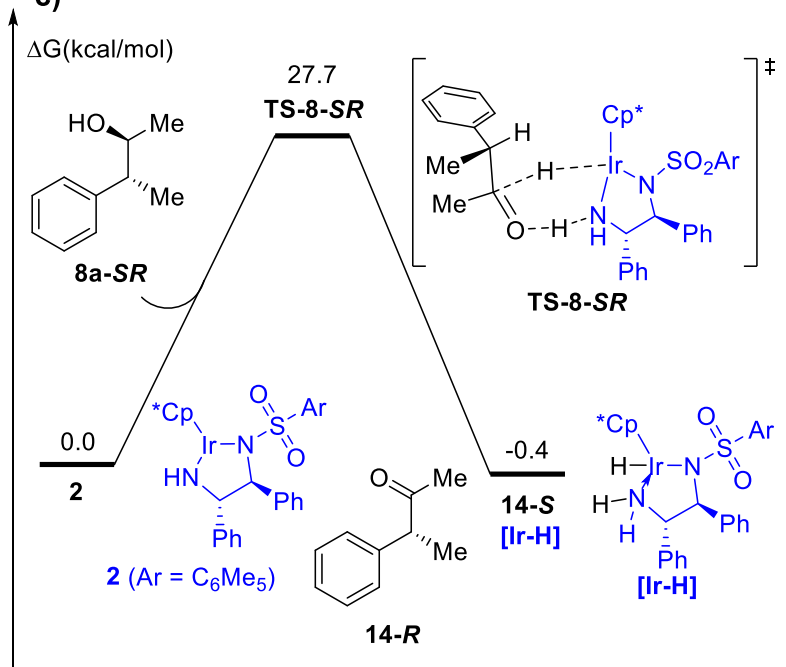

b)

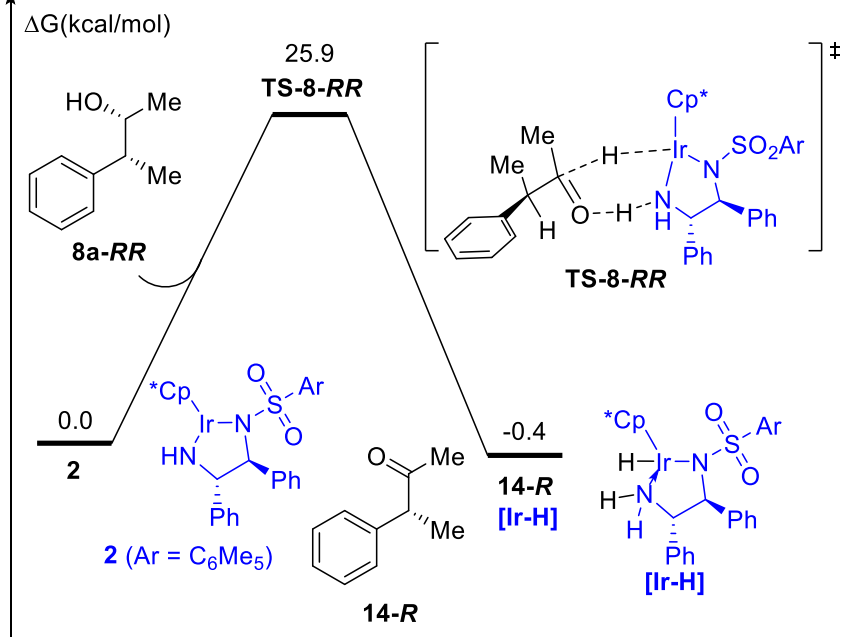

d)

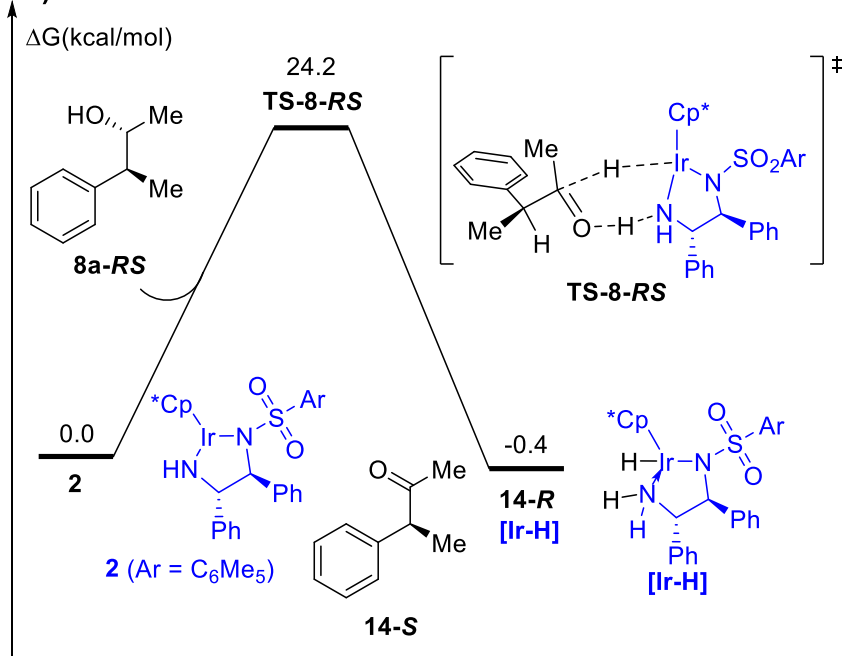

Figure S2. Free energy profiles of the first hydrogen transfer step of Ir-catalyzed dynamic kinetic asymmetric amination reaction of reactant 8a. The energy values are given in $\mathrm{kcal} / \mathrm{mol}$ and represent the relative free energies calculated by the M06-2X in toluene solvent. 


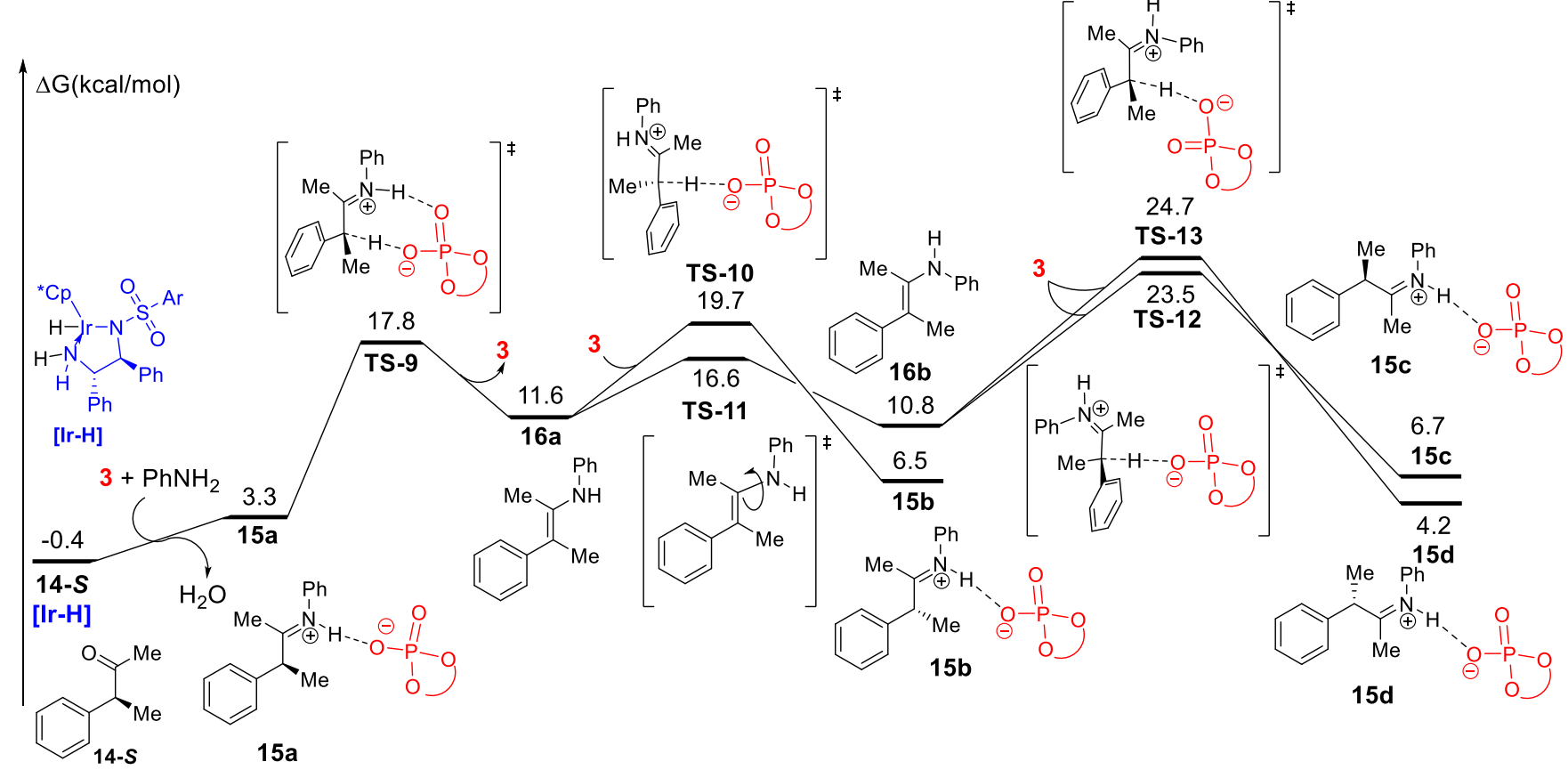

Figure S3. Free energy profiles of the tautomerization step of aminium intermediate. The energy values are given in $\mathrm{kcal} / \mathrm{mol}$ and represent the relative free energies calculated by the M06-2X in toluene solvent. 

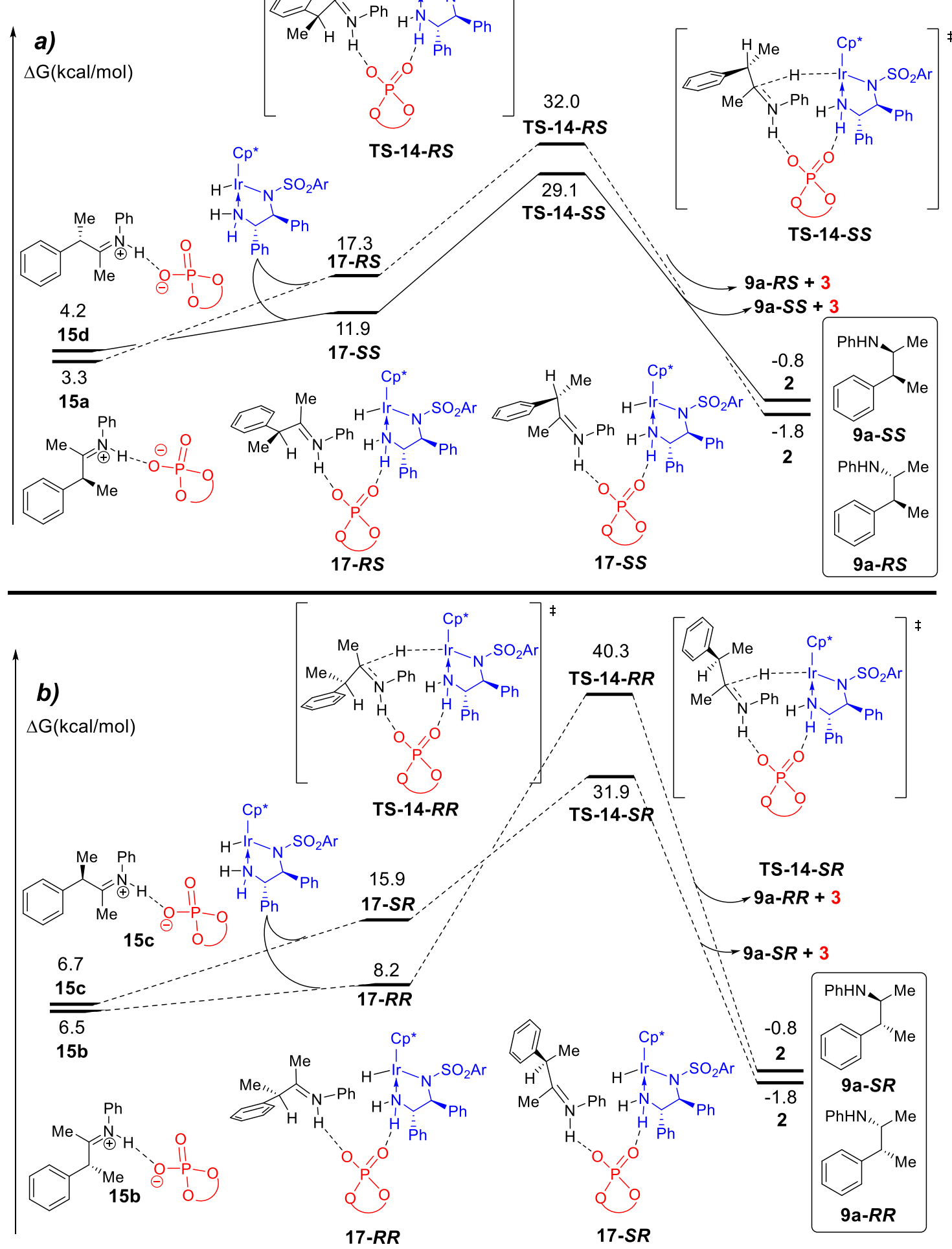

Figure S4. Free energy profiles of the second hydrogen transfer step of Ir-catalyzed dynamic kinetic asymmetric amination reaction. The energy values are given in $\mathrm{kcal} / \mathrm{mol}$ and represent the relative free energies calculated by the M06-2X in toluene solvent. 
4. B3LYP and M06-2X absolute calculation energies, enthalpies, and free energies.

\begin{tabular}{|c|c|c|c|c|c|}
\hline Geometry & $\mathrm{E}_{\mathrm{B} 3 \mathrm{LYP}(\mathrm{elec})}{ }^{1}$ & $\mathrm{E}_{\mathrm{M} 06-2 \mathrm{x}(\text { chloroform })^{2}}$ & $\begin{array}{c}\text { Correction of } \\
\mathrm{H}_{\text {(gas phase })^{3}}\end{array}$ & $\begin{array}{c}\text { Correction of } \\
\mathrm{G}_{\text {(gas phase })}{ }^{4}\end{array}$ & $\mathrm{IF}^{5}$ \\
\hline $1 \mathrm{a}-R R$ & -542.128749 & -542.048683 & 0.268559 & 0.218497 & - \\
\hline $1 \mathrm{a}-R S$ & -542.127786 & -542.047944 & 0.268355 & 0.218233 & - \\
\hline 1a-SR & -542.127786 & -542.047944 & 0.268355 & 0.218233 & - \\
\hline $1 \mathrm{a}-S S$ & -542.128749 & -542.048683 & 0.268560 & 0.218499 & - \\
\hline 2 & -2122.102102 & -2122.050669 & 0.750649 & 0.627783 & - \\
\hline 3 & -2468.895137 & -2468.580439 & 1.015113 & 0.875955 & - \\
\hline $4 \mathrm{a}-R R$ & -753.310875 & -753.186655 & 0.367051 & 0.304872 & - \\
\hline $4 \mathrm{a}-R S$ & -753.308356 & -753.183937 & 0.367006 & 0.304986 & - \\
\hline $4 \mathrm{a}-S R$ & -753.308356 & -753.183937 & 0.367006 & 0.304988 & - \\
\hline $4 \mathbf{a}-S S$ & -753.310875 & -753.186655 & 0.367049 & 0.304873 & - \\
\hline $8 \mathrm{a}-R S$ & -464.703999 & -464.633933 & 0.230595 & 0.181521 & - \\
\hline $8 \mathrm{a}-R S$ & -464.703999 & -464.633933 & 0.230595 & 0.181521 & - \\
\hline $8 \mathrm{a}-S R$ & -464.703999 & -464.633933 & 0.230595 & 0.181521 & - \\
\hline 8a-SS & -464.706379 & -464.636100 & 0.230628 & 0.181894 & - \\
\hline $9 \mathrm{a}-R R$ & -675.888795 & -675.774418 & 0.329144 & 0.268351 & - \\
\hline $9 \mathrm{a}-R S$ & -675.886983 & -675.772869 & 0.328801 & 0.266927 & - \\
\hline $9 \mathrm{a}-S R$ & -675.886983 & -675.772869 & 0.328800 & 0.266928 & - \\
\hline $9 \mathrm{a}-S S$ & -675.888783 & -675.774429 & 0.329147 & 0.268315 & - \\
\hline TS-1-RR & -2664.208941 & -2664.082156 & 1.015094 & 0.868125 & -722.1 \\
\hline TS-1-RS & -2664.205314 & -2664.078539 & 1.015079 & 0.868466 & -559.9 \\
\hline TS-1-SR & -2664.202275 & -2664.077764 & 1.015510 & 0.867609 & -492.3 \\
\hline TS-1-SS & -2664.206175 & -2664.080896 & 1.015324 & 0.868785 & -420.3 \\
\hline $10-R$ & -540.932181 & -540.846150 & 0.244575 & 0.193951 & - \\
\hline $10-S$ & -540.932181 & -540.846150 & 0.244575 & 0.193951 & - \\
\hline$[\mathbf{I r}-\mathbf{H}]$ & -2123.299698 & -2123.249233 & 0.772739 & 0.648974 & - \\
\hline $\mathrm{PhNH}_{2}$ & -287.595891 & -287.552459 & 0.124158 & 0.088280 & - \\
\hline $\mathrm{H}_{2} \mathrm{O}$ & -76.407024 & -76.416413 & 0.024918 & 0.002819 & - \\
\hline $11 \mathrm{a}$ & -3221.012295 & -3220.585131 & 1.359221 & 1.180055 & - \\
\hline
\end{tabular}




\begin{tabular}{|c|c|c|c|c|c|}
\hline TS-2 & -3220.982033 & -3220.557244 & 1.355456 & 1.180111 & -1234.2 \\
\hline $12 \mathbf{a}$ & -752.092222 & -751.967965 & 0.342860 & 0.281895 & - \\
\hline TS-3 & -3220.979866 & -3220.557012 & 1.355417 & 1.180793 & -1249.9 \\
\hline $11 b$ & -3221.013477 & -3220.585043 & 1.359880 & 1.182022 & - \\
\hline TS-4 & -752.087885 & -751.964618 & 0.341176 & 0.281731 & -56.5 \\
\hline $12 \mathrm{~b}$ & -752.092989 & -751.969807 & 0.342591 & 0.281915 & - \\
\hline TS-5 & -3220.970858 & -3220.549404 & 1.355485 & 1.180312 & -1263.6 \\
\hline $11 \mathrm{c}$ & -3221.015557 & -3220.585017 & 1.359378 & 1.177896 & - \\
\hline TS-6 & -3220.972487 & -3220.550294 & 1.355294 & 1.179821 & -1199.2 \\
\hline 11d & -3221.013074 & -3220.583227 & 1.359421 & 1.179792 & - \\
\hline $13-R R$ & -5344.315735 & -5343.848850 & 2.136087 & 1.856464 & - \\
\hline TS-7-RR & -5344.271477 & -5343.817444 & 2.135385 & 1.866975 & -740.1 \\
\hline $13-R S$ & -5344.310595 & -5343.844686 & 2.135819 & 1.857535 & - \\
\hline TS-7-RS & -5344.276157 & -5343.816933 & 2.135172 & 1.864831 & -656.1 \\
\hline $13-S R$ & -5344.315068 & -5343.844976 & 2.136649 & 1.858561 & - \\
\hline TS-7-SR & -5344.281538 & -5343.827605 & 2.135221 & 1.867790 & -646.9 \\
\hline $13-S S$ & -5344.321278 & -5343.850879 & 2.136103 & 1.857517 & - \\
\hline TS-7-SS & -5344.283951 & -5343.827009 & 2.134395 & 1.864042 & -735.4 \\
\hline TS-8-RR & -2586.783507 & -2586.666904 & 0.977106 & 0.831156 & -817.7 \\
\hline TS-8-RS & -2586.783287 & -2586.668892 & 0.977522 & 0.832167 & -761.4 \\
\hline TS-8-SR & -2586.776183 & -2586.662817 & 0.977606 & 0.831716 & -520.4 \\
\hline TS-8-SS & -2586.781723 & -2586.667335 & 0.977642 & 0.834084 & -516.1 \\
\hline $14-R$ & -463.510742 & -463.434801 & 0.206574 & 0.157389 & - \\
\hline $14-S$ & -463.510742 & -463.434801 & 0.206575 & 0.157389 & - \\
\hline $15 \mathbf{a}$ & -3143.585990 & -3143.169289 & 1.321398 & 1.142685 & - \\
\hline TS-9 & -3143.563082 & -3143.145066 & 1.317217 & 1.141527 & -1210.7 \\
\hline $16 a$ & -674.666554 & -674.553086 & 0.304660 & 0.244184 & - \\
\hline TS-10 & -3143.557632 & -3143.142822 & 1.317344 & 1.142331 & -1198.8 \\
\hline $15 b$ & -3143.586867 & -3143.167956 & 1.322384 & 1.146464 & - \\
\hline TS-11 & -674.664646 & -674.551514 & 0.303312 & 0.244845 & -19.2 \\
\hline $16 b$ & -674.667112 & -674.555240 & 0.304686 & 0.244975 & - \\
\hline
\end{tabular}




\begin{tabular}{cccccc}
\hline TS-12 & -3143.551354 & -3143.136857 & 1.317393 & 1.142399 & -1208.0 \\
$\mathbf{1 5 c}$ & -3143.585544 & -3143.165596 & 1.321911 & 1.144390 & - \\
$\mathbf{T S}-13$ & -3143.546330 & -3143.133188 & 1.317154 & 1.140600 & -1022.3 \\
$\mathbf{1 5 d}$ & -3143.587723 & -3143.168810 & 1.321409 & 1.143625 & - \\
$\mathbf{1 7 - R R}$ & -5266.890922 & -5266.438227 & 2.097527 & 1.819126 & - \\
TS-14-RR & -5266.846478 & -5266.399395 & 2.097784 & 1.831462 & -728.1 \\
$\mathbf{1 7 - R S}$ & -5266.888253 & -5266.427336 & 2.098764 & 1.822803 & - \\
TS-14-RS & -5266.860638 & -5266.409261 & 2.097167 & 1.828059 & -621.5 \\
$\mathbf{1 7 - S R}$ & -5266.886701 & -5266.427287 & 2.098476 & 1.820472 & - \\
TS-14-SR & -5266.856492 & -5266.412285 & 2.097530 & 1.830977 & -576.7 \\
$\mathbf{1 7 - S S}$ & -5266.892992 & -5266.434008 & 2.098337 & 1.820785 & - \\
TS-14-SS & -5266.858144 & -5266.411804 & 2.096691 & 1.826004 & -697.7 \\
\hline
\end{tabular}

\footnotetext{
${ }^{1}$ The electronic energy calculated by B3LYP in gas phase. ${ }^{2}$ The electronic energy calculated by M06-2X in toluene. ${ }^{3}$ The thermal correction to enthalpy calculated by B3LYP in gas phase. ${ }^{4}$ The thermal correction to Gibbs free energy calculated by B3LYP in gas phase. ${ }^{5}$ The B3LYP calculated imaginary frequencies for the transition states in gas phase.
} 
4. B3LYP geometries for all the optimized compounds and transition states.

1a-R
H
C
H
C
H
H
H
C
C
C
C
C
C
H
C
H
C
C
H
C
H
H
H
H
H
H
H
H
O

la- $R \boldsymbol{S}$
C
C
C
C
C
C
C
C
H
C
C
C
H
H
H
H
H
H
O
H

$\begin{array}{lll}5.65320800 & -9.77706000 & -1.18805600\end{array}$

$\begin{array}{llll}6.58865300 & -9.98630000 & -0.64421000\end{array}$

$\begin{array}{lll}5.85587300 & -11.35609600 & 0.54522900\end{array}$

$\begin{array}{lll}7.38127700 & -11.02724000 & -1.43691100\end{array}$

$6.80674000-11.96069200-1.50814600$

$\begin{array}{llll}8.32948800 & -11.25364400 & -0.93540800\end{array}$

$\begin{array}{llll}7.59028600 & -10.69235300 & -2.45643600\end{array}$

$\begin{array}{lll}7.33448700 & -8.64238500 & -0.41764000\end{array}$

$\begin{array}{llll}7.63060000 & -7.91998200 & -1.72811900\end{array}$

$\begin{array}{llll}6.54777500 & -7.72398800 & 0.54080600\end{array}$

$\begin{array}{llll}8.75719300 & -8.27341000 & -2.48946600\end{array}$

$\begin{array}{llll}6.80027000 & -6.88062300 & -2.19276600\end{array}$

$\begin{array}{llll}5.28801400 & -7.16893200 & -0.13332700\end{array}$

$\begin{array}{llll}7.19845200 & -6.88977800 & 0.83885400\end{array}$

$\begin{array}{llll}9.04243300 & -7.65819500 & -3.70569700\end{array}$

$\begin{array}{llll}9.42592800 & -9.04335800 & -2.11193600\end{array}$

$\begin{array}{llll}7.09340400 & -6.27303400 & -3.42351200\end{array}$

$\begin{array}{llll}5.64862700 & -6.32836300 & -1.36573300\end{array}$

$\begin{array}{llll}4.62978300 & -7.99831500 & -0.42058200\end{array}$

$\begin{array}{llll}8.19450800 & -6.65644300 & -4.18374600\end{array}$

$\begin{array}{llll}9.92132500 & -7.95374300 & -4.27272000\end{array}$

$\begin{array}{llll}6.44613300 & -5.47377500 & -3.78037500\end{array}$

$\begin{array}{llll}4.76726200 & -6.18618500 & -2.00515600\end{array}$

$\begin{array}{llll}8.39852300 & -6.16897800 & -5.13361000\end{array}$

$\begin{array}{llll}8.28906100 & -8.90417400 & 0.05994800\end{array}$

$\begin{array}{llll}6.29034900 & -8.27808500 & 1.44636600\end{array}$

$\begin{array}{llll}4.71492100 & -6.55669400 & 0.57351900\end{array}$

$\begin{array}{lll}5.93633000 & -5.31915600 & -1.03371400\end{array}$

$\begin{array}{llll}6.28549900 & -10.49457000 & 0.65996900\end{array}$

$2.82069400-3.88417000 \quad 2.06564400$

$\begin{array}{llll}1.47934500 & -3.30572600 & 1.53647900\end{array}$

$\begin{array}{lll}1.72693800 & -2.17779000 & 0.53483100\end{array}$

$\begin{array}{llll}0.52579700 & -2.83826900 & 2.65228100\end{array}$

$\begin{array}{llll}2.14944200 & -2.48426500 & -0.76953400\end{array}$

$\begin{array}{lll}1.54884400 & -0.82656400 & 0.89108800\end{array}$

$\begin{array}{llll}1.01089700 & -1.53261800 & 3.29362900\end{array}$

$\begin{array}{lll}2.40703200 & -1.48465000 & -1.70352100\end{array}$

$\begin{array}{llll}2.28775800 & -3.52564600 & -1.03956700\end{array}$

$\begin{array}{llll}1.81758700 & 0.17141500 & -0.05862900\end{array}$

$\begin{array}{lll}1.01441000 & -0.40717700 & 2.25207800\end{array}$

$\begin{array}{llll}2.24479000 & -0.14417800 & -1.34471000\end{array}$

$\begin{array}{lll}2.73222700 & -1.74954300 & -2.70639900\end{array}$

$\begin{array}{lll}1.67963600 & 1.21406700 & 0.22273800\end{array}$

$\begin{array}{lll}1.58095700 & 0.45733900 & 2.62213300\end{array}$

$\begin{array}{llll}2.44541400 & 0.64719600 & -2.06256500\end{array}$

$\begin{array}{lll}1.00385700 & -4.14138600 & 1.00409300\end{array}$

$\begin{array}{lll}-0.01830800 & -0.04948800 & 2.11912400\end{array}$

$\begin{array}{llll}3.42734900 & -4.54015200 & 0.94633200\end{array}$

$\begin{array}{lll}4.34315700 & -4.74940700 & 1.18528400\end{array}$
C

$\mathrm{H}$

$\mathrm{H}$

$\mathrm{H}$

$\mathrm{H}$

$\mathrm{H}$

$\mathrm{H}$

$\mathrm{H}$

$\mathrm{H}$

\section{1a-SR}

$\mathrm{C}$

$\mathrm{C}$

C

C

C

C

$\mathrm{C}$

$\mathrm{C}$

$\mathrm{H}$

C

C

C

$\mathrm{H}$

$\mathrm{H}$

$\mathrm{H}$

$\mathrm{H}$

$\mathrm{H}$

$\mathrm{H}$

$\mathrm{O}$

$\mathrm{H}$

$\mathrm{C}$

$\mathrm{H}$

$\mathrm{H}$

$\mathrm{H}$

$\mathrm{H}$

$\mathrm{H}$

$\mathrm{H}$

$\mathrm{H}$

$\mathrm{H}$

1a-SS
H
C
H
C
H
H
H
C
C
C

$2.67378500-4.88000700$

$2.33037400-4.40239300$

$1.97439200-5.68127200$

$3.64589600 \quad-5.34195000$

$3.46057700-3.04930600$

$0.36882100-1.25473400$

$2.02068500-1.66959200$

$-0.46560200-2.66802700$

$0.39345500-3.62193200$

3.22032100

4.14376900

2.95533900

3.43505600

2.39161200

4.13841200

3.70364400

2.20983900

3.40518400
$2.82069400-3.88417000-2.06564400$

$1.47934500-3.30572600-1.53647900$

$1.72693800-2.17779000-0.53483100$

$\begin{array}{llll}0.52579700 & -2.83826900 & -2.65228100\end{array}$

$\begin{array}{lll}2.14944200 & -2.48426500 & 0.76953400\end{array}$

$\begin{array}{lll}1.54884400 & -0.82656400 & -0.89108800\end{array}$

$\begin{array}{lll}1.01089700 & -1.53261800 & -3.29362900\end{array}$

$\begin{array}{lll}2.40703200 & -1.48465000 & 1.70352100\end{array}$

$2.28775800 \quad-3.52564600 \quad 1.03956700$

$\begin{array}{lll}1.81758700 & 0.17141500 & 0.05862900\end{array}$

$\begin{array}{lll}1.01441000 & -0.40717700 & -2.25207800\end{array}$

$\begin{array}{lll}2.24479000 & -0.14417800 & 1.34471000\end{array}$

$\begin{array}{lll}2.73222700 & -1.74954300 & 2.70639900\end{array}$

$\begin{array}{llll}1.67963600 & 1.21406700 & -0.22273800\end{array}$

$\begin{array}{lll}1.58095700 & 0.45733900 & -2.62213300\end{array}$

$\begin{array}{lll}2.44541400 & 0.64719600 & 2.06256500\end{array}$

$\begin{array}{lll}1.00385700 & -4.14138600 & -1.00409300\end{array}$

$-0.01830800 \quad-0.04948800 \quad-2.11912400$

$3.42734900-4.54015200 \quad-0.94633200$

$\begin{array}{lll}4.34315700 & -4.74940700 & -1.18528400\end{array}$

$2.67378500-4.88000700-3.22032100$

$2.33037400-4.40239300 \quad-4.14376900$

$\begin{array}{lll}1.97439200 & -5.68127200 & -2.95533900\end{array}$

$3.64589600-5.34195000 \quad-3.43505600$

$3.46057700 \quad-3.04930600 \quad-2.39161200$

$\begin{array}{llll}0.36882100 & -1.25473400 & -4.13841200\end{array}$

$2.02068500-1.66959200 \quad-3.70364400$

$\begin{array}{lll}-0.46560200 & -2.66802700 & -2.20983900\end{array}$

$\begin{array}{llll}0.39345500 & -3.62193200 & -3.40518400\end{array}$

$\begin{array}{lll}5.65320800 & -9.77706000 & 1.18805600\end{array}$

$\begin{array}{lll}6.58865300 & -9.98630000 & 0.64421000\end{array}$

$\begin{array}{llll}5.85587300 & -11.35609600 & -0.54522900\end{array}$

$\begin{array}{llll}7.38127700 & -11.02724000 & 1.43691100\end{array}$

$\begin{array}{llll}6.80674000 & -11.96069200 & 1.50814600\end{array}$

$8.32948800-11.25364400 \quad 0.93540800$

$\begin{array}{llll}7.59028600 & -10.69235300 & 2.45643600\end{array}$

$\begin{array}{lll}7.33448700 & -8.64238500 & 0.41764000\end{array}$

$\begin{array}{lll}7.63060000 & -7.91998200 & 1.72811900\end{array}$

$\begin{array}{llll}6.54777500 & -7.72398800 & -0.54080600\end{array}$ 
$\mathrm{C}$
$\mathrm{C}$
$\mathrm{C}$
$\mathrm{H}$
$\mathrm{C}$
$\mathrm{H}$
$\mathrm{C}$
$\mathrm{C}$
$\mathrm{H}$
$\mathrm{C}$
$\mathrm{H}$
$\mathrm{H}$
$\mathrm{H}$
$\mathrm{H}$
$\mathrm{H}$
$\mathrm{H}$
$\mathrm{H}$
$\mathrm{H}$
$\mathrm{O}$

C

C

$\mathrm{C}$

$\begin{array}{ccc}8.75719300 & -8.27341000 & 2.48946600 \\ 6.80027000 & -6.88062300 & 2.19276600 \\ 5.28801400 & -7.16893200 & 0.13332700 \\ 7.19845200 & -6.88977800 & -0.83885400 \\ 9.04243300 & -7.65819500 & 3.70569700 \\ 9.42592800 & -9.04335800 & 2.11193600 \\ 7.09340400 & -6.27303400 & 3.42351200 \\ 5.64862700 & -6.32836300 & 1.36573300 \\ 4.62978300 & -7.99831500 & 0.42058200 \\ 8.19450800 & -6.65644300 & 4.18374600 \\ 9.92132500 & -7.95374300 & 4.27272000 \\ 6.44613300 & -5.47377500 & 3.78037500 \\ 4.76726200 & -6.18618500 & 2.00515600 \\ 8.39852300 & -6.16897800 & 5.13361000 \\ 8.28906100 & -8.90417400 & -0.05994800 \\ 6.29034900 & -8.27808500 & -1.44636600 \\ 4.71492100 & -6.55669400 & -0.57351900 \\ 5.93633000 & -5.31915600 & 1.03371400 \\ 6.28549900 & -10.49457000 & -0.65996900\end{array}$

$4.00109500 \quad-0.28168100 \quad-1.18415400$ $\begin{array}{llll}4.31459000 & 0.31786700 & 0.08151500\end{array}$ $3.87399100 \quad-0.60946600 \quad 1.11229000$ $3.40498800 \quad-1.82300300 \quad 0.47450000$ $\begin{array}{lll}3.44666500 & -1.61828600 & -0.93257900\end{array}$ $2.15500800 \quad 0.02341400 \quad-0.06155800$ $\begin{array}{lll}0.16443200 & -0.30133300 & 0.34228400\end{array}$ $\begin{array}{lll}1.38297300 & 1.80025600 & -0.32285000\end{array}$ $\begin{array}{lll}-0.75500000 & 0.86469300 & 0.39057100\end{array}$

$\begin{array}{lll}0.14222900 & 2.14153500 & 0.36137000\end{array}$ 0.34935100 $-0.56440800$ $-0.65783000$

$-1.13948000$

$-1.31392600$

$-0.20105400$

$-1.79928200$

$-1.07373800$

$-1.88842400$

$-1.37539700$

$-2.23996200$

$-2.39786400$

$-1.36917900$

$-1.69679300$

$-3.03416500$

$-1.23266800$

$-3.89107500$

$-3.40754100$

$-2.08600300$

$-0.20562400$

$-3.41841200$

$-4.92567100$

$-1.71238300$
1.40996100

$\begin{array}{ll}2.41142900 & 1.40996100 \\ 3.32847100 & -0.28145100\end{array}$

$3.43935900-1.67666400$

$4.32927100 \quad 0.51126400$

$4.52208900-2.26249200$

$2.67273000-2.29672000$

$5.41319900-0.07233600$ $\begin{array}{ll}4.25428500 & 1.59399800\end{array}$

$5.51286000-1.46162200$

$4.59471100-3.34566900$

$6.18042500 \quad 0.55921200$

$6.35773700-1.91790500$

$0.86901700-0.51961800$

$\begin{array}{ll}0.94056200 & 1.58813400\end{array}$

$\begin{array}{ll}1.30839000 & 1.39964200\end{array}$

$\begin{array}{ll}0.73908500 & 2.89575900\end{array}$

$\begin{array}{ll}1.47896500 & 2.48987300\end{array}$

$1.46044800 \quad 0.39018400$

$0.90519300 \quad 3.98618300$

$0.42420500 \quad 3.05103700$

$1.27895400 \quad 3.78786700$

1.76814600

0.73562500
2.32294400

4.99287200

\begin{tabular}{|c|c|c|c|}
\hline $\mathrm{H}$ & -4.08275500 & 1.40831100 & 4.63856200 \\
\hline S & -0.46164600 & -1.84067500 & 0.50300300 \\
\hline $\mathrm{O}$ & 0.37708000 & -2.77083300 & -0.28439800 \\
\hline $\mathrm{O}$ & -0.59398900 & -2.17167100 & 1.93401200 \\
\hline $\mathrm{C}$ & -2.11114100 & -1.78364000 & -0.30842400 \\
\hline $\mathrm{C}$ & -3.28797500 & -2.08854600 & 0.41309200 \\
\hline C & -2.15343200 & -1.40972100 & -1.67291600 \\
\hline $\mathrm{C}$ & -4.53235700 & -1.92316400 & -0.23747000 \\
\hline $\mathrm{C}$ & -3.40813600 & -1.28099000 & -2.30254000 \\
\hline $\mathrm{C}$ & -4.59188500 & -1.56625700 & -1.59695500 \\
\hline$C$ & -3.30259800 & -2.62136200 & 1.83464500 \\
\hline $\mathrm{H}$ & -2.44581000 & -3.25713000 & 2.03900900 \\
\hline $\mathrm{H}$ & -3.27249200 & -1.80913900 & 2.57100700 \\
\hline $\mathrm{H}$ & -4.21007500 & -3.20239800 & 2.00985600 \\
\hline$C$ & -5.81711800 & -2.09979200 & 0.55440700 \\
\hline $\mathrm{H}$ & -6.64636900 & -1.55595900 & 0.09762900 \\
\hline $\mathrm{H}$ & -6.12330000 & -3.15234800 & 0.63818700 \\
\hline $\mathrm{H}$ & -5.70881300 & -1.71331500 & 1.57115200 \\
\hline $\mathrm{C}$ & -5.92830600 & -1.52087900 & -2.31691100 \\
\hline $\mathrm{H}$ & -6.64515600 & -2.21993800 & -1.88071600 \\
\hline $\mathrm{H}$ & -6.38665900 & -0.52186900 & -2.28404700 \\
\hline $\mathrm{H}$ & -5.82444000 & -1.79287900 & -3.36998700 \\
\hline $\mathrm{C}$ & -3.47342400 & -0.81641400 & -3.74635500 \\
\hline $\mathrm{H}$ & -4.42012300 & -0.31998600 & -3.96985600 \\
\hline $\mathrm{H}$ & -2.67925900 & -0.10115200 & -3.97331000 \\
\hline $\mathrm{H}$ & -3.36600500 & -1.64981100 & -4.45609000 \\
\hline $\mathrm{C}$ & -0.91736300 & -1.15640400 & -2.51843100 \\
\hline $\mathrm{H}$ & -0.77055400 & -0.08376100 & -2.70238700 \\
\hline $\mathrm{H}$ & -0.01187500 & -1.54395100 & -2.06342200 \\
\hline $\mathrm{H}$ & -1.03107800 & -1.63792300 & -3.49579700 \\
\hline $\mathrm{C}$ & 3.07939200 & -2.61831000 & -1.98708100 \\
\hline $\mathrm{H}$ & 2.21745800 & -3.20931700 & -1.67320800 \\
\hline $\mathrm{H}$ & 2.82654800 & -2.12631500 & -2.93094000 \\
\hline $\mathrm{H}$ & 3.92244300 & -3.29586500 & -2.18290300 \\
\hline $\mathrm{C}$ & 4.31436900 & 0.27985900 & -2.53990700 \\
\hline $\mathrm{H}$ & 4.40202600 & 1.36945400 & -2.51280800 \\
\hline $\mathrm{H}$ & 5.26521600 & -0.12386300 & -2.91425000 \\
\hline $\mathrm{H}$ & 3.53575200 & 0.02659500 & -3.26499500 \\
\hline $\mathrm{C}$ & 5.00110900 & 1.63240000 & 0.31522400 \\
\hline $\mathrm{H}$ & 6.08525900 & 1.49350400 & 0.42298100 \\
\hline $\mathrm{H}$ & 4.83992600 & 2.32495700 & -0.51660500 \\
\hline $\mathrm{H}$ & 4.63531400 & 2.11585500 & 1.22609800 \\
\hline $\mathrm{C}$ & 4.04306300 & -0.42890700 & 2.59147400 \\
\hline $\mathrm{H}$ & 5.01190900 & -0.83520400 & 2.91527600 \\
\hline $\mathrm{H}$ & 4.01109200 & 0.62612200 & 2.87768200 \\
\hline $\mathrm{H}$ & 3.25900900 & -0.95414500 & 3.14334400 \\
\hline $\mathrm{C}$ & 3.01288500 & -3.07194200 & 1.20825000 \\
\hline $\mathrm{H}$ & 2.33496900 & -2.85580800 & 2.03897300 \\
\hline $\mathrm{H}$ & 2.49723500 & -3.77371300 & 0.55425000 \\
\hline $\mathrm{H}$ & 3.91004900 & -3.55865200 & 1.61593200 \\
\hline $\mathrm{H}$ & 1.98475700 & 2.61250800 & -0.43657200 \\
\hline & & & \\
\hline & -0.32050700 & -0.96393200 & -1.82259100 \\
\hline
\end{tabular}




\begin{tabular}{|c|c|c|c|c|c|c|c|}
\hline $\mathrm{C}$ & -0.26859800 & 1.41520300 & -1.81007400 & $\mathrm{C}$ & 0.51050600 & 4.06492600 & 2.53976600 \\
\hline $\mathrm{C}$ & 0.06806200 & 0.24222600 & -2.70433900 & $\mathrm{H}$ & 0.75023100 & 3.33669800 & 1.76119000 \\
\hline $\mathrm{H}$ & -1.38917900 & -1.17786800 & -1.93908400 & $\mathrm{C}$ & -5.26797000 & 4.21773700 & -0.01589900 \\
\hline $\mathrm{H}$ & 0.23063000 & -1.87723900 & -2.06571300 & $\mathrm{H}$ & -5.79282600 & 4.03840800 & -0.96152400 \\
\hline $\mathrm{H}$ & 1.13732500 & 0.22558000 & -2.95775000 & $\mathrm{H}$ & -5.80242700 & 5.02193800 & 0.50262300 \\
\hline $\mathrm{H}$ & -0.47991700 & 0.26772200 & -3.65305500 & $\mathrm{H}$ & -5.35308200 & 3.30656200 & 0.58308800 \\
\hline $\mathrm{C}$ & -0.06020100 & -0.48211500 & -0.35334600 & $\mathrm{C}$ & -3.74474200 & 5.83336200 & -1.21035000 \\
\hline $\mathrm{C}$ & 1.45870500 & -0.65142200 & -0.01550800 & $\mathrm{H}$ & -4.29178500 & 5.65376800 & -2.14361300 \\
\hline $\mathrm{C}$ & -0.66776700 & -1.45829300 & 0.65446900 & $\mathrm{H}$ & -2.71661800 & 6.10955700 & -1.46715000 \\
\hline $\mathrm{C}$ & 1.60954600 & -2.14817500 & 0.33676800 & $\mathrm{H}$ & -4.20219200 & 6.69727000 & $-0.7132820 c$ \\
\hline $\mathrm{H}$ & 2.10886800 & -0.33003400 & -0.83531600 & $\mathrm{C}$ & -4.57222000 & 6.34670400 & 4.81041000 \\
\hline $\mathrm{H}$ & 1.69594400 & -0.03729900 & 0.86073900 & $\mathrm{H}$ & -4.93452300 & 7.00554900 & 5.60860000 \\
\hline $\mathrm{H}$ & 2.42454400 & -2.33416400 & 1.04556300 & $\mathrm{H}$ & -4.36795400 & 5.36290300 & 5.24673200 \\
\hline $\mathrm{H}$ & 1.82635900 & -2.75031500 & -0.55682100 & $\mathrm{H}$ & -5.38424000 & 6.22807600 & 4.08295000 \\
\hline $\mathrm{C}$ & -0.45619300 & 0.98591200 & -0.48591100 & $\mathrm{C}$ & -3.58768200 & 8.34386600 & 3.58695800 \\
\hline $\mathrm{C}$ & -0.91022400 & 1.90137700 & 0.45819600 & $\mathrm{H}$ & -3.93679500 & 9.01486400 & 4.38078500 \\
\hline $\mathrm{C}$ & -0.42189600 & 2.75484000 & -2.15030900 & $\mathrm{H}$ & -4.36159400 & 8.31810300 & 2.81055300 \\
\hline $\mathrm{C}$ & -1.11309700 & 3.26049900 & 0.14112700 & $\mathrm{H}$ & -2.68470800 & 8.77878200 & 3.14459100 \\
\hline $\mathrm{C}$ & -0.81859400 & 3.66617200 & -1.16978500 & $\mathrm{C}$ & 1.52385300 & 5.21949400 & 2.39809600 \\
\hline $\mathrm{H}$ & -0.25196200 & 3.09209200 & -3.16969700 & $\mathrm{H}$ & 1.35813100 & 5.99440000 & 3.15607600 \\
\hline $\mathrm{H}$ & -0.93627100 & 4.71386900 & -1.42799500 & $\mathrm{H}$ & 1.45016800 & 5.69466600 & 1.41329200 \\
\hline $\mathrm{C}$ & 0.25294200 & -2.48594300 & 0.91801400 & $\mathrm{H}$ & 2.54774400 & 4.84609700 & 2.52054300 \\
\hline $\mathrm{C}$ & -0.12963500 & -3.61122500 & 1.63952500 & $\mathrm{C}$ & 0.66804300 & 3.35158500 & 3.89616400 \\
\hline $\mathrm{C}$ & -1.92940100 & -1.52128300 & 1.24457700 & $\mathrm{H}$ & 1.68332800 & 2.94797100 & 3.99213700 \\
\hline $\mathrm{C}$ & -1.43806400 & -3.70093900 & 2.11708200 & $\mathrm{H}$ & -0.03918500 & 2.52270200 & 3.99237700 \\
\hline $\mathrm{H}$ & 0.57655000 & -4.41555400 & 1.83018500 & $\mathrm{H}$ & 0.50910400 & 4.03716100 & 4.73711800 \\
\hline $\mathrm{C}$ & -2.35953600 & -2.65198800 & 1.96495400 & $\mathrm{C}$ & -4.72518200 & -2.89769800 & 0.14259500 \\
\hline $\mathrm{H}$ & -1.75544200 & -4.59030600 & 2.65231000 & $\mathrm{H}$ & -3.67327800 & -2.74574100 & -0.11307700 \\
\hline $\mathrm{O}$ & -1.15024000 & 1.46856200 & 1.76331400 & $\mathrm{C}$ & -7.74923900 & -3.28147800 & 4.17405800 \\
\hline $\mathrm{O}$ & -2.79291900 & -0.41902300 & 1.11790600 & $\mathrm{H}$ & -7.64271900 & -3.30073500 & 5.26743000 \\
\hline $\mathrm{P}$ & -2.56697400 & 0.78431200 & 2.17955400 & $\mathrm{C}$ & -2.79550800 & -2.73496600 & 4.95107100 \\
\hline $\mathrm{O}$ & -2.55101200 & 0.46343500 & 3.61689200 & $\mathrm{H}$ & -1.89002500 & -2.43940400 & 4.41441000 \\
\hline $\mathrm{O}$ & -3.71744300 & 1.75108300 & 1.61489600 & $\mathrm{C}$ & -8.66754900 & -2.09562000 & 3.82175000 \\
\hline $\mathrm{H}$ & -3.59761700 & 2.67187200 & 1.91824500 & $\mathrm{H}$ & -9.64822300 & -2.20900100 & 4.29997200 \\
\hline $\mathrm{C}$ & -1.66522300 & 4.22686000 & 1.15038500 & $\mathrm{H}$ & -8.83042800 & -2.02720900 & 2.73947000 \\
\hline $\mathrm{C}$ & -0.91350100 & 4.56768700 & 2.30271400 & $\mathrm{H}$ & -8.23190300 & -1.14687900 & 4.15345000 \\
\hline $\mathrm{C}$ & -2.95331800 & 4.80864200 & 0.95847100 & $\mathrm{C}$ & -8.38668600 & -4.61850700 & 3.75009800 \\
\hline $\mathrm{C}$ & -1.46876200 & 5.44529600 & 3.24194800 & $\mathrm{H}$ & -9.36557100 & -4.75010700 & 4.2273060 \\
\hline $\mathrm{C}$ & -3.45679100 & 5.67276600 & 1.94009800 & $\mathrm{H}$ & -7.75234300 & -5.46627900 & 4.03169200 \\
\hline $\mathrm{C}$ & -2.73856800 & 6.00216300 & 3.09331500 & $\mathrm{H}$ & -8.53782400 & -4.66116000 & 2.66476600 \\
\hline $\mathrm{H}$ & -0.88830100 & 5.70476900 & 4.12403900 & $\mathrm{C}$ & -5.12919900 & -4.26523600 & -0.4445700 \\
\hline $\mathrm{H}$ & -4.43995500 & 6.11036900 & 1.78991400 & $\mathrm{H}$ & -6.18428000 & -4.49077700 & -0.2498130 \\
\hline $\mathrm{C}$ & -3.74271600 & -2.77151000 & 2.53617400 & $\mathrm{H}$ & -4.53045500 & -5.07471200 & -0.01189600 \\
\hline $\mathrm{C}$ & -4.85747400 & -2.88881400 & 1.66648400 & $\mathrm{H}$ & -4.98127500 & -4.27558500 & -1.53161900 \\
\hline $\mathrm{C}$ & -3.93919700 & -2.82941900 & 3.93852500 & $\mathrm{C}$ & -5.52139400 & -1.75477400 & -0.5162040 \\
\hline $\mathrm{C}$ & -6.13360300 & -3.04938000 & 2.21455200 & $\mathrm{H}$ & -5.21063000 & -0.78316900 & -0.1208730 \\
\hline $\mathrm{C}$ & -5.24053000 & -2.99965200 & 4.42805100 & $\mathrm{H}$ & -6.59926400 & -1.86294300 & -0.3461270 \\
\hline $\mathrm{C}$ & -6.35216100 & -3.10697100 & 3.59315100 & $\mathrm{H}$ & -5.35833900 & -1.75342400 & -1.60133700 \\
\hline $\mathrm{H}$ & -6.98154900 & -3.14075100 & 1.54014700 & $\mathrm{C}$ & -2.52877100 & -4.10311100 & 5.61326100 \\
\hline $\mathrm{H}$ & -5.39399700 & -3.04402100 & 5.50365600 & $\mathrm{H}$ & -3.40133400 & -4.44088200 & 6.18575200 \\
\hline $\mathrm{C}$ & -3.79810000 & 4.58491500 & -0.30319500 & $\mathrm{H}$ & -1.68098800 & -4.03515700 & 6.3061620 \\
\hline $\mathrm{H}$ & -3.36598400 & 3.74942900 & -0.85985200 & $\mathrm{H}$ & -2.30041700 & -4.88016100 & 4.87497800 \\
\hline $\mathrm{C}$ & -3.31124700 & 6.93701400 & 4.15042300 & $\mathrm{C}$ & -3.04118300 & -1.66017800 & 6.02769900 \\
\hline $\mathrm{H}$ & -2.54826000 & 7.04116800 & 4.93393000 & $\mathrm{H}$ & -3.20128900 & -0.68408300 & 5.56586800 \\
\hline
\end{tabular}




\begin{tabular}{|c|c|c|c|c|c|c|c|}
\hline $\mathrm{H}$ & -2.16549000 & -1.58993500 & 6.68496000 & $\mathrm{C}$ & 8.15185600 & -6.73646100 & -2.22522800 \\
\hline \multirow[t]{2}{*}{$\mathrm{H}$} & -3.90371800 & -1.90260700 & 6.66023200 & $\mathrm{H}$ & 8.24877700 & -5.61326900 & -4.05136500 \\
\hline & & & & $\mathrm{C}$ & 7.70349800 & -6.79651300 & -0.90574300 \\
\hline 4a- $R R$ & & & & $\mathrm{H}$ & 6.62198000 & -5.73630300 & 0.63321100 \\
\hline $\mathrm{H}$ & 5.47212000 & -9.63903000 & -1.12129000 & $\mathrm{H}$ & 8.71716900 & -7.56498400 & -2.64503000 \\
\hline $\mathrm{N}$ & 5.96680800 & -10.33227800 & 0.76870800 & $\mathrm{H}$ & 6.47452700 & -2.64592200 & -2.74382100 \\
\hline $\mathrm{C}$ & 6.38306800 & -9.85373700 & -0.55398700 & $\mathrm{C}$ & 8.96754300 & -2.50565800 & -4.07544500 \\
\hline $\mathrm{C}$ & 5.02979400 & -11.35056800 & 0.95681300 & $\mathrm{H}$ & 9.45793100 & -3.27258000 & -3.46768900 \\
\hline $\mathrm{C}$ & 5.02851200 & -12.06310600 & 2.17405700 & $\mathrm{H}$ & 8.90674700 & -1.59079800 & -3.47296000 \\
\hline $\mathrm{C}$ & 4.04217200 & -11.66839500 & 0.00468500 & $\mathrm{H}$ & 9.60602300 & -2.30399100 & -4.94102100 \\
\hline $\mathrm{C}$ & 4.07505500 & -13.04258800 & 2.43036200 & $\mathrm{H}$ & 7.91367600 & -7.66460500 & -0.28812100 \\
\hline $\mathrm{H}$ & 5.78496400 & -11.83342900 & 2.92258200 & $\mathrm{C}$ & 6.71040900 & -1.95013300 & -5.26760000 \\
\hline $\mathrm{C}$ & 3.09125800 & -12.65363900 & 0.27405900 & $\mathrm{C}$ & 5.58987500 & -2.58492300 & -6.11612100 \\
\hline $\mathrm{H}$ & 4.00817100 & -11.14606300 & -0.94562900 & $\mathrm{C}$ & 7.58323300 & -0.99998400 & -6.11202500 \\
\hline $\mathrm{C}$ & 3.09426400 & -13.34993200 & 1.48266800 & $\mathrm{C}$ & 4.87879000 & -3.70340500 & -5.64405000 \\
\hline $\mathrm{H}$ & 4.10214400 & -13.57294800 & 3.37917500 & $\mathrm{C}$ & 5.22553400 & -2.04890700 & -7.37094900 \\
\hline $\mathrm{H}$ & 2.34027500 & -12.87750600 & -0.47979700 & $\mathrm{C}$ & 6.72304800 & -0.04947400 & -6.94640000 \\
\hline $\mathrm{H}$ & 6.74163800 & -10.38929500 & 1.42140700 & $\mathrm{H}$ & 8.24666400 & -0.42796900 & -5.45580100 \\
\hline $\mathrm{C}$ & 7.17837700 & -10.92810600 & -1.31450300 & $\mathrm{C}$ & 3.85471800 & -4.28928100 & -6.38489800 \\
\hline $\mathrm{H}$ & 6.60256700 & -11.85867500 & -1.34724700 & $\mathrm{H}$ & 5.13416100 & -4.11209300 & -4.67349400 \\
\hline $\mathrm{H}$ & 8.12931300 & -11.14078800 & -0.80884100 & $\mathrm{C}$ & 4.18832100 & -2.64607300 & -8.10253500 \\
\hline $\mathrm{H}$ & 7.39858700 & -10.62474000 & -2.34143800 & $\mathrm{C}$ & 5.92786500 & -0.84747400 & -7.97820100 \\
\hline $\mathrm{H}$ & 2.35230800 & -14.11729100 & 1.68239700 & $\mathrm{H}$ & 7.35088900 & 0.69840700 & -7.44580100 \\
\hline $\mathrm{C}$ & 7.14625800 & -8.50098800 & -0.36802500 & $\mathrm{C}$ & 3.50368900 & -3.75948600 & -7.62672000 \\
\hline $\mathrm{C}$ & 7.61261200 & -7.90057200 & -1.69189200 & $\mathrm{H}$ & 3.33070600 & -5.15460700 & -5.98710700 \\
\hline $\mathrm{C}$ & 6.30518500 & -7.47204600 & 0.41644000 & $\mathrm{H}$ & 3.91882500 & -2.21845400 & -9.06662100 \\
\hline $\mathrm{C}$ & 8.80504900 & -8.34649900 & -2.28645100 & $\mathrm{H}$ & 6.61522400 & -1.19228200 & -8.76653700 \\
\hline $\mathrm{C}$ & 6.88111000 & -6.87984000 & -2.32990000 & $\mathrm{H}$ & 2.70231400 & -4.20194900 & -8.21287700 \\
\hline $\mathrm{C}$ & 5.14644100 & -6.93650800 & -0.43264500 & $\mathrm{H}$ & 6.22434400 & -1.31563600 & -4.50511700 \\
\hline $\mathrm{H}$ & 6.95752300 & -6.63688200 & 0.70756200 & $\mathrm{H}$ & 8.22876400 & -1.58267700 & -6.78586900 \\
\hline $\mathrm{C}$ & 9.25003500 & -7.84195100 & -3.50527100 & $\mathrm{H}$ & 6.03765400 & 0.50074400 & -6.28613000 \\
\hline $\mathrm{H}$ & 9.39507500 & -9.10391600 & -1.77632100 & $\mathrm{H}$ & 5.19192500 & -0.20797100 & -8.48203000 \\
\hline $\mathrm{C}$ & 7.33539700 & -6.38386900 & -3.56199300 & & & & \\
\hline $\mathrm{C}$ & 5.66646500 & -6.22529500 & -1.68971600 & $4 a-S R$ & & & \\
\hline $\mathrm{H}$ & 4.48169700 & -7.76320800 & -0.71231000 & $\mathrm{H}$ & 7.67872300 & -3.87210800 & 5.13085100 \\
\hline $\mathrm{C}$ & 8.50043900 & -6.85923800 & -4.15617700 & $\mathrm{~N}$ & 6.81016100 & -3.42785100 & 3.29588800 \\
\hline $\mathrm{H}$ & 10.17575000 & -8.20824600 & -3.94112700 & $\mathrm{C}$ & 7.56460800 & -2.99721600 & 4.48069000 \\
\hline $\mathrm{H}$ & 6.76356400 & -5.59816700 & -4.05257100 & $\mathrm{C}$ & 7.15988400 & -4.53444000 & 2.51776000 \\
\hline $\mathrm{H}$ & 4.86388500 & -6.12404800 & -2.43217500 & $\mathrm{C}$ & 6.71400500 & -4.60115500 & 1.18166600 \\
\hline $\mathrm{H}$ & 8.82956100 & -6.45709200 & -5.11079200 & $\mathrm{C}$ & 7.89015100 & -5.62506300 & 3.02756200 \\
\hline $\mathrm{H}$ & 8.04588300 & -8.73531800 & 0.22352200 & $\mathrm{C}$ & 6.98036900 & -5.71545100 & 0.39307300 \\
\hline $\mathrm{H}$ & 5.92393500 & -7.92657400 & 1.33482900 & $\mathrm{H}$ & 6.14809600 & -3.76749500 & 0.76948000 \\
\hline $\mathrm{H}$ & 4.53399200 & -6.24291800 & 0.15576900 & $\mathrm{C}$ & 8.15185600 & -6.73646100 & 2.22522800 \\
\hline $\mathrm{H}$ & 5.95023900 & -5.19607600 & -1.42229500 & $\mathrm{H}$ & 8.24877700 & -5.61326900 & 4.05136500 \\
\hline & & & & $\mathrm{C}$ & 7.70349800 & -6.79651300 & 0.90574300 \\
\hline 4a- $R S$ & & & & $\mathrm{H}$ & 6.62198000 & -5.73630300 & -0.63321100 \\
\hline $\mathrm{H}$ & 7.67872300 & -3.87210800 & -5.13085100 & $\mathrm{H}$ & 8.71716900 & -7.56498400 & 2.64503000 \\
\hline $\mathrm{N}$ & 6.81016100 & -3.42785100 & -3.29588800 & $\mathrm{H}$ & 6.47452700 & -2.64592200 & 2.74382100 \\
\hline $\mathrm{C}$ & 7.56460800 & -2.99721600 & -4.48069000 & $\mathrm{C}$ & 8.96754300 & -2.50565800 & 4.07544500 \\
\hline $\mathrm{C}$ & 7.15988400 & -4.53444000 & -2.51776000 & $\mathrm{H}$ & 9.45793100 & -3.27258000 & 3.46768900 \\
\hline $\mathrm{C}$ & 6.71400500 & -4.60115500 & -1.18166600 & $\mathrm{H}$ & 8.90674700 & -1.59079800 & 3.47296000 \\
\hline $\mathrm{C}$ & 7.89015100 & -5.62506300 & -3.02756200 & $\mathrm{H}$ & 9.60602300 & -2.30399100 & 4.94102100 \\
\hline $\mathrm{C}$ & 6.98036900 & -5.71545100 & -0.39307300 & $\mathrm{H}$ & 7.91367600 & -7.66460500 & 0.28812100 \\
\hline $\mathrm{H}$ & 6.14809600 & -3.76749500 & -0.76948000 & $\mathrm{C}$ & 6.71040900 & -1.95013300 & 5.26760000 \\
\hline
\end{tabular}


$$
\begin{aligned}
& \mathrm{C} \\
& \mathrm{C} \\
& \mathrm{C} \\
& \mathrm{C} \\
& \mathrm{C} \\
& \mathrm{H} \\
& \mathrm{C} \\
& \mathrm{H} \\
& \mathrm{C} \\
& \mathrm{C} \\
& \mathrm{H} \\
& \mathrm{C} \\
& \mathrm{H} \\
& \mathrm{H} \\
& \mathrm{H} \\
& \mathrm{H} \\
& \mathrm{H} \\
& \mathrm{H} \\
& \mathrm{H} \\
& \mathrm{H}
\end{aligned}
$$

$5.58987500-2.58492300$

$7.58323300-0.99998400$

$4.87879000-3.70340500$

$5.22553400-2.04890700$

$6.72304800-0.04947400$

$8.24666400 \quad-0.42796900$

$3.85471800-4.28928100$

$5.13416100-4.11209300$

$4.18832100-2.64607300$

$5.92786500 \quad-0.84747400$

$\begin{array}{ll}7.35088900 & 0.69840700\end{array}$

$3.50368900-3.75948600$

$3.33070600 \quad-5.15460700$

$3.91882500-2.21845400$

$6.61522400-1.19228200$

$2.70231400-4.20194900$

$6.22434400 \quad-1.31563600$

$8.22876400-1.58267700$

$6.03765400 \quad 0.50074400$

$5.19192500-0.20797100$

6.11612100

6.11202500

5.64405000

7.37094900

6.94640000

5.45580100

6.38489800

4.67349400

8.10253500

7.97820100

7.44580100

7.62672000

5.98710700

9.06662100

8.76653700

8.21287700

4.50511700

6.78586900

6.28613000

8.48203000

4a-SS
H
N
C
C
C
C
C
H
C
H
C
H
H
H
C
H
H
H
H
C
C
C
C
C
C
H
C
H

$\begin{array}{llll}5.47212000 & -9.63903000 & 1.12129000\end{array}$

$\begin{array}{llll}5.96680800 & -10.33227800 & -0.76870800\end{array}$

$\begin{array}{llll}6.38306800 & -9.85373700 & 0.55398700\end{array}$

$\begin{array}{llll}5.02979400 & -11.35056800 & -0.95681300\end{array}$

$5.02851200-12.06310600-2.17405700$

$\begin{array}{llll}4.04217200 & -11.66839500 & -0.00468500\end{array}$

$\begin{array}{llll}4.07505500 & -13.04258800 & -2.43036200\end{array}$

$\begin{array}{llll}5.78496400 & -11.83342900 & -2.92258200\end{array}$

$\begin{array}{llll}3.09125800 & -12.65363900 & -0.27405900\end{array}$

$\begin{array}{llll}4.00817100 & -11.14606300 & 0.94562900\end{array}$

$\begin{array}{llll}3.09426400 & -13.34993200 & -1.48266800\end{array}$

$\begin{array}{llll}4.10214400 & -13.57294800 & -3.37917500\end{array}$

$\begin{array}{llll}2.34027500 & -12.87750600 & 0.47979700\end{array}$

$\begin{array}{llll}6.74163800 & -10.38929500 & -1.42140700\end{array}$

$\begin{array}{llll}7.17837700 & -10.92810600 & 1.31450300\end{array}$

$6.60256700-11.85867500 \quad 1.34724700$

$8.12931300-11.14078800 \quad 0.80884100$

$\begin{array}{llll}7.39858700 & -10.62474000 & 2.34143800\end{array}$

$\begin{array}{llll}2.35230800 & -14.11729100 & -1.68239700\end{array}$

$\begin{array}{llll}7.14625800 & -8.50098800 & 0.36802500\end{array}$

$\begin{array}{llll}7.61261200 & -7.90057200 & 1.69189200\end{array}$

$\begin{array}{llll}6.30518500 & -7.47204600 & -0.41644000\end{array}$

$\begin{array}{llll}8.80504900 & -8.34649900 & 2.28645100\end{array}$

$\begin{array}{llll}6.88111000 & -6.87984000 & 2.32990000\end{array}$

$\begin{array}{llll}5.14644100 & -6.93650800 & 0.43264500\end{array}$

$6.95752300-6.63688200-0.70756200$

$\begin{array}{llll}9.25003500 & -7.84195100 & 3.50527100\end{array}$

$\begin{array}{llll}9.39507500 & -9.10391600 & 1.77632100\end{array}$

$\begin{array}{llll}7.33539700 & -6.38386900 & 3.56199300\end{array}$

$\begin{array}{llll}5.66646500 & -6.22529500 & 1.68971600\end{array}$

$4.48169700 \quad-7.76320800$

$8.50043900-6.85923800$

0.71231000

4.15617700 $\begin{array}{lll}10.17575000 & -8.20824600 & 3.94112700\end{array}$

$\begin{array}{llll}6.76356400 & -5.59816700 & 4.05257100\end{array}$

$\begin{array}{llll}4.86388500 & -6.12404800 & 2.43217500\end{array}$

$8.82956100 \quad-6.45709200 \quad 5.11079200$

$8.04588300 \quad-8.73531800 \quad-0.22352200$

$\begin{array}{llll}5.92393500 & -7.92657400 & -1.33482900\end{array}$

$\begin{array}{llll}4.53399200 & -6.24291800 & -0.15576900\end{array}$

$\begin{array}{llll}5.95023900 & -5.19607600 & 1.42229500\end{array}$

$\begin{array}{lrrr}\text { 8a- } \boldsymbol{R} \boldsymbol{R} & & & \\ \mathrm{H} & 3.39467900 & -3.05322800 & -2.49531800 \\ \mathrm{C} & 2.92911900 & -3.88918600 & -1.94487600 \\ \mathrm{C} & 3.88368200 & -4.35122900 & -0.84219600 \\ \mathrm{H} & 4.82860900 & -4.70411500 & -1.27644500 \\ \mathrm{H} & 3.43954600 & -5.18033300 & -0.27929300 \\ \mathrm{H} & 4.11942400 & -3.54125000 & -0.14555300 \\ \mathrm{C} & 1.55947700 & -3.41322000 & -1.40902200 \\ \mathrm{C} & 1.70310500 & -2.25931900 & -0.42642100 \\ \mathrm{C} & 0.59861200 & -3.06666400 & -2.56271900 \\ \mathrm{C} & 1.34444300 & -2.41851500 & 0.91908000 \\ \mathrm{C} & 2.18339800 & -1.00507200 & -0.83576200 \\ \mathrm{H} & 0.46529900 & -3.93048500 & -3.21796900 \\ \mathrm{C} & 1.46096000 & -1.36561300 & 1.82920300 \\ \mathrm{H} & 0.96718600 & -3.38122200 & 1.25709800 \\ \mathrm{C} & 2.30509000 & 0.04950900 & 0.06970800 \\ \mathrm{C} & 1.94359800 & -0.12623600 & 1.40740100 \\ \mathrm{H} & 1.17330500 & -1.51526300 & 2.86679700 \\ \mathrm{H} & 2.67987600 & 1.01142600 & -0.27106100 \\ \mathrm{H} & 2.03529600 & 0.69584800 & 2.11227400 \\ \mathrm{H} & 1.13316300 & -4.26775300 & -0.86559000 \\ \mathrm{H} & 0.98549300 & -2.23896700 & -3.16942100 \\ \mathrm{O} & 2.65418500 & -4.96294400 & -2.84935400 \\ \mathrm{H} & 3.50456500 & -5.30292100 & -3.16755000 \\ \mathrm{H} & -0.37801600 & -2.76367800 & -2.17002200 \\ \mathrm{H} & 2.46230400 & -0.84694600 & -1.87460100\end{array}$

\section{8a-RS}

C

C

C

C

C

C

$\mathrm{H}$

C

$\mathrm{H}$

C

C

$\mathrm{H}$

$\mathrm{H}$

$\mathrm{H}$

$\mathrm{H}$

$\mathrm{H}$

$\mathrm{O}$ $\begin{array}{rrr}2.82378300 & -3.95944100 & 1.96689900 \\ 1.46904200 & -3.39612000 & 1.48400500 \\ 1.67185600 & -2.28084800 & 0.46486600 \\ 0.57126500 & -2.94251800 & 2.65081400 \\ 1.37168900 & -2.48954800 & -0.88682400 \\ 2.15488600 & -1.02083700 & 0.84742100 \\ 0.27941200 & -3.78483100 & 3.28631200 \\ 1.54297900 & -1.47450900 & -1.82956300 \\ 1.00567600 & -3.46304700 & -1.20389600 \\ 2.33099600 & -0.00275400 & -0.09081000 \\ 2.02351400 & -0.22507000 & -1.43461300 \\ 1.30211100 & -1.66150300 & -2.87307100 \\ 2.70647700 & 0.96610000 & 0.22927400 \\ 2.15740800 & 0.56781700 & -2.16586200 \\ 0.96528100 & -4.22588700 & 0.96967200 \\ 1.07309800 & -2.20264200 & 3.28606000 \\ 3.48555700 & -4.43534600 & 0.79400100\end{array}$ 
$2.65418500-4.96294400$ 2.84935400

$-0.34356600-2.47997800$

2.26665900

$3.50456500-5.30292100$

3.16755000

$2.39728500 \quad-0.82734800$

1.88978200

$-0.37801600-2.76367800$

2.17002200

$2.46230400 \quad-0.84694600 \quad 1.87460100$

8a-SR
C
C
C
C
C
C
H
C
H
C
C
H
H
H
H
H
O
H
C
H
H
H
H
H
H

$\begin{array}{lll}2.82378300 & -3.95944100 & -1.96689900\end{array}$

$\begin{array}{llll}1.46904200 & -3.39612000 & -1.48400500\end{array}$

$\begin{array}{llll}1.67185600 & -2.28084800 & -0.46486600\end{array}$

$\begin{array}{llll}0.57126500 & -2.94251800 & -2.65081400\end{array}$

$\begin{array}{lll}1.37168900 & -2.48954800 & 0.88682400\end{array}$

$\begin{array}{lll}2.15488600 & -1.02083700 & -0.84742100\end{array}$

$\begin{array}{llll}0.27941200 & -3.78483100 & -3.28631200\end{array}$

$\begin{array}{llll}1.54297900 & -1.47450900 & 1.82956300\end{array}$

$\begin{array}{lll}1.00567600 & -3.46304700 & 1.20389600\end{array}$

$\begin{array}{lll}2.33099600 & -0.00275400 & 0.09081000\end{array}$

$\begin{array}{lll}2.02351400 & -0.22507000 & 1.43461300\end{array}$

$\begin{array}{lll}1.30211100 & -1.66150300 & 2.87307100\end{array}$

$\begin{array}{llll}2.70647700 & 0.96610000 & -0.22927400\end{array}$

$\begin{array}{llll}2.15740800 & 0.56781700 & 2.16586200\end{array}$

$\begin{array}{lll}0.96528100 & -4.22588700 & -0.96967200\end{array}$

$\begin{array}{llll}1.07309800 & -2.20264200 & -3.28606000\end{array}$

$\begin{array}{llll}3.48555700 & -4.43534600 & -0.79400100\end{array}$

$\begin{array}{llll}4.37049800 & -4.73451300 & -1.05361600\end{array}$

$\begin{array}{llll}2.69895100 & -5.08674500 & -2.99598900\end{array}$

$\begin{array}{llll}2.27395400 & -4.73976500 & -3.94344200\end{array}$

$\begin{array}{llll}2.07396000 & -5.89733900 & -2.60374800\end{array}$

$\begin{array}{llll}3.68994600 & -5.50408600 & -3.21759800\end{array}$

$\begin{array}{llll}3.39968900 & -3.13049000 & -2.41401300\end{array}$

$\begin{array}{lll}-0.34356600 & -2.47997800 & -2.26665900\end{array}$

$\begin{array}{lll}2.39728500 & -0.82734800 & -1.88978200\end{array}$

\section{8a-SS}

$\mathrm{H}$

C

C

$\mathrm{H}$

$\mathrm{H}$

$\mathrm{H}$

C

C

C

C

$\mathrm{C}$

$\mathrm{H}$

$\mathrm{C}$

$\mathrm{H}$

$\mathrm{C}$

C

$\mathrm{H}$ $\begin{array}{rrr}3.39467900 & -3.05322800 & 2.49531800 \\ 2.92911900 & -3.88918600 & 1.94487600 \\ 3.88368200 & -4.35122900 & 0.84219600 \\ 4.82860900 & -4.70411500 & 1.27644500 \\ 3.43954600 & -5.18033300 & 0.27929300 \\ 4.11942400 & -3.54125000 & 0.14555300 \\ 1.55947700 & -3.41322000 & 1.40902200 \\ 1.70310500 & -2.25931900 & 0.42642100 \\ 0.59861200 & -3.06666400 & 2.56271900 \\ 1.34444300 & -2.41851500 & -0.91908000 \\ 2.18339800 & -1.00507200 & 0.83576200 \\ 0.46529900 & -3.93048500 & 3.21796900 \\ 1.46096000 & -1.36561300 & -1.82920300 \\ 0.96718600 & -3.38122200 & -1.25709800 \\ 2.30509000 & 0.04950900 & -0.06970800 \\ 1.94359800 & -0.12623600 & -1.40740100 \\ 1.17330500 & -1.51526300 & -2.86679700\end{array}$

9a-R
H
N
C
C
C
C
C
H
C
H
C
H
H
H
C
H
H
H
H
C
C
C
C
C
H
C
H
C
C
H
H
C
H
H
H
H
H
9a-R
H
C
H

$\begin{array}{lll}5.42681700 & -9.53044400 & -1.02587100\end{array}$

$6.02299000-10.40455100 \quad 0.75330800$

$6.36474500-9.85841100-0.56266600$

$\begin{array}{llll}5.01995400 & -11.35590800 & 0.95227600\end{array}$

$\begin{array}{llll}5.04683300 & -12.14364700 & 2.12185200\end{array}$

$3.94302200-11.53150100 \quad 0.06237200$

$\begin{array}{lll}4.03525500 & -13.05864100 & 2.39318500\end{array}$

$\begin{array}{llll}5.87136500 & -12.02290600 & 2.82243700\end{array}$

$\begin{array}{llll}2.93411600 & -12.45284800 & 0.34644400\end{array}$

$3.88529600-10.94902900-0.85102300$

$\begin{array}{llll}2.96575300 & -13.22414500 & 1.50803600\end{array}$

$\begin{array}{llll}4.08578800 & -13.64958400 & 3.30448300\end{array}$

$2.11423000-12.56665900-0.35871900$

$6.84168900-10.57354200 \quad 1.32824400$

$\begin{array}{llll}7.02749800 & -10.90954500 & -1.46946400\end{array}$

$6.39533900-11.80041400-1.54100100$

$7.99763100-11.21594600-1.05704400$

$7.19736600-10.52289200-2.47864300$

$\begin{array}{llll}2.17754000 & -13.94054600 & 1.71950700\end{array}$

$\begin{array}{llll}7.24718100 & -8.59331700 & -0.36299800\end{array}$

$7.68023500-7.98003900-1.68874000$

$\begin{array}{lll}6.55968000 & -7.54518500 & 0.53366700\end{array}$

$9.03548700 \quad-7.91121500-2.03682200$

$6.74057100 \quad-7.45050900-2.58736700$

$7.22201800 \quad-6.68835600 \quad 0.69703000$

$9.44461900-7.33580100-3.24202300$

$9.78099400 \quad-8.31323900 \quad-1.35396400$

$\begin{array}{lll}7.14312900 & -6.87809600 & -3.79417700\end{array}$

$8.49857600-6.81780500-4.12682300$

$10.50268100 \quad-7.29406900 \quad-3.48769700$

$6.39695600 \quad-6.47620600-4.47487700$

$8.81266400-6.37025800-5.06592500$

$\begin{array}{lll}8.16249200 & -8.92676300 & 0.15119500\end{array}$

$6.28964100 \quad-7.97523200 \quad 1.50155000$

$\begin{array}{lll}5.64013100 & -7.17093100 & 0.06870600\end{array}$

$\begin{array}{llll}5.68133000 & -7.48252100 & -2.34339800\end{array}$

$\begin{array}{ccc}8.05703500 & -3.49140100 & -5.17034000 \\ 6.81813900 & -3.58666300 & -3.51575100 \\ 7.63497200 & -2.79730700 & -4.43174700 \\ 7.25855200 & -4.71950100 & -2.84378900 \\ 6.52077100 & -5.19368900 & -1.73791500\end{array}$ 


\begin{tabular}{|c|c|c|c|c|c|c|c|}
\hline $\mathrm{C}$ & 8.39343300 & -5.45354600 & -3.24376800 & $\mathrm{C}$ & 7.48306800 & -0.95797400 & 6.21877300 \\
\hline $\mathrm{C}$ & 6.89667500 & -6.35373100 & -1.07049900 & $\mathrm{C}$ & 4.21607500 & -2.16169000 & 5.52463700 \\
\hline $\mathrm{H}$ & 5.64030000 & -4.64200300 & -1.41338300 & $\mathrm{C}$ & 5.72141000 & -3.49780200 & 6.83907200 \\
\hline $\mathrm{C}$ & 8.75894000 & -6.61628300 & -2.56412000 & $\mathrm{H}$ & 8.23810800 & -0.32901300 & 5.73730200 \\
\hline $\mathrm{H}$ & 8.98877800 & -5.12456000 & -4.08905900 & $\mathrm{C}$ & 3.12368200 & -2.77427700 & 6.14320500 \\
\hline $\mathrm{C}$ & 8.02151100 & -7.07974200 & -1.47479100 & $\mathrm{H}$ & 4.04404800 & -1.39423600 & 4.77217700 \\
\hline $\mathrm{H}$ & 6.30493400 & -6.69243900 & -0.22344100 & $\mathrm{C}$ & 4.63494700 & -4.11445800 & 7.45854500 \\
\hline $\mathrm{H}$ & 9.63853900 & -7.16263900 & -2.89642900 & $\mathrm{C}$ & 3.32973300 & -3.75529800 & 7.11291800 \\
\hline $\mathrm{H}$ & 6.10195000 & -3.06274700 & -3.02940000 & $\mathrm{H}$ & 2.11359800 & -2.48381600 & 5.86558500 \\
\hline $\mathrm{C}$ & 8.79550500 & -2.08624100 & -3.70975900 & $\mathrm{H}$ & 4.80764800 & -4.87811800 & 8.21258200 \\
\hline $\mathrm{H}$ & 9.33548400 & -2.79744500 & -3.07727700 & $\mathrm{H}$ & 2.48326800 & -4.23625900 & 7.59565600 \\
\hline $\mathrm{H}$ & 8.41662900 & -1.28297300 & -3.06499800 & $\mathrm{H}$ & 6.29621400 & -1.12144900 & 4.44127300 \\
\hline $\mathrm{H}$ & 9.51334900 & -1.65132000 & -4.41287400 & $\mathrm{H}$ & 7.98845400 & -1.58925200 & 6.95947200 \\
\hline $\mathrm{H}$ & 8.31561900 & -7.98395100 & -0.95027000 & $\mathrm{H}$ & 6.72919600 & -3.79193800 & 7.12223900 \\
\hline $\mathrm{C}$ & 6.70896400 & -1.81127100 & -5.19447100 & $\mathrm{H}$ & 6.79359200 & -0.30193800 & 6.75970700 \\
\hline $\mathrm{C}$ & 5.53005400 & -2.51393300 & -5.85726600 & & & & \\
\hline $\mathrm{C}$ & 7.48316400 & -0.95810200 & -6.21899700 & 9a-SS & & & \\
\hline $\mathrm{C}$ & 4.21607800 & -2.16123800 & -5.52480700 & $\mathrm{H}$ & 5.41656200 & -9.51509300 & 1.01358000 \\
\hline $\mathrm{C}$ & 5.72130100 & -3.49810600 & -6.83857900 & $\mathrm{~N}$ & 6.04037400 & -10.42242100 & -0.73939400 \\
\hline $\mathrm{H}$ & 8.23832600 & -0.32917600 & -5.73765500 & $\mathrm{C}$ & 6.36014300 & -9.86168100 & 0.57596200 \\
\hline $\mathrm{C}$ & 3.12363700 & -2.77386100 & -6.14325400 & $\mathrm{C}$ & 5.03077900 & -11.36498300 & -0.94676000 \\
\hline $\mathrm{H}$ & 4.04410800 & -1.39342700 & -4.77269700 & $\mathrm{C}$ & 5.07272000 & -12.16845100 & -2.10513000 \\
\hline $\mathrm{C}$ & 4.63479700 & -4.11483000 & -7.45791500 & $\mathrm{C}$ & 3.93380400 & -11.51669800 & -0.07715000 \\
\hline $\mathrm{C}$ & 3.32961500 & -3.75530900 & -7.11256400 & $\mathrm{C}$ & 4.05649500 & -13.07552800 & -2.38545700 \\
\hline $\mathrm{H}$ & 2.11357600 & -2.48310800 & -5.86585200 & $\mathrm{H}$ & 5.91281800 & -12.06627600 & -2.78998500 \\
\hline $\mathrm{H}$ & 4.80745200 & -4.87882800 & -8.21162000 & $\mathrm{C}$ & 2.92039800 & -12.43029300 & -0.37012000 \\
\hline $\mathrm{H}$ & 2.48311500 & -4.23632000 & -7.59519400 & $\mathrm{H}$ & 3.86412100 & -10.92175400 & 0.82734300 \\
\hline $\mathrm{H}$ & 6.29640500 & -1.12124300 & -4.44138900 & $\mathrm{C}$ & 2.96711800 & -13.21724900 & -1.52064100 \\
\hline $\mathrm{H}$ & 7.98841100 & -1.58953000 & -6.95967400 & $\mathrm{H}$ & 4.11900900 & -13.67904200 & -3.28772900 \\
\hline $\mathrm{H}$ & 6.72906600 & -3.79254400 & -7.12152900 & $\mathrm{H}$ & 2.08489100 & -12.52551600 & 0.31928700 \\
\hline \multirow[t]{2}{*}{$\mathrm{H}$} & 6.79373200 & -0.30203600 & -6.75995400 & $\mathrm{H}$ & 6.86914100 & -10.60859000 & -1.29425000 \\
\hline & & & & $\mathrm{C}$ & 6.98925400 & -10.90774300 & 1.51199600 \\
\hline 9a-SR & & & & $\mathrm{H}$ & 6.34497400 & -11.79009700 & 1.58082900 \\
\hline $\mathrm{H}$ & 8.05680600 & -3.49158600 & 5.17039600 & $\mathrm{H}$ & 7.96490500 & -11.23099800 & 1.12635900 \\
\hline $\mathrm{N}$ & 6.81812100 & -3.58661900 & 3.51561800 & $\mathrm{H}$ & 7.14044400 & -10.50925400 & 2.51954100 \\
\hline $\mathrm{C}$ & 7.63491800 & -2.79741200 & 4.43177600 & $\mathrm{H}$ & 2.17522500 & -13.92746600 & -1.73911200 \\
\hline $\mathrm{C}$ & 7.25852100 & -4.71945100 & 2.84364600 & $\mathrm{C}$ & 7.26313100 & -8.61079800 & 0.37766700 \\
\hline $\mathrm{C}$ & 6.52096400 & -5.19336700 & 1.73751300 & $\mathrm{C}$ & 7.67527600 & -7.98422300 & 1.70389100 \\
\hline $\mathrm{C}$ & 8.39316500 & -5.45374200 & 3.24384700 & $\mathrm{C}$ & 6.60928800 & -7.56743300 & -0.54895200 \\
\hline $\mathrm{C}$ & 6.89684300 & -6.35337400 & 1.07003700 & $\mathrm{C}$ & 9.02313800 & -7.93067100 & 2.08201900 \\
\hline $\mathrm{H}$ & 5.64069200 & -4.64146900 & 1.41280500 & $\mathrm{C}$ & 6.72357000 & -7.42768100 & 2.57304400 \\
\hline $\mathrm{C}$ & 8.75864000 & -6.61647300 & 2.56414400 & $\mathrm{H}$ & 7.28643800 & -6.72191300 & -0.71049300 \\
\hline $\mathrm{H}$ & 8.98834400 & -5.12498300 & 4.08934000 & $\mathrm{C}$ & 9.41347100 & -7.34412300 & 3.28808800 \\
\hline $\mathrm{C}$ & 8.02142200 & -7.07965100 & 1.47454200 & $\mathrm{H}$ & 9.77773000 & -8.35367700 & 1.42222000 \\
\hline $\mathrm{H}$ & 6.30529300 & -6.69184300 & 0.22275400 & $\mathrm{C}$ & 7.10725800 & -6.84397400 & 3.78059800 \\
\hline $\mathrm{H}$ & 9.63803800 & -7.16304900 & 2.89664000 & $\mathrm{C}$ & 8.45553900 & -6.79932300 & 4.14350100 \\
\hline $\mathrm{H}$ & 6.10211600 & -3.06255500 & 3.02915700 & $\mathrm{H}$ & 10.46617300 & -7.31466900 & 3.55742600 \\
\hline $\mathrm{C}$ & 8.79561200 & -2.08641400 & 3.70997800 & $\mathrm{H}$ & 6.35203000 & -6.42106100 & 4.43818100 \\
\hline $\mathrm{H}$ & 9.33571700 & -2.79768100 & 3.07767800 & $\mathrm{H}$ & 8.75494900 & -6.34305100 & 5.08320000 \\
\hline $\mathrm{H}$ & 8.41686900 & -1.28322000 & 3.06504600 & $\mathrm{H}$ & 8.18500400 & -8.96359800 & -0.11134200 \\
\hline $\mathrm{H}$ & 9.51331200 & -1.65141600 & 4.41319300 & $\mathrm{H}$ & 6.35443100 & -8.00849200 & -1.51598900 \\
\hline $\mathrm{H}$ & 8.31551500 & -7.98384200 & 0.94998500 & $\mathrm{H}$ & 5.68496200 & -7.17417900 & -0.10988200 \\
\hline $\mathrm{C}$ & 6.70888700 & -1.81134200 & 5.19441900 & $\mathrm{H}$ & 5.66978200 & -7.44730600 & 2.30526800 \\
\hline $\mathrm{C}$ & 5.53007900 & -2.51403800 & 5.85736700 & & & & \\
\hline
\end{tabular}




\begin{tabular}{|c|c|c|c|c|c|c|c|}
\hline TS-1-RR & & & & $\mathrm{H}$ & -4.58017700 & -1.50218300 & 2.69192700 \\
\hline $\mathrm{C}$ & -2.85855500 & -2.69337200 & -1.03113300 & $\mathrm{H}$ & -4.72565400 & -0.09351000 & 1.62792100 \\
\hline $\mathrm{C}$ & -3.83042200 & -1.89417600 & -0.35074300 & $\mathrm{H}$ & -3.30992200 & -0.25584100 & 2.66936100 \\
\hline $\mathrm{C}$ & -3.29664500 & -1.56579300 & 0.97211200 & $\mathrm{C}$ & -1.23498100 & -2.27153300 & 2.37614300 \\
\hline $\mathrm{C}$ & -2.02919100 & -2.19170100 & 1.10721100 & $\mathrm{H}$ & -0.23463000 & -2.67018600 & 2.20160400 \\
\hline $\mathrm{C}$ & -1.70405800 & -2.84513800 & -0.15510600 & $\mathrm{H}$ & -1.74576600 & -2.94773100 & 3.07565600 \\
\hline Ir & -1.94374500 & -0.73899800 & -0.65227800 & $\mathrm{H}$ & -1.13542800 & -1.29084700 & 2.84404500 \\
\hline $\mathrm{N}$ & -0.09859200 & 0.33267100 & -0.31088400 & $\mathrm{H}$ & -1.70255000 & -0.85206700 & -3.28800100 \\
\hline $\mathrm{N}$ & -1.39562400 & -0.18931800 & -2.57473900 & $\mathrm{C}$ & -3.30225900 & 1.96990100 & -1.62107800 \\
\hline $\mathrm{C}$ & 0.46005500 & 0.96948400 & -1.55195400 & $\mathrm{O}$ & -2.57861900 & 1.98712000 & -2.74679300 \\
\hline $\mathrm{C}$ & 0.07221300 & 0.02450600 & -2.70489100 & $\mathrm{H}$ & -2.85968600 & 1.04276800 & -0.90938600 \\
\hline $\mathrm{S}$ & -0.02720100 & 1.31120400 & 1.03559700 & $\mathrm{C}$ & -4.77397300 & 1.59965900 & -1.87521700 \\
\hline $\mathrm{O}$ & -1.01652500 & 0.80896200 & 2.01879200 & $\mathrm{H}$ & -5.35433800 & 1.47730500 & -0.95458800 \\
\hline $\mathrm{O}$ & -0.14356700 & 2.72062200 & 0.60232400 & $\mathrm{H}$ & -4.82999400 & 0.67684400 & -2.45916100 \\
\hline $\mathrm{C}$ & 1.60886800 & 1.20392200 & 1.89229500 & $\mathrm{H}$ & -5.23499800 & 2.39942800 & -2.46406800 \\
\hline $\mathrm{C}$ & 1.96570400 & 2.34281900 & 2.65417900 & $\mathrm{C}$ & -3.11161400 & 3.21597300 & -0.70028000 \\
\hline $\mathrm{C}$ & 2.44443900 & 0.07272200 & 1.84427200 & $\mathrm{H}$ & -2.02784000 & 3.37724400 & -0.66807600 \\
\hline $\mathrm{C}$ & 3.26258400 & 2.40750500 & 3.20527800 & $\mathrm{C}$ & -3.56309800 & 2.94944800 & 0.74627200 \\
\hline $\mathrm{C}$ & 3.71499000 & 0.13608500 & 2.45912800 & $\mathrm{H}$ & -4.60103100 & 2.58321800 & 0.76185000 \\
\hline $\mathrm{C}$ & 4.13203200 & 1.30133700 & 3.12448000 & $\mathrm{H}$ & -2.93359100 & 2.16674200 & 1.17730000 \\
\hline $\mathrm{C}$ & 0.99438000 & 3.46431000 & 2.98844300 & $\mathrm{C}$ & -3.74641800 & 4.49184200 & -1.28116400 \\
\hline $\mathrm{H}$ & -0.03956900 & 3.12443900 & 2.98307700 & $\mathrm{C}$ & -3.66652700 & 4.76487200 & -2.65884200 \\
\hline $\mathrm{H}$ & 1.05886300 & 4.29931900 & 2.28257100 & $\mathrm{C}$ & -4.36069300 & 5.44987300 & -0.44517000 \\
\hline $\mathrm{H}$ & 1.20634300 & 3.84568500 & 3.99139200 & $\mathrm{C}$ & -4.19594300 & 5.93166800 & -3.20572100 \\
\hline $\mathrm{C}$ & 3.72282800 & 3.69184700 & 3.87580400 & $\mathrm{H}$ & -3.18780800 & 4.03317100 & -3.30039300 \\
\hline $\mathrm{H}$ & 4.77963100 & 3.88584200 & 3.67430800 & $\mathrm{C}$ & -4.88177100 & 6.62377000 & -1.01003400 \\
\hline $\mathrm{H}$ & 3.59894400 & 3.66746800 & 4.96810500 & $\mathrm{C}$ & -4.81190400 & 6.87147600 & -2.37753900 \\
\hline $\mathrm{H}$ & 3.17195300 & 4.55844100 & 3.50690100 & $\mathrm{H}$ & -4.12206800 & 6.10743300 & -4.27640400 \\
\hline $\mathrm{C}$ & 5.49601000 & 1.34048000 & 3.79150800 & $\mathrm{H}$ & -5.34736700 & 7.35653500 & -0.35269700 \\
\hline $\mathrm{H}$ & 5.60914600 & 2.19609000 & 4.45696300 & $\mathrm{H}$ & -5.22354500 & 7.78962100 & -2.78979100 \\
\hline $\mathrm{H}$ & 6.31094700 & 1.38679800 & 3.05619700 & $\mathrm{C}$ & -3.48518500 & 4.22046900 & 1.59325900 \\
\hline $\mathrm{H}$ & 5.66567200 & 0.44663500 & 4.40078300 & $\mathrm{C}$ & -4.47438000 & 5.25702000 & 1.05723300 \\
\hline $\mathrm{C}$ & 4.62182200 & -1.07487100 & 2.36115700 & $\mathrm{H}$ & -2.46129000 & 4.61520200 & 1.55605700 \\
\hline $\mathrm{H}$ & 5.60206600 & -0.90064100 & 2.80424900 & $\mathrm{H}$ & -3.70391800 & 3.99854500 & 2.64562200 \\
\hline $\mathrm{H}$ & 4.78852000 & -1.35793700 & 1.31426800 & $\mathrm{H}$ & -4.33936200 & 6.22383800 & 1.55988800 \\
\hline $\mathrm{H}$ & 4.18889500 & -1.95322900 & 2.85802100 & $\mathrm{H}$ & -5.49795700 & 4.93182100 & 1.30376900 \\
\hline $\mathrm{C}$ & 2.04303100 & -1.25339900 & 1.23861800 & $\mathrm{C}$ & 0.45806100 & 0.51377800 & -4.09183800 \\
\hline $\mathrm{H}$ & 2.76932100 & -1.58340900 & 0.48811400 & $\mathrm{C}$ & -0.01912000 & 1.73154700 & -4.60275200 \\
\hline $\mathrm{H}$ & 1.07093800 & -1.20862800 & 0.75790000 & $\mathrm{C}$ & 1.29951000 & -0.27044700 & -4.89171200 \\
\hline $\mathrm{H}$ & 2.02752200 & -2.02442000 & 2.02104600 & $\mathrm{C}$ & 0.34933400 & 2.14828900 & -5.88204900 \\
\hline $\mathrm{H}$ & -1.95641500 & 0.82917300 & -2.77775900 & $\mathrm{H}$ & -0.69414200 & 2.33866800 & -4.00704300 \\
\hline $\mathrm{C}$ & -0.52374500 & -3.73501100 & -0.41084100 & $\mathrm{C}$ & 1.66767900 & 0.14750900 & -6.17185700 \\
\hline $\mathrm{H}$ & 0.35819300 & -3.39821000 & 0.13885400 & $\mathrm{H}$ & 1.68013200 & -1.21362800 & -4.50464800 \\
\hline $\mathrm{H}$ & -0.26409500 & -3.76103900 & -1.47350700 & $\mathrm{C}$ & 1.19364900 & 1.36164300 & -6.66957600 \\
\hline $\mathrm{H}$ & -0.74227100 & -4.76565900 & -0.09658500 & $\mathrm{H}$ & -0.02738300 & 3.09307200 & -6.26514800 \\
\hline $\mathrm{C}$ & -3.02481300 & -3.33466200 & -2.37832500 & $\mathrm{H}$ & 2.32457200 & -0.47322000 & -6.77574700 \\
\hline $\mathrm{H}$ & -3.67885400 & -2.74661600 & -3.02910100 & $\mathrm{H}$ & 1.47804800 & 1.69249800 & -7.66509400 \\
\hline $\mathrm{H}$ & -3.47296700 & -4.33186300 & -2.27421700 & $\mathrm{C}$ & 1.96026400 & 1.24647000 & -1.52330800 \\
\hline $\mathrm{H}$ & -2.06427500 & -3.46204400 & -2.88720600 & $\mathrm{C}$ & 2.91814500 & 0.23167700 & -1.65986500 \\
\hline $\mathrm{C}$ & -5.23895100 & -1.64858400 & -0.80129400 & $\mathrm{C}$ & 2.40724200 & 2.56968500 & -1.41290400 \\
\hline $\mathrm{H}$ & -5.88392800 & -2.46255600 & -0.44091200 & $\mathrm{C}$ & 4.28104000 & 0.52861800 & -1.68242700 \\
\hline $\mathrm{H}$ & -5.32367000 & -1.61627900 & -1.89041000 & $\mathrm{H}$ & 2.60175400 & -0.80280500 & -1.75531400 \\
\hline $\mathrm{H}$ & -5.63644400 & -0.71168900 & -0.40815200 & $\mathrm{C}$ & 3.77011900 & 2.87129200 & -1.42991300 \\
\hline $\mathrm{C}$ & -4.01682600 & -0.81084800 & 2.04898000 & $\mathrm{H}$ & 1.67602300 & 3.36364100 & -1.29672300 \\
\hline
\end{tabular}




\begin{tabular}{|c|c|c|c|c|c|c|c|}
\hline $\mathrm{C}$ & 4.71254500 & 1.85185600 & -1.56577800 & $\mathrm{H}$ & -3.39725400 & -4.19360500 & -2.47210000 \\
\hline $\mathrm{H}$ & 5.00648300 & -0.27303600 & -1.79914200 & $\mathrm{H}$ & -1.96331000 & -3.32037500 & -3.01618600 \\
\hline $\mathrm{H}$ & 4.09319900 & 3.90517400 & -1.34020700 & $\mathrm{C}$ & -5.14573400 & -1.57587200 & -0.87992500 \\
\hline $\mathrm{H}$ & 5.77408600 & 2.08479300 & -1.58531800 & $\mathrm{H}$ & -5.80432500 & -2.40651600 & -0.58865900 \\
\hline $\mathrm{H}$ & 0.55634000 & -0.94488000 & -2.53394500 & $\mathrm{H}$ & -5.20962000 & -1.46598300 & -1.96532100 \\
\hline \multirow[t]{2}{*}{$\mathrm{H}$} & -0.04803900 & 1.92340300 & -1.72861600 & $\mathrm{H}$ & -5.54068100 & -0.66454800 & -0.42796000 \\
\hline & & & & $\mathrm{C}$ & -3.98025000 & -0.95338700 & 2.03816000 \\
\hline TS-1-RS & & & & $\mathrm{H}$ & -4.61690600 & -1.68431200 & 2.55751300 \\
\hline $\mathrm{C}$ & -2.77333300 & -2.63658500 & -1.13240700 & $\mathrm{H}$ & -4.62166500 & -0.14771700 & 1.67382900 \\
\hline $\mathrm{C}$ & -3.74724100 & -1.86477500 & -0.42365900 & $\mathrm{H}$ & -3.28856700 & -0.52237500 & 2.76484600 \\
\hline $\mathrm{C}$ & -3.23345400 & -1.61886300 & 0.92295700 & $\mathrm{C}$ & -1.19194300 & -2.40294900 & 2.31203700 \\
\hline $\mathrm{C}$ & -1.97041400 & -2.25639600 & 1.03892800 & $\mathrm{H}$ & -0.21515900 & -2.85651700 & 2.13707700 \\
\hline $\mathrm{C}$ & -1.63195600 & -2.84494300 & -0.25050700 & $\mathrm{H}$ & -1.74557100 & -3.05781500 & 2.99866100 \\
\hline Ir & -1.84198700 & -0.71298000 & -0.64803100 & $\mathrm{H}$ & -1.04060000 & -1.43577500 & 2.79500300 \\
\hline $\mathrm{N}$ & 0.02257700 & 0.30529900 & -0.22843200 & $\mathrm{H}$ & -1.49397100 & -0.82296800 & -3.26853200 \\
\hline $\mathrm{N}$ & -1.21243900 & -0.15939700 & -2.54562500 & $\mathrm{C}$ & -3.11621400 & 2.04003000 & -1.63572500 \\
\hline $\mathrm{C}$ & 0.60899400 & 0.97405700 & -1.43787600 & $\mathrm{O}$ & -2.40561300 & 2.01319500 & -2.75808200 \\
\hline $\mathrm{C}$ & 0.25963400 & 0.05060300 & -2.62053300 & $\mathrm{H}$ & -2.68773600 & 1.10489100 & -0.88772400 \\
\hline$S$ & 0.05598200 & 1.25044800 & 1.14364400 & $\mathrm{C}$ & -4.59795400 & 1.68304600 & -1.84556100 \\
\hline $\mathrm{O}$ & -0.94042100 & 0.70134900 & 2.09435500 & $\mathrm{H}$ & -5.13785500 & 1.55201800 & -0.90380100 \\
\hline $\mathrm{O}$ & -0.07898700 & 2.66779200 & 0.74336400 & $\mathrm{H}$ & -4.67704700 & 0.77520000 & -2.44915500 \\
\hline $\mathrm{C}$ & 1.67726600 & 1.14588200 & 2.02556700 & $\mathrm{H}$ & -5.08056500 & 2.49406800 & -2.40431200 \\
\hline $\mathrm{C}$ & 2.02521100 & 2.28020000 & 2.79804400 & $\mathrm{C}$ & -2.90686700 & 3.37074700 & -0.81868700 \\
\hline $\mathrm{C}$ & 2.50610400 & 0.00903100 & 1.99034400 & $\mathrm{H}$ & -1.88366900 & 3.33748800 & -0.43329500 \\
\hline $\mathrm{C}$ & 3.31160400 & 2.33328500 & 3.37534600 & $\mathrm{C}$ & -2.99882800 & 4.54477600 & -1.83665800 \\
\hline $\mathrm{C}$ & 3.76394100 & 0.06110400 & 2.63079900 & $\mathrm{H}$ & -2.02299300 & 4.66289000 & -2.31628400 \\
\hline $\mathrm{C}$ & 4.17392200 & 1.22087300 & 3.31030100 & $\mathrm{H}$ & -3.69529800 & 4.28641700 & -2.64398400 \\
\hline $\mathrm{C}$ & 1.05610500 & 3.40717200 & 3.12002500 & $\mathrm{C}$ & -3.83375300 & 3.52704200 & 0.38552100 \\
\hline $\mathrm{H}$ & 0.01976800 & 3.07545300 & 3.10198400 & $\mathrm{C}$ & -4.78336200 & 4.56580700 & 0.48213600 \\
\hline $\mathrm{H}$ & 1.13313100 & 4.24157400 & 2.41467100 & $\mathrm{C}$ & -3.71268100 & 2.63415600 & 1.46416000 \\
\hline $\mathrm{H}$ & 1.25894500 & 3.78821400 & 4.12487800 & $\mathrm{C}$ & -5.61420100 & 4.64277200 & 1.60892200 \\
\hline $\mathrm{C}$ & 3.76748500 & 3.61195800 & 4.05944500 & $\mathrm{C}$ & -4.54490700 & 2.72114400 & 2.57983000 \\
\hline $\mathrm{H}$ & 4.83292800 & 3.79098700 & 3.89302700 & $\mathrm{H}$ & -2.93413800 & 1.87905700 & 1.44268900 \\
\hline $\mathrm{H}$ & 3.60685500 & 3.59132600 & 5.14701900 & $\mathrm{C}$ & -5.51603700 & 3.72197500 & 2.64891600 \\
\hline $\mathrm{H}$ & 3.24063100 & 4.48527900 & 3.67136500 & $\mathrm{H}$ & -6.33703800 & 5.45443500 & 1.67218200 \\
\hline $\mathrm{C}$ & 5.52056800 & 1.24644100 & 4.01237200 & $\mathrm{H}$ & -4.41892900 & 2.02128400 & 3.40259700 \\
\hline $\mathrm{H}$ & 5.61288200 & 2.08540600 & 4.70216700 & $\mathrm{H}$ & -6.16682100 & 3.80375700 & 3.51616900 \\
\hline $\mathrm{H}$ & 6.35414000 & 1.31499400 & 3.29993500 & $\mathrm{C}$ & -3.47067200 & 5.85679900 & -1.20913400 \\
\hline $\mathrm{H}$ & 5.67781100 & 0.33792000 & 4.60266400 & $\mathrm{H}$ & -3.51373300 & 6.64362300 & -1.97356800 \\
\hline $\mathrm{C}$ & 4.66280700 & -1.15731900 & 2.55310700 & $\mathrm{H}$ & -2.76517200 & 6.19494200 & -0.43754600 \\
\hline $\mathrm{H}$ & 5.65229600 & -0.97365900 & 2.97123500 & $\mathrm{C}$ & -4.85150700 & 5.64526600 & -0.57914900 \\
\hline $\mathrm{H}$ & 4.80851800 & -1.47567100 & 1.51391300 & $\mathrm{H}$ & -5.56486800 & 5.36899900 & -1.37194900 \\
\hline $\mathrm{H}$ & 4.23549100 & -2.01720400 & 3.08702400 & $\mathrm{H}$ & -5.23279300 & 6.57310200 & -0.13416300 \\
\hline $\mathrm{C}$ & 2.10561600 & -1.31155800 & 1.37193600 & $\mathrm{C}$ & 2.10591500 & 1.25663100 & -1.36149400 \\
\hline $\mathrm{H}$ & 2.83482700 & -1.63643700 & 0.62152700 & $\mathrm{C}$ & 3.06951700 & 0.24467800 & -1.47684700 \\
\hline $\mathrm{H}$ & 1.13714600 & -1.26131900 & 0.88513200 & $\mathrm{C}$ & 2.54613600 & 2.57946700 & -1.22553200 \\
\hline $\mathrm{H}$ & 2.08370900 & -2.08909800 & 2.14739200 & $\mathrm{C}$ & 4.43161500 & 0.54425600 & -1.45596100 \\
\hline $\mathrm{H}$ & -1.75918400 & 0.83431600 & -2.76401600 & $\mathrm{H}$ & 2.75750700 & -0.78940000 & -1.58908300 \\
\hline $\mathrm{C}$ & -0.45379000 & -3.73000200 & -0.53245100 & $\mathrm{C}$ & 3.90833300 & 2.88386500 & -1.19917400 \\
\hline $\mathrm{H}$ & 0.43193400 & -3.40426700 & 0.01829400 & $\mathrm{H}$ & 1.80995700 & 3.37091500 & -1.12361900 \\
\hline $\mathrm{H}$ & -0.20097900 & -3.73485000 & -1.59699000 & $\mathrm{C}$ & 4.85654100 & 1.86743600 & -1.31593600 \\
\hline $\mathrm{H}$ & -0.66979800 & -4.76703000 & -0.23821700 & $\mathrm{H}$ & 5.16198100 & -0.25512300 & -1.55604400 \\
\hline $\mathrm{C}$ & -2.92903300 & -3.20120800 & -2.51486000 & $\mathrm{H}$ & 4.22654300 & 3.91744000 & -1.09043100 \\
\hline $\mathrm{H}$ & -3.56149500 & -2.56543800 & -3.14179300 & $\mathrm{H}$ & 5.91767600 & 2.10251900 & -1.30161500 \\
\hline
\end{tabular}




\begin{tabular}{|c|c|c|c|c|c|c|c|}
\hline $\mathrm{C}$ & 0.69541700 & 0.55729400 & -3.98617000 & $\mathrm{H}$ & -2.00709200 & 0.60379400 & -2.83447300 \\
\hline $\mathrm{C}$ & 0.21166700 & 1.76593100 & -4.51274700 & $\mathrm{C}$ & -0.58518600 & -3.78744700 & -0.19397400 \\
\hline $\mathrm{C}$ & 1.59138800 & -0.20210100 & -4.75021700 & $\mathrm{H}$ & 0.20901200 & -3.41957500 & 0.45916800 \\
\hline $\mathrm{C}$ & 0.62826800 & 2.19798500 & -5.77225200 & $\mathrm{H}$ & -0.18159500 & -3.84644200 & -1.20909800 \\
\hline $\mathrm{H}$ & -0.50862300 & 2.35105000 & -3.94801100 & $\mathrm{H}$ & -0.83717500 & -4.80952900 & 0.12327600 \\
\hline $\mathrm{C}$ & 2.00778300 & 0.23194400 & -6.00996500 & $\mathrm{C}$ & -2.69786600 & -3.33140600 & -2.57493000 \\
\hline $\mathrm{H}$ & 1.97609300 & -1.13828000 & -4.35061800 & $\mathrm{H}$ & -3.23480900 & -2.72446500 & -3.31077100 \\
\hline $\mathrm{C}$ & 1.52731800 & 1.43699400 & -6.52342800 & $\mathrm{H}$ & -3.16055600 & -4.32736400 & -2.57378300 \\
\hline $\mathrm{H}$ & 0.24497800 & 3.13446800 & -6.16907000 & $\mathrm{H}$ & -1.66484700 & -3.45518100 & -2.91537500 \\
\hline $\mathrm{H}$ & 2.70620700 & -0.36961200 & -6.58600200 & $\mathrm{C}$ & -5.13640200 & -1.66904000 & -1.43322800 \\
\hline $\mathrm{H}$ & 1.84840200 & 1.77987900 & -7.50362700 & $\mathrm{H}$ & -5.67929000 & -2.60702800 & -1.61338300 \\
\hline $\mathrm{H}$ & 0.09962700 & 1.92871000 & -1.61044600 & $\mathrm{H}$ & -4.95488600 & -1.18971800 & -2.40000400 \\
\hline \multirow[t]{2}{*}{$\mathrm{H}$} & 0.73226200 & -0.92338500 & -2.44600700 & $\mathrm{H}$ & -5.79535100 & -1.01653200 & -0.85696800 \\
\hline & & & & $\mathrm{C}$ & -4.50306900 & -0.99642400 & 1.67939600 \\
\hline TS-1-SR & & & & $\mathrm{H}$ & -5.18835200 & -1.73268100 & 2.12413900 \\
\hline $\mathrm{C}$ & -2.77018700 & -2.72873400 & -1.20213100 & $\mathrm{H}$ & -5.10573700 & -0.22195700 & 1.20121400 \\
\hline $\mathrm{C}$ & -3.85698900 & -1.94602300 & -0.69604300 & $\mathrm{H}$ & -3.94147200 & -0.52227200 & 2.48727400 \\
\hline $\mathrm{C}$ & -3.58251800 & -1.67704000 & 0.71409100 & $\mathrm{C}$ & -1.83985800 & -2.44435700 & 2.46574400 \\
\hline $\mathrm{C}$ & -2.35637400 & -2.29915900 & 1.06431500 & $\mathrm{H}$ & -0.88507700 & -2.97330700 & 2.48895400 \\
\hline $\mathrm{C}$ & -1.79705700 & -2.90595000 & -0.13597100 & $\mathrm{H}$ & -2.55762900 & -3.02993400 & 3.05600000 \\
\hline Ir & -1.95082300 & -0.78431900 & -0.64678100 & $\mathrm{H}$ & -1.70155100 & -1.47311800 & 2.94328300 \\
\hline $\mathrm{N}$ & -0.09922800 & 0.22804200 & -0.18747000 & $\mathrm{H}$ & -1.51931800 & -0.98329200 & -3.22760200 \\
\hline $\mathrm{N}$ & -1.31702900 & -0.25105700 & -2.54567000 & $\mathrm{C}$ & -3.22158700 & 1.96377700 & -1.69559000 \\
\hline $\mathrm{C}$ & 0.46686000 & 0.93714000 & -1.37541500 & $\mathrm{O}$ & -3.05309500 & 1.52250100 & -2.93957400 \\
\hline $\mathrm{C}$ & 0.13588800 & 0.06014100 & -2.60519800 & $\mathrm{H}$ & -2.70617100 & 1.08564300 & -0.92342200 \\
\hline S & -0.11762300 & 1.17635500 & 1.19773900 & $\mathrm{C}$ & -2.48700300 & 3.28619500 & -1.40777300 \\
\hline $\mathrm{O}$ & -0.99897700 & 0.50913900 & 2.18033400 & $\mathrm{H}$ & -2.34864600 & 3.49900300 & -0.34953500 \\
\hline $\mathrm{O}$ & -0.44973400 & 2.56579700 & 0.83178400 & $\mathrm{H}$ & -3.06909000 & 4.09956700 & -1.86042000 \\
\hline $\mathrm{C}$ & 1.57482000 & 1.17656600 & 1.93751600 & $\mathrm{H}$ & -1.50866100 & 3.27710200 & -1.89249500 \\
\hline $\mathrm{C}$ & 2.21412800 & 2.40237000 & 2.24118000 & $\mathrm{C}$ & -4.72916700 & 1.99406100 & -1.25831300 \\
\hline $\mathrm{C}$ & 2.18954200 & -0.06249400 & 2.22401200 & $\mathrm{H}$ & -5.04342500 & 0.94488800 & -1.21821700 \\
\hline $\mathrm{C}$ & 3.54053400 & 2.35838500 & 2.73018300 & $\mathrm{C}$ & -5.55973100 & 2.64013700 & -2.38694900 \\
\hline $\mathrm{C}$ & 3.48370200 & -0.07563200 & 2.77954300 & $\mathrm{H}$ & -5.17358400 & 3.64450400 & -2.61110600 \\
\hline $\mathrm{C}$ & 4.15418700 & 1.13288000 & 3.03920400 & $\mathrm{H}$ & -5.42649900 & 2.04501000 & -3.29363100 \\
\hline $\mathrm{C}$ & 1.56157800 & 3.76958700 & 2.12859200 & $\mathrm{C}$ & -5.03114700 & 2.61717400 & 0.11019600 \\
\hline $\mathrm{H}$ & 0.51223200 & 3.74499300 & 2.41168300 & $\mathrm{C}$ & -4.17645000 & 2.39555700 & 1.20538300 \\
\hline $\mathrm{H}$ & 1.59492700 & 4.15628100 & 1.10345400 & $\mathrm{C}$ & -6.19805900 & 3.38388000 & 0.32448300 \\
\hline $\mathrm{H}$ & 2.07033500 & 4.48419500 & 2.77752900 & $\mathrm{C}$ & -4.44748400 & 2.91744000 & 2.46885000 \\
\hline $\mathrm{C}$ & 4.32377000 & 3.64904700 & 2.90223800 & $\mathrm{H}$ & -3.26961100 & 1.81609300 & 1.07596100 \\
\hline $\mathrm{H}$ & 5.39967500 & 3.46921800 & 2.85563000 & $\mathrm{C}$ & -6.46394700 & 3.89532500 & 1.60339700 \\
\hline $\mathrm{H}$ & 4.11801500 & 4.14760200 & 3.86032100 & $\mathrm{C}$ & -5.60202300 & 3.67361300 & 2.67364100 \\
\hline $\mathrm{H}$ & 4.08974400 & 4.36384800 & 2.10929900 & $\mathrm{H}$ & -3.75135300 & 2.73336900 & 3.28290400 \\
\hline $\mathrm{C}$ & 5.53291500 & 1.11258000 & 3.67365200 & $\mathrm{H}$ & -7.36938600 & 4.48132700 & 1.75286600 \\
\hline $\mathrm{H}$ & 5.67589100 & 1.95610600 & 4.35409900 & $\mathrm{H}$ & -5.82953600 & 4.08421400 & 3.65433500 \\
\hline $\mathrm{H}$ & 6.33262000 & 1.16519200 & 2.92058400 & $\mathrm{C}$ & -7.03229000 & 2.75222400 & -1.99101900 \\
\hline $\mathrm{H}$ & 5.69859200 & 0.20387100 & 4.25561900 & $\mathrm{C}$ & -7.17677300 & 3.69598500 & -0.79499900 \\
\hline $\mathrm{C}$ & 4.17861400 & -1.39034100 & 3.08787700 & $\mathrm{H}$ & -7.42344000 & 1.75713300 & -1.73120600 \\
\hline $\mathrm{H}$ & 5.22753100 & -1.36956100 & 2.77477900 & $\mathrm{H}$ & -7.63413800 & 3.11683600 & -2.83328200 \\
\hline $\mathrm{H}$ & 3.71346600 & -2.23739100 & 2.58348100 & $\mathrm{H}$ & -8.20213400 & 3.67677600 & -0.40267300 \\
\hline $\mathrm{H}$ & 4.17055200 & -1.61410200 & 4.16422700 & $\mathrm{H}$ & -7.00739800 & 4.72832100 & -1.13915700 \\
\hline $\mathrm{C}$ & 1.51051800 & -1.39393100 & 1.98219200 & $\mathrm{C}$ & 0.54914400 & 0.65478500 & -3.94386900 \\
\hline $\mathrm{H}$ & 2.05201900 & -1.97928400 & 1.22774500 & $\mathrm{C}$ & -0.19308100 & 1.67541600 & -4.55977500 \\
\hline $\mathrm{H}$ & 0.49023100 & -1.28350700 & 1.63939100 & $\mathrm{C}$ & 1.70128800 & 0.17727200 & -4.58368900 \\
\hline $\mathrm{H}$ & 1.49532100 & -1.98597600 & 2.90513000 & $\mathrm{C}$ & 0.22135000 & 2.20327800 & -5.78420900 \\
\hline
\end{tabular}




\begin{tabular}{|c|c|c|c|c|c|c|c|}
\hline $\mathrm{H}$ & -1.10999800 & 2.03790200 & -4.10126300 & $\mathrm{H}$ & 5.03422300 & -1.34259200 & 3.20371100 \\
\hline $\mathrm{C}$ & 2.11590500 & 0.70987200 & -5.80481600 & $\mathrm{H}$ & 3.57097200 & -1.65999700 & 4.13482600 \\
\hline $\mathrm{H}$ & 2.28768500 & -0.60850800 & -4.11387700 & $\mathrm{H}$ & 4.76816200 & -0.53584300 & 4.75087400 \\
\hline $\mathrm{C}$ & 1.37566800 & 1.72728900 & -6.40900700 & $\mathrm{C}$ & 1.96524600 & -1.43367800 & 2.21563700 \\
\hline $\mathrm{H}$ & -0.36611800 & 2.98930600 & -6.25162300 & $\mathrm{H}$ & 2.84389900 & -2.06363900 & 2.03992900 \\
\hline $\mathrm{H}$ & 3.01434500 & 0.32824200 & -6.28290800 & $\mathrm{H}$ & 1.29123300 & -1.55598600 & 1.37206200 \\
\hline $\mathrm{H}$ & 1.69320700 & 2.14250700 & -7.36202100 & $\mathrm{H}$ & 1.45154600 & -1.81660500 & 3.10555600 \\
\hline $\mathrm{C}$ & 1.96730900 & 1.19841100 & -1.31574700 & $\mathrm{H}$ & -1.66206500 & 0.66926700 & -3.02187600 \\
\hline $\mathrm{C}$ & 2.88630700 & 0.16087700 & -1.10905500 & $\mathrm{C}$ & -0.97411300 & -3.97510300 & -1.49052800 \\
\hline $\mathrm{C}$ & 2.45597400 & 2.48935100 & -1.54687000 & $\mathrm{H}$ & 0.00698900 & -3.86430400 & -1.02124700 \\
\hline $\mathrm{C}$ & 4.25838100 & 0.40773000 & -1.13453000 & $\mathrm{H}$ & -0.84801600 & -3.82496100 & -2.56706200 \\
\hline $\mathrm{H}$ & 2.52072400 & -0.84348600 & -0.91444400 & $\mathrm{H}$ & -1.30314300 & -5.01274700 & -1.33840100 \\
\hline $\mathrm{C}$ & 3.82935300 & 2.74144900 & -1.57310700 & $\mathrm{C}$ & -3.55656900 & -2.76526200 & -3.00121100 \\
\hline $\mathrm{H}$ & 1.75247300 & 3.30156200 & -1.71043800 & $\mathrm{H}$ & -4.10053400 & -1.92227100 & -3.43671900 \\
\hline $\mathrm{C}$ & 4.73519300 & 1.70038300 & -1.36831100 & $\mathrm{H}$ & -4.20769000 & -3.64817800 & -3.05269600 \\
\hline $\mathrm{H}$ & 4.95740100 & -0.40851900 & -0.96961800 & $\mathrm{H}$ & -2.68975100 & -2.97011900 & -3.63791100 \\
\hline $\mathrm{H}$ & 4.18917900 & 3.75066500 & -1.75613400 & $\mathrm{C}$ & -5.26498900 & -1.16774200 & -0.85269100 \\
\hline $\mathrm{H}$ & 5.80474000 & 1.89298000 & -1.38922600 & $\mathrm{H}$ & -6.01906000 & -1.93782000 & -0.63577000 \\
\hline $\mathrm{H}$ & 0.66669700 & -0.89038900 & -2.46991600 & $\mathrm{H}$ & -5.40515700 & -0.84813000 & -1.88794700 \\
\hline \multirow[t]{2}{*}{$\mathrm{H}$} & -0.03267300 & 1.90236600 & -1.51063100 & $\mathrm{H}$ & -5.46873200 & -0.31619900 & -0.20255500 \\
\hline & & & & $\mathrm{C}$ & -3.69405100 & -1.24590300 & 1.96798900 \\
\hline TS-1-SS & & & & $\mathrm{H}$ & -4.15519000 & -2.03854100 & 2.57393700 \\
\hline $\mathrm{C}$ & -3.15274200 & -2.49173000 & -1.58066000 & $\mathrm{H}$ & -4.44433500 & -0.46549600 & 1.81843800 \\
\hline $\mathrm{C}$ & -3.89993900 & -1.73971000 & -0.62281600 & $\mathrm{H}$ & -2.86360000 & -0.81090700 & 2.52898100 \\
\hline $\mathrm{C}$ & -3.20651100 & -1.79675800 & 0.66004600 & $\mathrm{C}$ & -1.15508000 & -3.11685500 & 1.57603900 \\
\hline $\mathrm{C}$ & -2.05033200 & -2.61612200 & 0.48658400 & $\mathrm{H}$ & -0.17251600 & -3.40183900 & 1.19253700 \\
\hline $\mathrm{C}$ & -1.96922900 & -3.01406200 & -0.90928100 & $\mathrm{H}$ & -1.61002600 & -4.01262000 & 2.02319700 \\
\hline Ir & -1.84148600 & -0.84829300 & -0.89668100 & $\mathrm{H}$ & -1.01222600 & -2.36425500 & 2.34999500 \\
\hline $\mathrm{N}$ & 0.07073800 & -0.05728200 & -0.29294000 & $\mathrm{H}$ & -1.31719600 & -0.93751900 & -3.47051800 \\
\hline $\mathrm{N}$ & -1.07969200 & -0.26730900 & -2.73784500 & $\mathrm{C}$ & -2.79589600 & 2.08173300 & -1.88803000 \\
\hline $\mathrm{C}$ & 0.78006100 & 0.64028900 & -1.40420200 & $\mathrm{O}$ & -2.58446600 & 1.69361700 & -3.14796500 \\
\hline $\mathrm{C}$ & 0.39795500 & -0.11133300 & -2.70941400 & $\mathrm{H}$ & -2.43712800 & 1.12614000 & -1.14148300 \\
\hline S & -0.00080300 & 0.77094600 & 1.17444700 & $\mathrm{C}$ & -1.94780200 & 3.29832200 & -1.47487700 \\
\hline $\mathrm{O}$ & -0.60660200 & -0.15778700 & 2.15096100 & $\mathrm{H}$ & -1.90894000 & 3.45973100 & -0.39827800 \\
\hline $\mathrm{O}$ & -0.66882100 & 2.07855000 & 1.03991000 & $\mathrm{H}$ & -2.37081600 & 4.18306800 & -1.96588200 \\
\hline $\mathrm{C}$ & 1.70180400 & 1.08947100 & 1.80148600 & $\mathrm{H}$ & -0.92492900 & 3.17841100 & -1.83934900 \\
\hline $\mathrm{C}$ & 2.19584200 & 2.41051800 & 1.87462200 & $\mathrm{C}$ & -4.30115900 & 2.23664400 & -1.49419800 \\
\hline $\mathrm{C}$ & 2.39253600 & 0.00891800 & 2.39184700 & $\mathrm{H}$ & -4.77629300 & 1.31129100 & -1.84501400 \\
\hline $\mathrm{C}$ & 3.35888800 & 2.64675100 & 2.64191600 & $\mathrm{C}$ & -4.48959400 & 2.32557200 & 0.03001200 \\
\hline $\mathrm{C}$ & 3.55874400 & 0.26923200 & 3.13563600 & $\mathrm{H}$ & -4.11741300 & 1.40736500 & 0.49629000 \\
\hline $\mathrm{C}$ & 4.05357400 & 1.58233000 & 3.24277600 & $\mathrm{H}$ & -3.87030700 & 3.13428900 & 0.43681600 \\
\hline $\mathrm{C}$ & 1.59881700 & 3.59686300 & 1.13924300 & $\mathrm{C}$ & -5.01329200 & 3.38946900 & -2.22637900 \\
\hline $\mathrm{H}$ & 0.91972000 & 4.17385600 & 1.77859800 & $\mathrm{C}$ & -5.98825700 & 4.17817800 & -1.57721700 \\
\hline $\mathrm{H}$ & 1.02086900 & 3.30331700 & 0.26896900 & $\mathrm{C}$ & -4.75721000 & 3.63244300 & -3.58801900 \\
\hline $\mathrm{H}$ & 2.39849500 & 4.26547600 & 0.80823600 & $\mathrm{C}$ & -6.65627000 & 5.17914000 & -2.29865100 \\
\hline $\mathrm{C}$ & 3.84920600 & 4.07147100 & 2.83345500 & $\mathrm{C}$ & -5.42348900 & 4.63584600 & -4.28794600 \\
\hline $\mathrm{H}$ & 4.40405400 & 4.18185900 & 3.76766700 & $\mathrm{H}$ & -4.00918700 & 3.02371000 & -4.08381700 \\
\hline $\mathrm{H}$ & 3.01809000 & 4.77955100 & 2.87463100 & $\mathrm{C}$ & -6.38123400 & 5.41893700 & -3.64137000 \\
\hline $\mathrm{H}$ & 4.51218600 & 4.39948800 & 2.01945800 & $\mathrm{H}$ & -7.40942700 & 5.77668900 & -1.78758600 \\
\hline $\mathrm{C}$ & 5.35660000 & 1.84525100 & 3.97579000 & $\mathrm{H}$ & -5.19654700 & 4.80186100 & -5.33840000 \\
\hline $\mathrm{H}$ & 5.19494700 & 2.08502000 & 5.03675400 & $\mathrm{H}$ & -6.91322100 & 6.20085600 & -4.17792000 \\
\hline $\mathrm{H}$ & 5.90251000 & 2.68329800 & 3.53585300 & $\mathrm{C}$ & -5.94701300 & 2.59974400 & 0.40649900 \\
\hline $\mathrm{H}$ & 6.02255000 & 0.98027100 & 3.93834700 & $\mathrm{H}$ & -6.60392000 & 1.83450000 & -0.03337000 \\
\hline $\mathrm{C}$ & 4.26891300 & -0.87333900 & 3.83947800 & $\mathrm{H}$ & -6.08399000 & 2.55194900 & 1.49428800 \\
\hline
\end{tabular}


$$
\begin{aligned}
& \mathrm{C} \\
& \mathrm{H} \\
& \mathrm{H} \\
& \mathrm{C} \\
& \mathrm{C} \\
& \mathrm{C} \\
& \mathrm{C} \\
& \mathrm{H} \\
& \mathrm{C} \\
& \mathrm{H} \\
& \mathrm{C} \\
& \mathrm{H} \\
& \mathrm{H} \\
& \mathrm{H} \\
& \mathrm{C} \\
& \mathrm{C} \\
& \mathrm{C} \\
& \mathrm{C} \\
& \mathrm{H} \\
& \mathrm{C} \\
& \mathrm{H} \\
& \mathrm{C} \\
& \mathrm{H} \\
& \mathrm{H} \\
& \mathrm{H} \\
& \mathrm{H} \\
& \mathrm{H}
\end{aligned}
$$

\begin{abstract}
$-6.34731100$
$3.98153800-0.11439600$
\end{abstract}

$-5.83620700$

4.74588100

0.49154800

$-7.42195100$

4.15602000

0.02596900

2.30258300

$0.64167700-1.28996400$

3.03067200

3.00533300

$1.79816300-1.58968700$

4.42694300

$-0.53441600-0.99611600$

2.50079000

$1.78781600-1.58164700$

4.39981300

$2.71374300-1.83882800$

2.45018500

$-0.54984400-0.98741200$

5.11682200

4.97361800

4.92831600

6.20362100

0.94819100

0.36753500

2.06800500

0.90892000

$-0.52609500$

2.60909200

2.53003800

2.03060200

0.44569400

3.47978200

2.44718100

0.43652000

0.81738900

$-1.43648600-0.75717900$

$0.61354000-1.27904500$

$2.69775500-1.81514100$

$-1.47053400-0.75213700$

$0.60302900-1.27148500$

$0.50522200-3.98776300$

$1.64209800-4.57281300$

$-0.07302700-4.60185800$

$2.18440600-5.74013800$

$2.08515200-4.13983100$

$0.47305100-5.76610800$

$-0.94960300-4.15418600$

$1.60706300-6.33833200$

$3.06150100-6.18457300$

$0.01152200-6.22467100$

$2.03409700-7.24693100$

$1.67808000-1.50444400$

$-1.12034000-2.61055000$

\section{0-R}

$\mathrm{C}$

C

$\mathrm{H}$

$\mathrm{H}$

$\mathrm{H}$

C

$\mathrm{C}$

C

C

C

C

$\mathrm{H}$

C

$\mathrm{H}$

$\mathrm{C}$

$\mathrm{C}$

$\mathrm{H}$

C

$\mathrm{H}$

$\mathrm{H}$

$\mathrm{H}$

$\mathrm{H}$

$\mathrm{H}$

$\mathrm{H}$

$\mathrm{H}$
$2.92103600 \quad-3.78361500 \quad-1.80886500$

$\begin{array}{llll}3.72228700 & -4.60395600 & -0.81292200\end{array}$

$\begin{array}{llll}4.74082100 & -4.73780400 & -1.18264100\end{array}$

$\begin{array}{llll}3.25342500 & -5.58785800 & -0.68004400\end{array}$

$\begin{array}{llll}3.74207700 & -4.12019600 & 0.16921000\end{array}$

$\begin{array}{lll}1.48819300 & -3.37870600 & -1.38514700\end{array}$

$\begin{array}{lll}1.60684600 & -2.23848800 & -0.37892900\end{array}$

$\begin{array}{llll}0.61236400 & -3.02455800 & -2.61998200\end{array}$

$\begin{array}{lll}1.59386400 & -2.46703800 & 1.00180200\end{array}$

$\begin{array}{lll}1.76561100 & -0.92631200 & -0.86578500\end{array}$

$\begin{array}{llll}0.48151300 & -1.51013700 & -2.90537000\end{array}$

$\begin{array}{lll}-0.38602100 & -3.45075900 & -2.47303500\end{array}$

$\begin{array}{lll}1.75519300 & -1.41262200 & 1.90213900\end{array}$

$\begin{array}{lll}1.44836000 & -3.47856400 & 1.37603000\end{array}$

$\begin{array}{ccc}1.91821700 & 0.12349400 & 0.04546800\end{array}$

$\begin{array}{lll}1.67593800 & -0.70624300 & -2.35763000\end{array}$

$\begin{array}{lll}0.37779600 & -1.34666600 & -3.98410900\end{array}$

$1.92472700 \quad-0.11335200 \quad 1.42123500$

$\begin{array}{lll}1.74067400 & -1.60476600 & 2.97164800\end{array}$

$\begin{array}{lll}2.02792200 & 1.13856300 & -0.33027500\end{array}$

$\begin{array}{lll}2.59400900 & -1.04057900 & -2.85727700\end{array}$

$\begin{array}{lll}2.04737700 & 0.71482500 & 2.11423100\end{array}$

$1.06219600-4.24588200-0.86344600$

$\begin{array}{lll}1.05016100 & -3.52023400 & -3.49091700\end{array}$

$-0.43144800 \quad-1.12209400 \quad-2.43647000$
$\mathrm{H}$

$\mathrm{O}$

$\begin{array}{lll}1.55668200 & 0.36127100 & -2.57675000\end{array}$

$3.38790400 \quad-3.46396800 \quad-2.88627700$

10-S

$\mathrm{C}$

$\mathrm{C}$

$\mathrm{H}$

$\mathrm{H}$

$\mathrm{H}$

C

$\mathrm{C}$

C

C

C

$\mathrm{C}$

$\mathrm{H}$

C

$\mathrm{H}$

C

C

$\mathrm{H}$

C

$\mathrm{H}$

$\mathrm{H}$

$\mathrm{H}$

$\mathrm{H}$

$\mathrm{H}$

$\mathrm{H}$

$\mathrm{H}$

$\mathrm{H}$

$\mathrm{O}$

[Ir-H]

$\mathrm{C}$

C

C

C

$\mathrm{C}$

Ir

$\mathrm{N}$

$\mathrm{N}$

C

C

$\mathrm{H}$

$\mathrm{C}$

C

C

C

$\mathrm{H}$

$\mathrm{C}$

$\mathrm{H}$

$\mathrm{C}$

$\mathrm{H}$

$\mathrm{H}$

$\begin{array}{lll}2.92103600 & -3.78361500 & 1.80886500\end{array}$

$3.72228700 \quad-4.60395600 \quad 0.81292200$

$\begin{array}{lll}4.74082100 & -4.73780400 & 1.18264100\end{array}$

$3.25342500 \quad-5.58785800 \quad 0.68004400$

$3.74207700 \quad-4.12019600 \quad-0.16921000$

$\begin{array}{lll}1.48819300 & -3.37870600 & 1.38514700\end{array}$

$\begin{array}{lll}1.60684600 & -2.23848800 & 0.37892900\end{array}$

$\begin{array}{lll}0.61236400 & -3.02455800 & 2.61998200\end{array}$

$\begin{array}{lll}1.59386400 & -2.46703800 & -1.00180200\end{array}$

$\begin{array}{lll}1.76561100 & -0.92631200 & 0.86578500\end{array}$

$\begin{array}{lll}0.48151300 & -1.51013700 & 2.90537000\end{array}$

$\begin{array}{lll}-0.38602100 & -3.45075900 & 2.47303500\end{array}$

$\begin{array}{lll}1.75519300 & -1.41262200 & -1.90213900\end{array}$

$\begin{array}{lll}1.44836000 & -3.47856400 & -1.37603000\end{array}$

$\begin{array}{lll}1.91821700 & 0.12349400 & -0.04546800\end{array}$

$\begin{array}{lll}1.67593800 & -0.70624300 & 2.35763000\end{array}$

$\begin{array}{lll}0.37779600 & -1.34666600 & 3.98410900\end{array}$

$\begin{array}{lll}1.92472700 & -0.11335200 & -1.42123500\end{array}$

$1.74067400-1.60476600 \quad-2.97164800$

$\begin{array}{lll}2.02792200 & 1.13856300 & 0.33027500\end{array}$

$\begin{array}{lll}2.59400900 & -1.04057900 & 2.85727700\end{array}$

$2.04737700 \quad 0.71482500-2.11423100$

$\begin{array}{lll}1.06219600 & -4.24588200 & 0.86344600\end{array}$

$\begin{array}{lll}1.05016100 & -3.52023400 & 3.49091700\end{array}$

$\begin{array}{lll}-0.43144800 & -1.12209400 & 2.43647000\end{array}$

$\begin{array}{lll}1.55668200 & 0.36127100 & 2.57675000\end{array}$

$\begin{array}{lll}3.38790400 & -3.46396800 & 2.88627700\end{array}$

$4.12712200 \quad-1.20728300 \quad-0.65731900$

$\begin{array}{lll}4.31585400 & 0.18509900 & -0.34096400\end{array}$

$3.84224600 \quad 0.40604600 \quad 1.03191100$

$\begin{array}{lll}3.28810400 & -0.80204200 & 1.49747600\end{array}$

$3.40371400-1.81001700 \quad 0.43981100$

$\begin{array}{lll}2.16520600 & -0.25763800 & -0.52864200\end{array}$

$\begin{array}{lll}0.09032400 & -0.42389000 & 0.05118900\end{array}$

$\begin{array}{lll}1.20242900 & 1.54870800 & -1.20942200\end{array}$

$\begin{array}{lll}-0.72793600 & 0.79432400 & 0.13125400\end{array}$

$\begin{array}{lll}0.21110300 & 1.99402300 & -0.18124000\end{array}$

$\begin{array}{lll}0.80717200 & 2.19431100 & 0.71363500\end{array}$

$\begin{array}{lll}-0.51040700 & 3.26608800 & -0.58980600\end{array}$

$\begin{array}{llll}-1.28835700 & 3.32316900 & -1.75689300\end{array}$

$\begin{array}{lll}-0.38713900 & 4.42364400 & 0.18857900\end{array}$

$\begin{array}{lll}-1.91928000 & 4.50770300 & -2.13643800\end{array}$

$\begin{array}{lll}-1.41492600 & 2.43530500 & -2.37308100\end{array}$

$\begin{array}{llll}-1.02213400 & 5.60957000 & -0.18518200\end{array}$

$\begin{array}{lll}0.20178300 & 4.39148700 & 1.10214200\end{array}$

$\begin{array}{llll}-1.78759200 & 5.65550600 & -1.35087900\end{array}$

$\begin{array}{llll}-2.51823600 & 4.53299100 & -3.04281300\end{array}$

$\begin{array}{lll}-0.91825100 & 6.49547200 & 0.43540200\end{array}$ 


\begin{tabular}{|c|c|c|c|c|c|c|c|}
\hline $\mathrm{H}$ & -2.28121400 & 6.57764300 & -1.64507700 & $\mathrm{H}$ & 4.73000500 & 2.19657100 & -1.02579100 \\
\hline $\mathrm{H}$ & -1.50393600 & 0.77958900 & -0.65138600 & $\mathrm{C}$ & 3.98236600 & 1.69903500 & 1.78360700 \\
\hline $\mathrm{C}$ & -1.44846200 & 1.08369700 & 1.45221900 & $\mathrm{H}$ & 5.00698600 & 1.82863100 & 2.15979800 \\
\hline $\mathrm{C}$ & -2.77490000 & 1.52933900 & 1.43875900 & $\mathrm{H}$ & 3.76688400 & 2.56679600 & 1.14887000 \\
\hline $\mathrm{C}$ & -0.78894100 & 0.97168800 & 2.68307500 & $\mathrm{H}$ & 3.30952100 & 1.74245500 & 2.64470700 \\
\hline $\mathrm{C}$ & -3.42920300 & 1.86703600 & 2.62588000 & $\mathrm{C}$ & 2.67122500 & -1.07617500 & 2.83697800 \\
\hline $\mathrm{H}$ & -3.30208800 & 1.60657300 & 0.49110800 & $\mathrm{H}$ & 2.55080100 & -0.16127800 & 3.42510100 \\
\hline $\mathrm{C}$ & -1.43832600 & 1.30761200 & 3.87042400 & $\mathrm{H}$ & 1.68819500 & -1.54744100 & 2.73051400 \\
\hline $\mathrm{H}$ & 0.22410800 & 0.58554500 & 2.70715200 & $\mathrm{H}$ & 3.30871100 & -1.75881000 & 3.41521200 \\
\hline $\mathrm{C}$ & -2.76074500 & 1.75961200 & 3.84620400 & $\mathrm{H}$ & 0.72965000 & 1.33996100 & -2.08863700 \\
\hline $\mathrm{H}$ & -4.46015800 & 2.21047000 & 2.59548500 & $\mathrm{H}$ & 1.70812500 & -0.82776600 & -1.94279200 \\
\hline $\mathrm{H}$ & -0.91595800 & 1.20437600 & 4.81824400 & & & & \\
\hline $\mathrm{H}$ & -3.26755300 & 2.01750000 & 4.77262900 & $\mathbf{P h N H}_{2}$ & & & \\
\hline S & -0.56443700 & -1.88095800 & 0.43331700 & $\mathrm{C}$ & 0.08260400 & 0.06569200 & -0.01590400 \\
\hline $\mathrm{O}$ & 0.14958600 & -2.93415500 & -0.31643400 & $\mathrm{C}$ & 1.47558600 & 0.07254000 & -0.04765900 \\
\hline $\mathrm{O}$ & -0.58377700 & -2.06237100 & 1.90277600 & $\mathrm{C}$ & 2.18273500 & 1.28733000 & -0.04563500 \\
\hline $\mathrm{C}$ & -2.29775100 & -1.81613500 & -0.19323900 & $\mathrm{C}$ & 1.45482600 & 2.48914500 & -0.01241500 \\
\hline $\mathrm{C}$ & -3.40055500 & -1.98988600 & 0.67434100 & $\mathrm{C}$ & 0.06179000 & 2.47112600 & 0.01929700 \\
\hline $\mathrm{C}$ & -2.48053400 & -1.55303400 & -1.57181500 & $\mathrm{C}$ & -0.63768000 & 1.26213100 & 0.01719000 \\
\hline $\mathrm{C}$ & -4.70067400 & -1.78667100 & 0.15602500 & $\mathrm{H}$ & -0.44309900 & -0.88602600 & -0.01981100 \\
\hline $\mathrm{C}$ & -3.78967800 & -1.38859000 & -2.06846500 & $\mathrm{H}$ & 2.02576000 & -0.86604600 & -0.06903900 \\
\hline $\mathrm{C}$ & -4.89777700 & -1.52719500 & -1.21180900 & $\mathrm{H}$ & 1.98858000 & 3.43739700 & -0.00618300 \\
\hline $\mathrm{C}$ & -3.28505400 & -2.42755300 & 2.12367000 & $\mathrm{H}$ & -0.48004800 & 3.41346000 & 0.04320000 \\
\hline $\mathrm{H}$ & -2.42910500 & -3.07664700 & 2.28300200 & $\mathrm{H}$ & -1.72339400 & 1.25231900 & 0.04031600 \\
\hline $\mathrm{H}$ & -3.15663400 & -1.57158600 & 2.79772800 & $\mathrm{~N}$ & 3.58280200 & 1.29886700 & -0.01761500 \\
\hline $\mathrm{H}$ & -4.18752200 & -2.96333500 & 2.42630500 & $\mathrm{H}$ & 4.01941200 & 0.47322800 & -0.40929300 \\
\hline $\mathrm{C}$ & -5.89389600 & -1.82298300 & 1.09604700 & $\mathrm{H}$ & 4.00500200 & 2.14340000 & -0.38396900 \\
\hline $\mathrm{H}$ & -6.73038900 & -1.24053700 & 0.70387900 & & & & \\
\hline $\mathrm{H}$ & -6.26220400 & -2.84366300 & 1.27371800 & $\mathbf{H}_{2} \mathbf{O}$ & & & \\
\hline $\mathrm{H}$ & -5.64227400 & -1.39799300 & 2.07106900 & $\mathrm{O}$ & 2.84155600 & 1.68453800 & 0.00000000 \\
\hline $\mathrm{C}$ & -6.30553300 & -1.43429900 & -1.77364800 & $\mathrm{H}$ & 3.80896800 & 1.73204200 & 0.00000000 \\
\hline $\mathrm{H}$ & -6.99924500 & -2.07788400 & -1.22739400 & $\mathrm{H}$ & 2.56340200 & 2.61232300 & 0.00000000 \\
\hline $\mathrm{H}$ & -6.70756300 & -0.41157000 & -1.72691100 & & & & \\
\hline $\mathrm{H}$ & -6.34280500 & -1.74736600 & -2.81945400 & 11a & & & \\
\hline $\mathrm{C}$ & -4.00603500 & -1.05062400 & -3.53313000 & $\mathrm{C}$ & -2.03923700 & 1.61490900 & 2.05578500 \\
\hline $\mathrm{H}$ & -4.88947300 & -0.42243000 & -3.67448400 & $\mathrm{C}$ & -0.80029400 & 1.64085000 & 1.42121900 \\
\hline $\mathrm{H}$ & -3.15847400 & -0.50672600 & -3.95528000 & $\mathrm{C}$ & 0.31617500 & 2.24916000 & 2.01859000 \\
\hline $\mathrm{H}$ & -4.14636200 & -1.95040700 & -4.14990700 & $\mathrm{C}$ & 0.18807300 & 2.79444100 & 3.30903900 \\
\hline $\mathrm{C}$ & -1.33182700 & -1.47608200 & -2.56293800 & $\mathrm{C}$ & -1.06346100 & 2.75275500 & 3.94159200 \\
\hline $\mathrm{H}$ & -1.15510600 & -0.44271000 & -2.89628200 & $\mathrm{C}$ & -2.17400200 & 2.18395900 & 3.32440200 \\
\hline $\mathrm{H}$ & -0.40085400 & -1.86332100 & -2.16030900 & $\mathrm{H}$ & -2.88862000 & 1.14199100 & 1.57042300 \\
\hline $\mathrm{H}$ & -1.57407900 & -2.05692800 & -3.45986800 & $\mathrm{H}$ & -0.68871900 & 1.16200400 & 0.44999500 \\
\hline $\mathrm{H}$ & 1.85961900 & 2.30351600 & -1.40522800 & $\mathrm{C}$ & 1.64993300 & 2.23573300 & 1.27339300 \\
\hline $\mathrm{C}$ & 3.12390000 & -3.27493000 & 0.61937600 & $\mathrm{C}$ & 1.37995000 & 3.39076600 & 4.03438900 \\
\hline $\mathrm{H}$ & 2.22093100 & -3.44002700 & 1.20957200 & $\mathrm{H}$ & -1.15760700 & 3.16958000 & 4.94223200 \\
\hline $\mathrm{H}$ & 2.96325600 & -3.76813800 & -0.34180900 & $\mathrm{H}$ & -3.13232700 & 2.16517800 & 3.83637900 \\
\hline $\mathrm{H}$ & 3.96875800 & -3.76774500 & 1.12344900 & $\mathrm{C}$ & 2.70747300 & 2.85766900 & 3.48896500 \\
\hline $\mathrm{C}$ & 4.65736800 & -1.93100100 & -1.86215600 & $\mathrm{C}$ & 2.76972800 & 3.06131100 & 1.97462400 \\
\hline $\mathrm{H}$ & 4.74712900 & -1.26577500 & -2.72551200 & $\mathrm{H}$ & 1.29150000 & 3.18219600 & 5.10762100 \\
\hline $\mathrm{H}$ & 5.65247200 & -2.34701900 & -1.65080500 & $\mathrm{H}$ & 2.81059900 & 1.78936700 & 3.71594200 \\
\hline $\mathrm{H}$ & 4.00079600 & -2.75732800 & -2.14580100 & $\mathrm{H}$ & 2.65506600 & 4.13306100 & 1.75383800 \\
\hline $\mathrm{C}$ & 5.11458800 & 1.17659000 & -1.14092200 & $\mathrm{C}$ & 1.54239600 & 2.73464700 & -0.15293000 \\
\hline $\mathrm{H}$ & 6.16483500 & 1.19141400 & -0.81630200 & $\mathrm{C}$ & 0.63842000 & 3.88529300 & -0.48956900 \\
\hline $\mathrm{H}$ & 5.09992400 & 0.93410400 & -2.20731300 & $\mathrm{H}$ & 1.11790200 & 4.82147200 & -0.17421400 \\
\hline
\end{tabular}




\begin{tabular}{|c|c|c|c|c|c|c|c|}
\hline $\mathrm{H}$ & -0.29559200 & 3.80268100 & 0.06713400 & $\mathrm{C}$ & 6.92853200 & 1.31388300 & 2.87221100 \\
\hline $\mathrm{H}$ & 0.43347000 & 3.95362400 & -1.55892900 & $\mathrm{C}$ & 7.13952300 & 2.50034200 & 0.74210500 \\
\hline $\mathrm{N}$ & 2.30957900 & 2.17982500 & -1.04198600 & $\mathrm{C}$ & 6.72687700 & 2.54903800 & 3.50191600 \\
\hline $\mathrm{C}$ & 2.42867900 & 2.52188000 & -2.43002000 & $\mathrm{C}$ & 6.94398100 & 3.70578800 & 1.42653300 \\
\hline $\mathrm{C}$ & 2.33821100 & 1.47294100 & -3.35045400 & $\mathrm{C}$ & 6.74152400 & 3.75970900 & 2.80777900 \\
\hline $\mathrm{C}$ & 2.70752000 & 3.82429700 & -2.85621600 & $\mathrm{H}$ & 6.57989800 & 2.56906800 & 4.57992600 \\
\hline $\mathrm{C}$ & 2.48724700 & 1.74208600 & -4.70985000 & $\mathrm{H}$ & 6.96276600 & 4.63271800 & 0.85840600 \\
\hline $\mathrm{H}$ & 2.14960800 & 0.46431400 & -2.99825800 & $\mathrm{C}$ & 1.32597900 & -3.17127700 & -0.79694100 \\
\hline $\mathrm{C}$ & 2.86038800 & 4.08051800 & -4.21916600 & $\mathrm{C}$ & 1.24903800 & -2.91584200 & -2.18973600 \\
\hline $\mathrm{H}$ & 2.83623200 & 4.62030300 & -2.13040500 & $\mathrm{C}$ & 0.25571800 & -2.78294000 & 0.04561800 \\
\hline $\mathrm{C}$ & 2.74407500 & 3.04388000 & -5.14721500 & $\mathrm{C}$ & 0.13624600 & -2.23842900 & -2.69961500 \\
\hline $\mathrm{H}$ & 2.41211400 & 0.92875200 & -5.42542300 & $\mathrm{C}$ & -0.83876300 & -2.11510400 & -0.52110700 \\
\hline $\mathrm{H}$ & 3.08162000 & 5.09048300 & -4.55177400 & $\mathrm{C}$ & -0.91917900 & -1.82048400 & -1.88265500 \\
\hline $\mathrm{H}$ & 2.86609500 & 3.24783600 & -6.20708200 & $\mathrm{H}$ & 0.08255200 & -2.05603400 & -3.77076000 \\
\hline $\mathrm{H}$ & 3.00861100 & 1.38000900 & -0.73622700 & $\mathrm{H}$ & -1.66284500 & -1.81961300 & 0.12475200 \\
\hline $\mathrm{H}$ & 3.73695900 & 2.74676400 & 1.57141500 & $\mathrm{C}$ & 7.34578400 & 2.56941600 & -0.77307200 \\
\hline $\mathrm{H}$ & 3.55166200 & 3.36973700 & 3.96358200 & $\mathrm{H}$ & 7.45025600 & 1.55013600 & -1.15044300 \\
\hline $\mathrm{H}$ & 1.36386800 & 4.48793500 & 3.93712700 & $\mathrm{C}$ & 6.60690200 & 5.08358600 & 3.55118300 \\
\hline $\mathrm{H}$ & 2.00110700 & 1.19326200 & 1.24395700 & $\mathrm{H}$ & 6.30395800 & 4.84587400 & 4.58056900 \\
\hline $\mathrm{C}$ & 7.00934900 & -4.30699100 & -1.15246000 & $\mathrm{C}$ & 6.97200300 & 0.06390000 & 3.75127200 \\
\hline $\mathrm{C}$ & 8.25122000 & -2.36985100 & -0.53564200 & $\mathrm{H}$ & 7.14811800 & -0.80057500 & 3.10835700 \\
\hline $\mathrm{C}$ & 8.42847900 & -3.70482100 & -1.22611400 & $\mathrm{C}$ & 6.12257500 & 3.17392600 & -1.48564600 \\
\hline $\mathrm{H}$ & 6.41396000 & -3.96163500 & -2.00558900 & $\mathrm{H}$ & 6.28420600 & 3.19421400 & -2.57065300 \\
\hline $\mathrm{H}$ & 6.99957200 & -5.40148200 & -1.15930000 & $\mathrm{H}$ & 5.92715400 & 4.20361000 & -1.15947900 \\
\hline $\mathrm{H}$ & 9.15959600 & -4.33563000 & -0.70076100 & $\mathrm{H}$ & 5.24069800 & 2.56279000 & -1.28309300 \\
\hline $\mathrm{H}$ & 8.79040000 & -3.59764100 & -2.25563800 & $\mathrm{C}$ & 8.63098200 & 3.33659200 & -1.14229700 \\
\hline $\mathrm{C}$ & 6.39668200 & -3.70879800 & 0.15934200 & $\mathrm{H}$ & 8.78453700 & 3.32550000 & -2.22848400 \\
\hline $\mathrm{C}$ & 6.91488100 & -4.52872900 & 1.38949500 & $\mathrm{H}$ & 9.51622900 & 2.89441800 & -0.67128800 \\
\hline $\mathrm{C}$ & 4.90455200 & -4.00914800 & 0.25069500 & $\mathrm{H}$ & 8.57969100 & 4.38545500 & -0.82531100 \\
\hline $\mathrm{C}$ & 6.01366500 & -5.78259400 & 1.42755600 & $\mathrm{C}$ & 5.52876200 & 6.00748100 & 2.95649500 \\
\hline $\mathrm{H}$ & 7.98216000 & -4.76150600 & 1.31975200 & $\mathrm{H}$ & 5.42912400 & 6.91915800 & 3.55800500 \\
\hline $\mathrm{H}$ & 6.76083200 & -3.93369100 & 2.29695000 & $\mathrm{H}$ & 4.55183900 & 5.51222800 & 2.92556800 \\
\hline $\mathrm{H}$ & 5.88760300 & -6.18177100 & 2.44083800 & $\mathrm{H}$ & 5.78073100 & 6.31433500 & 1.93448100 \\
\hline $\mathrm{H}$ & 6.43319500 & -6.59659100 & 0.81925400 & $\mathrm{C}$ & 7.96374200 & 5.81273100 & 3.62917900 \\
\hline $\mathrm{C}$ & 7.01609000 & -2.31298900 & 0.12980700 & $\mathrm{H}$ & 7.87876500 & 6.73641900 & 4.21518500 \\
\hline $\mathrm{C}$ & 6.60126700 & -1.11013800 & 0.70781200 & $\mathrm{H}$ & 8.32286800 & 6.08041700 & 2.62806500 \\
\hline $\mathrm{C}$ & 9.12792100 & -1.29163000 & -0.50001200 & $\mathrm{H}$ & 8.72496300 & 5.17912200 & 4.09702600 \\
\hline $\mathrm{C}$ & 7.48022100 & -0.00676100 & 0.77978900 & $\mathrm{C}$ & 8.14825000 & 0.12247200 & 4.74729100 \\
\hline $\mathrm{C}$ & 8.74966100 & -0.14184800 & 0.19180100 & $\mathrm{H}$ & 8.03905900 & 0.95040800 & 5.45827300 \\
\hline $\mathrm{H}$ & 10.09630000 & -1.34225800 & -0.99203600 & $\mathrm{H}$ & 9.10328900 & 0.25448800 & 4.22595900 \\
\hline $\mathrm{H}$ & 9.44146800 & 0.69231900 & 0.26413700 & $\mathrm{H}$ & 8.20323700 & -0.80709200 & 5.32721000 \\
\hline $\mathrm{C}$ & 4.71489100 & -5.26722800 & 0.84508700 & $\mathrm{C}$ & 5.63642000 & -0.17835800 & 4.48042000 \\
\hline $\mathrm{C}$ & 3.46077900 & -5.86696400 & 0.85136900 & $\mathrm{H}$ & 5.68980300 & -1.10157600 & 5.07084800 \\
\hline $\mathrm{C}$ & 3.79984200 & -3.29583600 & -0.21369300 & $\mathrm{H}$ & 4.81443800 & -0.28028400 & 3.76600300 \\
\hline $\mathrm{C}$ & 2.38599000 & -5.18751400 & 0.27590900 & $\mathrm{H}$ & 5.40109400 & 0.64036000 & 5.17217100 \\
\hline $\mathrm{H}$ & 3.31409300 & -6.84967000 & 1.29309900 & $\mathrm{C}$ & 2.30208000 & -3.42817500 & -3.17443100 \\
\hline $\mathrm{C}$ & 2.51826500 & -3.88711800 & -0.23571000 & $\mathrm{H}$ & 3.08916900 & -3.92337900 & -2.60070900 \\
\hline $\mathrm{H}$ & 1.40765800 & -5.65804100 & 0.24591900 & $\mathrm{C}$ & -2.13399100 & -1.09981500 & -2.45398700 \\
\hline $\mathrm{O}$ & 5.34421000 & -1.02511900 & 1.27771900 & $\mathrm{H}$ & -2.79683900 & -0.86622000 & -1.60889000 \\
\hline $\mathrm{O}$ & 3.97557600 & -2.00395200 & -0.68661100 & $\mathrm{C}$ & 0.21700300 & -3.10766700 & 1.54042700 \\
\hline $\mathrm{P}$ & 3.97384200 & -0.74274800 & 0.39395900 & $\mathrm{H}$ & 1.20098200 & -3.48492900 & 1.82734300 \\
\hline $\mathrm{O}$ & 2.83956600 & -0.79772400 & 1.36211500 & $\mathrm{C}$ & -1.75701400 & 0.23461200 & -3.12472000 \\
\hline $\mathrm{O}$ & 4.16170700 & 0.48024100 & -0.49507500 & $\mathrm{H}$ & -2.65308500 & 0.76165200 & -3.47510800 \\
\hline $\mathrm{C}$ & 7.14095200 & 1.28155500 & 1.47110800 & $\mathrm{H}$ & -1.10482000 & 0.07578500 & -3.99173500 \\
\hline
\end{tabular}




\begin{abstract}
$\begin{array}{lll}-1.22517700 & 0.89285500 & -2.42772500\end{array}$
\end{abstract}
$\begin{array}{lll}-2.92836600 & -1.99498000 & -3.42472900\end{array}$

$\begin{array}{lll}-3.83034600 & -1.48087700 & -3.77926100\end{array}$

$\begin{array}{lll}-3.23462500 & -2.92762200 & -2.93873300\end{array}$

$-2.32762500 \quad-2.25928700 \quad-4.30321800$

$\begin{array}{lll}1.70188900 & -4.49073800 & -4.11787600\end{array}$

$\begin{array}{llll}0.91169300 & -4.06919800 & -4.75088100\end{array}$

$\begin{array}{llll}1.26633800 & -5.32095200 & -3.55076100\end{array}$

$\begin{array}{llll}2.47645700 & -4.89946400 & -4.77867900\end{array}$

$\begin{array}{llll}2.97354600 & -2.29577800 & -3.97244100\end{array}$

$\begin{array}{llll}3.45353900 & -1.57972800 & -3.29874100\end{array}$

$\begin{array}{llll}2.24847600 & -1.76093500 & -4.60027000\end{array}$

$\begin{array}{lll}3.74374900 & -2.70266000 & -4.63962700\end{array}$

$\begin{array}{lll}-0.81799700 & -4.21640200 & 1.82704300\end{array}$

$\begin{array}{lll}-1.83432100 & -3.88210000 & 1.58350800\end{array}$

$\begin{array}{lll}-0.80243900 & -4.48921500 & 2.88948300\end{array}$

$\begin{array}{lll}-0.62187800 & -5.12223400 & 1.24214200\end{array}$

$\begin{array}{lll}-0.05285400 & -1.87887700 & 2.42567700\end{array}$

$\begin{array}{llll}0.72070200 & -1.12544800 & 2.26780700\end{array}$

$\begin{array}{lll}-0.03486100 & -2.17524100 & 3.48223400\end{array}$

$\begin{array}{lll}-1.03522500 & -1.43221700 & 2.23121800\end{array}$

\section{TS-2}

C

C

C

C

C

C

$\mathrm{H}$

$\mathrm{H}$

C

C

$\mathrm{H}$

$\mathrm{H}$

C

C

$\mathrm{H}$

$\mathrm{H}$

$\mathrm{H}$

C

C

$\mathrm{H}$

$\mathrm{H}$

$\mathrm{H}$

$\mathrm{N}$

C

C

C

C

$\mathrm{H}$

C

$\mathrm{H}$

C

$\begin{array}{rrrl}2.98324500 & 0.05990900 & 1.02526300 & \mathrm{C} \\ 3.12403700 & -0.17074200 & -0.34359500 & \mathrm{C} \\ 2.03605300 & -0.03708300 & -1.22823000 & \mathrm{C} \\ 0.79802200 & 0.41087000 & -0.71119100 & \mathrm{C} \\ 0.67927800 & 0.65007300 & 0.66412000 & \mathrm{C} \\ 1.74942600 & 0.46239900 & 1.53645400 & \mathrm{H} \\ 3.84103300 & -0.05556400 & 1.68078300 & \mathrm{H} \\ 4.10598700 & -0.41430500 & -0.74205200 & \mathrm{C} \\ 2.16186100 & -0.39764100 & -2.69018600 & \mathrm{C} \\ -0.39914600 & 0.69348300 & -1.60537200 & \mathrm{C} \\ -0.27936300 & 0.98635600 & 1.05493700 & \mathrm{C} \\ 1.62503000 & 0.64383100 & 2.60069400 & \mathrm{H} \\ -0.28297900 & 0.10846700 & -3.01766500 & \mathrm{C} \\ 1.13456200 & 0.33209000 & -3.56434200 & \mathrm{H} \\ -0.50660900 & 1.78574200 & -1.68782600 & \mathrm{O} \\ -1.03254300 & 0.57198200 & -3.67110900 & \mathrm{O} \\ 1.20968300 & 0.02906700 & -4.61109600 & \mathrm{P} \\ 2.43007100 & -1.76922000 & -2.99692200 & \mathrm{O} \\ 2.18512400 & -2.31602500 & -4.37174700 & \mathrm{O} \\ 1.13339200 & -2.17271900 & -4.63657500 & \mathrm{C} \\ 2.41685700 & -3.37846300 & -4.43322200 & \mathrm{C} \\ 2.79548200 & -1.79193400 & -5.11004100 & \mathrm{C} \\ 2.90866300 & -2.60323500 & -2.05994900 & \mathrm{C} \\ 3.57727800 & -3.85430400 & -2.23233300 & \mathrm{C} \\ 3.20473600 & -4.92851700 & -1.41645800 & \mathrm{C} \\ 4.63354900 & -3.98292800 & -3.14319100 & \mathrm{H} \\ 3.88195500 & -6.14309200 & -1.52165100 & \mathrm{H} \\ 2.38384500 & -4.81102200 & -0.71388600 & \mathrm{C} \\ 5.29083200 & -5.20961500 & -3.24977100 & \mathrm{C} \\ 4.94156800 & -3.12813100 & -3.74096700 & \mathrm{C} \\ 4.92144200 & -6.28861800 & -2.44339100 & \mathrm{C}\end{array}$

$3.59135300 \quad-6.97637100 \quad-0.88795800$

$\begin{array}{lll}6.10809500 & -5.31191200 & -3.95797100\end{array}$

$\begin{array}{llll}5.44499100 & -7.23682300 & -2.52759900\end{array}$

$\begin{array}{lll}2.90271500 & -2.23946700 & -1.10957900\end{array}$

$\begin{array}{llll}1.36483500 & 1.40488500 & -3.54519800\end{array}$

$\begin{array}{lll}-0.50000600 & -0.96902000 & -2.99884300\end{array}$

$\begin{array}{llll}-1.31642500 & 0.34141700 & -1.11605900\end{array}$

$\begin{array}{llll}3.35240800 & 0.16777500 & -3.20219200\end{array}$

$\begin{array}{llll}8.87683800 & 1.95006600 & -7.42665700\end{array}$

$\begin{array}{llll}7.22103900 & 0.96533500 & -8.82713400\end{array}$

$\begin{array}{llll}8.65164300 & 1.45725600 & -8.87176000\end{array}$

$\begin{array}{llll}9.20261200 & 1.11639200 & -6.79407700\end{array}$

$\begin{array}{llll}9.62776300 & 2.74196500 & -7.34822500\end{array}$

$\begin{array}{lll}8.77622500 & 2.27253200 & -9.59829100\end{array}$

$\begin{array}{llll}9.35315200 & 0.66716400 & -9.16424500\end{array}$

$\begin{array}{llll}7.45973300 & 2.41693600 & -6.95108100\end{array}$

$\begin{array}{lll}7.16889900 & 3.84036400 & -7.53752900\end{array}$

$\begin{array}{llll}7.46480700 & 2.74035400 & -5.46011300\end{array}$

$\begin{array}{llll}7.93419800 & 4.80172900 & -6.60571200\end{array}$

$\begin{array}{llll}7.45833600 & 3.92792000 & -8.58913400\end{array}$

$\begin{array}{llll}6.09199700 & 4.03352600 & -7.47018300\end{array}$

$\begin{array}{llll}7.46791000 & 5.79195800 & -6.54212300\end{array}$

$\begin{array}{llll}8.96489700 & 4.96164600 & -6.95236200\end{array}$

$\begin{array}{llll}6.58985900 & 1.37035200 & -7.63866900\end{array}$

$\begin{array}{llll}5.31941200 & 0.87922000 & -7.33318200\end{array}$

$\begin{array}{llll}6.53445600 & 0.21865100 & -9.77602000\end{array}$

$\begin{array}{llll}4.59259200 & 0.12944200 & -8.28358500\end{array}$

$\begin{array}{lll}5.21624000 & -0.14814800 & -9.51220100\end{array}$

$\begin{array}{llll}7.00843500 & -0.07039300 & -10.71084100\end{array}$

$\begin{array}{llll}4.65304600 & -0.69875500 & -10.25954500\end{array}$

$\begin{array}{llll}7.90003800 & 4.06504100 & -5.28463200\end{array}$

$\begin{array}{llll}8.24576600 & 4.53983400 & -4.02566100\end{array}$

$\begin{array}{llll}7.22167300 & 1.94557600 & -4.33779400\end{array}$

$\begin{array}{llll}8.11292200 & 3.68930000 & -2.93046400\end{array}$

$\begin{array}{llll}8.61734400 & 5.55299600 & -3.89318700\end{array}$

$\begin{array}{lll}7.56247700 & 2.40117100 & -3.04607400\end{array}$

$\begin{array}{llll}8.40690400 & 4.03386200 & -1.94357500\end{array}$

$\begin{array}{llll}4.75304100 & 1.17382300 & -6.09413200\end{array}$

$\begin{array}{llll}6.72431900 & 0.65815100 & -4.49956000\end{array}$

$\begin{array}{llll}5.15661700 & 0.26602300 & -4.77584800\end{array}$

$\begin{array}{llll}4.27215900 & 0.87189500 & -3.65857900\end{array}$

$\begin{array}{llll}5.10542700 & -1.20244100 & -5.02301000\end{array}$

$\begin{array}{llll}3.19496200 & -0.37016900 & -8.06766800\end{array}$

$\begin{array}{llll}2.11974700 & 0.54359700 & -7.93953300\end{array}$

$\begin{array}{llll}2.92390800 & -1.76534700 & -8.11073200\end{array}$

$\begin{array}{llll}0.81281000 & 0.04697600 & -7.84556300\end{array}$

$\begin{array}{llll}1.59677900 & -2.20198500 & -8.01824900\end{array}$

$\begin{array}{lll}0.52163200 & -1.31724700 & -7.89188500\end{array}$

$\begin{array}{llll}-0.01176900 & 0.75247400 & -7.76587600\end{array}$

$\begin{array}{lll}1.39930200 & -3.27041500 & -8.06415500\end{array}$

$\begin{array}{llll}7.36062700 & 1.59460400 & -1.79922800\end{array}$

$\begin{array}{llll}8.07471300 & 0.38668200 & -1.59167800\end{array}$

$\begin{array}{llll}6.51395000 & 2.09130800 & -0.77479500\end{array}$

$\begin{array}{llll}7.91674900 & -0.29747000 & -0.38236500\end{array}$ 


\begin{tabular}{|c|c|c|c|c|c|c|c|}
\hline $\mathrm{C}$ & 6.40291300 & 1.36671600 & 0.41939500 & $\mathrm{H}$ & 8.73331000 & -2.30786200 & -2.23690500 \\
\hline $\mathrm{C}$ & 7.09029400 & 0.17249500 & 0.64162000 & $\mathrm{H}$ & 9.43740000 & -1.94066200 & -3.81693400 \\
\hline $\mathrm{H}$ & 8.47820000 & -1.21589100 & -0.22970800 & $\mathrm{C}$ & 6.17314900 & 4.43207200 & 0.15276400 \\
\hline $\mathrm{H}$ & 5.75848700 & 1.75247600 & 1.20624700 & $\mathrm{H}$ & 5.96605000 & 4.08693300 & 1.17289100 \\
\hline $\mathrm{C}$ & 4.01529800 & -2.82514500 & -8.29802600 & $\mathrm{H}$ & 5.63937300 & 5.37956300 & 0.00917700 \\
\hline $\mathrm{H}$ & 4.98119600 & -2.31741500 & -8.32874800 & $\mathrm{H}$ & 7.24835200 & 4.63543300 & 0.08701100 \\
\hline $\mathrm{C}$ & -0.92164500 & -1.80657100 & -7.85338800 & $\mathrm{C}$ & 4.19962500 & 3.17904300 & -0.82637700 \\
\hline $\mathrm{H}$ & -1.56128300 & -0.91483100 & -7.79874000 & $\mathrm{H}$ & 3.87172600 & 2.49902100 & -1.61673900 \\
\hline $\mathrm{C}$ & 2.30877600 & 2.06066900 & -7.98970000 & $\mathrm{H}$ & 3.68052500 & 4.13700200 & -0.95851100 \\
\hline $\mathrm{H}$ & 3.37752800 & 2.26969500 & -8.07238500 & $\mathrm{H}$ & 3.88647000 & 2.76130600 & 0.13651800 \\
\hline $\mathrm{C}$ & 4.09030700 & -3.82101600 & -7.12611100 & & & & \\
\hline $\mathrm{H}$ & 4.86441100 & -4.57309700 & -7.32425800 & $12 a$ & & & \\
\hline $\mathrm{H}$ & 3.14384600 & -4.35546700 & -6.97718800 & $\mathrm{C}$ & -1.97981400 & -1.59756600 & 0.32310300 \\
\hline $\mathrm{H}$ & 4.35229600 & -3.29881300 & -6.20356900 & $\mathrm{C}$ & -0.58859900 & -1.53844200 & 0.38813900 \\
\hline $\mathrm{C}$ & 3.84081100 & -3.57406400 & -9.63549700 & $\mathrm{C}$ & 0.11778600 & -0.35557000 & 0.08808200 \\
\hline $\mathrm{H}$ & 4.66990600 & -4.27495100 & -9.79178700 & $\mathrm{C}$ & -0.63459100 & 0.75934800 & -0.36598000 \\
\hline $\mathrm{H}$ & 3.81442600 & -2.88537300 & -10.48770800 & $\mathrm{C}$ & -2.02673200 & 0.68462900 & -0.43485200 \\
\hline $\mathrm{H}$ & 2.90945400 & -4.15287600 & -9.65311200 & $\mathrm{C}$ & -2.71012300 & -0.47776900 & -0.07429600 \\
\hline $\mathrm{C}$ & -1.22155600 & -2.66251800 & -6.60872600 & $\mathrm{H}$ & -2.48914000 & -2.52675600 & 0.56602500 \\
\hline $\mathrm{H}$ & -2.27132500 & -2.98039400 & -6.59990500 & $\mathrm{H}$ & -0.03950200 & -2.43778400 & 0.64226000 \\
\hline $\mathrm{H}$ & -1.03284600 & -2.10069600 & -5.68671100 & $\mathrm{C}$ & 1.58963500 & -0.24803700 & 0.17085100 \\
\hline $\mathrm{H}$ & -0.60020500 & -3.56577400 & -6.58627900 & $\mathrm{C}$ & 0.10866600 & 1.98123200 & -0.85117300 \\
\hline $\mathrm{C}$ & -1.29942800 & -2.56464000 & -9.14101300 & $\mathrm{H}$ & -2.58240300 & 1.55200500 & -0.78581200 \\
\hline $\mathrm{H}$ & -2.35893900 & -2.84800700 & -9.12710600 & $\mathrm{H}$ & -3.79466300 & -0.51586700 & -0.13094200 \\
\hline $\mathrm{H}$ & -0.71029700 & -3.48301400 & -9.24904500 & $\mathrm{C}$ & 1.31267700 & 1.51566200 & -1.68203200 \\
\hline $\mathrm{H}$ & -1.12167900 & -1.94825800 & -10.02892100 & $\mathrm{C}$ & 2.27792900 & 0.72308300 & -0.79124500 \\
\hline $\mathrm{C}$ & 1.64151500 & 2.65982800 & -9.24505100 & $\mathrm{H}$ & -0.55780500 & 2.62148200 & -1.44064100 \\
\hline $\mathrm{H}$ & 0.55376400 & 2.52185500 & -9.23109000 & $\mathrm{H}$ & 0.95262800 & 0.88807700 & -2.50688000 \\
\hline $\mathrm{H}$ & 2.02518000 & 2.19297600 & -10.15928400 & $\mathrm{H}$ & 2.85982000 & 1.46201600 & -0.21103400 \\
\hline $\mathrm{H}$ & 1.83908300 & 3.73717200 & -9.30468800 & $\mathrm{C}$ & 2.35676500 & -0.89984600 & 1.09841200 \\
\hline $\mathrm{C}$ & 1.80985600 & 2.75760800 & -6.71047200 & $\mathrm{C}$ & 1.81757100 & -1.71624500 & 2.25204500 \\
\hline $\mathrm{H}$ & 1.98652300 & 3.83849700 & -6.77410300 & $\mathrm{H}$ & 0.81348600 & -1.37599300 & 2.51394300 \\
\hline $\mathrm{H}$ & 2.33757600 & 2.37896700 & -5.83059800 & $\mathrm{H}$ & 1.75080900 & -2.79024500 & 2.04283300 \\
\hline $\mathrm{H}$ & 0.73307300 & 2.60855900 & -6.56226700 & $\mathrm{H}$ & 2.46533000 & -1.59926300 & 3.12618900 \\
\hline $\mathrm{C}$ & 9.07807900 & -0.16955900 & -2.60313700 & $\mathrm{~N}$ & 3.74146200 & -0.68219000 & 1.10188000 \\
\hline $\mathrm{H}$ & 9.06386200 & 0.47077600 & -3.48762800 & $\mathrm{C}$ & 4.76517400 & -1.40394100 & 1.72794300 \\
\hline $\mathrm{C}$ & 6.97281200 & -0.56438800 & 1.97047100 & $\mathrm{C}$ & 4.71217800 & -2.79484900 & 1.93031000 \\
\hline $\mathrm{H}$ & 6.22345300 & -0.02984800 & 2.57186000 & $\mathrm{C}$ & 5.93002900 & -0.71373500 & 2.11508100 \\
\hline $\mathrm{C}$ & 5.72159300 & 3.39747900 & -0.89807400 & $\mathrm{C}$ & 5.78073700 & -3.45880500 & 2.53150200 \\
\hline $\mathrm{H}$ & 5.92231100 & 3.82595500 & -1.88144400 & $\mathrm{H}$ & 3.85090500 & -3.35810200 & 1.59078400 \\
\hline $\mathrm{C}$ & 6.48100200 & -2.01400700 & 1.79887300 & $\mathrm{C}$ & 7.00035200 & -1.39077300 & 2.69258400 \\
\hline $\mathrm{H}$ & 6.36414100 & -2.50125000 & 2.77456900 & $\mathrm{H}$ & 5.98141000 & 0.36306300 & 1.96831700 \\
\hline $\mathrm{H}$ & 7.19050200 & -2.60989100 & 1.21319200 & $\mathrm{C}$ & 6.93139300 & -2.76831500 & 2.91653200 \\
\hline $\mathrm{H}$ & 5.51458600 & -2.04972100 & 1.28330500 & $\mathrm{H}$ & 5.71690200 & -4.53383200 & 2.67986100 \\
\hline $\mathrm{C}$ & 8.29877300 & -0.52558500 & 2.75581900 & $\mathrm{H}$ & 7.88859800 & -0.83441300 & 2.98077900 \\
\hline $\mathrm{H}$ & 8.18656500 & -1.00853700 & 3.73445900 & $\mathrm{H}$ & 7.76248200 & -3.29459400 & 3.37663400 \\
\hline $\mathrm{H}$ & 8.63047600 & 0.50566100 & 2.91863800 & $\mathrm{H}$ & 4.05642500 & 0.14398600 & 0.61495600 \\
\hline $\mathrm{H}$ & 9.09434400 & -1.04966400 & 2.21291000 & $\mathrm{H}$ & 3.00917900 & 0.18773700 & -1.41515900 \\
\hline $\mathrm{C}$ & 10.51352600 & -0.11922100 & -2.04033500 & $\mathrm{H}$ & 1.83975300 & 2.36668400 & -2.12982200 \\
\hline $\mathrm{H}$ & 10.62485300 & -0.76340000 & -1.15987100 & $\mathrm{H}$ & 0.46798800 & 2.58960000 & -0.00634400 \\
\hline $\mathrm{H}$ & 10.78827700 & 0.89963900 & -1.74441200 & & & & \\
\hline $\mathrm{H}$ & 11.23291600 & -0.46021400 & -2.79514900 & TS-3 & & & \\
\hline $\mathrm{C}$ & 8.71454100 & -1.59248700 & -3.06847100 & $\mathrm{C}$ & -0.75689500 & -1.64970400 & -4.27521100 \\
\hline $\mathrm{H}$ & 7.71969600 & -1.61483800 & -3.52212200 & $\mathrm{C}$ & 0.35188800 & -0.91990500 & -3.84444400 \\
\hline
\end{tabular}




\begin{tabular}{|c|c|c|c|c|c|c|c|}
\hline $\mathrm{C}$ & 1.31503600 & -1.49164000 & -2.99806800 & $\mathrm{C}$ & 0.10309300 & 6.95579600 & -5.46919900 \\
\hline $\mathrm{C}$ & 1.10784400 & -2.81394600 & -2.54389000 & $\mathrm{C}$ & -0.11523000 & 4.52821300 & -5.20842800 \\
\hline $\mathrm{C}$ & -0.01086200 & -3.53226400 & -2.97581500 & $\mathrm{C}$ & -0.20413900 & 5.69789700 & -5.98283800 \\
\hline $\mathrm{C}$ & -0.93746600 & -2.96555600 & -3.84977600 & $\mathrm{H}$ & 0.08872600 & 7.83135000 & -6.11367900 \\
\hline $\mathrm{H}$ & -1.48235000 & -1.17585900 & -4.92818900 & $\mathrm{H}$ & -0.48897200 & 5.59985500 & -7.02566000 \\
\hline $\mathrm{H}$ & 0.45769400 & 0.11234500 & -4.16371200 & $\mathrm{C}$ & 0.43867200 & 6.06201500 & 0.52396400 \\
\hline $\mathrm{C}$ & 2.52695800 & -0.71327600 & -2.53917500 & $\mathrm{C}$ & 0.93524400 & 5.68294400 & 1.76445500 \\
\hline $\mathrm{C}$ & 2.05558100 & -3.38745900 & -1.51788700 & $\mathrm{C}$ & 1.99217100 & 4.58077600 & -0.59492100 \\
\hline $\mathrm{H}$ & -0.15739100 & -4.54695800 & -2.61114700 & $\mathrm{C}$ & 1.98809100 & 4.77231300 & 1.81165700 \\
\hline $\mathrm{H}$ & -1.80251900 & -3.53712900 & -4.17583700 & $\mathrm{H}$ & 0.51481300 & 6.08608300 & 2.68246500 \\
\hline $\mathrm{C}$ & 2.36906700 & -2.28938600 & -0.49435800 & $\mathrm{C}$ & 2.50458400 & 4.16491700 & 0.65434300 \\
\hline $\mathrm{C}$ & 3.12993600 & -1.14421600 & -1.17437700 & $\mathrm{H}$ & 2.40472100 & 4.48310800 & 2.77183600 \\
\hline $\mathrm{H}$ & 2.99319500 & -3.72916700 & -1.98470300 & $\mathrm{O}$ & 0.32481900 & 3.54030600 & -3.02703500 \\
\hline $\mathrm{H}$ & 2.97590900 & -2.67728300 & 0.33338600 & $\mathrm{O}$ & 2.52574300 & 4.05754300 & -1.76581600 \\
\hline $\mathrm{H}$ & 3.20762700 & -0.29133600 & -0.49139800 & $\mathrm{P}$ & 1.82085500 & 2.92170600 & -2.72487500 \\
\hline $\mathrm{C}$ & 3.48905700 & -0.32618000 & -3.52345500 & $\mathrm{O}$ & 1.51983700 & 1.65770800 & -1.88408100 \\
\hline $\mathrm{C}$ & 3.20971600 & -0.34720000 & -4.99658000 & $\mathrm{O}$ & 2.66904100 & 2.79140100 & -3.94278000 \\
\hline $\mathrm{H}$ & 2.58160500 & -1.20270200 & -5.24992100 & $\mathrm{C}$ & -0.39040000 & 3.21834600 & -5.88515100 \\
\hline $\mathrm{H}$ & 4.13661800 & -0.38166300 & -5.57228200 & $\mathrm{C}$ & -1.53794300 & 2.46184800 & -5.54454500 \\
\hline $\mathrm{H}$ & 2.67236700 & 0.56296600 & -5.27946800 & $\mathrm{C}$ & 0.42957400 & 2.79480600 & -6.96616200 \\
\hline $\mathrm{N}$ & 4.69739900 & 0.10991500 & -3.12670800 & $\mathrm{C}$ & -1.83134000 & 1.30545500 & -6.27994800 \\
\hline $\mathrm{C}$ & 5.77242900 & 0.61979600 & -3.93469600 & $\mathrm{C}$ & 0.08956500 & 1.62793300 & -7.65908300 \\
\hline $\mathrm{C}$ & 6.97994100 & -0.08652300 & -3.95468500 & $\mathrm{C}$ & -1.03711500 & 0.86576300 & -7.33984100 \\
\hline $\mathrm{C}$ & 5.63588700 & 1.82289900 & -4.63334700 & $\mathrm{H}$ & -2.72788100 & 0.74023500 & -6.03286300 \\
\hline $\mathrm{C}$ & 8.05786400 & 0.40943900 & -4.68877400 & $\mathrm{H}$ & 0.71938600 & 1.31463900 & -8.48813000 \\
\hline $\mathrm{H}$ & 7.06503100 & -1.01996200 & -3.40525900 & $\mathrm{C}$ & 3.54253200 & 3.09584500 & 0.81378000 \\
\hline $\mathrm{C}$ & 6.71807300 & 2.30233900 & -5.37437600 & $\mathrm{C}$ & 4.85800700 & 3.27705500 & 0.31731600 \\
\hline $\mathrm{H}$ & 4.69967400 & 2.36923300 & -4.56712000 & $\mathrm{C}$ & 3.22738800 & 1.92180600 & 1.54696400 \\
\hline $\mathrm{C}$ & 7.92619700 & 1.60222000 & -5.40367500 & $\mathrm{C}$ & 5.81076900 & 2.27341500 & 0.52846200 \\
\hline $\mathrm{H}$ & 8.99532900 & -0.13937400 & -4.70633300 & $\mathrm{C}$ & 4.22658200 & 0.95808700 & 1.74038000 \\
\hline $\mathrm{H}$ & 6.61582200 & 3.23583500 & -5.92052300 & $\mathrm{C}$ & 5.52196100 & 1.10547200 & 1.24079300 \\
\hline $\mathrm{H}$ & 8.76451700 & 1.98605900 & -5.97858500 & $\mathrm{H}$ & 6.82014400 & 2.43022600 & 0.15534600 \\
\hline $\mathrm{H}$ & 4.86655200 & 0.13911900 & -2.12742100 & $\mathrm{H}$ & 3.98945200 & 0.06192500 & 2.31044900 \\
\hline $\mathrm{H}$ & 4.15544200 & -1.51301100 & -1.33422600 & $\mathrm{C}$ & 1.65770500 & 3.57530200 & -7.44623400 \\
\hline $\mathrm{H}$ & 1.42862400 & -1.92900200 & -0.06419000 & $\mathrm{H}$ & 1.81692600 & 4.41461300 & -6.76630100 \\
\hline $\mathrm{H}$ & 1.60690100 & -4.26413200 & -1.03642800 & $\mathrm{C}$ & -1.41803300 & -0.37076000 & -8.14443500 \\
\hline $\mathrm{H}$ & 2.01497900 & 0.57579300 & -2.27968800 & $\mathrm{H}$ & -2.28000200 & -0.83085500 & -7.64095700 \\
\hline $\mathrm{C}$ & 1.32108100 & 7.71141800 & -2.01591800 & $\mathrm{C}$ & -2.51992600 & 2.88984000 & -4.45313000 \\
\hline $\mathrm{C}$ & 0.41942300 & 7.06513500 & -4.12152000 & $\mathrm{H}$ & -2.14371100 & 3.80823000 & -3.99688600 \\
\hline $\mathrm{C}$ & 0.81583300 & 8.30352300 & -3.34789900 & $\mathrm{C}$ & 2.94535100 & 2.73367000 & -7.40670800 \\
\hline $\mathrm{H}$ & 2.38059300 & 7.44441900 & -2.10364500 & $\mathrm{H}$ & 3.79620000 & 3.33126800 & -7.75795700 \\
\hline $\mathrm{H}$ & 1.21863800 & 8.39517100 & -1.16776500 & $\mathrm{H}$ & 2.88041200 & 1.84663600 & -8.04849900 \\
\hline $\mathrm{H}$ & -0.04081900 & 8.97381300 & -3.19039000 & $\mathrm{H}$ & 3.15221400 & 2.41596600 & -6.38329700 \\
\hline $\mathrm{H}$ & 1.58150500 & 8.89077000 & -3.86828400 & $\mathrm{C}$ & 1.43666100 & 4.15139400 & -8.86033000 \\
\hline $\mathrm{C}$ & 0.48684400 & 6.39867700 & -1.83451700 & $\mathrm{H}$ & 2.29579800 & 4.76265800 & -9.16311900 \\
\hline $\mathrm{C}$ & -0.93336100 & 6.76840700 & -1.28674000 & $\mathrm{H}$ & 0.54050300 & 4.77975200 & -8.91078600 \\
\hline $\mathrm{C}$ & 1.01895800 & 5.58053700 & -0.66243400 & $\mathrm{H}$ & 1.31898500 & 3.35267200 & -9.60256600 \\
\hline $\mathrm{C}$ & -0.71242900 & 6.99627800 & 0.22227300 & $\mathrm{C}$ & -0.29274000 & -1.42154100 & -8.18320400 \\
\hline $\mathrm{H}$ & -1.37033300 & 7.63060200 & -1.79941300 & $\mathrm{H}$ & -0.62275600 & -2.31795700 & -8.72238700 \\
\hline $\mathrm{H}$ & -1.60147200 & 5.91348300 & -1.44195200 & $\mathrm{H}$ & 0.00481700 & -1.72075400 & -7.17267900 \\
\hline $\mathrm{H}$ & -1.60145100 & 6.76576700 & 0.82100800 & $\mathrm{H}$ & 0.59612100 & -1.03650700 & -8.69733200 \\
\hline $\mathrm{H}$ & -0.45159300 & 8.04208300 & 0.43746200 & $\mathrm{C}$ & -1.86784100 & 0.00586600 & -9.57016300 \\
\hline $\mathrm{C}$ & 0.39738900 & 5.93596000 & -3.28487200 & $\mathrm{H}$ & -2.19645300 & -0.88361300 & -10.1216750 \\
\hline $\mathrm{C}$ & 0.21418600 & 4.66896400 & -3.84251400 & $\mathrm{H}$ & -1.04697900 & 0.46524700 & -10.1339290 \\
\hline
\end{tabular}




\begin{tabular}{|c|c|c|c|c|c|c|c|}
\hline $\mathrm{H}$ & -2.69744400 & 0.72107900 & -9.54762500 & $\mathrm{C}$ & 3.16761600 & -0.68251100 & -1.02576700 \\
\hline $\mathrm{C}$ & -3.90257300 & 3.22539900 & -5.04863400 & $\mathrm{H}$ & 3.17178700 & -3.27453400 & 0.14032300 \\
\hline $\mathrm{H}$ & -4.37057100 & 2.34643900 & -5.50762900 & $\mathrm{H}$ & 2.82291300 & -1.05696900 & 1.07575700 \\
\hline $\mathrm{H}$ & -3.82551100 & 4.00210700 & -5.81803700 & $\mathrm{H}$ & 3.32891800 & 0.38705300 & -0.86772700 \\
\hline $\mathrm{H}$ & -4.57791000 & 3.58947400 & -4.26454300 & $\mathrm{C}$ & 3.32414900 & -0.49474000 & -3.50873700 \\
\hline $\mathrm{C}$ & -2.63708800 & 1.84163600 & -3.33081600 & $\mathrm{C}$ & 4.47196600 & -1.40138300 & -3.84920300 \\
\hline $\mathrm{H}$ & -3.31191600 & 2.20262500 & -2.54474200 & $\mathrm{H}$ & 4.23924500 & -2.42048500 & -3.5374030 \\
\hline $\mathrm{H}$ & -1.66209800 & 1.64184300 & -2.87732700 & $\mathrm{H}$ & 5.37494200 & -1.08494800 & -3.31310100 \\
\hline $\mathrm{H}$ & -3.04319000 & 0.89327400 & -3.70310400 & $\mathrm{H}$ & 4.69735200 & -1.38697400 & -4.91893600 \\
\hline $\mathrm{C}$ & 5.31233800 & 4.57035500 & -0.36068300 & $\mathrm{~N}$ & 3.08818700 & 0.61891300 & -4.13685600 \\
\hline $\mathrm{H}$ & 4.44534400 & 5.22763400 & -0.45690500 & $\mathrm{C}$ & 3.78962300 & 1.21363900 & -5.23097600 \\
\hline $\mathrm{C}$ & 6.58934900 & 0.05331600 & 1.51794700 & $\mathrm{C}$ & 5.18129300 & 1.16895900 & -5.38292800 \\
\hline $\mathrm{H}$ & 6.09845700 & -0.77198900 & 2.05195000 & $\mathrm{C}$ & 2.99939900 & 1.92290900 & -6.14758700 \\
\hline $\mathrm{C}$ & 1.84889900 & 1.66102300 & 2.16361500 & $\mathrm{C}$ & 5.77028600 & 1.79095200 & -6.48418700 \\
\hline $\mathrm{H}$ & 1.19779000 & 2.49915500 & 1.91178000 & $\mathrm{H}$ & 5.80657600 & 0.69695700 & -4.63582100 \\
\hline $\mathrm{C}$ & 7.19392500 & -0.53029500 & 0.22716400 & $\mathrm{C}$ & 3.60051500 & 2.53288700 & -7.24566900 \\
\hline $\mathrm{H}$ & 7.94173100 & -1.29753500 & 0.46128400 & $\mathrm{H}$ & 1.93146500 & 2.00199500 & -5.97542700 \\
\hline $\mathrm{H}$ & 7.68863900 & 0.24399000 & -0.37098600 & $\mathrm{C}$ & 4.98438600 & 2.46117500 & -7.42309700 \\
\hline $\mathrm{H}$ & 6.42093200 & -0.99636200 & -0.39593600 & $\mathrm{H}$ & 6.84992400 & 1.75892100 & -6.59848000 \\
\hline $\mathrm{C}$ & 7.69630500 & 0.60405500 & 2.43840700 & $\mathrm{H}$ & 2.98525400 & 3.07494500 & -7.95758600 \\
\hline $\mathrm{H}$ & 8.42456600 & -0.17888900 & 2.68295900 & $\mathrm{H}$ & 5.45055300 & 2.94193300 & -8.27825300 \\
\hline $\mathrm{H}$ & 7.27567700 & 0.98518400 & 3.37517000 & $\mathrm{H}$ & 2.19477800 & 1.19465200 & -3.84684500 \\
\hline $\mathrm{H}$ & 8.23785500 & 1.42701800 & 1.95715000 & $\mathrm{H}$ & 4.14884500 & -1.17784100 & -1.06375300 \\
\hline $\mathrm{C}$ & 6.34072300 & 5.31483200 & 0.51604900 & $\mathrm{H}$ & 1.35917600 & -0.79812600 & 0.13174100 \\
\hline $\mathrm{H}$ & 7.26122600 & 4.73211900 & 0.64162900 & $\mathrm{H}$ & 1.50302000 & -3.20522200 & 0.67460500 \\
\hline $\mathrm{H}$ & 5.93754200 & 5.52071900 & 1.51413600 & $\mathrm{H}$ & 1.57633900 & -0.15790800 & -2.34774500 \\
\hline $\mathrm{H}$ & 6.61307000 & 6.27223900 & 0.05512200 & $\mathrm{C}$ & -0.23430300 & 7.25574300 & -3.76061700 \\
\hline $\mathrm{C}$ & 5.86094500 & 4.33228300 & -1.77880600 & $\mathrm{C}$ & -1.80638900 & 5.71068000 & -4.67946200 \\
\hline $\mathrm{H}$ & 5.10525300 & 3.86363000 & -2.41435800 & $\mathrm{C}$ & -1.33338300 & 7.14050500 & -4.84100900 \\
\hline $\mathrm{H}$ & 6.75178700 & 3.69235900 & -1.76973300 & $\mathrm{H}$ & 0.72756400 & 6.92508700 & -4.16931600 \\
\hline $\mathrm{H}$ & 6.14756800 & 5.28630400 & -2.23855900 & $\mathrm{H}$ & -0.10407900 & 8.27417100 & -3.38067500 \\
\hline $\mathrm{C}$ & 1.92527500 & 1.58664000 & 3.70203300 & $\mathrm{H}$ & -2.14790500 & 7.85667300 & -4.66247800 \\
\hline $\mathrm{H}$ & 2.53258100 & 0.73653400 & 4.03541400 & $\mathrm{H}$ & -0.95568500 & 7.34335100 & -5.85041300 \\
\hline $\mathrm{H}$ & 0.92193900 & 1.46394000 & 4.12774500 & $\mathrm{C}$ & -0.67624500 & 6.25073900 & -2.64593200 \\
\hline $\mathrm{H}$ & 2.36669800 & 2.49384300 & 4.13043400 & $\mathrm{C}$ & -1.78806300 & 6.91569200 & -1.76201500 \\
\hline $\mathrm{C}$ & 1.16995400 & 0.40444900 & 1.59230700 & $\mathrm{C}$ & 0.41192800 & 6.05014600 & -1.59706500 \\
\hline $\mathrm{H}$ & 1.05128300 & 0.49023700 & 0.50812400 & $\mathrm{C}$ & -1.01483500 & 7.78593100 & -0.75017600 \\
\hline $\mathrm{H}$ & 0.17379900 & 0.28234300 & 2.03557800 & $\mathrm{H}$ & -2.51589400 & 7.47721500 & -2.35608300 \\
\hline \multirow[t]{2}{*}{$\mathrm{H}$} & \multirow[t]{3}{*}{1.74172400} & \multirow[t]{3}{*}{-0.50408400} & \multirow[t]{3}{*}{1.81386900} & $\mathrm{H}$ & -2.33042100 & 6.12516900 & -1.23061400 \\
\hline & & & & $\mathrm{H}$ & -1.55101800 & 7.91280600 & 0.19764600 \\
\hline $11 b$ & & & & $\mathrm{H}$ & -0.83312900 & 8.79526800 & -1.14663600 \\
\hline $\mathrm{C}$ & 0.85026700 & -3.96378900 & -4.01044100 & $\mathrm{C}$ & -1.28285700 & 5.14588600 & -3.50579400 \\
\hline $\mathrm{C}$ & 1.38385000 & -2.69397400 & -3.80592600 & $\mathrm{C}$ & -1.47775600 & 3.78738100 & -3.24986100 \\
\hline $\mathrm{C}$ & 1.84593400 & -2.28753500 & -2.54344000 & $\mathrm{C}$ & -2.64854200 & 4.97339700 & -5.50453800 \\
\hline $\mathrm{C}$ & 1.74204900 & -3.17729500 & -1.45795100 & $\mathrm{C}$ & -2.36995900 & 3.02928600 & -4.03813800 \\
\hline $\mathrm{C}$ & 1.19334400 & -4.44981400 & -1.67654500 & $\mathrm{C}$ & -2.96019700 & 3.66137000 & -5.14601300 \\
\hline $\mathrm{C}$ & 0.75767600 & -4.85142400 & -2.93632100 & $\mathrm{H}$ & -3.07720800 & 5.41292800 & -6.40215500 \\
\hline $\mathrm{H}$ & 0.50380200 & -4.25463700 & -4.99836500 & $\mathrm{H}$ & -3.66458700 & 3.09424800 & -5.74737800 \\
\hline $\mathrm{H}$ & 1.43212500 & -1.99969600 & -4.64129000 & $\mathrm{C}$ & 0.27750400 & 7.01641600 & -0.58697800 \\
\hline $\mathrm{C}$ & 2.40745100 & -0.87201500 & -2.36713700 & $\mathrm{C}$ & 1.25316200 & 7.16274600 & 0.39192900 \\
\hline $\mathrm{C}$ & 2.20228500 & -2.79330200 & -0.06368500 & $\mathrm{C}$ & 1.47684900 & 5.14835500 & -1.55267100 \\
\hline $\mathrm{H}$ & 1.10788100 & -5.13291800 & -0.83392700 & $\mathrm{C}$ & 2.36203500 & 6.31749600 & 0.36546900 \\
\hline $\mathrm{H}$ & 0.33804300 & -5.84377300 & -3.07729400 & $\mathrm{H}$ & 1.15955300 & 7.92143700 & 1.16519900 \\
\hline $\mathrm{C}$ & 2.34373100 & -1.28042600 & 0.11645000 & $\mathrm{C}$ & 2.48876500 & 5.28364900 & -0.57645300 \\
\hline
\end{tabular}




\begin{tabular}{|c|c|c|c|c|c|c|c|}
\hline $\mathrm{H}$ & 3.14677700 & 6.43715000 & 1.10688300 & $\mathrm{C}$ & 7.26538900 & 1.90274200 & -0.31635100 \\
\hline $\mathrm{O}$ & -0.83035900 & 3.19127100 & -2.18010600 & $\mathrm{H}$ & 7.17080700 & 1.28720000 & 0.58882800 \\
\hline $\mathrm{O}$ & 1.55338200 & 4.15303700 & -2.51374700 & $\mathrm{C}$ & 2.89418500 & 3.39226900 & 1.72539200 \\
\hline $\mathrm{P}$ & 0.74863100 & 2.71102600 & -2.30685500 & $\mathrm{H}$ & 2.01322100 & 3.98882500 & 1.47759200 \\
\hline $\mathrm{O}$ & 1.10411000 & 2.02862600 & -1.03096800 & $\mathrm{C}$ & 7.38694200 & 0.93921400 & -1.51245200 \\
\hline $\mathrm{O}$ & 0.96546500 & 2.01004200 & -3.64694500 & $\mathrm{H}$ & 8.27094100 & 0.29754400 & -1.41177800 \\
\hline $\mathrm{C}$ & -2.74638900 & 1.61330700 & -3.71475900 & $\mathrm{H}$ & 7.47918300 & 1.49033500 & -2.45657900 \\
\hline C & -3.46124000 & 1.31827000 & -2.52726100 & $\mathrm{H}$ & 6.50257400 & 0.29399900 & -1.58139100 \\
\hline $\mathrm{C}$ & -2.47721100 & 0.56954500 & -4.63759400 & $\mathrm{C}$ & 8.54376500 & 2.74939200 & -0.16408200 \\
\hline $\mathrm{C}$ & -3.88349300 & 0.00339400 & -2.29719100 & $\mathrm{H}$ & 9.42458000 & 2.10719500 & -0.03989200 \\
\hline $\mathrm{C}$ & -2.93209900 & -0.72391500 & -4.35964900 & $\mathrm{H}$ & 8.47563100 & 3.40888400 & 0.70772300 \\
\hline $\mathrm{C}$ & -3.63942900 & -1.03418300 & -3.19586600 & $\mathrm{H}$ & 8.71139400 & 3.37979000 & -1.04548100 \\
\hline $\mathrm{H}$ & -4.43513800 & -0.21795300 & -1.38602200 & $\mathrm{C}$ & 5.75005900 & 6.57042300 & -2.51574200 \\
\hline $\mathrm{H}$ & -2.72448900 & -1.51436500 & -5.07713700 & $\mathrm{H}$ & 6.73766600 & 6.10758500 & -2.63065500 \\
\hline $\mathrm{C}$ & 3.69293100 & 4.39081100 & -0.52501400 & $\mathrm{H}$ & 5.72497000 & 7.06193700 & -1.53671200 \\
\hline $\mathrm{C}$ & 4.69150000 & 4.49590600 & -1.52493800 & $\mathrm{H}$ & 5.65277700 & 7.34412800 & -3.28750200 \\
\hline $\mathrm{C}$ & 3.87728200 & 3.49626300 & 0.55747300 & $\mathrm{C}$ & 4.61294900 & 4.89009700 & -4.04536700 \\
\hline $\mathrm{C}$ & 5.82256200 & 3.67603500 & -1.44909900 & $\mathrm{H}$ & 3.76409400 & 4.20976200 & -4.15454200 \\
\hline $\mathrm{C}$ & 5.03430200 & 2.70592100 & 0.58825700 & $\mathrm{H}$ & 5.53442100 & 4.32740500 & -4.23838100 \\
\hline $\mathrm{C}$ & 6.01495900 & 2.76933900 & -0.40294100 & $\mathrm{H}$ & 4.53216700 & 5.66381700 & -4.81932900 \\
\hline $\mathrm{H}$ & 6.58549600 & 3.76711800 & -2.21931900 & $\mathrm{C}$ & 3.51113100 & 3.97763300 & 3.01289200 \\
\hline $\mathrm{H}$ & 5.18119300 & 2.02229400 & 1.42193900 & $\mathrm{H}$ & 4.38831500 & 3.40053500 & 3.33084600 \\
\hline $\mathrm{C}$ & -1.69583300 & 0.79119300 & -5.93459700 & $\mathrm{H}$ & 2.78177700 & 3.95396500 & 3.83194400 \\
\hline $\mathrm{H}$ & -1.33872900 & 1.82375700 & -5.94162200 & $\mathrm{H}$ & 3.83296800 & 5.01628200 & 2.87594800 \\
\hline $\mathrm{C}$ & -4.13763100 & -2.44513800 & -2.91090400 & $\mathrm{C}$ & 2.39498800 & 1.95679800 & 1.96653400 \\
\hline $\mathrm{H}$ & -4.66717900 & -2.41025500 & -1.94866000 & $\mathrm{H}$ & 1.89434100 & 1.58391200 & 1.07079600 \\
\hline $\mathrm{C}$ & -3.84011100 & 2.37697700 & -1.49096100 & $\mathrm{H}$ & 1.67716200 & 1.94696100 & 2.79658400 \\
\hline $\mathrm{H}$ & -3.44812900 & 3.34063500 & -1.82351300 & $\mathrm{H}$ & 3.21390000 & 1.27772600 & 2.23705100 \\
\hline $\mathrm{C}$ & -0.44795900 & -0.10762700 & -6.00634300 & & & & \\
\hline $\mathrm{H}$ & 0.13413100 & 0.11767900 & -6.90976700 & TS-4 & & & \\
\hline $\mathrm{H}$ & -0.71150100 & -1.17151900 & -6.04081100 & $\mathrm{~N}$ & 6.03498000 & -2.96152500 & -1.71095800 \\
\hline $\mathrm{H}$ & 0.18182100 & 0.06737700 & -5.13045700 & $\mathrm{C}$ & 6.17343800 & -4.14320700 & -0.89772100 \\
\hline $\mathrm{C}$ & -2.58034300 & 0.58892200 & -7.18093800 & $\mathrm{C}$ & 6.17903000 & -3.00318100 & -3.10210000 \\
\hline $\mathrm{H}$ & -2.00946000 & 0.78886900 & -8.09661400 & $\mathrm{C}$ & 5.48167300 & -2.08927200 & -3.91358200 \\
\hline $\mathrm{H}$ & -3.44962200 & 1.25565200 & -7.17344900 & $\mathrm{C}$ & 7.06309600 & -3.90886600 & -3.71754800 \\
\hline $\mathrm{H}$ & -2.95627400 & -0.43969200 & -7.24099000 & $\mathrm{C}$ & 5.66586300 & -2.08178200 & -5.29408900 \\
\hline $\mathrm{C}$ & -2.97779400 & -3.44696300 & -2.76159200 & $\mathrm{H}$ & 4.79385200 & -1.38267900 & -3.4522790 \\
\hline $\mathrm{H}$ & -3.35730700 & -4.44387200 & -2.50404100 & $\mathrm{C}$ & 7.23013300 & -3.89877800 & -5.10104000 \\
\hline $\mathrm{H}$ & -2.27980200 & -3.13290300 & -1.97843600 & $\mathrm{H}$ & 7.62695400 & -4.60348800 & -3.10191200 \\
\hline $\mathrm{H}$ & -2.40642300 & -3.53731000 & -3.69265800 & $\mathrm{C}$ & 6.53686600 & -2.98883400 & -5.90270600 \\
\hline $\mathrm{C}$ & -5.14684600 & -2.92285700 & -3.97263400 & $\mathrm{H}$ & 5.11622600 & -1.36436200 & -5.89857400 \\
\hline $\mathrm{H}$ & -5.53750800 & -3.91594300 & -3.71820900 & $\mathrm{H}$ & 7.91817200 & -4.60792600 & -5.55501000 \\
\hline $\mathrm{H}$ & -4.68008200 & -2.99247700 & -4.96265400 & $\mathrm{H}$ & 5.29746900 & -2.33408000 & -1.40440900 \\
\hline $\mathrm{H}$ & -5.99381400 & -2.23245500 & -4.05230600 & $\mathrm{C}$ & 5.23169300 & -5.27390300 & -1.24991500 \\
\hline $\mathrm{C}$ & -5.37041900 & 2.53617300 & -1.38392400 & $\mathrm{H}$ & 4.23879100 & -4.85655400 & -1.46269300 \\
\hline $\mathrm{H}$ & -5.85061300 & 1.61677300 & -1.02819100 & $\mathrm{H}$ & 5.54049000 & -5.80999500 & -2.15651300 \\
\hline $\mathrm{H}$ & -5.81208300 & 2.78895700 & -2.35470500 & $\mathrm{H}$ & 5.13327800 & -6.00076700 & -0.44175100 \\
\hline $\mathrm{H}$ & -5.62215500 & 3.33656900 & -0.67702200 & $\mathrm{H}$ & 6.67377700 & -2.98516200 & -6.98004000 \\
\hline $\mathrm{C}$ & -3.21235700 & 2.08120700 & -0.11509300 & $\mathrm{C}$ & 7.03908000 & -4.09465800 & 0.14407600 \\
\hline $\mathrm{H}$ & -3.45234300 & 2.88509800 & 0.59226100 & $\mathrm{C}$ & 7.29049200 & -5.23047700 & 1.0658990 \\
\hline $\mathrm{H}$ & -2.12418500 & 2.00580700 & -0.19206000 & $\mathrm{C}$ & 7.75967900 & -2.77690600 & 0.45537500 \\
\hline $\mathrm{H}$ & -3.59565500 & 1.14463200 & 0.30870800 & $\mathrm{C}$ & 7.34629900 & -6.56775500 & 0.63241400 \\
\hline $\mathrm{C}$ & 4.61729900 & 5.53231500 & -2.64736100 & $\mathrm{C}$ & 7.53640200 & -4.95684700 & 2.43294800 \\
\hline $\mathrm{H}$ & 3.67551100 & 6.07649600 & -2.54301100 & $\mathrm{C}$ & 8.48340800 & -2.76666700 & 1.81088200 \\
\hline
\end{tabular}




\begin{tabular}{|c|c|c|c|c|c|c|c|}
\hline $\mathrm{C}$ & 7.56465900 & -7.61137200 & 1.53057100 & & & & \\
\hline $\mathrm{H}$ & 7.24726200 & -6.79026600 & -0.42468200 & TS-5 & & & \\
\hline $\mathrm{C}$ & 7.75293300 & -6.01103100 & 3.32340400 & $\mathrm{C}$ & 3.32581300 & 1.29651800 & -2.63050300 \\
\hline $\mathrm{C}$ & 7.65095500 & -3.51441500 & 2.86509100 & $\mathrm{C}$ & 4.70105500 & 2.81804800 & -1.43148900 \\
\hline $\mathrm{H}$ & 8.66748800 & -1.73036700 & 2.11831100 & $\mathrm{C}$ & 3.91717500 & 2.71620600 & -2.71996900 \\
\hline $\mathrm{C}$ & 7.75163200 & -7.33613000 & 2.88573700 & $\mathrm{H}$ & 2.38442600 & 1.32409200 & -2.06976900 \\
\hline $\mathrm{H}$ & 7.60213800 & -8.63540400 & 1.16854900 & $\mathrm{H}$ & 3.11823200 & 0.84506400 & -3.60532600 \\
\hline $\mathrm{H}$ & 7.93856700 & -5.78701100 & 4.37170600 & $\mathrm{H}$ & 4.57538600 & 2.82260700 & -3.59417800 \\
\hline $\mathrm{H}$ & 6.65891800 & -3.04378800 & 2.94601000 & $\mathrm{H}$ & 3.14848200 & 3.49263000 & -2.80830300 \\
\hline $\mathrm{H}$ & 7.92277300 & -8.14388900 & 3.59236700 & $\mathrm{C}$ & 4.37552800 & 0.47364600 & -1.80336700 \\
\hline $\mathrm{H}$ & 7.02343000 & -1.96435000 & 0.44710400 & $\mathrm{C}$ & 5.50166900 & -0.04026200 & -2.75907500 \\
\hline $\mathrm{H}$ & 9.46266700 & -3.25535100 & 1.72997200 & $\mathrm{C}$ & 3.75203900 & -0.85169200 & -1.38269000 \\
\hline $\mathrm{H}$ & 8.11298200 & -3.45082800 & 3.85690200 & $\mathrm{C}$ & 4.88093400 & -1.27407100 & -3.45685200 \\
\hline \multirow[t]{2}{*}{$\mathrm{H}$} & 8.45926100 & -2.54133000 & -0.35577100 & $\mathrm{H}$ & 5.84022400 & 0.72929000 & -3.46031100 \\
\hline & & & & $\mathrm{H}$ & 6.36386400 & -0.34575300 & -2.15491900 \\
\hline $12 \mathrm{~b}$ & & & & $\mathrm{H}$ & 5.63106900 & -2.03001200 & -3.71829100 \\
\hline $\mathrm{N}$ & 6.37766800 & -3.57975700 & -2.17533600 & $\mathrm{H}$ & 4.38012500 & -0.99461500 & -4.39429600 \\
\hline $\mathrm{C}$ & 6.52045600 & -4.56739700 & -1.17427600 & $\mathrm{C}$ & 4.87283500 & 1.55296900 & -0.84758300 \\
\hline $\mathrm{C}$ & 7.39294000 & -2.99954700 & -2.95281000 & $\mathrm{C}$ & 5.49071600 & 1.44548000 & 0.40124000 \\
\hline $\mathrm{C}$ & 7.03559800 & -2.01057200 & -3.88878800 & $\mathrm{C}$ & 5.21422900 & 3.95702000 & -0.82354600 \\
\hline $\mathrm{C}$ & 8.74284400 & -3.37761400 & -2.85602500 & $\mathrm{C}$ & 6.06658000 & 2.57799000 & 1.01751800 \\
\hline $\mathrm{C}$ & 7.99929900 & -1.42057700 & -4.70100100 & $\mathrm{C}$ & 5.91522900 & 3.81823200 & 0.37214700 \\
\hline $\mathrm{H}$ & 5.99412700 & -1.70496000 & -3.96799200 & $\mathrm{H}$ & 5.08366000 & 4.93817500 & -1.27308600 \\
\hline $\mathrm{C}$ & 9.70061800 & -2.77204300 & -3.67028500 & $\mathrm{H}$ & 6.35696700 & 4.69351400 & 0.83851100 \\
\hline $\mathrm{H}$ & 9.03013400 & -4.15923300 & -2.16112300 & $\mathrm{C}$ & 3.88924400 & -1.77732500 & -2.42747000 \\
\hline $\mathrm{C}$ & 9.34307400 & -1.79127400 & -4.59622600 & $\mathrm{C}$ & 3.13653300 & -2.94680600 & -2.43122300 \\
\hline $\mathrm{H}$ & 7.69711700 & -0.66085000 & -5.41750500 & $\mathrm{C}$ & 2.98568900 & -1.18291800 & -0.26617600 \\
\hline $\mathrm{H}$ & 10.73875800 & -3.08249000 & -3.58092000 & $\mathrm{C}$ & 2.23741200 & -3.17161000 & -1.38780200 \\
\hline $\mathrm{H}$ & 5.47849200 & -3.58872000 & -2.64132500 & $\mathrm{H}$ & 3.21621400 & -3.66113100 & -3.24716400 \\
\hline $\mathrm{C}$ & 5.67982700 & -5.79451400 & -1.43956800 & $\mathrm{C}$ & 2.14937700 & -2.31850700 & -0.27290100 \\
\hline $\mathrm{H}$ & 4.67409600 & -5.48472600 & -1.76240600 & $\mathrm{H}$ & 1.58879400 & -4.04126200 & -1.42025200 \\
\hline $\mathrm{H}$ & 6.09094500 & -6.40899400 & -2.25181400 & $\mathrm{O}$ & 5.55150500 & 0.18903500 & 1.00433600 \\
\hline $\mathrm{H}$ & 5.56565700 & -6.42072800 & -0.55407000 & $\mathrm{O}$ & 3.00355400 & -0.34096600 & 0.84212900 \\
\hline $\mathrm{H}$ & 10.09518100 & -1.32453500 & -5.22542000 & $\mathrm{P}$ & 4.25660200 & -0.33292000 & 1.90058600 \\
\hline $\mathrm{C}$ & 7.25933000 & -4.33168900 & -0.05506500 & $\mathrm{O}$ & 4.64757000 & -1.79875200 & 2.21711200 \\
\hline $\mathrm{C}$ & 7.52006100 & -5.37108800 & 0.96605900 & $\mathrm{O}$ & 3.91633100 & 0.60133800 & 3.00671600 \\
\hline $\mathrm{C}$ & 7.77241900 & -2.92512000 & 0.24206500 & $\mathrm{C}$ & 6.88676500 & 2.54695900 & 2.27847900 \\
\hline $\mathrm{C}$ & 7.84566300 & -6.70112100 & 0.64687800 & $\mathrm{C}$ & 8.22297300 & 2.06887800 & 2.22682000 \\
\hline $\mathrm{C}$ & 7.46515700 & -4.99764800 & 2.32994300 & $\mathrm{C}$ & 6.39687200 & 3.13436600 & 3.47270800 \\
\hline $\mathrm{C}$ & 8.11477700 & -2.69212000 & 1.72807800 & $\mathrm{C}$ & 9.01296500 & 2.14273800 & 3.38264200 \\
\hline $\mathrm{C}$ & 8.05999100 & -7.65092500 & 1.64528200 & $\mathrm{C}$ & 7.23956900 & 3.19801000 & 4.59189100 \\
\hline $\mathrm{H}$ & 7.95185300 & -6.98572300 & -0.39592600 & $\mathrm{C}$ & 8.54843100 & 2.71056000 & 4.57472600 \\
\hline $\mathrm{C}$ & 7.68867200 & -5.95544000 & 3.32075200 & $\mathrm{H}$ & 10.04174100 & 1.79576600 & 3.34298100 \\
\hline $\mathrm{C}$ & 7.22349000 & -3.54235100 & 2.64847800 & $\mathrm{H}$ & 6.86244400 & 3.66543600 & 5.49810100 \\
\hline $\mathrm{H}$ & 8.01257200 & -1.62589100 & 1.96239500 & $\mathrm{C}$ & 1.14147100 & -2.60496600 & 0.80150400 \\
\hline $\mathrm{C}$ & 7.97304400 & -7.28169200 & 2.98855100 & $\mathrm{C}$ & 0.07041200 & -1.70023300 & 1.03281200 \\
\hline $\mathrm{H}$ & 8.30946200 & -8.67333500 & 1.37332500 & $\mathrm{C}$ & 1.18751700 & -3.82082800 & 1.52766200 \\
\hline $\mathrm{H}$ & 7.64341700 & -5.65632200 & 4.36594000 & $\mathrm{C}$ & -0.90243100 & -2.02557700 & 1.98307700 \\
\hline $\mathrm{H}$ & 6.16869200 & -3.27673400 & 2.47882400 & $\mathrm{C}$ & 0.17584600 & -4.09906900 & 2.45532600 \\
\hline $\mathrm{H}$ & 8.14416600 & -8.01512100 & 3.77210600 & $\mathrm{C}$ & -0.87539500 & -3.21968400 & 2.70704000 \\
\hline $\mathrm{H}$ & 7.00023100 & -2.21525500 & -0.08179700 & $\mathrm{H}$ & -1.71559300 & -1.32434400 & 2.15317200 \\
\hline $\mathrm{H}$ & 9.16092300 & -2.96073000 & 1.92136700 & $\mathrm{H}$ & 0.21470900 & -5.03307800 & 3.01121700 \\
\hline $\mathrm{H}$ & 7.43731200 & -3.33895100 & 3.70416400 & $\mathrm{C}$ & 5.01622000 & 3.79132100 & 3.56658600 \\
\hline $\mathrm{H}$ & 8.65455100 & -2.69029300 & -0.36882700 & $\mathrm{H}$ & 4.45639200 & 3.52802500 & 2.66582700 \\
\hline
\end{tabular}




\begin{tabular}{|c|c|c|c|}
\hline $\mathrm{C}$ & 9.47899800 & 2.85781200 & 5.77447300 \\
\hline $\mathrm{H}$ & 10.43315700 & 2.38606600 & 5.50103900 \\
\hline $\mathrm{C}$ & 8.85896700 & 1.60086600 & 0.91266200 \\
\hline $\mathrm{H}$ & 8.09377900 & 1.06625700 & 0.34029000 \\
\hline $\mathrm{C}$ & 4.17736700 & 3.30536100 & 4.76286600 \\
\hline $\mathrm{H}$ & 3.22575200 & 3.85024000 & 4.78846000 \\
\hline $\mathrm{H}$ & 4.67844000 & 3.48244000 & 5.72249900 \\
\hline $\mathrm{H}$ & 3.95004200 & 2.24326300 & 4.66247300 \\
\hline $\mathrm{C}$ & 5.15685100 & 5.32871900 & 3.61049100 \\
\hline $\mathrm{H}$ & 4.16756800 & 5.80194600 & 3.60216400 \\
\hline $\mathrm{H}$ & 5.72296500 & 5.71547700 & 2.75633000 \\
\hline $\mathrm{H}$ & 5.67389100 & 5.65304200 & 4.52212200 \\
\hline $\mathrm{C}$ & 8.95622300 & 2.14009900 & 7.03225100 \\
\hline $\mathrm{H}$ & 9.66124300 & 2.25500900 & 7.86427300 \\
\hline $\mathrm{H}$ & 8.82305900 & 1.06654900 & 6.85400600 \\
\hline $\mathrm{H}$ & 7.99178800 & 2.54869700 & 7.35626000 \\
\hline $\mathrm{C}$ & 9.77183000 & 4.34132400 & 6.07464900 \\
\hline $\mathrm{H}$ & 10.49820700 & 4.43562800 & 6.89098400 \\
\hline $\mathrm{H}$ & 8.86073400 & 4.87242400 & 6.37456800 \\
\hline $\mathrm{H}$ & 10.17880300 & 4.84899800 & 5.19353300 \\
\hline $\mathrm{C}$ & 9.31386500 & 2.81580900 & 0.07224000 \\
\hline $\mathrm{H}$ & 10.07705300 & 3.38916600 & 0.61295400 \\
\hline $\mathrm{H}$ & 8.48473900 & 3.48836000 & -0.16095100 \\
\hline $\mathrm{H}$ & 9.75346200 & 2.47947300 & -0.87491900 \\
\hline $\mathrm{C}$ & 10.05181900 & 0.64473200 & 1.08276800 \\
\hline $\mathrm{H}$ & 10.33981600 & 0.24224500 & 0.10502600 \\
\hline $\mathrm{H}$ & 9.81983200 & -0.19984000 & 1.73575200 \\
\hline $\mathrm{H}$ & 10.93172500 & 1.16013100 & 1.48694800 \\
\hline $\mathrm{C}$ & -0.11615500 & -0.39869900 & 0.25031700 \\
\hline $\mathrm{H}$ & 0.72044600 & -0.28797000 & -0.44291600 \\
\hline $\mathrm{C}$ & -1.94155900 & -3.55488100 & 3.74164300 \\
\hline $\mathrm{H}$ & -1.71521900 & -4.56057100 & 4.12216600 \\
\hline $\mathrm{C}$ & 2.30505200 & -4.85112800 & 1.36358300 \\
\hline $\mathrm{H}$ & 3.07177000 & -4.42167500 & 0.71352200 \\
\hline $\mathrm{C}$ & -1.88987300 & -2.58812800 & 4.94039100 \\
\hline $\mathrm{H}$ & -2.61929300 & -2.87897200 & 5.70662800 \\
\hline $\mathrm{H}$ & -2.12089400 & -1.56145600 & 4.63158300 \\
\hline $\mathrm{H}$ & -0.89381600 & -2.58422600 & 5.39619100 \\
\hline $\mathrm{C}$ & -3.35357600 & -3.60226200 & 3.12819100 \\
\hline $\mathrm{H}$ & -4.09273700 & -3.90403000 & 3.88039600 \\
\hline $\mathrm{H}$ & -3.40099900 & -4.31530800 & 2.29759800 \\
\hline $\mathrm{H}$ & -3.65475300 & -2.62095100 & 2.74264700 \\
\hline $\mathrm{C}$ & -1.39592300 & -0.44530900 & -0.60964100 \\
\hline $\mathrm{H}$ & -2.29690600 & -0.52713000 & 0.00983000 \\
\hline $\mathrm{H}$ & -1.38314500 & -1.30143900 & -1.29383500 \\
\hline $\mathrm{H}$ & -1.48734600 & 0.46864000 & -1.20981400 \\
\hline $\mathrm{C}$ & -0.11053600 & 0.83706000 & 1.17078400 \\
\hline $\mathrm{H}$ & 0.81198800 & 0.88582600 & 1.75586600 \\
\hline $\mathrm{H}$ & -0.95885900 & 0.82513700 & 1.86592100 \\
\hline $\mathrm{H}$ & -0.18824100 & 1.75484700 & 0.57432000 \\
\hline $\mathrm{C}$ & 1.79831600 & -6.15096700 & 0.70599200 \\
\hline $\mathrm{H}$ & 1.03572200 & -6.63689500 & 1.32673600 \\
\hline $\mathrm{H}$ & 2.62339300 & -6.86197300 & 0.57373700 \\
\hline $\mathrm{H}$ & 1.35141200 & -5.96644100 & -0.27718800 \\
\hline
\end{tabular}

$\begin{array}{ccc}2.98527600 & -5.16532800 & 2.70777800 \\ 3.31054700 & -4.24437200 & 3.19715100 \\ 3.86045000 & -5.80737900 & 2.54629100 \\ 2.31378500 & -5.69245400 & 3.39557200 \\ 7.56062300 & -1.15545800 & 4.17975900 \\ 6.36841900 & -1.37185500 & 4.77799600 \\ 8.34853800 & -1.96748900 & 3.28961700 \\ 9.60925900 & -2.38913000 & 3.72371800 \\ 7.90964300 & -2.25994900 & 1.99355300 \\ 10.42940100 & -3.12393000 & 2.86561000 \\ 9.93593800 & -2.14889300 & 4.73161600 \\ 8.73184100 & -3.00712700 & 1.14910100 \\ 6.93426100 & -1.91981000 & 1.66504600 \\ 9.98926300 & -3.43896300 & 1.57866100 \\ 11.40698600 & -3.45320200 & 3.20645900 \\ 8.38700500 & -3.24437900 & 0.14663600 \\ 7.92247500 & -0.21082800 & 4.28390000 \\ 5.89169500 & -0.16806700 & 5.55093500 \\ 5.36424100 & -0.47036300 & 6.45662800 \\ 6.72795700 & 0.48281700 & 5.81646400 \\ 5.20030100 & 0.41000300 & 4.92987900 \\ 10.62473000 & -4.01561600 & 0.91230400 \\ 5.54801300 & -2.52548800 & 4.62067800 \\ 4.33009700 & -2.64609000 & 5.51961400 \\ 6.19024200 & -3.90132200 & 4.38606500 \\ 3.25462000 & -1.74415800 & 5.41171400 \\ 4.23200700 & -3.70371300 & 6.45156000 \\ 6.55355800 & -4.52508200 & 5.74302700 \\ 7.06934700 & -3.85532300 & 3.74922500 \\ 2.13540800 & -1.84594100 & 6.23488100 \\ 3.29301100 & -0.95097100 & 4.67140000 \\ 3.09788300 & -3.79150300 & 7.27112400 \\ 5.28879200 & -4.79137700 & 6.56670000 \\ 7.10677800 & -5.46211500 & 5.60066500 \\ 2.05732700 & -2.87123000 & 7.17922600 \\ 1.32543200 & -1.12920300 & 6.12998200 \\ 3.03715900 & -4.60419800 & 7.99311900 \\ 4.83867500 & -5.73494500 & 6.22340100 \\ 1.19130500 & -2.95898400 & 7.83039700 \\ 5.04464500 & -2.08427400 & 3.36077400 \\ 5.46935300 & -4.54609900 & 3.87175100 \\ 7.22416300 & -3.84077100 & 6.28073100 \\ 5.54344000 & -4.95303400 & 7.62269500\end{array}$

$11 \mathrm{c}$

$\begin{array}{llll}\text { C } & -1.48335900 & 0.75512700 & 2.58654800\end{array}$

$\begin{array}{lll}-0.47681000 & 1.21537600 & 1.74084100\end{array}$

$\begin{array}{lll}0.12568100 & 2.46846700 & 1.93454600\end{array}$

$\begin{array}{lll}-0.29625900 & 3.27462300 & 3.00858700\end{array}$

$\begin{array}{lll}-1.30643700 & 2.79567000 & 3.85528900\end{array}$

$\begin{array}{lll}-1.90042600 & 1.55245400 & 3.65383100\end{array}$

$\begin{array}{lll}-1.93714400 & -0.21594400 & 2.41117100\end{array}$

$\begin{array}{lll}-0.16094700 & 0.59054700 & 0.90789900\end{array}$

$\begin{array}{lll}1.27531500 & 2.90205700 & 1.02404300\end{array}$ 


\begin{tabular}{|c|c|c|c|c|c|c|c|}
\hline $\mathrm{C}$ & 0.29721300 & 4.64955700 & 3.26162100 & $\mathrm{C}$ & 3.27237800 & 4.96683900 & -5.80324000 \\
\hline $\mathrm{H}$ & -1.62919200 & 3.41714700 & 4.68798500 & $\mathrm{C}$ & 4.02936100 & 7.25505200 & -5.88373000 \\
\hline $\mathrm{H}$ & -2.68114300 & 1.20681500 & 4.32578800 & $\mathrm{H}$ & 3.47008200 & 8.39771500 & -7.62733500 \\
\hline $\mathrm{C}$ & 1.65079100 & 4.85477300 & 2.57527900 & $\mathrm{C}$ & 3.93943900 & 6.04357400 & -5.18013100 \\
\hline $\mathrm{C}$ & 1.57163200 & 4.42574100 & 1.10733700 & $\mathrm{H}$ & 4.56071200 & 8.08198100 & -5.42226300 \\
\hline $\mathrm{H}$ & 0.38615700 & 4.81641100 & 4.34214700 & $\mathrm{O}$ & 0.60774200 & 3.40739300 & -5.55621100 \\
\hline $\mathrm{H}$ & 2.42665700 & 4.27092100 & 3.08902700 & $\mathrm{O}$ & 3.12980700 & 3.76039900 & -5.13234500 \\
\hline $\mathrm{H}$ & 0.78460400 & 4.99752800 & 0.60127100 & $\mathrm{P}$ & 1.70812700 & 3.47310600 & -4.32474000 \\
\hline $\mathrm{C}$ & 1.04432200 & 2.56873300 & -0.43099500 & $\mathrm{O}$ & 1.29280400 & 4.61742700 & -3.45829600 \\
\hline $\mathrm{C}$ & -0.17921000 & 3.07920900 & -1.12826000 & $\mathrm{O}$ & 1.90777400 & 2.08714400 & -3.72724700 \\
\hline $\mathrm{H}$ & 0.12087600 & 3.82651900 & -1.88093100 & $\mathrm{C}$ & -0.94450800 & 0.94355700 & -5.37002400 \\
\hline $\mathrm{H}$ & -0.88379100 & 3.51435000 & -0.41859900 & $\mathrm{C}$ & -2.09904000 & 1.72751300 & -5.12572300 \\
\hline $\mathrm{H}$ & -0.67442000 & 2.26859200 & -1.67439300 & $\mathrm{C}$ & -0.69862100 & -0.20114000 & -4.56495900 \\
\hline $\mathrm{N}$ & 1.92641600 & 1.93982100 & -1.14946300 & $\mathrm{C}$ & -2.96847500 & 1.35639900 & -4.09188200 \\
\hline $\mathrm{C}$ & 3.14115500 & 1.31138300 & -0.71785700 & $\mathrm{C}$ & -1.60826000 & -0.52944400 & -3.55310800 \\
\hline $\mathrm{C}$ & 4.31649800 & 1.62866100 & -1.40635600 & $\mathrm{C}$ & -2.75348700 & 0.22997900 & -3.29711500 \\
\hline $\mathrm{C}$ & 3.14165700 & 0.33407000 & 0.28463300 & $\mathrm{H}$ & -3.85952800 & 1.95557700 & -3.91630000 \\
\hline $\mathrm{C}$ & 5.50457000 & 0.98761800 & -1.05631600 & $\mathrm{H}$ & -1.41849000 & -1.41684900 & -2.95395600 \\
\hline $\mathrm{H}$ & 4.29352400 & 2.36557500 & -2.20146000 & $\mathrm{C}$ & 4.58264200 & 5.92742900 & -3.82841400 \\
\hline $\mathrm{C}$ & 4.33705300 & -0.29862100 & 0.62473600 & $\mathrm{C}$ & 5.75519400 & 5.14479000 & -3.67267300 \\
\hline $\mathrm{H}$ & 2.21527100 & 0.05671400 & 0.77786800 & $\mathrm{C}$ & 4.08001100 & 6.65798300 & -2.72343400 \\
\hline $\mathrm{C}$ & 5.52017700 & 0.02871300 & -0.04110600 & $\mathrm{C}$ & 6.38387400 & 5.09539000 & -2.42306100 \\
\hline $\mathrm{H}$ & 6.41728800 & 1.23746500 & -1.58833200 & $\mathrm{C}$ & 4.75686500 & 6.57958900 & -1.49870500 \\
\hline $\mathrm{H}$ & 4.33778800 & -1.05742100 & 1.40191700 & $\mathrm{C}$ & 5.90557400 & 5.80810000 & -1.31961300 \\
\hline $\mathrm{H}$ & 6.44814100 & -0.47039800 & 0.22273000 & $\mathrm{H}$ & 7.28961300 & 4.50188900 & -2.31845400 \\
\hline $\mathrm{H}$ & 1.81781900 & 1.97575200 & -2.24236500 & $\mathrm{H}$ & 4.38056700 & 7.15223600 & -0.65357700 \\
\hline $\mathrm{H}$ & 2.50700500 & 4.63828100 & 0.57865100 & $\mathrm{C}$ & 0.51012000 & -1.12119800 & -4.76102600 \\
\hline $\mathrm{H}$ & 1.94952500 & 5.90723000 & 2.63941200 & $\mathrm{H}$ & 1.15482600 & -0.67889200 & -5.52332900 \\
\hline $\mathrm{H}$ & -0.40672200 & 5.41279200 & 2.89664200 & $\mathrm{C}$ & -3.76839000 & -0.17854100 & -2.23618500 \\
\hline $\mathrm{H}$ & 2.18346500 & 2.39136000 & 1.35906700 & $\mathrm{H}$ & -4.50830800 & 0.63093300 & -2.16847700 \\
\hline $\mathrm{C}$ & 3.38819800 & 3.45240100 & -8.95969700 & $\mathrm{C}$ & -2.48340800 & 2.93421400 & -5.98255200 \\
\hline $\mathrm{C}$ & 1.71235200 & 1.78464500 & -8.66362900 & $\mathrm{H}$ & -1.69921200 & 3.08929100 & -6.72657600 \\
\hline $\mathrm{C}$ & 2.74539100 & 2.24171000 & -9.67128100 & $\mathrm{C}$ & 1.36643100 & -1.25081900 & -3.48767700 \\
\hline $\mathrm{H}$ & 4.19511100 & 3.10975200 & -8.30158100 & $\mathrm{H}$ & 2.22003400 & -1.91439300 & -3.67481300 \\
\hline $\mathrm{H}$ & 3.81040300 & 4.18977800 & -9.64989900 & $\mathrm{H}$ & 0.79901300 & -1.67512200 & -2.64986000 \\
\hline $\mathrm{H}$ & 2.27756500 & 2.53770500 & -10.62103400 & $\mathrm{H}$ & 1.75407400 & -0.27281800 & -3.19649300 \\
\hline $\mathrm{H}$ & 3.47266300 & 1.45714800 & -9.91141100 & $\mathrm{C}$ & 0.07706800 & -2.51373900 & -5.26408600 \\
\hline $\mathrm{C}$ & 2.23865900 & 4.04528600 & -8.07527600 & $\mathrm{H}$ & 0.95558100 & -3.14160200 & -5.45773500 \\
\hline $\mathrm{C}$ & 1.31681200 & 4.94685900 & -8.96376900 & $\mathrm{H}$ & -0.50372600 & -2.44848000 & -6.19094100 \\
\hline $\mathrm{C}$ & 2.77565400 & 5.09171100 & -7.10187200 & $\mathrm{H}$ & -0.54473400 & -3.03105200 & -4.52298100 \\
\hline $\mathrm{C}$ & 2.06404100 & 6.29507900 & -9.05384300 & $\mathrm{C}$ & -3.14140700 & -0.34426200 & -0.84023100 \\
\hline $\mathrm{H}$ & 1.10675700 & 4.49846600 & -9.94005800 & $\mathrm{H}$ & -3.91021900 & -0.59789400 & -0.09986300 \\
\hline $\mathrm{H}$ & 0.36073700 & 5.08655700 & -8.44636700 & $\mathrm{H}$ & -2.64815500 & 0.57715000 & -0.51152200 \\
\hline $\mathrm{H}$ & 1.38652100 & 7.14650900 & -9.18791600 & $\mathrm{H}$ & -2.39570600 & -1.14866600 & -0.83073700 \\
\hline $\mathrm{H}$ & 2.76086500 & 6.31067900 & -9.90411500 & $\mathrm{C}$ & -4.52312500 & -1.45745900 & -2.65117200 \\
\hline $\mathrm{C}$ & 1.52673900 & 2.75831900 & -7.67011700 & $\mathrm{H}$ & -5.29408300 & -1.71279700 & -1.91353600 \\
\hline $\mathrm{C}$ & 0.71775500 & 2.47262900 & -6.57005300 & $\mathrm{H}$ & -3.83859700 & -2.31047100 & -2.73143500 \\
\hline $\mathrm{C}$ & 0.99507300 & 0.59392900 & -8.63063200 & $\mathrm{H}$ & -5.00932500 & -1.32888300 & -3.62425300 \\
\hline $\mathrm{C}$ & -0.02620800 & 1.27459500 & -6.50978400 & $\mathrm{C}$ & -3.78774900 & 2.66688400 & -6.76152700 \\
\hline $\mathrm{C}$ & 0.10940200 & 0.36953400 & -7.57612800 & $\mathrm{H}$ & -4.64086800 & 2.52511200 & -6.08710300 \\
\hline $\mathrm{H}$ & 1.11630100 & -0.15277100 & -9.41175400 & $\mathrm{H}$ & -3.70112700 & 1.76821400 & -7.38297800 \\
\hline $\mathrm{H}$ & -0.47797300 & -0.54373300 & -7.55562200 & $\mathrm{H}$ & -4.02093500 & 3.51385700 & -7.41864100 \\
\hline $\mathrm{C}$ & 2.80084500 & 6.34647800 & -7.73232300 & $\mathrm{C}$ & -2.58559100 & 4.22866800 & -5.15346900 \\
\hline $\mathrm{C}$ & 3.43327100 & 7.42978300 & -7.13326900 & $\mathrm{H}$ & -2.81318900 & 5.07952700 & -5.80770000 \\
\hline
\end{tabular}




\begin{tabular}{|c|c|c|c|c|c|c|c|}
\hline $\mathrm{H}$ & -1.64380800 & 4.43863000 & -4.63876400 & $\mathrm{H}$ & 4.94282300 & 4.07665800 & 3.33682000 \\
\hline $\mathrm{H}$ & -3.38634900 & 4.16737200 & -4.40575700 & $\mathrm{H}$ & 5.79748900 & 2.04350100 & 2.20674400 \\
\hline $\mathrm{C}$ & 6.40508700 & 4.39888600 & -4.83966000 & $\mathrm{C}$ & -0.56050700 & 5.07220700 & -0.70194300 \\
\hline $\mathrm{H}$ & 5.79108500 & 4.55984600 & -5.72885400 & $\mathrm{C}$ & -1.85629500 & 5.02581800 & -1.20096600 \\
\hline $\mathrm{C}$ & 6.62760300 & 5.79146900 & 0.02224600 & $\mathrm{C}$ & -0.80095600 & 2.84823000 & 0.22066200 \\
\hline $\mathrm{H}$ & 6.00511000 & 6.35852700 & 0.72868600 & $\mathrm{C}$ & -2.63897400 & 3.90520600 & -0.92840400 \\
\hline $\mathrm{C}$ & 2.85130800 & 7.56474600 & -2.81747600 & $\mathrm{H}$ & -2.26099500 & 5.84980400 & -1.7835080 \\
\hline $\mathrm{H}$ & 2.36229200 & 7.36766000 & -3.77414800 & $\mathrm{C}$ & -2.13200300 & 2.78680500 & -0.24498300 \\
\hline $\mathrm{C}$ & 6.78941800 & 4.37021900 & 0.59062600 & $\mathrm{H}$ & -3.66863100 & 3.87293600 & -1.27169600 \\
\hline $\mathrm{H}$ & 7.24882900 & 4.40150400 & 1.58636900 & $\mathrm{O}$ & 2.05004300 & 1.67067300 & -0.37015100 \\
\hline $\mathrm{H}$ & 7.43228700 & 3.75465000 & -0.04981700 & $\mathrm{O}$ & -0.23157400 & 1.74865000 & 0.86212300 \\
\hline $\mathrm{H}$ & 5.82301400 & 3.86121900 & 0.67374300 & $\mathrm{P}$ & 0.73815800 & 0.74496500 & -0.02554400 \\
\hline $\mathrm{C}$ & 7.99088400 & 6.50599900 & -0.06304400 & $\mathrm{O}$ & 0.12340700 & 0.33766100 & -1.31958100 \\
\hline $\mathrm{H}$ & 8.47574900 & 6.54089700 & 0.92047200 & $\mathrm{O}$ & 1.13781900 & -0.34061100 & 0.99770300 \\
\hline $\mathrm{H}$ & 7.87570300 & 7.53360700 & -0.42466100 & $\mathrm{C}$ & 4.61957200 & 0.47899500 & 0.39458400 \\
\hline $\mathrm{H}$ & 8.66659600 & 5.98478200 & -0.75171000 & $\mathrm{C}$ & 4.96060200 & 0.40634200 & -0.97803900 \\
\hline $\mathrm{C}$ & 7.80398700 & 4.96628300 & -5.15470200 & $\mathrm{C}$ & 4.88644300 & -0.63120500 & 1.24023100 \\
\hline $\mathrm{H}$ & 8.49905600 & 4.81800500 & -4.31937100 & $\mathrm{C}$ & 5.53022200 & -0.77210400 & -1.47538600 \\
\hline $\mathrm{H}$ & 7.75732100 & 6.04119800 & -5.36219700 & $\mathrm{C}$ & 5.46236000 & -1.78088200 & 0.68713300 \\
\hline $\mathrm{H}$ & 8.23051700 & 4.46839100 & -6.03431200 & $\mathrm{C}$ & 5.79279400 & -1.87826800 & -0.66752100 \\
\hline $\mathrm{C}$ & 6.46491600 & 2.87802100 & -4.60455300 & $\mathrm{H}$ & 5.80125800 & -0.82143600 & -2.52804100 \\
\hline $\mathrm{H}$ & 5.46169500 & 2.46787600 & -4.45420800 & $\mathrm{H}$ & 5.67017000 & -2.62348500 & 1.34270900 \\
\hline $\mathrm{H}$ & 7.08148000 & 2.63012400 & -3.73076000 & $\mathrm{C}$ & -3.05392100 & 1.62566300 & -0.01275200 \\
\hline $\mathrm{H}$ & 6.91007900 & 2.37417500 & -5.47153800 & $\mathrm{C}$ & -3.52257000 & 1.34296300 & 1.29683000 \\
\hline $\mathrm{C}$ & 3.25754500 & 9.05289800 & -2.78171500 & $\mathrm{C}$ & -3.57549100 & 0.89637900 & -1.11117500 \\
\hline $\mathrm{H}$ & 3.73002300 & 9.31201600 & -1.82581400 & $\mathrm{C}$ & -4.48468600 & 0.34189500 & 1.47559000 \\
\hline $\mathrm{H}$ & 2.37564300 & 9.69415000 & -2.90289600 & $\mathrm{C}$ & -4.54993600 & -0.08226700 & -0.87282600 \\
\hline $\mathrm{H}$ & 3.96743100 & 9.30281500 & -3.57754900 & $\mathrm{C}$ & -5.02591100 & -0.37655400 & 0.40570600 \\
\hline $\mathrm{C}$ & 1.80650700 & 7.27132100 & -1.72527000 & $\mathrm{H}$ & -4.84574900 & 0.14195500 & 2.48158100 \\
\hline $\mathrm{H}$ & 1.48771400 & 6.22943300 & -1.78966800 & $\mathrm{H}$ & -4.96529700 & -0.62612400 & -1.71916300 \\
\hline $\mathrm{H}$ & 0.92618900 & 7.90917600 & -1.87380700 & $\mathrm{C}$ & 4.61055100 & -0.62144300 & 2.74758100 \\
\hline \multirow[t]{2}{*}{$\mathrm{H}$} & 2.19211200 & 7.47793500 & -0.71853900 & $\mathrm{H}$ & 4.11937900 & 0.32082900 & 2.99894600 \\
\hline & & & & $\mathrm{C}$ & 6.44885300 & -3.12569000 & -1.24641000 \\
\hline TS-6 & & & & $\mathrm{H}$ & 6.62728200 & -2.92889700 & -2.31272700 \\
\hline $\mathrm{C}$ & 1.01080300 & 5.20006500 & 2.05668300 & $\mathrm{C}$ & 4.81367700 & 1.59102800 & -1.93406800 \\
\hline $\mathrm{C}$ & 3.13173500 & 4.12106300 & 2.15990800 & $\mathrm{H}$ & 4.34502500 & 2.41451000 & -1.39095300 \\
\hline $\mathrm{C}$ & 2.41071900 & 5.34114600 & 2.69068000 & $\mathrm{C}$ & 3.65012100 & -1.73937900 & 3.18916900 \\
\hline $\mathrm{H}$ & 0.37669300 & 4.56998400 & 2.69105900 & $\mathrm{H}$ & 3.48376000 & -1.68723600 & 4.27218500 \\
\hline $\mathrm{H}$ & 0.49677400 & 6.15571100 & 1.91484900 & $\mathrm{H}$ & 4.04965400 & -2.73674300 & 2.96756700 \\
\hline $\mathrm{H}$ & 2.90363200 & 6.27032800 & 2.37172500 & $\mathrm{H}$ & 2.68156200 & -1.63233800 & 2.69606300 \\
\hline $\mathrm{H}$ & 2.37877400 & 5.36369900 & 3.78631400 & $\mathrm{C}$ & 5.92423000 & -0.68732300 & 3.55360300 \\
\hline $\mathrm{C}$ & 1.26686000 & 4.45097300 & 0.70515100 & $\mathrm{H}$ & 5.71840900 & -0.61025600 & 4.62829800 \\
\hline $\mathrm{C}$ & 1.76267700 & 5.47810300 & -0.36836000 & $\mathrm{H}$ & 6.61043500 & 0.12259900 & 3.28088600 \\
\hline $\mathrm{C}$ & -0.05068100 & 4.01611200 & 0.07158800 & $\mathrm{H}$ & 6.45142400 & -1.63383800 & 3.38328500 \\
\hline $\mathrm{C}$ & 0.47462200 & 6.16283800 & -0.87153600 & $\mathrm{C}$ & 5.53347300 & -4.36115600 & -1.15599900 \\
\hline $\mathrm{H}$ & 2.50165400 & 6.17954300 & 0.03109300 & $\mathrm{H}$ & 6.01581700 & -5.23443900 & -1.61266700 \\
\hline $\mathrm{H}$ & 2.23501500 & 4.92576100 & -1.18883000 & $\mathrm{H}$ & 4.58299400 & -4.18678400 & -1.67183200 \\
\hline $\mathrm{H}$ & 0.55412800 & 6.50355600 & -1.91051400 & $\mathrm{H}$ & 5.30928600 & -4.61292100 & -0.11238100 \\
\hline $\mathrm{H}$ & 0.22817400 & 7.04840300 & -0.26878700 & $\mathrm{C}$ & 7.81740000 & -3.40616000 & -0.59632000 \\
\hline $\mathrm{C}$ & 2.39677900 & 3.51821800 & 1.12624700 & $\mathrm{H}$ & 8.30258000 & -4.26842400 & -1.07007000 \\
\hline $\mathrm{C}$ & 2.82572800 & 2.29997000 & 0.59753000 & $\mathrm{H}$ & 7.71213800 & -3.62948500 & 0.47209000 \\
\hline $\mathrm{C}$ & 4.35535500 & 3.59650800 & 2.55805200 & $\mathrm{H}$ & 8.48506600 & -2.54282400 & -0.69171200 \\
\hline $\mathrm{C}$ & 4.06929000 & 1.74801100 & 0.97330700 & $\mathrm{C}$ & 6.19406500 & 2.09565100 & -2.40251200 \\
\hline $\mathrm{C}$ & 4.82618700 & 2.44342100 & 1.93219500 & $\mathrm{H}$ & 6.72721400 & 1.33276700 & -2.98233400 \\
\hline
\end{tabular}




\begin{tabular}{|c|c|c|c|c|}
\hline $\mathrm{H}$ & 6.82620000 & 2.36816700 & -1.54986300 & C \\
\hline $\mathrm{H}$ & 6.08190900 & 2.98126300 & -3.04031300 & $\mathrm{C}$ \\
\hline $\mathrm{C}$ & 3.90592300 & 1.26905700 & -3.13593800 & $\mathrm{C}$ \\
\hline $\mathrm{H}$ & 3.79953400 & 2.15181900 & -3.77862700 & $\mathrm{C}$ \\
\hline $\mathrm{H}$ & 2.90822800 & 0.97108400 & -2.80085200 & $\mathrm{C}$ \\
\hline $\mathrm{H}$ & 4.32542000 & 0.46367500 & -3.75217000 & $\mathrm{C}$ \\
\hline $\mathrm{C}$ & -3.08074700 & 2.14381900 & 2.52333800 & $\mathrm{C}$ \\
\hline $\mathrm{H}$ & -2.37371900 & 2.90871000 & 2.19360400 & $\mathrm{C}$ \\
\hline $\mathrm{C}$ & -6.13527000 & -1.40181000 & 0.60732400 & $\mathrm{H}$ \\
\hline $\mathrm{H}$ & -6.30927600 & -1.88366100 & -0.36503400 & $\mathrm{H}$ \\
\hline $\mathrm{C}$ & -3.14664600 & 1.14704600 & -2.55927700 & $\mathrm{H}$ \\
\hline $\mathrm{H}$ & -2.35074200 & 1.89492200 & -2.55313200 & $\mathrm{H}$ \\
\hline $\mathrm{C}$ & -5.75569900 & -2.50824900 & 1.60864900 & $\mathrm{H}$ \\
\hline $\mathrm{H}$ & -6.56234700 & -3.24676000 & 1.68939200 & $\mathrm{C}$ \\
\hline $\mathrm{H}$ & -5.58049100 & -2.10054200 & 2.61105000 & $\mathrm{H}$ \\
\hline $\mathrm{H}$ & -4.84522400 & -3.03321100 & 1.29935600 & $\mathrm{H}$ \\
\hline $\mathrm{C}$ & -7.45333100 & -0.71795300 & 1.02299700 & $\mathrm{C}$ \\
\hline $\mathrm{H}$ & -8.26406700 & -1.45219100 & 1.10580600 & $\mathrm{H}$ \\
\hline $\mathrm{H}$ & -7.75204700 & 0.04112600 & 0.29182500 & $\mathrm{H}$ \\
\hline $\mathrm{H}$ & -7.34925900 & -0.22155300 & 1.99520100 & $\mathrm{H}$ \\
\hline $\mathrm{C}$ & -4.27081300 & 2.88741500 & 3.16348500 & $\mathrm{H}$ \\
\hline $\mathrm{H}$ & -5.01893800 & 2.19165900 & 3.56160800 & \\
\hline $\mathrm{H}$ & -4.77169300 & 3.53507400 & 2.43513800 & \\
\hline $\mathrm{H}$ & -3.92568600 & 3.51417700 & 3.99481300 & $\mathrm{C}$ \\
\hline $\mathrm{C}$ & -2.35016800 & 1.26994800 & 3.55971500 & $\mathrm{C}$ \\
\hline $\mathrm{H}$ & -1.44571400 & 0.83182200 & 3.12754100 & $\mathrm{C}$ \\
\hline $\mathrm{H}$ & -2.99014600 & 0.45823300 & 3.92737800 & $\mathrm{C}$ \\
\hline $\mathrm{H}$ & -2.05194500 & 1.87400400 & 4.42557800 & $\mathrm{C}$ \\
\hline $\mathrm{C}$ & -4.31124400 & 1.70351100 & -3.40365200 & $\mathrm{C}$ \\
\hline $\mathrm{H}$ & -5.13053800 & 0.97889000 & -3.48625000 & $\mathrm{H}$ \\
\hline $\mathrm{H}$ & -3.96963200 & 1.93616100 & -4.41961400 & $\mathrm{H}$ \\
\hline $\mathrm{H}$ & -4.72618100 & 2.61989700 & -2.96918800 & $\mathrm{C}$ \\
\hline $\mathrm{C}$ & -2.55125100 & -0.11166800 & -3.21854700 & $\mathrm{C}$ \\
\hline $\mathrm{H}$ & -1.63980000 & -0.39924900 & -2.68833800 & $\mathrm{H}$ \\
\hline $\mathrm{H}$ & -2.28258500 & 0.09769100 & -4.26118700 & $\mathrm{H}$ \\
\hline $\mathrm{H}$ & -3.26487600 & -0.94644200 & -3.22543100 & $\mathrm{C}$ \\
\hline $\mathrm{N}$ & -0.53074500 & -2.85420600 & -1.58662700 & $\mathrm{C}$ \\
\hline $\mathrm{C}$ & -0.56893400 & -2.77621400 & -0.24236200 & $\mathrm{H}$ \\
\hline $\mathrm{C}$ & 0.52332300 & -3.16086600 & -2.52373300 & $\mathrm{H}$ \\
\hline $\mathrm{C}$ & 1.29547800 & -2.12679700 & -3.06105700 & $\mathrm{H}$ \\
\hline $\mathrm{C}$ & 0.67497500 & -4.47661800 & -2.96908900 & $\mathrm{C}$ \\
\hline $\mathrm{C}$ & 2.25448500 & -2.43090600 & -4.02899100 & $\mathrm{C}$ \\
\hline $\mathrm{H}$ & 1.14034000 & -1.11278300 & -2.70277400 & $\mathrm{H}$ \\
\hline $\mathrm{C}$ & 1.63958300 & -4.76934400 & -3.93434300 & $\mathrm{H}$ \\
\hline $\mathrm{H}$ & 0.04211200 & -5.25817100 & -2.55983400 & $\mathrm{H}$ \\
\hline $\mathrm{C}$ & 2.43172600 & -3.74725300 & -4.46289800 & $\mathrm{~N}$ \\
\hline $\mathrm{H}$ & 2.86417400 & -1.63268800 & -4.44210000 & $\mathrm{C}$ \\
\hline $\mathrm{H}$ & 1.76562500 & -5.79258500 & -4.27701600 & $\mathrm{C}$ \\
\hline $\mathrm{H}$ & -1.35019100 & -2.46223400 & -2.03839800 & $\mathrm{C}$ \\
\hline $\mathrm{C}$ & -1.94332100 & -2.39150900 & 0.25291700 & $\mathrm{C}$ \\
\hline $\mathrm{H}$ & -2.19238500 & -2.92151900 & 1.17277100 & $\mathrm{H}$ \\
\hline $\mathrm{H}$ & -1.98783700 & -1.31751400 & 0.46210100 & $\mathrm{C}$ \\
\hline $\mathrm{H}$ & -2.70622500 & -2.60929500 & -0.49874800 & $\mathrm{H}$ \\
\hline $\mathrm{H}$ & 3.18014400 & -3.97581900 & -5.21679000 & $\mathrm{C}$ \\
\hline
\end{tabular}

$\begin{array}{rrr}0.52656400 & -2.89580100 & 0.66346100 \\ 0.21046400 & -3.23919100 & 2.11139300 \\ 1.83659500 & -3.55759600 & 0.22516700 \\ 0.66851200 & -4.45635800 & 2.66239600 \\ -0.48043000 & -2.34440600 & 2.94838200 \\ 0.35917100 & -4.76127700 & 3.99590600 \\ -0.77707500 & -2.66021200 & 4.27174500 \\ -0.36564200 & -3.88530800 & 4.79890600 \\ 0.70496900 & -5.70768800 & 4.40828500 \\ -1.31360200 & -1.94460700 & 4.88902200 \\ -0.59182400 & -4.14816000 & 5.82903400 \\ 0.80128100 & -1.52847500 & 0.73256700 \\ -0.76857100 & -1.37433700 & 2.55975900 \\ 1.54904000 & -5.42690300 & 1.89035800 \\ 1.16337500 & -6.44917800 & 2.00191200 \\ 2.53738000 & -5.43076100 & 2.37392000 \\ 1.73009300 & -5.07915700 & 0.40750800 \\ 2.62272900 & -5.58169300 & 0.01536200 \\ 0.87743200 & -5.45274000 & -0.17469900 \\ 2.64409700 & -3.17083900 & 0.85614300 \\ 2.10836800 & -3.30203300 & -0.79668500\end{array}$

$2.76810800-4.54853500 \quad-3.80889000$ $3.05435100 \quad-3.20097700-3.60024300$ $3.65587000-2.76071300-2.40938300$ $3.98177700-3.70038300-1.41325500$ $3.67755200-5.05097200-1.63522700$ $3.07713900-5.47979100-2.81639300$ $\begin{array}{lll}2.31371700 & -4.86343100 & -4.74359200\end{array}$ $2.82087900-2.49050900-4.39016900$ $3.90356500-1.26384600-2.18503300$ $\begin{array}{lll}4.68341100 & -3.30214400 & -0.12535000\end{array}$ $3.92428300 \quad-5.77629700 \quad-0.86253700$ $2.85557800 \quad-6.53334300 \quad-2.96279900$ $\begin{array}{llll}4.62652300 & -1.79813000 & 0.16172300\end{array}$ $\begin{array}{lll}4.97569000 & -0.99911100 & -1.09778600\end{array}$ $5.73708900 \quad-3.61302300 \quad-0.19011900$ $\begin{array}{lll}5.31960200 & -1.54463400 & 0.97206600\end{array}$ $\begin{array}{llll}5.01208200 & 0.07501800 & -0.88247400\end{array}$ $\begin{array}{lll}4.29388200 & -0.56887200 & -3.46719600\end{array}$ $\begin{array}{llll}5.57274100 & -0.95291800 & -4.15142100\end{array}$ $\begin{array}{llll}5.96530800 & -1.88537300 & -3.74289200\end{array}$ $6.32200300-0.16161700-4.01610900$ $5.41288400-1.05584900 \quad-5.23355800$ $3.59699600 \quad 0.39302300 \quad-3.99485200$ $\begin{array}{llll}2.35050800 & 0.95805100 & -3.57125700\end{array}$ $\begin{array}{lll}1.24132300 & 0.17754800 & -3.22531400\end{array}$ $\begin{array}{llll}2.24889100 & 2.35428500 & -3.61223000\end{array}$ $\begin{array}{llll}0.04874200 & 0.80414600 & -2.86248900\end{array}$ $\begin{array}{lll}1.29239200 & -0.90451000 & -3.27162200\end{array}$ $\begin{array}{lll}1.05247200 & 2.96800100 & -3.24601400\end{array}$ $\begin{array}{lll}3.10018700 & 2.94153300 & -3.93897000\end{array}$ $\begin{array}{llll}-0.04696200 & 2.19701800 & -2.86177200\end{array}$ 


\begin{tabular}{|c|c|c|c|c|c|c|c|}
\hline $\mathrm{H}$ & -0.81026900 & 0.19589700 & -2.59496100 & $\mathrm{C}$ & 0.97761800 & -2.77975200 & -7.81950000 \\
\hline $\mathrm{H}$ & 0.97861700 & 4.05115100 & -3.27192000 & $\mathrm{C}$ & -0.11466700 & -2.15654100 & -7.21557500 \\
\hline $\mathrm{H}$ & -0.97945000 & 2.67833100 & -2.58147800 & $\mathrm{H}$ & -1.12018400 & -0.27237700 & -6.97802100 \\
\hline $\mathrm{H}$ & 4.02977700 & 0.89527100 & -4.88200200 & $\mathrm{H}$ & 1.12337700 & -3.84578200 & -7.65648600 \\
\hline $\mathrm{H}$ & 5.97017700 & -1.29160700 & -1.45590600 & $\mathrm{C}$ & 7.06747800 & 3.86961200 & -4.43125400 \\
\hline $\mathrm{H}$ & 3.62156600 & -1.51686500 & 0.50493300 & $\mathrm{H}$ & 6.45299400 & 4.21565300 & -5.26547000 \\
\hline $\mathrm{H}$ & 4.25787900 & -3.86572300 & 0.71420700 & $\mathrm{C}$ & 10.44166000 & 0.26178200 & -3.34848300 \\
\hline $\mathrm{H}$ & 2.97632400 & -0.81575900 & -1.81436000 & $\mathrm{H}$ & 11.01349400 & -0.50243100 & -3.8926290 \\
\hline $\mathrm{C}$ & 4.17775400 & 4.28523300 & -10.70142800 & $\mathrm{C}$ & 9.26206000 & 1.04346000 & -8.18253200 \\
\hline $\mathrm{C}$ & 6.03021900 & 4.80020500 & -9.28607200 & $\mathrm{H}$ & 8.61891600 & 1.66169400 & -8.81231600 \\
\hline $\mathrm{C}$ & 5.14702000 & 5.43430000 & -10.34020500 & $\mathrm{C}$ & 6.09592900 & 3.35018400 & -3.35595500 \\
\hline $\mathrm{H}$ & 3.32185000 & 4.28987000 & -10.01675600 & $\mathrm{H}$ & 5.39103900 & 4.14112300 & -3.06933000 \\
\hline $\mathrm{H}$ & 3.78942800 & 4.35029600 & -11.72292300 & $\mathrm{H}$ & 6.61665800 & 3.03275800 & -2.44410700 \\
\hline $\mathrm{H}$ & 5.72991600 & 5.75677600 & -11.21451900 & $\mathrm{H}$ & 5.52723300 & 2.50420000 & -3.74773300 \\
\hline $\mathrm{H}$ & 4.62610200 & 6.32398300 & -9.96678100 & $\mathrm{C}$ & 7.86378700 & 5.07494900 & -3.89139700 \\
\hline $\mathrm{C}$ & 5.00876300 & 2.98189300 & -10.45660600 & $\mathrm{H}$ & 7.18389900 & 5.86942200 & -3.55894300 \\
\hline $\mathrm{C}$ & 5.95254400 & 2.73382200 & -11.68381400 & $\mathrm{H}$ & 8.52865200 & 5.49750100 & -4.65224800 \\
\hline $\mathrm{C}$ & 4.14219400 & 1.72668600 & -10.53173400 & $\mathrm{H}$ & 8.48609800 & 4.78782900 & -3.03484700 \\
\hline $\mathrm{C}$ & 5.05896200 & 2.03510000 & -12.72927900 & $\mathrm{C}$ & 9.55649000 & -0.47265500 & -2.32361700 \\
\hline $\mathrm{H}$ & 6.41510500 & 3.65569100 & -12.05009300 & $\mathrm{H}$ & 10.17043300 & -1.05654900 & -1.6269440 \\
\hline $\mathrm{H}$ & 6.75687900 & 2.05654500 & -11.37429200 & $\mathrm{H}$ & 8.86268900 & -1.15832500 & -2.82352300 \\
\hline $\mathrm{H}$ & 5.62505000 & 1.37837800 & -13.40018200 & $\mathrm{H}$ & 8.96360300 & 0.23499500 & -1.73116000 \\
\hline $\mathrm{H}$ & 4.54172900 & 2.76653400 & -13.36682500 & $\mathrm{C}$ & 11.45776800 & 1.17849600 & -2.64017600 \\
\hline $\mathrm{C}$ & 5.82474300 & 3.41249700 & -9.24005600 & $\mathrm{H}$ & 12.08199500 & 0.60416000 & -1.94437700 \\
\hline $\mathrm{C}$ & 6.42551600 & 2.66486100 & -8.22691000 & $\mathrm{H}$ & 10.95200100 & 1.96338000 & -2.06497500 \\
\hline $\mathrm{C}$ & 6.94581200 & 5.40833500 & -8.43422900 & $\mathrm{H}$ & 12.11638500 & 1.66961700 & -3.36466700 \\
\hline $\mathrm{C}$ & 7.38019700 & 3.24742000 & -7.36521300 & $\mathrm{C}$ & 10.71634000 & 1.35645300 & -8.59028000 \\
\hline $\mathrm{C}$ & 7.64257700 & 4.61898300 & -7.51751600 & $\mathrm{H}$ & 11.43474600 & 0.75552600 & -8.01982900 \\
\hline $\mathrm{H}$ & 7.12875900 & 6.47911800 & -8.48393100 & $\mathrm{H}$ & 10.95747500 & 2.41253600 & -8.42291000 \\
\hline $\mathrm{H}$ & 8.39456900 & 5.07558300 & -6.88098000 & $\mathrm{H}$ & 10.87031900 & 1.13715500 & -9.65402500 \\
\hline $\mathrm{C}$ & 4.08425600 & 1.27275800 & -11.85901500 & $\mathrm{C}$ & 8.90504800 & -0.42701700 & -8.47315000 \\
\hline $\mathrm{C}$ & 3.22273300 & 0.24358200 & -12.22090000 & $\mathrm{H}$ & 9.04130000 & -0.64527000 & -9.53979500 \\
\hline $\mathrm{C}$ & 3.41168700 & 1.06058100 & -9.54540000 & $\mathrm{H}$ & 7.86374100 & -0.63395100 & -8.21139100 \\
\hline $\mathrm{C}$ & 2.43454700 & -0.35144200 & -11.23638500 & $\mathrm{H}$ & 9.54830800 & -1.11700500 & -7.91288500 \\
\hline $\mathrm{H}$ & 3.16202300 & -0.09714200 & -13.25170600 & $\mathrm{C}$ & 0.26781900 & 1.42287600 & -8.50252500 \\
\hline $\mathrm{C}$ & 2.52805400 & 0.01302600 & -9.88278400 & $\mathrm{H}$ & 1.01170000 & 1.81962100 & -9.19743900 \\
\hline $\mathrm{H}$ & 1.73926600 & -1.13964900 & -11.50918100 & $\mathrm{C}$ & -1.10166400 & -2.95951300 & -6.37624900 \\
\hline $\mathrm{O}$ & 6.11201500 & 1.32394000 & -8.09197200 & $\mathrm{H}$ & -0.77750500 & -4.00893100 & -6.41544800 \\
\hline $\mathrm{O}$ & 3.53542500 & 1.47200200 & -8.22820000 & $\mathrm{C}$ & 3.04100200 & -2.88469000 & -9.25161300 \\
\hline $\mathrm{P}$ & 4.74740600 & 0.86009900 & -7.28058200 & $\mathrm{H}$ & 3.70461200 & -2.17602200 & -9.75292100 \\
\hline $\mathrm{O}$ & 4.76391800 & -0.63163500 & -7.23971800 & $\mathrm{C}$ & -1.09354300 & -2.53396300 & -4.89602100 \\
\hline $\mathrm{O}$ & 4.61298900 & 1.65928800 & -5.98727300 & $\mathrm{H}$ & -1.80507000 & -3.13303100 & -4.31397400 \\
\hline $\mathrm{C}$ & 8.12190300 & 2.45227300 & -6.33007100 & $\mathrm{H}$ & -1.37295900 & -1.47910800 & -4.78642100 \\
\hline $\mathrm{C}$ & 9.00788100 & 1.41760900 & -6.72209400 & $\mathrm{H}$ & -0.09919400 & -2.66762300 & -4.45465500 \\
\hline $\mathrm{C}$ & 7.99413800 & 2.76808500 & -4.95125000 & $\mathrm{C}$ & -2.52827100 & -2.89981500 & -6.95502500 \\
\hline $\mathrm{C}$ & 9.72628500 & 0.72584300 & -5.73876700 & $\mathrm{H}$ & -3.20818900 & -3.54084200 & -6.38025200 \\
\hline $\mathrm{C}$ & 8.74720800 & 2.05134500 & -4.01344100 & $\mathrm{H}$ & -2.54388300 & -3.23280000 & -7.99841000 \\
\hline $\mathrm{C}$ & 9.61872900 & 1.02219300 & -4.38061200 & $\mathrm{H}$ & -2.92815400 & -1.87927700 & -6.92565100 \\
\hline $\mathrm{H}$ & 10.40614200 & -0.06612700 & -6.04588400 & $\mathrm{C}$ & -1.10913000 & 1.60937200 & -9.17076400 \\
\hline $\mathrm{H}$ & 8.65332700 & 2.31229800 & -2.96187700 & $\mathrm{H}$ & -1.92533300 & 1.26597800 & -8.52374900 \\
\hline $\mathrm{C}$ & 1.67247000 & -0.71322400 & -8.88417200 & $\mathrm{H}$ & -1.17191600 & 1.05033500 & -10.11120800 \\
\hline $\mathrm{C}$ & 0.58119400 & -0.05470000 & -8.26373100 & $\mathrm{H}$ & -1.28584300 & 2.66922300 & -9.39257900 \\
\hline $\mathrm{C}$ & 1.88190300 & -2.09386700 & -8.64081200 & $\mathrm{C}$ & 0.37413200 & 2.24905700 & -7.20676600 \\
\hline $\mathrm{C}$ & -0.27907800 & -0.78661300 & -7.43726900 & $\mathrm{H}$ & 1.37640200 & 2.16794400 & -6.77721800 \\
\hline
\end{tabular}




\begin{tabular}{|c|c|c|c|c|c|c|c|}
\hline $\mathrm{H}$ & -0.35200400 & 1.91739000 & -6.45427800 & $\mathrm{C}$ & 5.38916400 & 4.13933500 & -2.09121500 \\
\hline $\mathrm{H}$ & 0.17627800 & 3.30867800 & -7.41358900 & $\mathrm{H}$ & 4.73132700 & 4.56580600 & -1.32665000 \\
\hline $\mathrm{C}$ & 2.54167400 & -3.89497300 & -10.30504700 & $\mathrm{H}$ & 5.31301100 & 4.78424400 & -2.97918900 \\
\hline $\mathrm{H}$ & 1.89090100 & -4.65360300 & -9.85215500 & $\mathrm{H}$ & 6.41689300 & 4.21964300 & -1.72410700 \\
\hline $\mathrm{H}$ & 3.38918700 & -4.41698400 & -10.76616000 & $\mathrm{C}$ & 2.47579000 & 2.80728900 & -1.84136700 \\
\hline $\mathrm{H}$ & 1.97190800 & -3.40682500 & -11.10349000 & $\mathrm{H}$ & 2.70829900 & 3.56857700 & -1.08934700 \\
\hline $\mathrm{C}$ & 3.88986900 & -3.59800100 & -8.18179700 & $\mathrm{H}$ & 1.78411000 & 2.08724000 & -1.39309700 \\
\hline $\mathrm{H}$ & 4.29043700 & -2.86820800 & -7.47581800 & $\mathrm{H}$ & 1.94894100 & 3.31387900 & -2.66080300 \\
\hline $\mathrm{H}$ & 4.73218600 & -4.11479100 & -8.65906500 & $\mathrm{C}$ & 2.58089500 & -0.08268500 & -3.19709200 \\
\hline \multirow[t]{2}{*}{$\mathrm{H}$} & 3.31159800 & -4.35471000 & -7.63574600 & $\mathrm{H}$ & 2.12310200 & 0.19102700 & -4.15812000 \\
\hline & & & & $\mathrm{H}$ & 1.81620900 & -0.00851100 & -2.41803900 \\
\hline 13-RR & & & & $\mathrm{H}$ & 2.88453400 & -1.13187400 & -3.26619500 \\
\hline $\mathrm{C}$ & 3.71940900 & 2.13529600 & -2.35015700 & $\mathrm{C}$ & 5.67247600 & -0.67300600 & -3.99847000 \\
\hline $\mathrm{C}$ & 3.76582800 & 0.78979200 & -2.89466300 & $\mathrm{H}$ & 5.71885300 & -0.49399200 & -5.08253200 \\
\hline $\mathrm{C}$ & 5.14470100 & 0.53473100 & -3.27702100 & $\mathrm{H}$ & 5.03258900 & -1.54429200 & -3.83210300 \\
\hline $\mathrm{C}$ & 5.91450900 & 1.70074700 & -2.93677300 & $\mathrm{H}$ & 6.67473400 & -0.93129700 & -3.64723200 \\
\hline $\mathrm{C}$ & 5.02375500 & 2.71843600 & -2.41125800 & $\mathrm{C}$ & 7.37609600 & 1.86933800 & -3.23137700 \\
\hline Ir & 5.02750300 & 0.75938400 & -1.09438900 & $\mathrm{H}$ & 7.92573200 & 0.95521900 & -2.99688900 \\
\hline $\mathrm{N}$ & 6.68488700 & 0.89444700 & 0.31262900 & $\mathrm{H}$ & 7.81345800 & 2.68132600 & -2.64474000 \\
\hline $\mathrm{N}$ & 4.06633700 & 0.84713400 & 0.82330000 & $\mathrm{H}$ & 7.52719200 & 2.10776900 & -4.29376500 \\
\hline $\mathrm{H}$ & 3.06434400 & 1.04984500 & 0.76019600 & $\mathrm{H}$ & 6.33006600 & 0.55630100 & 2.39863600 \\
\hline $\mathrm{C}$ & 6.30055900 & 1.37425900 & 1.66443900 & $\mathrm{C}$ & 7.19690200 & 2.49763000 & 2.18118700 \\
\hline $\mathrm{C}$ & 4.81547300 & 1.85026600 & 1.63253200 & $\mathrm{C}$ & 7.58070200 & 3.55024400 & 1.34028400 \\
\hline S & 7.56955400 & -0.52107900 & 0.35609400 & $\mathrm{C}$ & 7.61564000 & 2.52172500 & 3.51647800 \\
\hline $\mathrm{O}$ & 8.04152700 & -0.80270300 & -1.01268400 & $\mathrm{C}$ & 8.35848100 & 4.60298100 & 1.82328100 \\
\hline $\mathrm{O}$ & 6.82850300 & -1.61585800 & 1.03122800 & $\mathrm{H}$ & 7.28260400 & 3.52317500 & 0.29722700 \\
\hline $\mathrm{C}$ & 9.08438700 & -0.18393600 & 1.35770900 & $\mathrm{C}$ & 8.39683800 & 3.57158600 & 4.00362100 \\
\hline $\mathrm{C}$ & 9.30438700 & -0.82172200 & 2.60025400 & $\mathrm{H}$ & 7.32760900 & 1.71166300 & 4.18189400 \\
\hline $\mathrm{C}$ & 10.08339500 & 0.61561700 & 0.75711200 & $\mathrm{C}$ & 8.76938000 & 4.61792200 & 3.15872500 \\
\hline $\mathrm{C}$ & 10.58085100 & -0.70679400 & 3.19798200 & $\mathrm{H}$ & 8.64893700 & 5.41046800 & 1.15532500 \\
\hline $\mathrm{C}$ & 11.34043500 & 0.72650400 & 1.38206700 & $\mathrm{H}$ & 8.71677800 & 3.56833500 & 5.04254900 \\
\hline $\mathrm{C}$ & 11.58019400 & 0.09103100 & 2.61412900 & $\mathrm{H}$ & 9.37914000 & 5.43539800 & 3.53499600 \\
\hline $\mathrm{C}$ & 8.25368500 & -1.59597200 & 3.37843100 & $\mathrm{H}$ & 4.76787400 & 2.78032200 & 1.05926700 \\
\hline $\mathrm{H}$ & 8.32661300 & -2.67557900 & 3.19446400 & $\mathrm{C}$ & 4.25895700 & 2.10563200 & 3.02252800 \\
\hline $\mathrm{H}$ & 7.24044300 & -1.31457400 & 3.11181300 & $\mathrm{C}$ & 3.97351700 & 1.05594700 & 3.90702700 \\
\hline $\mathrm{H}$ & 8.38963100 & -1.43283700 & 4.45159300 & $\mathrm{C}$ & 4.09112700 & 3.42243800 & 3.47027300 \\
\hline $\mathrm{C}$ & 10.88211900 & -1.47773300 & 4.47237800 & $\mathrm{C}$ & 3.53758500 & 1.31711300 & 5.20657100 \\
\hline $\mathrm{H}$ & 11.94840400 & -1.69471600 & 4.56565300 & $\mathrm{H}$ & 4.09965500 & 0.02457100 & 3.58944000 \\
\hline $\mathrm{H}$ & 10.36254800 & -2.43891900 & 4.49167100 & $\mathrm{C}$ & 3.66221000 & 3.68770800 & 4.77220600 \\
\hline $\mathrm{H}$ & 10.58087600 & -0.93134400 & 5.37840000 & $\mathrm{H}$ & 4.32290500 & 4.24657400 & 2.80013500 \\
\hline $\mathrm{C}$ & 12.90611600 & 0.29282800 & 3.32666800 & $\mathrm{C}$ & 3.38559300 & 2.63463400 & 5.64554600 \\
\hline $\mathrm{H}$ & 13.64988200 & -0.46745300 & 3.04682500 & $\mathrm{H}$ & 3.31934100 & 0.49039000 & 5.87725400 \\
\hline $\mathrm{H}$ & 12.79124000 & 0.24741900 & 4.41229900 & $\mathrm{H}$ & 3.55075100 & 4.71657700 & 5.10407900 \\
\hline $\mathrm{H}$ & 13.34033500 & 1.26848100 & 3.09654300 & $\mathrm{H}$ & 3.05257600 & 2.83751300 & 6.65980100 \\
\hline $\mathrm{C}$ & 12.44518400 & 1.51663500 & 0.70353100 & $\mathrm{~N}$ & -0.20407200 & -3.22181800 & -1.91394900 \\
\hline $\mathrm{H}$ & 13.43505500 & 1.14801500 & 0.98236000 & $\mathrm{C}$ & 0.95432200 & -3.80448400 & -2.02861300 \\
\hline $\mathrm{H}$ & 12.41022100 & 2.58569100 & 0.95976400 & $\mathrm{C}$ & -1.18349600 & -2.96053900 & -2.92147600 \\
\hline $\mathrm{H}$ & 12.37616500 & 1.44601100 & -0.38463000 & $\mathrm{C}$ & -1.96921100 & -1.81114000 & -2.76545500 \\
\hline $\mathrm{C}$ & 9.85285600 & 1.39629000 & -0.52085100 & $\mathrm{C}$ & -1.43713100 & -3.85603100 & -3.96966300 \\
\hline $\mathrm{H}$ & 10.34019400 & 2.37419000 & -0.45550300 & $\mathrm{C}$ & -2.96845500 & -1.53303200 & -3.69570200 \\
\hline $\mathrm{H}$ & 8.79562400 & 1.56208600 & -0.70571000 & $\mathrm{H}$ & -1.80883500 & -1.15727100 & -1.91589700 \\
\hline $\mathrm{H}$ & 10.26280200 & 0.87068900 & -1.39202000 & $\mathrm{C}$ & -2.44296100 & -3.56786100 & -4.89041600 \\
\hline $\mathrm{H}$ & 4.14179800 & -0.07839800 & 1.24588800 & $\mathrm{H}$ & -0.88714600 & -4.78565800 & -4.04957500 \\
\hline $\mathrm{H}$ & 5.03239900 & -0.80787300 & -0.81145600 & $\mathrm{C}$ & -3.20099500 & -2.40250300 & -4.76352300 \\
\hline
\end{tabular}




\begin{tabular}{|c|c|c|c|c|c|c|c|}
\hline $\mathrm{H}$ & -3.56458900 & -0.63536200 & -3.57417900 & $\mathrm{C}$ & -1.65772400 & -2.26610700 & 5.13304300 \\
\hline $\mathrm{H}$ & -2.63919000 & -4.26457300 & -5.70030000 & $\mathrm{H}$ & -2.99017100 & -1.28932100 & 6.52133200 \\
\hline $\mathrm{H}$ & -0.44518900 & -2.77986500 & -0.95431900 & $\mathrm{C}$ & -0.83597400 & -2.16874500 & 3.99543500 \\
\hline $\mathrm{C}$ & 1.54794500 & -4.18575900 & -3.35341500 & $\mathrm{H}$ & -1.75524900 & -3.23762000 & 5.60840100 \\
\hline $\mathrm{H}$ & 1.30216900 & -5.22057500 & -3.61301000 & $\mathrm{O}$ & -1.73994100 & 0.27812300 & 0.78476500 \\
\hline $\mathrm{H}$ & 1.19839600 & -3.53167000 & -4.15556700 & $\mathrm{O}$ & 0.15484700 & -0.70892500 & 2.3146720 \\
\hline $\mathrm{H}$ & 2.63726500 & -4.13653300 & -3.28072800 & $\mathrm{P}$ & -0.33374500 & -0.63172500 & 0.73547200 \\
\hline $\mathrm{H}$ & -3.98109300 & -2.18076400 & -5.48601600 & $\mathrm{O}$ & -0.83445500 & -2.00175500 & 0.30862900 \\
\hline $\mathrm{C}$ & 1.74880900 & -4.07659800 & -0.77072400 & $\mathrm{O}$ & 0.76983900 & 0.06205000 & -0.0034890 \\
\hline $\mathrm{C}$ & 2.24603700 & -5.52840200 & -0.79833400 & $\mathrm{C}$ & -2.90495000 & 2.11821400 & -1.28805300 \\
\hline $\mathrm{C}$ & 2.88764600 & -3.02707800 & -0.62874600 & $\mathrm{C}$ & -4.22742600 & 1.67821800 & -1.00480200 \\
\hline $\mathrm{C}$ & 1.31565900 & -6.55580000 & -1.02794200 & $\mathrm{C}$ & -2.54883000 & 2.37251300 & -2.64041900 \\
\hline $\mathrm{C}$ & 3.59881000 & -5.85605600 & -0.59266800 & $\mathrm{C}$ & -5.14597100 & 1.55184600 & -2.05563900 \\
\hline $\mathrm{C}$ & 3.97025200 & -3.53305300 & 0.32608800 & $\mathrm{C}$ & -3.51597800 & 2.24937600 & $-3.6454740 c$ \\
\hline $\mathrm{H}$ & 2.44768900 & -2.08235100 & -0.29612300 & $\mathrm{C}$ & -4.83129700 & 1.86182100 & -3.37839900 \\
\hline $\mathrm{C}$ & 1.71116900 & -7.88887700 & -1.10221000 & $\mathrm{H}$ & -6.16053300 & 1.23499000 & -1.82427900 \\
\hline $\mathrm{H}$ & 0.26220500 & -6.30468000 & -1.14079500 & $\mathrm{H}$ & -3.23167400 & 2.47763200 & -4.66981600 \\
\hline $\mathrm{C}$ & 3.98239800 & -7.20433700 & -0.66852500 & $\mathrm{C}$ & -0.14999600 & -3.43600500 & 3.56230800 \\
\hline $\mathrm{C}$ & 4.62316800 & -4.79034200 & -0.25316000 & $\mathrm{C}$ & 1.16837400 & -3.70345800 & 3.99995400 \\
\hline $\mathrm{H}$ & 4.73212700 & -2.76161600 & 0.47456800 & $\mathrm{C}$ & -0.88307900 & -4.44711100 & 2.89111600 \\
\hline $\mathrm{C}$ & 3.06006700 & -8.21352600 & -0.93252700 & $\mathrm{C}$ & 1.71798800 & -4.97469000 & 3.78127200 \\
\hline $\mathrm{H}$ & 0.97486000 & -8.66694300 & -1.28482100 & $\mathrm{C}$ & -0.28124200 & -5.69448400 & 2.69092400 \\
\hline $\mathrm{H}$ & 5.02801200 & -7.45812000 & -0.50807100 & $\mathrm{C}$ & 1.00774600 & -5.99299100 & 3.14369000 \\
\hline $\mathrm{H}$ & 5.19356800 & -4.51415600 & -1.15307600 & $\mathrm{H}$ & 2.71999300 & -5.17755800 & 4.14216700 \\
\hline $\mathrm{H}$ & 3.38663500 & -9.24855600 & -0.98800700 & $\mathrm{H}$ & -0.84675100 & -6.47696200 & 2.18902900 \\
\hline $\mathrm{H}$ & 1.07659200 & -3.95440000 & 0.08712200 & $\mathrm{C}$ & -1.13702800 & 2.77618100 & -3.06571400 \\
\hline $\mathrm{H}$ & 3.35351100 & -2.82575100 & -1.60167500 & $\mathrm{H}$ & -0.50142900 & 2.76213500 & -2.17675000 \\
\hline $\mathrm{H}$ & 3.52399500 & -3.76328200 & 1.30299200 & $\mathrm{C}$ & -5.89845100 & 1.82236000 & -4.46621100 \\
\hline $\mathrm{H}$ & 5.35811900 & -5.20223100 & 0.44860600 & $\mathrm{H}$ & -6.83217500 & 1.49517400 & -3.98849700 \\
\hline $\mathrm{C}$ & -0.07125000 & 2.46258200 & 4.19699300 & $\mathrm{C}$ & -4.74780500 & 1.38763600 & 0.40419900 \\
\hline $\mathrm{C}$ & -0.70202900 & 3.52890000 & 2.13996400 & $\mathrm{H}$ & -3.94827900 & 1.59076100 & 1.11706300 \\
\hline $\mathrm{C}$ & -0.07589800 & 3.84018800 & 3.48590600 & $\mathrm{C}$ & -0.54452800 & 1.76626200 & -4.06774600 \\
\hline $\mathrm{H}$ & 0.86453800 & 1.93498300 & 3.98904400 & $\mathrm{H}$ & 0.48512300 & 2.04214000 & -4.32216400 \\
\hline $\mathrm{H}$ & -0.17554700 & 2.54121700 & 5.28423600 & $\mathrm{H}$ & -1.11787500 & 1.73802700 & -5.00184000 \\
\hline $\mathrm{H}$ & -0.66621600 & 4.58188000 & 4.04173700 & $\mathrm{H}$ & -0.53163500 & 0.75448600 & -3.65130600 \\
\hline $\mathrm{H}$ & 0.93462700 & 4.25297900 & 3.38792300 & $\mathrm{C}$ & -1.08795600 & 4.19799300 & -3.66120800 \\
\hline $\mathrm{C}$ & -1.25249600 & 1.68738800 & 3.53935700 & $\mathrm{H}$ & -0.05605700 & 4.47345200 & -3.91214400 \\
\hline $\mathrm{C}$ & -2.60998500 & 2.21012800 & 4.14097300 & $\mathrm{H}$ & -1.47561800 & 4.94785100 & -2.96373000 \\
\hline $\mathrm{C}$ & -1.33640500 & 0.21703400 & 3.93473600 & $\mathrm{H}$ & -1.68052300 & 4.26610000 & -4.58132200 \\
\hline $\mathrm{C}$ & -2.82819100 & 1.37837200 & 5.41584400 & $\mathrm{C}$ & -5.57863700 & 0.81219800 & -5.58379200 \\
\hline $\mathrm{H}$ & -2.59801500 & 3.28984400 & 4.31594000 & $\mathrm{H}$ & -6.37165900 & 0.81103300 & -6.34126200 \\
\hline $\mathrm{H}$ & -3.41428900 & 1.99933700 & 3.42686700 & $\mathrm{H}$ & -5.49428900 & -0.20484500 & -5.1843990 \\
\hline $\mathrm{H}$ & -3.88637100 & 1.27195200 & 5.68174200 & $\mathrm{H}$ & -4.63654200 & 1.05653600 & -6.08893600 \\
\hline $\mathrm{H}$ & -2.32813600 & 1.83550700 & 6.28210400 & $\mathrm{C}$ & -6.14983100 & 3.22507300 & -5.05366100 \\
\hline $\mathrm{C}$ & -1.15299200 & 2.20241600 & 2.10559000 & $\mathrm{H}$ & -6.96628800 & 3.19827600 & -5.78562000 \\
\hline $\mathrm{C}$ & -1.61762600 & 1.65575200 & 0.90661700 & $\mathrm{H}$ & -5.25716400 & 3.60802500 & -5.56243500 \\
\hline $\mathrm{C}$ & -0.91510400 & 4.36528200 & 1.04814400 & $\mathrm{H}$ & -6.41769500 & 3.93975200 & -4.26799400 \\
\hline $\mathrm{C}$ & -1.99460400 & 2.51089600 & -0.15465000 & $\mathrm{C}$ & -5.91896500 & 2.31636900 & 0.78379200 \\
\hline $\mathrm{C}$ & -1.63041100 & 3.86707500 & -0.04211300 & $\mathrm{H}$ & -6.79885700 & 2.14776600 & 0.15184700 \\
\hline $\mathrm{H}$ & -0.59427500 & 5.40415300 & 1.06358700 & $\mathrm{H}$ & -5.63563400 & 3.37063400 & 0.68797800 \\
\hline $\mathrm{H}$ & -1.92329700 & 4.54341600 & -0.83875100 & $\mathrm{H}$ & -6.22146000 & 2.13884900 & 1.82320500 \\
\hline $\mathrm{C}$ & -2.18141100 & 0.06480500 & 5.04534300 & $\mathrm{C}$ & -5.13280900 & -0.09523600 & 0.57057200 \\
\hline $\mathrm{C}$ & -2.34321300 & -1.17243700 & 5.65540700 & $\mathrm{H}$ & -5.46484200 & -0.28911100 & 1.59792700 \\
\hline $\mathrm{C}$ & -0.69264900 & -0.89992300 & 3.39256100 & $\mathrm{H}$ & -4.27698700 & -0.74430900 & 0.3634280 \\
\hline
\end{tabular}




\begin{tabular}{|c|c|c|c|c|c|c|c|}
\hline $\mathrm{H}$ & -5.95277700 & -0.37660700 & -0.10188500 & $\mathrm{H}$ & 8.87400700 & -3.19579000 & 1.35338300 \\
\hline $\mathrm{C}$ & 1.97021000 & -2.67691400 & 4.80325700 & $\mathrm{H}$ & 7.89194500 & -1.88729000 & 1.96515500 \\
\hline $\mathrm{H}$ & 1.58986500 & -1.68180900 & 4.55160200 & $\mathrm{H}$ & 9.51062400 & -2.13645400 & 2.62155800 \\
\hline $\mathrm{C}$ & 1.54822000 & -7.41169500 & 2.98496100 & $\mathrm{C}$ & 11.75769800 & -1.99091700 & 1.57743400 \\
\hline $\mathrm{H}$ & 1.27239000 & -7.74894200 & 1.97608900 & $\mathrm{H}$ & 12.75929000 & -2.13975100 & 1.16807900 \\
\hline $\mathrm{C}$ & -2.33521600 & -4.24857800 & 2.44853700 & $\mathrm{H}$ & 11.30836200 & -2.98182500 & 1.67777900 \\
\hline $\mathrm{H}$ & -2.55225200 & -3.17735200 & 2.48368300 & $\mathrm{H}$ & 11.87777100 & -1.58629800 & 2.5931410 \\
\hline $\mathrm{C}$ & 3.07442900 & -7.52746300 & 3.10348500 & $\mathrm{C}$ & 13.05964000 & 0.07915900 & $-0.0189760 \mathrm{C}$ \\
\hline $\mathrm{H}$ & 3.39213000 & -8.55021800 & 2.87070100 & $\mathrm{H}$ & 13.62371000 & -0.57152000 & -0.7028150 \\
\hline $\mathrm{H}$ & 3.41958600 & -7.30358000 & 4.12037900 & $\mathrm{H}$ & 13.42153900 & -0.12404200 & 0.9916420 \\
\hline $\mathrm{H}$ & 3.58594600 & -6.85127300 & 2.41123700 & $\mathrm{H}$ & 13.33700300 & 1.10977600 & -0.2515990 \\
\hline $\mathrm{C}$ & 0.86426200 & -8.36180200 & 3.99198500 & $\mathrm{C}$ & 11.49155700 & 1.62631200 & -1.95326000 \\
\hline $\mathrm{H}$ & 1.19766400 & -9.39595600 & 3.83945200 & $\mathrm{H}$ & 12.50432100 & 1.30214600 & -2.20359900 \\
\hline $\mathrm{H}$ & -0.22609100 & -8.33698100 & 3.89113500 & $\mathrm{H}$ & 11.57071500 & 2.63141500 & -1.5133600 \\
\hline $\mathrm{H}$ & 1.10979400 & -8.07509900 & 5.02198500 & $\mathrm{H}$ & 10.95171100 & 1.73122900 & -2.89721900 \\
\hline $\mathrm{C}$ & 1.75564400 & -2.88695000 & 6.31764500 & $\mathrm{C}$ & 8.62739100 & 1.43548300 & -1.97915500 \\
\hline $\mathrm{H}$ & 2.10777000 & -3.87903100 & 6.62596400 & $\mathrm{H}$ & 9.04058100 & 2.44831900 & -1.92076400 \\
\hline $\mathrm{H}$ & 0.69789400 & -2.80477800 & 6.58556400 & $\mathrm{H}$ & 7.57879900 & 1.48380500 & -1.70004800 \\
\hline $\mathrm{H}$ & 2.31080800 & -2.13757600 & 6.89672900 & $\mathrm{H}$ & 8.67758500 & 1.11264000 & -3.02566600 \\
\hline $\mathrm{C}$ & 3.47478400 & -2.67925000 & 4.48121900 & $\mathrm{H}$ & 3.61594700 & -0.28473900 & 1.52138800 \\
\hline $\mathrm{H}$ & 3.66107700 & -2.55206200 & 3.40925000 & $\mathrm{H}$ & 3.46301800 & -1.16350100 & -0.70594400 \\
\hline $\mathrm{H}$ & 3.96696200 & -3.60594900 & 4.79701500 & $\mathrm{C}$ & 5.16578600 & 3.69732200 & -1.60911000 \\
\hline $\mathrm{H}$ & 3.97400900 & -1.86260700 & 5.01604400 & $\mathrm{H}$ & 4.97475000 & 4.10132600 & -0.61088800 \\
\hline $\mathrm{C}$ & -3.30859000 & -4.96386100 & 3.40954300 & $\mathrm{H}$ & 5.03327400 & 4.52105600 & -2.32518300 \\
\hline $\mathrm{H}$ & -3.12964700 & -6.04626700 & 3.41599300 & $\mathrm{H}$ & 6.21161600 & 3.38519500 & -1.65469400 \\
\hline $\mathrm{H}$ & -4.34721500 & -4.79692600 & 3.09804400 & $\mathrm{C}$ & 2.21343200 & 3.27523300 & -0.45053800 \\
\hline $\mathrm{H}$ & -3.20451500 & -4.60314100 & 4.43743100 & $\mathrm{H}$ & 2.93183600 & 3.67672500 & 0.27226100 \\
\hline $\mathrm{C}$ & -2.59921700 & -4.70841000 & 1.00378000 & $\mathrm{H}$ & 1.43940600 & 2.73451700 & 0.10071800 \\
\hline $\mathrm{H}$ & -1.94483100 & -4.17897200 & 0.30956700 & $\mathrm{H}$ & 1.74997100 & 4.13317100 & -0.95114600 \\
\hline $\mathrm{H}$ & -3.63645100 & -4.48505300 & 0.72576900 & $\mathrm{C}$ & 0.86670400 & 0.84466200 & -2.09668200 \\
\hline \multirow[t]{2}{*}{$\mathrm{H}$} & -2.45584400 & -5.78892900 & 0.88015200 & $\mathrm{H}$ & 0.30241500 & 1.26437600 & -2.93843400 \\
\hline & & & & $\mathrm{H}$ & 0.39125400 & 1.16416700 & -1.17019700 \\
\hline TS-7-RR & & & & $\mathrm{H}$ & 0.78158800 & -0.23741800 & -2.15706600 \\
\hline $\mathrm{C}$ & 2.88969400 & 2.39774300 & -1.46297600 & $\mathrm{C}$ & 3.09890800 & -0.28090300 & -4.12863600 \\
\hline $\mathrm{C}$ & 2.29884000 & 1.27036400 & -2.14821800 & $\mathrm{H}$ & 2.87331800 & 0.20667300 & -5.08829900 \\
\hline $\mathrm{C}$ & 3.30223700 & 0.76450300 & -3.07559300 & $\mathrm{H}$ & 2.26714100 & -0.94523600 & -3.89588000 \\
\hline $\mathrm{C}$ & 4.48114200 & 1.55048400 & -2.95242000 & $\mathrm{H}$ & 4.00106600 & -0.88284000 & -4.27117900 \\
\hline $\mathrm{C}$ & 4.24126800 & 2.56588300 & -1.94049500 & $\mathrm{C}$ & 5.66980800 & 1.44756300 & -3.86374100 \\
\hline Ir & 4.01271200 & 0.56393100 & -0.94288900 & $\mathrm{H}$ & 6.15301200 & 0.47169900 & -3.78244800 \\
\hline $\mathrm{N}$ & 6.03847500 & 0.41696500 & -0.05588100 & $\mathrm{H}$ & 6.41665600 & 2.20932900 & -3.63203300 \\
\hline $\mathrm{N}$ & 3.63871300 & 0.66130600 & 1.14416100 & $\mathrm{H}$ & 5.34999100 & 1.60094900 & -4.90325800 \\
\hline $\mathrm{H}$ & 2.69170300 & 1.03546200 & 1.32848600 & $\mathrm{H}$ & 6.06342200 & -0.28797800 & 1.96530400 \\
\hline $\mathrm{C}$ & 6.06943400 & 0.66242300 & 1.40962800 & $\mathrm{C}$ & 7.26479200 & 1.48134700 & 1.89768100 \\
\hline $\mathrm{C}$ & 4.75813600 & 1.41237500 & 1.78076600 & $\mathrm{C}$ & 7.63119000 & 2.67306500 & 1.25924800 \\
\hline S & 6.99991500 & -0.83101200 & -0.58701900 & $\mathrm{C}$ & 7.96708200 & 1.09748600 & 3.04582500 \\
\hline $\mathrm{O}$ & 6.86285700 & -0.88684600 & -2.05509700 & $\mathrm{C}$ & 8.67266100 & 3.45821300 & 1.75290100 \\
\hline $\mathrm{O}$ & 6.70134000 & -2.09980900 & 0.12348300 & $\mathrm{H}$ & 7.10412400 & 2.97536600 & 0.36122400 \\
\hline $\mathrm{C}$ & 8.77562500 & -0.45317000 & -0.24913300 & $\mathrm{C}$ & 9.01202800 & 1.87880800 & 3.54301200 \\
\hline $\mathrm{C}$ & 9.51697800 & -1.22554600 & 0.67400200 & $\mathrm{H}$ & 7.69342000 & 0.17930300 & 3.55872000 \\
\hline $\mathrm{C}$ & 9.40342500 & 0.50701000 & -1.07111800 & $\mathrm{C}$ & 9.36805300 & 3.06368900 & 2.89838500 \\
\hline $\mathrm{C}$ & 10.92347500 & -1.08651900 & 0.68614400 & $\mathrm{H}$ & 8.94323800 & 4.37917500 & 1.24214500 \\
\hline $\mathrm{C}$ & 10.80339100 & 0.64498600 & -1.02169100 & $\mathrm{H}$ & 9.54575800 & 1.56030500 & 4.43466800 \\
\hline $\mathrm{C}$ & 11.56091700 & -0.13446300 & -0.12898900 & $\mathrm{H}$ & 10.18059500 & 3.67416300 & 3.28345200 \\
\hline $\mathrm{C}$ & 8.91076800 & -2.15842400 & 1.70768700 & $\mathrm{H}$ & 4.78611000 & 2.38534100 & 1.28339400 \\
\hline
\end{tabular}




\begin{tabular}{|c|c|c|c|c|c|c|c|}
\hline $\mathrm{C}$ & 4.57024900 & 1.64986300 & 3.26966000 & $\mathrm{H}$ & -2.20066600 & 2.36391200 & 5.91885100 \\
\hline $\mathrm{C}$ & 4.60953600 & 0.60542100 & 4.20394300 & $\mathrm{H}$ & -2.58072800 & 4.50274900 & 4.82192400 \\
\hline $\mathrm{C}$ & 4.36492400 & 2.95487000 & 3.73520100 & $\mathrm{H}$ & -0.83187400 & 4.45312500 & 4.64925200 \\
\hline $\mathrm{C}$ & 4.46244900 & 0.86384200 & 5.56682100 & $\mathrm{C}$ & -2.53390200 & 1.66311600 & 3.83678500 \\
\hline $\mathrm{H}$ & 4.76547800 & -0.41842200 & 3.87442300 & $\mathrm{C}$ & -4.07154300 & 1.98364900 & 3.97755100 \\
\hline $\mathrm{C}$ & 4.20869700 & 3.21635300 & 5.09690300 & $\mathrm{C}$ & -2.56076700 & 0.15121600 & 4.03302400 \\
\hline $\mathrm{H}$ & 4.33697800 & 3.77715000 & 3.02399400 & $\mathrm{C}$ & -4.60324800 & 0.97114100 & 5.00169300 \\
\hline $\mathrm{C}$ & 4.26139900 & 2.16995500 & 6.01798700 & $\mathrm{H}$ & -4.25534200 & 3.02606800 & 4.25202200 \\
\hline $\mathrm{H}$ & 4.50563900 & 0.04251200 & 6.27602700 & $\mathrm{H}$ & -4.55342600 & 1.80929700 & 3.00870400 \\
\hline $\mathrm{H}$ & 4.05193000 & 4.23670900 & 5.43610200 & $\mathrm{H}$ & -5.66693200 & 0.74290200 & 4.86591200 \\
\hline $\mathrm{H}$ & 4.14677000 & 2.36894700 & 7.08001900 & $\mathrm{H}$ & -4.48783000 & 1.34208000 & 6.03066600 \\
\hline $\mathrm{N}$ & 1.60876300 & -2.52884700 & -1.26588500 & $\mathrm{C}$ & -2.05745200 & 2.38415300 & 2.57683300 \\
\hline $\mathrm{C}$ & 2.89482300 & -2.62598900 & -0.80861300 & $\mathrm{C}$ & -2.00420400 & 1.96609100 & 1.24443100 \\
\hline $\mathrm{C}$ & 0.97222800 & -2.82495100 & -2.49245400 & $\mathrm{C}$ & -1.99623100 & 4.69498300 & 1.88932000 \\
\hline $\mathrm{C}$ & -0.38475100 & -2.44494100 & -2.57850700 & $\mathrm{C}$ & -2.23351700 & 2.90886900 & 0.20326700 \\
\hline $\mathrm{C}$ & 1.54202000 & -3.53042000 & -3.56818000 & $\mathrm{C}$ & -2.25550300 & 4.26612300 & 0.58606900 \\
\hline $\mathrm{C}$ & -1.13489200 & -2.74180200 & -3.71151700 & $\mathrm{H}$ & -1.93849300 & 5.75781900 & 2.11219300 \\
\hline $\mathrm{H}$ & -0.83796200 & -1.92350800 & -1.74032100 & $\mathrm{H}$ & -2.48286500 & 5.01236600 & -0.16246800 \\
\hline $\mathrm{C}$ & 0.77625200 & -3.81500100 & -4.70025500 & $\mathrm{C}$ & -3.71130200 & -0.21949200 & 4.74958300 \\
\hline $\mathrm{H}$ & 2.56534500 & -3.87051400 & -3.53668100 & $\mathrm{C}$ & -3.92503400 & -1.53519100 & 5.13792200 \\
\hline $\mathrm{C}$ & -0.55964500 & -3.42400400 & -4.78712700 & $\mathrm{C}$ & -1.63388100 & -0.82760100 & 3.65432200 \\
\hline $\mathrm{H}$ & -2.17726800 & -2.43769400 & -3.74686500 & $\mathrm{C}$ & -2.97313400 & -2.48942000 & 4.79486100 \\
\hline $\mathrm{H}$ & 1.23842000 & -4.36045500 & -5.51882700 & $\mathrm{H}$ & -4.81274600 & -1.81533000 & 5.69967100 \\
\hline $\mathrm{H}$ & 0.94589400 & -2.16388600 & -0.55988100 & $\mathrm{C}$ & -1.82529000 & -2.17119100 & 4.04774900 \\
\hline $\mathrm{C}$ & 4.00319500 & -3.13114600 & -1.71048200 & $\mathrm{H}$ & -3.10668300 & -3.52109200 & 5.10731400 \\
\hline $\mathrm{H}$ & 3.86071800 & -4.19378500 & -1.93181500 & $\mathrm{O}$ & -1.79027900 & 0.62519300 & 0.96576100 \\
\hline $\mathrm{H}$ & 4.04821700 & -2.56866400 & -2.64263100 & $\mathrm{O}$ & -0.48515900 & -0.43612700 & 2.99365500 \\
\hline $\mathrm{H}$ & 4.96169000 & -3.00996700 & -1.20414800 & $\mathrm{P}$ & -0.33198700 & -0.09600100 & 1.37375500 \\
\hline $\mathrm{H}$ & -1.14490400 & -3.65483300 & -5.67244500 & $\mathrm{O}$ & -0.29828200 & -1.37662700 & 0.57707100 \\
\hline $\mathrm{C}$ & 2.88433900 & -3.19521400 & 0.65629000 & $\mathrm{O}$ & 0.78540600 & 0.90992700 & 1.29804300 \\
\hline $\mathrm{C}$ & 2.91763300 & -4.72708700 & 0.48168200 & $\mathrm{C}$ & -2.55916500 & 2.50908700 & -1.21276000 \\
\hline $\mathrm{C}$ & 3.97468500 & -2.65122500 & 1.61018500 & $\mathrm{C}$ & -3.54554700 & 1.50729500 & -1.45137000 \\
\hline $\mathrm{C}$ & 1.80289500 & -5.38633300 & -0.05853800 & $\mathrm{C}$ & -1.97504100 & 3.15526100 & -2.34894900 \\
\hline $\mathrm{C}$ & 4.06340200 & -5.48085100 & 0.80468900 & $\mathrm{C}$ & -3.82168000 & 1.10711000 & -2.76484100 \\
\hline $\mathrm{C}$ & 4.71364700 & -3.71864600 & 2.42652400 & $\mathrm{C}$ & -2.30891300 & 2.71762700 & -3.63602500 \\
\hline $\mathrm{H}$ & 3.49014600 & -1.95951000 & 2.31143000 & $\mathrm{C}$ & -3.20737500 & 1.67739000 & -3.87560100 \\
\hline $\mathrm{C}$ & 1.82822700 & -6.75443400 & -0.32420800 & $\mathrm{H}$ & -4.57033600 & 0.33524500 & -2.92730900 \\
\hline $\mathrm{H}$ & 0.90288400 & -4.82192700 & -0.27568900 & $\mathrm{H}$ & -1.85422400 & 3.21694700 & -4.48667900 \\
\hline $\mathrm{C}$ & 4.07411200 & -6.85711100 & 0.54018600 & $\mathrm{C}$ & -0.87842000 & -3.31062700 & 3.79834700 \\
\hline $\mathrm{C}$ & 5.23308200 & -4.82010900 & 1.49790100 & $\mathrm{C}$ & 0.23952000 & -3.48794500 & 4.64174000 \\
\hline $\mathrm{H}$ & 5.54628100 & -3.24692300 & 2.96294900 & $\mathrm{C}$ & -1.22044400 & -4.32349900 & 2.86838800 \\
\hline $\mathrm{C}$ & 2.97750100 & -7.49428000 & -0.03706700 & $\mathrm{C}$ & 0.98325900 & -4.67409600 & 4.55695100 \\
\hline $\mathrm{H}$ & 0.95356200 & -7.23817400 & -0.75090600 & $\mathrm{C}$ & -0.44869800 & -5.48813000 & 2.82755800 \\
\hline $\mathrm{H}$ & 4.96023900 & -7.43262700 & 0.79966500 & $\mathrm{C}$ & 0.64348300 & -5.70182700 & 3.67656300 \\
\hline $\mathrm{H}$ & 5.92675500 & -4.37182200 & 0.77411600 & $\mathrm{H}$ & 1.82888600 & -4.80396600 & 5.22561300 \\
\hline $\mathrm{H}$ & 3.01202600 & -8.56110200 & -0.24265100 & $\mathrm{H}$ & -0.71828600 & -6.27283800 & 2.12364800 \\
\hline $\mathrm{H}$ & 1.90428700 & -2.94006200 & 1.07109200 & $\mathrm{C}$ & -1.05615200 & 4.38331200 & -2.27620300 \\
\hline $\mathrm{H}$ & 4.72564000 & -2.10731900 & 1.03640200 & $\mathrm{H}$ & -0.66459100 & 4.46510800 & -1.25905800 \\
\hline $\mathrm{H}$ & 4.04475600 & -4.15492700 & 3.17953500 & $\mathrm{C}$ & -3.53895900 & 1.20993700 & -5.28604200 \\
\hline $\mathrm{H}$ & 5.80076400 & -5.57276900 & 2.05856900 & $\mathrm{H}$ & -4.26296300 & 0.38876100 & -5.19033600 \\
\hline $\mathrm{C}$ & -1.75092700 & 2.45848100 & 4.92478400 & $\mathrm{C}$ & -4.44675200 & 0.90642100 & -0.36696100 \\
\hline $\mathrm{C}$ & -1.90706800 & 3.73995600 & 2.89492400 & $\mathrm{H}$ & -4.11186800 & 1.25695600 & 0.60851500 \\
\hline $\mathrm{C}$ & -1.75489200 & 3.91795500 & 4.39468300 & $\mathrm{C}$ & 0.15889600 & 4.33494500 & -3.22578800 \\
\hline $\mathrm{H}$ & -0.72648600 & 2.07426100 & 4.97914100 & $\mathrm{H}$ & 0.80843700 & 5.19843700 & -3.03444900 \\
\hline
\end{tabular}




\begin{tabular}{|c|c|c|c|c|c|c|c|}
\hline $\mathrm{H}$ & -0.13713700 & 4.39133500 & -4.27902800 & $\mathrm{C}$ & 5.43389800 & -3.99378000 & -2.88257200 \\
\hline $\mathrm{H}$ & 0.75301100 & 3.42841200 & -3.09386200 & $\mathrm{C}$ & 4.23566300 & -3.87246500 & -3.61292400 \\
\hline $\mathrm{C}$ & -1.85645100 & 5.66910700 & -2.59464500 & $\mathrm{C}$ & 3.26147700 & -4.81666200 & -3.05789900 \\
\hline $\mathrm{H}$ & -1.23361900 & 6.56077000 & -2.44896300 & $\mathrm{C}$ & 3.93108800 & -5.57283100 & -2.02755000 \\
\hline $\mathrm{H}$ & -2.75217900 & 5.77366600 & -1.97478900 & $\mathrm{C}$ & 5.24470900 & -5.01175400 & -1.84670800 \\
\hline $\mathrm{H}$ & -2.19021700 & 5.65935900 & -3.63918900 & Ir & 3.68069900 & -3.51236800 & -1.33645800 \\
\hline $\mathrm{C}$ & -2.30110000 & 0.65146800 & -6.01378900 & $\mathrm{~N}$ & 4.60948200 & -1.95850600 & -0.10616600 \\
\hline $\mathrm{H}$ & -2.57453200 & 0.26764900 & -7.00445800 & $\mathrm{~N}$ & 2.28942200 & -1.88322000 & -1.37037800 \\
\hline $\mathrm{H}$ & -1.83951900 & -0.16386300 & -5.44592800 & $\mathrm{H}$ & 1.50759400 & -1.95869500 & -2.03106300 \\
\hline $\mathrm{H}$ & -1.54108200 & 1.42907100 & -6.15796200 & $\mathrm{C}$ & 4.04458500 & -0.59198400 & -0.31160900 \\
\hline $\mathrm{C}$ & -4.21148400 & 2.32022700 & -6.11645600 & $\mathrm{C}$ & 3.09664100 & -0.64719200 & -1.55046100 \\
\hline $\mathrm{H}$ & -4.49949500 & 1.94423900 & -7.10584100 & $\mathrm{~S}$ & 4.72440600 & -2.37214300 & 1.49574000 \\
\hline $\mathrm{H}$ & -3.53385700 & 3.16903000 & -6.26804200 & $\mathrm{O}$ & 5.33728700 & -3.71391400 & 1.59172500 \\
\hline $\mathrm{H}$ & -5.11155100 & 2.69672300 & -5.61820200 & $\mathrm{O}$ & 3.42395500 & -2.22058000 & 2.2025050 \\
\hline $\mathrm{C}$ & -5.89394300 & 1.41747200 & -0.53843600 & $\mathrm{C}$ & 5.94213900 & -1.22885600 & 2.28090400 \\
\hline $\mathrm{H}$ & -6.33064500 & 1.08539100 & -1.48772700 & $\mathrm{C}$ & 5.55588500 & -0.30472100 & 3.27665700 \\
\hline $\mathrm{H}$ & -5.93319700 & 2.51262800 & -0.51818200 & $\mathrm{C}$ & 7.30231900 & -1.42532100 & 1.95019100 \\
\hline $\mathrm{H}$ & -6.52967000 & 1.03861700 & 0.27169300 & $\mathrm{C}$ & 6.56758000 & 0.36666600 & 4.00082100 \\
\hline $\mathrm{C}$ & -4.40735200 & -0.63267000 & -0.33073400 & $\mathrm{C}$ & 8.28974200 & -0.72685100 & 2.67150600 \\
\hline $\mathrm{H}$ & -5.01371400 & -1.00319900 & 0.50484900 & $\mathrm{C}$ & 7.92341100 & 0.18764400 & 3.67619200 \\
\hline $\mathrm{H}$ & -3.38463600 & -0.99275700 & -0.19423600 & $\mathrm{C}$ & 4.11847000 & 0.06188900 & 3.59697000 \\
\hline $\mathrm{H}$ & -4.81557100 & -1.07330600 & -1.24878500 & $\mathrm{H}$ & 3.72226700 & -0.52980300 & 4.43201800 \\
\hline $\mathrm{C}$ & 0.63360200 & -2.44930100 & 5.68922700 & $\mathrm{H}$ & 3.44949800 & -0.10871100 & 2.76026000 \\
\hline $\mathrm{H}$ & -0.10379200 & -1.64309900 & 5.65774900 & $\mathrm{H}$ & 4.05506700 & 1.11707300 & 3.87684500 \\
\hline $\mathrm{C}$ & 1.35360100 & -7.05317300 & 3.65143100 & $\mathrm{C}$ & 6.18255800 & 1.27250700 & 5.15847700 \\
\hline $\mathrm{H}$ & 1.49323900 & -7.32306500 & 2.59616000 & $\mathrm{H}$ & 6.98254700 & 1.33597900 & 5.89938900 \\
\hline $\mathrm{C}$ & -2.43448400 & -4.20695000 & 1.94552000 & $\mathrm{H}$ & 5.29794700 & 0.90006100 & 5.68086200 \\
\hline $\mathrm{H}$ & -2.85626100 & -3.20588500 & 2.07272800 & $\mathrm{H}$ & 5.95683500 & 2.29904400 & 4.83430600 \\
\hline $\mathrm{C}$ & 2.73856800 & -7.06059100 & 4.31286700 & $\mathrm{C}$ & 8.98980300 & 1.00353400 & 4.38510300 \\
\hline $\mathrm{H}$ & 3.21481100 & -8.03824000 & 4.17524600 & $\mathrm{H}$ & 9.36859400 & 0.50091900 & 5.28695700 \\
\hline $\mathrm{H}$ & 2.67308500 & -6.87925400 & 5.39286100 & $\mathrm{H}$ & 8.61016900 & 1.97987800 & 4.69513400 \\
\hline $\mathrm{H}$ & 3.39871900 & -6.30295700 & 3.87855300 & $\mathrm{H}$ & 9.84815100 & 1.19158600 & 3.73601800 \\
\hline $\mathrm{C}$ & 0.45893200 & -8.13755900 & 4.29105600 & $\mathrm{C}$ & 9.75549200 & -0.98706200 & 2.37297700 \\
\hline $\mathrm{H}$ & 0.93231900 & -9.12485600 & 4.21928100 & $\mathrm{H}$ & 10.38659900 & -0.79365300 & 3.2432170 \\
\hline $\mathrm{H}$ & -0.51842300 & -8.19276600 & 3.79999900 & $\mathrm{H}$ & 10.13041100 & -0.35928600 & $1.5513540 \mathrm{C}$ \\
\hline $\mathrm{H}$ & 0.28598400 & -7.92028900 & 5.35242400 & $\mathrm{H}$ & 9.92645700 & -2.02689000 & 2.08352000 \\
\hline $\mathrm{C}$ & 0.60473200 & -3.02653800 & 7.11772800 & $\mathrm{C}$ & 7.75780000 & -2.33283800 & 0.82453300 \\
\hline $\mathrm{H}$ & 1.34362400 & -3.82578400 & 7.25072700 & $\mathrm{H}$ & 8.57861300 & -1.86135400 & 0.27415100 \\
\hline $\mathrm{H}$ & -0.38129200 & -3.44125800 & 7.35548500 & $\mathrm{H}$ & 6.95624500 & -2.53900700 & 0.12053200 \\
\hline $\mathrm{H}$ & 0.82922400 & -2.24245600 & 7.85165300 & $\mathrm{H}$ & 8.11821400 & -3.29555000 & 1.20770800 \\
\hline $\mathrm{C}$ & 1.99860900 & -1.81703300 & 5.36993100 & $\mathrm{H}$ & 1.83037700 & -1.87380800 & -0.45809100 \\
\hline $\mathrm{H}$ & 1.97775600 & -1.34254600 & 4.38356300 & $\mathrm{H}$ & 2.83874700 & -3.95106100 & -0.05969800 \\
\hline $\mathrm{H}$ & 2.80204000 & -2.56507900 & 5.38016700 & $\mathrm{C}$ & 6.32568900 & -5.57374100 & -0.96788200 \\
\hline $\mathrm{H}$ & 2.24641000 & -1.04550200 & 6.10814800 & $\mathrm{H}$ & 7.09375800 & -4.82704600 & -0.75434400 \\
\hline $\mathrm{C}$ & -3.53065500 & -5.22289200 & 2.32435100 & $\mathrm{H}$ & 6.81605100 & -6.43351900 & -1.44871100 \\
\hline $\mathrm{H}$ & -3.17434500 & -6.25421500 & 2.21188600 & $\mathrm{H}$ & 5.92338000 & -5.89458300 & -0.00474900 \\
\hline $\mathrm{H}$ & -4.40814600 & -5.10012000 & 1.67742500 & $\mathrm{C}$ & 6.71699700 & -3.25203500 & -3.11439600 \\
\hline $\mathrm{H}$ & -3.85631400 & -5.09520800 & 3.36257400 & $\mathrm{H}$ & 6.56866200 & -2.36598000 & -3.73849800 \\
\hline $\mathrm{C}$ & -2.05330100 & -4.34187400 & 0.46080100 & $\mathrm{H}$ & 7.44909900 & -3.89462700 & -3.62339500 \\
\hline $\mathrm{H}$ & -1.32064600 & -3.57693500 & 0.19282000 & $\mathrm{H}$ & 7.16895300 & -2.92979800 & -2.17170700 \\
\hline $\mathrm{H}$ & -2.93913300 & -4.20384000 & -0.17186700 & $\mathrm{C}$ & 3.96271400 & -2.96704500 & -4.77864800 \\
\hline \multirow[t]{2}{*}{$\mathrm{H}$} & -1.63878100 & -5.33182200 & 0.23270000 & $\mathrm{H}$ & 4.02573600 & -3.51895600 & -5.7267390 \\
\hline & & & & $\mathrm{H}$ & 4.67701900 & -2.13985600 & -4.83266100 \\
\hline $13-R S$ & & & & $\mathrm{H}$ & 2.95598600 & -2.53914000 & -4.72626400 \\
\hline
\end{tabular}




\begin{tabular}{|c|c|c|c|c|c|c|c|}
\hline $\mathrm{C}$ & 1.92359600 & -5.12278500 & -3.66239000 & $\mathrm{C}$ & 1.56381000 & -5.10040400 & 5.61070800 \\
\hline $\mathrm{H}$ & 1.26648400 & -5.60486500 & -2.93435100 & $\mathrm{C}$ & -0.77555500 & -4.76460000 & 4.60850500 \\
\hline $\mathrm{H}$ & 2.02286200 & -5.79714400 & -4.52439100 & $\mathrm{C}$ & 4.35578300 & -5.23593000 & 5.28435700 \\
\hline $\mathrm{H}$ & 1.41041200 & -4.22317000 & -4.01151600 & $\mathrm{H}$ & 3.98563900 & -4.70797200 & 3.23663200 \\
\hline $\mathrm{C}$ & 3.40118800 & -6.79349100 & -1.33077000 & $\mathrm{C}$ & 2.40591300 & -5.40773300 & 6.69060700 \\
\hline $\mathrm{H}$ & 3.68419000 & -7.70087100 & -1.88336800 & $\mathrm{C}$ & 0.07640000 & -4.91884300 & 5.87480300 \\
\hline $\mathrm{H}$ & 2.31043600 & -6.77541600 & -1.25772000 & $\mathrm{C}$ & 3.78723200 & -5.48632100 & 6.53551500 \\
\hline $\mathrm{H}$ & 3.80934000 & -6.87805900 & -0.31963800 & $\mathrm{H}$ & 5.43375800 & -5.26032300 & 5.15153700 \\
\hline $\mathrm{H}$ & 3.40211000 & -0.30062500 & 0.53206000 & $\mathrm{H}$ & 1.96260700 & -5.57422100 & 7.67078600 \\
\hline $\mathrm{C}$ & 5.11809900 & 0.47710400 & -0.48116200 & $\mathrm{H}$ & -0.29857300 & -5.74473900 & 6.49375300 \\
\hline $\mathrm{C}$ & 6.22526500 & 0.25971000 & -1.31210700 & $\mathrm{H}$ & 4.41704700 & -5.72501200 & 7.38878900 \\
\hline $\mathrm{C}$ & 4.99512800 & 1.71621700 & 0.15780100 & $\mathrm{H}$ & 1.82160000 & -3.90985500 & 2.46097900 \\
\hline $\mathrm{C}$ & 7.18118300 & 1.25784100 & -1.50323600 & $\mathrm{H}$ & -0.04318600 & -4.01370600 & 6.48879300 \\
\hline $\mathrm{H}$ & 6.34239200 & -0.70596900 & -1.79259300 & $\mathrm{C}$ & -6.33062400 & -4.00012500 & -0.38467600 \\
\hline $\mathrm{C}$ & 5.95165900 & 2.71670100 & -0.02714400 & $\mathrm{C}$ & -5.73492700 & -4.45670000 & -2.61781700 \\
\hline $\mathrm{H}$ & 4.14033400 & 1.90103100 & 0.80358600 & $\mathrm{C}$ & -6.92125700 & -4.54793700 & -1.69023200 \\
\hline $\mathrm{C}$ & 7.04778500 & 2.49164900 & -0.86062500 & $\mathrm{H}$ & -6.36626500 & -2.90491600 & -0.40253400 \\
\hline $\mathrm{H}$ & 8.03456500 & 1.07125800 & -2.15096100 & $\mathrm{H}$ & -6.85772400 & -4.33275800 & 0.51406700 \\
\hline $\mathrm{H}$ & 5.83908500 & 3.67086200 & 0.48130500 & $\mathrm{H}$ & -7.25619300 & -5.58908200 & -1.57295300 \\
\hline $\mathrm{H}$ & 7.79426600 & 3.26830300 & -1.00607300 & $\mathrm{H}$ & -7.78482000 & -3.97419100 & -2.04559700 \\
\hline $\mathrm{H}$ & 3.71951800 & -0.82072400 & -2.43382300 & $\mathrm{C}$ & -4.82066400 & -4.45243900 & -0.39517000 \\
\hline $\mathrm{C}$ & 2.28544900 & 0.61868100 & -1.78978300 & $\mathrm{C}$ & -4.72127200 & -5.86663400 & 0.25327700 \\
\hline $\mathrm{C}$ & 0.97746600 & 0.77363500 & -1.31425900 & $\mathrm{C}$ & -4.10936300 & -3.61731400 & 0.66562700 \\
\hline $\mathrm{C}$ & 2.85875900 & 1.66526200 & -2.53015900 & $\mathrm{C}$ & -4.87934700 & -5.59279300 & 1.77540300 \\
\hline $\mathrm{C}$ & 0.26461200 & 1.94933600 & -1.56302400 & $\mathrm{H}$ & -5.47501400 & -6.56054300 & -0.13429600 \\
\hline $\mathrm{H}$ & 0.47574100 & -0.03674500 & -0.79848900 & $\mathrm{H}$ & -3.73433400 & -6.29159200 & 0.04326300 \\
\hline $\mathrm{C}$ & 2.15126300 & 2.84158000 & -2.77254700 & $\mathrm{H}$ & -4.29236400 & -6.29289900 & 2.38285900 \\
\hline $\mathrm{H}$ & 3.86974400 & 1.55849000 & -2.91427100 & $\mathrm{H}$ & -5.92346300 & -5.70244500 & 2.09878000 \\
\hline $\mathrm{C}$ & 0.84952100 & 2.98825400 & -2.28718900 & $\mathrm{C}$ & -4.53087400 & -4.36878800 & -1.89673700 \\
\hline $\mathrm{H}$ & -0.75413700 & 2.03762000 & -1.19700700 & $\mathrm{C}$ & -3.32141700 & -4.20753600 & -2.59089800 \\
\hline $\mathrm{H}$ & 2.61414300 & 3.63891000 & -3.34840400 & $\mathrm{C}$ & -5.75327800 & -4.41600400 & -4.00491500 \\
\hline $\mathrm{H}$ & 0.29086000 & 3.89929500 & -2.48604200 & $\mathrm{C}$ & -3.31884900 & -4.13995500 & -4.00489500 \\
\hline $\mathrm{N}$ & 0.03977300 & -5.79308500 & 1.38190200 & $\mathrm{C}$ & -4.54681900 & -4.25596800 & -4.67856600 \\
\hline $\mathrm{C}$ & 0.95580700 & -5.84582500 & 2.30966700 & $\mathrm{H}$ & -6.68953500 & -4.48125300 & -4.55353600 \\
\hline C & -0.54593600 & -6.91138900 & 0.69773800 & $\mathrm{H}$ & -4.54026700 & -4.19599900 & -5.76300200 \\
\hline $\mathrm{C}$ & -0.75241800 & -6.82105400 & -0.68055000 & $\mathrm{C}$ & -4.38808300 & -4.16291700 & 1.92464900 \\
\hline $\mathrm{C}$ & -1.00173200 & -8.02311700 & 1.41797300 & $\mathrm{C}$ & -4.22888300 & -3.38508400 & 3.06982800 \\
\hline $\mathrm{C}$ & -1.37551900 & -7.87920900 & -1.34351400 & $\mathrm{C}$ & -3.45080900 & -2.39029700 & 0.57118000 \\
\hline $\mathrm{H}$ & -0.46497300 & -5.92236600 & -1.21189700 & $\mathrm{C}$ & -3.82134100 & -2.05501100 & 2.92425400 \\
\hline $\mathrm{C}$ & -1.62008400 & -9.07406200 & 0.74335300 & $\mathrm{H}$ & -4.46880500 & -3.77852500 & 4.05509000 \\
\hline $\mathrm{H}$ & -0.89534300 & -8.05416300 & 2.49746500 & $\mathrm{C}$ & -3.40634300 & -1.52726700 & 1.68656700 \\
\hline $\mathrm{C}$ & -1.80035500 & -9.00776200 & -0.64005900 & $\mathrm{H}$ & -3.79618200 & -1.40353200 & 3.79364500 \\
\hline $\mathrm{H}$ & -1.53801400 & -7.81045700 & -2.41290300 & $\mathrm{O}$ & -2.13263200 & -4.19772900 & -1.88672300 \\
\hline $\mathrm{H}$ & -1.97287500 & -9.93568100 & 1.30262500 & $\mathrm{O}$ & -2.82819900 & -2.03442700 & -0.61589400 \\
\hline $\mathrm{H}$ & -0.33163200 & -4.85820600 & 1.03680700 & $\mathrm{P}$ & -1.46996800 & -2.92299800 & -1.03467000 \\
\hline $\mathrm{C}$ & 1.70662300 & -7.11126700 & 2.58201500 & $\mathrm{O}$ & -0.86868500 & -3.55613900 & 0.20413200 \\
\hline $\mathrm{H}$ & 1.44294300 & -7.51284300 & 3.56703000 & $\mathrm{O}$ & -0.63285100 & -2.06906100 & -1.93229300 \\
\hline $\mathrm{H}$ & 1.54316600 & -7.87209600 & 1.81975400 & $\mathrm{C}$ & -2.11092900 & -3.93817100 & -4.88192000 \\
\hline $\mathrm{H}$ & 2.77048000 & -6.86194200 & 2.64324900 & $\mathrm{C}$ & -1.48557100 & -5.05668300 & -5.48698400 \\
\hline $\mathrm{H}$ & -2.28734700 & -9.82462800 & -1.16476500 & $\mathrm{C}$ & -1.73072500 & -2.63054500 & -5.26631300 \\
\hline $\mathrm{C}$ & 1.26318800 & -4.59140500 & 3.12328500 & $\mathrm{C}$ & -0.49176800 & -4.84036400 & -6.44701400 \\
\hline $\mathrm{C}$ & 2.13904100 & -4.88847300 & 4.34261300 & $\mathrm{C}$ & -0.72742900 & -2.47181300 & -6.2335330 \\
\hline $\mathrm{C}$ & -0.05048500 & -3.90390900 & 3.56854100 & $\mathrm{C}$ & -0.09966000 & -3.55815300 & -6.84429500 \\
\hline $\mathrm{C}$ & 3.53527700 & -4.93619000 & 4.19916400 & $\mathrm{H}$ & -0.01767800 & -5.69622400 & -6.92285000 \\
\hline
\end{tabular}




\begin{tabular}{|c|c|c|c|c|c|c|c|}
\hline $\mathrm{H}$ & -0.45012100 & -1.46547300 & -6.52801400 & $\mathrm{H}$ & -6.42438700 & 2.03811500 & 0.48643900 \\
\hline $\mathrm{C}$ & -3.04826100 & -0.07104800 & 1.57773700 & $\mathrm{H}$ & -6.53482100 & 0.72146600 & 1.66491500 \\
\hline $\mathrm{C}$ & -3.88419300 & 0.80388300 & 0.83100400 & $\mathrm{H}$ & -7.28007400 & 0.54836000 & 0.06312900 \\
\hline $\mathrm{C}$ & -1.95874100 & 0.47393600 & 2.29758800 & $\mathrm{C}$ & -4.95972500 & 0.60390100 & -1.46180600 \\
\hline $\mathrm{C}$ & -3.61358200 & 2.17643200 & 0.83500700 & $\mathrm{H}$ & -4.05597700 & 0.12690800 & -1.85237400 \\
\hline $\mathrm{C}$ & -1.73838800 & 1.85727200 & 2.27063700 & $\mathrm{H}$ & -4.89965300 & 1.67872300 & -1.67219200 \\
\hline $\mathrm{C}$ & -2.55437100 & 2.73317900 & 1.55728900 & $\mathrm{H}$ & -5.82303700 & 0.20667100 & -2.01010000 \\
\hline $\mathrm{H}$ & -4.26806900 & 2.83451500 & 0.26853200 & $\mathrm{C}$ & -0.94184500 & -0.00899400 & 4.58185500 \\
\hline $\mathrm{H}$ & -0.89920700 & 2.26609600 & 2.82941300 & $\mathrm{H}$ & -0.58003400 & 1.01491400 & 4.73258700 \\
\hline $\mathrm{C}$ & -2.45790800 & -1.39000700 & -4.74386000 & $\mathrm{H}$ & -0.26751600 & -0.67788400 & 5.13145500 \\
\hline $\mathrm{H}$ & -2.94933900 & -1.66182200 & -3.80506400 & $\mathrm{H}$ & -1.93586800 & -0.07809900 & 5.03929100 \\
\hline $\mathrm{C}$ & 0.92589900 & -3.40569600 & -7.96492300 & $\mathrm{C}$ & 0.42662100 & -0.33624200 & 2.46648100 \\
\hline $\mathrm{H}$ & 1.67957500 & -4.19394900 & -7.81926700 & $\mathrm{H}$ & 0.39618000 & -0.68678300 & 1.43039300 \\
\hline $\mathrm{C}$ & -1.93384000 & -6.49025800 & -5.19146100 & $\mathrm{H}$ & 1.12997600 & -0.97429800 & 3.01098500 \\
\hline $\mathrm{H}$ & -2.49222000 & -6.47475200 & -4.24827000 & $\mathrm{H}$ & 0.82907200 & 0.68383300 & 2.46573800 \\
\hline $\mathrm{C}$ & -1.52669000 & -0.20679800 & -4.43252200 & $\mathrm{H}$ & -1.00119800 & -5.75260100 & 4.17958100 \\
\hline $\mathrm{H}$ & -2.10761400 & 0.60539300 & -3.97899200 & $\mathrm{H}$ & -1.74397500 & -4.31755100 & 4.85772700 \\
\hline $\mathrm{H}$ & -1.05771000 & 0.20214300 & -5.33603700 & $\mathrm{H}$ & -0.69051900 & -3.67819400 & 2.71284900 \\
\hline $\mathrm{H}$ & -0.75281900 & -0.50121200 & -3.72216400 & $\mathrm{H}$ & 0.22438000 & -2.94262500 & 4.01500800 \\
\hline $\mathrm{C}$ & -3.55589900 & -0.95296100 & -5.73830800 & & & & \\
\hline $\mathrm{H}$ & -4.09228000 & -0.07755300 & -5.35195300 & TS-7-RS & & & \\
\hline $\mathrm{H}$ & -4.28877600 & -1.74715300 & -5.91334500 & $\mathrm{C}$ & 4.58853000 & -3.51227500 & -2.70898400 \\
\hline $\mathrm{H}$ & -3.11846300 & -0.67920600 & -6.70670800 & $\mathrm{C}$ & 3.19676500 & -3.52951000 & -3.06511700 \\
\hline $\mathrm{C}$ & 1.66453200 & -2.05901400 & -7.97367500 & $\mathrm{C}$ & 2.60927000 & -4.75442000 & -2.56086400 \\
\hline $\mathrm{H}$ & 2.45846900 & -2.06933100 & -8.72943800 & $\mathrm{C}$ & 3.67370900 & -5.50634500 & -1.93629500 \\
\hline $\mathrm{H}$ & 2.12146100 & -1.83859700 & -7.00300800 & $\mathrm{C}$ & 4.88157000 & -4.74158900 & -1.98493900 \\
\hline $\mathrm{H}$ & 0.99089700 & -1.23062400 & -8.22285300 & Ir & 3.33011300 & -3.53982500 & -0.84602100 \\
\hline $\mathrm{C}$ & 0.26338600 & -3.65719500 & -9.33666800 & $\mathrm{~N}$ & 4.48346800 & -2.00524900 & 0.27403900 \\
\hline $\mathrm{H}$ & 1.00529300 & -3.61212900 & -10.14377200 & $\mathrm{~N}$ & 1.93099300 & -1.95545200 & -0.59069200 \\
\hline $\mathrm{H}$ & -0.50393400 & -2.89967800 & -9.53744200 & $\mathrm{H}$ & 1.12693100 & -2.00548200 & -1.24512200 \\
\hline $\mathrm{H}$ & -0.22094400 & -4.63894400 & -9.37256800 & $\mathrm{C}$ & 3.77010600 & -0.70092500 & 0.38253100 \\
\hline $\mathrm{C}$ & -2.89377600 & -7.00401800 & -6.28581800 & $\mathrm{C}$ & 2.70746800 & -0.69348400 & -0.75491600 \\
\hline $\mathrm{H}$ & -2.39602700 & -7.01673700 & -7.26308500 & S & 5.24464700 & -2.48107700 & 1.67368500 \\
\hline $\mathrm{H}$ & -3.78210800 & -6.37160700 & -6.36934100 & $\mathrm{O}$ & 5.76283000 & -3.84690200 & 1.44083800 \\
\hline $\mathrm{H}$ & -3.22571400 & -8.02595500 & -6.06234600 & $\mathrm{O}$ & 4.35425700 & -2.32173900 & 2.84736900 \\
\hline $\mathrm{C}$ & -0.76735000 & -7.48169400 & -5.02641900 & $\mathrm{C}$ & 6.71953300 & -1.42271200 & 2.01522800 \\
\hline $\mathrm{H}$ & -1.14925900 & -8.46909400 & -4.73732900 & $\mathrm{C}$ & 6.73322200 & -0.53781300 & 3.11837000 \\
\hline $\mathrm{H}$ & -0.05568600 & -7.15167800 & -4.26379700 & $\mathrm{C}$ & 7.88132800 & -1.65493400 & 1.24834800 \\
\hline $\mathrm{H}$ & -0.21218700 & -7.61985300 & -5.96122400 & $\mathrm{C}$ & 7.96731000 & 0.04331700 & 3.49191700 \\
\hline $\mathrm{C}$ & -5.10965800 & 0.33114200 & 0.04728700 & $\mathrm{C}$ & 9.09230200 & -1.04703100 & 1.62828200 \\
\hline $\mathrm{H}$ & -5.20020100 & -0.75016200 & 0.16944100 & $\mathrm{C}$ & 9.13056800 & -0.17855500 & 2.73404400 \\
\hline $\mathrm{C}$ & -2.31088900 & 4.23696500 & 1.59462800 & $\mathrm{C}$ & 5.51167000 & -0.12223400 & 3.91886900 \\
\hline $\mathrm{H}$ & -1.43141600 & 4.40425500 & 2.23177000 & $\mathrm{H}$ & 5.37767300 & -0.74833800 & 4.80946800 \\
\hline $\mathrm{C}$ & -0.97988300 & -0.38821600 & 3.08866500 & $\mathrm{H}$ & 4.59186800 & -0.19874300 & 3.34984700 \\
\hline $\mathrm{H}$ & -1.31947000 & -1.42328700 & 3.02313900 & $\mathrm{H}$ & 5.62069100 & 0.91447300 & 4.24738900 \\
\hline $\mathrm{C}$ & -1.98703500 & 4.81321500 & 0.20362200 & $\mathrm{C}$ & 8.04055100 & 0.89992400 & 4.74446500 \\
\hline $\mathrm{H}$ & -1.79392100 & 5.89122100 & 0.26693000 & $\mathrm{H}$ & 9.04643600 & 0.90259100 & 5.16939400 \\
\hline $\mathrm{H}$ & -2.82127800 & 4.66496400 & -0.49264400 & $\mathrm{H}$ & 7.37027000 & 0.52827900 & 5.52338200 \\
\hline $\mathrm{H}$ & -1.10094000 & 4.33334900 & -0.22492100 & $\mathrm{H}$ & 7.76383500 & 1.94702400 & 4.55383100 \\
\hline $\mathrm{C}$ & -3.49388100 & 4.98669100 & 2.23791700 & $\mathrm{C}$ & 10.41800800 & 0.54288200 & 3.08973200 \\
\hline $\mathrm{H}$ & -3.27417100 & 6.05811300 & 2.32169100 & $\mathrm{H}$ & 11.01064800 & -0.00778200 & 3.83450300 \\
\hline $\mathrm{H}$ & -3.70809700 & 4.60191300 & 3.24113200 & $\mathrm{H}$ & 10.22073900 & 1.53448000 & 3.50436600 \\
\hline $\mathrm{H}$ & -4.40558700 & 4.87963800 & 1.63808300 & $\mathrm{H}$ & 11.05420500 & 0.68791700 & 2.21400500 \\
\hline $\mathrm{C}$ & -6.41046400 & 0.94772600 & 0.59980300 & $\mathrm{C}$ & 10.35993200 & -1.35488300 & 0.85127700 \\
\hline
\end{tabular}




\begin{tabular}{|c|c|c|c|c|c|c|c|}
\hline $\mathrm{H}$ & 11.24709300 & -1.28803300 & 1.48517200 & $\mathrm{C}$ & 1.95179400 & -5.10810800 & 1.71407900 \\
\hline $\mathrm{H}$ & 10.51026300 & -0.66543700 & 0.00741400 & $\mathrm{C}$ & 0.37388600 & -6.83042000 & 0.69545800 \\
\hline $\mathrm{H}$ & 10.34347500 & -2.36649200 & 0.43903600 & $\mathrm{C}$ & -0.65874100 & -6.84338500 & -0.26428700 \\
\hline $\mathrm{C}$ & 7.87361000 & -2.50564400 & -0.00207100 & $\mathrm{C}$ & 0.84656500 & -8.05835200 & 1.19565700 \\
\hline $\mathrm{H}$ & 8.47200900 & -2.02878000 & -0.78655000 & $\mathrm{C}$ & -1.16970000 & -8.05094900 & -0.73348400 \\
\hline $\mathrm{H}$ & 6.86670200 & -2.64650100 & -0.38172800 & $\mathrm{H}$ & -1.05719300 & -5.89996500 & -0.62357300 \\
\hline $\mathrm{H}$ & 8.29463400 & -3.50128200 & 0.18114000 & $\mathrm{C}$ & 0.33493000 & -9.25924200 & 0.70320700 \\
\hline $\mathrm{H}$ & 1.50863400 & -1.99317000 & 0.33854900 & $\mathrm{H}$ & 1.57989400 & -8.08601700 & 1.98920500 \\
\hline $\mathrm{H}$ & 2.55913500 & -4.27820500 & 0.64630900 & $\mathrm{C}$ & -0.66914700 & -9.26859000 & -0.26581600 \\
\hline $\mathrm{C}$ & 6.23648000 & -5.24342500 & -1.56616800 & $\mathrm{H}$ & -1.96496000 & -8.03414200 & -1.47319800 \\
\hline $\mathrm{H}$ & 7.02348300 & -4.54903200 & -1.86851600 & $\mathrm{H}$ & 0.71546400 & -10.19546500 & 1.1032450 \\
\hline $\mathrm{H}$ & 6.44906800 & -6.20919400 & -2.04281800 & $\mathrm{H}$ & 0.09658400 & -4.83123400 & 0.94099900 \\
\hline $\mathrm{H}$ & 6.30362400 & -5.36279600 & -0.48191700 & $\mathrm{C}$ & 3.09810800 & -6.08930300 & 1.87306500 \\
\hline $\mathrm{C}$ & 5.59267100 & -2.49525800 & -3.15694900 & $\mathrm{H}$ & 2.96743400 & -6.69609200 & 2.77271500 \\
\hline $\mathrm{H}$ & 5.13114400 & -1.52093300 & -3.33777000 & $\mathrm{H}$ & 3.16902500 & -6.74908500 & 1.01204300 \\
\hline $\mathrm{H}$ & 6.05508500 & -2.81902400 & -4.10015100 & $\mathrm{H}$ & 4.03367700 & -5.53677100 & 1.96760900 \\
\hline $\mathrm{H}$ & 6.39032200 & -2.36142000 & -2.42493100 & $\mathrm{H}$ & -1.06691000 & -10.20858100 & -0.6373540 \\
\hline $\mathrm{C}$ & 2.46345700 & -2.51124200 & -3.88420000 & $\mathrm{C}$ & 1.78709200 & -4.06265200 & 2.87952000 \\
\hline $\mathrm{H}$ & 2.42230600 & -2.83918700 & -4.92940400 & $\mathrm{C}$ & 2.07215100 & -4.69306400 & 4.25809500 \\
\hline $\mathrm{H}$ & 2.95435100 & -1.53419100 & -3.86440100 & $\mathrm{C}$ & 0.42317400 & -3.34566200 & 2.91018400 \\
\hline $\mathrm{H}$ & 1.43024200 & -2.39125500 & -3.54815600 & $\mathrm{C}$ & 3.40692500 & -4.89211200 & 4.65951700 \\
\hline $\mathrm{C}$ & 1.23297100 & -5.24167900 & -2.89231800 & $\mathrm{C}$ & 1.04407800 & -5.00157200 & 5.17116300 \\
\hline $\mathrm{H}$ & 0.91114700 & -6.02786700 & -2.20743800 & $\mathrm{C}$ & -0.71405800 & -4.19899000 & 3.49634000 \\
\hline $\mathrm{H}$ & 1.21178700 & -5.64768400 & -3.91210500 & $\mathrm{C}$ & 3.72329700 & -5.46063900 & 5.88944400 \\
\hline $\mathrm{H}$ & 0.49605500 & -4.43659000 & -2.84967300 & $\mathrm{H}$ & 4.20558600 & -4.57223400 & 3.99985300 \\
\hline $\mathrm{C}$ & 3.59337800 & -6.93710600 & -1.50269800 & $\mathrm{C}$ & 1.37807700 & -5.58555600 & 6.40445800 \\
\hline $\mathrm{H}$ & 3.79115900 & -7.57634700 & -2.37550500 & $\mathrm{C}$ & -0.40358700 & -4.61206100 & 4.93532500 \\
\hline $\mathrm{H}$ & 2.60698000 & -7.20594100 & -1.12067000 & $\mathrm{C}$ & 2.69847600 & -5.83244400 & 6.76336800 \\
\hline $\mathrm{H}$ & 4.34570200 & -7.17477100 & -0.74717900 & $\mathrm{H}$ & 4.76441400 & -5.60305300 & 6.16733900 \\
\hline $\mathrm{H}$ & 3.21705400 & -0.63316400 & 1.33289500 & $\mathrm{H}$ & 0.57564500 & -5.83023300 & 7.09834000 \\
\hline $\mathrm{C}$ & 4.67766600 & 0.52113300 & 0.27797300 & $\mathrm{H}$ & -1.06802300 & -5.42605800 & 5.25448000 \\
\hline $\mathrm{C}$ & 5.72972200 & 0.57212700 & -0.64547900 & $\mathrm{H}$ & 2.92834100 & -6.28670200 & 7.72402300 \\
\hline $\mathrm{C}$ & 4.41890300 & 1.65350300 & 1.05945200 & $\mathrm{H}$ & 2.57162400 & -3.32131900 & 2.72993100 \\
\hline $\mathrm{C}$ & 6.50188200 & 1.72558700 & -0.78510200 & $\mathrm{H}$ & -0.62685700 & -3.76892600 & 5.60800000 \\
\hline $\mathrm{H}$ & 5.95114900 & -0.30708900 & -1.24064100 & $\mathrm{C}$ & -6.46024600 & -3.55121200 & -0.81613900 \\
\hline $\mathrm{C}$ & 5.19109500 & 2.80889100 & 0.92552700 & $\mathrm{C}$ & -5.77111400 & -3.96094600 & -3.04008200 \\
\hline $\mathrm{H}$ & 3.60019300 & 1.63431000 & 1.77425800 & $\mathrm{C}$ & -7.01708700 & -3.95543900 & -2.18914200 \\
\hline $\mathrm{C}$ & 6.23509200 & 2.84964800 & 0.00050100 & $\mathrm{H}$ & -6.40858300 & -2.45860600 & -0.75307700 \\
\hline $\mathrm{H}$ & 7.31681700 & 1.74544700 & -1.50454200 & $\mathrm{H}$ & -7.06668800 & -3.89942100 & 0.02450300 \\
\hline $\mathrm{H}$ & 4.97361800 & 3.67668300 & 1.54273400 & $\mathrm{H}$ & -7.47632800 & -4.95441800 & -2.15866300 \\
\hline $\mathrm{H}$ & 6.83845900 & 3.74740300 & -0.10550300 & $\mathrm{H}$ & -7.78454900 & -3.26592800 & -2.55971100 \\
\hline $\mathrm{H}$ & 3.26150900 & -0.80744400 & -1.68995900 & $\mathrm{C}$ & -4.99306800 & -4.12668000 & -0.77107500 \\
\hline $\mathrm{C}$ & 1.84766400 & 0.55329000 & -0.89680700 & $\mathrm{C}$ & -5.03268300 & -5.57347300 & -0.19247300 \\
\hline $\mathrm{C}$ & 0.55959000 & 0.65041300 & -0.36076600 & $\mathrm{C}$ & -4.27642600 & -3.39907800 & 0.36485700 \\
\hline $\mathrm{C}$ & 2.33951000 & 1.62574900 & -1.65825900 & $\mathrm{C}$ & -5.25254000 & -5.36202900 & 1.33061400 \\
\hline $\mathrm{C}$ & -0.21655700 & 1.79293100 & -0.57015500 & $\mathrm{H}$ & -5.81120500 & -6.18957900 & -0.65519300 \\
\hline $\mathrm{H}$ & 0.12295000 & -0.17742300 & 0.18735800 & $\mathrm{H}$ & -4.06655000 & -6.05869300 & -0.37057500 \\
\hline $\mathrm{C}$ & 1.57180900 & 2.76992600 & -1.86228500 & $\mathrm{H}$ & -4.74355500 & -6.12551200 & 1.93116200 \\
\hline $\mathrm{H}$ & 3.33384400 & 1.56360100 & -2.09181500 & $\mathrm{H}$ & -6.31677900 & -5.41522600 & 1.59737200 \\
\hline $\mathrm{C}$ & 0.28796100 & 2.85544000 & -1.31904600 & $\mathrm{C}$ & -4.61500000 & -4.01614100 & -2.24465600 \\
\hline $\mathrm{H}$ & -1.22007100 & 1.83641700 & -0.16193100 & $\mathrm{C}$ & -3.35210300 & -3.99816600 & -2.85019800 \\
\hline $\mathrm{H}$ & 1.97176800 & 3.58748600 & -2.45645000 & $\mathrm{C}$ & -5.68867400 & -3.91654800 & -4.42538500 \\
\hline $\mathrm{H}$ & -0.32215400 & 3.73766300 & -1.49309600 & $\mathrm{C}$ & -3.24314100 & -3.98446300 & -4.25959400 \\
\hline $\mathrm{N}$ & 0.76521500 & -5.56968700 & 1.19330700 & $\mathrm{C}$ & -4.42868200 & -3.93950700 & -5.01509700 \\
\hline
\end{tabular}




\begin{tabular}{|c|c|c|c|c|}
\hline $\mathrm{H}$ & -6.58543000 & -3.86873800 & -5.03804400 & $\mathrm{H}$ \\
\hline $\mathrm{H}$ & -4.34131900 & -3.92550800 & -6.09773900 & $\mathrm{H}$ \\
\hline $\mathrm{C}$ & -4.67932900 & -3.97744000 & 1.57413900 & $\mathrm{C}$ \\
\hline $\mathrm{C}$ & -4.57422000 & -3.24954400 & 2.75534500 & $\mathrm{H}$ \\
\hline $\mathrm{C}$ & -3.54037800 & -2.21095300 & 0.36419600 & $\mathrm{H}$ \\
\hline $\mathrm{C}$ & -4.10983100 & -1.93459300 & 2.68366400 & $\mathrm{H}$ \\
\hline $\mathrm{H}$ & -4.90937900 & -3.66039400 & 3.70467100 & $\mathrm{C}$ \\
\hline $\mathrm{C}$ & -3.56526200 & -1.37616300 & 1.50878500 & $\mathrm{H}$ \\
\hline $\mathrm{H}$ & -4.16620200 & -1.30838500 & 3.56672400 & $\mathrm{C}$ \\
\hline $\mathrm{O}$ & -2.23239300 & -4.09508800 & -2.04592400 & $\mathrm{H}$ \\
\hline $\mathrm{O}$ & -2.81114700 & -1.86604900 & -0.76991100 & $\mathrm{C}$ \\
\hline $\mathrm{P}$ & -1.52157500 & -2.86092100 & -1.17487000 & $\mathrm{H}$ \\
\hline $\mathrm{O}$ & -0.96179200 & -3.52086400 & 0.05738400 & $\mathrm{C}$ \\
\hline $\mathrm{O}$ & -0.61217900 & -2.06572600 & -2.07242100 & $\mathrm{H}$ \\
\hline $\mathrm{C}$ & -1.97055200 & -4.08764100 & -5.05768500 & $\mathrm{H}$ \\
\hline $\mathrm{C}$ & -1.51339900 & -5.36851700 & -5.45116100 & $\mathrm{H}$ \\
\hline C & -1.36555200 & -2.92697400 & -5.59945200 & $\mathrm{C}$ \\
\hline $\mathrm{C}$ & -0.47020900 & -5.46013600 & -6.38191100 & $\mathrm{H}$ \\
\hline $\mathrm{C}$ & -0.32284700 & -3.07699000 & -6.52325800 & $\mathrm{H}$ \\
\hline $\mathrm{C}$ & 0.13114000 & -4.33180800 & -6.94284700 & $\mathrm{H}$ \\
\hline $\mathrm{H}$ & -0.13956000 & -6.44250500 & -6.71198100 & $\mathrm{C}$ \\
\hline $\mathrm{H}$ & 0.11913600 & -2.18350100 & -6.95700900 & $\mathrm{H}$ \\
\hline $\mathrm{C}$ & -3.24372500 & 0.09635900 & 1.51320200 & $\mathrm{H}$ \\
\hline $\mathrm{C}$ & -3.94737400 & 0.96969900 & 0.63124200 & $\mathrm{H}$ \\
\hline $\mathrm{C}$ & -2.41217300 & 0.67483900 & 2.51234100 & $\mathrm{C}$ \\
\hline $\mathrm{C}$ & -3.81948800 & 2.35515000 & 0.78602000 & $\mathrm{H}$ \\
\hline $\mathrm{C}$ & -2.34194500 & 2.06981700 & 2.62654600 & $\mathrm{H}$ \\
\hline $\mathrm{C}$ & -3.04037300 & 2.93535400 & 1.78861800 & $\mathrm{H}$ \\
\hline $\mathrm{H}$ & -4.37607600 & 3.00113200 & 0.11185500 & $\mathrm{C}$ \\
\hline $\mathrm{H}$ & -1.71883000 & 2.49969400 & 3.40697800 & $\mathrm{H}$ \\
\hline $\mathrm{C}$ & -1.89789300 & -1.52435400 & -5.29623400 & $\mathrm{H}$ \\
\hline $\mathrm{H}$ & -2.54380800 & -1.59685500 & -4.41620800 & $\mathrm{H}$ \\
\hline $\mathrm{C}$ & 1.19232400 & -4.48406500 & -8.02760300 & $\mathrm{C}$ \\
\hline $\mathrm{H}$ & 1.34731000 & -5.56198600 & -8.17461300 & $\mathrm{H}$ \\
\hline $\mathrm{C}$ & -2.20141300 & -6.65233700 & -4.98032800 & $\mathrm{H}$ \\
\hline $\mathrm{H}$ & -2.80691200 & -6.40420500 & -4.10288400 & $\mathrm{H}$ \\
\hline $\mathrm{C}$ & -0.79922300 & -0.50293300 & -4.95410700 & $\mathrm{H}$ \\
\hline $\mathrm{H}$ & -1.25592400 & 0.47232800 & -4.74639800 & $\mathrm{H}$ \\
\hline $\mathrm{H}$ & -0.09277000 & -0.35931500 & -5.78137600 & $\mathrm{H}$ \\
\hline $\mathrm{H}$ & -0.25671100 & -0.81101300 & -4.05862800 & $\mathrm{H}$ \\
\hline $\mathrm{C}$ & -2.75819800 & -1.01183100 & -6.47102300 & \\
\hline $\mathrm{H}$ & -3.17746800 & -0.02585500 & -6.23621700 & 13-SK \\
\hline $\mathrm{H}$ & -3.59031000 & -1.68897000 & -6.68993600 & $\mathrm{C}$ \\
\hline $\mathrm{H}$ & -2.15766600 & -0.91299800 & -7.38404900 & $\mathrm{C}$ \\
\hline $\mathrm{C}$ & 2.55121600 & -3.87983600 & -7.62925100 & $\mathrm{C}$ \\
\hline $\mathrm{H}$ & 3.29344900 & -4.03950600 & -8.42101700 & $\mathrm{H}$ \\
\hline $\mathrm{H}$ & 2.93465900 & -4.33902200 & -6.71064500 & $\mathrm{H}$ \\
\hline $\mathrm{H}$ & 2.47435900 & -2.79857800 & -7.46193800 & $\mathrm{H}$ \\
\hline $\mathrm{C}$ & 0.71140000 & -3.90336600 & -9.37194800 & $\mathrm{H}$ \\
\hline $\mathrm{H}$ & 1.45253800 & -4.08374100 & -10.16031400 & $\mathrm{C}$ \\
\hline $\mathrm{H}$ & 0.55355200 & -2.82050100 & -9.30344400 & $\mathrm{C}$ \\
\hline $\mathrm{H}$ & -0.23552300 & -4.35873900 & -9.68138800 & $\mathrm{C}$ \\
\hline $\mathrm{C}$ & -3.16044100 & -7.18435200 & -6.06621200 & $\mathrm{C}$ \\
\hline $\mathrm{H}$ & -2.61160700 & -7.44123500 & -6.98059500 & $\mathrm{H}$ \\
\hline
\end{tabular}

$\begin{array}{rrr}-3.91963100 & -6.44032300 & -6.32623900 \\ -3.67610200 & -8.08770900 & -5.71720400 \\ -1.22107100 & -7.76044100 & -4.55586600 \\ -1.77917800 & -8.62495900 & -4.17584900 \\ -0.54590900 & -7.42206100 & -3.76468100 \\ -0.61104900 & -8.11536200 & -5.39481200 \\ -4.90955000 & 0.49563800 & -0.46067100 \\ -4.94457700 & -0.59296100 & -0.44724300 \\ -2.96667800 & 4.44410400 & 1.98329600 \\ -2.28479800 & 4.62598600 & 2.82556000 \\ -1.57011600 & -0.13511800 & 3.50290700 \\ -1.63281300 & -1.18986600 & 3.22130600 \\ -2.38107900 & 5.16533400 & 0.75507100 \\ -2.29702100 & 6.24273300 & 0.94285800 \\ -3.01825800 & 5.03126400 & -0.12749000 \\ -1.38402300 & 4.78253800 & 0.51219300 \\ -4.33839000 & 5.03344500 & 2.36588100 \\ -4.25698300 & 6.10951100 & 2.56235800 \\ -4.74009500 & 4.55117200 & 3.26381700 \\ -5.06814400 & 4.89591800 & 1.55908800 \\ -6.34988300 & 0.98043900 & -0.19645900 \\ -6.42975000 & 2.07313100 & -0.23710600 \\ -6.70225100 & 0.65713800 & 0.78970600 \\ -7.03247300 & 0.57220100 & -0.95226300 \\ -4.42779200 & 0.90722300 & -1.86496200 \\ -3.42077100 & 0.52544400 & -2.05661200 \\ -4.41268700 & 1.99766200 & -1.98424400 \\ -5.09914100 & 0.49971100 & -2.63074500 \\ -2.08400500 & 0.00394300 & 4.95239100 \\ -1.99418700 & 1.03865200 & 5.30396400 \\ -1.49632900 & -0.62908900 & 5.62864100 \\ -3.13549100 & -0.28362000 & 5.05127900 \\ -0.08263600 & 0.27291300 & 3.45812100 \\ 0.33692600 & 0.16487100 & 2.45371700 \\ 0.50439600 & -0.34762200 & 4.14464600 \\ 0.05966300 & 1.31575000 & 3.76308500 \\ -0.87370200 & -5.09297200 & 2.88813600 \\ -1.65311300 & -3.63676800 & 3.46139600 \\ 0.13186400 & -2.98260600 & 1.92035500 \\ 0.55478900 & -2.46137600 & 3.54458100\end{array}$

$\begin{array}{lll}4.66342500 & -6.13316800 & -2.81377500\end{array}$ $\begin{array}{lll}6.92440900 & -6.28750600 & -3.48381500\end{array}$

$\begin{array}{llll}5.59365100 & -6.96116900 & -3.71196200\end{array}$

$\begin{array}{llll}4.32389200 & -5.24730700 & -3.36206300\end{array}$

$\begin{array}{lll}3.77334600 & -6.67484000 & -2.48115300\end{array}$

$\begin{array}{llll}5.62522700 & -8.01433200 & -3.39631600\end{array}$

$\begin{array}{llll}5.28799500 & -6.95597700 & -4.76466100\end{array}$

$\begin{array}{llll}5.56078900 & -5.66150000 & -1.60734400\end{array}$

$\begin{array}{llll}5.51418200 & -6.74427200 & -0.48589400\end{array}$

$\begin{array}{llll}4.81196500 & -4.52405100 & -0.91949400\end{array}$

$\begin{array}{llll}4.12622400 & -6.53843500 & 0.17970800\end{array}$

$\begin{array}{llll}5.65514100 & -7.75831700 & -0.87504600\end{array}$ 


\begin{tabular}{|c|c|c|c|c|c|c|c|}
\hline $\mathrm{H}$ & 6.31316800 & -6.54767100 & 0.23777900 & $\mathrm{H}$ & 14.65107800 & -1.64244000 & -0.63235300 \\
\hline $\mathrm{H}$ & 4.14391200 & -6.76481900 & 1.25285600 & $\mathrm{H}$ & 14.46415400 & -0.80156700 & -2.18039500 \\
\hline $\mathrm{H}$ & 3.36559400 & -7.19570600 & -0.26327600 & $\mathrm{C}$ & 15.29121100 & -2.96694400 & -3.80146000 \\
\hline $\mathrm{C}$ & 6.90612500 & -5.50872000 & -2.31530700 & $\mathrm{H}$ & 16.36648700 & -2.75010400 & -3.79501600 \\
\hline $\mathrm{C}$ & 8.04083200 & -4.76740700 & -1.95561800 & $\mathrm{H}$ & 14.80140100 & -2.20621600 & -4.42081100 \\
\hline $\mathrm{C}$ & 8.06037300 & -6.36367500 & -4.27837900 & $\mathrm{H}$ & 15.14126000 & -3.93801000 & -4.28602200 \\
\hline $\mathrm{C}$ & 9.21810500 & -4.84957100 & -2.73329800 & $\mathrm{C}$ & 11.63563800 & -7.49617200 & -1.92238900 \\
\hline $\mathrm{C}$ & 9.19117600 & -5.65421300 & -3.88745600 & $\mathrm{H}$ & 12.72424100 & -7.48263200 & -2.05627400 \\
\hline $\mathrm{H}$ & 8.06979000 & -6.96258000 & -5.18561700 & $\mathrm{H}$ & 11.17376100 & -7.46911200 & -2.91411100 \\
\hline $\mathrm{H}$ & 10.09577500 & -5.71289800 & -4.48576800 & $\mathrm{H}$ & 11.36604400 & -8.44895900 & -1.44919400 \\
\hline $\mathrm{C}$ & 3.82707400 & -5.07467400 & -0.09085400 & $\mathrm{C}$ & 11.74979400 & -6.43427500 & 0.36651100 \\
\hline $\mathrm{C}$ & 2.74684900 & -4.29438500 & 0.31272600 & $\mathrm{H}$ & 11.41134800 & -7.37106100 & 0.82719000 \\
\hline $\mathrm{C}$ & 4.84006500 & -3.14791100 & -1.15505000 & $\mathrm{H}$ & 11.43592300 & -5.60217900 & 1.00523000 \\
\hline $\mathrm{C}$ & 2.65447100 & -2.98511800 & -0.16549400 & $\mathrm{H}$ & 12.84550900 & -6.45874700 & 0.36552500 \\
\hline $\mathrm{H}$ & 1.95447800 & -4.70664000 & 0.93325900 & $\mathrm{C}$ & 3.79377100 & -1.91780000 & -3.88060300 \\
\hline $\mathrm{C}$ & 3.69114100 & -2.36560500 & -0.89248800 & $\mathrm{H}$ & 4.04327700 & -2.82411700 & -3.32781700 \\
\hline $\mathrm{H}$ & 1.75509600 & -2.41095600 & 0.03284900 & $\mathrm{C}$ & 2.16592900 & 2.80457600 & -3.21751900 \\
\hline $\mathrm{O}$ & 8.00661700 & -4.03124500 & -0.78343100 & $\mathrm{H}$ & 1.90086200 & 3.46360700 & -2.37905900 \\
\hline $\mathrm{O}$ & 6.00211400 & -2.56971700 & -1.64862800 & $\mathrm{C}$ & 3.06155600 & 0.04362100 & 0.88696000 \\
\hline $\mathrm{P}$ & 7.31313000 & -2.52322700 & -0.60510500 & $\mathrm{H}$ & 3.46892000 & -0.92280000 & 1.19405100 \\
\hline $\mathrm{O}$ & 6.81146300 & -2.51438800 & 0.82351600 & $\mathrm{C}$ & 3.26443900 & 3.51542300 & -4.02940200 \\
\hline $\mathrm{O}$ & 8.22952100 & -1.44657300 & -1.09232200 & $\mathrm{H}$ & 2.89804100 & 4.47295600 & -4.41941100 \\
\hline $\mathrm{C}$ & 10.55469000 & -4.21941400 & -2.43484300 & $\mathrm{H}$ & 3.58341500 & 2.90834800 & -4.88538300 \\
\hline $\mathrm{C}$ & 11.51728800 & -4.97227700 & -1.72122700 & $\mathrm{H}$ & 4.14531200 & 3.71292200 & -3.40986800 \\
\hline $\mathrm{C}$ & 10.93810200 & -3.01282900 & -3.07049600 & $\mathrm{C}$ & 0.89446200 & 2.60588900 & -4.06517900 \\
\hline $\mathrm{C}$ & 12.84508900 & -4.52708600 & -1.69389500 & $\mathrm{H}$ & 0.52182800 & 3.56862300 & -4.43584200 \\
\hline $\mathrm{C}$ & 12.27798600 & -2.60982200 & -3.00361800 & $\mathrm{H}$ & 0.09811700 & 2.13327500 & -3.47966900 \\
\hline $\mathrm{C}$ & 13.25477300 & -3.36244900 & -2.34431200 & $\mathrm{H}$ & 1.09184200 & 1.96817600 & -4.93508000 \\
\hline $\mathrm{H}$ & 13.59374900 & -5.12303600 & -1.17671400 & $\mathrm{C}$ & 2.58082300 & -2.26732100 & -4.76689500 \\
\hline $\mathrm{H}$ & 12.57160900 & -1.69681400 & -3.51473800 & $\mathrm{H}$ & 2.27659600 & -1.42194300 & -5.39501000 \\
\hline $\mathrm{C}$ & 3.45648800 & -0.99337400 & -1.46447900 & $\mathrm{H}$ & 1.71673000 & -2.55960700 & -4.15934400 \\
\hline $\mathrm{C}$ & 3.45486000 & -0.81219800 & -2.87790100 & $\mathrm{H}$ & 2.82633100 & -3.10289600 & -5.43415200 \\
\hline $\mathrm{C}$ & 3.06880500 & 0.09664000 & -0.64165900 & $\mathrm{C}$ & 5.02547200 & -1.56011300 & -4.73442500 \\
\hline $\mathrm{C}$ & 3.06398500 & 0.42131900 & -3.41121100 & $\mathrm{H}$ & 5.89164100 & -1.35655800 & -4.09848100 \\
\hline $\mathrm{C}$ & 2.67133800 & 1.30156500 & -1.23565900 & $\mathrm{H}$ & 4.84141200 & -0.67883300 & -5.36110200 \\
\hline $\mathrm{C}$ & 2.64936100 & 1.49152700 & -2.61518800 & $\mathrm{H}$ & 5.27939100 & -2.39259900 & -5.40216900 \\
\hline $\mathrm{H}$ & 3.05715800 & 0.53615100 & -4.49222600 & $\mathrm{C}$ & 1.63540400 & 0.15451400 & 1.46556200 \\
\hline $\mathrm{H}$ & 2.36206600 & 2.12441100 & -0.59553900 & $\mathrm{H}$ & 1.17933600 & 1.11918600 & 1.21399200 \\
\hline $\mathrm{C}$ & 9.95516800 & -2.19022500 & -3.90502800 & $\mathrm{H}$ & 1.65637900 & 0.07395100 & 2.55975100 \\
\hline $\mathrm{H}$ & 8.94363700 & -2.50097300 & -3.62873100 & $\mathrm{H}$ & 0.97502700 & -0.63036200 & 1.08130100 \\
\hline $\mathrm{C}$ & 14.72710100 & -2.96820900 & -2.36714300 & $\mathrm{C}$ & 3.95777900 & 1.14104200 & 1.49523300 \\
\hline $\mathrm{H}$ & 15.27342800 & -3.73656000 & -1.80243100 & $\mathrm{H}$ & 4.97621200 & 1.08806900 & 1.09842300 \\
\hline $\mathrm{C}$ & 11.17346800 & -6.30622400 & -1.05571800 & $\mathrm{H}$ & 4.00911700 & 1.04199100 & 2.58523000 \\
\hline $\mathrm{H}$ & 10.08321200 & -6.36608900 & -0.97192500 & $\mathrm{H}$ & 3.57268000 & 2.14329100 & 1.27571200 \\
\hline $\mathrm{C}$ & 10.04220800 & -0.67894100 & -3.62932900 & $\mathrm{C}$ & 11.80540200 & -0.26053100 & 4.13865400 \\
\hline $\mathrm{H}$ & 9.26482300 & -0.15440800 & -4.19717400 & $\mathrm{C}$ & 12.26650000 & 0.62956000 & 3.07128300 \\
\hline $\mathrm{H}$ & 11.00780700 & -0.25673900 & -3.93430600 & $\mathrm{C}$ & 12.09927100 & -0.02797700 & 1.83541100 \\
\hline $\mathrm{H}$ & 9.87152400 & -0.47271300 & -2.57110300 & $\mathrm{C}$ & 11.52888700 & -1.35465900 & 2.09450800 \\
\hline $\mathrm{C}$ & 10.14491400 & -2.47285400 & -5.41037000 & $\mathrm{C}$ & 11.43355100 & -1.51033100 & 3.52645200 \\
\hline $\mathrm{H}$ & 9.42260400 & -1.89622300 & -6.00114900 & Ir & 9.99299700 & 0.01040800 & 2.87199200 \\
\hline $\mathrm{H}$ & 10.00236000 & -3.53278600 & -5.64558100 & $\mathrm{~N}$ & 8.97859700 & 1.87352900 & 3.45541000 \\
\hline $\mathrm{H}$ & 11.15221100 & -2.18855600 & -5.74021400 & $\mathrm{~N}$ & 8.70643800 & 0.44615700 & 1.21878000 \\
\hline $\mathrm{C}$ & 14.98984000 & -1.61959300 & -1.67380200 & $\mathrm{H}$ & 8.89040500 & -0.09811800 & 0.36459200 \\
\hline $\mathrm{H}$ & 16.06084800 & -1.38246300 & -1.67895600 & $\mathrm{C}$ & 8.29322600 & 2.56638000 & 2.32775400 \\
\hline
\end{tabular}




\begin{tabular}{|c|c|c|c|c|c|c|c|}
\hline $\mathrm{C}$ & 8.80491300 & 1.91457600 & 1.00441900 & $\mathrm{C}$ & 9.80176600 & 4.60427000 & 2.55342600 \\
\hline S & 8.06260100 & 1.75001000 & 4.83100500 & $\mathrm{C}$ & 7.47937200 & 4.95383600 & 2.02313800 \\
\hline $\mathrm{O}$ & 8.86220300 & 1.06330200 & 5.86331400 & $\mathrm{C}$ & 10.02439100 & 5.98005600 & 2.49022200 \\
\hline $\mathrm{O}$ & 6.72463400 & 1.14902000 & 4.56879800 & $\mathrm{H}$ & 10.61433500 & 3.93029500 & 2.80504600 \\
\hline $\mathrm{C}$ & 7.75920300 & 3.44706400 & 5.49134400 & $\mathrm{C}$ & 7.69664800 & 6.33200400 & 1.96356100 \\
\hline $\mathrm{C}$ & 6.46526400 & 4.01348000 & 5.50191600 & $\mathrm{H}$ & 6.48507900 & 4.55681300 & 1.83381600 \\
\hline $\mathrm{C}$ & 8.84430500 & 4.08544800 & 6.13198200 & $\mathrm{C}$ & 8.97115100 & 6.85019500 & 2.19566000 \\
\hline $\mathrm{C}$ & 6.25509100 & 5.19461200 & 6.24956700 & $\mathrm{H}$ & 11.02049800 & 6.37486800 & $2.6757730 \mathrm{C}$ \\
\hline $\mathrm{C}$ & 8.61300300 & 5.27279500 & 6.85263100 & $\mathrm{H}$ & 6.86919400 & 6.99909800 & 1.73560000 \\
\hline $\mathrm{C}$ & 7.32614000 & 5.84020300 & 6.89174200 & $\mathrm{H}$ & 9.14306800 & 7.92256700 & 2.15114500 \\
\hline $\mathrm{C}$ & 5.28105100 & 3.47370900 & 4.71920500 & $\mathrm{H}$ & 9.87566000 & 2.13253900 & 0.93814600 \\
\hline $\mathrm{H}$ & 4.65434700 & 2.81115200 & 5.32955400 & $\mathrm{C}$ & 8.15359300 & 2.41329500 & -0.27850300 \\
\hline $\mathrm{H}$ & 5.58393000 & 2.89447700 & 3.85294500 & $\mathrm{C}$ & 7.10809600 & 1.72749400 & -0.90819800 \\
\hline $\mathrm{H}$ & 4.65474100 & 4.30116600 & 4.37462400 & $\mathrm{C}$ & 8.65316700 & 3.57633200 & -0.88696500 \\
\hline $\mathrm{C}$ & 4.85041300 & 5.75971200 & 6.37658300 & $\mathrm{C}$ & 6.56834600 & 2.19486800 & -2.10946200 \\
\hline $\mathrm{H}$ & 4.73097300 & 6.33087300 & 7.29984300 & $\mathrm{H}$ & 6.73743900 & 0.79239900 & -0.50384400 \\
\hline $\mathrm{H}$ & 4.09879100 & 4.96666900 & 6.39782000 & $\mathrm{C}$ & 8.11224300 & 4.04845700 & -2.08112200 \\
\hline $\mathrm{H}$ & 4.58907000 & 6.42898900 & 5.54372400 & $\mathrm{H}$ & 9.47296100 & 4.11541300 & -0.42033800 \\
\hline $\mathrm{C}$ & 7.10137900 & 7.16632800 & 7.59620000 & $\mathrm{C}$ & 7.06653400 & 3.35714300 & -2.69770100 \\
\hline $\mathrm{H}$ & 6.81405500 & 7.03548400 & 8.64971000 & $\mathrm{H}$ & 5.76936300 & 1.63503600 & -2.58587500 \\
\hline $\mathrm{H}$ & 6.31011300 & 7.74734200 & 7.11630400 & $\mathrm{H}$ & 8.51643800 & 4.94934100 & -2.53569700 \\
\hline $\mathrm{H}$ & 8.00007800 & 7.78706900 & 7.58194200 & $\mathrm{H}$ & 6.65541700 & 3.71346300 & -3.63861300 \\
\hline $\mathrm{C}$ & 9.75759200 & 5.92453800 & 7.60790300 & $\mathrm{~N}$ & 6.58567600 & -3.43772500 & 3.34234800 \\
\hline $\mathrm{H}$ & 9.40231200 & 6.47303800 & 8.48345900 & $\mathrm{C}$ & 6.05007700 & -2.50715800 & 4.08748900 \\
\hline $\mathrm{H}$ & 10.31728700 & 6.63555500 & 6.98254300 & $\mathrm{C}$ & 7.24403200 & -4.67811300 & 3.60552200 \\
\hline $\mathrm{H}$ & 10.47398800 & 5.18277600 & 7.96857400 & $\mathrm{C}$ & 8.05238300 & -5.15669600 & 2.56212700 \\
\hline $\mathrm{C}$ & 10.26714500 & 3.57079400 & 6.04544200 & $\mathrm{C}$ & 7.05847700 & -5.44292700 & 4.76275100 \\
\hline $\mathrm{H}$ & 10.96025400 & 4.40832800 & 5.91341900 & $\mathrm{C}$ & 8.70011300 & -6.38277800 & 2.70146500 \\
\hline $\mathrm{H}$ & 10.39809500 & 2.89111900 & 5.20781600 & $\mathrm{H}$ & 8.15586700 & -4.57682200 & 1.65003200 \\
\hline $\mathrm{H}$ & 10.55758300 & 3.03283100 & 6.95608800 & $\mathrm{C}$ & 7.71468900 & -6.66746000 & 4.88572900 \\
\hline $\mathrm{H}$ & 7.75443200 & 0.18421400 & 1.47657200 & $\mathrm{H}$ & 6.39269600 & -5.11121600 & 5.54668700 \\
\hline $\mathrm{H}$ & 8.73018300 & -0.77988000 & 3.44082200 & $\mathrm{C}$ & 8.54041800 & -7.13929000 & 3.86432100 \\
\hline $\mathrm{C}$ & 11.10467200 & -2.77944300 & 4.25858600 & $\mathrm{H}$ & 9.32624400 & -6.74520500 & 1.89320600 \\
\hline $\mathrm{H}$ & 10.62150600 & -2.57192100 & 5.21687700 & $\mathrm{H}$ & 7.56517300 & -7.25803900 & 5.78503900 \\
\hline $\mathrm{H}$ & 12.02318900 & -3.34840400 & 4.46157700 & $\mathrm{H}$ & 6.60303700 & -3.19485200 & 2.31526000 \\
\hline $\mathrm{H}$ & 10.43714800 & -3.42008400 & 3.67708000 & $\mathrm{C}$ & 5.38895300 & -1.38348800 & 3.36656600 \\
\hline $\mathrm{C}$ & 11.98606900 & -0.01459700 & 5.60867500 & $\mathrm{H}$ & 5.39500000 & -1.51621200 & 2.28539800 \\
\hline $\mathrm{H}$ & 11.29339900 & -0.61774000 & 6.19965900 & $\mathrm{H}$ & 4.35884200 & -1.29102800 & 3.73666400 \\
\hline $\mathrm{H}$ & 11.79207800 & 1.02844200 & 5.86601600 & $\mathrm{H}$ & 5.89587600 & -0.44800400 & 3.64390800 \\
\hline $\mathrm{H}$ & 13.01150000 & -0.26050100 & 5.92353700 & $\mathrm{H}$ & 9.04560500 & -8.09528000 & 3.96809500 \\
\hline $\mathrm{C}$ & 12.85393800 & 1.99146900 & 3.29393600 & $\mathrm{C}$ & 6.08062600 & -2.37711000 & 5.60063200 \\
\hline $\mathrm{H}$ & 13.90360500 & 1.91305200 & 3.61082900 & $\mathrm{C}$ & 5.14121100 & -3.31755500 & 6.36759400 \\
\hline $\mathrm{H}$ & 12.31844100 & 2.53486000 & 4.07711500 & $\mathrm{C}$ & 7.52763200 & -2.33938500 & 6.15285100 \\
\hline $\mathrm{H}$ & 12.83142400 & 2.59839400 & 2.38397100 & $\mathrm{C}$ & 3.92100500 & -3.72068500 & 5.80219200 \\
\hline $\mathrm{C}$ & 12.44621600 & 0.50635000 & 0.47551600 & $\mathrm{C}$ & 5.45671000 & -3.71642000 & 7.68182500 \\
\hline $\mathrm{H}$ & 13.46564000 & 0.21769400 & 0.18884800 & $\mathrm{C}$ & 7.47520100 & -2.15614300 & 7.67206300 \\
\hline $\mathrm{H}$ & 12.39596300 & 1.59939200 & 0.44072800 & $\mathrm{H}$ & 8.05859000 & -1.50283400 & 5.68937500 \\
\hline $\mathrm{H}$ & 11.77621200 & 0.11238500 & -0.29496800 & $\mathrm{C}$ & 3.02089900 & -4.51256600 & 6.51149500 \\
\hline $\mathrm{C}$ & 11.32633100 & -2.43017600 & 1.06676100 & $\mathrm{H}$ & 3.66973500 & -3.41744800 & 4.78873300 \\
\hline $\mathrm{H}$ & 10.88872600 & -2.04624500 & 0.14093600 & $\mathrm{C}$ & 4.53603700 & -4.50818000 & 8.38388400 \\
\hline $\mathrm{H}$ & 10.64799100 & -3.20207300 & 1.43998400 & $\mathrm{C}$ & 6.77383900 & -3.34213700 & 8.34164700 \\
\hline $\mathrm{H}$ & 12.27542800 & -2.91560600 & 0.80375400 & $\mathrm{H}$ & 8.49164500 & -2.04861100 & 8.06689500 \\
\hline $\mathrm{H}$ & 7.20699000 & 2.38718700 & 2.35089800 & $\mathrm{C}$ & 3.32982700 & -4.90806000 & 7.81406600 \\
\hline $\mathrm{C}$ & 8.52609000 & 4.07360200 & 2.31914200 & $\mathrm{H}$ & 2.08472700 & -4.81464700 & 6.05034600 \\
\hline
\end{tabular}




\begin{tabular}{|c|c|c|c|c|c|c|c|}
\hline $\mathrm{H}$ & 4.77803800 & -4.81189700 & 9.40033700 & $\mathrm{C}$ & 10.74889000 & -7.82224900 & -0.69797400 \\
\hline $\mathrm{H}$ & 7.44210500 & -4.21691700 & 8.31100100 & $\mathrm{C}$ & 12.71185200 & -6.52058900 & -0.36707100 \\
\hline $\mathrm{H}$ & 2.63497200 & -5.52034000 & 8.38234400 & $\mathrm{C}$ & 11.98109600 & -7.66644100 & -0.06217700 \\
\hline $\mathrm{H}$ & 5.69245800 & -1.36400600 & 5.76955900 & $\mathrm{H}$ & 10.16956000 & -8.72289900 & -0.51065900 \\
\hline $\mathrm{H}$ & 8.06611500 & -3.26144200 & 5.90704700 & $\mathrm{H}$ & 13.68767200 & -6.39543500 & 0.09642000 \\
\hline $\mathrm{H}$ & 6.95078200 & -1.22065900 & 7.90552400 & $\mathrm{C}$ & 3.87208500 & -4.79696700 & 0.34051900 \\
\hline \multirow[t]{2}{*}{$\mathrm{H}$} & 6.60059500 & -3.13693700 & 9.40545300 & $\mathrm{H}$ & 4.56603200 & -4.90316300 & -0.49766700 \\
\hline & & & & $\mathrm{C}$ & 1.50052200 & -1.10257200 & 2.93364700 \\
\hline TS-7-SR & & & & $\mathrm{H}$ & 0.77265700 & -0.40103300 & 2.49913500 \\
\hline $\mathrm{C}$ & 6.98483900 & -4.38842300 & -5.47412300 & $\mathrm{C}$ & 3.77143200 & 0.05096300 & -1.40292900 \\
\hline $\mathrm{C}$ & 4.95881000 & -3.81257400 & -4.40716300 & $\mathrm{H}$ & 4.64907000 & -0.27147400 & -1.97367700 \\
\hline $\mathrm{C}$ & 5.47283000 & -4.32623500 & -5.72962500 & $\mathrm{C}$ & 4.57891000 & -5.38312500 & 1.57465600 \\
\hline $\mathrm{H}$ & 7.23632100 & -5.33579800 & -4.98340400 & $\mathrm{H}$ & 4.83788700 & -6.43330800 & 1.39329200 \\
\hline $\mathrm{H}$ & 7.58934100 & -4.31719000 & -6.38255100 & $\mathrm{H}$ & 3.94234900 & -5.35551500 & 2.46779100 \\
\hline $\mathrm{H}$ & 5.23202800 & -3.63210600 & -6.54793700 & $\mathrm{H}$ & 5.50416400 & -4.84171900 & 1.77801900 \\
\hline $\mathrm{H}$ & 5.04191100 & -5.29686900 & -6.00148000 & $\mathrm{C}$ & 2.60627400 & -5.61579300 & 0.01228000 \\
\hline $\mathrm{C}$ & 7.27490900 & -3.21362900 & -4.46811800 & $\mathrm{H}$ & 2.86091400 & -6.67437900 & -0.12130800 \\
\hline $\mathrm{C}$ & 7.46762300 & -1.89271800 & -5.27682700 & $\mathrm{H}$ & 2.12407600 & -5.26697200 & -0.90655100 \\
\hline $\mathrm{C}$ & 8.69312800 & -3.41952000 & -3.94424800 & $\mathrm{H}$ & 1.86999400 & -5.54889800 & 0.82274600 \\
\hline $\mathrm{C}$ & 8.88332900 & -2.02952500 & -5.89186300 & $\mathrm{C}$ & 2.31691100 & -0.31322300 & 3.97857600 \\
\hline $\mathrm{H}$ & 6.68566200 & -1.74012700 & -6.02821100 & $\mathrm{H}$ & 1.65492500 & 0.10824000 & 4.74533300 \\
\hline $\mathrm{H}$ & 7.44209500 & -1.04590700 & -4.58343600 & $\mathrm{H}$ & 2.86928400 & 0.51254600 & 3.51698900 \\
\hline $\mathrm{H}$ & 9.39372100 & -1.06264800 & -5.97844900 & $\mathrm{H}$ & 3.03977300 & -0.96460000 & 4.48165800 \\
\hline $\mathrm{H}$ & 8.84677500 & -2.45489000 & -6.90417800 & $\mathrm{C}$ & 0.69946800 & -2.22237200 & 3.61436500 \\
\hline $\mathrm{C}$ & 5.99256800 & -3.25263600 & -3.63649300 & $\mathrm{H}$ & -0.00308500 & -1.79407100 & 4.33854700 \\
\hline $\mathrm{C}$ & 5.72616000 & -2.79927300 & -2.33476000 & $\mathrm{H}$ & 1.35396500 & -2.90753400 & 4.16552700 \\
\hline $\mathrm{C}$ & 3.65457700 & -3.86043600 & -3.93672100 & $\mathrm{H}$ & 0.12308700 & -2.80754100 & 2.88874700 \\
\hline $\mathrm{C}$ & 4.40133000 & -2.83416400 & -1.83575200 & $\mathrm{C}$ & 2.58914600 & 0.14908200 & -2.39095500 \\
\hline $\mathrm{C}$ & 3.39306900 & -3.35431800 & -2.66934500 & $\mathrm{H}$ & 1.67405000 & 0.45120700 & -1.86695200 \\
\hline $\mathrm{H}$ & 2.85623700 & -4.28596200 & -4.53957500 & $\mathrm{H}$ & 2.39764300 & -0.80834500 & -2.88385100 \\
\hline $\mathrm{H}$ & 2.37758600 & -3.36814500 & -2.28531900 & $\mathrm{H}$ & 2.79570000 & 0.89630200 & -3.16777700 \\
\hline $\mathrm{C}$ & 9.58697200 & -2.95553700 & -4.91923900 & $\mathrm{C}$ & 4.07757100 & 1.44562100 & -0.82628300 \\
\hline $\mathrm{C}$ & 10.89786800 & -3.41589900 & -4.93260600 & $\mathrm{H}$ & 4.43875600 & 2.11016600 & -1.61861100 \\
\hline $\mathrm{C}$ & 9.16741700 & -4.14084800 & -2.84724900 & $\mathrm{H}$ & 4.84576600 & 1.40373500 & -0.04655900 \\
\hline $\mathrm{C}$ & 11.28912800 & -4.33926600 & -3.96326400 & $\mathrm{H}$ & 3.18508900 & 1.91074600 & -0.39193000 \\
\hline $\mathrm{H}$ & 11.59506100 & -3.10946800 & -5.70882800 & $\mathrm{C}$ & 8.94442300 & -7.26463900 & -2.32376400 \\
\hline $\mathrm{C}$ & 10.47012300 & -4.69843800 & -2.87455600 & $\mathrm{H}$ & 8.65316400 & -6.44505600 & -2.98073900 \\
\hline $\mathrm{H}$ & 12.26967100 & -4.79259300 & -4.04034200 & $\mathrm{C}$ & 12.53596900 & -8.71664600 & 0.89082800 \\
\hline $\mathrm{O}$ & 6.76138400 & -2.24498500 & -1.59843300 & $\mathrm{H}$ & 13.50387900 & -8.34282300 & 1.25327800 \\
\hline $\mathrm{O}$ & 8.32217600 & -4.35434700 & -1.76640800 & $\mathrm{C}$ & 13.21058900 & -4.37159200 & -1.53241600 \\
\hline $\mathrm{P}$ & 7.92519700 & -3.10022100 & -0.75214400 & $\mathrm{H}$ & 12.67094500 & -3.60638600 & -2.09534100 \\
\hline $\mathrm{O}$ & 9.07765400 & -2.13780200 & -0.60744200 & $\mathrm{C}$ & 11.63284300 & -8.92092900 & 2.12137200 \\
\hline $\mathrm{O}$ & 7.28745300 & -3.74009900 & 0.45317600 & $\mathrm{H}$ & 12.08200700 & -9.63997800 & 2.81759000 \\
\hline $\mathrm{C}$ & 3.89671400 & -2.35824100 & -0.49619800 & $\mathrm{H}$ & 10.64944500 & -9.31073700 & 1.83110900 \\
\hline $\mathrm{C}$ & 3.50502300 & -1.00486900 & -0.32920500 & $\mathrm{H}$ & 11.47515300 & -7.97861800 & 2.65721500 \\
\hline $\mathrm{C}$ & 3.54557700 & -3.30898400 & 0.49106400 & $\mathrm{C}$ & 12.79949600 & -10.05543100 & 0.17427900 \\
\hline $\mathrm{C}$ & 2.76077800 & -0.64842200 & 0.79873000 & $\mathrm{H}$ & 13.25257400 & -10.78102500 & 0.86098800 \\
\hline $\mathrm{C}$ & 2.79991500 & -2.89408500 & 1.60707100 & $\mathrm{H}$ & 13.47579300 & -9.92141400 & -0.67710600 \\
\hline $\mathrm{C}$ & 2.37546300 & -1.57478500 & 1.77366300 & $\mathrm{H}$ & 11.86837300 & -10.49233300 & -0.20582600 \\
\hline $\mathrm{H}$ & 2.42891300 & 0.38096700 & 0.90903100 & $\mathrm{C}$ & 9.17127800 & -8.49187900 & -3.23212800 \\
\hline $\mathrm{H}$ & 2.51094100 & -3.63955500 & 2.34114300 & $\mathrm{H}$ & 9.44134800 & -9.38214200 & -2.65189400 \\
\hline $\mathrm{C}$ & 10.97917800 & -5.69536700 & -1.87092700 & $\mathrm{H}$ & 9.97535200 & -8.30833900 & -3.95401000 \\
\hline $\mathrm{C}$ & 10.23925100 & -6.88251600 & -1.60035500 & $\mathrm{H}$ & 8.25723800 & -8.72485200 & -3.79258300 \\
\hline $\mathrm{C}$ & 12.25324100 & -5.53652400 & -1.25270500 & $\mathrm{C}$ & 7.77296600 & -7.50448500 & -1.35252300 \\
\hline
\end{tabular}




\begin{tabular}{|c|c|c|c|c|c|c|c|}
\hline 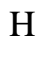 & 7.59340800 & -6.62270400 & -0.73179300 & $\mathrm{H}$ & 10.60161300 & -1.40170700 & 6.21654200 \\
\hline $\mathrm{H}$ & 7.96124300 & -8.36246000 & -0.69529700 & $\mathrm{H}$ & 11.95628600 & -2.38755800 & 5.65048400 \\
\hline $\mathrm{H}$ & 6.85540500 & -7.71888000 & -1.91443900 & $\mathrm{H}$ & 10.30700800 & -2.80874400 & 5.17961300 \\
\hline $\mathrm{C}$ & 14.41700400 & -4.83438000 & -2.37921600 & $\mathrm{C}$ & 11.71677200 & 1.10926800 & 5.41225000 \\
\hline $\mathrm{H}$ & 15.02800100 & -5.55383400 & -1.82102300 & $\mathrm{H}$ & 10.99261300 & 0.86036100 & 6.18987300 \\
\hline $\mathrm{H}$ & 15.05690700 & -3.98145300 & -2.63814900 & $\mathrm{H}$ & 11.60289900 & 2.17266400 & 5.2005130 \\
\hline $\mathrm{H}$ & 14.11290100 & -5.32425300 & -3.30984200 & $\mathrm{H}$ & 12.72797400 & 0.94574500 & 5.8121060 \\
\hline C & 13.73287800 & -3.69589000 & -0.25175000 & $\mathrm{C}$ & 12.42121600 & 1.99905300 & 2.42258700 \\
\hline $\mathrm{H}$ & 12.90890600 & -3.36224700 & 0.38026500 & $\mathrm{H}$ & 13.50676100 & 2.00488600 & 2.59536200 \\
\hline $\mathrm{H}$ & 14.34384500 & -2.82220800 & -0.51236300 & $\mathrm{H}$ & 11.98839200 & 2.81252200 & 3.0096190 \\
\hline $\mathrm{H}$ & 14.36535300 & -4.36589200 & 0.34210800 & $\mathrm{H}$ & 12.25844100 & 2.21788600 & 1.3636120 \\
\hline $\mathrm{C}$ & 11.51416000 & 0.27724700 & 4.18126900 & $\mathrm{C}$ & 11.95537300 & -0.53849500 & 0.49723900 \\
\hline $\mathrm{C}$ & 11.84275200 & 0.67527500 & 2.81809400 & $\mathrm{H}$ & 12.98846200 & -0.87107600 & 0.3372890 \\
\hline $\mathrm{C}$ & 11.66950100 & -0.47536700 & 1.96877300 & $\mathrm{H}$ & 11.85373700 & 0.44075500 & 0.0199830 \\
\hline $\mathrm{C}$ & 11.20886300 & -1.58041900 & 2.78433600 & $\mathrm{H}$ & 11.29181800 & -1.24067400 & -0.0160470 \\
\hline $\mathrm{C}$ & 11.16617900 & -1.10601300 & 4.15633500 & $\mathrm{C}$ & 11.04619500 & -3.00314800 & 2.34053100 \\
\hline Ir & 9.69950800 & 0.03465100 & 2.84938100 & $\mathrm{H}$ & 10.66078400 & -3.06175800 & 1.31912600 \\
\hline $\mathrm{N}$ & 8.79926200 & 2.07210700 & 2.85675200 & $\mathrm{H}$ & 10.35323800 & -3.54339400 & 2.98611500 \\
\hline $\mathrm{N}$ & 8.70289700 & 0.16687500 & 0.97527800 & $\mathrm{H}$ & 12.00837800 & -3.53085200 & 2.38029400 \\
\hline $\mathrm{H}$ & 8.99654700 & -0.57695500 & 0.30872500 & $\mathrm{H}$ & 7.13982800 & 2.22272900 & 1.49516300 \\
\hline $\mathrm{C}$ & 8.21576400 & 2.46094200 & 1.54435500 & $\mathrm{C}$ & 8.38348700 & 3.93532000 & 1.19149600 \\
\hline $\mathrm{C}$ & 8.90710400 & 1.55620800 & 0.48131100 & $\mathrm{C}$ & 9.61063000 & 4.58974100 & 1.36094100 \\
\hline S & 7.84545100 & 2.40437000 & 4.17872500 & $\mathrm{C}$ & 7.32490500 & 4.64231200 & 0.61008200 \\
\hline $\mathrm{O}$ & 8.64782600 & 2.07413900 & 5.37329900 & $\mathrm{C}$ & 9.77424600 & 5.91595700 & 0.96042400 \\
\hline $\mathrm{O}$ & 6.52623800 & 1.73195600 & 4.07894700 & $\mathrm{H}$ & 10.43611800 & 4.05652300 & 1.81959800 \\
\hline $\mathrm{C}$ & 7.51674100 & 4.21358500 & 4.28995500 & $\mathrm{C}$ & 7.48250000 & 5.97088400 & 0.21126700 \\
\hline $\mathrm{C}$ & 6.21520900 & 4.72348200 & 4.08675000 & $\mathrm{H}$ & 6.36822900 & 4.14757200 & 0.46296100 \\
\hline $\mathrm{C}$ & 8.56273200 & 5.03145000 & 4.77059200 & $\mathrm{C}$ & 8.70922900 & 6.61279600 & 0.38398700 \\
\hline $\mathrm{C}$ & 5.95307400 & 6.05815100 & 4.47057000 & $\mathrm{H}$ & 10.73397900 & 6.40747500 & 1.10051600 \\
\hline $\mathrm{C}$ & 8.27996600 & 6.36366300 & 5.12614300 & $\mathrm{H}$ & 6.64624000 & 6.50205300 & -0.23570200 \\
\hline $\mathrm{C}$ & 6.98310900 & 6.88315600 & 4.95627500 & $\mathrm{H}$ & 8.83510200 & 7.64698500 & 0.07451300 \\
\hline $\mathrm{C}$ & 5.07962100 & 3.95699900 & 3.43136100 & $\mathrm{H}$ & 9.97991600 & 1.75240700 & 0.55458100 \\
\hline $\mathrm{H}$ & 4.42964400 & 3.47501800 & 4.17182500 & $\mathrm{C}$ & 8.49869000 & 1.78970200 & -0.96520200 \\
\hline $\mathrm{H}$ & 5.43055400 & 3.16599400 & 2.77563000 & $\mathrm{C}$ & 7.47946500 & 1.06668300 & -1.59231400 \\
\hline $\mathrm{H}$ & 4.46493300 & 4.64124300 & 2.83995500 & $\mathrm{C}$ & 9.18773100 & 2.76055600 & -1.71025300 \\
\hline $\mathrm{C}$ & 4.53652100 & 6.59843700 & 4.37619300 & $\mathrm{C}$ & 7.14070100 & 1.32757800 & -2.92308900 \\
\hline $\mathrm{H}$ & 4.36377200 & 7.39652200 & 5.10143900 & $\mathrm{H}$ & 6.96091900 & 0.26846000 & -1.07338900 \\
\hline $\mathrm{H}$ & 3.79631600 & 5.82097400 & 4.58039000 & $\mathrm{C}$ & 8.84947000 & 3.02285700 & -3.03600200 \\
\hline $\mathrm{H}$ & 4.30832400 & 7.00759900 & 3.38107400 & $\mathrm{H}$ & 9.98816400 & 3.32634800 & -1.24186100 \\
\hline $\mathrm{C}$ & 6.70497800 & 8.34358300 & 5.26287100 & $\mathrm{C}$ & 7.81760600 & 2.30800600 & -3.64777800 \\
\hline $\mathrm{H}$ & 6.35974200 & 8.49252300 & 6.29622300 & $\mathrm{H}$ & 6.34813200 & 0.75176500 & -3.39181900 \\
\hline $\mathrm{H}$ & 5.93554900 & 8.75318200 & 4.60394500 & $\mathrm{H}$ & 9.39462400 & 3.78196600 & -3.59063000 \\
\hline $\mathrm{H}$ & 7.59641900 & 8.96018500 & 5.12838700 & $\mathrm{H}$ & 7.55151200 & 2.50612500 & -4.68266900 \\
\hline $\mathrm{C}$ & 9.37841800 & 7.22965600 & 5.71638100 & $\mathrm{~N}$ & 7.42017400 & -3.06664600 & 3.26332200 \\
\hline $\mathrm{H}$ & 8.97598400 & 7.97599700 & 6.40526400 & $\mathrm{C}$ & 7.05934300 & -1.78770400 & 3.61408800 \\
\hline $\mathrm{H}$ & 9.94248300 & 7.77116500 & 4.94274100 & $\mathrm{C}$ & 7.93265800 & -4.19496000 & 3.93931900 \\
\hline $\mathrm{H}$ & 10.09930300 & 6.63443500 & 6.28146900 & $\mathrm{C}$ & 8.31865100 & -5.27037900 & 3.10904500 \\
\hline $\mathrm{C}$ & 9.99648200 & 4.55286600 & 4.87690700 & $\mathrm{C}$ & 8.03621600 & -4.35216700 & 5.33070300 \\
\hline $\mathrm{H}$ & 10.67836600 & 5.33808200 & 4.53386700 & $\mathrm{C}$ & 8.80028700 & -6.45317500 & 3.65942000 \\
\hline $\mathrm{H}$ & 10.16826700 & 3.66833700 & 4.26943600 & $\mathrm{H}$ & 8.22572300 & -5.16180600 & 2.03241000 \\
\hline $\mathrm{H}$ & 10.26361200 & 4.29988400 & 5.90992400 & $\mathrm{C}$ & 8.53021800 & -5.54255300 & 5.86776400 \\
\hline $\mathrm{H}$ & 7.70400900 & 0.01050000 & 1.11143300 & $\mathrm{H}$ & 7.70490800 & -3.57765200 & 6.00179300 \\
\hline $\mathrm{H}$ & 8.22948800 & -0.89979900 & 3.42097400 & $\mathrm{C}$ & 8.91752800 & -6.59905700 & 5.04451500 \\
\hline $\mathrm{C}$ & 10.97964600 & -1.97016600 & 5.36527900 & $\mathrm{H}$ & 9.08527900 & -7.26425600 & 2.99580800 \\
\hline
\end{tabular}




\begin{tabular}{|c|c|c|c|c|c|c|c|}
\hline $\mathrm{H}$ & 8.59538700 & -5.64015400 & 6.94829600 & $\mathrm{C}$ & 4.77197700 & -2.99878200 & -1.11651300 \\
\hline $\mathrm{H}$ & 7.38945700 & -3.22974400 & 2.24368800 & $\mathrm{C}$ & 2.61526400 & -2.86951100 & -0.06025500 \\
\hline $\mathrm{C}$ & 6.14270800 & -1.18565300 & 2.54913200 & $\mathrm{H}$ & 1.93090800 & -4.63692700 & 0.97332100 \\
\hline $\mathrm{H}$ & 6.40456100 & -1.52446900 & 1.54363300 & $\mathrm{C}$ & 3.63616800 & -2.22384800 & -0.78653300 \\
\hline $\mathrm{H}$ & 5.11718400 & -1.52626500 & 2.72610500 & $\mathrm{H}$ & 1.72703200 & -2.29854300 & 0.19123200 \\
\hline $\mathrm{H}$ & 6.14185700 & -0.09761100 & 2.62534600 & $\mathrm{O}$ & 7.92304100 & -3.89695200 & -0.81594900 \\
\hline $\mathrm{H}$ & 9.29440400 & -7.52322600 & 5.47324000 & $\mathrm{O}$ & 5.92011200 & -2.40463600 & -1.62362100 \\
\hline $\mathrm{C}$ & 6.60607500 & -1.37548600 & 5.04581700 & $\mathrm{P}$ & 7.25464600 & -2.38166600 & -0.61105500 \\
\hline $\mathrm{C}$ & 5.57742400 & -2.32076100 & 5.70543000 & $\mathrm{O}$ & 6.78285300 & -2.37326800 & 0.82971200 \\
\hline $\mathrm{C}$ & 7.69818200 & -0.95231300 & 6.04771600 & $\mathrm{O}$ & 8.17346400 & -1.31156300 & -1.10503000 \\
\hline $\mathrm{C}$ & 4.80048800 & -3.22928400 & 4.96728500 & $\mathrm{C}$ & 10.47600200 & -4.04678900 & -2.48164400 \\
\hline $\mathrm{C}$ & 5.36191500 & -2.24071400 & 7.10134500 & $\mathrm{C}$ & 11.43309000 & -4.82678300 & -1.78974300 \\
\hline $\mathrm{C}$ & 7.03378600 & -0.31391200 & 7.26820400 & $\mathrm{C}$ & 10.87113500 & -2.82974700 & -3.09045300 \\
\hline $\mathrm{H}$ & 8.35330300 & -0.22608400 & 5.56763900 & $\mathrm{C}$ & 12.76579800 & -4.39676200 & -1.75657100 \\
\hline $\mathrm{C}$ & 3.84149200 & -4.03912700 & 5.57356400 & $\mathrm{C}$ & 12.21593400 & -2.44382600 & -3.01933800 \\
\hline $\mathrm{H}$ & 4.95503400 & -3.33389800 & 3.90101100 & $\mathrm{C}$ & 13.18672000 & -3.22277200 & -2.38228700 \\
\hline $\mathrm{C}$ & 4.38274100 & -3.05258800 & 7.69220300 & $\mathrm{H}$ & 13.51014200 & -5.01371100 & -1.25826000 \\
\hline $\mathrm{C}$ & 6.18111300 & -1.34202900 & 8.01469400 & $\mathrm{H}$ & 12.51806200 & -1.52310300 & -3.51130000 \\
\hline $\mathrm{H}$ & 7.79321800 & 0.10408000 & 7.93928400 & $\mathrm{C}$ & 3.39958400 & -0.82271600 & -1.28128700 \\
\hline $\mathrm{C}$ & 3.62296500 & -3.94890000 & 6.94767600 & $\mathrm{C}$ & 3.35303000 & -0.56896600 & -2.68224300 \\
\hline $\mathrm{H}$ & 3.27586500 & -4.74381500 & 4.96972800 & $\mathrm{C}$ & 3.05754700 & 0.22699900 & -0.38951500 \\
\hline $\mathrm{H}$ & 4.22578900 & -2.97625000 & 8.76663800 & $\mathrm{C}$ & 2.96112000 & 0.69523700 & -3.13699800 \\
\hline $\mathrm{H}$ & 6.84004400 & -1.98232300 & 8.62211200 & $\mathrm{C}$ & 2.66205000 & 1.46808100 & -0.90567100 \\
\hline $\mathrm{H}$ & 2.87508300 & -4.57054600 & 7.43278200 & $\mathrm{C}$ & 2.59351400 & 1.72886500 & -2.27217500 \\
\hline $\mathrm{H}$ & 6.07583700 & -0.43701300 & 4.84748500 & $\mathrm{H}$ & 2.91700700 & 0.86675300 & -4.20957000 \\
\hline $\mathrm{H}$ & 8.31295400 & -1.80050600 & 6.36675900 & $\mathrm{H}$ & 2.39183100 & 2.26160800 & -0.21259500 \\
\hline $\mathrm{H}$ & 6.41862700 & 0.52931100 & 6.93217700 & $\mathrm{C}$ & 9.89674900 & -1.97624800 & -3.90398700 \\
\hline \multirow[t]{2}{*}{$\mathrm{H}$} & 5.51352800 & -0.84491100 & 8.73062500 & $\mathrm{H}$ & 8.88205500 & -2.28062100 & -3.63306700 \\
\hline & & & & $\mathrm{C}$ & 14.66448200 & -2.84949900 & -2.40868900 \\
\hline 13-SS & & & & $\mathrm{H}$ & 15.20331800 & -3.63528800 & -1.86101900 \\
\hline $\mathrm{C}$ & 4.56744700 & -5.89957500 & -2.90798900 & $\mathrm{C}$ & 11.07928900 & -6.17594000 & -1.16073000 \\
\hline $\mathrm{C}$ & 6.82476500 & -6.04556000 & -3.59688800 & $\mathrm{H}$ & 9.98931800 & -6.22533600 & -1.06673100 \\
\hline $\mathrm{C}$ & 5.48581800 & -6.69566800 & -3.84738800 & $\mathrm{C}$ & 10.00329900 & -0.47300800 & -3.59360900 \\
\hline $\mathrm{H}$ & 4.23243000 & -4.98737000 & -3.41438600 & $\mathrm{H}$ & 9.22965700 & 0.07375600 & -4.14521300 \\
\hline $\mathrm{H}$ & 3.67512800 & -6.44826600 & -2.59278500 & $\mathrm{H}$ & 10.97259900 & -0.05553800 & -3.89333500 \\
\hline $\mathrm{H}$ & 5.50826300 & -7.76205800 & -3.57889200 & $\mathrm{H}$ & 9.84012400 & -0.28929700 & -2.53020400 \\
\hline $\mathrm{H}$ & 5.17512000 & -6.64080600 & -4.89717000 & $\mathrm{C}$ & 10.08122700 & -2.22501000 & -5.41607800 \\
\hline $\mathrm{C}$ & 5.47677700 & -5.49046800 & -1.68949500 & $\mathrm{H}$ & 9.36661400 & -1.62380500 & -5.99154400 \\
\hline $\mathrm{C}$ & 5.44038100 & -6.62750500 & -0.62252800 & $\mathrm{H}$ & 9.92311100 & -3.27656800 & -5.67725300 \\
\hline $\mathrm{C}$ & 4.74505300 & -4.38378600 & -0.93758900 & $\mathrm{H}$ & 11.09207600 & -1.94699800 & -5.74040700 \\
\hline $\mathrm{C}$ & 4.06891300 & -6.44386600 & 0.08173100 & $\mathrm{C}$ & 14.95322600 & -1.51678700 & -1.69569900 \\
\hline $\mathrm{H}$ & 5.56320500 & -7.62180100 & -1.06522000 & $\mathrm{H}$ & 16.02745300 & -1.29506600 & -1.70721900 \\
\hline $\mathrm{H}$ & 6.25621100 & -6.47558700 & 0.09238200 & $\mathrm{H}$ & 14.62417900 & -1.55275700 & -0.65167500 \\
\hline $\mathrm{H}$ & 4.10813000 & -6.72318600 & 1.14187700 & $\mathrm{H}$ & 14.43470300 & -0.68260500 & -2.18292000 \\
\hline $\mathrm{H}$ & 3.29289500 & -7.07220500 & -0.37651300 & $\mathrm{C}$ & 15.21758000 & -2.83324200 & -3.84727100 \\
\hline $\mathrm{C}$ & 6.81747600 & -5.31356900 & -2.39878400 & $\mathrm{H}$ & 16.29599500 & -2.63250300 & -3.84582200 \\
\hline $\mathrm{C}$ & 7.95711300 & -4.59324700 & -2.01346600 & $\mathrm{H}$ & 14.73433800 & -2.05531800 & -4.45017100 \\
\hline $\mathrm{C}$ & 7.95929100 & -6.10196100 & -4.39511800 & $\mathrm{H}$ & 15.04937100 & -3.79391000 & -4.34634600 \\
\hline $\mathrm{C}$ & 9.13296600 & -4.65632000 & -2.79534500 & $\mathrm{C}$ & 11.51851400 & -7.34444100 & -2.06780800 \\
\hline $\mathrm{C}$ & 9.09707800 & -5.41903200 & -3.97778700 & $\mathrm{H}$ & 12.60591000 & -7.33964000 & -2.21157400 \\
\hline $\mathrm{H}$ & 7.96301000 & -6.66550500 & -5.32477400 & $\mathrm{H}$ & 11.04793800 & -7.28190600 & -3.05371800 \\
\hline $\mathrm{H}$ & 10.00072900 & -5.46463100 & -4.57856300 & $\mathrm{H}$ & 11.24178300 & -8.30789100 & -1.62094900 \\
\hline $\mathrm{C}$ & 3.77632900 & -4.96664800 & -0.11151400 & $\mathrm{C}$ & 11.66976900 & -6.35528400 & 0.24995800 \\
\hline $\mathrm{C}$ & 2.71097400 & -4.20015600 & 0.35384200 & $\mathrm{H}$ & 11.33284600 & -7.30653500 & 0.68120200 \\
\hline
\end{tabular}




\begin{tabular}{|c|c|c|c|c|c|c|c|}
\hline $\mathrm{H}$ & 11.36768800 & -5.54380000 & 0.92040500 & $\mathrm{H}$ & 5.57260800 & 2.97985300 & 3.85631900 \\
\hline $\mathrm{H}$ & 12.76515100 & -6.38504400 & 0.23763900 & $\mathrm{H}$ & 4.66359800 & 4.40828100 & 4.35637900 \\
\hline $\mathrm{C}$ & 3.64835500 & -1.62488100 & -3.74945500 & $\mathrm{C}$ & 4.84809500 & 5.85854700 & 6.36879900 \\
\hline $\mathrm{H}$ & 3.90144200 & -2.56123100 & -3.25068200 & $\mathrm{H}$ & 4.72253200 & 6.43265200 & 7.28943600 \\
\hline $\mathrm{C}$ & 2.10775200 & 3.07726700 & -2.78887100 & $\mathrm{H}$ & 4.08096500 & 5.08012900 & 6.37581200 \\
\hline $\mathrm{H}$ & 1.88838800 & 3.69758000 & -1.90881400 & $\mathrm{H}$ & 4.61527000 & 6.53210100 & 5.53093100 \\
\hline $\mathrm{C}$ & 3.10047300 & 0.09005000 & 1.13283900 & $\mathrm{C}$ & 7.10163900 & 7.21631200 & 7.63666300 \\
\hline $\mathrm{H}$ & 3.48269600 & -0.90527100 & 1.37228700 & $\mathrm{H}$ & 6.78026900 & 7.08803800 & 8.68062700 \\
\hline $\mathrm{C}$ & 3.18168000 & 3.81211100 & -3.61251100 & $\mathrm{H}$ & 6.33873200 & 7.81810600 & 7.13664800 \\
\hline $\mathrm{H}$ & 2.81443600 & 4.79350000 & -3.93689600 & $\mathrm{H}$ & 8.01469500 & 7.81554100 & 7.65167700 \\
\hline $\mathrm{H}$ & 3.45086800 & 3.24491500 & -4.51191400 & $\mathrm{C}$ & 9.73269700 & 5.92012300 & 7.69683400 \\
\hline $\mathrm{H}$ & 4.09351300 & 3.96485800 & -3.02594400 & $\mathrm{H}$ & 9.37110800 & 6.47453800 & 8.56604400 \\
\hline $\mathrm{C}$ & 0.79920700 & 2.93693300 & -3.59113400 & $\mathrm{H}$ & 10.32147500 & 6.61972900 & 7.08545900 \\
\hline $\mathrm{H}$ & 0.42789800 & 3.92107700 & -3.90213300 & $\mathrm{H}$ & 10.42462600 & 5.16190200 & 8.07082100 \\
\hline $\mathrm{H}$ & 0.01948800 & 2.44963200 & -2.99534200 & $\mathrm{C}$ & 10.22641800 & 3.56307700 & 6.13645900 \\
\hline $\mathrm{H}$ & 0.95118600 & 2.33675500 & -4.49620200 & $\mathrm{H}$ & 10.93869700 & 4.38735400 & 6.02439000 \\
\hline $\mathrm{C}$ & 2.40713000 & -1.91508100 & -4.61786100 & $\mathrm{H}$ & 10.36311600 & 2.88383600 & 5.29956800 \\
\hline $\mathrm{H}$ & 2.09615200 & -1.03543300 & -5.19350700 & $\mathrm{H}$ & 10.48528800 & 3.01500100 & 7.05055800 \\
\hline $\mathrm{H}$ & 1.55656500 & -2.22858200 & -4.00182800 & $\mathrm{H}$ & 7.72239100 & 0.26051900 & 1.51324400 \\
\hline $\mathrm{H}$ & 2.62360600 & -2.71799000 & -5.33353700 & $\mathrm{H}$ & 8.65664500 & -0.72803800 & 3.47921400 \\
\hline $\mathrm{C}$ & 4.85990200 & -1.23627000 & -4.61829500 & $\mathrm{C}$ & 10.95758600 & -2.76856900 & 4.33044800 \\
\hline $\mathrm{H}$ & 5.74605100 & -1.07542400 & -3.99788900 & $\mathrm{H}$ & 10.48080400 & -2.54437000 & 5.28871600 \\
\hline $\mathrm{H}$ & 4.66823600 & -0.32113000 & -5.19205400 & $\mathrm{H}$ & 11.85245900 & -3.37332000 & 4.53558200 \\
\hline $\mathrm{H}$ & 5.08487900 & -2.03492600 & -5.33595800 & $\mathrm{H}$ & 10.26778800 & -3.38178400 & 3.74450200 \\
\hline $\mathrm{C}$ & 1.69975100 & 0.21371600 & 1.76744100 & $\mathrm{C}$ & 11.87487000 & -0.04127500 & 5.70518700 \\
\hline $\mathrm{H}$ & 1.26870600 & 1.20630500 & 1.59106000 & $\mathrm{H}$ & 11.04080000 & -0.47458900 & 6.26141800 \\
\hline $\mathrm{H}$ & 1.75595800 & 0.06527800 & 2.85301900 & $\mathrm{H}$ & 11.89095000 & 1.02428600 & 5.94383700 \\
\hline $\mathrm{H}$ & 0.99999900 & -0.52461100 & 1.36047000 & $\mathrm{H}$ & 12.81189100 & -0.48335900 & 6.07581000 \\
\hline $\mathrm{C}$ & 4.05733400 & 1.11953200 & 1.76445500 & $\mathrm{C}$ & 12.83538000 & 1.96235200 & 3.40710400 \\
\hline $\mathrm{H}$ & 5.06273700 & 1.03610500 & 1.34063200 & $\mathrm{H}$ & 13.87551600 & 1.86451600 & 3.74885600 \\
\hline $\mathrm{H}$ & 4.13388000 & 0.97315600 & 2.84704700 & $\mathrm{H}$ & 12.29203800 & 2.51950800 & 4.17511100 \\
\hline $\mathrm{H}$ & 3.71346000 & 2.14610200 & 1.59410400 & $\mathrm{H}$ & 12.84670100 & 2.56676000 & 2.49522600 \\
\hline $\mathrm{C}$ & 11.72334700 & -0.26966000 & 4.22833700 & $\mathrm{C}$ & 12.44562800 & 0.49543000 & 0.57982300 \\
\hline $\mathrm{C}$ & 12.22597500 & 0.61197700 & 3.17273500 & $\mathrm{H}$ & 13.46829200 & 0.20055800 & 0.31195200 \\
\hline C & 12.06739600 & -0.03802800 & 1.93173800 & $\mathrm{H}$ & 12.40351200 & 1.58893900 & 0.54596300 \\
\hline $\mathrm{C}$ & 11.46551400 & -1.35345500 & 2.17548900 & $\mathrm{H}$ & 11.78698600 & 0.10834200 & -0.20397800 \\
\hline $\mathrm{C}$ & 11.33483600 & -1.50886500 & 3.60463200 & $\mathrm{C}$ & 11.27007800 & -2.42384600 & 1.14093100 \\
\hline Ir & 9.94060100 & 0.03910800 & 2.92835100 & $\mathrm{H}$ & 10.85767300 & -2.03269300 & 0.20666100 \\
\hline $\mathrm{N}$ & 8.96017700 & 1.91698800 & 3.50891400 & $\mathrm{H}$ & 10.57481200 & -3.18797300 & 1.49920700 \\
\hline $\mathrm{N}$ & 8.68066000 & 0.50665000 & 1.26235800 & $\mathrm{H}$ & 12.21826700 & -2.92123800 & 0.89667000 \\
\hline $\mathrm{H}$ & 8.85858100 & -0.03260000 & 0.40372800 & $\mathrm{H}$ & 7.21341100 & 2.47908200 & 2.38988200 \\
\hline $\mathrm{C}$ & 8.30357800 & 2.63125900 & 2.37797100 & $\mathrm{C}$ & 8.57393300 & 4.13238700 & 2.37611400 \\
\hline $\mathrm{C}$ & 8.81028800 & 1.97409100 & 1.05655000 & $\mathrm{C}$ & 9.85739300 & 4.63169100 & 2.63480400 \\
\hline$S$ & 8.01313000 & 1.79333000 & 4.86467900 & $\mathrm{C}$ & 7.55498000 & 5.03800800 & 2.05990300 \\
\hline $\mathrm{O}$ & 8.78012600 & 1.08982900 & 5.90936900 & $\mathrm{C}$ & 10.11501300 & 6.00157800 & 2.57550800 \\
\hline $\mathrm{O}$ & 6.67380700 & 1.21344300 & 4.56257900 & $\mathrm{H}$ & 10.64763300 & 3.93776800 & 2.90249400 \\
\hline $\mathrm{C}$ & 7.72699200 & 3.49176700 & 5.53056900 & $\mathrm{C}$ & 7.80730200 & 6.41025300 & 2.00371300 \\
\hline $\mathrm{C}$ & 6.44453100 & 4.08335400 & 5.51994500 & $\mathrm{H}$ & 6.55521500 & 4.66491700 & 1.85124500 \\
\hline $\mathrm{C}$ & 8.81235800 & 4.10611800 & 6.19460600 & $\mathrm{C}$ & 9.08959000 & 6.89711700 & 2.25995100 \\
\hline $\mathrm{C}$ & 6.24347700 & 5.26602300 & 6.26767200 & $\mathrm{H}$ & 11.11676500 & 6.37192400 & 2.78028100 \\
\hline $\mathrm{C}$ & 8.59041400 & 5.29514000 & 6.91572600 & $\mathrm{H}$ & 7.00106500 & 7.09731300 & 1.75953200 \\
\hline $\mathrm{C}$ & 7.31457600 & 5.88817900 & 6.93223400 & $\mathrm{H}$ & 9.28876100 & 7.96489100 & 2.21814800 \\
\hline $\mathrm{C}$ & 5.26506000 & 3.56809000 & 4.71453400 & $\mathrm{H}$ & 9.88570200 & 2.17018500 & 0.99660600 \\
\hline $\mathrm{H}$ & 4.61178400 & 2.92052300 & 5.31314000 & $\mathrm{C}$ & 8.17315300 & 2.49507500 & -0.22356700 \\
\hline
\end{tabular}




\begin{tabular}{|c|c|c|c|c|c|c|c|}
\hline $\mathrm{C}$ & 7.07385500 & 1.87243100 & -0.82648800 & $\mathrm{H}$ & 4.50093900 & -4.85539300 & -4.00363500 \\
\hline $\mathrm{C}$ & 8.73025300 & 3.62167900 & -0.84961400 & $\mathrm{H}$ & 3.94120700 & -6.43038700 & -3.43358100 \\
\hline $\mathrm{C}$ & 6.53940900 & 2.36565700 & -2.01929000 & $\mathrm{H}$ & 5.89009700 & -7.54448600 & -4.43520400 \\
\hline $\mathrm{H}$ & 6.64974000 & 0.96809400 & -0.40477800 & $\mathrm{H}$ & 5.63511600 & -6.25281200 & -5.60630200 \\
\hline $\mathrm{C}$ & 8.19439200 & 4.12101800 & -2.03513800 & $\mathrm{C}$ & 5.62221800 & -5.54636600 & -2.26068600 \\
\hline $\mathrm{H}$ & 9.58954500 & 4.11294500 & -0.40107000 & $\mathrm{C}$ & 5.56068900 & -6.81677800 & -1.35728000 \\
\hline $\mathrm{C}$ & 7.09525100 & 3.49258500 & -2.62518400 & $\mathrm{C}$ & 4.79533900 & -4.57318900 & -1.42711900 \\
\hline $\mathrm{H}$ & 5.69694700 & 1.85423500 & -2.47467400 & $\mathrm{C}$ & 4.14048300 & -6.77542900 & -0.73762900 \\
\hline $\mathrm{H}$ & 8.64280900 & 4.99383500 & -2.50279800 & $\mathrm{H}$ & 5.76009200 & -7.73779100 & -1.91549000 \\
\hline $\mathrm{H}$ & 6.68488500 & 3.87006000 & -3.55822200 & $\mathrm{H}$ & 6.31646700 & -6.72974500 & -0.56866600 \\
\hline $\mathrm{N}$ & 6.42226800 & -3.62724100 & 3.13135200 & $\mathrm{H}$ & 4.11671500 & -7.19424800 & 0.27559300 \\
\hline $\mathrm{C}$ & 5.99162900 & -2.80742200 & 4.05103200 & $\mathrm{H}$ & 3.42356200 & -7.35772700 & -1.33272000 \\
\hline $\mathrm{C}$ & 6.93760500 & -4.95764600 & 3.24692000 & $\mathrm{C}$ & 7.00689000 & -5.24683800 & -2.81995200 \\
\hline $\mathrm{C}$ & 7.99283500 & -5.29776900 & 2.39153700 & $\mathrm{C}$ & 8.07944900 & -4.56952300 & -2.22681600 \\
\hline $\mathrm{C}$ & 6.36654500 & -5.92030800 & 4.08953900 & $\mathrm{C}$ & 8.36085400 & -5.76429200 & -4.76711500 \\
\hline $\mathrm{C}$ & 8.51237400 & -6.59151600 & 2.42768400 & $\mathrm{C}$ & 9.33773900 & -4.54284900 & -2.87150400 \\
\hline $\mathrm{H}$ & 8.37601000 & -4.56077000 & 1.69296400 & $\mathrm{C}$ & 9.43816700 & -5.14603400 & -4.14029100 \\
\hline $\mathrm{C}$ & 6.89799300 & -7.20968200 & 4.11498000 & $\mathrm{H}$ & 8.47392200 & -6.20186600 & -5.75586500 \\
\hline $\mathrm{H}$ & 5.50188900 & -5.68177000 & 4.69863900 & $\mathrm{H}$ & 10.40455200 & -5.12370900 & -4.63538500 \\
\hline $\mathrm{C}$ & 7.97582100 & -7.54625700 & 3.29422500 & $\mathrm{C}$ & 3.80379200 & -5.29668700 & -0.75190300 \\
\hline $\mathrm{H}$ & 9.33491200 & -6.85066800 & 1.77006900 & $\mathrm{C}$ & 2.67935100 & -4.64266200 & -0.26052700 \\
\hline $\mathrm{H}$ & 6.45511100 & -7.95401300 & 4.77037400 & $\mathrm{C}$ & 4.76887500 & -3.17604000 & -1.43072800 \\
\hline $\mathrm{H}$ & 6.49091800 & -3.22152900 & 2.14825200 & $\mathrm{C}$ & 2.55015000 & -3.27463300 & -0.50214800 \\
\hline $\mathrm{C}$ & 5.43566000 & -1.50404400 & 3.59465600 & $\mathrm{H}$ & 1.88585200 & -5.18710000 & 0.24572100 \\
\hline $\mathrm{H}$ & 5.32438700 & -1.48001900 & 2.51135300 & $\mathrm{C}$ & 3.57835800 & -2.49961500 & -1.07274800 \\
\hline $\mathrm{H}$ & 4.46909000 & -1.33660500 & 4.08281700 & $\mathrm{H}$ & 1.62103000 & -2.77799000 & -0.24572200 \\
\hline $\mathrm{H}$ & 6.08575500 & -0.67327400 & 3.90304700 & $\mathrm{O}$ & 7.87708600 & -4.01125400 & -0.97411200 \\
\hline $\mathrm{H}$ & 8.38380700 & -8.55262000 & 3.31652600 & $\mathrm{O}$ & 5.91090500 & -2.47860200 & -1.81662100 \\
\hline $\mathrm{C}$ & 6.08895800 & -3.09087400 & 5.53018500 & $\mathrm{P}$ & 7.19538500 & -2.49709800 & -0.74390300 \\
\hline $\mathrm{C}$ & 4.71416600 & -3.20187000 & 6.20273600 & $\mathrm{O}$ & 6.66290200 & -2.50778000 & 0.66787100 \\
\hline $\mathrm{C}$ & 6.98480200 & -2.01109500 & 6.19756200 & $\mathrm{O}$ & 8.17459400 & -1.44145500 & -1.16241800 \\
\hline $\mathrm{C}$ & 3.63891400 & -3.79791100 & 5.52332500 & $\mathrm{C}$ & 10.64273900 & -4.02463900 & -2.32212000 \\
\hline $\mathrm{C}$ & 4.52861100 & -2.75895600 & 7.52677300 & $\mathrm{C}$ & 11.46775700 & -4.91048600 & -1.58618600 \\
\hline $\mathrm{C}$ & 2.39731700 & -3.96709900 & 6.13317200 & $\mathrm{C}$ & 11.16221000 & -2.77760200 & -2.75104300 \\
\hline $\mathrm{H}$ & 3.76496100 & -4.12287000 & 4.49267600 & $\mathrm{C}$ & 12.79442400 & -4.54475500 & -1.32315300 \\
\hline $\mathrm{C}$ & 3.27523500 & -2.94287100 & 8.12810200 & $\mathrm{C}$ & 12.49407100 & -2.45975400 & -2.45178400 \\
\hline $\mathrm{C}$ & 2.21573600 & -3.53928700 & 7.44868700 & $\mathrm{C}$ & 13.33748700 & -3.33621100 & -1.76221300 \\
\hline $\mathrm{H}$ & 1.58073500 & -4.42635600 & 5.58312500 & $\mathrm{H}$ & 13.44011500 & -5.23986900 & -0.79146100 \\
\hline $\mathrm{H}$ & 3.13380900 & -2.60408300 & 9.15236900 & $\mathrm{H}$ & 12.89373000 & -1.51385800 & -2.80853800 \\
\hline $\mathrm{H}$ & 1.25567100 & -3.66714700 & 7.94125100 & $\mathrm{C}$ & 3.26720900 & -1.06741900 & -1.41650000 \\
\hline $\mathrm{H}$ & 6.60723900 & -4.04633900 & 5.66372700 & $\mathrm{C}$ & 3.22215200 & -0.67483300 & -2.78494700 \\
\hline $\mathrm{C}$ & 5.62848200 & -2.06011100 & 8.30807800 & $\mathrm{C}$ & 2.83339700 & -0.14332500 & -0.42877200 \\
\hline $\mathrm{H}$ & 5.40494700 & -0.98339900 & 8.33396300 & $\mathrm{C}$ & 2.75022800 & 0.59945500 & -3.11972000 \\
\hline $\mathrm{H}$ & 5.60544800 & -2.39791100 & 9.35185500 & $\mathrm{C}$ & 2.36064700 & 1.11465000 & -0.82811600 \\
\hline $\mathrm{C}$ & 7.02348000 & -2.25717500 & 7.70704600 & $\mathrm{C}$ & 2.30191600 & 1.51213800 & -2.16237500 \\
\hline $\mathrm{H}$ & 7.73193600 & -1.56248100 & 8.17061100 & $\mathrm{H}$ & 2.71651100 & 0.87756600 & -4.17016300 \\
\hline $\mathrm{H}$ & 7.38478100 & -3.27458900 & 7.91437400 & $\mathrm{H}$ & 2.01734300 & 1.81214900 & -0.06771900 \\
\hline $\mathrm{H}$ & 7.98804400 & -2.05096000 & 5.76236800 & $\mathrm{C}$ & 10.34786800 & -1.81238200 & -3.61476800 \\
\hline \multirow[t]{2}{*}{$\mathrm{H}$} & 6.60593900 & -1.00431100 & 6.00042800 & $\mathrm{H}$ & 9.29844000 & -2.10981200 & -3.54723000 \\
\hline & & & & $\mathrm{C}$ & 14.81615600 & -3.03295500 & -1.54714300 \\
\hline TS-7-SS & & & & $\mathrm{H}$ & 15.24181200 & -3.88558700 & -0.99997600 \\
\hline $\mathrm{C}$ & 4.83230700 & -5.81659400 & -3.59498500 & $\mathrm{C}$ & 10.99092700 & -6.30160300 & -1.16182800 \\
\hline $\mathrm{C}$ & 7.14835000 & -5.81961300 & -4.09259000 & $\mathrm{H}$ & 9.89647500 & -6.30306000 & -1.19187600 \\
\hline $\mathrm{C}$ & 5.85588800 & -6.45134500 & -4.55098000 & $\mathrm{C}$ & 10.42050600 & -0.35503200 & -3.12691800 \\
\hline
\end{tabular}




\begin{tabular}{|c|c|c|c|c|c|c|c|}
\hline $\mathrm{H}$ & 9.77447300 & 0.27647900 & -3.74749100 & $\mathrm{C}$ & 11.73010500 & 0.15657300 & 3.47880800 \\
\hline $\mathrm{H}$ & 11.43659000 & 0.05517600 & -3.18976300 & $\mathrm{C}$ & 11.53638500 & -0.45680800 & 2.19558200 \\
\hline $\mathrm{H}$ & 10.05652000 & -0.27893400 & -2.10083700 & $\mathrm{C}$ & 11.01200500 & -1.80004900 & 2.41552300 \\
\hline $\mathrm{C}$ & 10.78028900 & -1.90161700 & -5.09354800 & $\mathrm{C}$ & 10.91157200 & -1.98827400 & 3.83837100 \\
\hline $\mathrm{H}$ & 10.17284100 & -1.22702400 & -5.70926400 & Ir & 9.54205100 & -0.34146900 & 3.18037000 \\
\hline $\mathrm{H}$ & 10.66396000 & -2.91522500 & -5.49060800 & $\mathrm{~N}$ & 8.82576200 & 1.75924800 & 3.47845700 \\
\hline $\mathrm{H}$ & 11.83179600 & -1.61200000 & -5.21579600 & $\mathrm{~N}$ & 8.35986300 & 0.03834600 & 1.42918900 \\
\hline $\mathrm{C}$ & 15.05086700 & -1.77741100 & -0.68836300 & $\mathrm{H}$ & 8.57068600 & -0.55168000 & 0.60712000 \\
\hline $\mathrm{H}$ & 16.12321900 & -1.60805000 & -0.53138700 & $\mathrm{C}$ & 8.08234300 & 2.29763700 & 2.30892700 \\
\hline $\mathrm{H}$ & 14.57747800 & -1.87961500 & 0.29497900 & $\mathrm{C}$ & 8.54267400 & 1.47702900 & 1.07702500 \\
\hline $\mathrm{H}$ & 14.64035900 & -0.88271100 & -1.17179600 & S & 8.13984600 & 2.11480900 & 4.95627200 \\
\hline $\mathrm{C}$ & 15.57046000 & -2.92681800 & -2.88710000 & $\mathrm{O}$ & 9.08846500 & 1.64545400 & 5.98595000 \\
\hline $\mathrm{H}$ & 16.64430000 & -2.77969200 & -2.71811800 & $\mathrm{O}$ & 6.75674300 & 1.58902200 & 5.05426000 \\
\hline $\mathrm{H}$ & 15.20664100 & -2.08077400 & -3.48208900 & $\mathrm{C}$ & 7.98546200 & 3.93960200 & 5.21405500 \\
\hline $\mathrm{H}$ & 15.43928000 & -3.83495800 & -3.48528700 & $\mathrm{C}$ & 6.71327700 & 4.55510200 & 5.21856100 \\
\hline $\mathrm{C}$ & 11.48231500 & -7.37642500 & -2.15462100 & $\mathrm{C}$ & 9.14191600 & 4.64320200 & 5.61219600 \\
\hline $\mathrm{H}$ & 12.57848900 & -7.41542400 & -2.17499900 & $\mathrm{C}$ & 6.60853700 & 5.86919300 & 5.72839000 \\
\hline $\mathrm{H}$ & 11.13214500 & -7.17062200 & -3.17057300 & $\mathrm{C}$ & 9.01394200 & 5.95784300 & 6.09845800 \\
\hline $\mathrm{H}$ & 11.11497900 & -8.36858900 & -1.86368100 & $\mathrm{C}$ & 7.75221700 & 6.57858700 & 6.13618400 \\
\hline $\mathrm{C}$ & 11.40858100 & -6.68054100 & 0.27071000 & $\mathrm{C}$ & 5.44461100 & 3.93229300 & 4.66291000 \\
\hline $\mathrm{H}$ & 10.96923500 & -7.64713100 & 0.54513700 & $\mathrm{H}$ & 4.84166000 & 3.46416200 & 5.45027900 \\
\hline $\mathrm{H}$ & 11.07156100 & -5.93854600 & 1.00103400 & $\mathrm{H}$ & 5.64197200 & 3.15728000 & 3.92947700 \\
\hline $\mathrm{H}$ & 12.49541700 & -6.78462700 & 0.36892100 & $\mathrm{H}$ & 4.83393500 & 4.70427400 & 4.18589000 \\
\hline $\mathrm{C}$ & 3.61269100 & -1.59320000 & -3.94468400 & $\mathrm{C}$ & 5.23849800 & 6.51292100 & 5.85611500 \\
\hline $\mathrm{H}$ & 3.90840900 & -2.55998200 & -3.53580700 & $\mathrm{H}$ & 5.22124200 & 7.26258900 & 6.65010100 \\
\hline $\mathrm{C}$ & 1.75636000 & 2.88064400 & -2.54916200 & $\mathrm{H}$ & 4.47008300 & 5.77584700 & 6.10182600 \\
\hline $\mathrm{H}$ & 1.50865300 & 3.40356400 & -1.61499000 & $\mathrm{H}$ & 4.92100900 & 7.01270500 & 4.92921500 \\
\hline $\mathrm{C}$ & 2.83295800 & -0.44627100 & 1.07273600 & $\mathrm{C}$ & 7.62937100 & 8.02423400 & 6.58247900 \\
\hline $\mathrm{H}$ & 3.37576700 & -1.38355800 & 1.23082600 & $\mathrm{H}$ & 7.42227500 & 8.11019300 & 7.65903000 \\
\hline $\mathrm{C}$ & 2.80048600 & 3.73553800 & -3.29214600 & $\mathrm{H}$ & 6.82183300 & 8.53966500 & 6.05697500 \\
\hline $\mathrm{H}$ & 2.40393700 & 4.73728100 & -3.49801000 & $\mathrm{H}$ & 8.54414900 & 8.58677200 & 6.38373100 \\
\hline $\mathrm{H}$ & 3.07229900 & 3.28341300 & -4.25379200 & $\mathrm{C}$ & 10.24439100 & 6.69124700 & 6.60143700 \\
\hline $\mathrm{H}$ & 3.71712700 & 3.84402200 & -2.70242400 & $\mathrm{H}$ & 9.99222500 & 7.41260700 & 7.38218700 \\
\hline $\mathrm{C}$ & 0.45733000 & 2.76221300 & -3.36990600 & $\mathrm{H}$ & 10.75629200 & 7.24403300 & 5.79998100 \\
\hline $\mathrm{H}$ & 0.04737600 & 3.75477500 & -3.59362900 & $\mathrm{H}$ & 10.97456300 & 6.00231900 & 7.03263900 \\
\hline $\mathrm{H}$ & -0.30405100 & 2.19237600 & -2.82593300 & $\mathrm{C}$ & 10.53132600 & 4.05787500 & 5.48712300 \\
\hline $\mathrm{H}$ & 0.63702500 & 2.25222900 & -4.32390500 & $\mathrm{H}$ & 11.23090600 & 4.82039200 & 5.12904400 \\
\hline $\mathrm{C}$ & 2.41845600 & -1.86242300 & -4.88258400 & $\mathrm{H}$ & 10.54697700 & 3.23309400 & 4.78252400 \\
\hline $\mathrm{H}$ & 2.07679600 & -0.94909400 & -5.38376900 & $\mathrm{H}$ & 10.90389000 & 3.67967000 & 6.44692400 \\
\hline $\mathrm{H}$ & 1.56767900 & -2.27902400 & -4.33149000 & $\mathrm{H}$ & 7.37500000 & -0.16126500 & 1.61113200 \\
\hline $\mathrm{H}$ & 2.70235700 & -2.57978100 & -5.66254200 & $\mathrm{H}$ & 8.10914700 & -1.32311600 & 3.83008800 \\
\hline $\mathrm{C}$ & 4.82427000 & -1.04449200 & -4.72276400 & $\mathrm{C}$ & 10.59694900 & -3.27655500 & 4.53295600 \\
\hline $\mathrm{H}$ & 5.68100000 & -0.90438100 & -4.05755600 & $\mathrm{H}$ & 10.07372700 & -3.11142300 & 5.47719700 \\
\hline $\mathrm{H}$ & 4.59499300 & -0.08295100 & -5.19870900 & $\mathrm{H}$ & 11.53835600 & -3.79438700 & 4.76470600 \\
\hline $\mathrm{H}$ & 5.11719100 & -1.74428200 & -5.51534700 & $\mathrm{H}$ & 9.99241500 & -3.94138800 & 3.91702100 \\
\hline $\mathrm{C}$ & 1.40143300 & -0.61781400 & 1.62582200 & $\mathrm{C}$ & 11.48456200 & -0.56581300 & 5.98384400 \\
\hline $\mathrm{H}$ & 0.82844300 & 0.31105200 & 1.51840200 & $\mathrm{H}$ & 10.94914800 & -1.32837200 & 6.55525800 \\
\hline $\mathrm{H}$ & 1.43102700 & -0.87060300 & 2.69280500 & $\mathrm{H}$ & 11.09534300 & 0.40594100 & 6.29563700 \\
\hline $\mathrm{H}$ & 0.84670300 & -1.40665000 & 1.10894500 & $\mathrm{H}$ & 12.54686100 & -0.63309800 & 6.25811600 \\
\hline $\mathrm{C}$ & 3.54826000 & 0.65077200 & 1.88675700 & $\mathrm{C}$ & 12.38960300 & 1.47524400 & 3.72556700 \\
\hline $\mathrm{H}$ & 4.56878400 & 0.81932500 & 1.53041800 & $\mathrm{H}$ & 13.48055700 & 1.34539100 & 3.68564300 \\
\hline $\mathrm{H}$ & 3.59920500 & 0.36905500 & 2.94379800 & $\mathrm{H}$ & 12.13397800 & 1.86738600 & 4.70966200 \\
\hline $\mathrm{H}$ & 3.01873000 & 1.60850100 & 1.83113700 & $\mathrm{H}$ & 12.11915900 & 2.22110500 & 2.97416400 \\
\hline C & 11.30657200 & -0.76744700 & 4.50623100 & $\mathrm{C}$ & 11.94660900 & 0.11602500 & 0.87296900 \\
\hline
\end{tabular}




\begin{tabular}{|c|c|c|c|c|c|c|c|}
\hline 4 & 12.97901700 & -0.17615400 & 0.64333800 & $\mathrm{C}$ & 7.79959100 & -1.50409200 & 7.91613700 \\
\hline $\mathrm{H}$ & 11.90955900 & 1.20914800 & 0.87586800 & $\mathrm{H}$ & 8.59533100 & -0.88255100 & 5.99686400 \\
\hline $\mathrm{H}$ & 11.32175600 & -0.25178400 & 0.05468400 & $\mathrm{C}$ & 3.67169800 & -4.14510700 & 6.24971700 \\
\hline $\mathrm{C}$ & 10.79346900 & -2.83109100 & 1.34977500 & $\mathrm{H}$ & 4.99748500 & -3.89503900 & 4.59719700 \\
\hline $\mathrm{H}$ & 10.34341600 & -2.40748100 & 0.44757500 & $\mathrm{C}$ & 4.34797500 & -3.09863200 & 8.30075100 \\
\hline $\mathrm{H}$ & 10.13390100 & -3.62875600 & 1.69793900 & $\mathrm{C}$ & 6.42562700 & -1.72615800 & 8.54816600 \\
\hline $\mathrm{H}$ & 11.74656300 & -3.28517400 & 1.05170100 & $\mathrm{H}$ & 8.32149400 & -0.68682500 & 8.42626700 \\
\hline $\mathrm{H}$ & 6.99670000 & 2.13485500 & 2.41093800 & $\mathrm{C}$ & 3.42762800 & -3.86528300 & 7.59343100 \\
\hline $\mathrm{C}$ & 8.32202300 & 3.78211500 & 2.03950900 & $\mathrm{H}$ & 2.96958800 & -4.73944800 & 5.67124900 \\
\hline $\mathrm{C}$ & 9.61439000 & 4.32127400 & 2.06781400 & $\mathrm{H}$ & 4.16606600 & -2.87143700 & 9.34947800 \\
\hline $\mathrm{C}$ & 7.26084000 & 4.61250300 & 1.66226500 & $\mathrm{H}$ & 5.93989500 & -0.74867200 & 8.6866380 \\
\hline $\mathrm{C}$ & 9.83985300 & 5.65701800 & 1.73525900 & $\mathrm{H}$ & 2.53312000 & -4.23929100 & 8.08457100 \\
\hline $\mathrm{H}$ & 10.44337200 & 3.68936100 & 2.37030800 & $\mathrm{H}$ & 7.82754300 & -3.17822300 & 5.79560600 \\
\hline $\mathrm{C}$ & 7.48070600 & 5.95040300 & 1.33019200 & $\mathrm{H}$ & 6.98363900 & -0.30663500 & 6.31948100 \\
\hline $\mathrm{H}$ & 6.25362300 & 4.20683200 & 1.61948800 & $\mathrm{H}$ & 8.41901200 & -2.40618100 & 8.03784100 \\
\hline $\mathrm{C}$ & 8.77193800 & 6.47803400 & 1.36522500 & $\mathrm{H}$ & 6.51782500 & -2.16100500 & 9.55200300 \\
\hline $\mathrm{H}$ & 10.85012000 & 6.05800500 & 1.76531300 & & & & \\
\hline $\mathrm{H}$ & 6.64188400 & 6.57837900 & 1.04152100 & TS-8-RR & & & \\
\hline $\mathrm{H}$ & 8.94578400 & 7.51927900 & 1.10676500 & $\mathrm{C}$ & -2.87858900 & -2.64557500 & -0.95731900 \\
\hline $\mathrm{H}$ & 9.62209000 & 1.62363300 & 0.98962800 & $\mathrm{C}$ & -3.83133700 & -1.83338000 & -0.26499000 \\
\hline $\mathrm{C}$ & 7.91972700 & 1.88583000 & -0.24639200 & $\mathrm{C}$ & -3.26885200 & -1.49761000 & 1.04425500 \\
\hline $\mathrm{C}$ & 6.65158100 & 1.45687700 & -0.64943500 & $\mathrm{C}$ & -2.00330900 & -2.13107300 & 1.15939700 \\
\hline $\mathrm{C}$ & 8.64699600 & 2.72703500 & -1.10025700 & $\mathrm{C}$ & -1.70825500 & -2.79873900 & -0.10314900 \\
\hline $\mathrm{C}$ & 6.11813400 & 1.86357300 & -1.87281000 & Ir & -1.94312200 & -0.69383000 & -0.61407700 \\
\hline $\mathrm{H}$ & 6.07729900 & 0.77117300 & -0.03458100 & $\mathrm{~N}$ & -0.07852100 & 0.35905900 & -0.32276500 \\
\hline $\mathrm{C}$ & 8.11547700 & 3.14315100 & -2.31984400 & $\mathrm{~N}$ & -1.43326000 & -0.15926700 & -2.55119600 \\
\hline $\mathrm{H}$ & 9.63410800 & 3.06924000 & -0.80012700 & $\mathrm{C}$ & 0.46253300 & 0.97592700 & -1.58226900 \\
\hline $\mathrm{C}$ & 6.84677800 & 2.71055100 & -2.70930900 & $\mathrm{C}$ & 0.03369800 & 0.02683200 & -2.71767700 \\
\hline $\mathrm{H}$ & 5.14066000 & 1.50387600 & -2.17312000 & $S$ & 0.02746600 & 1.35219800 & 1.00985600 \\
\hline $\mathrm{H}$ & 8.69530500 & 3.79697900 & -2.96609300 & $\mathrm{O}$ & -0.96815200 & 0.88862500 & 2.00552900 \\
\hline $\mathrm{H}$ & 6.43107300 & 3.02179900 & -3.66406300 & $\mathrm{O}$ & -0.05194100 & 2.75834800 & 0.55933400 \\
\hline $\mathrm{N}$ & 6.95324200 & -3.26462600 & 3.33263400 & $\mathrm{C}$ & 1.66583900 & 1.21855000 & 1.85850500 \\
\hline $\mathrm{C}$ & 6.92982200 & -2.15990900 & 4.15811000 & $\mathrm{C}$ & 2.03459700 & 2.35017100 & 2.62590900 \\
\hline $\mathrm{C}$ & 7.45616700 & -4.58378200 & 3.45122200 & $\mathrm{C}$ & 2.49517000 & 0.08360500 & 1.79527600 \\
\hline $\mathrm{C}$ & 7.84034000 & -5.19817500 & 2.24057600 & $\mathrm{C}$ & 3.33388400 & 2.40201600 & 3.17229700 \\
\hline $\mathrm{C}$ & 7.49248200 & -5.35401100 & 4.63026200 & $\mathrm{C}$ & 3.77061400 & 0.13579900 & 2.40129600 \\
\hline $\mathrm{C}$ & 8.27667100 & -6.52008400 & 2.21973200 & $\mathrm{C}$ & 4.19655200 & 1.29161900 & 3.07710000 \\
\hline $\mathrm{H}$ & 7.77494400 & -4.63433900 & 1.31662000 & $\mathrm{C}$ & 1.07352700 & 3.47802700 & 2.96822600 \\
\hline $\mathrm{C}$ & 7.95086000 & -6.67193000 & 4.59483200 & $\mathrm{H}$ & 0.03617900 & 3.14889700 & 2.95918200 \\
\hline $\mathrm{H}$ & 7.12274500 & -4.96659700 & 5.56766000 & $\mathrm{H}$ & 1.14765400 & 4.31791500 & 2.26913400 \\
\hline $\mathrm{C}$ & 8.35078500 & -7.26590900 & 3.39830100 & $\mathrm{H}$ & 1.28791600 & 3.84933600 & 3.97447600 \\
\hline $\mathrm{H}$ & 8.56068400 & -6.96449500 & 1.27040200 & $\mathrm{C}$ & 3.80386000 & 3.67663400 & 3.85433900 \\
\hline $\mathrm{H}$ & 7.96929500 & -7.24229800 & 5.51985300 & $\mathrm{H}$ & 4.86335300 & 3.86214600 & 3.65937400 \\
\hline $\mathrm{H}$ & 6.75884600 & -3.02823800 & 2.34566100 & $\mathrm{H}$ & 3.67442600 & 3.64517900 & 4.94582300 \\
\hline $\mathrm{C}$ & 5.86204600 & -1.16657900 & 3.72139900 & $\mathrm{H}$ & 3.26260000 & 4.55115100 & 3.48970000 \\
\hline $\mathrm{H}$ & 5.80867400 & -1.12673900 & 2.63063600 & $\mathrm{C}$ & 5.56184200 & 1.31493000 & 3.74239000 \\
\hline $\mathrm{H}$ & 4.88803300 & -1.51924500 & 4.07879800 & $\mathrm{H}$ & 5.67598300 & 2.15597500 & 4.42625200 \\
\hline $\mathrm{H}$ & 6.05184300 & -0.17501300 & 4.13094600 & $\mathrm{H}$ & 6.37606900 & 1.37749000 & 3.00748200 \\
\hline $\mathrm{H}$ & 8.69656100 & -8.29550600 & 3.38162700 & $\mathrm{H}$ & 5.73171500 & 0.40782700 & 4.33165100 \\
\hline $\mathrm{C}$ & 7.07965300 & -2.38588600 & 5.67678300 & $\mathrm{C}$ & 4.67108100 & -1.07872700 & 2.28901000 \\
\hline $\mathrm{C}$ & 5.76916000 & -2.88331300 & 6.34150300 & $\mathrm{H}$ & 5.66916400 & -0.89638600 & 2.68668600 \\
\hline $\mathrm{C}$ & 7.63896800 & -1.17314100 & 6.43341800 & $\mathrm{H}$ & 4.79384500 & -1.38372400 & 1.24281300 \\
\hline $\mathrm{C}$ & 4.82895100 & -3.66171800 & 5.64223700 & $\mathrm{H}$ & 4.25910000 & -1.94645800 & 2.82222000 \\
\hline $\mathrm{C}$ & 5.51232400 & -2.59546700 & 7.70167800 & $\mathrm{C}$ & 2.08144600 & -1.23705500 & 1.18606000 \\
\hline
\end{tabular}




\begin{tabular}{|c|c|c|c|c|c|c|c|}
\hline $\mathrm{H}$ & 2.79318800 & -1.56251000 & 0.41984900 & $\mathrm{C}$ & 1.08412000 & 1.31475800 & -6.71907000 \\
\hline $\mathrm{H}$ & 1.10138800 & -1.18661700 & 0.72235700 & $\mathrm{H}$ & -0.15919400 & 3.03328000 & -6.32781600 \\
\hline $\mathrm{H}$ & 2.07905400 & -2.01406700 & 1.96269000 & $\mathrm{H}$ & 2.24013900 & -0.50527900 & -6.80965000 \\
\hline $\mathrm{H}$ & -1.98473900 & 0.88007900 & -2.73735700 & $\mathrm{H}$ & 1.35033900 & 1.63277900 & -7.72376500 \\
\hline $\mathrm{C}$ & -0.54113400 & -3.70183600 & -0.37286900 & $\mathrm{C}$ & 1.96665700 & 1.23317700 & -1.58723700 \\
\hline $\mathrm{H}$ & 0.35650000 & -3.36334200 & 0.14965700 & $\mathrm{C}$ & 2.90811500 & 0.20562700 & -1.74199900 \\
\hline $\mathrm{H}$ & -0.30764600 & -3.74733300 & -1.44094600 & $\mathrm{C}$ & 2.43409400 & 2.55039200 & -1.4888240 \\
\hline $\mathrm{H}$ & -0.75963600 & -4.72548700 & -0.03640700 & $\mathrm{C}$ & 4.27427900 & 0.48398300 & -1.79201900 \\
\hline $\mathrm{C}$ & -3.07785800 & -3.29823500 & -2.29459100 & $\mathrm{H}$ & 2.57616900 & -0.82460900 & -1.82961900 \\
\hline $\mathrm{H}$ & -3.73433500 & -2.70745400 & -2.94050600 & $\mathrm{C}$ & 3.80024800 & 2.83354600 & -1.53403500 \\
\hline $\mathrm{H}$ & -3.53937400 & -4.28718100 & -2.17162200 & $\mathrm{H}$ & 1.71641500 & 3.35414000 & -1.35812400 \\
\hline $\mathrm{H}$ & -2.12867400 & -3.44568300 & -2.81899900 & $\mathrm{C}$ & 4.72606000 & 1.80128400 & -1.68632500 \\
\hline $\mathrm{C}$ & -5.24853200 & -1.58667600 & -0.68768200 & $\mathrm{H}$ & 4.98622400 & -0.32773800 & -1.92155100 \\
\hline $\mathrm{H}$ & -5.88763500 & -2.39700700 & -0.30899300 & $\mathrm{H}$ & 4.13889100 & 3.86313300 & -1.4525360 \\
\hline $\mathrm{H}$ & -5.35659500 & -1.56093800 & -1.77495200 & $\mathrm{H}$ & 5.79008800 & 2.01968100 & -1.72692000 \\
\hline $\mathrm{H}$ & -5.63600900 & -0.64674900 & -0.29181100 & $\mathrm{H}$ & 0.50792400 & -0.94828100 & -2.55028900 \\
\hline $\mathrm{C}$ & -3.95922500 & -0.72618500 & 2.12907500 & $\mathrm{H}$ & -0.03741200 & 1.93398500 & -1.7591860 \\
\hline $\mathrm{H}$ & -4.49511200 & -1.40800100 & 2.80475400 & $\mathrm{H}$ & -5.45560900 & 4.63105500 & -0.2316040 \\
\hline $\mathrm{H}$ & -4.68818200 & -0.02339100 & 1.71780400 & $\mathrm{H}$ & -3.38893000 & 3.99604400 & 1.3860710 \\
\hline $\mathrm{H}$ & -3.23608200 & -0.15345500 & 2.71361900 & & & & \\
\hline $\mathrm{C}$ & -1.18268600 & -2.19989700 & 2.41218000 & TS-8-RS & & & \\
\hline $\mathrm{H}$ & -0.19045100 & -2.61149400 & 2.22245600 & $\mathrm{C}$ & -2.63635700 & -2.53739500 & -1.28053500 \\
\hline $\mathrm{H}$ & -1.68458000 & -2.85955900 & 3.13357000 & $\mathrm{C}$ & -3.73401700 & -1.78232800 & -0.75355300 \\
\hline $\mathrm{H}$ & -1.06371900 & -1.21291500 & 2.86245400 & $\mathrm{C}$ & -3.45167300 & -1.52205900 & 0.65743200 \\
\hline $\mathrm{H}$ & -1.77012900 & -0.81744900 & -3.25494200 & $\mathrm{C}$ & -2.22363500 & -2.14523500 & 0.99600400 \\
\hline $\mathrm{C}$ & -3.27135100 & 2.01627900 & -1.54316100 & $\mathrm{C}$ & -1.65718800 & -2.71863800 & -0.21915500 \\
\hline $\mathrm{O}$ & -2.57462000 & 2.03883500 & -2.68111300 & Ir & -1.84944000 & -0.59107400 & -0.67685500 \\
\hline $\mathrm{H}$ & -2.82297700 & 1.08536200 & -0.83748300 & $\mathrm{~N}$ & 0.05589500 & 0.35472500 & -0.16564800 \\
\hline $\mathrm{C}$ & -4.74953300 & 1.65583900 & -1.76261600 & $\mathrm{~N}$ & -1.17839400 & 0.07926500 & -2.51839900 \\
\hline $\mathrm{H}$ & -5.30915100 & 1.53811700 & -0.82821900 & $\mathrm{C}$ & 0.68376700 & 1.04731300 & -1.33480800 \\
\hline $\mathrm{H}$ & -4.82362500 & 0.73162700 & -2.34214000 & $\mathrm{C}$ & 0.30076900 & 0.20809100 & -2.56917100 \\
\hline $\mathrm{H}$ & -5.21707500 & 2.45657800 & -2.34509800 & $\mathrm{~S}$ & 0.08242200 & 1.28580200 & 1.22370000 \\
\hline $\mathrm{C}$ & -3.05100500 & 3.27334300 & -0.63417800 & $\mathrm{O}$ & -0.80480800 & 0.63720800 & 2.21249400 \\
\hline $\mathrm{H}$ & -1.96598400 & 3.38717300 & -0.57047000 & $\mathrm{O}$ & -0.21310000 & 2.69129800 & 0.87706000 \\
\hline $\mathrm{C}$ & -3.56420500 & 3.08679600 & 0.80022800 & $\mathrm{C}$ & 1.77870700 & 1.24389500 & 1.96332500 \\
\hline $\mathrm{H}$ & -4.63767000 & 2.86770900 & 0.84730500 & $\mathrm{C}$ & 2.45499000 & 2.45180700 & 2.26249100 \\
\hline $\mathrm{H}$ & -3.02471500 & 2.27059800 & 1.28827700 & $\mathrm{C}$ & 2.34577700 & -0.01116200 & 2.27895900 \\
\hline $\mathrm{C}$ & -3.59871600 & 4.51882200 & -1.32741700 & $\mathrm{C}$ & 3.76543700 & 2.36848500 & 2.78970400 \\
\hline $\mathrm{C}$ & -2.83779000 & 5.14129300 & -2.33018100 & $\mathrm{C}$ & 3.61723000 & -0.06005000 & 2.88232400 \\
\hline $\mathrm{C}$ & -4.84287000 & 5.08110700 & -1.00701700 & $\mathrm{C}$ & 4.32070000 & 1.12825300 & 3.14616700 \\
\hline $\mathrm{C}$ & -3.29976400 & 6.28182000 & -2.98548400 & $\mathrm{C}$ & 1.85878600 & 3.84041000 & 2.10662700 \\
\hline $\mathrm{H}$ & -1.87484400 & 4.71608600 & -2.59473900 & $\mathrm{H}$ & 0.81522800 & 3.86943000 & 2.41071100 \\
\hline $\mathrm{C}$ & -5.31099000 & 6.22396300 & -1.66123100 & $\mathrm{H}$ & 1.88508300 & 4.18476000 & 1.06671700 \\
\hline $\mathrm{C}$ & -4.54144900 & 6.83019500 & -2.65392100 & $\mathrm{H}$ & 2.40738200 & 4.55893100 & 2.71660800 \\
\hline $\mathrm{H}$ & -2.68734600 & 6.74653000 & -3.75466900 & $\mathrm{C}$ & 4.59704100 & 3.62994700 & 2.95487400 \\
\hline $\mathrm{H}$ & -6.27766600 & 6.64132900 & -1.38889900 & $\mathrm{H}$ & 5.66492600 & 3.40291900 & 2.94790100 \\
\hline $\mathrm{H}$ & -4.90181900 & 7.72186800 & -3.16085800 & $\mathrm{H}$ & 4.38320500 & 4.16302700 & 3.89232200 \\
\hline $\mathrm{C}$ & 0.39444200 & 0.49915500 & -4.11764100 & $\mathrm{H}$ & 4.42021500 & 4.33204400 & 2.13616900 \\
\hline $\mathrm{C}$ & -0.10733700 & 1.70122600 & -4.64258400 & $\mathrm{C}$ & 5.67182100 & 1.06576300 & 3.83474200 \\
\hline $\mathrm{C}$ & 1.23641800 & -0.28599900 & -4.91615300 & $\mathrm{H}$ & 5.83766000 & 1.93206500 & 4.47997700 \\
\hline $\mathrm{C}$ & 0.23805100 & 2.10151300 & -5.93367200 & $\mathrm{H}$ & 6.50104400 & 1.03480800 & 3.11296500 \\
\hline $\mathrm{H}$ & -0.78470300 & 2.30905100 & -4.04991000 & $\mathrm{H}$ & 5.76222600 & 0.17869200 & 4.46510500 \\
\hline $\mathrm{C}$ & 1.58239300 & 0.11619500 & -6.20743200 & $\mathrm{C}$ & 4.25324800 & -1.39038700 & 3.24541200 \\
\hline $\mathrm{H}$ & 1.63477800 & -1.21767700 & -4.51948900 & $\mathrm{H}$ & 5.31120500 & -1.41655100 & 2.96425000 \\
\hline
\end{tabular}




\begin{tabular}{|c|c|c|c|c|c|c|c|}
\hline $\mathrm{H}$ & 3.77168200 & -2.23460500 & 2.75241600 & $\mathrm{H}$ & 2.61282600 & -0.87520300 & -0.89970900 \\
\hline $\mathrm{H}$ & 4.20520700 & -1.58177000 & 4.32693900 & $\mathrm{C}$ & 4.15692100 & 2.62789900 & -1.48666400 \\
\hline $\mathrm{C}$ & 1.63907700 & -1.32360200 & 2.01607000 & $\mathrm{H}$ & 2.12125500 & 3.32454000 & -1.62182100 \\
\hline $\mathrm{H}$ & 2.23058100 & -1.95416500 & 1.34002800 & $\mathrm{C}$ & 4.99156300 & 1.52617200 & -1.29704400 \\
\hline $\mathrm{H}$ & 0.66580600 & -1.18701300 & 1.56441300 & $\mathrm{H}$ & 5.07361600 & -0.59987000 & -0.93916300 \\
\hline $\mathrm{H}$ & 1.50733800 & -1.88211700 & 2.95073400 & $\mathrm{H}$ & 4.58314700 & 3.61428900 & -1.6510060 \\
\hline $\mathrm{H}$ & -1.66689700 & 1.14696300 & -2.65353300 & $\mathrm{H}$ & 6.07155200 & 1.64851300 & -1.3109350 \\
\hline $\mathrm{C}$ & -0.42997800 & -3.57630900 & -0.30253800 & $\mathrm{C}$ & 0.78255600 & 0.76343000 & -3.90003500 \\
\hline $\mathrm{H}$ & 0.34815900 & -3.22827500 & 0.37971300 & $\mathrm{C}$ & 0.33103200 & 1.99952600 & -4.39158800 \\
\hline $\mathrm{H}$ & -0.01091900 & -3.57422400 & -1.31317800 & $\mathrm{C}$ & 1.69399100 & 0.02461700 & -4.66565700 \\
\hline $\mathrm{H}$ & -0.66818700 & -4.61763600 & -0.04243400 & $\mathrm{C}$ & 0.78916000 & 2.47467200 & -5.6209690 \\
\hline $\mathrm{C}$ & -2.55094600 & -3.11912700 & -2.66194000 & $\mathrm{H}$ & -0.38766300 & 2.58055800 & -3.82089900 \\
\hline $\mathrm{H}$ & -3.09946800 & -2.51530800 & -3.39157100 & $\mathrm{C}$ & 2.15300400 & 0.50255600 & -5.89443200 \\
\hline $\mathrm{H}$ & -2.98901400 & -4.12610400 & -2.67579300 & $\mathrm{H}$ & 2.05827000 & -0.92902900 & -4.2894290 \\
\hline $\mathrm{H}$ & -1.51529300 & -3.20908200 & -3.00338300 & $\mathrm{C}$ & 1.70023900 & 1.73146000 & -6.37548500 \\
\hline $\mathrm{C}$ & -5.05408700 & -1.55896000 & -1.42987500 & $\mathrm{H}$ & 0.43038800 & 3.43181900 & -5.99069400 \\
\hline $\mathrm{H}$ & -5.70391300 & -2.42910200 & -1.25913700 & $\mathrm{H}$ & 2.86289600 & -0.08400000 & -6.4719150 \\
\hline $\mathrm{H}$ & -4.95031100 & -1.43254200 & -2.51074300 & $\mathrm{H}$ & 2.05342500 & 2.10774600 & -7.3321350 \\
\hline $\mathrm{H}$ & -5.56828100 & -0.68039600 & -1.03828000 & $\mathrm{H}$ & 0.23655700 & 2.03983100 & -1.4646790 \\
\hline $\mathrm{C}$ & -4.36731000 & -0.83048900 & 1.62142200 & $\mathrm{H}$ & 0.73022000 & -0.79319900 & -2.4364780 \\
\hline $\mathrm{H}$ & -5.06303100 & -1.55493800 & 2.06935400 & $\mathrm{H}$ & -5.80791700 & 3.93334100 & -0.3900540 \\
\hline $\mathrm{H}$ & -4.95891500 & -0.05241400 & 1.13413300 & $\mathrm{H}$ & -3.18373100 & 5.56513600 & -0.6454160 \\
\hline $\mathrm{H}$ & -3.80178800 & -0.35463500 & 2.42479400 & & & & \\
\hline $\mathrm{C}$ & -1.70403400 & -2.30543500 & 2.39460100 & TS-8-SR & & & \\
\hline $\mathrm{H}$ & -0.76918100 & -2.86888700 & 2.41228700 & $\mathrm{C}$ & -2.82866100 & -2.67671000 & -1.36240300 \\
\hline $\mathrm{H}$ & -2.43950600 & -2.86352600 & 2.98920500 & $\mathrm{C}$ & -3.90732400 & -1.91438900 & -0.8092180 \\
\hline $\mathrm{H}$ & -1.52801400 & -1.33834000 & 2.86825100 & $\mathrm{C}$ & -3.62283300 & -1.71971900 & 0.6112290 \\
\hline $\mathrm{H}$ & -1.49265500 & -0.50661900 & -3.29295500 & $\mathrm{C}$ & -2.39129900 & -2.35438900 & 0.91827600 \\
\hline $\mathrm{C}$ & -3.08873200 & 2.18641900 & -1.52772900 & $\mathrm{C}$ & -1.84466700 & -2.90350700 & -0.3159730 \\
\hline $\mathrm{O}$ & -2.22283600 & 2.33194400 & -2.53097500 & Ir & -1.98981000 & -0.76640600 & -0.71754200 \\
\hline $\mathrm{H}$ & -2.70443600 & 1.21542300 & -0.82506800 & $\mathrm{~N}$ & -0.08692000 & 0.13492200 & -0.22932800 \\
\hline $\mathrm{C}$ & -4.48693400 & 1.77331700 & -2.01714600 & $\mathrm{~N}$ & -1.36374100 & -0.16050100 & -2.5924880 \\
\hline $\mathrm{H}$ & -5.17379000 & 1.52879200 & -1.20256300 & $\mathrm{C}$ & 0.50012600 & 0.87703000 & -1.38279400 \\
\hline $\mathrm{H}$ & -4.39822800 & 0.91714600 & -2.69114200 & $\mathrm{C}$ & 0.09873000 & 0.09251100 & -2.65813300 \\
\hline $\mathrm{H}$ & -4.91709400 & 2.60048100 & -2.59417600 & $\mathrm{~S}$ & -0.08506800 & 0.98576000 & 1.22256600 \\
\hline $\mathrm{C}$ & -3.07540500 & 3.39313600 & -0.52636200 & $\mathrm{O}$ & -0.78098200 & 0.13657400 & 2.20809100 \\
\hline $\mathrm{H}$ & -2.06137100 & 3.41473900 & -0.12288700 & $\mathrm{O}$ & -0.61893300 & 2.34913300 & 1.0461420 \\
\hline $\mathrm{C}$ & -3.27693900 & 4.70364600 & -1.31617400 & $\mathrm{C}$ & 1.64412000 & 1.13584700 & 1.84711500 \\
\hline $\mathrm{H}$ & -2.51647700 & 4.77579100 & -2.09732200 & $\mathrm{C}$ & 2.25057400 & 2.40378200 & 1.99361400 \\
\hline $\mathrm{H}$ & -4.25919200 & 4.76381600 & -1.79919200 & $\mathrm{C}$ & 2.25786800 & -0.03465300 & 2.34562900 \\
\hline $\mathrm{C}$ & -4.03447300 & 3.26456100 & 0.65106800 & $\mathrm{C}$ & 3.45748600 & 2.48641500 & 2.72668700 \\
\hline $\mathrm{C}$ & -5.40055800 & 3.58258300 & 0.55397600 & $\mathrm{C}$ & 3.46879900 & 0.07344800 & 3.05383300 \\
\hline $\mathrm{C}$ & -3.55145500 & 2.84195400 & 1.89997100 & $\mathrm{C}$ & 4.08209200 & 1.32887500 & 3.22164700 \\
\hline $\mathrm{C}$ & -6.25323600 & 3.46667400 & 1.65335200 & $\mathrm{C}$ & 1.72829800 & 3.69144600 & 1.38202500 \\
\hline $\mathrm{C}$ & -4.40072200 & 2.72882600 & 3.00377700 & $\mathrm{H}$ & 1.10183700 & 4.25124600 & 2.08782400 \\
\hline $\mathrm{H}$ & -2.49961600 & 2.60019900 & 2.00527000 & $\mathrm{H}$ & 1.11790200 & 3.51755000 & 0.50289100 \\
\hline $\mathrm{C}$ & -5.75703900 & 3.03684400 & 2.88631900 & $\mathrm{H}$ & 2.56637300 & 4.33183500 & 1.09519600 \\
\hline $\mathrm{H}$ & -7.30508800 & 3.72262800 & 1.54785600 & $\mathrm{C}$ & 4.07138100 & 3.84883200 & 2.99944700 \\
\hline $\mathrm{H}$ & -3.99440200 & 2.40652700 & 3.95982000 & $\mathrm{H}$ & 4.69560900 & 3.83577300 & 3.89528900 \\
\hline $\mathrm{H}$ & -6.41808600 & 2.95445300 & 3.74569500 & $\mathrm{H}$ & 3.30225700 & 4.60835300 & 3.16099100 \\
\hline $\mathrm{C}$ & 2.19660700 & 1.20626700 & -1.26105700 & $\mathrm{H}$ & 4.69993500 & 4.19934900 & 2.16791200 \\
\hline $\mathrm{C}$ & 3.04462300 & 0.10673300 & -1.07264400 & $\mathrm{C}$ & 5.43348400 & 1.42571800 & 3.90659500 \\
\hline $\mathrm{C}$ & 2.76996600 & 2.46598100 & -1.46904400 & $\mathrm{H}$ & 5.34079200 & 1.60380800 & 4.98779200 \\
\hline $\mathrm{C}$ & 4.42998400 & 0.26352600 & -1.08915500 & $\mathrm{H}$ & 6.03419600 & 2.24075000 & 3.49586100 \\
\hline
\end{tabular}




\begin{tabular}{|c|c|c|c|c|c|c|c|}
\hline $\mathrm{H}$ & 6.01547600 & 0.51018100 & 3.77927500 & $\mathrm{C}$ & -0.18624900 & 1.82805700 & -4.51367300 \\
\hline $\mathrm{C}$ & 4.10038200 & -1.16889900 & 3.65652300 & $\mathrm{C}$ & 1.63419200 & 0.24670700 & -4.65749200 \\
\hline $\mathrm{H}$ & 4.79993600 & -1.65989000 & 2.96385200 & $\mathrm{C}$ & 0.23083600 & 2.40287600 & -5.71586100 \\
\hline $\mathrm{H}$ & 3.34778500 & -1.91135900 & 3.93131100 & $\mathrm{H}$ & -1.07750200 & 2.20536900 & -4.01831500 \\
\hline $\mathrm{H}$ & 4.65829700 & -0.93504600 & 4.56636900 & $\mathrm{C}$ & 2.05131100 & 0.82563400 & -5.85646100 \\
\hline $\mathrm{C}$ & 1.69391200 & -1.42169300 & 2.11399900 & $\mathrm{H}$ & 2.19147500 & -0.58934900 & -4.24182700 \\
\hline $\mathrm{H}$ & 2.50015600 & -2.11595300 & 1.85184700 & $\mathrm{C}$ & 1.34958100 & 1.90820800 & -6.3892510 \\
\hline $\mathrm{H}$ & 0.96618100 & -1.43280600 & 1.30652500 & $\mathrm{H}$ & -0.32682900 & 3.23986900 & -6.12799700 \\
\hline $\mathrm{H}$ & 1.20296100 & -1.80841700 & 3.01514000 & $\mathrm{H}$ & 2.92185000 & 0.42962700 & -6.37289100 \\
\hline $\mathrm{H}$ & -2.03177100 & 0.73261500 & -2.83152600 & $\mathrm{H}$ & 1.66916600 & 2.35989800 & -7.3248260 \\
\hline $\mathrm{C}$ & -0.62261700 & -3.76583300 & -0.42607400 & $\mathrm{C}$ & 2.01995900 & 1.00779600 & -1.3545420 \\
\hline $\mathrm{H}$ & 0.16905800 & -3.41827400 & 0.24206800 & $\mathrm{C}$ & 2.83893200 & -0.09704600 & -1.08408500 \\
\hline $\mathrm{H}$ & -0.22312800 & -3.76599900 & -1.44469800 & $\mathrm{C}$ & 2.62529300 & 2.21988700 & -1.70424200 \\
\hline $\mathrm{H}$ & -0.85656300 & -4.80622100 & -0.15903600 & $\mathrm{C}$ & 4.22760500 & 0.01282100 & -1.14683400 \\
\hline $\mathrm{C}$ & -2.77097200 & -3.21201500 & -2.76361400 & $\mathrm{H}$ & 2.37993900 & -1.04121400 & -0.80544900 \\
\hline $\mathrm{H}$ & -3.30377400 & -2.56385000 & -3.46670500 & $\mathrm{C}$ & 4.01556600 & 2.33416100 & -1.76909500 \\
\hline $\mathrm{H}$ & -3.24794900 & -4.20022800 & -2.80745200 & $\mathrm{H}$ & 2.00256900 & 3.08025600 & -1.93498900 \\
\hline $\mathrm{H}$ & -1.74177100 & -3.33419700 & -3.11607800 & $\mathrm{C}$ & 4.82168700 & 1.23064700 & -1.48859000 \\
\hline $\mathrm{C}$ & -5.19376400 & -1.59708900 & -1.51808800 & $\mathrm{H}$ & 4.84820200 & -0.85214700 & -0.92596900 \\
\hline $\mathrm{H}$ & -5.76040600 & -2.51970000 & -1.70502300 & $\mathrm{H}$ & 4.46595500 & 3.28524700 & -2.0410590 \\
\hline $\mathrm{H}$ & -5.02112500 & -1.10441600 & -2.47974200 & $\mathrm{H}$ & 5.90414200 & 1.31680500 & -1.5360700 \\
\hline $\mathrm{H}$ & -5.82870300 & -0.94101700 & -0.91928400 & $\mathrm{H}$ & 0.59132600 & -0.88531200 & -2.58489200 \\
\hline $\mathrm{C}$ & -4.55087100 & -1.09415600 & 1.60675700 & $\mathrm{H}$ & 0.06534500 & 1.88220000 & -1.45900300 \\
\hline $\mathrm{H}$ & -5.26140000 & -1.84713800 & 1.97699800 & $\mathrm{H}$ & -6.62248500 & 2.56749200 & -2.08309700 \\
\hline $\mathrm{H}$ & -5.12717000 & -0.27292200 & 1.17570300 & $\mathrm{H}$ & -6.63944400 & 3.89320700 & -0.3441560 \\
\hline $\mathrm{H}$ & -4.00335200 & -0.69631700 & 2.46362600 & & & & \\
\hline $\mathrm{C}$ & -1.84773200 & -2.59095600 & 2.29337200 & TS-8-SS & & & \\
\hline $\mathrm{H}$ & -0.77142100 & -2.76162500 & 2.27920200 & $\mathrm{C}$ & -3.20286100 & -2.43821400 & -1.59784100 \\
\hline $\mathrm{H}$ & -2.32805600 & -3.48604900 & 2.71545100 & $\mathrm{C}$ & -3.91511300 & -1.71675600 & -0.5905640 \\
\hline $\mathrm{H}$ & -2.03444700 & -1.74530200 & 2.95413200 & $\mathrm{C}$ & -3.18196500 & -1.81412500 & 0.66508700 \\
\hline $\mathrm{H}$ & -1.60185400 & -0.85212500 & -3.30445600 & $\mathrm{C}$ & -2.03565200 & -2.63434000 & 0.42809500 \\
\hline $\mathrm{C}$ & -3.19511200 & 2.05236400 & -1.61561800 & $\mathrm{C}$ & -2.00407500 & -2.99072400 & -0.98086700 \\
\hline $\mathrm{O}$ & -3.03739800 & 1.67704000 & -2.88113700 & Ir & -1.85199800 & -0.82895700 & -0.89776500 \\
\hline $\mathrm{H}$ & -2.68610500 & 1.13040200 & -0.89056100 & $\mathrm{~N}$ & 0.06714200 & -0.06022900 & -0.27925000 \\
\hline $\mathrm{C}$ & -2.44084000 & 3.34604200 & -1.26392200 & $\mathrm{~N}$ & -1.08770400 & -0.20893400 & $-2.7242030 c$ \\
\hline $\mathrm{H}$ & -2.32364500 & 3.51468900 & -0.19541300 & $\mathrm{C}$ & 0.77095500 & 0.66521800 & -1.37602800 \\
\hline $\mathrm{H}$ & -2.99869000 & 4.18538900 & -1.70028400 & $\mathrm{C}$ & 0.39052800 & -0.06407100 & -2.69325300 \\
\hline $\mathrm{H}$ & -1.45186900 & 3.33343700 & -1.72797500 & $\mathrm{~S}$ & -0.00103000 & 0.72692700 & 1.21094500 \\
\hline $\mathrm{C}$ & -4.70373600 & 2.07126200 & -1.15411900 & $\mathrm{O}$ & -0.57827100 & -0.23918100 & 2.16818600 \\
\hline $\mathrm{H}$ & -5.00336000 & 1.01849000 & -1.11281800 & $\mathrm{O}$ & -0.69441900 & 2.02505900 & 1.12055500 \\
\hline $\mathrm{C}$ & -5.55198700 & 2.71386600 & -2.26631600 & $\mathrm{C}$ & 1.70230400 & 1.05821900 & 1.82869200 \\
\hline $\mathrm{H}$ & -5.36989100 & 3.79180500 & -2.34908900 & $\mathrm{C}$ & 2.17492900 & 2.38506000 & 1.92912500 \\
\hline $\mathrm{H}$ & -5.29297900 & 2.25245500 & -3.22059700 & $\mathrm{C}$ & 2.41530800 & -0.02445300 & 2.38798200 \\
\hline $\mathrm{C}$ & -4.94871000 & 2.67587900 & 0.23233800 & $\mathrm{C}$ & 3.34019300 & 2.62267200 & 2.69253600 \\
\hline $\mathrm{C}$ & -4.14309400 & 2.32189400 & 1.33042900 & $\mathrm{C}$ & 3.58270300 & 0.23787100 & 3.12911900 \\
\hline $\mathrm{C}$ & -5.99565800 & 3.58056700 & 0.47067400 & $\mathrm{C}$ & 4.05663500 & 1.55620400 & 3.26342700 \\
\hline $\mathrm{C}$ & -4.37407700 & 2.84571100 & 2.60246100 & $\mathrm{C}$ & 1.55253500 & 3.57791700 & 1.22618300 \\
\hline $\mathrm{H}$ & -3.30001400 & 1.65407200 & 1.19354200 & $\mathrm{H}$ & 0.87328500 & 4.13170900 & 1.88556900 \\
\hline $\mathrm{C}$ & -6.23544100 & 4.10225900 & 1.74500400 & $\mathrm{H}$ & 0.96813400 & 3.29488400 & 0.35670900 \\
\hline $\mathrm{C}$ & -5.42661700 & 3.73712900 & 2.81983400 & $\mathrm{H}$ & 2.33891900 & 4.26472600 & 0.90031200 \\
\hline $\mathrm{H}$ & -3.71699000 & 2.56101300 & 3.42029800 & $\mathrm{C}$ & 3.80854600 & 4.05062800 & 2.91249800 \\
\hline $\mathrm{H}$ & -7.05629600 & 4.80060500 & 1.89084500 & $\mathrm{H}$ & 4.37310400 & 4.14866900 & 3.84221400 \\
\hline $\mathrm{H}$ & -5.60775900 & 4.14607200 & 3.81067300 & $\mathrm{H}$ & 2.96577000 & 4.74277600 & 2.98072800 \\
\hline $\mathrm{C}$ & 0.51735700 & 0.74130700 & -3.96968000 & $\mathrm{H}$ & 4.45524100 & 4.40977000 & 2.09853300 \\
\hline
\end{tabular}




\begin{tabular}{|c|c|c|c|c|}
\hline $\mathrm{C}$ & 5.36078400 & 1.82336300 & 3.99288200 & $\mathrm{H}$ \\
\hline $\mathrm{H}$ & 5.20300600 & 2.04062700 & 5.05926300 & $\mathrm{H}$ \\
\hline $\mathrm{H}$ & 5.89196400 & 2.67726300 & 3.56546800 & $\mathrm{C}$ \\
\hline $\mathrm{H}$ & 6.03834700 & 0.96866100 & 3.93459400 & $\mathrm{C}$ \\
\hline $\mathrm{C}$ & 4.31715800 & -0.90837000 & 3.80133000 & $\mathrm{C}$ \\
\hline $\mathrm{H}$ & 5.09117600 & -1.34458700 & 3.15270000 & $\mathrm{C}$ \\
\hline $\mathrm{H}$ & 3.63614500 & -1.71684200 & 4.07626400 & $\mathrm{H}$ \\
\hline $\mathrm{H}$ & 4.81069400 & -0.58488100 & 4.72096600 & $\mathrm{C}$ \\
\hline $\mathrm{C}$ & 2.01030700 & -1.46925500 & 2.17979500 & $\mathrm{H}$ \\
\hline $\mathrm{H}$ & 2.89778500 & -2.08011700 & 1.98271500 & $\mathrm{C}$ \\
\hline $\mathrm{H}$ & 1.33259200 & -1.58154300 & 1.33790900 & $\mathrm{H}$ \\
\hline $\mathrm{H}$ & 1.50854300 & -1.88183600 & 3.06324100 & $\mathrm{H}$ \\
\hline $\mathrm{H}$ & -1.65764000 & 0.75837900 & -2.97988000 & $\mathrm{H}$ \\
\hline $\mathrm{C}$ & -1.03974700 & -3.94452300 & -1.62260400 & $\mathrm{C}$ \\
\hline $\mathrm{H}$ & -0.04453000 & -3.86600600 & -1.17741200 & $\mathrm{C}$ \\
\hline $\mathrm{H}$ & -0.94158000 & -3.75973100 & -2.69652600 & $\mathrm{C}$ \\
\hline $\mathrm{H}$ & -1.38165900 & -4.98157000 & -1.49686500 & $\mathrm{C}$ \\
\hline $\mathrm{C}$ & -3.65203000 & -2.66135700 & -3.01358600 & $\mathrm{H}$ \\
\hline $\mathrm{H}$ & -4.20734400 & -1.80221500 & -3.40044700 & $\mathrm{C}$ \\
\hline $\mathrm{H}$ & -4.30535000 & -3.54180100 & -3.07828300 & $\mathrm{H}$ \\
\hline $\mathrm{H}$ & -2.80549800 & -2.84099100 & -3.68409600 & $\mathrm{C}$ \\
\hline $\mathrm{C}$ & -5.28813900 & -1.13840300 & -0.74640200 & $\mathrm{H}$ \\
\hline $\mathrm{H}$ & -6.02815700 & -1.89729600 & -0.45453000 & $\mathrm{H}$ \\
\hline $\mathrm{H}$ & -5.49948100 & -0.85206700 & -1.77910800 & $\mathrm{H}$ \\
\hline $\mathrm{H}$ & -5.44344000 & -0.26654700 & -0.10985600 & $\mathrm{H}$ \\
\hline $\mathrm{C}$ & -3.63241300 & -1.29862600 & 2.00051400 & $\mathrm{H}$ \\
\hline $\mathrm{H}$ & -4.14259400 & -2.08837500 & 2.57005600 & $\mathrm{H}$ \\
\hline $\mathrm{H}$ & -4.33168300 & -0.46551100 & 1.89020900 & $\mathrm{H}$ \\
\hline $\mathrm{H}$ & -2.77674500 & -0.94076800 & 2.57699300 & \\
\hline $\mathrm{C}$ & -1.10381700 & -3.17282700 & 1.46750300 & 14- $R$ \\
\hline $\mathrm{H}$ & -0.12185400 & -3.40387400 & 1.04793700 & $\mathrm{C}$ \\
\hline $\mathrm{H}$ & -1.52292200 & -4.10695200 & 1.86874100 & $\mathrm{C}$ \\
\hline $\mathrm{H}$ & -0.96553300 & -2.46338400 & 2.28158600 & $\mathrm{H}$ \\
\hline $\mathrm{H}$ & -1.33311400 & -0.85356700 & -3.47693000 & $\mathrm{H}$ \\
\hline $\mathrm{C}$ & -2.79471800 & 2.11525700 & -1.81278700 & $\mathrm{H}$ \\
\hline $\mathrm{O}$ & -2.51218800 & 1.80888700 & -3.07794700 & $\mathrm{C}$ \\
\hline $\mathrm{H}$ & -2.44573800 & 1.13914700 & -1.09535700 & $\mathrm{C}$ \\
\hline $\mathrm{C}$ & -2.02561800 & 3.33668400 & -1.28604500 & $\mathrm{C}$ \\
\hline $\mathrm{H}$ & -2.07674400 & 3.44848000 & -0.20310300 & $\mathrm{C}$ \\
\hline $\mathrm{H}$ & -2.44427900 & 4.22727000 & -1.77087100 & $\mathrm{C}$ \\
\hline $\mathrm{H}$ & -0.97374300 & 3.26774000 & -1.57243600 & $\mathrm{H}$ \\
\hline $\mathrm{C}$ & -4.33182200 & 2.19642100 & -1.50690400 & $\mathrm{C}$ \\
\hline $\mathrm{H}$ & -4.73094100 & 1.24108000 & -1.86273500 & $\mathrm{H}$ \\
\hline $\mathrm{C}$ & -4.62093400 & 2.31311800 & -0.00489400 & $\mathrm{C}$ \\
\hline $\mathrm{H}$ & -4.09714600 & 1.52567600 & 0.54634500 & $\mathrm{C}$ \\
\hline $\mathrm{H}$ & -4.27679700 & 3.26335000 & 0.41347600 & $\mathrm{H}$ \\
\hline $\mathrm{C}$ & -5.00309100 & 3.28090100 & -2.35033100 & $\mathrm{H}$ \\
\hline $\mathrm{C}$ & -5.46874800 & 4.48889300 & -1.81187500 & $\mathrm{H}$ \\
\hline $\mathrm{C}$ & -5.17763000 & 3.06855500 & -3.72882200 & $\mathrm{H}$ \\
\hline $\mathrm{C}$ & -6.08778500 & 5.44972400 & -2.61721000 & $\mathrm{H}$ \\
\hline $\mathrm{C}$ & -5.79339500 & 4.02425000 & -4.53492100 & $\mathrm{O}$ \\
\hline $\mathrm{H}$ & -4.80168800 & 2.14977400 & -4.16635900 & $\mathrm{H}$ \\
\hline $\mathrm{C}$ & -6.25433900 & 5.22221800 & -3.98224800 & $\mathrm{H}$ \\
\hline $\mathrm{H}$ & -6.44113500 & 6.37607900 & -2.17054600 & \\
\hline
\end{tabular}

$\begin{array}{rrr}-5.91474200 & 3.83315100 & -5.59860300 \\ -6.73833800 & 5.96711700 & -4.60893800 \\ 2.29349000 & 0.67488600 & -1.26542600 \\ 3.01168500 & 1.84650500 & -1.52780300 \\ 3.00670800 & -0.50505300 & -1.01510900 \\ 4.40800400 & 1.84700800 & -1.52486400 \\ 2.47404700 & 2.76601500 & -1.74354300 \\ 4.40127400 & -0.50957400 & -1.01111800 \\ 2.46052400 & -1.42009500 & -0.80673600 \\ 5.10821500 & 0.66869600 & -1.26479300 \\ 4.94660900 & 2.76874500 & -1.72906300 \\ 4.93765200 & -1.43375000 & -0.80979700 \\ 6.19508700 & 0.66654400 & -1.26131100 \\ 0.94594600 & 0.57169300 & -3.95976700 \\ 0.39305400 & 1.74092500 & -4.50682100 \\ 2.04243200 & -0.02142400 & -4.60092400 \\ 0.93737000 & 2.29799000 & -5.66570900 \\ -0.48100400 & 2.19846700 & -4.04951200 \\ 2.58723200 & 0.53986400 & -5.75626800 \\ 2.48413400 & -0.92206200 & -4.18119200 \\ 2.03531400 & 1.70456200 & -6.29192000 \\ 0.49526500 & 3.19980600 & -6.08131000 \\ 3.43994900 & 0.06658700 & -6.23619900 \\ 2.45441000 & 2.14363900 & -7.19365400 \\ 0.41928700 & 1.70252200 & -1.45698200 \\ 0.80612200 & -1.07606600 & -2.61012700 \\ -5.35837500 & 4.69187000 & -0.75143400 \\ -5.69575100 & 2.23282400 & 0.19889300\end{array}$

$\begin{array}{ccc}2.93145700 & -3.83298700 & -1.85885000 \\ 3.84989800 & -4.61627600 & -0.93832700 \\ 4.85659900 & -4.65587400 & -1.35942800 \\ 3.46913600 & -5.64003300 & -0.82355800 \\ 3.87585300 & -4.16812000 & 0.06132400 \\ 1.54471800 & -3.46754500 & -1.29897100 \\ 1.68821300 & -2.26169400 & -0.37155600 \\ 0.53242900 & -3.22119000 & -2.42806000 \\ 1.25048500 & -2.32200800 & 0.95783100 \\ 2.25287800 & -1.06516100 & -0.83910900 \\ 0.41487100 & -4.11812700 & -3.04584900 \\ 1.36278700 & -1.21347400 & 1.79966500 \\ 0.81080000 & -3.24280500 & 1.33528200 \\ 2.36751100 & 0.04273400 & 0.00091000 \\ 1.92188100 & -0.02682000 & 1.32308200 \\ 1.01333600 & -1.27879100 & 2.82682300 \\ 2.80859400 & 0.96121500 & -0.37746600 \\ 2.01133100 & 0.83678500 & 1.97655600 \\ 1.20347900 & -4.31249000 & -0.68554200 \\ 0.86889600 & -2.41230900 & -3.08142600 \\ 3.27509100 & -3.49611900 & -2.97640500 \\ -0.44390200 & -2.95285700 & -2.01196300 \\ 2.61081300 & -1.00728100 & -1.86370800\end{array}$




\begin{tabular}{|c|c|c|c|c|c|c|c|}
\hline $14-S$ & & & & $\mathrm{H}$ & 3.16214900 & 1.34053700 & -0.83145200 \\
\hline $\mathrm{C}$ & 2.93145700 & -3.83298700 & 1.85885000 & $\mathrm{H}$ & 2.05783800 & 1.23350500 & 1.19996000 \\
\hline $\mathrm{C}$ & 3.84989800 & -4.61627600 & 0.93832700 & $\mathrm{C}$ & 7.08124500 & -4.31829100 & -1.09480800 \\
\hline $\mathrm{H}$ & 4.85659900 & -4.65587400 & 1.35942800 & $\mathrm{C}$ & 8.30540300 & -2.38298400 & -0.43435100 \\
\hline $\mathrm{H}$ & 3.46913600 & -5.64003300 & 0.82355800 & $\mathrm{C}$ & 8.49863400 & -3.70794100 & -1.14011000 \\
\hline $\mathrm{H}$ & 3.87585300 & -4.16812000 & -0.06132400 & $\mathrm{H}$ & 6.49673900 & -3.96692800 & -1.95294100 \\
\hline $\mathrm{C}$ & 1.54471800 & -3.46754500 & 1.29897100 & $\mathrm{H}$ & 7.07780700 & -5.41273000 & -1.11314500 \\
\hline $\mathrm{C}$ & 1.68821300 & -2.26169400 & 0.37155600 & $\mathrm{H}$ & 9.22577700 & -4.34259400 & -0.61398400 \\
\hline $\mathrm{C}$ & 0.53242900 & -3.22119000 & 2.42806000 & $\mathrm{H}$ & 8.87388600 & -3.58437600 & -2.16298900 \\
\hline $\mathrm{C}$ & 1.25048500 & -2.32200800 & -0.95783100 & $\mathrm{C}$ & 6.44720400 & -3.73694100 & 0.21366100 \\
\hline $\mathrm{C}$ & 2.25287800 & -1.06516100 & 0.83910900 & $\mathrm{C}$ & 6.96108900 & -4.56037000 & 1.44390100 \\
\hline $\mathrm{H}$ & 0.41487100 & -4.11812700 & 3.04584900 & $\mathrm{C}$ & 4.95666800 & -4.04877700 & 0.29023100 \\
\hline $\mathrm{C}$ & 1.36278700 & -1.21347400 & -1.79966500 & $\mathrm{C}$ & 6.06944600 & -5.82046400 & 1.46758300 \\
\hline $\mathrm{H}$ & 0.81080000 & -3.24280500 & -1.33528200 & $\mathrm{H}$ & 8.03066300 & -4.78424600 & 1.38217500 \\
\hline $\mathrm{C}$ & 2.36751100 & 0.04273400 & -0.00091000 & $\mathrm{H}$ & 6.79477200 & -3.97156600 & 2.35327300 \\
\hline $\mathrm{C}$ & 1.92188100 & -0.02682000 & -1.32308200 & $\mathrm{H}$ & 5.94054600 & -6.22883800 & 2.47682000 \\
\hline $\mathrm{H}$ & 1.01333600 & -1.27879100 & -2.82682300 & $\mathrm{H}$ & 6.49768700 & -6.62644800 & 0.85467800 \\
\hline $\mathrm{H}$ & 2.80859400 & 0.96121500 & 0.37746600 & $\mathrm{C}$ & 7.05691400 & -2.33692500 & 0.20635000 \\
\hline $\mathrm{H}$ & 2.01133100 & 0.83678500 & -1.97655600 & $\mathrm{C}$ & 6.62494100 & -1.14161100 & 0.78703500 \\
\hline $\mathrm{H}$ & 1.20347900 & -4.31249000 & 0.68554200 & $\mathrm{C}$ & 9.17928700 & -1.30430300 & -0.36394800 \\
\hline $\mathrm{H}$ & 0.86889600 & -2.41230900 & 3.08142600 & $\mathrm{C}$ & 7.50019400 & -0.03830700 & 0.89511000 \\
\hline $\mathrm{O}$ & 3.27509100 & -3.49611900 & 2.97640500 & $\mathrm{C}$ & 8.78394900 & -0.16565500 & 0.33658900 \\
\hline $\mathrm{H}$ & -0.44390200 & -2.95285700 & 2.01196300 & $\mathrm{H}$ & 10.15857900 & -1.34623900 & -0.83474000 \\
\hline \multirow[t]{2}{*}{$\mathrm{H}$} & 2.61081300 & -1.00728100 & 1.86370800 & $\mathrm{H}$ & 9.47364100 & 0.66705000 & 0.43834200 \\
\hline & & & & $\mathrm{C}$ & 4.77102700 & -5.30886100 & 0.88204000 \\
\hline $15 \mathbf{a}$ & & & & $\mathrm{C}$ & 3.52007000 & -5.91475000 & 0.88530200 \\
\hline $\mathrm{C}$ & -1.69937600 & 1.81387800 & 2.52614300 & $\mathrm{C}$ & 3.85064400 & -3.34012200 & -0.17955600 \\
\hline $\mathrm{C}$ & -0.50411800 & 1.63338800 & 1.83050000 & $\mathrm{C}$ & 2.44255600 & -5.23624100 & 0.31513300 \\
\hline $\mathrm{C}$ & 0.55383600 & 2.54622900 & 1.96599800 & $\mathrm{H}$ & 3.37770500 & -6.89962700 & 1.32354700 \\
\hline $\mathrm{C}$ & 0.38511000 & 3.63967000 & 2.82471600 & $\mathrm{C}$ & 2.56976300 & -3.93401200 & -0.19362100 \\
\hline $\mathrm{C}$ & -0.81029000 & 3.82048900 & 3.52573300 & $\mathrm{H}$ & 1.46479200 & -5.70820700 & 0.28781500 \\
\hline $\mathrm{C}$ & -1.85724800 & 2.91092300 & 3.37660400 & $\mathrm{O}$ & 5.34929100 & -1.06304000 & 1.31855600 \\
\hline $\mathrm{H}$ & -2.50273400 & 1.09083400 & 2.41312100 & $\mathrm{O}$ & 4.02748300 & -2.05469600 & -0.66955500 \\
\hline $\mathrm{H}$ & -0.37964200 & 0.76548200 & 1.18781200 & $\mathrm{P}$ & 4.01839500 & -0.76934200 & 0.38099400 \\
\hline $\mathrm{C}$ & 1.83106300 & 2.30957100 & 1.16554100 & $\mathrm{O}$ & 2.84327700 & -0.77496500 & 1.30118200 \\
\hline $\mathrm{H}$ & -0.91877800 & 4.67265200 & 4.19157700 & $\mathrm{O}$ & 4.27329100 & 0.42975100 & -0.52574000 \\
\hline $\mathrm{H}$ & -2.78535700 & 3.05001300 & 3.92435800 & $\mathrm{C}$ & 7.14666300 & 1.24191300 & 1.59444100 \\
\hline $\mathrm{C}$ & 1.66912500 & 2.69287700 & -0.29749100 & $\mathrm{C}$ & 6.89302700 & 1.25636800 & 2.98897300 \\
\hline $\mathrm{C}$ & 0.66479900 & 3.73398200 & -0.68618400 & $\mathrm{C}$ & 7.17943600 & 2.47223100 & 0.88414900 \\
\hline $\mathrm{H}$ & 0.82834000 & 4.63872700 & -0.08908200 & $\mathrm{C}$ & 6.69290100 & 2.48479500 & 3.63204600 \\
\hline $\mathrm{H}$ & -0.33436600 & 3.37520000 & -0.41791000 & $\mathrm{C}$ & 6.98118600 & 3.66951200 & 1.58192700 \\
\hline $\mathrm{H}$ & 0.69286200 & 3.98184400 & -1.74611900 & $\mathrm{C}$ & 6.74600300 & 3.70527100 & 2.95884300 \\
\hline $\mathrm{N}$ & 2.43635500 & 2.11120800 & -1.16637200 & $\mathrm{H}$ & 6.51399900 & 2.49033900 & 4.70519100 \\
\hline $\mathrm{C}$ & 2.49296000 & 2.39047300 & -2.57648800 & $\mathrm{H}$ & 7.02448000 & 4.60491900 & 1.02878100 \\
\hline $\mathrm{C}$ & 2.16639900 & 1.36374700 & -3.46493100 & $\mathrm{C}$ & 1.36589500 & -3.22099900 & -0.73225100 \\
\hline $\mathrm{C}$ & 2.94350700 & 3.62850700 & -3.04390000 & $\mathrm{C}$ & 1.26381200 & -2.95355600 & -2.12055200 \\
\hline $\mathrm{C}$ & 2.26558100 & 1.59433500 & -4.83732000 & $\mathrm{C}$ & 0.30566300 & -2.85136100 & 0.13145800 \\
\hline $\mathrm{H}$ & 1.83771300 & 0.40312900 & -3.08204300 & $\mathrm{C}$ & 0.13587000 & -2.28188800 & -2.60571000 \\
\hline $\mathrm{C}$ & 3.03924800 & 3.84698600 & -4.41862300 & $\mathrm{C}$ & -0.80521900 & -2.19084000 & -0.41076800 \\
\hline $\mathrm{H}$ & 3.23641900 & 4.39911700 & -2.33752900 & $\mathrm{C}$ & -0.91111600 & -1.88395900 & -1.76797700 \\
\hline $\mathrm{C}$ & 2.69754000 & 2.83319600 & -5.31607600 & $\mathrm{H}$ & 0.06208100 & -2.09161100 & -3.67433500 \\
\hline $\mathrm{H}$ & 2.01103700 & 0.79877000 & -5.53128700 & $\mathrm{H}$ & -1.62388200 & -1.91343600 & 0.25020700 \\
\hline $\mathrm{H}$ & 3.39108800 & 4.80698000 & -4.78508700 & $\mathrm{C}$ & 7.42781100 & 2.56139200 & -0.62418100 \\
\hline $\mathrm{H}$ & 2.77734700 & 3.00521300 & -6.38557500 & $\mathrm{H}$ & 7.52895600 & 1.54670200 & -1.01448400 \\
\hline
\end{tabular}




\begin{tabular}{|c|c|c|c|c|c|c|c|}
\hline $\mathrm{C}$ & 6.60443900 & 5.01962300 & 3.71712200 & $\mathrm{C}$ & 0.05132700 & -1.96074600 & 2.52150500 \\
\hline $\mathrm{H}$ & 6.36056800 & 4.76590000 & 4.75801500 & $\mathrm{H}$ & 0.82311900 & -1.20897200 & 2.34595200 \\
\hline $\mathrm{C}$ & 6.88461000 & -0.00619900 & 3.85058600 & $\mathrm{H}$ & 0.09327000 & -2.25896900 & 3.57668500 \\
\hline $\mathrm{H}$ & 7.07059500 & -0.86469700 & 3.20264300 & $\mathrm{H}$ & -0.93723500 & -1.51522500 & 2.35191200 \\
\hline $\mathrm{C}$ & 6.23397300 & 3.19289800 & -1.36330000 & $\mathrm{H}$ & 1.19130100 & 4.35390900 & 2.96201900 \\
\hline $\mathrm{H}$ & 6.43061400 & 3.23101500 & -2.44203400 & $\mathrm{C}$ & 3.08356600 & 3.03380100 & 1.71734000 \\
\hline $\mathrm{H}$ & 6.03913700 & 4.21846700 & -1.02380100 & $\mathrm{H}$ & 3.19652200 & 2.79747500 & 2.77898800 \\
\hline $\mathrm{H}$ & 5.33939200 & 2.58723900 & -1.20247900 & $\mathrm{H}$ & 3.02216400 & 4.12291900 & 1.61042200 \\
\hline $\mathrm{C}$ & 8.73170400 & 3.31811800 & -0.94681400 & $\mathrm{H}$ & 3.97945100 & 2.68408600 & 1.20006300 \\
\hline $\mathrm{H}$ & 8.91688900 & 3.31842300 & -2.02812000 & & & & \\
\hline $\mathrm{H}$ & 9.59778400 & 2.86024100 & -0.45589200 & TS-9 & & & \\
\hline $\mathrm{H}$ & 8.68293300 & 4.36346000 & -0.61812200 & $\mathrm{C}$ & 0.93996300 & -2.63939600 & 5.08304200 \\
\hline $\mathrm{C}$ & 5.46152100 & 5.90090200 & 3.18097700 & $\mathrm{C}$ & 0.66361900 & -2.23675100 & 3.77501800 \\
\hline $\mathrm{H}$ & 5.36724700 & 6.81645300 & 3.77768700 & $\mathrm{C}$ & 0.56242900 & -3.17613400 & 2.73591500 \\
\hline $\mathrm{H}$ & 4.50350400 & 5.37084600 & 3.21735500 & $\mathrm{C}$ & 0.72657700 & -4.53408800 & 3.05721400 \\
\hline $\mathrm{H}$ & 5.64055100 & 6.20099800 & 2.14140600 & $\mathrm{C}$ & 1.00740800 & -4.93980400 & 4.36269000 \\
\hline $\mathrm{C}$ & 7.93357900 & 5.80087200 & 3.73415600 & $\mathrm{C}$ & 1.11702900 & -3.99157800 & 5.38152700 \\
\hline $\mathrm{H}$ & 7.84140900 & 6.71426500 & 4.33490700 & $\mathrm{H}$ & 1.01785400 & -1.89291500 & 5.86939400 \\
\hline $\mathrm{H}$ & 8.23200500 & 6.09475700 & 2.72054100 & $\mathrm{H}$ & 0.52754600 & -1.18219900 & 3.55116300 \\
\hline $\mathrm{H}$ & 8.74201600 & 5.19389600 & 4.15591900 & $\mathrm{C}$ & 0.17675300 & -2.73801500 & 1.34144100 \\
\hline $\mathrm{C}$ & 8.02303400 & 0.01746600 & 4.89089200 & $\mathrm{H}$ & 1.13332900 & -5.99666600 & 4.58425600 \\
\hline $\mathrm{H}$ & 7.90070600 & 0.83570700 & 5.61085500 & $\mathrm{H}$ & 1.33347200 & -4.30454500 & 6.39951600 \\
\hline $\mathrm{H}$ & 8.99923100 & 0.14145300 & 4.40810100 & $\mathrm{C}$ & 1.00056800 & -3.12257100 & 0.25230200 \\
\hline $\mathrm{H}$ & 8.04090500 & -0.92175100 & 5.45749100 & $\mathrm{C}$ & 2.40430500 & -3.62789300 & 0.46200500 \\
\hline $\mathrm{C}$ & 5.51740000 & -0.23306500 & 4.52449200 & $\mathrm{H}$ & 2.76301300 & -3.31382900 & 1.44238000 \\
\hline $\mathrm{H}$ & 5.52818400 & -1.16944600 & 5.09620200 & $\mathrm{H}$ & 3.08077800 & -3.25470900 & -0.31065000 \\
\hline $\mathrm{H}$ & 4.71968400 & -0.29975200 & 3.77927100 & $\mathrm{H}$ & 2.43989900 & -4.72298900 & 0.44396400 \\
\hline $\mathrm{H}$ & 5.27486500 & 0.57624400 & 5.22479500 & $\mathrm{~N}$ & 0.57816500 & -2.90209300 & -1.01140700 \\
\hline $\mathrm{C}$ & 2.30528800 & -3.45125100 & -3.12455800 & $\mathrm{C}$ & 1.25074900 & -3.16695200 & -2.23464500 \\
\hline $\mathrm{H}$ & 3.10957500 & -3.93436200 & -2.56468100 & $\mathrm{C}$ & 1.23841300 & -2.17302900 & -3.22389100 \\
\hline $\mathrm{C}$ & -2.14161700 & -1.16642100 & -2.30929500 & $\mathrm{C}$ & 1.83951100 & -4.40957400 & -2.50281300 \\
\hline $\mathrm{H}$ & -2.81217600 & -0.99072400 & -1.45662400 & $\mathrm{C}$ & 1.83635100 & -2.41763400 & -4.45847700 \\
\hline $\mathrm{C}$ & 0.29568500 & -3.18642700 & 1.62471700 & $\mathrm{H}$ & 0.76302400 & -1.22100300 & -3.00944300 \\
\hline $\mathrm{H}$ & 1.28368900 & -3.56885100 & 1.88963000 & $\mathrm{C}$ & 2.44830900 & -4.63783100 & -3.73733800 \\
\hline $\mathrm{C}$ & -1.79082800 & 0.20905300 & -2.90779200 & $\mathrm{H}$ & 1.79927900 & -5.20131100 & -1.76261000 \\
\hline $\mathrm{H}$ & -2.69546100 & 0.73252400 & -3.24109800 & $\mathrm{C}$ & 2.45150600 & -3.64491400 & -4.71912300 \\
\hline $\mathrm{H}$ & -1.12603500 & 0.10866600 & -3.77404300 & $\mathrm{H}$ & 1.82501600 & -1.64024200 & -5.21734800 \\
\hline $\mathrm{H}$ & -1.28286300 & 0.84175300 & -2.17063700 & $\mathrm{H}$ & 2.90449400 & -5.60385400 & -3.93588400 \\
\hline $\mathrm{C}$ & -2.91224200 & -2.02768200 & -3.32820700 & $\mathrm{H}$ & 2.91949200 & -3.83014200 & -5.68181200 \\
\hline $\mathrm{H}$ & -3.82483800 & -1.51606400 & -3.65804500 & $\mathrm{H}$ & -0.19175300 & -2.23164300 & -1.10251100 \\
\hline $\mathrm{H}$ & -3.19851100 & -2.99122900 & -2.89293600 & $\mathrm{H}$ & 0.43021300 & -1.33078800 & 1.21393300 \\
\hline $\mathrm{H}$ & -2.30486700 & -2.23174700 & -4.21803900 & $\mathrm{C}$ & -0.93353700 & 5.24503000 & -1.09412200 \\
\hline $\mathrm{C}$ & 1.70172100 & -4.52407500 & -4.05420400 & $\mathrm{C}$ & -3.01006800 & 4.07888500 & -1.12458800 \\
\hline $\mathrm{H}$ & 0.89217500 & -4.11502800 & -4.67084500 & $\mathrm{C}$ & -2.41042500 & 5.41385700 & -1.50916700 \\
\hline $\mathrm{H}$ & 1.29048300 & -5.36002900 & -3.47740700 & $\mathrm{H}$ & -0.37076500 & 4.77504300 & -1.90901500 \\
\hline $\mathrm{H}$ & 2.46839400 & -4.92203900 & -4.73057300 & $\mathrm{H}$ & -0.43824500 & 6.18931500 & -0.84806900 \\
\hline $\mathrm{C}$ & 2.94633500 & -2.31194400 & -3.93657000 & $\mathrm{H}$ & -2.88836400 & 6.23999300 & -0.96382100 \\
\hline $\mathrm{H}$ & 3.41585700 & -1.57881300 & -3.27416600 & $\mathrm{H}$ & -2.52749000 & 5.63362700 & -2.57674500 \\
\hline $\mathrm{H}$ & 2.20630600 & -1.79810200 & -4.56439900 & $\mathrm{C}$ & -0.97448500 & 4.25583700 & 0.12092800 \\
\hline $\mathrm{H}$ & 3.71954000 & -2.70892400 & -4.60613900 & $\mathrm{C}$ & -1.33307300 & 5.04823200 & 1.42246000 \\
\hline $\mathrm{C}$ & -0.73654000 & -4.29284100 & 1.92805800 & $\mathrm{C}$ & 0.43377900 & 3.78505300 & 0.47345200 \\
\hline $\mathrm{H}$ & -1.75682100 & -3.95533900 & 1.70664700 & $\mathrm{C}$ & -0.00222300 & 5.69839100 & 1.85869000 \\
\hline $\mathrm{H}$ & -0.70000100 & -4.57024200 & 2.98876100 & $\mathrm{H}$ & -2.13980800 & 5.77049900 & 1.26350700 \\
\hline $\mathrm{H}$ & -0.55442300 & -5.19657200 & 1.33553300 & $\mathrm{H}$ & -1.66722900 & 4.33556700 & 2.18526800 \\
\hline
\end{tabular}




\begin{tabular}{|c|c|c|c|c|}
\hline $\mathrm{H}$ & 0.06435500 & 5.83539900 & 2.94437800 & $\mathrm{H}$ \\
\hline $\mathrm{H}$ & 0.12640500 & 6.69297300 & 1.40893200 & $\mathrm{C}$ \\
\hline $\mathrm{C}$ & -2.12598400 & 3.35408300 & -0.30914900 & $\mathrm{H}$ \\
\hline $\mathrm{C}$ & -2.44111300 & 2.04310000 & 0.04504800 & $\mathrm{H}$ \\
\hline $\mathrm{C}$ & -4.24691000 & 3.54897600 & -1.47246700 & $\mathrm{H}$ \\
\hline $\mathrm{C}$ & -3.69939900 & 1.48760700 & -0.26491400 & $\mathrm{C}$ \\
\hline $\mathrm{C}$ & -4.59144600 & 2.28048200 & -1.00616600 & $\mathrm{H}$ \\
\hline $\mathrm{H}$ & -4.94258100 & 4.11345800 & -2.08850300 & $\mathrm{H}$ \\
\hline $\mathrm{H}$ & -5.57129900 & 1.87270700 & -1.23427700 & $\mathrm{H}$ \\
\hline $\mathrm{C}$ & 1.02792500 & 4.71856500 & 1.33872500 & $\mathrm{C}$ \\
\hline $\mathrm{C}$ & 2.39153000 & 4.67049100 & 1.60363000 & $\mathrm{H}$ \\
\hline $\mathrm{C}$ & 1.19253600 & 2.71309900 & 0.00307400 & $\mathrm{H}$ \\
\hline $\mathrm{C}$ & 3.15994600 & 3.67480200 & 1.00086500 & $\mathrm{H}$ \\
\hline $\mathrm{H}$ & 2.85999400 & 5.40529600 & 2.25380200 & $\mathrm{C}$ \\
\hline $\mathrm{C}$ & 2.58669800 & 2.66089100 & 0.21452700 & $\mathrm{H}$ \\
\hline $\mathrm{H}$ & 4.23452600 & 3.66046500 & 1.15426900 & $\mathrm{C}$ \\
\hline $\mathrm{O}$ & -1.50147100 & 1.29658500 & 0.75825500 & $\mathrm{H}$ \\
\hline $\mathrm{O}$ & 0.57339900 & 1.71068700 & -0.74328900 & $\mathrm{C}$ \\
\hline $\mathrm{P}$ & -0.30614000 & 0.51689800 & -0.04969100 & $\mathrm{H}$ \\
\hline $\mathrm{O}$ & 0.51473500 & -0.12090600 & 1.10115700 & $\mathrm{C}$ \\
\hline $\mathrm{O}$ & -0.79684300 & -0.36664300 & -1.15168300 & $\mathrm{H}$ \\
\hline $\mathrm{C}$ & -4.14387900 & 0.12931900 & 0.19330400 & $\mathrm{H}$ \\
\hline $\mathrm{C}$ & -4.39725200 & -0.10358800 & 1.56947300 & $\mathrm{H}$ \\
\hline $\mathrm{C}$ & -4.41596000 & -0.89276700 & -0.74969300 & $\mathrm{C}$ \\
\hline $\mathrm{C}$ & -4.90524700 & -1.34468100 & 1.96832500 & $\mathrm{H}$ \\
\hline $\mathrm{C}$ & -4.92593400 & -2.11499100 & -0.29203200 & $\mathrm{H}$ \\
\hline $\mathrm{C}$ & -5.18183700 & -2.36710600 & 1.05627800 & $\mathrm{H}$ \\
\hline $\mathrm{H}$ & -5.10690500 & -1.50884300 & 3.02412900 & $\mathrm{C}$ \\
\hline $\mathrm{H}$ & -5.14001800 & -2.89885700 & -1.01553700 & $\mathrm{H}$ \\
\hline $\mathrm{C}$ & 3.47104500 & 1.61474700 & -0.39831300 & $\mathrm{H}$ \\
\hline $\mathrm{C}$ & 3.63427300 & 1.55967400 & -1.80523100 & $\mathrm{H}$ \\
\hline $\mathrm{C}$ & 4.22191500 & 0.73388300 & 0.42370300 & $\mathrm{C}$ \\
\hline $\mathrm{C}$ & 4.51141100 & 0.61481600 & -2.35088800 & $\mathrm{H}$ \\
\hline $\mathrm{C}$ & 5.09192400 & -0.18267500 & -0.17825200 & $\mathrm{H}$ \\
\hline $\mathrm{C}$ & 5.25269300 & -0.26592200 & -1.56358900 & $\mathrm{H}$ \\
\hline $\mathrm{H}$ & 4.63819700 & 0.57771600 & -3.43062500 & $\mathrm{C}$ \\
\hline $\mathrm{H}$ & 5.66963800 & -0.84595000 & 0.46082500 & $\mathrm{H}$ \\
\hline $\mathrm{C}$ & -4.21107800 & -0.71483500 & -2.25615800 & $\mathrm{H}$ \\
\hline $\mathrm{H}$ & -3.70437200 & 0.23967500 & -2.41882300 & $\mathrm{H}$ \\
\hline $\mathrm{C}$ & -5.78141300 & -3.69597200 & 1.49966100 & $\mathrm{C}$ \\
\hline $\mathrm{H}$ & -5.81584400 & -4.34462900 & 0.61328600 & $\mathrm{H}$ \\
\hline $\mathrm{C}$ & -4.19532500 & 0.96838300 & 2.64166700 & $\mathrm{H}$ \\
\hline $\mathrm{H}$ & -3.80906500 & 1.86860400 & 2.15768200 & $\mathrm{H}$ \\
\hline $\mathrm{C}$ & -3.30767900 & -1.80232000 & -2.86700500 & $\mathrm{C}$ \\
\hline $\mathrm{H}$ & -3.18094500 & -1.62290300 & -3.94185600 & $\mathrm{H}$ \\
\hline $\mathrm{H}$ & -3.73248900 & -2.80641700 & -2.75028100 & $\mathrm{H}$ \\
\hline $\mathrm{H}$ & -2.32016800 & -1.77885700 & -2.40224500 & $\mathrm{H}$ \\
\hline $\mathrm{C}$ & -5.56342700 & -0.66596000 & -2.99749600 & $\mathrm{H}$ \\
\hline $\mathrm{H}$ & -5.40795700 & -0.48766700 & -4.06859700 & \\
\hline $\mathrm{H}$ & -6.21194800 & 0.12908200 & -2.61312500 & 16a \\
\hline $\mathrm{H}$ & -6.10658400 & -1.61287500 & -2.89203100 & $\mathrm{C}$ \\
\hline $\mathrm{C}$ & -7.23068500 & -3.51964900 & 1.99499400 & $\mathrm{C}$ \\
\hline $\mathrm{H}$ & -7.67417300 & -4.48942800 & 2.25244200 & $\mathrm{C}$ \\
\hline $\mathrm{H}$ & -7.85622600 & -3.05027000 & 1.22774400 & $\mathrm{C}$ \\
\hline
\end{tabular}

$\begin{array}{rrr}-7.26882400 & -2.88633800 & 2.88953600 \\ -4.92293200 & -4.40973200 & 2.55968900 \\ -5.35382800 & -5.38670100 & 2.81031200 \\ -4.86357300 & -3.82700900 & 3.48656200 \\ -3.90015100 & -4.56899500 & 2.20162400 \\ -5.53312700 & 1.35967900 & 3.30177500 \\ -5.97749000 & 0.51849900 & 3.84708400 \\ -6.26102100 & 1.69346800 & 2.55370000 \\ -5.38235000 & 2.17688700 & 4.01789800 \\ -3.15797600 & 0.54259700 & 3.69806600 \\ -3.00987800 & 1.34377900 & 4.43310700 \\ -2.19256100 & 0.32867500 & 3.22964200 \\ -3.48119800 & -0.35125500 & 4.24532000 \\ 2.95297300 & 2.53872900 & -2.76310600 \\ 2.31303300 & 3.20348300 & -2.17798900 \\ 6.22697000 & -1.24905200 & -2.20031800 \\ 6.14890200 & -1.11994300 & -3.28857200 \\ 4.14905300 & 0.75509400 & 1.95261000 \\ 3.35347100 & 1.44433300 & 2.24613500 \\ 7.68390300 & -0.93998000 & -1.80320300 \\ 8.37831300 & -1.61535700 & -2.31789800 \\ 7.83607400 & -1.06397600 & -0.72430200 \\ 7.95410600 & 0.08991300 & -2.06108800 \\ 5.87083200 & -2.71389600 & -1.88602400 \\ 6.57039200 & -3.39697800 & -2.38353500 \\ 4.85905800 & -2.95743700 & -2.22783900 \\ 5.92415300 & -2.91357100 & -0.80874400 \\ 3.99171700 & 3.43424800 & -3.46917800 \\ 4.66328700 & 2.84834000 & -4.10783600 \\ 4.60947100 & 3.97322700 & -2.74199900 \\ 3.48990700 & 4.17454200 & -4.10442300 \\ 2.04841600 & 1.82672200 & -3.78610300 \\ 1.27183000 & 1.24795400 & -3.27813600 \\ 2.62167500 & 1.14914300 & -4.43085800 \\ 1.55663300 & 2.56176000 & -4.43555000 \\ 5.46670500 & 1.26586200 & 2.57067800 \\ 6.30276300 & 0.59876800 & 2.32838000 \\ 5.38460900 & 1.31505900 & 3.66344700 \\ 5.72868000 & 2.26512700 & 2.20569000 \\ 3.78140400 & -0.61779700 & 2.54160800 \\ 2.82919500 & -0.96489800 & 2.13516100 \\ 3.67805800 & -0.55008300 & 3.63118800 \\ 4.54749000 & -1.37413000 & 2.33150800 \\ -1.35126600 & -2.73028300 & 1.16102500 \\ -1.69138600 & -2.23141700 & 0.25256000 \\ -1.81979700 & -2.20975500 & 2.00003500 \\ -1.73858000 & -3.75820200 & 1.15481300 \\ 0.62352200 & -5.28274900 & 2.27467900\end{array}$

6a

$\mathrm{C}$

$\begin{array}{lll}-2.77763700 & -0.57012600 & 0.46699100\end{array}$

$\begin{array}{lll}-1.87704400 & 0.07869000 & 1.31291500\end{array}$

$\begin{array}{lll}-0.50267700 & -0.03589400 & 1.09740300\end{array}$

$\begin{array}{lll}0.00617700 & -0.80586700 & 0.03737100\end{array}$ 


\begin{tabular}{|c|c|c|c|c|c|c|c|}
\hline $\mathrm{C}$ & -0.91737900 & -1.44449900 & -0.81076600 & $\mathrm{C}$ & 6.64326700 & 2.14689500 & -5.50527800 \\
\hline $\mathrm{C}$ & -2.29037900 & -1.33305600 & -0.59725200 & $\mathrm{H}$ & 4.66285800 & 2.21613800 & -4.61600000 \\
\hline $\mathrm{H}$ & -3.84796500 & -0.48036400 & 0.63219200 & $\mathrm{C}$ & 7.83533100 & 1.42812900 & -5.61735100 \\
\hline $\mathrm{H}$ & -2.24316500 & 0.68290400 & 2.13920400 & $\mathrm{H}$ & 8.89529300 & -0.36542400 & -5.04963200 \\
\hline $\mathrm{C}$ & 1.47099200 & -0.91981800 & -0.21342300 & $\mathrm{H}$ & 6.53916800 & 3.11022700 & -5.99646400 \\
\hline $\mathrm{H}$ & -0.55230000 & -2.05090000 & -1.63604400 & $\mathrm{H}$ & 8.65924300 & 1.82651300 & -6.20299400 \\
\hline $\mathrm{H}$ & -2.98171500 & -1.84604000 & -1.26111300 & $\mathrm{H}$ & 4.91061000 & -0.26300700 & -2.3294960 \\
\hline $\mathrm{C}$ & 2.34230000 & -1.33520400 & 0.74101300 & $\mathrm{H}$ & 1.95443000 & 0.62265600 & -2.37702200 \\
\hline $\mathrm{C}$ & 1.94836800 & -1.84563300 & 2.10978500 & $\mathrm{C}$ & 1.47728600 & 7.73048500 & -2.05249800 \\
\hline $\mathrm{H}$ & 2.09115800 & -1.06429600 & 2.86794900 & $\mathrm{C}$ & 0.54620400 & 7.12085700 & -4.16024200 \\
\hline $\mathrm{H}$ & 0.90494400 & -2.16379700 & 2.13672300 & $\mathrm{C}$ & 1.00258500 & 8.34088400 & -3.38921100 \\
\hline $\mathrm{H}$ & 2.57650500 & -2.68858700 & 2.41197300 & $\mathrm{H}$ & 2.52423900 & 7.41664600 & -2.13533900 \\
\hline $\mathrm{N}$ & 3.73507700 & -1.20344700 & 0.53871500 & $\mathrm{H}$ & 1.40191700 & 8.42141500 & -1.20719500 \\
\hline $\mathrm{C}$ & 4.70264100 & -2.20719600 & 0.69407700 & $\mathrm{H}$ & 0.18068300 & 9.05418000 & -3.2359730 \\
\hline $\mathrm{C}$ & 4.37181800 & -3.57286200 & 0.73342200 & $\mathrm{H}$ & 1.79759900 & 8.88705200 & -3.91035900 \\
\hline $\mathrm{C}$ & 6.05701600 & -1.83825300 & 0.78909300 & $\mathrm{C}$ & 0.58432600 & 6.45781900 & -1.87043000 \\
\hline $\mathrm{C}$ & 5.37059700 & -4.53330800 & 0.89605700 & $\mathrm{C}$ & -0.82466200 & 6.89494100 & -1.34090800 \\
\hline $\mathrm{H}$ & 3.33844600 & -3.87880100 & 0.60387200 & $\mathrm{C}$ & 1.05905600 & 5.61068700 & -0.69292100 \\
\hline $\mathrm{C}$ & 7.04732000 & -2.80691400 & 0.92607900 & $\mathrm{C}$ & -0.61851600 & 7.10139200 & 0.17251000 \\
\hline $\mathrm{H}$ & 6.32231800 & -0.78347600 & 0.76431100 & $\mathrm{H}$ & -1.20937800 & 7.78047700 & -1.85545400 \\
\hline $\mathrm{C}$ & 6.71272000 & -4.16262600 & 0.99210700 & $\mathrm{H}$ & -1.53196600 & 6.07531300 & -1.51216600 \\
\hline $\mathrm{H}$ & 5.09330600 & -5.58414700 & 0.92570600 & $\mathrm{H}$ & -1.52977300 & 6.91986100 & 0.75429900 \\
\hline $\mathrm{H}$ & 8.08750400 & -2.49823400 & 0.99380200 & $\mathrm{H}$ & -0.30064500 & 8.12910100 & 0.39854400 \\
\hline $\mathrm{H}$ & 7.48608400 & -4.91599300 & 1.10983600 & $\mathrm{C}$ & 0.47995500 & 5.99631500 & -3.32026000 \\
\hline $\mathrm{H}$ & 4.03063900 & -0.41272900 & -0.01574200 & $\mathrm{C}$ & 0.24448500 & 4.73882500 & -3.87615800 \\
\hline $\mathrm{H}$ & 0.19018300 & 0.48797500 & 1.75002500 & $\mathrm{C}$ & 0.21588200 & 7.01993900 & -5.50579600 \\
\hline $\mathrm{C}$ & 1.94995500 & -0.48496600 & -1.58449100 & $\mathrm{C}$ & -0.10477300 & 4.60560900 & -5.23731100 \\
\hline $\mathrm{H}$ & 1.20328100 & -0.71776200 & -2.35058400 & $\mathrm{C}$ & -0.15174100 & 5.77502700 & -6.01482400 \\
\hline $\mathrm{H}$ & 2.11117300 & 0.60297800 & -1.63751600 & $\mathrm{H}$ & 0.23572200 & 7.89311800 & -6.15336900 \\
\hline \multirow[t]{2}{*}{$\mathrm{H}$} & 2.88633400 & -0.97780400 & -1.86601600 & $\mathrm{H}$ & -0.44923700 & 5.68818300 & -7.05527200 \\
\hline & & & & $\mathrm{C}$ & 0.47182300 & 6.10123600 & 0.48632000 \\
\hline TS-10 & & & & $\mathrm{C}$ & 0.90718000 & 5.66960300 & 1.73272800 \\
\hline $\mathrm{C}$ & -1.03324900 & -1.69058800 & -3.88682300 & $\mathrm{C}$ & 1.98233300 & 4.56493100 & -0.61500600 \\
\hline $\mathrm{C}$ & 0.08320300 & -0.91821500 & -3.55589700 & $\mathrm{C}$ & 1.90540300 & 4.70031500 & 1.79295600 \\
\hline $\mathrm{C}$ & 1.23193000 & -1.51069700 & -3.01146700 & $\mathrm{H}$ & 0.47907100 & 6.07572600 & 2.64584400 \\
\hline $\mathrm{C}$ & 1.22069200 & -2.90137400 & -2.80221000 & $\mathrm{C}$ & 2.43060400 & 4.09382400 & 0.63898300 \\
\hline $\mathrm{C}$ & 0.10589300 & -3.67351500 & -3.12829700 & $\mathrm{H}$ & 2.27191800 & 4.36603000 & 2.75892400 \\
\hline $\mathrm{C}$ & -1.02897400 & -3.06948000 & -3.67350100 & $\mathrm{O}$ & 0.32940900 & 3.60462300 & -3.06568500 \\
\hline $\mathrm{H}$ & -1.90989800 & -1.20354300 & -4.30402300 & $\mathrm{O}$ & 2.53128400 & 4.04521000 & -1.78112100 \\
\hline $\mathrm{H}$ & 0.05199300 & 0.15507000 & -3.71255600 & $\mathrm{P}$ & 1.81319100 & 2.95493200 & -2.77707000 \\
\hline $\mathrm{C}$ & 2.44932800 & -0.69939400 & -2.61855500 & $\mathrm{O}$ & 1.47185800 & 1.67502500 & -1.97155500 \\
\hline $\mathrm{H}$ & 0.12549800 & -4.74720000 & -2.95831300 & $\mathrm{O}$ & 2.67105000 & 2.83290400 & -3.98807200 \\
\hline $\mathrm{H}$ & -1.90085100 & -3.66726300 & -3.92592800 & $\mathrm{C}$ & -0.43098200 & 3.29088500 & -5.88036300 \\
\hline $\mathrm{C}$ & 3.38867900 & -0.38517200 & -3.63720300 & $\mathrm{C}$ & -1.58406700 & 2.56816400 & -5.48668300 \\
\hline $\mathrm{C}$ & 2.99457800 & -0.30143600 & -5.08215600 & $\mathrm{C}$ & 0.36636600 & 2.80423500 & -6.95106600 \\
\hline $\mathrm{H}$ & 2.32889200 & -1.13061100 & -5.33188400 & $\mathrm{C}$ & -1.89360300 & 1.37160300 & -6.14628800 \\
\hline $\mathrm{H}$ & 3.86520900 & -0.31714300 & -5.73970500 & $\mathrm{C}$ & 0.00805600 & 1.60167400 & -7.57042200 \\
\hline $\mathrm{H}$ & 2.44915600 & 0.63062900 & -5.25807500 & $\mathrm{C}$ & -1.11412100 & 0.86319100 & -7.18617400 \\
\hline $\mathrm{N}$ & 4.66600000 & -0.12526700 & -3.30319400 & $\mathrm{H}$ & -2.78946200 & 0.82768800 & -5.85332000 \\
\hline $\mathrm{C}$ & 5.71784300 & 0.40775200 & -4.11994000 & $\mathrm{H}$ & 0.62137100 & 1.23855900 & -8.39143700 \\
\hline $\mathrm{C}$ & 6.91056200 & -0.31698900 & -4.22181500 & $\mathrm{C}$ & 3.41423000 & 2.97558100 & 0.81390400 \\
\hline $\mathrm{C}$ & 5.58039000 & 1.64963400 & -4.74835500 & $\mathrm{C}$ & 4.75846000 & 3.11498800 & 0.38491700 \\
\hline $\mathrm{C}$ & 7.96986400 & 0.19800500 & -4.96897300 & $\mathrm{C}$ & 3.01979300 & 1.80150200 & 1.50802700 \\
\hline $\mathrm{H}$ & 6.99811900 & -1.28028500 & -3.72640900 & $\mathrm{C}$ & 5.66446900 & 2.07818600 & 0.63533000 \\
\hline
\end{tabular}




\begin{tabular}{|c|c|c|c|c|c|c|c|}
\hline $\mathrm{C}$ & 3.97551100 & 0.80440600 & 1.74542000 & $\mathrm{H}$ & 6.75469200 & 3.45754700 & -1.61275000 \\
\hline $\mathrm{C}$ & 5.30038800 & 0.91518100 & 1.32017400 & $\mathrm{H}$ & 6.23177200 & 5.07141400 & -2.11044700 \\
\hline $\mathrm{H}$ & 6.69518000 & 2.20068200 & 0.31098900 & $\mathrm{C}$ & 1.57616900 & 1.46419000 & 3.57177200 \\
\hline $\mathrm{H}$ & 3.67561600 & -0.09273600 & 2.28339400 & $\mathrm{H}$ & 2.12957300 & 0.58495500 & 3.92281500 \\
\hline $\mathrm{C}$ & 1.58318500 & 3.55570900 & -7.50036300 & $\mathrm{H}$ & 0.54450000 & 1.37127000 & 3.93225100 \\
\hline $\mathrm{H}$ & 1.76443800 & 4.42403100 & -6.86385300 & $\mathrm{H}$ & 2.02294500 & 2.34509200 & 4.04681700 \\
\hline $\mathrm{C}$ & -1.50517000 & -0.42476800 & -7.89983600 & $\mathrm{C}$ & 0.91531500 & 0.34931800 & 1.39409600 \\
\hline $\mathrm{H}$ & -2.37709400 & -0.83465900 & -7.37123800 & $\mathrm{H}$ & 0.84870700 & 0.46789800 & 0.30915300 \\
\hline $\mathrm{C}$ & -2.55884500 & 3.08000500 & -4.42502400 & $\mathrm{H}$ & -0.10275700 & 0.24131800 & 1.7882950 \\
\hline $\mathrm{H}$ & -2.15808800 & 4.00959300 & -4.01423400 & $\mathrm{H}$ & 1.45293100 & -0.58103200 & 1.6143380 \\
\hline $\mathrm{C}$ & 2.87223000 & 2.71638300 & -7.46395500 & $\mathrm{C}$ & 3.02482500 & -1.04643400 & -1.24165700 \\
\hline $\mathrm{H}$ & 3.71002400 & 3.29906200 & -7.86756900 & $\mathrm{H}$ & 3.52731700 & -0.19304100 & -0.7736650 \\
\hline $\mathrm{H}$ & 2.79020600 & 1.80220400 & -8.06443500 & $\mathrm{H}$ & 3.73615500 & -1.88722200 & -1.2774590 \\
\hline $\mathrm{H}$ & 3.11172400 & 2.44315200 & -6.43480700 & $\mathrm{H}$ & 2.21866100 & -1.33355000 & -0.5654380 \\
\hline $\mathrm{C}$ & 1.31524100 & 4.06983600 & -8.93031200 & $\mathrm{H}$ & 2.09750400 & -3.38642200 & -2.3807380 \\
\hline $\mathrm{H}$ & 2.16540500 & 4.66223800 & -9.29046900 & & & & \\
\hline $\mathrm{H}$ & 0.41965400 & 4.69969700 & -8.97673300 & $15 b$ & & & \\
\hline $\mathrm{H}$ & 1.16759600 & 3.23905800 & -9.63090300 & $\mathrm{C}$ & -0.02437500 & -2.61408100 & -2.27618000 \\
\hline $\mathrm{C}$ & -0.39236100 & -1.48781700 & -7.84233600 & $\mathrm{C}$ & 0.66856000 & -1.41580400 & -2.10819500 \\
\hline $\mathrm{H}$ & -0.72217100 & -2.41755900 & -8.32191500 & $\mathrm{C}$ & 1.84089500 & -1.37159200 & -1.34053600 \\
\hline $\mathrm{H}$ & -0.11993800 & -1.71604800 & -6.80649700 & $\mathrm{C}$ & 2.29744400 & -2.55038700 & -0.73460300 \\
\hline $\mathrm{H}$ & 0.51050400 & -1.14894500 & -8.36460900 & $\mathrm{C}$ & 1.60157400 & -3.75014000 & -0.90113100 \\
\hline $\mathrm{C}$ & -1.93574900 & -0.15395700 & -9.35495300 & $\mathrm{C}$ & 0.44185100 & -3.78612000 & -1.67673300 \\
\hline $\mathrm{H}$ & -2.27363900 & -1.07867100 & -9.83879700 & $\mathrm{H}$ & -0.93118000 & -2.62264900 & -2.87174100 \\
\hline $\mathrm{H}$ & -1.10342600 & 0.24727400 & -9.94561100 & $\mathrm{H}$ & 0.29253500 & -0.50191600 & -2.55992300 \\
\hline $\mathrm{H}$ & -2.75438100 & 0.57300700 & -9.39650300 & $\mathrm{C}$ & 2.56927900 & -0.02919800 & -1.21251900 \\
\hline $\mathrm{C}$ & -3.92490300 & 3.42605000 & -5.05272600 & $\mathrm{H}$ & 1.96490100 & -4.65368100 & -0.41840400 \\
\hline $\mathrm{H}$ & -4.41283900 & 2.53873800 & -5.47341600 & $\mathrm{H}$ & -0.10016900 & -4.71924200 & -1.80567300 \\
\hline $\mathrm{H}$ & -3.81605700 & 4.16024100 & -5.85901000 & $\mathrm{C}$ & 3.50382500 & 0.13653300 & -2.39856700 \\
\hline $\mathrm{H}$ & -4.59749700 & 3.84841900 & -4.29594100 & $\mathrm{C}$ & 4.73027500 & -0.72317200 & -2.47984700 \\
\hline $\mathrm{C}$ & -2.72262500 & 2.09989000 & -3.24872300 & $\mathrm{H}$ & 4.51496300 & -1.69448800 & -2.02813600 \\
\hline $\mathrm{H}$ & -3.39163100 & 2.52639600 & -2.49094500 & $\mathrm{H}$ & 5.55543400 & -0.27328600 & -1.91435400 \\
\hline $\mathrm{H}$ & -1.75966700 & 1.89257900 & -2.77332800 & $\mathrm{H}$ & 5.06192400 & -0.86703400 & -3.51022100 \\
\hline $\mathrm{H}$ & -3.16130100 & 1.14788400 & -3.57204300 & $\mathrm{~N}$ & 3.18779100 & 0.99932500 & -3.31767600 \\
\hline $\mathrm{C}$ & 5.28842400 & 4.39115200 & -0.27020900 & $\mathrm{C}$ & 3.91262200 & 1.36290000 & -4.49597500 \\
\hline $\mathrm{H}$ & 4.45127600 & 5.07923500 & -0.40475700 & $\mathrm{C}$ & 5.30344200 & 1.52625700 & -4.51047900 \\
\hline $\mathrm{C}$ & 6.31502100 & -0.17983800 & 1.62776800 & $\mathrm{C}$ & 3.15177200 & 1.64448200 & -5.63897500 \\
\hline $\mathrm{H}$ & 5.77799800 & -0.97983900 & 2.15564600 & $\mathrm{C}$ & 5.93492600 & 1.91954600 & -5.69026000 \\
\hline $\mathrm{C}$ & 1.59815000 & 1.57239600 & 2.03356700 & $\mathrm{H}$ & 5.88417800 & 1.39287000 & -3.60529500 \\
\hline $\mathrm{H}$ & 0.99061000 & 2.43715200 & 1.76137500 & $\mathrm{C}$ & 3.79693900 & 2.03067200 & -6.81181300 \\
\hline $\mathrm{C}$ & 6.91695600 & -0.79598000 & 0.35029300 & $\mathrm{H}$ & 2.07103000 & 1.57643300 & -5.58559300 \\
\hline $\mathrm{H}$ & 7.62644300 & -1.59408900 & 0.60004600 & $\mathrm{C}$ & 5.18744400 & 2.16038600 & -6.84458500 \\
\hline $\mathrm{H}$ & 7.45568000 & -0.04527700 & -0.24018200 & $\mathrm{H}$ & 7.01306600 & 2.05051500 & -5.69957200 \\
\hline $\mathrm{H}$ & 6.13484300 & -1.23076200 & -0.28396200 & $\mathrm{H}$ & 3.20746300 & 2.24187200 & -7.69904700 \\
\hline $\mathrm{C}$ & 7.42813700 & 0.32597000 & 2.56613800 & $\mathrm{H}$ & 5.68515700 & 2.46707000 & -7.75999600 \\
\hline $\mathrm{H}$ & 8.11367400 & -0.48829200 & 2.83114200 & $\mathrm{H}$ & 2.19618000 & 1.46046800 & -3.27557300 \\
\hline $\mathrm{H}$ & 7.00749100 & 0.73412800 & 3.49144500 & $\mathrm{H}$ & 1.81436600 & 0.76281800 & -1.25754700 \\
\hline $\mathrm{H}$ & 8.01806400 & 1.11828100 & 2.09028400 & $\mathrm{C}$ & -0.67399300 & 7.07022700 & -4.76446100 \\
\hline $\mathrm{C}$ & 6.30416900 & 5.10019400 & 0.64950900 & $\mathrm{C}$ & -1.83580100 & 5.27007400 & -5.81525000 \\
\hline $\mathrm{H}$ & 7.19486100 & 4.48340400 & 0.81939100 & $\mathrm{C}$ & -1.44719700 & 6.71400400 & -6.05326900 \\
\hline $\mathrm{H}$ & 5.86404300 & 5.32672000 & 1.62734100 & $\mathrm{H}$ & 0.38235300 & 6.79958000 & -4.87585800 \\
\hline $\mathrm{H}$ & 6.63407700 & 6.04406400 & 0.19829700 & $\mathrm{H}$ & -0.72152500 & 8.13360900 & -4.50858700 \\
\hline $\mathrm{C}$ & 5.88960100 & 4.12984400 & -1.66332200 & $\mathrm{H}$ & -2.33382300 & 7.35194200 & -6.17718700 \\
\hline $\mathrm{H}$ & 5.14689900 & 3.68783800 & -2.33300700 & $\mathrm{H}$ & -0.84180400 & 6.84114000 & -6.95876800 \\
\hline
\end{tabular}




\begin{tabular}{|c|c|c|c|c|c|c|c|}
\hline $\mathrm{C}$ & -1.30991200 & 6.16000100 & -3.66151400 & $\mathrm{H}$ & -0.86345300 & -0.04116100 & -8.42130800 \\
\hline $\mathrm{C}$ & -2.64771500 & 6.80649400 & -3.16148800 & $\mathrm{H}$ & -2.50395200 & 0.48849600 & -8.00670900 \\
\hline $\mathrm{C}$ & -0.49727600 & 6.19799500 & -2.37362100 & $\mathrm{H}$ & -2.02292100 & -1.19000700 & -7.73535600 \\
\hline $\mathrm{C}$ & -2.21045900 & 7.85908500 & -2.12047700 & $\mathrm{C}$ & -3.57586600 & -3.76444300 & -3.35933200 \\
\hline $\mathrm{H}$ & -3.24334500 & 7.22366700 & -3.97962300 & $\mathrm{H}$ & -4.10533600 & -4.70954800 & -3.1869380 \\
\hline $\mathrm{H}$ & -3.24889100 & 6.03098200 & -2.67316000 & $\mathrm{H}$ & -3.08941600 & -3.47272200 & -2.4223130 \\
\hline $\mathrm{H}$ & -2.96760400 & 8.02746100 & -1.34562200 & $\mathrm{H}$ & -2.79335700 & -3.95655800 & -4.1038460 \\
\hline $\mathrm{H}$ & -2.02055900 & 8.83431800 & -2.59143800 & $\mathrm{C}$ & -5.29667200 & -3.13290900 & -5.11067500 \\
\hline $\mathrm{C}$ & -1.59972700 & 4.90541200 & -4.48075100 & $\mathrm{H}$ & -5.83282300 & -4.07287600 & -4.930397 \\
\hline $\mathrm{C}$ & -1.77093100 & 3.57528400 & -4.08806400 & $\mathrm{H}$ & -4.59923100 & -3.29961100 & -5.9404670 \\
\hline $\mathrm{C}$ & -2.37813600 & 4.35732400 & -6.71283800 & $\mathrm{H}$ & -6.02415700 & -2.38023400 & -5.4334560 \\
\hline $\mathrm{C}$ & -2.38259900 & 2.64514700 & -4.95658700 & $\mathrm{C}$ & -5.95259800 & 2.37864300 & -3.09298000 \\
\hline $\mathrm{C}$ & -2.69132300 & 3.07646700 & -6.25907800 & $\mathrm{H}$ & -6.51613100 & 1.49973300 & -2.7582090 \\
\hline $\mathrm{H}$ & -2.58389800 & 4.63998000 & -7.74257400 & $\mathrm{H}$ & -6.13646600 & 2.50468700 & -4.1661670 \\
\hline $\mathrm{H}$ & -3.18151600 & 2.37636400 & -6.92863000 & $\mathrm{H}$ & -6.36420500 & 3.25230900 & -2.57266900 \\
\hline $\mathrm{C}$ & -0.93741500 & 7.25774700 & -1.56571700 & $\mathrm{C}$ & -4.18442800 & 2.10811800 & -1.28287800 \\
\hline $\mathrm{C}$ & -0.22564300 & 7.62438300 & -0.42926900 & $\mathrm{H}$ & -4.60476300 & 2.96940400 & -0.7485270 \\
\hline $\mathrm{C}$ & 0.59537900 & 5.42853900 & -1.97317900 & $\mathrm{H}$ & -3.11180500 & 2.06780400 & -1.07383700 \\
\hline $\mathrm{C}$ & 0.93012300 & 6.91476100 & -0.10321700 & $\mathrm{H}$ & -4.65213500 & 1.20447500 & -0.87253800 \\
\hline $\mathrm{H}$ & -0.55377900 & 8.45364800 & 0.19274700 & $\mathrm{C}$ & 3.82308100 & 6.08673300 & -2.48461600 \\
\hline $\mathrm{C}$ & 1.35418400 & 5.79771400 & -0.84191100 & $\mathrm{H}$ & 2.81393600 & 6.46029700 & -2.67223600 \\
\hline $\mathrm{H}$ & 1.51898600 & 7.21564000 & 0.75828200 & $\mathrm{C}$ & 6.37974700 & 3.36363800 & 0.92745000 \\
\hline $\mathrm{O}$ & -1.39524200 & 3.18669700 & -2.81277700 & $\mathrm{H}$ & 6.15546900 & 2.78382800 & 1.83353300 \\
\hline $\mathrm{O}$ & 0.94657200 & 4.32178200 & -2.73111900 & $\mathrm{C}$ & 1.46136800 & 4.20827100 & 1.71259700 \\
\hline $\mathrm{P}$ & 0.18659700 & 2.86670200 & -2.44495100 & $\mathrm{H}$ & 0.58173400 & 4.57857500 & 1.18080700 \\
\hline $\mathrm{O}$ & 0.23187700 & 2.47447600 & -1.00870200 & $\mathrm{C}$ & 7.02581300 & 2.39972500 & -0.08485800 \\
\hline $\mathrm{O}$ & 0.78714800 & 1.95772600 & -3.51529200 & $\mathrm{H}$ & 7.94443600 & 1.96246600 & 0.32489800 \\
\hline $\mathrm{C}$ & -2.80125700 & 1.26110600 & -4.54891800 & $\mathrm{H}$ & 7.29110600 & 2.91548900 & -1.01559600 \\
\hline $\mathrm{C}$ & -3.82057800 & 1.08571500 & -3.57856000 & $\mathrm{H}$ & 6.34281600 & 1.57924000 & -0.33614300 \\
\hline $\mathrm{C}$ & -2.29945800 & 0.12448600 & -5.23627500 & $\mathrm{C}$ & 7.36891900 & 4.47614000 & 1.32763400 \\
\hline $\mathrm{C}$ & -4.32785200 & -0.19932000 & -3.35024000 & $\mathrm{H}$ & 8.28954000 & 4.04947800 & 1.74485800 \\
\hline $\mathrm{C}$ & -2.85321400 & -1.13518300 & -4.97430600 & $\mathrm{H}$ & 6.92870400 & 5.14044300 & 2.07894700 \\
\hline $\mathrm{C}$ & -3.88397900 & -1.32177700 & -4.04860100 & $\mathrm{H}$ & 7.64551900 & 5.09001400 & 0.46206500 \\
\hline $\mathrm{H}$ & -5.12299900 & -0.32511400 & -2.61870900 & $\mathrm{C}$ & 4.72850800 & 7.32235500 & -2.30462800 \\
\hline $\mathrm{H}$ & -2.47907600 & -1.99246100 & -5.52969900 & $\mathrm{H}$ & 5.77408000 & 7.03780700 & -2.13476200 \\
\hline $\mathrm{C}$ & 2.61337300 & 5.09343400 & -0.42878700 & $\mathrm{H}$ & 4.40524200 & 7.92718500 & -1.44993500 \\
\hline $\mathrm{C}$ & 3.78711900 & 5.24179200 & -1.21019300 & $\mathrm{H}$ & 4.69711000 & 7.95530700 & -3.20023700 \\
\hline $\mathrm{C}$ & 2.67018600 & 4.37178800 & 0.78868500 & $\mathrm{C}$ & 4.23599600 & 5.26906300 & -3.72122400 \\
\hline $\mathrm{C}$ & 4.97927200 & 4.65569100 & -0.77106500 & $\mathrm{H}$ & 3.54225300 & 4.43973600 & -3.88589900 \\
\hline $\mathrm{C}$ & 3.89519900 & 3.81770900 & 1.18678000 & $\mathrm{H}$ & 5.24770000 & 4.85760400 & -3.61956100 \\
\hline $\mathrm{C}$ & 5.06084900 & 3.94235600 & 0.42814700 & $\mathrm{H}$ & 4.22712900 & 5.90236900 & -4.61712000 \\
\hline $\mathrm{H}$ & 5.87566800 & 4.78856700 & -1.37288100 & $\mathrm{C}$ & 1.63119400 & 5.04710100 & 2.99678000 \\
\hline $\mathrm{H}$ & 3.94396700 & 3.27801100 & 2.13034600 & $\mathrm{H}$ & 2.48893800 & 4.70144100 & 3.58749500 \\
\hline $\mathrm{C}$ & -1.17796100 & 0.21208800 & -6.27504000 & $\mathrm{H}$ & 0.73770000 & 4.96427600 & 3.62761900 \\
\hline $\mathrm{H}$ & -0.82385400 & 1.24606800 & -6.30064200 & $\mathrm{H}$ & 1.79042400 & 6.10834800 & 2.77528400 \\
\hline $\mathrm{C}$ & -4.55213800 & -2.67621600 & -3.84045900 & $\mathrm{C}$ & 1.17004800 & 2.73792000 & 2.06563100 \\
\hline $\mathrm{H}$ & -5.30465100 & -2.54269200 & -3.05112800 & $\mathrm{H}$ & 0.95809100 & 2.17091500 & 1.15711200 \\
\hline $\mathrm{C}$ & -4.44547200 & 2.24126600 & -2.79601300 & $\mathrm{H}$ & 0.28756500 & 2.68172700 & 2.71510300 \\
\hline $\mathrm{H}$ & -3.97736100 & 3.17173400 & -3.12243300 & $\mathrm{H}$ & 2.00278300 & 2.27110600 & 2.60719400 \\
\hline $\mathrm{C}$ & 0.02887400 & -0.66433200 & -5.89092700 & $\mathrm{H}$ & 3.18641700 & -2.53924500 & -0.11012800 \\
\hline $\mathrm{H}$ & 0.83208400 & -0.55139500 & -6.63074300 & $\mathrm{C}$ & 3.33792400 & 0.15464500 & 0.11106300 \\
\hline $\mathrm{H}$ & -0.23207300 & -1.72854000 & -5.85248700 & $\mathrm{H}$ & 4.15871600 & -0.55939600 & 0.23572700 \\
\hline $\mathrm{H}$ & 0.41819800 & -0.37174100 & -4.91255400 & $\mathrm{H}$ & 2.64126300 & 0.02073100 & 0.94319300 \\
\hline $\mathrm{C}$ & -1.67356100 & -0.15148000 & -7.68948100 & $\mathrm{H}$ & 3.74354500 & 1.16844300 & 0.17726700 \\
\hline
\end{tabular}




\section{TS-11}

$\mathrm{N}$

C

C

C

C

C

$\mathrm{H}$

C

$\mathrm{H}$

C

$\mathrm{H}$

$\mathrm{H}$

$\mathrm{H}$

C

$\mathrm{H}$

$\mathrm{H}$

$\mathrm{H}$

$\mathrm{H}$

C

C

C

C

C

C

$\mathrm{H}$

C

C

$\mathrm{H}$

$\mathrm{H}$

$\mathrm{H}$

$\mathrm{H}$

$\mathrm{H}$

$\mathrm{H}$

$\mathrm{H}$

16b

$\mathrm{N}$

C

C

C

C

C

$\mathrm{H}$

C

$\mathrm{H}$

C

$\mathrm{H}$

$\mathrm{H}$

$\mathrm{H}$

C

$\mathrm{H}$

$\mathrm{H}$
$6.78809400 \quad-3.21323800 \quad-1.97514500$

$\begin{array}{lll}6.84817600 & -4.35199900 & -1.09583200\end{array}$

$\begin{array}{llll}7.19489600 & -3.27976100 & -3.31224700\end{array}$

$\begin{array}{llll}6.59194600 & -2.45241600 & -4.27737400\end{array}$

$8.24939800 \quad-4.12072200 \quad-3.71287500$

$\begin{array}{llll}7.03206800 & -2.46629700 & -5.59867800\end{array}$

$\begin{array}{llll}5.77462800 & -1.79626300 & -3.98296500\end{array}$

$\begin{array}{llll}8.67219700 & -4.13497300 & -5.04090600\end{array}$

$\begin{array}{llll}8.74353300 & -4.74434800 & -2.97358200\end{array}$

$\begin{array}{llll}8.07194100 & -3.31083200 & -5.99548700\end{array}$

$\begin{array}{llll}6.55077800 & -1.81579900 & -6.32488000\end{array}$

$\begin{array}{llll}9.48834700 & -4.79354100 & -5.32816900\end{array}$

$\begin{array}{llll}5.97618300 & -2.62284500 & -1.83010500\end{array}$

$\begin{array}{llll}6.08927800 & -5.57198300 & -1.56702500\end{array}$

$\begin{array}{lll}5.02831000 & -5.31868200 & -1.70335800\end{array}$

$\begin{array}{llll}6.45502100 & -5.91334700 & -2.54237200\end{array}$

$6.15450500-6.40243100 \quad-0.86252600$

$\begin{array}{llll}8.40903500 & -3.32431700 & -7.02775700\end{array}$

$\begin{array}{llll}7.47358800 & -4.23419000 & 0.09495000\end{array}$

$\begin{array}{lll}7.52948300 & -5.35569800 & 1.08340600\end{array}$

$\begin{array}{llll}8.15973900 & -2.96102500 & 0.53559900\end{array}$

$\begin{array}{lll}8.26031300 & -6.52437800 & 0.81665100\end{array}$

$\begin{array}{lll}6.89718200 & -5.23825600 & 2.33202200\end{array}$

$\begin{array}{lll}8.34407300 & -7.54966600 & 1.76038900\end{array}$

$\begin{array}{lll}8.77102500 & -6.62244500 & -0.13763300\end{array}$

$\begin{array}{llll}6.97613700 & -6.26333100 & 3.27513200\end{array}$

$\begin{array}{lll}7.70066200 & -7.42340500 & 2.99271400\end{array}$

$\begin{array}{llll}8.91604700 & -8.44555300 & 1.53287300\end{array}$

$\begin{array}{llll}6.47041800 & -6.15596900 & 4.23133600\end{array}$

$\begin{array}{llll}7.76506500 & -8.22078500 & 3.72822400\end{array}$

$\begin{array}{llll}8.17287800 & -2.22087900 & -0.26639400\end{array}$

$\begin{array}{llll}9.19028300 & -3.17128100 & 0.84874500\end{array}$

$\begin{array}{llll}7.65536500 & -2.52294400 & 1.40813500\end{array}$

$\begin{array}{lll}6.32810400 & -4.34048900 & 2.56052200\end{array}$

$6.34515100 \quad-3.52404100 \quad-2.14294900$

$6.47335700-4.48700300 \quad-1.11488700$

$\begin{array}{lll}7.36651000 & -2.99902000 & -2.94912500\end{array}$

$\begin{array}{lll}7.02438900 & -2.03807500 & -3.91995400\end{array}$

$\begin{array}{llll}8.70938500 & -3.40030500 & -2.84552900\end{array}$

$\begin{array}{lll}7.99495100 & -1.49816900 & -4.75832900\end{array}$

$\begin{array}{llll}5.98896000 & -1.71395300 & -4.00525200\end{array}$

$\begin{array}{llll}9.67376000 & -2.84526900 & -3.68713900\end{array}$

$\begin{array}{llll}8.98793400 & -4.15747200 & -2.12121200\end{array}$

$\begin{array}{llll}9.33141400 & -1.89227600 & -4.64733900\end{array}$

$\begin{array}{lll}7.70362400 & -0.75919400 & -5.50062500\end{array}$

$\begin{array}{lll}10.70603200 & -3.17309300 & -3.59132200\end{array}$

$\begin{array}{llll}5.43612300 & -3.50857300 & -2.58845200\end{array}$

$\begin{array}{lll}5.60891200 & -5.70554700 & -1.33874900\end{array}$

$\begin{array}{llll}4.58501300 & -5.39014800 & -1.59131300\end{array}$

$\begin{array}{llll}5.96746400 & -6.30852700 & -2.18348100\end{array}$

\section{TS-12}

C

C

C

$\mathrm{H}$

$\mathrm{H}$

$\mathrm{H}$

$\mathrm{H}$

C

C

C

C

$\mathrm{H}$

$\mathrm{H}$

$\mathrm{H}$

$\mathrm{H}$

C

C

C

C

C

$\mathrm{H}$

$\mathrm{H}$

C

C

C

C

$\mathrm{H}$

C

$\mathrm{H}$

$\mathrm{O}$

$\mathrm{O}$

$\mathrm{P}$

$\mathrm{O}$

O $\begin{array}{lll}5.55086700 & -6.34248600 & -0.45527800\end{array}$

$10.08923300 \quad-1.46496800 \quad-5.29733200$

$\begin{array}{lll}7.23499900 & -4.25864800 & -0.01400300\end{array}$

$\begin{array}{lll}7.49600900 & -5.32512300 & 0.99368300\end{array}$

$\begin{array}{lll}7.87489900 & -2.91305700 & 0.24298100\end{array}$

$8.02588700 \quad-6.57231300 \quad 0.61774700$

$\begin{array}{lll}7.25316500 & -5.10298000 & 2.36176500\end{array}$

$8.28586400 \quad-7.56354600 \quad 1.56516500$

$8.24817200 \quad-6.75628300 \quad-0.42991200$

$\begin{array}{llll}7.51104200 & -6.09174700 & 3.31006400\end{array}$

$\begin{array}{lll}8.02906900 & -7.32815800 & 2.91663100\end{array}$

$\begin{array}{lll}8.69880700 & -8.51722700 & 1.24610700\end{array}$

$\begin{array}{lll}7.30364600 & -5.89731600 & 4.35933400\end{array}$

$8.23412100 \quad-8.09720700 \quad 3.65651200$

$\begin{array}{llll}7.35261400 & -2.11654400 & -0.29385000\end{array}$

$8.92726600-2.88721000 \quad-0.07252100$

$\begin{array}{lll}7.86290500 & -2.68083900 & 1.31323900\end{array}$

$6.83846900 \quad-4.15131600 \quad 2.68373200$

$\begin{array}{lll}1.39412900 & 2.88134600 & 0.42921700\end{array}$

$\begin{array}{llll}2.95834700 & 4.18224000 & 1.66856800\end{array}$

$\begin{array}{lll}1.49956700 & 4.17384000 & 1.26564700\end{array}$

$\begin{array}{lll}1.19637500 & 2.02671400 & 1.08653800\end{array}$

$\begin{array}{llll}0.60213300 & 2.91236000 & -0.32538200\end{array}$

$\begin{array}{lll}1.24039700 & 5.06066900 & 0.67033700\end{array}$

$\begin{array}{lll}0.82862000 & 4.17243500 & 2.13278600\end{array}$

$\begin{array}{lll}2.81683400 & 2.70815800 & -0.20153800\end{array}$

$2.94454900 \quad 3.64952300-1.44752500$

$\begin{array}{llll}2.94586600 & 1.34885700 & -0.88034300\end{array}$

$\begin{array}{llll}2.23055300 & 2.88618600 & -2.58254500\end{array}$

$\begin{array}{llll}2.53108700 & 4.64578900 & -1.26372000\end{array}$

$\begin{array}{llll}4.00699200 & 3.76810700 & -1.68951100\end{array}$

$2.63654200 \quad 3.12184600-3.57322700$

$\begin{array}{lll}1.15879400 & 3.12792500 & -2.61638700\end{array}$

$\begin{array}{llll}3.68239000 & 3.21831800 & 0.94732000\end{array}$

$\begin{array}{lll}5.01473400 & 2.96777000 & 1.28239700\end{array}$

$\begin{array}{lll}3.59404300 & 4.99907200 & 2.59541000\end{array}$

$\begin{array}{lll}5.69207500 & 3.79071100 & 2.20953700\end{array}$

$\begin{array}{lll}4.95841900 & 4.82353300 & 2.81879400\end{array}$

$3.04510800 \quad 5.76984300 \quad 3.13093000$

$\begin{array}{lll}5.47664300 & 5.48365700 & 3.50727000\end{array}$

$\begin{array}{llll}2.46007500 & 1.44155300 & -2.19498700\end{array}$

$\begin{array}{lll}2.25201300 & 0.29772800 & -2.95577700\end{array}$

$\begin{array}{llll}3.35771600 & 0.11119200 & -0.38371900\end{array}$

$\begin{array}{llll}2.55351000 & -0.94269200 & -2.39646100\end{array}$

$\begin{array}{llll}1.85447200 & 0.36214300 & -3.96569800\end{array}$

$3.13921500-1.07013000-1.12482600$

$2.36033100 \quad-1.84810200 \quad-2.96414700$

$\begin{array}{lll}5.70274500 & 1.94939000 & 0.63218400\end{array}$

$\begin{array}{lll}3.95372200 & 0.05316100 & 0.87424600\end{array}$

$\begin{array}{llll}5.57003000 & 0.35594300 & 1.00833400\end{array}$

$\begin{array}{lll}6.40063500 & -0.40130800 & 0.03178300\end{array}$

$\begin{array}{lll}5.81600800 & 0.16415200 & 2.52453500\end{array}$ 


\begin{tabular}{|c|c|c|c|c|c|c|c|}
\hline $\mathrm{C}$ & 7.14081600 & 3.62565400 & 2.56024200 & $\mathrm{H}$ & 3.51957900 & -6.57843000 & 2.56170200 \\
\hline $\mathrm{C}$ & 8.14375300 & 3.80613100 & 1.57570700 & $\mathrm{H}$ & 5.24179600 & -6.37395400 & 2.21198000 \\
\hline $\mathrm{C}$ & 7.52430800 & 3.37387600 & 3.90580400 & $\mathrm{C}$ & 2.75932700 & -7.45263300 & -0.00353800 \\
\hline $\mathrm{C}$ & 9.48911200 & 3.69670400 & 1.94773500 & $\mathrm{H}$ & 2.90275000 & -8.52429100 & 0.18173000 \\
\hline $\mathrm{C}$ & 8.88519500 & 3.28041200 & 4.21812000 & $\mathrm{H}$ & 2.54732700 & -7.31451800 & -1.06936800 \\
\hline $\mathrm{C}$ & 9.88862800 & 3.43423300 & 3.25794700 & $\mathrm{H}$ & 1.87368400 & -7.13010700 & 0.55701800 \\
\hline $\mathrm{H}$ & 10.25672500 & 3.84495600 & 1.19121100 & $\mathrm{C}$ & 0.27351900 & -2.87031800 & 1.04869500 \\
\hline $\mathrm{H}$ & 9.16803600 & 3.08929600 & 5.25055400 & $\mathrm{H}$ & 0.22831500 & -3.88188500 & 1.46930700 \\
\hline $\mathrm{C}$ & 3.45833600 & -2.45279000 & -0.63566700 & $\mathrm{H}$ & -0.00124300 & -2.93675300 & -0.01010900 \\
\hline $\mathrm{C}$ & 2.73352600 & -3.01569000 & 0.44570100 & $\mathrm{H}$ & -0.48507600 & -2.26475800 & 1.55959800 \\
\hline $\mathrm{C}$ & 4.38960400 & -3.25730300 & -1.34193800 & $\mathrm{C}$ & 2.03516400 & -2.09896100 & 2.71337300 \\
\hline $\mathrm{C}$ & 2.94783700 & -4.35610800 & 0.78772700 & $\mathrm{H}$ & 2.99585300 & -1.58892800 & 2.83031200 \\
\hline $\mathrm{C}$ & 4.55720200 & -4.59520700 & -0.95868800 & $\mathrm{H}$ & 2.09795600 & -3.07590100 & 3.20817700 \\
\hline $\mathrm{C}$ & 3.84459600 & -5.17261100 & 0.09388300 & $\mathrm{H}$ & 1.27199000 & -1.51115800 & 3.23844000 \\
\hline $\mathrm{H}$ & 2.37236400 & -4.77989700 & 1.60690600 & $\mathrm{C}$ & 4.86849400 & -3.48539600 & -3.82855300 \\
\hline $\mathrm{H}$ & 5.25989000 & -5.21599600 & -1.51152200 & $\mathrm{H}$ & 5.12957400 & -4.54886600 & -3.76889300 \\
\hline $\mathrm{C}$ & 6.51960800 & 3.23711900 & 5.05486800 & $\mathrm{H}$ & 5.42149700 & -3.05503100 & -4.67226000 \\
\hline $\mathrm{H}$ & 5.51230500 & 3.27318900 & 4.63515300 & $\mathrm{H}$ & 3.79884600 & -3.42161500 & -4.05797500 \\
\hline $\mathrm{C}$ & 11.36635300 & 3.36116300 & 3.62173300 & $\mathrm{C}$ & 6.72976800 & -2.80764600 & -2.24398600 \\
\hline $\mathrm{H}$ & 11.93302600 & 3.52708300 & 2.69487300 & $\mathrm{H}$ & 6.96840200 & -2.14943900 & -1.40406300 \\
\hline $\mathrm{C}$ & 7.83186500 & 4.20730500 & 0.13298000 & $\mathrm{H}$ & 7.29385900 & -2.45708300 & -3.11712500 \\
\hline $\mathrm{H}$ & 6.74726500 & 4.22439000 & 0.00746100 & $\mathrm{H}$ & 7.06263500 & -3.83111300 & -2.02723900 \\
\hline $\mathrm{C}$ & 6.63303100 & 1.89169600 & 5.79144900 & $\mathrm{~N}$ & 8.42970600 & -2.37794800 & 1.20586700 \\
\hline $\mathrm{H}$ & 5.88148600 & 1.83162900 & 6.58850400 & $\mathrm{C}$ & 7.58876600 & -2.43269600 & 2.25440400 \\
\hline $\mathrm{H}$ & 7.61620300 & 1.75653400 & 6.25828300 & $\mathrm{C}$ & 9.68160000 & -1.69973600 & 1.04377300 \\
\hline $\mathrm{H}$ & 6.46056700 & 1.06252500 & 5.10219100 & $\mathrm{C}$ & 9.76783100 & -0.63976500 & 0.13554300 \\
\hline $\mathrm{C}$ & 6.64936500 & 4.40775900 & 6.05149100 & $\mathrm{C}$ & 10.81783200 & -2.15741100 & 1.71674400 \\
\hline $\mathrm{H}$ & 5.88115200 & 4.33401700 & 6.83110300 & $\mathrm{C}$ & 11.00233800 & -0.02744700 & -0.08274100 \\
\hline $\mathrm{H}$ & 6.53788600 & 5.37920100 & 5.55663000 & $\mathrm{H}$ & 8.86307900 & -0.29204600 & -0.35402900 \\
\hline $\mathrm{H}$ & 7.62769200 & 4.40358600 & 6.54703400 & $\mathrm{C}$ & 12.04522700 & -1.52954900 & 1.50097000 \\
\hline $\mathrm{C}$ & 11.76807700 & 1.97627900 & 4.16268200 & $\mathrm{H}$ & 10.73578700 & -3.00189000 & 2.39454300 \\
\hline $\mathrm{H}$ & 12.84230400 & 1.94577300 & 4.38415300 & $\mathrm{C}$ & 12.14024600 & -0.46633200 & 0.59958300 \\
\hline $\mathrm{H}$ & 11.54691400 & 1.18754700 & 3.43577200 & $\mathrm{H}$ & 11.07033200 & 0.80227700 & -0.7805160 \\
\hline $\mathrm{H}$ & 11.23169300 & 1.73940500 & 5.08926300 & $\mathrm{H}$ & 12.92846900 & -1.88015300 & 2.02756100 \\
\hline $\mathrm{C}$ & 11.76539700 & 4.47543900 & 4.60870800 & $\mathrm{H}$ & 8.03232100 & -2.71607800 & 0.33578300 \\
\hline $\mathrm{H}$ & 12.84315900 & 4.44676800 & 4.81104900 & $\mathrm{C}$ & 6.46977900 & -3.42607400 & 2.06477300 \\
\hline $\mathrm{H}$ & 11.24392600 & 4.36367800 & 5.56677000 & $\mathrm{H}$ & 6.77429800 & -4.39828900 & 2.47218800 \\
\hline $\mathrm{H}$ & 11.51862500 & 5.46521500 & 4.20898600 & $\mathrm{H}$ & 5.56661500 & -3.11889800 & 2.59110200 \\
\hline $\mathrm{C}$ & 8.33443000 & 5.63656200 & -0.15874200 & $\mathrm{H}$ & 6.22397400 & -3.55931100 & 1.00800200 \\
\hline $\mathrm{H}$ & 9.42593700 & 5.70511900 & -0.07882400 & $\mathrm{H}$ & 13.09855400 & 0.01583100 & 0.42766200 \\
\hline $\mathrm{H}$ & 7.90338500 & 6.35881800 & 0.54383000 & $\mathrm{C}$ & 7.59266900 & -1.60499600 & 3.40359100 \\
\hline $\mathrm{H}$ & 8.05455400 & 5.94172800 & -1.17480000 & $\mathrm{C}$ & 6.93827900 & -2.20184600 & 4.63936400 \\
\hline $\mathrm{C}$ & 8.38896500 & 3.20412000 & -0.89414800 & $\mathrm{C}$ & 8.73929300 & -0.64981100 & 3.74318500 \\
\hline $\mathrm{H}$ & 8.11039400 & 3.50851900 & -1.91074200 & $\mathrm{C}$ & 7.60701900 & -3.20986900 & 5.35341900 \\
\hline $\mathrm{H}$ & 7.98645700 & 2.20289300 & -0.71686400 & $\mathrm{C}$ & 5.70239000 & -1.75159400 & 5.12703600 \\
\hline $\mathrm{H}$ & 9.48449600 & 3.15350300 & -0.85594800 & $\mathrm{H}$ & 9.02757000 & -0.02199700 & 2.89856700 \\
\hline $\mathrm{C}$ & 1.66997300 & -2.23947600 & 1.22350900 & $\mathrm{C}$ & 7.06127700 & -3.75450300 & 6.51631100 \\
\hline $\mathrm{H}$ & 1.61277000 & -1.23019600 & 0.81008700 & $\mathrm{H}$ & 8.56899500 & -3.56854700 & 4.99246500 \\
\hline $\mathrm{C}$ & 4.00605400 & -6.65169800 & 0.42401100 & $\mathrm{C}$ & 5.15335600 & -2.29662000 & 6.29063100 \\
\hline $\mathrm{H}$ & 4.85295600 & -7.02361600 & -0.16958500 & $\mathrm{C}$ & 5.82914600 & -3.29772400 & 6.98938000 \\
\hline $\mathrm{C}$ & 5.21592000 & -2.74153000 & -2.52342300 & $\mathrm{H}$ & 7.59695800 & -4.53477200 & 7.05130500 \\
\hline $\mathrm{H}$ & 4.97782700 & -1.68595400 & -2.66959900 & $\mathrm{H}$ & 4.19271000 & -1.93584500 & 6.64912200 \\
\hline $\mathrm{C}$ & 4.33461800 & -6.90638700 & 1.90610500 & $\mathrm{H}$ & 5.40031200 & -3.71957400 & 7.89459700 \\
\hline $\mathrm{H}$ & 4.49207100 & -7.97660500 & 2.08621400 & $\mathrm{H}$ & 6.64712900 & -0.72116900 & 2.84697100 \\
\hline
\end{tabular}




\begin{tabular}{|c|c|c|c|c|c|c|c|}
\hline $\mathrm{H}$ & 9.63320400 & -1.17931800 & 4.09728900 & $\mathrm{C}$ & 6.39373200 & 2.68585100 & -8.22770100 \\
\hline $\mathrm{H}$ & 5.16676600 & -0.97713200 & 4.58723000 & $\mathrm{C}$ & 6.87533600 & 5.43999700 & -8.38337300 \\
\hline \multirow[t]{2}{*}{$\mathrm{H}$} & 8.41389400 & 0.01536600 & 4.54547800 & $\mathrm{C}$ & 7.32940100 & 3.26792700 & -7.34508800 \\
\hline & & & & $\mathrm{C}$ & 7.57171800 & 4.64597700 & -7.47066400 \\
\hline $15 c$ & & & & $\mathrm{H}$ & 7.04326500 & 6.51395100 & -8.41353100 \\
\hline $\mathrm{C}$ & 2.78934200 & -4.62784000 & -3.59324300 & $\mathrm{H}$ & 8.30932100 & 5.10265900 & -6.81769400 \\
\hline $\mathrm{C}$ & 3.34804400 & -3.34774500 & -3.52654800 & $\mathrm{C}$ & 4.10516400 & 1.32043600 & -11.90502800 \\
\hline $\mathrm{C}$ & 3.59038500 & -2.73648700 & -2.28800800 & $\mathrm{C}$ & 3.25339700 & 0.29122900 & $-12.2896850 \mathrm{C}$ \\
\hline $\mathrm{C}$ & 3.24051100 & -3.43298100 & -1.12010300 & $\mathrm{C}$ & 3.41077800 & 1.07155300 & -9.60248200 \\
\hline $\mathrm{C}$ & 2.68244400 & -4.70835600 & -1.18684300 & $\mathrm{C}$ & 2.45490900 & -0.31927000 & -11.32246600 \\
\hline $\mathrm{C}$ & 2.45899900 & -5.31403300 & -2.42595600 & $\mathrm{H}$ & 3.20644200 & -0.03658300 & -13.325396 \\
\hline $\mathrm{H}$ & 2.61496300 & -5.08059900 & -4.56511200 & $\mathrm{C}$ & 2.53149800 & 0.02837500 & -9.96369300 \\
\hline $\mathrm{H}$ & 3.58286000 & -2.83632000 & -4.45589700 & $\mathrm{H}$ & 1.76486700 & -1.10549600 & -11.6139070 \\
\hline $\mathrm{C}$ & 4.19200700 & -1.32962800 & -2.15550100 & $\mathrm{O}$ & 6.10130700 & 1.33790900 & -8.12141300 \\
\hline $\mathrm{H}$ & 2.41898800 & -5.22789600 & -0.26949700 & $\mathrm{O}$ & 3.52439300 & 1.45831600 & -8.27623000 \\
\hline $\mathrm{H}$ & 2.02557900 & -6.30876000 & -2.47869100 & $\mathrm{P}$ & 4.73492700 & 0.83076500 & -7.33829200 \\
\hline $\mathrm{C}$ & 5.48818500 & -1.32563600 & -1.30676400 & $\mathrm{O}$ & 4.77746600 & -0.66108700 & -7.34406200 \\
\hline $\mathrm{H}$ & 5.90805700 & -0.31629900 & -1.23971000 & $\mathrm{O}$ & 4.57728700 & 1.57977100 & -6.01805900 \\
\hline $\mathrm{C}$ & 4.45536800 & -0.68354000 & -3.49982300 & $\mathrm{C}$ & 8.08064500 & 2.46889200 & -6.31951600 \\
\hline $\mathrm{C}$ & 5.69000100 & -1.05251400 & -4.26946200 & $\mathrm{C}$ & 8.99318500 & 1.46270700 & -6.72519600 \\
\hline $\mathrm{H}$ & 6.04695100 & -2.04049700 & -3.97182400 & $\mathrm{C}$ & 7.94291900 & 2.76074300 & -4.93635700 \\
\hline $\mathrm{H}$ & 6.48788400 & -0.32669000 & -4.05851900 & $\mathrm{C}$ & 9.73140500 & 0.77860900 & -5.75120700 \\
\hline $\mathrm{H}$ & 5.50374800 & -1.03448400 & -5.35028400 & $\mathrm{C}$ & 8.71719900 & 2.05384900 & -4.00819600 \\
\hline $\mathrm{N}$ & 3.68517500 & 0.23049400 & -4.00984600 & $\mathrm{C}$ & 9.61973900 & 1.05681500 & -4.38944000 \\
\hline $\mathrm{C}$ & 2.45885100 & 0.78117000 & -3.52343600 & $\mathrm{H}$ & 10.43365900 & 0.01078300 & -6.06915600 \\
\hline $\mathrm{C}$ & 1.45949000 & 0.01285500 & -2.91284200 & $\mathrm{H}$ & 8.61800200 & 2.29955900 & -2.95341900 \\
\hline $\mathrm{C}$ & 2.25615500 & 2.14730400 & -3.76646500 & $\mathrm{C}$ & 1.66579100 & -0.69946300 & -8.97593600 \\
\hline $\mathrm{C}$ & 0.28092700 & 0.63221900 & -2.49561500 & $\mathrm{C}$ & 0.57781300 & -0.03398000 & -8.35695600 \\
\hline $\mathrm{H}$ & 1.57584200 & -1.05886300 & -2.80139900 & $\mathrm{C}$ & 1.87174100 & -2.07916900 & -8.72569000 \\
\hline $\mathrm{C}$ & 1.07663700 & 2.75246900 & -3.33891300 & $\mathrm{C}$ & -0.27357600 & -0.75495600 & -7.51271800 \\
\hline $\mathrm{H}$ & 3.01555700 & 2.71096100 & -4.29764800 & $\mathrm{C}$ & 0.97651100 & -2.75379800 & -7.88586400 \\
\hline $\mathrm{C}$ & 0.09063400 & 2.00064400 & -2.69515200 & $\mathrm{C}$ & -0.10285900 & -2.12028200 & -7.27045700 \\
\hline $\mathrm{H}$ & -0.49481200 & 0.03454800 & -2.02603300 & $\mathrm{H}$ & -1.10829400 & -0.23450000 & -7.04898800 \\
\hline $\mathrm{H}$ & 0.92467200 & 3.81215300 & -3.52098700 & $\mathrm{H}$ & 1.12451200 & -3.81702900 & -7.70843200 \\
\hline $\mathrm{H}$ & -0.83023100 & 2.47514100 & -2.36847300 & $\mathrm{C}$ & 6.98407500 & 3.82704400 & -4.40172800 \\
\hline $\mathrm{H}$ & 4.05132300 & 0.73237400 & -4.92083500 & $\mathrm{H}$ & 6.36173100 & 4.16794100 & -5.23242400 \\
\hline $\mathrm{H}$ & 6.24986100 & -1.99391300 & -1.71601800 & $\mathrm{C}$ & 10.47887900 & 0.31963800 & $-3.3697740 \mathrm{C}$ \\
\hline $\mathrm{H}$ & 3.46290800 & -0.70473600 & -1.62747900 & $\mathrm{H}$ & 11.05894900 & -0.43288900 & -3.9214910 \\
\hline $\mathrm{C}$ & 4.15598900 & 4.31506900 & -10.70696700 & $\mathrm{C}$ & 9.25556000 & 1.11238900 & -8.19011500 \\
\hline $\mathrm{C}$ & 5.98093600 & 4.83236600 & -9.25783800 & $\mathrm{H}$ & 8.60379000 & 1.72857400 & -8.81283900 \\
\hline $\mathrm{C}$ & 5.10567500 & 5.47045200 & -10.31605200 & $\mathrm{C}$ & 6.02625200 & 3.26380500 & -3.33588700 \\
\hline $\mathrm{H}$ & 3.29080500 & 4.29945200 & -10.03420100 & $\mathrm{H}$ & 5.29468700 & 4.02702100 & -3.04225600 \\
\hline $\mathrm{H}$ & 3.78077700 & 4.38990200 & -11.73266000 & $\mathrm{H}$ & 6.55536200 & 2.95320200 & -2.42633700 \\
\hline $\mathrm{H}$ & 5.69774600 & 5.81110600 & -11.17717600 & $\mathrm{H}$ & 5.48514400 & 2.40465600 & -3.73763000 \\
\hline $\mathrm{H}$ & 4.56885600 & 6.34900900 & -9.93884100 & $\mathrm{C}$ & 7.74348100 & 5.04684400 & -3.84124800 \\
\hline $\mathrm{C}$ & 4.99955900 & 3.01821300 & -10.46926200 & $\mathrm{H}$ & 7.03938000 & 5.81576100 & -3.49935300 \\
\hline $\mathrm{C}$ & 5.95931200 & 2.79597200 & -11.68887800 & $\mathrm{H}$ & 8.39828000 & 5.50020800 & -4.59294600 \\
\hline $\mathrm{C}$ & 4.14641600 & 1.75633400 & -10.57110200 & $\mathrm{H}$ & 8.37087300 & 4.76493500 & -2.98668500 \\
\hline $\mathrm{C}$ & 5.08421500 & 2.10191400 & -12.75357300 & $\mathrm{C}$ & 9.63481500 & -0.43058800 & -2.32255500 \\
\hline $\mathrm{H}$ & 6.41625000 & 3.72713900 & -12.03843500 & $\mathrm{H}$ & 10.27857100 & -0.99157100 & -1.6341390 \\
\hline $\mathrm{H}$ & 6.76719100 & 2.12294700 & -11.37932100 & $\mathrm{H}$ & 8.95069700 & -1.13970000 & -2.80255100 \\
\hline $\mathrm{H}$ & 5.66450000 & 1.45840700 & -13.42515800 & $\mathrm{H}$ & 9.03449400 & 0.26432000 & -1.72259000 \\
\hline $\mathrm{H}$ & 4.56860800 & 2.83648500 & -13.38881100 & $\mathrm{C}$ & 11.48557800 & 1.26608800 & -2.68695300 \\
\hline $\mathrm{C}$ & 5.79450500 & 3.44139200 & -9.23619400 & $\mathrm{H}$ & 12.13894700 & 0.71165400 & -2.00190200 \\
\hline
\end{tabular}




\begin{tabular}{|c|c|c|c|c|c|c|c|}
\hline $\mathrm{H}$ & 10.97081800 & 2.04018800 & -2.10515100 & $\mathrm{C}$ & 10.16658100 & -1.07154200 & -3.01362000 \\
\hline $\mathrm{H}$ & 12.11566200 & 1.77077900 & -3.42728400 & $\mathrm{H}$ & 8.93977700 & 0.72814400 & -3.44598300 \\
\hline $\mathrm{C}$ & 10.70504600 & 1.45258200 & -8.59268700 & $\mathrm{H}$ & 9.55519600 & 0.62349600 & -1.78967300 \\
\hline $\mathrm{H}$ & 11.43263100 & 0.85428200 & -8.03127100 & $\mathrm{H}$ & 11.20911800 & -0.76118200 & -2.87589300 \\
\hline $\mathrm{H}$ & 10.93032300 & 2.50962900 & -8.41012100 & $\mathrm{H}$ & 10.06227500 & -1.35816700 & -4.06950100 \\
\hline $\mathrm{H}$ & 10.86201000 & 1.25095900 & -9.65945500 & $\mathrm{C}$ & 6.85776200 & 0.19549800 & -1.52294300 \\
\hline $\mathrm{C}$ & 8.92093700 & -0.35970800 & -8.49933100 & $\mathrm{C}$ & 6.67845100 & 0.64787100 & -0.21107300 \\
\hline $\mathrm{H}$ & 9.06531400 & -0.56394000 & -9.56764300 & $\mathrm{C}$ & 5.16022500 & 1.75307100 & -2.30215800 \\
\hline $\mathrm{H}$ & 7.88125500 & -0.58429400 & -8.24569400 & $\mathrm{C}$ & 5.79123200 & 1.71244600 & 0.06469600 \\
\hline $\mathrm{H}$ & 9.57135700 & -1.04653800 & -7.94338800 & $\mathrm{C}$ & 5.07525100 & 2.26677400 & -1.01110800 \\
\hline $\mathrm{C}$ & 0.25893600 & 1.43943200 & -8.61409900 & $\mathrm{H}$ & 4.55153300 & 2.16899400 & -3.10125400 \\
\hline $\mathrm{H}$ & 1.00934800 & 1.83461000 & -9.30290400 & $\mathrm{H}$ & 4.41478000 & 3.10477500 & -0.80908700 \\
\hline $\mathrm{C}$ & -1.05594400 & -2.90205900 & -6.37483800 & $\mathrm{C}$ & 9.77927700 & -2.20384700 & -2.08552500 \\
\hline $\mathrm{H}$ & -0.74982300 & -3.95652800 & -6.42271800 & $\mathrm{C}$ & 10.45278700 & -3.39330600 & -1.83374700 \\
\hline $\mathrm{C}$ & 3.01858600 & -2.87938600 & -9.34693400 & $\mathrm{C}$ & 8.11774200 & -2.75940300 & -0.41987300 \\
\hline $\mathrm{H}$ & 3.68592500 & -2.17585600 & -9.85072600 & $\mathrm{C}$ & 9.91088800 & -4.29046100 & -0.91303600 \\
\hline $\mathrm{C}$ & -0.94912200 & -2.46059200 & -4.90289600 & $\mathrm{H}$ & 11.38047000 & -3.63066800 & -2.34894400 \\
\hline $\mathrm{H}$ & -1.62001800 & -3.05291200 & -4.26770900 & $\mathrm{C}$ & 8.76471400 & -3.98613400 & -0.15891100 \\
\hline $\mathrm{H}$ & -1.21890900 & -1.40410400 & -4.78624100 & $\mathrm{H}$ & 10.40403600 & -5.24231400 & -0.73844400 \\
\hline $\mathrm{H}$ & 0.07370200 & -2.58720600 & -4.53079900 & $\mathrm{O}$ & 7.44550500 & 0.11481900 & 0.81019700 \\
\hline $\mathrm{C}$ & -2.51295100 & -2.82633900 & -6.86897700 & $\mathrm{O}$ & 7.00271500 & -2.39065700 & 0.32399400 \\
\hline $\mathrm{H}$ & -3.16622500 & -3.45195600 & -6.24815500 & $\mathrm{P}$ & 7.18115600 & -1.32487700 & 1.57374100 \\
\hline $\mathrm{H}$ & -2.59594300 & -3.16921800 & -7.90609400 & $\mathrm{O}$ & 8.37174600 & -1.56675900 & 2.43315600 \\
\hline $\mathrm{H}$ & -2.89746000 & -1.80041200 & -6.82609200 & $\mathrm{O}$ & 5.77381300 & -1.31738700 & 2.20597200 \\
\hline $\mathrm{C}$ & -1.11030700 & 1.60724000 & -9.30299200 & $\mathrm{C}$ & 5.55990000 & 2.30513300 & 1.42431900 \\
\hline $\mathrm{H}$ & -1.93084200 & 1.25780200 & -8.66475700 & $\mathrm{C}$ & 6.59240800 & 2.99631000 & 2.10447800 \\
\hline $\mathrm{H}$ & -1.15370300 & 1.04146300 & -10.24050500 & $\mathrm{C}$ & 4.24831900 & 2.29838500 & 1.97486400 \\
\hline $\mathrm{H}$ & -1.29611200 & 2.66358200 & -9.53429500 & $\mathrm{C}$ & 6.28778000 & 3.67738400 & 3.29173000 \\
\hline $\mathrm{C}$ & 0.34039900 & 2.28098100 & -7.32656000 & $\mathrm{C}$ & 3.99995000 & 3.01063500 & 3.15441600 \\
\hline $\mathrm{H}$ & 1.33255000 & 2.19946900 & -6.87391500 & $\mathrm{C}$ & 4.99855100 & 3.72394100 & 3.82469600 \\
\hline $\mathrm{H}$ & -0.40361500 & 1.96193400 & -6.58615300 & $\mathrm{H}$ & 7.07800500 & 4.22980900 & 3.79637000 \\
\hline $\mathrm{H}$ & 0.15131500 & 3.33867800 & -7.55093700 & $\mathrm{H}$ & 2.98833300 & 3.01694600 & 3.55357300 \\
\hline $\mathrm{C}$ & 2.49877700 & -3.87959600 & -10.40015200 & $\mathrm{C}$ & 8.27849300 & -4.96171400 & 0.87058600 \\
\hline $\mathrm{H}$ & 1.84282400 & -4.63197400 & -9.94429900 & $\mathrm{C}$ & 7.01958300 & -5.59902400 & 0.71809700 \\
\hline $\mathrm{H}$ & 3.33584000 & -4.40953400 & -10.87132900 & $\mathrm{C}$ & 9.10424600 & -5.30898800 & 1.96948000 \\
\hline $\mathrm{H}$ & 1.92729100 & -3.38113400 & -11.19103000 & $\mathrm{C}$ & 6.61018800 & -6.53791900 & 1.67076700 \\
\hline $\mathrm{C}$ & 3.86947200 & -3.60671300 & -8.28806600 & $\mathrm{C}$ & 8.64272900 & -6.25907700 & 2.89024600 \\
\hline $\mathrm{H}$ & 4.28496900 & -2.88282000 & -7.58425400 & $\mathrm{C}$ & 7.40287800 & -6.88607400 & 2.76821400 \\
\hline $\mathrm{H}$ & 4.70165100 & -4.13075200 & -8.77516000 & $\mathrm{H}$ & 5.64734400 & -7.02573700 & 1.53886200 \\
\hline $\mathrm{H}$ & 3.28770500 & -4.35953700 & -7.74015200 & $\mathrm{H}$ & 9.27709300 & -6.52135400 & 3.73435600 \\
\hline $\mathrm{H}$ & 5.26145200 & -1.66005700 & -0.29084600 & $\mathrm{C}$ & 3.07692400 & 1.54608000 & 1.33346000 \\
\hline \multirow[t]{2}{*}{$\mathrm{H}$} & 3.39810600 & -2.97290600 & -0.14788800 & $\mathrm{H}$ & 3.43600200 & 1.07751000 & 0.41536000 \\
\hline & & & & $\mathrm{C}$ & 4.69600400 & 4.57761900 & 5.05128000 \\
\hline TS-13 & & & & $\mathrm{H}$ & 5.65575800 & 4.97324400 & 5.41198000 \\
\hline $\mathrm{C}$ & 7.09329400 & -1.24991500 & -3.38799400 & $\mathrm{C}$ & 8.02000900 & 3.10266000 & 1.56702100 \\
\hline $\mathrm{C}$ & 6.03985800 & 0.70569600 & -2.54568000 & $\mathrm{H}$ & 8.07264800 & 2.55360500 & 0.62523300 \\
\hline $\mathrm{C}$ & 6.27794800 & -0.02621000 & -3.84738400 & $\mathrm{C}$ & 2.56570000 & 0.40357100 & 2.23079900 \\
\hline $\mathrm{H}$ & 6.41499100 & -2.04880800 & -3.06655400 & $\mathrm{H}$ & 1.73537700 & -0.11995200 & 1.74080500 \\
\hline $\mathrm{H}$ & 7.74455300 & -1.65979800 & -4.16576700 & $\mathrm{H}$ & 2.19598700 & 0.77481100 & 3.19493300 \\
\hline $\mathrm{H}$ & 6.84477300 & 0.59093900 & -4.55878200 & $\mathrm{H}$ & 3.36147500 & -0.32355300 & 2.41325000 \\
\hline $\mathrm{H}$ & 5.34212700 & -0.30169200 & -4.34777800 & $\mathrm{C}$ & 1.92468600 & 2.49320600 & 0.94395900 \\
\hline $\mathrm{C}$ & 7.89430100 & -0.74330800 & -2.13925100 & $\mathrm{H}$ & 1.13389200 & 1.93609100 & 0.42718100 \\
\hline $\mathrm{C}$ & 9.16090300 & 0.03644200 & -2.62708400 & $\mathrm{H}$ & 2.26557400 & 3.29365800 & 0.27745300 \\
\hline $\mathrm{C}$ & 8.56265700 & -1.92327000 & -1.44288900 & $\mathrm{H}$ & 1.47266800 & 2.96670700 & 1.82375400 \\
\hline
\end{tabular}




\begin{tabular}{|c|c|c|c|}
\hline $\mathrm{C}$ & 4.06554000 & 3.77861100 & 6.20642600 \\
\hline $\mathrm{H}$ & 3.88802800 & 4.42752400 & 7.07249500 \\
\hline $\mathrm{H}$ & 4.71819700 & 2.95942100 & 6.52819100 \\
\hline $\mathrm{H}$ & 3.10163300 & 3.34461700 & 5.91557700 \\
\hline $\mathrm{C}$ & 3.81289900 & 5.78625100 & 4.68128500 \\
\hline $\mathrm{H}$ & 3.65717700 & 6.43466300 & 5.55219000 \\
\hline $\mathrm{H}$ & 2.82818200 & 5.46177000 & 4.32419300 \\
\hline $\mathrm{H}$ & 4.27482800 & 6.38339100 & 3.88773500 \\
\hline $\mathrm{C}$ & 8.39529900 & 4.56514400 & 1.25184700 \\
\hline $\mathrm{H}$ & 8.41030900 & 5.18692500 & 2.15500900 \\
\hline $\mathrm{H}$ & 7.68410500 & 5.01426400 & 0.54940500 \\
\hline $\mathrm{H}$ & 9.39396400 & 4.61250600 & 0.80073200 \\
\hline $\mathrm{C}$ & 9.04466700 & 2.45995400 & 2.52217900 \\
\hline $\mathrm{H}$ & 10.05540400 & 2.54097700 & 2.10373200 \\
\hline $\mathrm{H}$ & 8.82773800 & 1.39751000 & 2.66896400 \\
\hline $\mathrm{H}$ & 9.05322500 & 2.96127300 & 3.49894600 \\
\hline $\mathrm{C}$ & 6.10815200 & -5.36036300 & -0.48705000 \\
\hline $\mathrm{H}$ & 6.57567700 & -4.60889700 & -1.12758700 \\
\hline $\mathrm{C}$ & 6.95589800 & -7.93777800 & 3.77603100 \\
\hline $\mathrm{H}$ & 7.72233400 & -7.97643800 & 4.56267000 \\
\hline $\mathrm{C}$ & 10.49301900 & -4.70820500 & 2.19895100 \\
\hline $\mathrm{H}$ & 10.68198900 & -3.97456900 & 1.41296300 \\
\hline $\mathrm{C}$ & 5.61999500 & -7.57595500 & 4.45126900 \\
\hline $\mathrm{H}$ & 5.35474600 & -8.32453300 & 5.20796100 \\
\hline $\mathrm{H}$ & 4.80151600 & -7.53743600 & 3.72279200 \\
\hline $\mathrm{H}$ & 5.67359900 & -6.59807300 & 4.94159900 \\
\hline $\mathrm{C}$ & 6.88448500 & -9.33804900 & 3.13521000 \\
\hline $\mathrm{H}$ & 6.61810900 & -10.09525100 & 3.88310200 \\
\hline $\mathrm{H}$ & 7.84562000 & -9.61920500 & 2.69080900 \\
\hline $\mathrm{H}$ & 6.12810900 & -9.37121200 & 2.34185900 \\
\hline $\mathrm{C}$ & 5.96569600 & -6.64018100 & -1.33626800 \\
\hline $\mathrm{H}$ & 5.47591300 & -7.44527500 & -0.77561700 \\
\hline $\mathrm{H}$ & 6.94435300 & -7.00972100 & -1.66284100 \\
\hline $\mathrm{H}$ & 5.36106600 & -6.44164500 & -2.22998600 \\
\hline $\mathrm{C}$ & 4.72889400 & -4.81082700 & -0.07648100 \\
\hline $\mathrm{H}$ & 4.83757600 & -3.86851300 & 0.46804300 \\
\hline $\mathrm{H}$ & 4.18361700 & -5.52094900 & 0.55761300 \\
\hline $\mathrm{H}$ & 4.11371700 & -4.62351400 & -0.96559800 \\
\hline $\mathrm{C}$ & 11.59322100 & -5.78407900 & 2.10074400 \\
\hline $\mathrm{H}$ & 11.49427200 & -6.53621200 & 2.89288000 \\
\hline $\mathrm{H}$ & 12.58519000 & -5.32623700 & 2.20035100 \\
\hline $\mathrm{H}$ & 11.55922500 & -6.31259100 & 1.14076400 \\
\hline $\mathrm{C}$ & 10.58306400 & -3.94459800 & 3.53115500 \\
\hline $\mathrm{H}$ & 9.87427000 & -3.11346900 & 3.53365700 \\
\hline $\mathrm{H}$ & 11.59335700 & -3.53448700 & 3.66033800 \\
\hline $\mathrm{H}$ & 10.37736200 & -4.59047400 & 4.39339800 \\
\hline $\mathrm{N}$ & 7.21080700 & -0.60326100 & 5.64039000 \\
\hline $\mathrm{C}$ & 5.98545000 & -0.98012700 & 5.23670500 \\
\hline $\mathrm{C}$ & 8.28285800 & -1.35849000 & 6.20849800 \\
\hline $\mathrm{C}$ & 9.58301900 & -1.13800000 & 5.74605400 \\
\hline $\mathrm{C}$ & 8.04846300 & -2.20925700 & 7.29396000 \\
\hline $\mathrm{C}$ & 10.65160000 & -1.77276900 & 6.37833800 \\
\hline $\mathrm{H}$ & 9.74008200 & -0.51012500 & 4.87455800 \\
\hline $\mathrm{C}$ & 9.12229200 & -2.85684000 & 7.90445000 \\
\hline
\end{tabular}

$\begin{array}{ccc}7.03637300 & -2.34511600 & 7.66313000 \\ 10.42549800 & -2.63431100 & 7.45454500 \\ 11.66144700 & -1.60632900 & 6.01536100 \\ 8.93961400 & -3.52164200 & 8.74397600 \\ 7.38742100 & 0.39509600 & 5.59546100 \\ 5.00777100 & 0.16325700 & 5.17172700 \\ 4.71372500 & 0.46275900 & 6.18491500 \\ 5.46641000 & 1.03180200 & 4.68498700 \\ 4.11187800 & -0.10085200 & 4.61514500 \\ 11.26096900 & -3.13054900 & 7.93990600 \\ 5.63116300 & -2.25165100 & 4.70301200 \\ 4.18679600 & -2.67922200 & 4.85050000 \\ 6.60735800 & -3.42678300 & 4.65615800 \\ 3.48087200 & -3.19204300 & 3.75078000 \\ 3.54599900 & -2.65010100 & 6.09902000 \\ 7.60102400 & -3.11616900 & 4.33279400 \\ 2.17165300 & -3.65177400 & 3.89641600 \\ 3.95968400 & -3.21592400 & 2.77647900 \\ 2.23479000 & -3.10598100 & 6.24565600 \\ 1.54294400 & -3.60927000 & 5.14295200 \\ 1.64027800 & -4.03900600 & 3.03107600 \\ 1.75771900 & -3.07302100 & 7.22190900 \\ 0.52284800 & -3.96707300 & 5.25436200 \\ 5.73180400 & -1.74987700 & 3.47149300 \\ 6.24037200 & -4.15414100 & 3.92597400 \\ 4.08267000 & -2.27303900 & 6.96742300 \\ 6.68509000 & -3.93871700 & 5.62335200\end{array}$

15d

$\begin{array}{lll}-1.27687200 & 0.66891700 & 3.04290800\end{array}$

$\begin{array}{lll}-0.18558300 & 1.09389200 & 2.28486000\end{array}$

$\begin{array}{lll}-0.09913100 & 2.41325100 & 1.81657100\end{array}$

$\begin{array}{lll}-1.13461600 & 3.30168100 & 2.13797000\end{array}$

$\begin{array}{lll}-2.22922500 & 2.87901400 & 2.89518500\end{array}$

$\begin{array}{lll}-2.30604600 & 1.56153500 & 3.34795500\end{array}$

$\begin{array}{lll}-1.31948300 & -0.35693200 & 3.39843400\end{array}$

$\begin{array}{lll}0.61840500 & 0.39523700 & 2.06672900\end{array}$

$\begin{array}{lll}1.11910100 & 2.82801800 & 0.99064300\end{array}$

$\begin{array}{lll}-3.01975600 & 3.58503000 & 3.13434500\end{array}$

$\begin{array}{lll}-3.15697400 & 1.23462700 & 3.93893900\end{array}$

$\begin{array}{lll}1.51352900 & 4.31889400 & 1.14039200\end{array}$

$\begin{array}{lll}0.76329800 & 4.99485600 & 0.72125600\end{array}$

$\begin{array}{lll}0.93702900 & 2.56170100 & -0.49428100\end{array}$

$\begin{array}{llll}-0.24169000 & 3.12161600 & -1.22545200\end{array}$

$\begin{array}{llll}0.11269200 & 3.86634800 & -1.95644600\end{array}$

$\begin{array}{lll}-0.96192600 & 3.57129600 & -0.54245100\end{array}$

$\begin{array}{lll}-0.74136600 & 2.33316300 & -1.79979300\end{array}$

$\begin{array}{lll}1.83483100 & 1.93872900 & -1.19486200\end{array}$

$3.02606200 \quad 1.29376500-0.71669000$

$\begin{array}{lll}4.26667700 & 1.80188800 & -1.11124800\end{array}$

$\begin{array}{llll}2.93857600 & 0.11412100 & 0.02938400\end{array}$

$\begin{array}{llll}5.42813400 & 1.13685500 & -0.71580800\end{array}$

$\begin{array}{lll}4.32009500 & 2.69978900 & -1.71854600\end{array}$

$\begin{array}{lll}4.10735900 & -0.54011500 & 0.41979100\end{array}$ 


\begin{tabular}{|c|c|c|c|c|c|c|c|}
\hline $\mathrm{H}$ & 1.96345400 & -0.29712000 & 0.27129000 & $\mathrm{C}$ & 4.87785400 & 6.60036200 & -1.54305400 \\
\hline $\mathrm{C}$ & 5.35293900 & -0.02785400 & 0.05150900 & $\mathrm{C}$ & 6.02018200 & 5.81805600 & -1.36944600 \\
\hline $\mathrm{H}$ & 6.39301800 & 1.53239500 & -1.01690200 & $\mathrm{H}$ & 7.35088700 & 4.45164500 & -2.36133000 \\
\hline $\mathrm{H}$ & 4.04111900 & -1.45611400 & 0.99978300 & $\mathrm{H}$ & 4.52774100 & 7.19876200 & -0.70455800 \\
\hline $\mathrm{H}$ & 6.26192500 & -0.54132500 & 0.35136100 & $\mathrm{C}$ & 0.57694700 & -1.09561400 & -4.83012000 \\
\hline $\mathrm{H}$ & 1.77289700 & 1.98640600 & -2.29877700 & $\mathrm{H}$ & 1.17679300 & -0.64546400 & -5.62358600 \\
\hline $\mathrm{H}$ & 2.45831600 & 4.51736300 & 0.62532900 & $\mathrm{C}$ & -3.53205100 & -0.17859700 & -2.0326460 \\
\hline $\mathrm{H}$ & 1.96854400 & 2.23462800 & 1.33739200 & $\mathrm{H}$ & -4.27496000 & 0.62335000 & -1.91958200 \\
\hline $\mathrm{C}$ & 3.31485200 & 3.50661000 & -9.01912000 & $\mathrm{C}$ & -2.48921300 & 2.96563000 & -5.83314200 \\
\hline $\mathrm{C}$ & 1.62173700 & 1.86062900 & -8.70605000 & $\mathrm{H}$ & -1.74340500 & 3.13620000 & -6.6125620 \\
\hline $\mathrm{C}$ & 2.63315700 & 2.31973000 & -9.73427200 & $\mathrm{C}$ & 1.50764700 & -1.24257000 & -3.61176700 \\
\hline $\mathrm{H}$ & 4.13308900 & 3.13880900 & -8.38916700 & $\mathrm{H}$ & 2.34292600 & -1.91097000 & -3.85594200 \\
\hline $\mathrm{H}$ & 3.73127800 & 4.24880900 & -9.70755200 & $\mathrm{H}$ & 0.98823400 & -1.67083500 & -2.74524700 \\
\hline $\mathrm{H}$ & 2.14427300 & 2.64083500 & -10.66502600 & $\mathrm{H}$ & 1.92004100 & -0.27023700 & -3.3355220 \\
\hline $\mathrm{H}$ & 3.34046900 & 1.52821000 & -10.00859500 & $\mathrm{C}$ & 0.11126600 & -2.48077300 & -5.32489200 \\
\hline $\mathrm{C}$ & 2.19944300 & 4.10306600 & -8.09424300 & $\mathrm{H}$ & 0.97495700 & -3.10859700 & -5.57642900 \\
\hline $\mathrm{C}$ & 1.27180000 & 5.03728000 & -8.94198600 & $\mathrm{H}$ & -0.52136200 & -2.40252200 & -6.2162560 \\
\hline $\mathrm{C}$ & 2.78110600 & 5.12329700 & -7.11732300 & $\mathrm{H}$ & -0.46923700 & -3.00557600 & -4.5560960 \\
\hline $\mathrm{C}$ & 2.04482900 & 6.37085800 & -9.03233300 & $\mathrm{C}$ & -2.81277700 & -0.33219600 & -0.68022000 \\
\hline $\mathrm{H}$ & 1.02422000 & 4.60977500 & -9.91887600 & $\mathrm{H}$ & -3.52804300 & -0.58545500 & 0.1116860 \\
\hline $\mathrm{H}$ & 0.33418700 & 5.18846200 & -8.39495500 & $\mathrm{H}$ & -2.30389800 & 0.59297300 & -0.38891700 \\
\hline $\mathrm{H}$ & 1.38225300 & 7.23819500 & -9.13543400 & $\mathrm{H}$ & -2.06259200 & -1.13143400 & -0.71487700 \\
\hline $\mathrm{H}$ & 2.71902600 & 6.38512200 & -9.90064500 & $\mathrm{C}$ & -4.29873400 & -1.46669700 & -2.39311800 \\
\hline $\mathrm{C}$ & 1.47632100 & 2.82071100 & -7.69260000 & $\mathrm{H}$ & -5.01883800 & -1.72498500 & -1.6067120 \\
\hline $\mathrm{C}$ & 0.69546500 & 2.52626400 & -6.57458600 & $\mathrm{H}$ & -3.61267800 & -2.31403300 & -2.51163800 \\
\hline $\mathrm{C}$ & 0.89292000 & 0.67699000 & -8.67034300 & $\mathrm{H}$ & -4.84714200 & -1.34871800 & -3.33399800 \\
\hline $\mathrm{C}$ & -0.05020500 & 1.32951500 & -6.50531200 & $\mathrm{C}$ & -3.82746800 & 2.69134800 & -6.54984200 \\
\hline $\mathrm{C}$ & 0.04226000 & 0.44111200 & -7.58990200 & $\mathrm{H}$ & -4.64403600 & 2.53174100 & -5.83534200 \\
\hline $\mathrm{H}$ & 0.98240900 & -0.05787800 & -9.46681000 & $\mathrm{H}$ & -3.76180100 & 1.80074500 & -7.18522600 \\
\hline $\mathrm{H}$ & -0.54756000 & -0.47044500 & -7.56197400 & $\mathrm{H}$ & -4.10274800 & 3.54254100 & -7.18484700 \\
\hline $\mathrm{C}$ & 2.81615800 & 6.38661400 & -7.72987400 & $\mathrm{C}$ & -2.56485500 & 4.25187500 & -4.98875800 \\
\hline $\mathrm{C}$ & 3.48244200 & 7.44861000 & -7.12957200 & $\mathrm{H}$ & -2.83727300 & 5.10461700 & -5.62302900 \\
\hline $\mathrm{C}$ & 3.30170500 & 4.97143200 & -5.83072900 & $\mathrm{H}$ & -1.60008000 & 4.47091900 & -4.52272100 \\
\hline $\mathrm{C}$ & 4.09700400 & 7.24612100 & -5.89345200 & $\mathrm{H}$ & -3.32489200 & 4.17437100 & -4.20115600 \\
\hline $\mathrm{H}$ & 3.52853700 & 8.42240600 & -7.61118100 & $\mathrm{C}$ & 6.39693300 & 4.27651100 & -4.84877000 \\
\hline $\mathrm{C}$ & 3.99534500 & 6.02797000 & -5.20227600 & $\mathrm{H}$ & 5.76815800 & 4.42328100 & -5.72981000 \\
\hline $\mathrm{H}$ & 4.65042800 & 8.05748100 & -5.43040900 & $\mathrm{C}$ & 6.78557900 & 5.85114800 & -0.05191800 \\
\hline $\mathrm{O}$ & 0.61807700 & 3.45052300 & -5.54798700 & $\mathrm{H}$ & 6.16430600 & 6.40507200 & 0.66576600 \\
\hline $\mathrm{O}$ & 3.15149400 & 3.75780000 & -5.17476400 & $\mathrm{C}$ & 2.95130800 & 7.57290600 & -2.83513900 \\
\hline $\mathrm{P}$ & 1.74561700 & 3.48945000 & -4.34066700 & $\mathrm{H}$ & 2.44765200 & 7.37558400 & -3.78402900 \\
\hline $\mathrm{O}$ & 1.36621100 & 4.63263800 & -3.45624800 & $\mathrm{C}$ & 7.03084800 & 4.45170000 & 0.53909000 \\
\hline $\mathrm{O}$ & 1.93122800 & 2.09611900 & -3.75510200 & $\mathrm{H}$ & 7.51307100 & 4.52624500 & 1.52144200 \\
\hline $\mathrm{C}$ & -0.91157100 & 0.97664700 & -5.32838000 & $\mathrm{H}$ & 7.69016100 & 3.85608500 & -0.10407600 \\
\hline $\mathrm{C}$ & -2.05182600 & 1.75390900 & -5.00907000 & $\mathrm{H}$ & 6.09336300 & 3.89809400 & 0.66024200 \\
\hline $\mathrm{C}$ & -0.61768900 & -0.17754700 & -4.55307100 & $\mathrm{C}$ & 8.11314800 & 6.62147600 & -0.19943700 \\
\hline $\mathrm{C}$ & -2.85749600 & 1.37045200 & -3.92839200 & $\mathrm{H}$ & 8.63165100 & 6.69534400 & 0.76464800 \\
\hline $\mathrm{C}$ & -1.46487400 & -0.51831500 & -3.49263400 & $\mathrm{H}$ & 7.94031500 & 7.63621900 & -0.57398000 \\
\hline $\mathrm{C}$ & -2.59193300 & 0.23758900 & -3.15771700 & $\mathrm{H}$ & 8.78419700 & 6.11625800 & -0.90453100 \\
\hline $\mathrm{H}$ & -3.73829300 & 1.96437700 & -3.69298600 & $\mathrm{C}$ & 7.80654900 & 4.78140800 & -5.21765600 \\
\hline $\mathrm{H}$ & -1.23822200 & -1.41150700 & -2.91522100 & $\mathrm{H}$ & 8.51576300 & 4.64377700 & -4.39247000 \\
\hline $\mathrm{C}$ & 4.64639900 & 5.89891700 & -3.85470000 & $\mathrm{H}$ & 7.79168700 & 5.84769300 & -5.46983000 \\
\hline $\mathrm{C}$ & 5.79843200 & 5.08677100 & -3.69794200 & $\mathrm{H}$ & 8.19494500 & 4.23224100 & -6.08421600 \\
\hline $\mathrm{C}$ & 4.17706700 & 6.66050900 & -2.75484500 & $\mathrm{C}$ & 6.40833700 & 2.76538100 & -4.55039500 \\
\hline $\mathrm{C}$ & 6.45486100 & 5.06038100 & -2.46095900 & $\mathrm{H}$ & 5.39660100 & 2.39952300 & -4.35148300 \\
\hline
\end{tabular}




\begin{tabular}{|c|c|c|c|c|c|c|c|}
\hline $\mathrm{H}$ & 7.04189300 & 2.53028800 & -3.68541300 & $\mathrm{H}$ & 1.96452900 & -1.79517900 & -0.17375000 \\
\hline $\mathrm{H}$ & 6.80765700 & 2.21045100 & -5.40853100 & $\mathrm{H}$ & 3.25271700 & -3.70398800 & 0.24129900 \\
\hline $\mathrm{C}$ & 3.35863600 & 9.06063800 & -2.80612100 & $\mathrm{C}$ & 6.79797500 & -4.97012200 & -0.98261400 \\
\hline $\mathrm{H}$ & 3.84423700 & 9.32099200 & -1.85722900 & $\mathrm{H}$ & 7.46886600 & -4.11043000 & -0.91974700 \\
\hline $\mathrm{H}$ & 2.47522600 & 9.70191500 & -2.91543800 & $\mathrm{H}$ & 7.34771200 & -5.79306200 & -1.46373600 \\
\hline $\mathrm{H}$ & 4.05705300 & 9.30991600 & -3.61221800 & $\mathrm{H}$ & 6.56164700 & -5.25774100 & 0.04380200 \\
\hline $\mathrm{C}$ & 1.92324900 & 7.27984300 & -1.72624200 & $\mathrm{C}$ & 6.60470600 & -2.83567300 & -3.3469910 \\
\hline $\mathrm{H}$ & 1.60463500 & 6.23742500 & -1.78432600 & $\mathrm{H}$ & 6.25389400 & -2.01030900 & -3.97380400 \\
\hline $\mathrm{H}$ & 1.04009900 & 7.91673800 & -1.86200600 & $\mathrm{H}$ & 7.31953700 & -3.42057400 & -3.94315600 \\
\hline $\mathrm{H}$ & 2.32457400 & 7.48743600 & -0.72603800 & $\mathrm{H}$ & 7.15884100 & -2.40983200 & -2.5053940 \\
\hline $\mathrm{H}$ & 1.63679500 & 4.55388500 & 2.20222300 & $\mathrm{C}$ & 3.66185800 & -3.04656200 & -4.6324760 \\
\hline \multirow[t]{2}{*}{$\mathrm{H}$} & -1.08965400 & 4.33531300 & 1.80893900 & $\mathrm{H}$ & 3.82100800 & -3.62281500 & -5.55447500 \\
\hline & & & & $\mathrm{H}$ & 4.16041700 & -2.08039100 & -4.7615000 \\
\hline 17-RR & & & & $\mathrm{H}$ & 2.58567400 & -2.86540100 & -4.5522140 \\
\hline $\mathrm{C}$ & 5.47241000 & -3.70031600 & -2.87710200 & $\mathrm{C}$ & 2.06476900 & -5.31140600 & -3.07700200 \\
\hline $\mathrm{C}$ & 4.18604500 & -3.79650400 & -3.44319600 & $\mathrm{H}$ & 1.62366000 & -5.89183000 & -2.26103900 \\
\hline $\mathrm{C}$ & 3.42193600 & -4.79690700 & -2.69181000 & $\mathrm{H}$ & 2.12151700 & -5.96414400 & -3.9592230 \\
\hline $\mathrm{C}$ & 4.31273800 & -5.36569600 & -1.70742500 & $\mathrm{H}$ & 1.36625400 & -4.50311300 & -3.31207800 \\
\hline $\mathrm{C}$ & 5.55254500 & -4.63528500 & -1.75279000 & $\mathrm{C}$ & 4.04034200 & -6.56271300 & -0.84154300 \\
\hline Ir & 3.88296400 & -3.29378000 & -1.15335500 & $\mathrm{H}$ & 4.36345300 & -7.48403900 & -1.34690300 \\
\hline $\mathrm{N}$ & 4.76032100 & -1.57992100 & -0.11147700 & $\mathrm{H}$ & 2.97383400 & -6.66168900 & -0.61920100 \\
\hline $\mathrm{N}$ & 2.32606500 & -1.82176300 & -1.12832600 & $\mathrm{H}$ & 4.57962300 & -6.49356800 & 0.10717300 \\
\hline $\mathrm{H}$ & 1.49831000 & -2.01081600 & -1.70771500 & $\mathrm{H}$ & 3.45139800 & -0.01677100 & 0.56504900 \\
\hline $\mathrm{C}$ & 4.03218100 & -0.29675500 & -0.32649400 & $\mathrm{C}$ & 4.95144800 & 0.86948500 & -0.67613900 \\
\hline $\mathrm{C}$ & 2.97981300 & -0.52583700 & -1.45394400 & $\mathrm{C}$ & 5.99027500 & 0.71396500 & -1.60357200 \\
\hline S & 5.07195200 & -1.89397800 & 1.49419500 & $\mathrm{C}$ & 4.74119900 & 2.13399400 & -0.11443500 \\
\hline $\mathrm{O}$ & 5.87257800 & -3.12861300 & 1.57967600 & $\mathrm{C}$ & 6.79481800 & 1.79597600 & -1.96245200 \\
\hline $\mathrm{O}$ & 3.83488200 & -1.86847800 & 2.31650900 & $\mathrm{H}$ & 6.17598700 & -0.26765900 & -2.02663600 \\
\hline $\mathrm{C}$ & 6.18166300 & -0.54681700 & 2.09613100 & $\mathrm{C}$ & 5.54591000 & 3.21898000 & -0.46805800 \\
\hline $\mathrm{C}$ & 5.76181400 & 0.37887000 & 3.07700700 & $\mathrm{H}$ & 3.93772600 & 2.27159700 & 0.60492700 \\
\hline $\mathrm{C}$ & 7.51931000 & -0.57506900 & 1.63967200 & $\mathrm{C}$ & 6.57529900 & 3.05413400 & -1.39547700 \\
\hline $\mathrm{C}$ & 6.72857400 & 1.23512500 & 3.65284500 & $\mathrm{H}$ & 7.59789400 & 1.65628400 & -2.68240000 \\
\hline $\mathrm{C}$ & 8.45940100 & 0.29854700 & 2.21925700 & $\mathrm{H}$ & 5.36791600 & 4.19191500 & -0.01699000 \\
\hline $\mathrm{C}$ & 8.05981000 & 1.22258000 & 3.20244500 & $\mathrm{H}$ & 7.20422100 & 3.89668300 & -1.67170000 \\
\hline C & 4.32504100 & 0.55903500 & 3.53503000 & $\mathrm{H}$ & 3.52704800 & -0.69173800 & -2.38698900 \\
\hline $\mathrm{H}$ & 4.11747200 & -0.00457700 & 4.45390200 & $\mathrm{C}$ & 2.01898600 & 0.63039700 & -1.68108400 \\
\hline $\mathrm{H}$ & 3.60619400 & 0.21195700 & 2.80037000 & $\mathrm{C}$ & 0.81107600 & 0.75098700 & -0.98404900 \\
\hline $\mathrm{H}$ & 4.12892300 & 1.61489600 & 3.74326900 & $\mathrm{C}$ & 2.34109800 & 1.60335500 & -2.63960400 \\
\hline $\mathrm{C}$ & 6.32385600 & 2.16214000 & 4.78677100 & $\mathrm{C}$ & -0.05608000 & 1.81457100 & -1.24262000 \\
\hline $\mathrm{H}$ & 7.17439200 & 2.41231900 & 5.42453600 & $\mathrm{H}$ & 0.51278200 & 0.00009900 & -0.26056300 \\
\hline $\mathrm{H}$ & 5.57115500 & 1.70065000 & 5.43118700 & $\mathrm{C}$ & 1.48325400 & 2.67290600 & -2.89138300 \\
\hline $\mathrm{H}$ & 5.89852900 & 3.10983800 & 4.42480200 & $\mathrm{H}$ & 3.27578700 & 1.52198600 & -3.18864300 \\
\hline $\mathrm{C}$ & 9.05835800 & 2.22681400 & 3.75046400 & $\mathrm{C}$ & 0.27796800 & 2.78002500 & -2.19388100 \\
\hline $\mathrm{H}$ & 9.57156800 & 1.85758000 & 4.65042400 & $\mathrm{H}$ & -0.99587700 & 1.87550500 & -0.70312600 \\
\hline $\mathrm{H}$ & 8.57453200 & 3.16912100 & 4.01882700 & $\mathrm{H}$ & 1.75225100 & 3.41546600 & -3.63825000 \\
\hline $\mathrm{H}$ & 9.82957200 & 2.46771600 & 3.01537800 & $\mathrm{H}$ & -0.40032000 & 3.60489900 & -2.39652000 \\
\hline $\mathrm{C}$ & 9.91496000 & 0.22058200 & 1.79368400 & $\mathrm{~N}$ & -0.73993200 & -6.15204900 & 1.38884100 \\
\hline $\mathrm{H}$ & 10.58720300 & 0.53598100 & 2.59509100 & $\mathrm{C}$ & 0.19170900 & -6.27189200 & 2.28977000 \\
\hline $\mathrm{H}$ & 10.12785400 & 0.85414300 & 0.92014400 & $\mathrm{C}$ & -1.57612200 & -7.20232800 & 0.88374600 \\
\hline $\mathrm{H}$ & 10.19909900 & -0.79979400 & 1.52561600 & $\mathrm{C}$ & -1.76285300 & -7.30104800 & -0.49751700 \\
\hline $\mathrm{C}$ & 7.99035500 & -1.48423900 & 0.52085300 & $\mathrm{C}$ & -2.25228600 & -8.05839600 & 1.76169100 \\
\hline $\mathrm{H}$ & 8.69709100 & -0.94965200 & -0.12204400 & $\mathrm{C}$ & -2.59740300 & -8.30077000 & -0.99878600 \\
\hline $\mathrm{H}$ & 7.16338500 & -1.82371900 & -0.09770200 & $\mathrm{H}$ & -1.28356000 & -6.58958200 & -1.16079500 \\
\hline $\mathrm{H}$ & 8.49655800 & -2.37418900 & 0.91461000 & $\mathrm{C}$ & -3.08495100 & -9.05147200 & 1.24741700 \\
\hline
\end{tabular}




\begin{tabular}{|c|c|c|c|c|c|c|c|}
\hline$H$ & -2.14724600 & -7.92969100 & 2.83430600 & $\mathrm{C}$ & -2.76575300 & -0.28189900 & 1.88329200 \\
\hline $\mathrm{C}$ & -3.25104700 & -9.18052100 & -0.13326900 & $\mathrm{C}$ & -3.44116300 & 0.79176000 & 1.24190700 \\
\hline $\mathrm{H}$ & -2.74367800 & -8.38143800 & -2.07060600 & $\mathrm{C}$ & -1.62859300 & -0.00294700 & 2.67650800 \\
\hline $\mathrm{H}$ & -3.60984000 & -9.71583100 & 1.92762500 & $\mathrm{C}$ & -2.97611700 & 2.09858000 & 1.42736400 \\
\hline $\mathrm{H}$ & -0.90519500 & -5.17266000 & 0.94984400 & $\mathrm{C}$ & -1.20623900 & 1.32522500 & 2.82491500 \\
\hline $\mathrm{C}$ & 0.64805000 & -7.60285800 & 2.80829100 & $\mathrm{C}$ & -1.86762100 & 2.39591700 & 2.22595600 \\
\hline $\mathrm{H}$ & 0.33370100 & -7.73016100 & 3.84989100 & $\mathrm{H}$ & -3.51422800 & 2.91201700 & 0.94691300 \\
\hline $\mathrm{H}$ & 0.27156000 & -8.43750300 & 2.21761100 & $\mathrm{H}$ & -0.33389900 & 1.53225700 & 3.44082900 \\
\hline $\mathrm{H}$ & 1.74188700 & -7.62140900 & 2.80602700 & $\mathrm{C}$ & -1.98334400 & -1.55375800 & -4.93289600 \\
\hline $\mathrm{H}$ & -3.90105800 & -9.95360600 & -0.53255000 & $\mathrm{H}$ & -2.63802200 & -1.68478300 & -4.06632000 \\
\hline $\mathrm{C}$ & 0.81197100 & -5.01034200 & 2.85421500 & $\mathrm{C}$ & 1.30212700 & -4.26014300 & -7.71847800 \\
\hline $\mathrm{H}$ & 0.42774900 & -4.17063500 & 2.26618700 & $\mathrm{H}$ & 2.09454500 & -4.92159200 & -7.33619000 \\
\hline $\mathrm{C}$ & -6.48061200 & -3.40383000 & -0.68780000 & $\mathrm{C}$ & -1.86898200 & -6.69652800 & -4.64969600 \\
\hline $\mathrm{C}$ & -5.75923000 & -3.97101300 & -2.86377200 & $\mathrm{H}$ & -2.46824100 & -6.49866000 & -3.75379000 \\
\hline $\mathrm{C}$ & -7.02930200 & -3.82030200 & -2.06110300 & $\mathrm{C}$ & -0.91969300 & -0.51556000 & -4.53294200 \\
\hline $\mathrm{H}$ & -6.30102900 & -2.32277700 & -0.68051000 & $\mathrm{H}$ & -1.40568700 & 0.42206400 & -4.23829600 \\
\hline $\mathrm{H}$ & -7.15178400 & -3.63442100 & 0.14480000 & $\mathrm{H}$ & -0.23977400 & -0.28027900 & -5.36124100 \\
\hline $\mathrm{H}$ & -7.58073300 & -4.77002000 & -2.00155400 & $\mathrm{H}$ & -0.33985500 & -0.87191800 & -3.67967600 \\
\hline $\mathrm{H}$ & -7.71618000 & -3.08187600 & -2.49035400 & $\mathrm{C}$ & -2.84564600 & -1.02056600 & -6.09632500 \\
\hline $\mathrm{C}$ & -5.09649600 & -4.14196700 & -0.56540600 & $\mathrm{H}$ & -3.29037900 & -0.05401700 & -5.8295990 \\
\hline $\mathrm{C}$ & -5.34354700 & -5.57675600 & -0.00422000 & $\mathrm{H}$ & -3.65961200 & -1.70878400 & -6.34778100 \\
\hline $\mathrm{C}$ & -4.34431500 & -3.51403000 & 0.60355000 & $\mathrm{H}$ & -2.24065900 & -0.87238400 & -6.99960600 \\
\hline $\mathrm{C}$ & -5.57961100 & -5.35665600 & 1.51283800 & $\mathrm{C}$ & 1.95459100 & -2.91732600 & -8.07668900 \\
\hline $\mathrm{H}$ & -6.18116900 & -6.08113900 & -0.49749000 & $\mathrm{H}$ & 2.77653900 & -3.07560100 & -8.78428400 \\
\hline $\mathrm{H}$ & -4.44787900 & -6.18475400 & -0.16303800 & $\mathrm{H}$ & 2.36032700 & -2.40943700 & -7.19587200 \\
\hline $\mathrm{H}$ & -5.21552600 & -6.19845400 & 2.11454400 & $\mathrm{H}$ & 1.23817100 & -2.23912400 & -8.55576800 \\
\hline $\mathrm{H}$ & -6.64842200 & -5.24947600 & 1.74342000 & $\mathrm{C}$ & 0.73343400 & -4.92063000 & -8.99339100 \\
\hline $\mathrm{C}$ & -4.63903900 & -4.08858100 & -2.02325400 & $\mathrm{H}$ & 1.51606100 & -5.03912400 & -9.75289200 \\
\hline $\mathrm{C}$ & -3.35374700 & -4.13949100 & -2.58211200 & $\mathrm{H}$ & -0.06513900 & -4.30276900 & -9.42166600 \\
\hline $\mathrm{C}$ & -5.62465300 & -3.96931200 & -4.24521500 & $\mathrm{H}$ & 0.31229300 & -5.90940100 & -8.78380000 \\
\hline $\mathrm{C}$ & -3.18999400 & -4.11066200 & -3.98596900 & $\mathrm{C}$ & -2.81283600 & -7.30950000 & -5.70530500 \\
\hline $\mathrm{C}$ & -4.34533700 & -4.04815800 & -4.78618800 & $\mathrm{H}$ & -2.27272300 & -7.51565100 & -6.63728200 \\
\hline $\mathrm{H}$ & -6.49450800 & -3.88708300 & -4.89211500 & $\mathrm{H}$ & -3.63983600 & -6.63121200 & -5.93754000 \\
\hline $\mathrm{H}$ & -4.21488900 & -4.03641600 & -5.86434500 & $\mathrm{H}$ & -3.23996700 & -8.25508500 & -5.34670400 \\
\hline C & -4.81754800 & -4.07568300 & 1.79718800 & $\mathrm{C}$ & -0.77691600 & -7.70915000 & -4.26003500 \\
\hline $\mathrm{C}$ & -4.59989200 & -3.42640800 & 3.01012100 & $\mathrm{H}$ & -1.22944600 & -8.62240000 & -3.85428000 \\
\hline $\mathrm{C}$ & -3.48402200 & -2.41411800 & 0.64603900 & $\mathrm{H}$ & -0.09770200 & -7.29930700 & -3.50545600 \\
\hline $\mathrm{C}$ & -3.94182300 & -2.19328500 & 2.99971200 & $\mathrm{H}$ & -0.17196900 & -8.01355200 & -5.12166200 \\
\hline $\mathrm{H}$ & -4.98504700 & -3.83432000 & 3.94225400 & $\mathrm{C}$ & -4.69431000 & 0.60128800 & 0.38576600 \\
\hline $\mathrm{C}$ & -3.36156400 & -1.66137800 & 1.83333700 & $\mathrm{H}$ & -4.95311100 & -0.45973000 & 0.38961000 \\
\hline $\mathrm{H}$ & -3.85344000 & -1.62302700 & 3.92028600 & $\mathrm{C}$ & -1.42163000 & 3.83003300 & 2.48500000 \\
\hline $\mathrm{O}$ & -2.26432800 & -4.29773100 & -1.74056500 & $\mathrm{H}$ & -0.48382800 & 3.77525900 & 3.05501000 \\
\hline $\mathrm{O}$ & -2.75751700 & -2.06418700 & -0.48390500 & $\mathrm{C}$ & -0.82507800 & -1.09141500 & 3.38327300 \\
\hline $\mathrm{P}$ & -1.49388100 & -3.07545800 & -0.90477500 & $\mathrm{H}$ & -1.30398800 & -2.05217600 & 3.17775500 \\
\hline $\mathrm{O}$ & -0.97141900 & -3.76681200 & 0.34487800 & $\mathrm{C}$ & -1.12828800 & 4.61367100 & 1.19325200 \\
\hline $\mathrm{O}$ & -0.57307200 & -2.30434700 & -1.79082200 & $\mathrm{H}$ & -0.76043500 & 5.61928100 & 1.43097000 \\
\hline $\mathrm{C}$ & -1.89023800 & -4.12621100 & -4.74711400 & $\mathrm{H}$ & -2.03115400 & 4.73070100 & 0.58153100 \\
\hline $\mathrm{C}$ & -1.30933300 & -5.35842800 & -5.13548900 & $\mathrm{H}$ & -0.37291800 & 4.10789100 & 0.58349100 \\
\hline $\mathrm{C}$ & -1.38225500 & -2.91791700 & -5.27703600 & $\mathrm{C}$ & -2.44841300 & 4.57798300 & 3.35926200 \\
\hline $\mathrm{C}$ & -0.26170100 & -5.35390700 & -6.06074200 & $\mathrm{H}$ & -2.09566500 & 5.58989200 & 3.59382900 \\
\hline $\mathrm{C}$ & -0.33497100 & -2.97138900 & -6.20956200 & $\mathrm{H}$ & -2.62524400 & 4.04952400 & 4.30262700 \\
\hline $\mathrm{C}$ & 0.22784500 & -4.17470600 & -6.63476200 & $\mathrm{H}$ & -3.41181700 & 4.67008500 & 2.84322000 \\
\hline $\mathrm{H}$ & 0.17172700 & -6.30145900 & -6.37267900 & $\mathrm{C}$ & -5.90688400 & 1.35257200 & 0.97098500 \\
\hline $\mathrm{H}$ & 0.02810000 & -2.03797500 & -6.62606000 & $\mathrm{H}$ & -5.75308000 & 2.43810600 & 0.97177100 \\
\hline
\end{tabular}




\begin{tabular}{|c|c|c|c|c|c|c|c|}
\hline $\mathrm{H}$ & -6.10393000 & 1.04301000 & 2.00365100 & $\mathrm{H}$ & 9.19843000 & -2.49224800 & 2.64309700 \\
\hline $\mathrm{H}$ & -6.80572100 & 1.14706200 & 0.37647000 & $\mathrm{C}$ & 11.46001900 & -2.49511800 & 1.62771000 \\
\hline $\mathrm{C}$ & -4.44551800 & 1.00000600 & -1.08183000 & $\mathrm{H}$ & 12.45659600 & -2.70732900 & 1.23463800 \\
\hline $\mathrm{H}$ & -3.61661600 & 0.42514600 & -1.50559300 & $\mathrm{H}$ & 10.94452200 & -3.45484400 & 1.71268200 \\
\hline $\mathrm{H}$ & -4.20585900 & 2.06656200 & -1.17297200 & $\mathrm{H}$ & 11.58865300 & -2.10603900 & 2.64840300 \\
\hline $\mathrm{H}$ & -5.34069100 & 0.80941500 & -1.68720400 & $\mathrm{C}$ & 12.92328800 & -0.52127600 & 0.04904900 \\
\hline $\mathrm{C}$ & -0.81177900 & -0.89548200 & 4.91208100 & $\mathrm{H}$ & 13.44802000 & -1.21814700 & -0.62043800 \\
\hline $\mathrm{H}$ & -0.31026700 & 0.03780900 & 5.19438400 & $\mathrm{H}$ & 13.25726200 & -0.73831300 & 1.06648600 \\
\hline $\mathrm{H}$ & -0.27955800 & -1.72035700 & 5.39852800 & $\mathrm{H}$ & 13.27704200 & 0.48407300 & -0.19004200 \\
\hline $\mathrm{H}$ & -1.82856200 & -0.85970300 & 5.32081000 & $\mathrm{C}$ & 11.48854200 & 1.13032000 & -1.90639800 \\
\hline $\mathrm{C}$ & 0.60747400 & -1.17265600 & 2.82863200 & $\mathrm{H}$ & 12.48301000 & 0.74164400 & -2.13687100 \\
\hline $\mathrm{H}$ & 0.59178200 & -1.43746400 & 1.76604600 & $\mathrm{H}$ & 11.62207900 & 2.13216300 & -1.47231100 \\
\hline $\mathrm{H}$ & 1.19512500 & -1.93118400 & 3.35514700 & $\mathrm{H}$ & 10.97178800 & 1.26079100 & -2.86013200 \\
\hline $\mathrm{H}$ & 1.14066400 & -0.22172600 & 2.93480400 & $\mathrm{C}$ & 8.62052700 & 1.14316300 & -1.96434200 \\
\hline $\mathrm{C}$ & 2.34555700 & -5.00660300 & 2.74024800 & $\mathrm{H}$ & 9.10788200 & 2.12228400 & -1.90508600 \\
\hline $\mathrm{H}$ & 2.74415400 & -4.03465100 & 3.04354000 & $\mathrm{H}$ & 7.57568500 & 1.27096200 & -1.69555000 \\
\hline $\mathrm{H}$ & 2.82101300 & -5.78306900 & 3.34805300 & $\mathrm{H}$ & 8.65594900 & 0.81327600 & -3.00943800 \\
\hline $\mathrm{H}$ & 2.64856000 & -5.14628000 & 1.69933800 & $\mathrm{H}$ & 3.52984000 & -0.24619100 & 1.52544400 \\
\hline $\mathrm{C}$ & 0.25881300 & -4.88721400 & 4.28686800 & $\mathrm{H}$ & 3.32396600 & -1.15156800 & -0.71324500 \\
\hline $\mathrm{C}$ & 1.07394800 & -4.99748700 & 5.42072900 & $\mathrm{C}$ & 5.18948500 & 3.66502200 & -1.57133100 \\
\hline $\mathrm{C}$ & -1.11931500 & -4.68158100 & 4.46668600 & $\mathrm{H}$ & 5.01743000 & 4.05997000 & -0.56605400 \\
\hline $\mathrm{C}$ & 0.52484600 & -4.90254600 & 6.70250700 & $\mathrm{H}$ & 5.07790800 & 4.50316200 & -2.27408200 \\
\hline $\mathrm{H}$ & 2.14426300 & -5.13638300 & 5.31373900 & $\mathrm{H}$ & 6.22518200 & 3.32181600 & -1.62865600 \\
\hline $\mathrm{C}$ & -1.66704000 & -4.59237500 & 5.74611100 & $\mathrm{C}$ & 2.22075200 & 3.31657900 & -0.41413200 \\
\hline $\mathrm{H}$ & -1.76881200 & -4.56742500 & 3.60222400 & $\mathrm{H}$ & 2.95119900 & 3.68604700 & 0.31333600 \\
\hline $\mathrm{C}$ & -0.84646600 & -4.70722700 & 6.87051000 & $\mathrm{H}$ & 1.43247500 & 2.78798500 & 0.12904900 \\
\hline $\mathrm{H}$ & 1.17540900 & -4.97857700 & 7.56962500 & $\mathrm{H}$ & 1.77962600 & 4.19494600 & -0.89886400 \\
\hline $\mathrm{H}$ & -2.73407400 & -4.42250800 & 5.86066400 & $\mathrm{C}$ & 0.80330400 & 0.95012200 & -2.09049500 \\
\hline \multirow[t]{2}{*}{$\mathrm{H}$} & -1.27074600 & -4.63502500 & 7.86819700 & $\mathrm{H}$ & 0.24352500 & 1.41127000 & -2.91322800 \\
\hline & & & & $\mathrm{H}$ & 0.34548800 & 1.25184800 & -1.14882900 \\
\hline TS-14-RR & & & & $\mathrm{H}$ & 0.68838100 & -0.12707900 & -2.18426400 \\
\hline $\mathrm{C}$ & 2.87078400 & 2.43625200 & -1.44161400 & $\mathrm{C}$ & 2.98820800 & -0.19191600 & -4.15947000 \\
\hline $\mathrm{C}$ & 2.24623500 & 1.33770600 & -2.14201800 & $\mathrm{H}$ & 2.74859700 & 0.33351800 & -5.09555800 \\
\hline $\mathrm{C}$ & 3.23313600 & 0.81500000 & -3.07832700 & $\mathrm{H}$ & 2.14965200 & -0.84937900 & -3.93091100 \\
\hline $\mathrm{C}$ & 4.43818600 & 1.55693500 & -2.93824800 & $\mathrm{H}$ & 3.87525600 & -0.80379100 & -4.34665800 \\
\hline $\mathrm{C}$ & 4.22951000 & 2.56704200 & -1.91421500 & $\mathrm{C}$ & 5.63263500 & 1.41838100 & -3.83735300 \\
\hline Ir & 3.93493500 & 0.56615400 & -0.94062100 & $\mathrm{H}$ & 6.06471400 & 0.41727200 & -3.77484100 \\
\hline $\mathrm{N}$ & 5.95708100 & 0.32496900 & -0.06960800 & $\mathrm{H}$ & 6.41407900 & 2.13473900 & -3.57633400 \\
\hline $\mathrm{N}$ & 3.58775500 & 0.69721600 & 1.14857300 & $\mathrm{H}$ & 5.33659100 & 1.61360000 & -4.87691100 \\
\hline $\mathrm{H}$ & 2.66059800 & 1.10931000 & 1.34333800 & $\mathrm{H}$ & 5.95970500 & -0.36451200 & 1.95752800 \\
\hline $\mathrm{C}$ & 6.01271900 & 0.57968400 & 1.39345900 & $\mathrm{C}$ & 7.25670500 & 1.33454900 & 1.86252200 \\
\hline $\mathrm{C}$ & 4.74643600 & 1.39920000 & 1.77047100 & $\mathrm{C}$ & 7.67301700 & 2.50821300 & 1.22142500 \\
\hline S & 6.82268300 & -0.99556400 & -0.58670600 & $\mathrm{C}$ & 7.96019800 & 0.90861100 & 2.99487900 \\
\hline $\mathrm{O}$ & 6.69836000 & -1.04202900 & -2.05640200 & $\mathrm{C}$ & 8.76432600 & 3.23490200 & 1.69693000 \\
\hline $\mathrm{O}$ & 6.41685500 & -2.23542800 & 0.12327200 & $\mathrm{H}$ & 7.14480800 & 2.84289200 & 0.33573800 \\
\hline $\mathrm{C}$ & 8.61580100 & -0.75001100 & -0.23009100 & $\mathrm{C}$ & 9.05471000 & 1.63110700 & 3.47391400 \\
\hline $\mathrm{C}$ & 9.28985600 & -1.57271900 & 0.70085300 & $\mathrm{H}$ & 7.64777200 & 0.00412500 & 3.51002000 \\
\hline $\mathrm{C}$ & 9.31829000 & 0.16271900 & -1.04609500 & $\mathrm{C}$ & 9.46043900 & 2.79856000 & 2.82676800 \\
\hline $\mathrm{C}$ & 10.70233400 & -1.53277000 & 0.72872400 & $\mathrm{H}$ & 9.07300100 & 4.14271500 & 1.18422300 \\
\hline $\mathrm{C}$ & 10.72390800 & 0.20154800 & -0.98062500 & $\mathrm{H}$ & 9.58840800 & 1.28030800 & 4.35337300 \\
\hline $\mathrm{C}$ & 11.41451100 & -0.62821300 & -0.07881200 & $\mathrm{H}$ & 10.31178500 & 3.36315500 & 3.19773600 \\
\hline $\mathrm{C}$ & 8.60676500 & -2.46320200 & 1.72416500 & $\mathrm{H}$ & 4.81753900 & 2.36696500 & 1.26757800 \\
\hline $\mathrm{H}$ & 8.49577000 & -3.49287800 & 1.36286600 & $\mathrm{C}$ & 4.59338500 & 1.65457900 & 3.26000900 \\
\hline $\mathrm{H}$ & 7.60851500 & -2.11857100 & 1.97518600 & $\mathrm{C}$ & 4.57315600 & 0.61323800 & 4.19825200 \\
\hline
\end{tabular}




\begin{tabular}{|c|c|c|c|c|c|c|c|}
\hline C & 4.49773900 & 2.97321100 & 3.72254900 & $\mathrm{H}$ & -4.76497200 & 1.63260400 & 2.87592900 \\
\hline $\mathrm{C}$ & 4.47696700 & 0.88764300 & 5.56262300 & $\mathrm{H}$ & -5.89892800 & 0.47372600 & 4.66426200 \\
\hline $\mathrm{H}$ & 4.64158300 & -0.42106600 & 3.87092000 & $\mathrm{H}$ & -4.82237000 & 1.13472400 & 5.89306400 \\
\hline $\mathrm{C}$ & 4.39258600 & 3.25109400 & 5.08584700 & $\mathrm{C}$ & -2.28301000 & 2.36978200 & 2.58075700 \\
\hline $\mathrm{H}$ & 4.51886900 & 3.79268900 & 3.00788100 & $\mathrm{C}$ & -2.14805300 & 1.98106900 & 1.24420200 \\
\hline $\mathrm{C}$ & 4.38661500 & 2.20704800 & 6.01097200 & $\mathrm{C}$ & -2.29007900 & 4.69566600 & 1.93920100 \\
\hline $\mathrm{H}$ & 4.47018900 & 0.06815200 & 6.27487500 & $\mathrm{C}$ & -2.37020200 & 2.93587300 & 0.21193100 \\
\hline $\mathrm{H}$ & 4.32188400 & 4.28172000 & 5.42283200 & $\mathrm{C}$ & -2.46515400 & 4.28310700 & 0.61743600 \\
\hline $\mathrm{H}$ & 4.31175700 & 2.41804600 & 7.07423600 & $\mathrm{H}$ & -2.28991800 & 5.75505000 & 2.18481400 \\
\hline $\mathrm{N}$ & 1.44265500 & -2.49322200 & -1.28653500 & $\mathrm{H}$ & -2.68671900 & 5.03411000 & -0.12798500 \\
\hline $\mathrm{C}$ & 2.74591200 & -2.60029500 & -0.87916800 & $\mathrm{C}$ & -3.88143100 & -0.36116100 & 4.64414700 \\
\hline $\mathrm{C}$ & 0.76757200 & -2.70656600 & -2.51008900 & $\mathrm{C}$ & -4.01805300 & -1.68989400 & 5.02373900 \\
\hline $\mathrm{C}$ & -0.57790600 & -2.28032600 & -2.54234100 & $\mathrm{C}$ & -1.71996500 & -0.81947100 & 3.64206300 \\
\hline $\mathrm{C}$ & 1.28583100 & -3.38019300 & -3.63155500 & $\mathrm{C}$ & -2.98222900 & -2.57200700 & 4.73182800 \\
\hline $\mathrm{C}$ & -1.36616300 & -2.50031600 & -3.66675700 & $\mathrm{H}$ & -4.90814400 & -2.03469700 & 5.54424700 \\
\hline $\mathrm{H}$ & -0.99048700 & -1.78196800 & -1.66991300 & $\mathrm{C}$ & -1.82778800 & -2.17200400 & 4.03684800 \\
\hline $\mathrm{C}$ & 0.48218200 & -3.58864700 & -4.75386300 & $\mathrm{H}$ & -3.05173400 & -3.60873000 & 5.0478860 \\
\hline $\mathrm{H}$ & 2.29722000 & -3.75564300 & -3.64205500 & $\mathrm{O}$ & -1.87574600 & 0.65560800 & 0.94912600 \\
\hline $\mathrm{C}$ & -0.84176300 & -3.15116800 & -4.78699200 & $\mathrm{O}$ & -0.58275100 & -0.34199800 & 3.01841700 \\
\hline $\mathrm{H}$ & -2.39711400 & -2.15782400 & -3.66098600 & $\mathrm{P}$ & -0.41485300 & -0.04373300 & 1.39046000 \\
\hline $\mathrm{H}$ & 0.90447900 & -4.11159600 & -5.60792800 & $\mathrm{O}$ & -0.37731200 & -1.34421200 & 0.6273790 \\
\hline $\mathrm{H}$ & 0.80601100 & -2.14783300 & -0.54721500 & $\mathrm{O}$ & 0.69880900 & 0.96478200 & 1.30216000 \\
\hline $\mathrm{C}$ & 3.81142200 & -3.05057400 & -1.85875700 & $\mathrm{C}$ & -2.62715900 & 2.55876700 & -1.22444800 \\
\hline $\mathrm{H}$ & 3.65651500 & -4.09723000 & -2.13766700 & $\mathrm{C}$ & -3.57275500 & 1.53454000 & -1.52524300 \\
\hline $\mathrm{H}$ & 3.81032100 & -2.43332300 & -2.75571400 & $\mathrm{C}$ & -2.02562600 & 3.25506700 & -2.32110200 \\
\hline $\mathrm{H}$ & 4.79129200 & -2.96596200 & -1.38991300 & $\mathrm{C}$ & -3.79586500 & 1.16801000 & -2.85870100 \\
\hline $\mathrm{H}$ & -1.45687900 & -3.32289400 & -5.66552300 & $\mathrm{C}$ & -2.30849200 & 2.85047400 & -3.63101200 \\
\hline $\mathrm{C}$ & 2.77808600 & -3.26181800 & 0.55215700 & $\mathrm{C}$ & -3.16740900 & 1.79294100 & -3.93152900 \\
\hline $\mathrm{C}$ & 3.13059100 & -4.73877400 & 0.33447700 & $\mathrm{H}$ & -4.51577000 & 0.38008800 & -3.06818200 \\
\hline $\mathrm{C}$ & 3.62488500 & -2.61619300 & 1.65391000 & $\mathrm{H}$ & -1.84344500 & 3.38966400 & -4.45113100 \\
\hline $\mathrm{C}$ & 2.12775700 & -5.64725100 & -0.03180100 & $\mathrm{C}$ & -0.76960600 & -3.22011100 & 3.84933700 \\
\hline $\mathrm{C}$ & 4.44793200 & -5.20662500 & 0.45572900 & $\mathrm{C}$ & 0.33003200 & -3.26362900 & 4.73562300 \\
\hline $\mathrm{H}$ & 3.05768100 & -1.79755300 & 2.10798200 & $\mathrm{C}$ & -0.96217500 & -4.27123500 & 2.92026900 \\
\hline $\mathrm{C}$ & 2.42776500 & -6.99179500 & -0.26151500 & $\mathrm{C}$ & 1.22138300 & -4.34312500 & 4.67029700 \\
\hline $\mathrm{H}$ & 1.10440400 & -5.29880900 & -0.13511300 & $\mathrm{C}$ & -0.04771200 & -5.33019400 & 2.90296600 \\
\hline $\mathrm{C}$ & 4.74785700 & -6.55151700 & 0.23071800 & $\mathrm{C}$ & 1.04588600 & -5.39830400 & 3.77328000 \\
\hline $\mathrm{C}$ & 3.74030200 & -7.44961300 & -0.12800600 & $\mathrm{H}$ & 2.05509600 & -4.36951000 & 5.36643600 \\
\hline $\mathrm{H}$ & 1.63470300 & -7.68018800 & -0.54252600 & $\mathrm{H}$ & -0.20116700 & -6.14834900 & 2.20238100 \\
\hline $\mathrm{H}$ & 5.77429800 & -6.89529300 & 0.32937900 & $\mathrm{C}$ & -1.13259500 & 4.49681900 & -2.18280700 \\
\hline $\mathrm{H}$ & 3.97629700 & -8.49562200 & -0.30542200 & $\mathrm{H}$ & -0.77725800 & 4.55659900 & -1.15071800 \\
\hline $\mathrm{H}$ & 1.74443600 & -3.23583600 & 0.90694200 & $\mathrm{C}$ & -3.44287000 & 1.36521700 & -5.36660600 \\
\hline $\mathrm{H}$ & 4.59750000 & -2.27453300 & 1.29763600 & $\mathrm{H}$ & -4.13311300 & 0.51134300 & -5.32103100 \\
\hline $\mathrm{C}$ & -2.10506200 & 2.41642700 & 4.94365700 & $\mathrm{C}$ & -4.48609900 & 0.87025800 & -0.48905900 \\
\hline $\mathrm{C}$ & -2.21243700 & 3.72460400 & 2.93002000 & $\mathrm{H}$ & -4.20095000 & 1.21192000 & 0.50499300 \\
\hline $\mathrm{C}$ & -2.15515300 & 3.88255800 & 4.43813800 & $\mathrm{C}$ & 0.11395400 & 4.49043300 & -3.09225300 \\
\hline $\mathrm{H}$ & -1.06648800 & 2.08378400 & 5.04788200 & $\mathrm{H}$ & 0.74792400 & 5.35339900 & -2.85253500 \\
\hline $\mathrm{H}$ & -2.60196600 & 2.27999200 & 5.91003300 & $\mathrm{H}$ & -0.14852200 & 4.57764700 & -4.15231000 \\
\hline $\mathrm{H}$ & -3.03485500 & 4.41480000 & 4.82484100 & $\mathrm{H}$ & 0.71174900 & 3.58507100 & -2.97023400 \\
\hline $\mathrm{H}$ & -1.27856500 & 4.46073700 & 4.75513300 & $\mathrm{C}$ & -1.93963000 & 5.77918900 & -2.49779500 \\
\hline $\mathrm{C}$ & -2.78484500 & 1.60092400 & 3.80187600 & $\mathrm{H}$ & -1.33554800 & 6.67499700 & -2.30610100 \\
\hline $\mathrm{C}$ & -4.34343400 & 1.82480400 & 3.86916800 & $\mathrm{H}$ & -2.85952400 & 5.85638600 & -1.9104690 \\
\hline $\mathrm{C}$ & -2.72778800 & 0.08932200 & 3.98105500 & $\mathrm{H}$ & -2.23344100 & 5.79203500 & -3.55424900 \\
\hline $\mathrm{C}$ & -4.86050200 & 0.76792600 & 4.85689700 & $\mathrm{C}$ & -2.16376700 & 0.88799700 & $-6.0808880 \mathrm{C}$ \\
\hline $\mathrm{H}$ & -4.60472900 & 2.85047200 & 4.14434000 & $\mathrm{H}$ & -2.39383700 & 0.53814100 & -7.09487900 \\
\hline
\end{tabular}




\begin{tabular}{|c|c|c|c|c|c|c|c|}
\hline $\mathrm{H}$ & -1.68701600 & 0.06652400 & -5.53496900 & $\mathrm{~N}$ & 4.59248300 & -2.01059100 & -0.01843300 \\
\hline $\mathrm{H}$ & -1.43254700 & 1.70082900 & -6.16848800 & $\mathrm{~N}$ & 2.25806300 & -1.91463800 & -1.25510600 \\
\hline $\mathrm{C}$ & -4.14134200 & 2.47780000 & -6.17219900 & $\mathrm{H}$ & 1.46872400 & -1.98067000 & -1.90974100 \\
\hline $\mathrm{H}$ & -4.38615200 & 2.12970600 & -7.18320300 & $\mathrm{C}$ & 4.01827900 & -0.64207900 & -0.18275400 \\
\hline $\mathrm{H}$ & -3.49802500 & 3.36041300 & -6.27117700 & $\mathrm{C}$ & 3.06583900 & -0.67637600 & -1.41960800 \\
\hline $\mathrm{H}$ & -5.07008500 & 2.79508900 & -5.68545200 & $S$ & 4.77300900 & -2.45666600 & 1.56441300 \\
\hline $\mathrm{C}$ & -5.94690200 & 1.32383900 & -0.70117400 & $\mathrm{O}$ & 5.32791300 & -3.82359700 & 1.61768100 \\
\hline $\mathrm{H}$ & -6.33783700 & 0.99340100 & -1.67083800 & $\mathrm{O}$ & 3.51531200 & -2.26275600 & 2.34537500 \\
\hline $\mathrm{H}$ & -6.03282200 & 2.41581100 & -0.66171500 & $\mathrm{C}$ & 6.05563600 & -1.36992200 & 2.32689500 \\
\hline $\mathrm{H}$ & -6.59304100 & 0.90281300 & 0.07935100 & $\mathrm{C}$ & 5.73338700 & -0.43331400 & 3.33435300 \\
\hline $\mathrm{C}$ & -4.38200700 & -0.66635800 & -0.48458500 & $\mathrm{C}$ & 7.39725300 & -1.62278900 & 1.96266600 \\
\hline $\mathrm{H}$ & -5.01460900 & -1.08201600 & 0.30935400 & $\mathrm{C}$ & 6.79151800 & 0.18577500 & 4.03889900 \\
\hline $\mathrm{H}$ & -3.35365400 & -0.98785600 & -0.30067300 & $\mathrm{C}$ & 8.43215100 & -0.98215300 & 2.67041400 \\
\hline $\mathrm{H}$ & -4.72358200 & -1.10109100 & -1.43235200 & $\mathrm{C}$ & 8.13063900 & -0.05956800 & 3.68908100 \\
\hline $\mathrm{C}$ & 0.54455500 & -2.20128200 & 5.81189900 & $\mathrm{C}$ & 4.32402900 & 0.00988000 & 3.68608500 \\
\hline $\mathrm{H}$ & -0.28960000 & -1.49687700 & 5.76147000 & $\mathrm{H}$ & 3.93173000 & -0.52570000 & 4.56053800 \\
\hline $\mathrm{C}$ & 1.93211200 & -6.63987500 & 3.77360000 & $\mathrm{H}$ & 3.61721400 & -0.16127100 & 2.88152500 \\
\hline $\mathrm{H}$ & 1.94470500 & -7.02495400 & 2.74561700 & $\mathrm{H}$ & 4.32016000 & 1.07712900 & 3.92555000 \\
\hline $\mathrm{C}$ & -2.16842600 & -4.31775600 & 1.98013800 & $\mathrm{C}$ & 6.47533300 & 1.11294400 & 5.20023200 \\
\hline $\mathrm{H}$ & -2.68632200 & -3.35728800 & 2.05291000 & $\mathrm{H}$ & 7.28780000 & 1.13062900 & 5.93003800 \\
\hline $\mathrm{C}$ & 3.39072100 & -6.37832700 & 4.17884500 & $\mathrm{H}$ & 5.57695100 & 0.79582600 & 5.73535600 \\
\hline $\mathrm{H}$ & 3.98306700 & -7.29299700 & 4.06037400 & $\mathrm{H}$ & 6.30674300 & 2.15031100 & 4.87575000 \\
\hline $\mathrm{H}$ & 3.47430400 & -6.07095400 & 5.22830200 & $\mathrm{C}$ & 9.24849800 & 0.69656500 & 4.38441800 \\
\hline $\mathrm{H}$ & 3.84692500 & -5.60214100 & 3.55595300 & $\mathrm{H}$ & 9.60178400 & 0.17926100 & 5.28828700 \\
\hline $\mathrm{C}$ & 1.31469700 & -7.73473400 & 4.67034000 & $\mathrm{H}$ & 8.92907400 & 1.69603700 & 4.68906800 \\
\hline $\mathrm{H}$ & 1.91123400 & -8.65482100 & 4.62963100 & $\mathrm{H}$ & 10.11246400 & 0.82831500 & 3.72928800 \\
\hline $\mathrm{H}$ & 0.29287900 & -7.97590400 & 4.35751400 & $\mathrm{C}$ & 9.87724900 & -1.31193400 & 2.34139600 \\
\hline $\mathrm{H}$ & 1.27325500 & -7.40328600 & 5.71533600 & $\mathrm{H}$ & 10.52862200 & -1.18019000 & 3.20839000 \\
\hline $\mathrm{C}$ & 0.52939000 & -2.80851900 & 7.22853800 & $\mathrm{H}$ & 10.27581300 & -0.67927700 & 1.53478600 \\
\hline $\mathrm{H}$ & 1.36483000 & -3.50131000 & 7.38538600 & $\mathrm{H}$ & 9.98752100 & -2.34979900 & 2.01823300 \\
\hline $\mathrm{H}$ & -0.39953600 & -3.36015400 & 7.41180600 & $\mathrm{C}$ & 7.77850600 & -2.52722900 & 0.80794600 \\
\hline $\mathrm{H}$ & 0.60924400 & -2.01710500 & 7.98415100 & $\mathrm{H}$ & 8.61611500 & -2.09145500 & 0.25358900 \\
\hline $\mathrm{C}$ & 1.82771900 & -1.39197900 & 5.56506800 & $\mathrm{H}$ & 6.95199900 & -2.66287900 & 0.11556800 \\
\hline $\mathrm{H}$ & 1.78969200 & -0.89700700 & 4.58999500 & $\mathrm{H}$ & 8.08391100 & -3.52101800 & 1.15704300 \\
\hline $\mathrm{H}$ & 2.71836300 & -2.03307700 & 5.59463300 & $\mathrm{H}$ & 1.80724200 & -1.92258500 & -0.33884000 \\
\hline $\mathrm{H}$ & 1.94744600 & -0.61592900 & 6.33057400 & $\mathrm{H}$ & 2.83980700 & -4.02540700 & -0.00579100 \\
\hline $\mathrm{C}$ & -3.16319100 & -5.41863900 & 2.40230500 & $\mathrm{C}$ & 6.34336500 & -5.58002400 & -1.10248800 \\
\hline $\mathrm{H}$ & -2.70100500 & -6.41212600 & 2.34922900 & $\mathrm{H}$ & 7.11942300 & -4.83956300 & -0.89727800 \\
\hline $\mathrm{H}$ & -4.03758600 & -5.42066800 & 1.73963000 & $\mathrm{H}$ & 6.81395500 & -6.41996600 & -1.63504100 \\
\hline $\mathrm{H}$ & -3.51836600 & -5.27239500 & 3.42802800 & $\mathrm{H}$ & 5.98545600 & -5.93835700 & -0.13471800 \\
\hline $\mathrm{C}$ & -1.76785400 & -4.49318900 & 0.50545000 & $\mathrm{C}$ & 6.61564400 & -3.14712800 & -3.15437200 \\
\hline $\mathrm{H}$ & -1.10259600 & -3.68395100 & 0.19731400 & $\mathrm{H}$ & 6.42130200 & -2.21666300 & -3.69576300 \\
\hline $\mathrm{H}$ & -2.65858600 & -4.46372800 & -0.13422600 & $\mathrm{H}$ & 7.32064400 & -3.73731000 & -3.75662500 \\
\hline $\mathrm{H}$ & -1.27172200 & -5.45534100 & 0.32712300 & $\mathrm{H}$ & 7.12238100 & -2.89351400 & -2.21881000 \\
\hline $\mathrm{H}$ & 3.78319300 & -3.34162900 & 2.45787400 & $\mathrm{C}$ & 3.79825100 & -2.83804800 & -4.69586300 \\
\hline \multirow[t]{2}{*}{$\mathrm{H}$} & 5.24771800 & -4.51485700 & 0.70259900 & $\mathrm{H}$ & 3.85064500 & -3.34187100 & -5.67077200 \\
\hline & & & & $\mathrm{H}$ & 4.48815800 & -1.98926700 & -4.72823800 \\
\hline 17-RS & & & & $\mathrm{H}$ & 2.78161500 & -2.44311400 & -4.59598400 \\
\hline $\mathrm{C}$ & 5.35593300 & -3.92257400 & -2.90458900 & $\mathrm{C}$ & 1.83816900 & -5.08788700 & -3.60844300 \\
\hline $\mathrm{C}$ & 4.12889000 & -3.79188800 & -3.58518700 & $\mathrm{H}$ & 1.21351200 & -5.60234400 & -2.87393000 \\
\hline $\mathrm{C}$ & 3.19222100 & -4.77907200 & -3.04108200 & $\mathrm{H}$ & 1.91906400 & -5.73354100 & -4.49396900 \\
\hline $\mathrm{C}$ & 3.91430400 & -5.56771300 & -2.07237300 & $\mathrm{H}$ & 1.29975900 & -4.18667800 & -3.91265900 \\
\hline $\mathrm{C}$ & 5.22312400 & -4.99176900 & -1.91229400 & $\mathrm{C}$ & 3.43653600 & -6.83842300 & -1.43215300 \\
\hline Ir & 3.65270000 & -3.53670800 & -1.28588900 & $\mathrm{H}$ & 3.69572200 & -7.70010400 & -2.06396900 \\
\hline
\end{tabular}




\begin{tabular}{|c|c|c|c|c|c|c|c|}
\hline$H$ & 2.35200000 & -6.84079700 & -1.29653700 & $\mathrm{H}$ & 0.92128100 & -6.60040300 & 7.34253600 \\
\hline $\mathrm{H}$ & 3.90644800 & -6.99003800 & -0.45661700 & $\mathrm{H}$ & 3.09243000 & -5.71412700 & 8.17542100 \\
\hline $\mathrm{H}$ & 3.37741900 & -0.37996100 & 0.67161800 & $\mathrm{H}$ & 2.04828400 & -3.63646900 & 2.81160600 \\
\hline $\mathrm{C}$ & 5.08174000 & 0.44072300 & -0.32719900 & $\mathrm{C}$ & -6.35505800 & -3.89607400 & -0.23203700 \\
\hline $\mathrm{C}$ & 6.20154600 & 0.24619400 & -1.14663700 & $\mathrm{C}$ & -5.81065100 & -4.41720500 & -2.46444000 \\
\hline $\mathrm{C}$ & 4.93487400 & 1.67145200 & 0.32311000 & $\mathrm{C}$ & -6.97665000 & -4.47576200 & -1.50900000 \\
\hline $\mathrm{C}$ & 7.14715800 & 1.25814700 & -1.31498600 & $\mathrm{H}$ & -6.38730700 & -2.80166800 & -0.2784740 \\
\hline $\mathrm{H}$ & 6.33622100 & -0.71256400 & -1.63588600 & $\mathrm{H}$ & -6.86398800 & -4.20184300 & 0.68633500 \\
\hline $\mathrm{C}$ & 5.88121800 & 2.68550100 & 0.16106200 & $\mathrm{H}$ & -7.31409900 & -5.51148800 & -1.35622300 \\
\hline $\mathrm{H}$ & 4.06910400 & 1.83938300 & 0.95874500 & $\mathrm{H}$ & -7.84505500 & -3.90710200 & -1.86079400 \\
\hline $\mathrm{C}$ & 6.99055900 & 2.48300500 & -0.66070000 & $\mathrm{C}$ & -4.84637600 & -4.35370300 & -0.26164800 \\
\hline $\mathrm{H}$ & 8.01073600 & 1.08914700 & -1.95389300 & $\mathrm{C}$ & -4.73519400 & -5.74665700 & 0.42971600 \\
\hline $\mathrm{H}$ & 5.74994300 & 3.63293100 & 0.67757300 & $\mathrm{C}$ & -4.11220800 & -3.48691500 & 0.75908400 \\
\hline $\mathrm{H}$ & 7.72906700 & 3.27032300 & -0.78815600 & $\mathrm{C}$ & -4.86810900 & -5.42299000 & 1.94480600 \\
\hline $\mathrm{H}$ & 3.68847600 & -0.83949800 & -2.30477100 & $\mathrm{H}$ & -5.49418600 & -6.45371000 & 0.07766500 \\
\hline $\mathrm{C}$ & 2.25568200 & 0.59205200 & -1.64874900 & $\mathrm{H}$ & -3.75026800 & -6.17704400 & 0.21712900 \\
\hline $\mathrm{C}$ & 0.93627000 & 0.73264300 & -1.20160100 & $\mathrm{H}$ & -4.27396400 & -6.10408200 & 2.56673000 \\
\hline $\mathrm{C}$ & 2.83904500 & 1.65175400 & -2.36245100 & $\mathrm{H}$ & -5.90738900 & -5.52034400 & 2.28694800 \\
\hline $\mathrm{C}$ & 0.22143700 & 1.90755300 & -1.44985000 & $\mathrm{C}$ & -4.59079200 & -4.31770200 & -1.77162700 \\
\hline $\mathrm{H}$ & 0.42722600 & -0.09004200 & -0.71292200 & $\mathrm{C}$ & -3.39734200 & -4.19079300 & -2.4988800 \\
\hline $\mathrm{C}$ & 2.12952800 & 2.82644500 & -2.60545300 & $\mathrm{C}$ & -5.85899600 & -4.41741100 & -3.85167700 \\
\hline $\mathrm{H}$ & 3.85832800 & 1.55597700 & -2.72688300 & $\mathrm{C}$ & -3.42431400 & -4.16593400 & -3.91318800 \\
\hline $\mathrm{C}$ & 0.81624100 & 2.95854500 & -2.14775900 & $\mathrm{C}$ & -4.66675500 & -4.28874500 & -4.5574540 \\
\hline $\mathrm{H}$ & -0.80527600 & 1.98598600 & -1.10437300 & $\mathrm{H}$ & -6.80791600 & -4.49154400 & -4.37693900 \\
\hline $\mathrm{H}$ & 2.59958300 & 3.63367500 & -3.16136400 & $\mathrm{H}$ & -4.68362800 & -4.26244600 & -5.64321100 \\
\hline $\mathrm{H}$ & 0.25607800 & 3.86788800 & -2.34979800 & $\mathrm{C}$ & -4.37280900 & -3.98975700 & 2.03920900 \\
\hline $\mathrm{N}$ & 0.23408000 & -5.70600200 & 1.54792900 & $\mathrm{C}$ & -4.19592400 & -3.17355600 & 3.15424600 \\
\hline $\mathrm{C}$ & 1.25344500 & -5.51285400 & 2.34370800 & $\mathrm{C}$ & -3.44756800 & -2.26796000 & 0.61383800 \\
\hline $\mathrm{C}$ & -0.09558900 & -6.89779400 & 0.82344900 & $\mathrm{C}$ & -3.79593400 & -1.84857800 & 2.95561100 \\
\hline $\mathrm{C}$ & -0.69026500 & -6.76625400 & -0.43546000 & $\mathrm{H}$ & -4.41593100 & -3.53314900 & 4.15676300 \\
\hline $\mathrm{C}$ & 0.05373300 & -8.15814400 & 1.41678200 & $\mathrm{C}$ & -3.39334200 & -1.36182200 & 1.69642100 \\
\hline $\mathrm{C}$ & -1.10012400 & -7.91474400 & -1.11360000 & $\mathrm{H}$ & -3.76741000 & -1.16752000 & 3.80127500 \\
\hline $\mathrm{H}$ & -0.85032500 & -5.78112800 & -0.85753100 & $\mathrm{O}$ & -2.18994000 & -4.17524900 & -1.83093800 \\
\hline $\mathrm{C}$ & -0.34887700 & -9.29647400 & 0.72080100 & $\mathrm{O}$ & -2.82779600 & -1.96912700 & -0.59105900 \\
\hline $\mathrm{H}$ & 0.44887900 & -8.24826000 & 2.42278400 & $\mathrm{P}$ & -1.49562700 & -2.90935800 & -0.98870000 \\
\hline $\mathrm{C}$ & -0.92214400 & -9.17871200 & -0.54717500 & $\mathrm{O}$ & -0.91910800 & -3.55557500 & 0.25208600 \\
\hline $\mathrm{H}$ & -1.56794500 & -7.81218500 & -2.08657600 & $\mathrm{O}$ & -0.63160000 & -2.09149100 & -1.89689400 \\
\hline $\mathrm{H}$ & -0.23167600 & -10.27313900 & 1.18132900 & $\mathrm{C}$ & -2.22718700 & -4.00486500 & -4.81230800 \\
\hline $\mathrm{H}$ & -0.37304300 & -4.88872500 & 1.30720000 & $\mathrm{C}$ & -1.61643900 & -5.14859500 & -5.38326400 \\
\hline $\mathrm{C}$ & 2.43500100 & -6.43235000 & 2.36385200 & $\mathrm{C}$ & -1.83744500 & -2.71445500 & -5.24095600 \\
\hline $\mathrm{H}$ & 2.47919700 & -7.01217800 & 3.29062200 & $\mathrm{C}$ & -0.63470800 & -4.97356900 & -6.3638680 \\
\hline $\mathrm{H}$ & 2.44705700 & -7.10728600 & 1.50880700 & $\mathrm{C}$ & -0.84672300 & -2.59676800 & -6.22671200 \\
\hline $\mathrm{H}$ & 3.33092800 & -5.79802400 & 2.34205000 & $\mathrm{C}$ & -0.23850200 & -3.70878100 & -6.81036800 \\
\hline $\mathrm{H}$ & -1.24459800 & -10.06687800 & -1.08291600 & $\mathrm{H}$ & -0.17245600 & -5.84887500 & -6.81547500 \\
\hline $\mathrm{C}$ & 1.28986700 & -4.30651000 & 3.24938100 & $\mathrm{H}$ & -0.56307600 & -1.60307400 & -6.55641500 \\
\hline $\mathrm{C}$ & 1.80618400 & -4.72126700 & 4.63348600 & $\mathrm{C}$ & -3.05355600 & 0.09572200 & 1.53777100 \\
\hline $\mathrm{C}$ & -0.04006200 & -3.54579600 & 3.35743400 & $\mathrm{C}$ & -3.87717900 & 0.92366700 & 0.72430700 \\
\hline $\mathrm{C}$ & 3.02563800 & -4.22455500 & 5.11020700 & $\mathrm{C}$ & -2.00942200 & 0.69857100 & 2.28303800 \\
\hline $\mathrm{C}$ & 1.05479300 & -5.57838900 & 5.45220100 & $\mathrm{C}$ & -3.63867300 & 2.30191800 & 0.68665400 \\
\hline $\mathrm{C}$ & 3.48380800 & -4.58061200 & 6.38129800 & $\mathrm{C}$ & -1.82503800 & 2.08564000 & 2.21491800 \\
\hline $\mathrm{H}$ & 3.60696400 & -3.55755300 & 4.48035400 & $\mathrm{C}$ & -2.62815200 & 2.91337200 & 1.43320300 \\
\hline $\mathrm{C}$ & 1.51509600 & -5.93645500 & 6.71954700 & $\mathrm{H}$ & -4.28436700 & 2.92098800 & 0.06866600 \\
\hline $\mathrm{C}$ & 2.73350600 & -5.43767000 & 7.18761400 & $\mathrm{H}$ & -1.02561400 & 2.53726000 & 2.79844700 \\
\hline $\mathrm{H}$ & 4.43164800 & -4.18676000 & 6.73850300 & $\mathrm{C}$ & -2.53801600 & -1.45005700 & -4.74052600 \\
\hline
\end{tabular}




\begin{tabular}{|c|c|c|c|c|c|c|c|}
\hline$H$ & -3.03451900 & -1.69481800 & -3.79678600 & $\mathrm{H}$ & -2.19371600 & 0.15453900 & 5.02023500 \\
\hline $\mathrm{C}$ & 0.77322900 & -3.60196600 & -7.94873800 & $\mathrm{C}$ & 0.40790200 & 0.04794500 & 2.68418900 \\
\hline $\mathrm{H}$ & 1.51653800 & -4.39801600 & -7.79270700 & $\mathrm{H}$ & 0.50281600 & -0.23377600 & 1.63119000 \\
\hline $\mathrm{C}$ & -2.06358800 & -6.56668600 & -5.02094500 & $\mathrm{H}$ & 1.07793100 & -0.59482500 & 3.26601900 \\
\hline $\mathrm{H}$ & -2.60754700 & -6.50886800 & -4.07092300 & $\mathrm{H}$ & 0.76521100 & 1.07970900 & 2.78385100 \\
\hline $\mathrm{C}$ & -1.57968800 & -0.28309600 & -4.44877100 & $\mathrm{H}$ & -0.34334300 & -3.11319900 & 2.40144500 \\
\hline $\mathrm{H}$ & -2.14304400 & 0.55365900 & -4.01812600 & $\mathrm{H}$ & 0.08445700 & -2.73577600 & 4.07936800 \\
\hline $\mathrm{H}$ & -1.09410500 & 0.09311700 & -5.35773200 & $\mathrm{H}$ & 0.09849500 & -5.96336100 & 5.10312700 \\
\hline $\mathrm{H}$ & -0.81830700 & -0.58173000 & -3.72609600 & $\mathrm{H}$ & -0.85231400 & -4.18781300 & 3.71786400 \\
\hline $\mathrm{C}$ & -3.62888100 & -1.00863400 & -5.74039600 & & & & \\
\hline $\mathrm{H}$ & -4.15009900 & -0.11851400 & -5.36709900 & TS-14-RS & & & \\
\hline $\mathrm{H}$ & -4.37501500 & -1.79353400 & -5.90237300 & $\mathrm{C}$ & 4.61299900 & -3.50553300 & -2.72821500 \\
\hline $\mathrm{H}$ & -3.18832700 & -0.75750100 & -6.71351100 & $\mathrm{C}$ & 3.22204200 & -3.52729900 & -3.08670000 \\
\hline $\mathrm{C}$ & 1.53207400 & -2.26761800 & -8.00194500 & $\mathrm{C}$ & 2.63247000 & -4.74421300 & -2.56623700 \\
\hline $\mathrm{H}$ & 2.31660700 & -2.30978700 & -8.76642900 & $\mathrm{C}$ & 3.69633800 & -5.48869600 & -1.92803800 \\
\hline $\mathrm{H}$ & 2.00338500 & -2.02933600 & -7.04252800 & $\mathrm{C}$ & 4.90447600 & -4.72696200 & -1.98937700 \\
\hline $\mathrm{H}$ & 0.86798100 & -1.43579200 & -8.26497500 & Ir & 3.34897200 & -3.51226800 & -0.86710600 \\
\hline $\mathrm{C}$ & 0.08783600 & -3.87829700 & -9.30429300 & $\mathrm{~N}$ & 4.50683100 & -1.98486500 & 0.26641800 \\
\hline $\mathrm{H}$ & 0.81883100 & -3.86511600 & -10.12245700 & $\mathrm{~N}$ & 1.97008500 & -1.90943700 & -0.63982300 \\
\hline $\mathrm{H}$ & -0.67090200 & -3.11456500 & -9.51395600 & $\mathrm{H}$ & 1.17100500 & -1.95330300 & -1.30055200 \\
\hline $\mathrm{H}$ & -0.41140600 & -4.85319700 & -9.30803700 & $\mathrm{C}$ & 3.80542700 & -0.67383900 & 0.36544800 \\
\hline $\mathrm{C}$ & -3.04048300 & -7.12447300 & -6.07782300 & $\mathrm{C}$ & 2.75903500 & -0.65487700 & -0.78850400 \\
\hline $\mathrm{H}$ & -2.55713500 & -7.18106100 & -7.06082200 & S & 5.27454400 & -2.46715100 & 1.65944900 \\
\hline $\mathrm{H}$ & -3.92838000 & -6.49328500 & -6.17592800 & $\mathrm{O}$ & 5.79799600 & -3.82727900 & 1.41285300 \\
\hline $\mathrm{H}$ & -3.37178000 & -8.13468100 & -5.80551900 & $\mathrm{O}$ & 4.39283200 & -2.32255600 & 2.84416000 \\
\hline $\mathrm{C}$ & -0.89515000 & -7.55078700 & -4.82872100 & $\mathrm{C}$ & 6.74595900 & -1.40365400 & 2.00278100 \\
\hline $\mathrm{H}$ & -1.27337400 & -8.52268800 & -4.48727400 & $\mathrm{C}$ & 6.75753700 & -0.51841700 & 3.10527000 \\
\hline $\mathrm{H}$ & -0.17278200 & -7.18599300 & -4.09267700 & $\mathrm{C}$ & 7.90942800 & -1.63478300 & 1.23806800 \\
\hline $\mathrm{H}$ & -0.35549900 & -7.73254500 & -5.76517900 & $\mathrm{C}$ & 7.99034600 & 0.06294500 & 3.48219600 \\
\hline $\mathrm{C}$ & -5.06682800 & 0.40148200 & -0.08352000 & $\mathrm{C}$ & 9.12017900 & -1.02938600 & 1.62303000 \\
\hline $\mathrm{H}$ & -5.14052600 & -0.67675100 & 0.06778300 & $\mathrm{C}$ & 9.15628000 & -0.16152400 & 2.72933500 \\
\hline $\mathrm{C}$ & -2.42449600 & 4.42319600 & 1.42633100 & $\mathrm{C}$ & 5.53406800 & -0.09908800 & 3.90073400 \\
\hline $\mathrm{H}$ & -1.57227900 & 4.63533600 & 2.08669900 & $\mathrm{H}$ & 5.41325300 & -0.70437800 & 4.80771400 \\
\hline $\mathrm{C}$ & -1.04923300 & -0.09776800 & 3.16269500 & $\mathrm{H}$ & 4.61244100 & -0.20004600 & 3.33876900 \\
\hline $\mathrm{H}$ & -1.31493100 & -1.15181600 & 3.07306900 & $\mathrm{H}$ & 5.63279900 & 0.94627600 & 4.20496300 \\
\hline $\mathrm{C}$ & -2.06667500 & 4.95993100 & 0.02801600 & $\mathrm{C}$ & 8.05826300 & 0.92391400 & 4.73193900 \\
\hline $\mathrm{H}$ & -1.89931800 & 6.04354800 & 0.06068500 & $\mathrm{H}$ & 9.06096300 & 0.92384700 & 5.16439900 \\
\hline $\mathrm{H}$ & -2.87301000 & 4.76916100 & -0.69033300 & $\mathrm{H}$ & 7.38040200 & 0.55908700 & 5.50750000 \\
\hline $\mathrm{H}$ & -1.15631400 & 4.48568900 & -0.35321100 & $\mathrm{H}$ & 7.78778400 & 1.97144700 & 4.53469700 \\
\hline $\mathrm{C}$ & -3.64784400 & 5.16258500 & 2.00260500 & $\mathrm{C}$ & 10.44376100 & 0.55726000 & 3.09015100 \\
\hline $\mathrm{H}$ & -3.45802700 & 6.24158100 & 2.05773900 & $\mathrm{H}$ & 11.03294900 & 0.00450500 & 3.83611600 \\
\hline $\mathrm{H}$ & -3.88769700 & 4.80556200 & 3.01025700 & $\mathrm{H}$ & 10.24700800 & 1.54869100 & 3.50538700 \\
\hline $\mathrm{H}$ & -4.53471100 & 5.01218300 & 1.37545300 & $\mathrm{H}$ & 11.08312700 & 0.70228200 & 2.21673700 \\
\hline $\mathrm{C}$ & -6.39604900 & 1.00648300 & 0.41188100 & $\mathrm{C}$ & 10.38975100 & -1.33912000 & 0.84995700 \\
\hline $\mathrm{H}$ & -6.42843200 & 2.09277400 & 0.26729500 & $\mathrm{H}$ & 11.27516000 & -1.27223900 & 1.48625700 \\
\hline $\mathrm{H}$ & -6.54825400 & 0.80742400 & 1.47880200 & $\mathrm{H}$ & 10.54300500 & -0.65093400 & 0.00560000 \\
\hline $\mathrm{H}$ & -7.24067400 & 0.57416200 & -0.13899500 & $\mathrm{H}$ & 10.37344800 & -2.35128600 & 0.43901300 \\
\hline $\mathrm{C}$ & -4.87672200 & 0.63155400 & -1.59485000 & $\mathrm{C}$ & 7.90405800 & -2.47988000 & -0.01631500 \\
\hline $\mathrm{H}$ & -3.95075400 & 0.16408800 & -1.94203700 & $\mathrm{H}$ & 8.50822800 & -2.00148200 & -0.79537300 \\
\hline $\mathrm{H}$ & -4.83611400 & 1.70031000 & -1.83854000 & $\mathrm{H}$ & 6.89825600 & -2.61435200 & -0.40152000 \\
\hline $\mathrm{H}$ & -5.71293800 & 0.19582300 & -2.15564200 & $\mathrm{H}$ & 8.31968100 & -3.47824000 & 0.16399600 \\
\hline $\mathrm{C}$ & -1.17029600 & 0.28585200 & 4.65087200 & $\mathrm{H}$ & 1.53814600 & -1.94644900 & 0.28397600 \\
\hline $\mathrm{H}$ & -0.89082500 & 1.33265500 & 4.81923600 & $\mathrm{H}$ & 2.55257300 & -4.22522500 & 0.60914400 \\
\hline $\mathrm{H}$ & -0.50610500 & -0.33592000 & 5.26434700 & $\mathrm{C}$ & 6.25877000 & -5.22478500 & -1.56480400 \\
\hline
\end{tabular}




\begin{tabular}{|c|c|c|c|c|c|c|c|}
\hline $\mathrm{H}$ & 7.04533000 & -4.52912900 & -1.86513400 & $\mathrm{H}$ & 0.79156100 & -10.13909900 & 1.09667700 \\
\hline $\mathrm{H}$ & 6.47458400 & -6.19071700 & -2.03981500 & $\mathrm{H}$ & 0.06678000 & -4.77984800 & 0.97526600 \\
\hline $\mathrm{H}$ & 6.32198100 & -5.34236500 & -0.48029000 & $\mathrm{C}$ & 3.10409000 & -5.98345400 & 1.92592900 \\
\hline $\mathrm{C}$ & 5.61818400 & -2.49354700 & -3.18569900 & $\mathrm{H}$ & 2.94704000 & -6.58326500 & 2.82705700 \\
\hline $\mathrm{H}$ & 5.15638100 & -1.52155200 & -3.37854100 & $\mathrm{H}$ & 3.24828400 & -6.65165600 & 1.08024500 \\
\hline $\mathrm{H}$ & 6.08381500 & -2.82688200 & -4.12398800 & $\mathrm{H}$ & 4.01062700 & -5.39043500 & 2.06474000 \\
\hline $\mathrm{H}$ & 6.41373400 & -2.35089500 & -2.45284000 & $\mathrm{H}$ & -0.99811000 & -10.17696700 & -0.635371( \\
\hline $\mathrm{C}$ & 2.49205200 & -2.51590300 & -3.91770700 & $\mathrm{C}$ & 1.77938900 & -3.99366500 & 2.84013900 \\
\hline $\mathrm{H}$ & 2.48515800 & -2.83568900 & -4.96636200 & $\mathrm{C}$ & 1.80188800 & -4.71345000 & 4.19606200 \\
\hline $\mathrm{H}$ & 2.96515800 & -1.53057500 & -3.87765700 & $\mathrm{C}$ & 0.53584700 & -3.09988600 & 2.76387300 \\
\hline $\mathrm{H}$ & 1.44901000 & -2.41521700 & -3.60665900 & $\mathrm{C}$ & 2.85650400 & -4.47203500 & 5.08776500 \\
\hline $\mathrm{C}$ & 1.25664300 & -5.23717500 & -2.89203500 & $\mathrm{C}$ & 0.77598500 & -5.58726000 & 4.58795100 \\
\hline $\mathrm{H}$ & 0.93050300 & -6.00517600 & -2.18870200 & $\mathrm{C}$ & 2.88067900 & -5.08351600 & 6.34357000 \\
\hline $\mathrm{H}$ & 1.24016300 & -5.66925600 & -3.90122100 & $\mathrm{H}$ & 3.65715000 & -3.80073300 & 4.78926400 \\
\hline $\mathrm{H}$ & 0.51975400 & -4.43114100 & -2.87300900 & $\mathrm{C}$ & 0.80375800 & -6.20318200 & 5.84004800 \\
\hline $\mathrm{C}$ & 3.61204600 & -6.91018300 & -1.46484300 & $\mathrm{C}$ & 1.85620800 & -5.95194900 & 6.72373600 \\
\hline $\mathrm{H}$ & 3.80118400 & -7.57014300 & -2.32396800 & $\mathrm{H}$ & 3.70407200 & -4.88098900 & 7.02398900 \\
\hline $\mathrm{H}$ & 2.62731100 & -7.16572100 & -1.06964900 & $\mathrm{H}$ & 0.00049000 & -6.87753700 & 6.12647200 \\
\hline $\mathrm{H}$ & 4.36789900 & -7.13422300 & -0.70840700 & $\mathrm{H}$ & 1.87621600 & -6.42954100 & 7.69997600 \\
\hline $\mathrm{H}$ & 3.24013400 & -0.59902800 & 1.30834500 & $\mathrm{H}$ & 2.66535600 & -3.36243700 & 2.80482600 \\
\hline $\mathrm{C}$ & 4.72393000 & 0.54080500 & 0.26845300 & $\mathrm{C}$ & -6.38721500 & -3.59421500 & -0.69003700 \\
\hline $\mathrm{C}$ & 5.77737900 & 0.58605300 & -0.65382300 & $\mathrm{C}$ & -5.75168900 & -4.00978300 & -2.92955600 \\
\hline $\mathrm{C}$ & 4.47211900 & 1.67345800 & 1.05158300 & $\mathrm{C}$ & -6.97463400 & -4.01501900 & -2.04548400 \\
\hline $\mathrm{C}$ & 6.55893600 & 1.73356900 & -0.78915900 & $\mathrm{H}$ & -6.34991300 & -2.50065900 & -0.63479800 \\
\hline $\mathrm{H}$ & 5.99184700 & -0.29276800 & -1.25234000 & $\mathrm{H}$ & -6.96565100 & -3.94593000 & 0.16882000 \\
\hline $\mathrm{C}$ & 5.25360400 & 2.82315000 & 0.92169600 & $\mathrm{H}$ & -7.41967800 & -5.01967900 & -1.99660100 \\
\hline $\mathrm{H}$ & 3.65152400 & 1.65924500 & 1.76434100 & $\mathrm{H}$ & -7.76075300 & -3.33816800 & -2.39995400 \\
\hline $\mathrm{C}$ & 6.29976500 & 2.85773800 & -0.00105800 & $\mathrm{C}$ & -4.91131100 & -4.14784300 & -0.68059400 \\
\hline $\mathrm{H}$ & 7.37485600 & 1.74898300 & -1.50758400 & $\mathrm{C}$ & -4.91379000 & -5.59159100 & -0.09287100 \\
\hline $\mathrm{H}$ & 5.04138900 & 3.69145300 & 1.54003100 & $\mathrm{C}$ & -4.17320300 & -3.40276600 & 0.42997100 \\
\hline $\mathrm{H}$ & 6.91029100 & 3.75099900 & -0.10396200 & $\mathrm{C}$ & -5.08759900 & -5.37516000 & 1.43534700 \\
\hline $\mathrm{H}$ & 3.32683700 & -0.76930200 & -1.71555700 & $\mathrm{H}$ & -5.69836600 & -6.22027500 & -0.52763800 \\
\hline $\mathrm{C}$ & 1.90910100 & 0.59835700 & -0.93633500 & $\mathrm{H}$ & -3.94773900 & -6.06564200 & -0.29902900 \\
\hline $\mathrm{C}$ & 0.61718500 & 0.70276100 & -0.41025900 & $\mathrm{H}$ & -4.55282000 & -6.13118800 & 2.02291900 \\
\hline $\mathrm{C}$ & 2.41378600 & 1.66941100 & -1.69125500 & $\mathrm{H}$ & -6.14257300 & -5.43795500 & 1.73514300 \\
\hline $\mathrm{C}$ & -0.15028900 & 1.85042600 & -0.62354600 & $\mathrm{C}$ & -4.57429500 & -4.04235000 & -2.16471200 \\
\hline $\mathrm{H}$ & 0.17073300 & -0.12251700 & 0.13368100 & $\mathrm{C}$ & -3.32862800 & -4.00992200 & -2.80432800 \\
\hline $\mathrm{C}$ & 1.65469000 & 2.81874400 & -1.89901700 & $\mathrm{C}$ & -5.70648200 & -3.97490200 & -4.31701000 \\
\hline $\mathrm{H}$ & 3.41136000 & 1.60224800 & -2.11651700 & $\mathrm{C}$ & -3.25706000 & -4.00602700 & -4.21608100 \\
\hline $\mathrm{C}$ & 0.36699100 & 2.91125600 & -1.36610800 & $\mathrm{C}$ & -4.46241600 & -3.98433300 & -4.94032700 \\
\hline $\mathrm{H}$ & -1.15671000 & 1.89917500 & -0.22256000 & $\mathrm{H}$ & -6.61981200 & -3.94446600 & -4.90585300 \\
\hline $\mathrm{H}$ & 2.06460800 & 3.63516200 & -2.48794300 & $\mathrm{H}$ & -4.40411700 & -3.97711000 & -6.02506000 \\
\hline $\mathrm{H}$ & -0.23594700 & 3.79778900 & -1.54330400 & $\mathrm{C}$ & -4.52317800 & -3.98297400 & 1.65439800 \\
\hline $\mathrm{N}$ & 0.74095900 & -5.51662900 & 1.22812300 & $\mathrm{C}$ & -4.38170300 & -3.25102600 & 2.82929800 \\
\hline $\mathrm{C}$ & 1.93470900 & -5.03978700 & 1.70883800 & $\mathrm{C}$ & -3.46027200 & -2.20116200 & 0.39933300 \\
\hline $\mathrm{C}$ & 0.37223800 & -6.78039500 & 0.71655300 & $\mathrm{C}$ & -3.94526100 & -1.92819500 & 2.73706900 \\
\hline $\mathrm{C}$ & -0.65960800 & -6.80654300 & -0.24226900 & $\mathrm{H}$ & -4.66995600 & -3.66546600 & 3.79233400 \\
\hline $\mathrm{C}$ & 0.87596800 & -7.99966400 & 1.20553000 & $\mathrm{C}$ & -3.45981300 & -1.36123400 & 1.54041500 \\
\hline $\mathrm{C}$ & -1.14633400 & -8.02179900 & -0.71820200 & $\mathrm{H}$ & -3.97842300 & -1.30232500 & 3.62123800 \\
\hline $\mathrm{H}$ & -1.07250600 & -5.86831600 & -0.59898200 & $\mathrm{O}$ & -2.18544200 & -4.08222600 & -2.0319940 \\
\hline $\mathrm{C}$ & 0.38884200 & -9.20812400 & 0.70628700 & $\mathrm{O}$ & -2.77312000 & -1.85312400 & -0.7603320 \\
\hline $\mathrm{H}$ & 1.61471900 & -8.01475300 & 1.99463100 & $\mathrm{P}$ & -1.47601600 & -2.83189800 & -1.18105300 \\
\hline $\mathrm{C}$ & -0.61902000 & -9.23129200 & -0.25869400 & $\mathrm{O}$ & -0.89006200 & -3.47490000 & 0.04668500 \\
\hline $\mathrm{H}$ & -1.94268400 & -8.01781600 & -1.45690700 & $\mathrm{O}$ & -0.59140700 & -2.03192700 & -2.0988540 \\
\hline
\end{tabular}




\begin{tabular}{|c|c|c|c|c|c|c|c|}
\hline $\mathrm{C}$ & -2.00171200 & -4.09810800 & -5.04230000 & $\mathrm{H}$ & -3.01026100 & 5.01606500 & -0.21704300 \\
\hline $\mathrm{C}$ & -1.54001900 & -5.37530900 & -5.44216100 & $\mathrm{H}$ & -1.37253900 & 4.80590200 & 0.42756000 \\
\hline $\mathrm{C}$ & -1.41644100 & -2.93282000 & -5.59558700 & $\mathrm{C}$ & -4.32741000 & 5.06082900 & 2.27923900 \\
\hline $\mathrm{C}$ & -0.51278700 & -5.45904000 & -6.39123200 & $\mathrm{H}$ & -4.25732000 & 6.14151900 & 2.45344900 \\
\hline $\mathrm{C}$ & -0.38899900 & -3.07485800 & -6.53744600 & $\mathrm{H}$ & -4.72260400 & 4.59281400 & 3.18754900 \\
\hline $\mathrm{C}$ & 0.06820900 & -4.32616500 & -6.96391400 & $\mathrm{H}$ & -5.05684600 & 4.89911900 & 1.47667000 \\
\hline $\mathrm{H}$ & -0.17895000 & -6.43891200 & -6.72558000 & $\mathrm{C}$ & -6.33088400 & 0.92674500 & -0.11382900 \\
\hline $\mathrm{H}$ & 0.03865200 & -2.17830500 & -6.97898900 & $\mathrm{H}$ & -6.42969800 & 2.01729500 & -0.16756800 \\
\hline $\mathrm{C}$ & -3.16982600 & 0.11782000 & 1.52176900 & $\mathrm{H}$ & -6.64633500 & 0.61259000 & 0.88767900 \\
\hline $\mathrm{C}$ & -3.90585100 & 0.96546900 & 0.64005400 & $\mathrm{H}$ & -7.03024700 & 0.49644200 & -0.84159900 \\
\hline $\mathrm{C}$ & -2.33160500 & 0.72791100 & 2.49697200 & $\mathrm{C}$ & -4.46133700 & 0.86110600 & -1.84073700 \\
\hline $\mathrm{C}$ & -3.79474200 & 2.35516900 & 0.76638500 & $\mathrm{H}$ & -3.45461600 & 0.49312000 & -2.05847600 \\
\hline $\mathrm{C}$ & -2.28116000 & 2.12575100 & 2.58451500 & $\mathrm{H}$ & -4.46860100 & 1.94982900 & -1.97523100 \\
\hline $\mathrm{C}$ & -3.00588600 & 2.96548400 & 1.74291100 & $\mathrm{H}$ & -5.14946100 & 0.43191800 & -2.57929200 \\
\hline $\mathrm{H}$ & -4.37441600 & 2.98053200 & 0.09227700 & $\mathrm{C}$ & -1.96185400 & 0.11328100 & 4.94535900 \\
\hline $\mathrm{H}$ & -1.65223200 & 2.57902900 & 3.34677800 & $\mathrm{H}$ & -1.88035900 & 1.15565900 & 5.27583600 \\
\hline $\mathrm{C}$ & -1.95495800 & -1.53490000 & -5.28244900 & $\mathrm{H}$ & -1.35938700 & -0.50015200 & 5.62633700 \\
\hline $\mathrm{H}$ & -2.57223300 & -1.61064800 & -4.38234000 & $\mathrm{H}$ & -3.00892300 & -0.18377400 & 5.06243200 \\
\hline $\mathrm{C}$ & 1.11279800 & -4.46972200 & -8.06573700 & $\mathrm{C}$ & 0.01620100 & 0.38552400 & 3.42178800 \\
\hline $\mathrm{H}$ & 1.27028200 & -5.54635900 & -8.21943700 & $\mathrm{H}$ & 0.42378000 & 0.27157000 & 2.41317300 \\
\hline $\mathrm{C}$ & -2.20556200 & -6.66506700 & -4.95553600 & $\mathrm{H}$ & 0.62338900 & -0.21555000 & 4.10820600 \\
\hline $\mathrm{H}$ & -2.80193400 & -6.41990300 & -4.07102600 & $\mathrm{H}$ & 0.14408900 & 1.43452900 & 3.71152600 \\
\hline $\mathrm{C}$ & -0.85757400 & -0.49984200 & -4.97983800 & $\mathrm{H}$ & 0.44894300 & -2.56244900 & 1.81759500 \\
\hline $\mathrm{H}$ & -1.31889700 & 0.46909600 & -4.75361400 & $\mathrm{H}$ & 0.59156600 & -2.37218400 & 3.57632800 \\
\hline $\mathrm{H}$ & -0.18491500 & -0.34575100 & -5.83295900 & $\mathrm{H}$ & -0.04924600 & -5.78988100 & 3.91067300 \\
\hline $\mathrm{H}$ & -0.27766400 & -0.80162000 & -4.10589400 & $\mathrm{H}$ & -0.39664300 & -3.65932600 & 2.88211700 \\
\hline $\mathrm{C}$ & -2.85701100 & -1.03758700 & -6.43224200 & & & & \\
\hline $\mathrm{H}$ & -3.27738200 & -0.05378900 & -6.19031300 & $17-S R$ & & & \\
\hline $\mathrm{H}$ & -3.68956400 & -1.72333900 & -6.62034100 & $\mathrm{C}$ & 4.69832000 & -6.17435500 & -2.77735100 \\
\hline $\mathrm{H}$ & -2.28643200 & -0.93956900 & -7.36433600 & $\mathrm{C}$ & 6.94756000 & -6.31150300 & -3.49144700 \\
\hline $\mathrm{C}$ & 2.47444700 & -3.86090400 & -7.68421500 & $\mathrm{C}$ & 5.61957800 & -7.00098300 & -3.68674200 \\
\hline $\mathrm{H}$ & 3.20590800 & -4.01129800 & -8.48777500 & $\mathrm{H}$ & 4.33686500 & -5.29835000 & -3.32741800 \\
\hline $\mathrm{H}$ & 2.87361600 & -4.32404300 & -6.77434000 & $\mathrm{H}$ & 3.82194000 & -6.72319600 & -2.42067300 \\
\hline $\mathrm{H}$ & 2.39361300 & -2.78128300 & -7.50845200 & $\mathrm{H}$ & 5.66864800 & -8.05075400 & -3.36222800 \\
\hline $\mathrm{C}$ & 0.60958300 & -3.88569400 & -9.40032600 & $\mathrm{H}$ & 5.29264600 & -7.00870700 & -4.73300200 \\
\hline $\mathrm{H}$ & 1.33961700 & -4.05981000 & -10.20038700 & $\mathrm{C}$ & 5.61519700 & -5.67972800 & -1.59502200 \\
\hline $\mathrm{H}$ & 0.44824400 & -2.80379900 & -9.32522700 & $\mathrm{C}$ & 5.60595200 & -6.75015600 & -0.46131300 \\
\hline $\mathrm{H}$ & -0.33992700 & -4.34386900 & -9.69747800 & $\mathrm{C}$ & 4.86970900 & -4.54137300 & -0.90468800 \\
\hline $\mathrm{C}$ & -3.17267200 & -7.21503600 & -6.02495500 & $\mathrm{C}$ & 4.23368400 & -6.54926600 & 0.23731900 \\
\hline $\mathrm{H}$ & -2.63361400 & -7.47063200 & -6.94550000 & $\mathrm{H}$ & 5.74514300 & -7.76688300 & -0.84388000 \\
\hline $\mathrm{H}$ & -3.94406100 & -6.48139400 & -6.27864200 & $\mathrm{H}$ & 6.42229600 & -6.54019100 & 0.23870400 \\
\hline $\mathrm{H}$ & -3.67286300 & -8.12233700 & -5.66381300 & $\mathrm{H}$ & 4.28254500 & -6.75923200 & 1.31298300 \\
\hline $\mathrm{C}$ & -1.20426200 & -7.75735500 & -4.53883100 & $\mathrm{H}$ & 3.46860400 & -7.22100600 & -0.17524600 \\
\hline $\mathrm{H}$ & -1.74392000 & -8.62870000 & -4.14780200 & $\mathrm{C}$ & 6.94325500 & -5.52134500 & -2.33108500 \\
\hline $\mathrm{H}$ & -0.52369100 & -7.40516800 & -3.75836500 & $\mathrm{C}$ & 8.07457800 & -4.76408300 & -1.99720100 \\
\hline $\mathrm{H}$ & -0.59974800 & -8.10806500 & -5.38352100 & $\mathrm{C}$ & 8.06990800 & -6.38212700 & -4.30557300 \\
\hline $\mathrm{C}$ & -4.89167300 & 0.46103400 & -0.41677700 & $\mathrm{C}$ & 9.23966800 & -4.84119800 & -2.79398900 \\
\hline $\mathrm{H}$ & -4.90942300 & -0.62744200 & -0.38792600 & $\mathrm{C}$ & 9.19946500 & -5.65711500 & -3.94033700 \\
\hline $\mathrm{C}$ & -2.94997800 & 4.47870400 & 1.90654600 & $\mathrm{H}$ & 8.07041900 & -6.98877700 & -5.20769000 \\
\hline $\mathrm{H}$ & -2.26855000 & 4.68617500 & 2.74323500 & $\mathrm{H}$ & 10.09385500 & -5.71195300 & -4.5541020 \\
\hline $\mathrm{C}$ & -1.46308800 & -0.04764400 & 3.49260800 & $\mathrm{C}$ & 3.91054700 & -5.09323100 & -0.04705600 \\
\hline $\mathrm{H}$ & -1.51094800 & -1.10917800 & 3.23480000 & $\mathrm{C}$ & 2.82509600 & -4.32353300 & 0.36266200 \\
\hline $\mathrm{C}$ & -2.37495300 & 5.18003200 & 0.66180400 & $\mathrm{C}$ & 4.87252200 & -3.16837500 & -1.16406900 \\
\hline $\mathrm{H}$ & -2.30607800 & 6.26260700 & 0.82453100 & $\mathrm{C}$ & 2.70255700 & -3.02506800 & -0.13691400 \\
\hline
\end{tabular}




\begin{tabular}{|c|c|c|c|c|c|c|c|}
\hline$H$ & 2.04954900 & -4.73770700 & 1.00290400 & $\mathrm{C}$ & 3.80864400 & -1.97239600 & -3.88745300 \\
\hline $\mathrm{C}$ & 3.71566300 & -2.39931400 & -0.89216100 & $\mathrm{H}$ & 4.07977300 & -2.86925100 & -3.33009600 \\
\hline $\mathrm{H}$ & 1.79601700 & -2.46471100 & 0.06631700 & $\mathrm{C}$ & 2.09620100 & 2.72320000 & -3.26585500 \\
\hline $\mathrm{O}$ & 8.04046000 & -4.01457900 & -0.83193100 & $\mathrm{H}$ & 1.81248100 & 3.38285700 & -2.43402000 \\
\hline $\mathrm{O}$ & 6.01369200 & -2.58499500 & -1.69804500 & $\mathrm{C}$ & 2.98886000 & 0.00644200 & 0.86481900 \\
\hline $\mathrm{P}$ & 7.35542100 & -2.49906100 & -0.69643300 & $\mathrm{H}$ & 3.40585600 & -0.94950300 & 1.18918100 \\
\hline $\mathrm{O}$ & 6.88970000 & -2.42446300 & 0.74552300 & $\mathrm{C}$ & 3.18732900 & 3.44875700 & -4.07457800 \\
\hline $\mathrm{O}$ & 8.26009900 & -1.44335700 & -1.24089300 & $\mathrm{H}$ & 2.80612200 & 4.39624700 & -4.47475300 \\
\hline $\mathrm{C}$ & 10.57683100 & -4.19822300 & -2.52602200 & $\mathrm{H}$ & 3.52518500 & 2.84152100 & -4.92306700 \\
\hline $\mathrm{C}$ & 11.55552900 & -4.93526200 & -1.81699900 & $\mathrm{H}$ & 4.05875800 & 3.66809200 & -3.44908700 \\
\hline $\mathrm{C}$ & 10.94324400 & -2.99889700 & -3.18545800 & $\mathrm{C}$ & 0.83488800 & 2.49539200 & -4.12138300 \\
\hline $\mathrm{C}$ & 12.87952600 & -4.47800800 & -1.81089000 & $\mathrm{H}$ & 0.44656700 & 3.44880000 & -4.49992100 \\
\hline $\mathrm{C}$ & 12.27987200 & -2.58274800 & -3.13762200 & $\mathrm{H}$ & 0.04359700 & 2.01095300 & -3.53870300 \\
\hline $\mathrm{C}$ & 13.27041400 & -3.31638000 & -2.47809000 & $\mathrm{H}$ & 1.05026800 & 1.85689500 & -4.98644600 \\
\hline $\mathrm{H}$ & 13.63995700 & -5.06097100 & -1.29617700 & $\mathrm{C}$ & 2.59698900 & -2.35314700 & -4.76293400 \\
\hline $\mathrm{H}$ & 12.55966300 & -1.67448600 & -3.66454900 & $\mathrm{H}$ & 2.27065700 & -1.51821100 & -5.39384700 \\
\hline $\mathrm{C}$ & 3.44849200 & -1.03796700 & -1.47648500 & $\mathrm{H}$ & 1.74342700 & -2.65984000 & -4.14759300 \\
\hline $\mathrm{C}$ & 3.45115300 & -0.86612200 & -2.89158900 & $\mathrm{H}$ & 2.85560100 & -3.18747600 & -5.42682500 \\
\hline $\mathrm{C}$ & 3.02670900 & 0.04899200 & -0.66423500 & $\mathrm{C}$ & 5.02689700 & -1.59692400 & -4.75265300 \\
\hline $\mathrm{C}$ & 3.04014400 & 0.35597800 & -3.43606400 & $\mathrm{H}$ & 5.89157800 & -1.36469900 & -4.12475600 \\
\hline $\mathrm{C}$ & 2.61187400 & 1.24194100 & -1.27032700 & $\mathrm{H}$ & 4.81859000 & -0.72900300 & -5.39028800 \\
\hline $\mathrm{C}$ & 2.59939400 & 1.42352800 & -2.65102700 & $\mathrm{H}$ & 5.29627700 & -2.43222400 & -5.41067800 \\
\hline $\mathrm{H}$ & 3.03794400 & 0.46266300 & -4.51786600 & $\mathrm{C}$ & 1.54766900 & 0.09438100 & 1.40975500 \\
\hline $\mathrm{H}$ & 2.27895500 & 2.06190800 & -0.63847800 & $\mathrm{H}$ & 1.08333100 & 1.05238400 & 1.14819300 \\
\hline $\mathrm{C}$ & 9.94806900 & -2.19998800 & -4.02854700 & $\mathrm{H}$ & 1.54426900 & 0.01194800 & 2.50384900 \\
\hline $\mathrm{H}$ & 8.94097300 & -2.51363700 & -3.74048400 & $\mathrm{H}$ & 0.90788100 & -0.69905900 & 1.00859300 \\
\hline $\mathrm{C}$ & 14.73771700 & -2.90555700 & -2.52062000 & $\mathrm{C}$ & 3.84954600 & 1.12233700 & 1.49044600 \\
\hline $\mathrm{H}$ & 15.29653900 & -3.64823600 & -1.93417600 & $\mathrm{H}$ & 4.87904600 & 1.08683400 & 1.12183000 \\
\hline $\mathrm{C}$ & 11.23251700 & -6.26775700 & -1.13773400 & $\mathrm{H}$ & 3.87050600 & 1.02502100 & 2.58215100 \\
\hline $\mathrm{H}$ & 10.14544200 & -6.32811100 & -1.01999600 & $\mathrm{H}$ & 3.45194900 & 2.11688300 & 1.25825800 \\
\hline $\mathrm{C}$ & 10.02370300 & -0.68291100 & -3.78265100 & $\mathrm{C}$ & 11.97745400 & -0.30561700 & 4.10279200 \\
\hline $\mathrm{H}$ & 9.23939200 & -0.17701200 & -4.35795700 & $\mathrm{C}$ & 12.38438100 & 0.63153400 & 3.05358900 \\
\hline $\mathrm{H}$ & 10.98409500 & -0.25827600 & -4.10064400 & $\mathrm{C}$ & 12.22675400 & 0.00069000 & 1.80299000 \\
\hline $\mathrm{H}$ & 9.85553500 & -0.45773600 & -2.72813300 & $\mathrm{C}$ & 11.71492900 & -1.35478500 & 2.03402700 \\
\hline C & 10.13244100 & -2.50881300 & -5.52952400 & $\mathrm{C}$ & 11.64848200 & -1.55321400 & 3.46224600 \\
\hline $\mathrm{H}$ & 9.40136200 & -1.94991400 & -6.12652200 & Ir & 10.13411800 & -0.08078600 & 2.86787300 \\
\hline $\mathrm{H}$ & 9.99900700 & -3.57405700 & -5.74512400 & $\mathrm{~N}$ & 9.04762000 & 1.71579500 & 3.52127600 \\
\hline $\mathrm{H}$ & 11.13504400 & -2.22081800 & -5.87026200 & $\mathrm{~N}$ & 8.81078700 & 0.33379200 & 1.23580900 \\
\hline $\mathrm{C}$ & 14.98699800 & -1.53125400 & -1.87415500 & $\mathrm{H}$ & 9.03439000 & -0.14638200 & 0.35513200 \\
\hline $\mathrm{H}$ & 16.05478800 & -1.28093000 & -1.89389200 & $\mathrm{C}$ & 8.28068100 & 2.38445800 & 2.43229200 \\
\hline $\mathrm{H}$ & 14.65473600 & -1.52283900 & -0.83043600 & $\mathrm{C}$ & 8.80486600 & 1.81341500 & 1.07914900 \\
\hline $\mathrm{H}$ & 14.44802100 & -0.73707200 & -2.40417700 & $S$ & 8.19648000 & 1.51998700 & 4.93055300 \\
\hline $\mathrm{C}$ & 15.29366000 & -2.94451000 & -3.95768200 & $\mathrm{O}$ & 9.07332600 & 0.87299700 & 5.92186800 \\
\hline $\mathrm{H}$ & 16.36585800 & -2.71284800 & -3.96541100 & $\mathrm{O}$ & 6.88672200 & 0.84006900 & 4.71243300 \\
\hline $\mathrm{H}$ & 14.78909900 & -2.21169100 & -4.59845600 & $\mathrm{C}$ & 7.81562200 & 3.18635100 & 5.62815600 \\
\hline $\mathrm{H}$ & 15.15420500 & -3.93317500 & -4.40863100 & $\mathrm{C}$ & 6.49236700 & 3.67578300 & 5.69065900 \\
\hline $\mathrm{C}$ & 11.67004100 & -7.45917500 & -2.01549800 & $\mathrm{C}$ & 8.88201700 & 3.87513000 & 6.24763900 \\
\hline $\mathrm{H}$ & 12.75440600 & -7.44441600 & -2.18026900 & $\mathrm{C}$ & 6.23817400 & 4.82907600 & 6.46745100 \\
\hline $\mathrm{H}$ & 11.18001800 & -7.43477600 & -2.99346200 & $\mathrm{C}$ & 8.60561900 & 5.03310100 & 6.99942600 \\
\hline $\mathrm{H}$ & 11.41512600 & -8.41124800 & -1.53277700 & $\mathrm{C}$ & 7.29011900 & 5.52398500 & 7.08952800 \\
\hline $\mathrm{C}$ & 11.85242300 & -6.39678900 & 0.26582800 & $\mathrm{C}$ & 5.31688700 & 3.08079300 & 4.93540900 \\
\hline $\mathrm{H}$ & 11.52212500 & -7.33083300 & 0.73784000 & $\mathrm{H}$ & 4.74752100 & 2.37514700 & 5.55365800 \\
\hline $\mathrm{H}$ & 11.56624400 & -5.56092000 & 0.91277600 & $\mathrm{H}$ & 5.62518400 & 2.53288400 & 4.05079400 \\
\hline $\mathrm{H}$ & 12.94734200 & -6.42950700 & 0.23144400 & $\mathrm{H}$ & 4.63474500 & 3.87612400 & 4.62266100 \\
\hline
\end{tabular}




\begin{tabular}{|c|c|c|c|c|c|c|c|}
\hline C & 4.80783300 & 5.30876400 & 6.64786300 & $\mathrm{C}$ & 6.45908300 & 2.08484300 & -1.95303100 \\
\hline $\mathrm{H}$ & 4.68543700 & 5.86001600 & 7.58274600 & $\mathrm{H}$ & 6.69618500 & 0.67786200 & -0.35877500 \\
\hline $\mathrm{H}$ & 4.10502900 & 4.47243000 & 6.68123900 & $\mathrm{C}$ & 7.97560400 & 3.96226400 & -1.95912500 \\
\hline $\mathrm{H}$ & 4.48048400 & 5.97202900 & 5.83372900 & $\mathrm{H}$ & 9.38507000 & 4.03783300 & -0.33853700 \\
\hline $\mathrm{C}$ & 7.01176200 & 6.82171100 & 7.82692400 & $\mathrm{C}$ & 6.92318600 & 3.25771500 & -2.54841700 \\
\hline $\mathrm{H}$ & 6.76604000 & 6.65514400 & 8.88587300 & $\mathrm{H}$ & 5.65423400 & 1.51675600 & -2.40908800 \\
\hline $\mathrm{H}$ & 6.17351100 & 7.36411700 & 7.38302600 & $\mathrm{H}$ & 8.35067000 & 4.87303800 & -2.41891000 \\
\hline $\mathrm{H}$ & 7.87232600 & 7.49382500 & 7.79755300 & $\mathrm{H}$ & 6.47998800 & 3.61232800 & -3.47518800 \\
\hline $\mathrm{C}$ & 9.73500500 & 5.73617200 & 7.73124400 & $\mathrm{~N}$ & 6.61086000 & -3.46588000 & 3.16376600 \\
\hline $\mathrm{H}$ & 9.37721700 & 6.25149700 & 8.62566500 & $\mathrm{C}$ & 6.12783500 & -2.54748800 & 3.95436500 \\
\hline $\mathrm{H}$ & 10.23581600 & 6.48584400 & 7.10116400 & $\mathrm{C}$ & 7.23974800 & -4.72282100 & 3.42068400 \\
\hline $\mathrm{H}$ & 10.50157800 & 5.02986500 & 8.05861500 & $\mathrm{C}$ & 8.23499300 & -5.12244000 & 2.51938600 \\
\hline $\mathrm{C}$ & 10.32886600 & 3.44835100 & 6.10367400 & $\mathrm{C}$ & 6.80394500 & -5.58740200 & 4.43062000 \\
\hline $\mathrm{H}$ & 10.96600200 & 4.32804300 & 5.96384500 & $\mathrm{C}$ & 8.83345500 & -6.37234900 & 2.67497300 \\
\hline $\mathrm{H}$ & 10.46997600 & 2.79168200 & 5.24953800 & $\mathrm{H}$ & 8.51148900 & -4.46663400 & 1.69969500 \\
\hline $\mathrm{H}$ & 10.68272700 & 2.91345400 & 6.99340100 & $\mathrm{C}$ & 7.41110800 & -6.83471300 & 4.57295900 \\
\hline $\mathrm{H}$ & 7.88111100 & -0.01784000 & 1.46966600 & $\mathrm{H}$ & 5.98965400 & -5.30248200 & 5.08628700 \\
\hline $\mathrm{H}$ & 8.91632100 & -0.93658300 & 3.44097800 & $\mathrm{C}$ & 8.43134000 & -7.22673200 & 3.70446500 \\
\hline $\mathrm{C}$ & 11.38553300 & -2.85388000 & 4.16522600 & $\mathrm{H}$ & 9.61039000 & -6.67855200 & 1.98287800 \\
\hline $\mathrm{H}$ & 10.92887300 & -2.68986800 & 5.14478300 & $\mathrm{H}$ & 7.07348400 & -7.50402200 & 5.35896900 \\
\hline $\mathrm{H}$ & 12.32813600 & -3.39734200 & 4.32257600 & $\mathrm{H}$ & 6.60866800 & -3.20262100 & 2.13546200 \\
\hline $\mathrm{H}$ & 10.72028600 & -3.50056200 & 3.58726200 & $\mathrm{C}$ & 5.35917500 & -1.44754700 & 3.29925200 \\
\hline $\mathrm{C}$ & 12.17265400 & -0.09182800 & 5.57602600 & $\mathrm{H}$ & 5.25154100 & -1.60362400 & 2.22703800 \\
\hline $\mathrm{H}$ & 11.50595200 & -0.72803700 & 6.16200400 & $\mathrm{H}$ & 4.37232100 & -1.38633700 & 3.77560000 \\
\hline $\mathrm{H}$ & 11.95339200 & 0.93851300 & 5.86211900 & $\mathrm{H}$ & 5.86304400 & -0.49625200 & 3.50435600 \\
\hline $\mathrm{H}$ & 13.20960100 & -0.31601900 & 5.86865600 & $\mathrm{H}$ & 8.89837400 & -8.20087100 & 3.81752200 \\
\hline $\mathrm{C}$ & 12.91559100 & 2.01150200 & 3.30511500 & $\mathrm{C}$ & 6.31133000 & -2.41108000 & 5.45376300 \\
\hline $\mathrm{H}$ & 13.96839100 & 1.96933300 & 3.61815800 & $\mathrm{C}$ & 5.06475700 & -2.80656500 & 6.25306900 \\
\hline $\mathrm{H}$ & 12.36058700 & 2.51504100 & 4.10108200 & $\mathrm{C}$ & 7.62378200 & -2.96617400 & 6.04932100 \\
\hline $\mathrm{H}$ & 12.86465700 & 2.63719300 & 2.40907200 & $\mathrm{C}$ & 4.05310900 & -3.63522600 & 5.74881700 \\
\hline $\mathrm{C}$ & 12.53482000 & 0.58247100 & 0.45316700 & $\mathrm{C}$ & 4.92769600 & -2.30874800 & 7.55857900 \\
\hline $\mathrm{H}$ & 13.55585200 & 0.32799400 & 0.14006800 & $\mathrm{H}$ & 8.46972500 & -2.70550900 & 5.40705900 \\
\hline $\mathrm{H}$ & 12.45660700 & 1.67417800 & 0.45144300 & $\mathrm{C}$ & 2.94388900 & -3.97150800 & 6.53129300 \\
\hline $\mathrm{H}$ & 11.86130300 & 0.19392700 & -0.31713900 & $\mathrm{H}$ & 4.11147600 & -4.01637900 & 4.73206700 \\
\hline $\mathrm{C}$ & 11.54576200 & -2.40905200 & 0.97890700 & $\mathrm{C}$ & 3.82588700 & -2.64731000 & 8.34157200 \\
\hline $\mathrm{H}$ & 11.08037000 & -2.01887800 & 0.06889900 & $\mathrm{C}$ & 2.82869600 & -3.48337800 & 7.83142200 \\
\hline $\mathrm{H}$ & 10.90911600 & -3.22224700 & 1.33802100 & $\mathrm{H}$ & 2.17106300 & -4.61344700 & 6.11707800 \\
\hline $\mathrm{H}$ & 12.51109700 & -2.84510100 & 0.69007500 & $\mathrm{H}$ & 3.74130400 & -2.24998000 & 9.34945500 \\
\hline $\mathrm{H}$ & 7.21176700 & 2.12154400 & 2.47576400 & $\mathrm{H}$ & 1.96778200 & -3.74407000 & 8.44063500 \\
\hline $\mathrm{C}$ & 8.40179700 & 3.90498000 & 2.45419400 & $\mathrm{H}$ & 6.40172200 & -1.31865500 & 5.54999800 \\
\hline $\mathrm{C}$ & 9.64101100 & 4.52088300 & 2.67489300 & $\mathrm{H}$ & 7.61217600 & -4.04431000 & 6.21195700 \\
\hline $\mathrm{C}$ & 7.28997900 & 4.71305500 & 2.19210900 & $\mathrm{H}$ & 7.78950600 & -2.47930300 & 7.01474800 \\
\hline $\mathrm{C}$ & 9.76437300 & 5.90980600 & 2.63375700 & $\mathrm{H}$ & 5.68884100 & -1.64457100 & 7.9599460 \\
\hline $\mathrm{H}$ & 10.50383200 & 3.90159900 & 2.89868300 & & & & \\
\hline $\mathrm{C}$ & 7.40820700 & 6.10401800 & 2.15375600 & TS-14-SR & & & \\
\hline $\mathrm{H}$ & 6.32351100 & 4.24973700 & 2.01028700 & $\mathrm{C}$ & 6.92628800 & -4.62589900 & -5.45967900 \\
\hline $\mathrm{C}$ & 8.64682200 & 6.70753900 & 2.37349400 & $\mathrm{C}$ & 4.90017200 & -4.08533100 & -4.37199500 \\
\hline $\mathrm{H}$ & 10.73329900 & 6.37124700 & 2.80913800 & $\mathrm{C}$ & 5.40727700 & -4.64245400 & -5.67950600 \\
\hline $\mathrm{H}$ & 6.53163300 & 6.71429600 & 1.95166200 & $\mathrm{H}$ & 7.23205600 & -5.53865400 & -4.93528700 \\
\hline $\mathrm{H}$ & 8.74142000 & 7.79003500 & 2.34557300 & $\mathrm{H}$ & 7.50556200 & -4.56789700 & -6.3853770 \\
\hline $\mathrm{H}$ & 9.85692100 & 2.10480800 & 0.99927400 & $\mathrm{H}$ & 5.11617100 & -4.00227500 & -6.52501100 \\
\hline $\mathrm{C}$ & 8.09965400 & 2.31326200 & -0.17381800 & $\mathrm{H}$ & 5.01467500 & -5.64373100 & -5.89202700 \\
\hline $\mathrm{C}$ & 7.04296700 & 1.61776400 & -0.77278800 & $\mathrm{C}$ & 7.18701900 & -3.39714800 & -4.51215100 \\
\hline $\mathrm{C}$ & 8.56079800 & 3.48928800 & -0.78642700 & $\mathrm{C}$ & 7.30079900 & -2.10396000 & -5.37858700 \\
\hline
\end{tabular}




\begin{tabular}{|c|c|c|c|c|c|c|c|}
\hline $\mathrm{C}$ & 8.62461200 & -3.51864700 & -4.01570300 & $\mathrm{H}$ & 1.83780300 & -5.56382100 & 1.01523700 \\
\hline $\mathrm{C}$ & 8.70615700 & -2.20362700 & -6.02292700 & $\mathrm{C}$ & 2.23766200 & -0.13664200 & 3.79438800 \\
\hline $\mathrm{H}$ & 6.49451900 & -2.01822600 & -6.11449700 & $\mathrm{H}$ & 1.57263100 & 0.31538600 & 4.54084600 \\
\hline $\mathrm{H}$ & 7.25258400 & -1.23086500 & -4.71904500 & $\mathrm{H}$ & 2.77042300 & 0.67142900 & 3.28135100 \\
\hline $\mathrm{H}$ & 9.17130700 & -1.22008700 & -6.16123300 & $\mathrm{H}$ & 2.97845400 & -0.74070900 & 4.32807900 \\
\hline $\mathrm{H}$ & 8.66494700 & -2.67160100 & -7.01604200 & $\mathrm{C}$ & 0.63731900 & -2.08144100 & 3.54887300 \\
\hline $\mathrm{C}$ & 5.92643100 & -3.45137500 & -3.65067800 & $\mathrm{H}$ & -0.06858400 & -1.61458400 & 4.24551400 \\
\hline $\mathrm{C}$ & 5.66875500 & -2.94310700 & -2.36812100 & $\mathrm{H}$ & 1.29600400 & -2.72749000 & 4.14031600 \\
\hline $\mathrm{C}$ & 3.60676900 & -4.15252800 & -3.87465000 & $\mathrm{H}$ & 0.06537500 & -2.71265300 & 2.85915100 \\
\hline $\mathrm{C}$ & 4.35336700 & -2.98723300 & -1.84694100 & $\mathrm{C}$ & 2.57603800 & -0.05297500 & -2.58343600 \\
\hline $\mathrm{C}$ & 3.34939900 & -3.58436600 & -2.63313900 & $\mathrm{H}$ & 1.64660600 & 0.26426100 & -2.09467700 \\
\hline $\mathrm{H}$ & 2.81282200 & -4.63499400 & -4.43923600 & $\mathrm{H}$ & 2.40707500 & -1.03867900 & -3.02632800 \\
\hline $\mathrm{H}$ & 2.34051500 & -3.60434300 & -2.23251200 & $\mathrm{H}$ & 2.78806100 & 0.65352100 & -3.39610600 \\
\hline $\mathrm{C}$ & 9.47272800 & -3.05725200 & -5.03202700 & $\mathrm{C}$ & 4.01537600 & 1.34995400 & -1.06515300 \\
\hline $\mathrm{C}$ & 10.80075500 & -3.46351200 & -5.06343300 & $\mathrm{H}$ & 4.38045000 & 1.97433800 & -1.88755000 \\
\hline $\mathrm{C}$ & 9.15604300 & -4.17334300 & -2.90278000 & $\mathrm{H}$ & 4.77166500 & 1.36399200 & -0.27290600 \\
\hline $\mathrm{C}$ & 11.25545500 & -4.32787300 & -4.06818500 & $\mathrm{H}$ & 3.10894800 & 1.82548400 & -0.67291600 \\
\hline $\mathrm{H}$ & 11.46358900 & -3.16270400 & -5.87130300 & $\mathrm{C}$ & 9.08898400 & -7.27293300 & -2.22079300 \\
\hline $\mathrm{C}$ & 10.48246700 & -4.67368200 & -2.94191500 & $\mathrm{H}$ & 8.74393100 & -6.50066900 & -2.90788400 \\
\hline $\mathrm{H}$ & 12.25098000 & -4.74429100 & -4.15587800 & $\mathrm{C}$ & 12.84213400 & -8.42090300 & 0.93118700 \\
\hline $\mathrm{O}$ & 6.70187000 & -2.32742000 & -1.67780200 & $\mathrm{H}$ & 13.79715700 & -7.98325300 & 1.25406800 \\
\hline $\mathrm{O}$ & 8.34383800 & -4.38388200 & -1.79635500 & $\mathrm{C}$ & 13.26644500 & -4.18767200 & -1.72953000 \\
\hline $\mathrm{P}$ & 7.90218900 & -3.11518300 & -0.81781500 & $\mathrm{H}$ & 12.68337000 & -3.46776800 & -2.30852400 \\
\hline $\mathrm{O}$ & 9.02210600 & -2.10908000 & -0.71684600 & $\mathrm{C}$ & 11.98623200 & -8.63558200 & 2.19278400 \\
\hline $\mathrm{O}$ & 7.30176900 & -3.74373900 & 0.41247300 & $\mathrm{H}$ & 12.49336600 & -9.30808800 & 2.89557500 \\
\hline $\mathrm{C}$ & 3.85048400 & -2.43915000 & -0.53546700 & $\mathrm{H}$ & 11.01800900 & -9.08703400 & 1.94361400 \\
\hline $\mathrm{C}$ & 3.46310400 & -1.07697700 & -0.44233600 & $\mathrm{H}$ & 11.79303700 & -7.68761600 & 2.70662500 \\
\hline $\mathrm{C}$ & 3.49140600 & -3.33495900 & 0.49885400 & $\mathrm{C}$ & 13.16121000 & -9.76608300 & 0.24941600 \\
\hline $\mathrm{C}$ & 2.71167800 & -0.66139500 & 0.65959100 & $\mathrm{H}$ & 13.67444700 & -10.44199400 & 0.94448300 \\
\hline $\mathrm{C}$ & 2.73926500 & -2.86104500 & 1.58657700 & $\mathrm{H}$ & 13.80413400 & -9.62230500 & -0.62584600 \\
\hline $\mathrm{C}$ & 2.31526000 & -1.53482500 & 1.67825100 & $\mathrm{H}$ & 12.24589800 & -10.26653400 & -0.08832500 \\
\hline $\mathrm{H}$ & 2.38149200 & 0.37278800 & 0.71381600 & $\mathrm{C}$ & 9.34902400 & -8.53160000 & -3.07594400 \\
\hline $\mathrm{H}$ & 2.44672000 & -3.56653700 & 2.35777500 & $\mathrm{H}$ & 9.67605900 & -9.37926400 & -2.46218100 \\
\hline $\mathrm{C}$ & 11.06674700 & -5.59912300 & -1.91068400 & $\mathrm{H}$ & 10.12377600 & -8.34701900 & -3.82897700 \\
\hline $\mathrm{C}$ & 10.38465500 & -6.79915600 & -1.55511800 & $\mathrm{H}$ & 8.43216100 & -8.83307800 & -3.59776100 \\
\hline $\mathrm{C}$ & 12.35535800 & -5.36210900 & -1.34839400 & $\mathrm{C}$ & 7.95532500 & -7.51811300 & -1.20716100 \\
\hline $\mathrm{C}$ & 10.96040000 & -7.67018000 & -0.62357600 & $\mathrm{H}$ & 7.74911500 & -6.61665600 & -0.62424000 \\
\hline $\mathrm{C}$ & 12.88113300 & -6.28105600 & -0.43045600 & $\mathrm{H}$ & 8.19970100 & -8.33404900 & -0.51568100 \\
\hline $\mathrm{C}$ & 12.20678300 & -7.43621700 & -0.04185000 & $\mathrm{H}$ & 7.03525400 & -7.80158300 & -1.73310500 \\
\hline $\mathrm{H}$ & 10.42513600 & -8.58211600 & -0.37140000 & $\mathrm{C}$ & 14.44816700 & -4.66710500 & -2.60231600 \\
\hline $\mathrm{H}$ & 13.86792100 & -6.09702400 & -0.01204700 & $\mathrm{H}$ & 15.10249200 & -5.33703300 & -2.03174200 \\
\hline $\mathrm{C}$ & 3.82157400 & -4.82872000 & 0.43512000 & $\mathrm{H}$ & 15.05171400 & -3.81405600 & -2.93705100 \\
\hline $\mathrm{H}$ & 4.49541500 & -4.98663000 & -0.41140400 & $\mathrm{H}$ & 14.11853600 & -5.21827300 & -3.48877100 \\
\hline $\mathrm{C}$ & 1.43071200 & -0.99919700 & 2.80225200 & $\mathrm{C}$ & 13.82829300 & -3.42807400 & -0.51401400 \\
\hline $\mathrm{H}$ & 0.69763600 & -0.33388000 & 2.32172800 & $\mathrm{H}$ & 13.02641400 & -3.07526500 & 0.13537000 \\
\hline $\mathrm{C}$ & 3.73913700 & -0.07902800 & -1.56827200 & $\mathrm{H}$ & 14.40478900 & -2.55802000 & -0.85312700 \\
\hline $\mathrm{H}$ & 4.63313600 & -0.41945700 & -2.10191600 & $\mathrm{H}$ & 14.50336100 & -4.04807200 & 0.08729400 \\
\hline $\mathrm{C}$ & 4.56282200 & -5.32971800 & 1.68683100 & $\mathrm{C}$ & 11.47734000 & 0.09397000 & 4.13679500 \\
\hline $\mathrm{H}$ & 4.80514300 & -6.39382400 & 1.57796200 & $\mathrm{C}$ & 11.79948000 & 0.60888600 & 2.81120900 \\
\hline $\mathrm{H}$ & 3.95603400 & -5.22367700 & 2.59487000 & $\mathrm{C}$ & 11.63333300 & -0.46422200 & 1.86689400 \\
\hline $\mathrm{H}$ & 5.49936500 & -4.78554400 & 1.81744400 & $\mathrm{C}$ & 11.19060100 & -1.64236500 & 2.58777000 \\
\hline $\mathrm{C}$ & 2.55458000 & -5.67492600 & 0.19219400 & $\mathrm{C}$ & 11.14955800 & -1.28826700 & 3.99206900 \\
\hline $\mathrm{H}$ & 2.81499000 & -6.73796400 & 0.11811200 & $\mathrm{Ir}$ & 9.65932500 & -0.05671100 & 2.78836900 \\
\hline $\mathrm{H}$ & 2.04533500 & -5.38826100 & -0.73368800 & $\mathrm{~N}$ & 8.73763500 & 1.97362000 & 2.86466800 \\
\hline
\end{tabular}




\begin{tabular}{|c|c|c|c|c|c|c|c|}
\hline $\mathrm{N}$ & 8.63447400 & 0.12653800 & 0.93848000 & $\mathrm{H}$ & 12.02827400 & -3.53378000 & 2.01785800 \\
\hline $\mathrm{H}$ & 8.91943200 & -0.60322600 & 0.25167200 & $\mathrm{H}$ & 7.07681800 & 2.17311600 & 1.51569600 \\
\hline $\mathrm{C}$ & 8.15349100 & 2.40512800 & 1.56462300 & $\mathrm{C}$ & 8.32619900 & 3.88957800 & 1.26104600 \\
\hline $\mathrm{C}$ & 8.83591600 & 1.52643800 & 0.47440000 & $\mathrm{C}$ & 9.54019400 & 4.54630300 & 1.50101200 \\
\hline S & 7.77301800 & 2.27339200 & 4.18601300 & $\mathrm{C}$ & 7.28617400 & 4.60748300 & 0.65919200 \\
\hline $\mathrm{O}$ & 8.51044600 & 1.79659600 & 5.37210600 & $\mathrm{C}$ & 9.70973400 & 5.88530000 & 1.14826900 \\
\hline $\mathrm{O}$ & 6.41272000 & 1.70886500 & 3.99813500 & $\mathrm{H}$ & 10.34743400 & 4.00456400 & 1.98144300 \\
\hline $\mathrm{C}$ & 7.56037300 & 4.08877100 & 4.42896300 & $\mathrm{C}$ & 7.44990000 & 5.94880100 & 0.30799800 \\
\hline $\mathrm{C}$ & 6.29966000 & 4.70002100 & 4.24621500 & $\mathrm{H}$ & 6.34015400 & 4.11174300 & 0.45706600 \\
\hline $\mathrm{C}$ & 8.64787500 & 4.79721900 & 4.98516800 & $\mathrm{C}$ & 8.66384100 & 6.59270500 & 0.55002200 \\
\hline $\mathrm{C}$ & 6.12008000 & 6.01753100 & 4.72601300 & $\mathrm{H}$ & 10.65839500 & 6.37896700 & 1.34465300 \\
\hline $\mathrm{C}$ & 8.44910200 & 6.11626600 & 5.43349100 & $\mathrm{H}$ & 6.62845100 & 6.48817200 & -0.15632600 \\
\hline $\mathrm{C}$ & 7.19334200 & 6.73347500 & 5.28482700 & $\mathrm{H}$ & 8.79402600 & 7.63691200 & 0.27822400 \\
\hline $\mathrm{C}$ & 5.12664300 & 4.06418300 & 3.52125700 & $\mathrm{H}$ & 9.90892000 & 1.72222200 & 0.54611700 \\
\hline $\mathrm{H}$ & 4.43091900 & 3.57814600 & 4.21619000 & $\mathrm{C}$ & 8.42391400 & 1.78253500 & -0.96827300 \\
\hline $\mathrm{H}$ & 5.43554700 & 3.29937600 & 2.81606800 & $\mathrm{C}$ & 7.46606500 & 1.00855300 & -1.63113000 \\
\hline $\mathrm{H}$ & 4.57276200 & 4.82983500 & 2.97119900 & $\mathrm{C}$ & 9.06425900 & 2.81029700 & -1.68051800 \\
\hline $\mathrm{C}$ & 4.74367700 & 6.65675900 & 4.65697100 & $\mathrm{C}$ & 7.14048300 & 1.27171100 & -2.96486500 \\
\hline $\mathrm{H}$ & 4.60939600 & 7.40190300 & 5.44414700 & $\mathrm{H}$ & 6.98886200 & 0.16786900 & -1.14049100 \\
\hline $\mathrm{H}$ & 3.95058900 & 5.91624900 & 4.78532300 & $\mathrm{C}$ & 8.73644200 & 3.07602400 & -3.00819900 \\
\hline $\mathrm{H}$ & 4.56195000 & 7.16097700 & 3.69659200 & $\mathrm{H}$ & 9.82104100 & 3.41386500 & -1.18741500 \\
\hline $\mathrm{C}$ & 7.00939600 & 8.18315400 & 5.69578500 & $\mathrm{C}$ & 7.76699700 & 2.30737900 & -3.65638500 \\
\hline $\mathrm{H}$ & 6.68284300 & 8.28027900 & 6.74136200 & $\mathrm{H}$ & 6.39833500 & 0.65311900 & -3.46093000 \\
\hline $\mathrm{H}$ & 6.26297800 & 8.68583100 & 5.07648000 & $\mathrm{H}$ & 9.24328900 & 3.87888600 & -3.53702800 \\
\hline $\mathrm{H}$ & 7.93763400 & 8.75012400 & 5.59414700 & $\mathrm{H}$ & 7.51240400 & 2.50690300 & -4.69393200 \\
\hline $\mathrm{C}$ & 9.59076000 & 6.86204700 & 6.10067000 & $\mathrm{~N}$ & 7.33466800 & -3.13332500 & 3.2422420 \\
\hline $\mathrm{H}$ & 9.22590600 & 7.57991600 & 6.83908900 & $\mathrm{C}$ & 7.01348600 & -1.84154200 & 3.58851800 \\
\hline $\mathrm{H}$ & 10.20145400 & 7.42200600 & 5.37710600 & $\mathrm{C}$ & 7.85259100 & -4.27171700 & 3.90546500 \\
\hline $\mathrm{H}$ & 10.26262600 & 6.18148900 & 6.62873200 & $\mathrm{C}$ & 8.44446800 & -5.24387300 & 3.07076300 \\
\hline $\mathrm{C}$ & 10.04207700 & 4.21344400 & 5.08287000 & $\mathrm{C}$ & 7.72105400 & -4.55612000 & 5.27514100 \\
\hline $\mathrm{H}$ & 10.78536400 & 4.96944200 & 4.80727800 & $\mathrm{C}$ & 8.93622400 & -6.43182700 & 3.60282300 \\
\hline $\mathrm{H}$ & 10.16572000 & 3.36060100 & 4.42128400 & $\mathrm{H}$ & 8.48683100 & -5.06164800 & 2.00111800 \\
\hline $\mathrm{H}$ & 10.26822000 & 3.87432900 & 6.10091600 & $\mathrm{C}$ & 8.23182400 & -5.74611100 & 5.79708700 \\
\hline $\mathrm{H}$ & 7.63698600 & -0.03036000 & 1.08672000 & $\mathrm{H}$ & 7.18181300 & -3.89061900 & 5.92979600 \\
\hline $\mathrm{H}$ & 8.20181800 & -0.98957300 & 3.33563300 & $\mathrm{C}$ & 8.85065000 & -6.68650700 & 4.97399000 \\
\hline $\mathrm{C}$ & 10.98935200 & -2.26623400 & 5.11578700 & $\mathrm{H}$ & 9.38426000 & -7.16131000 & 2.93492500 \\
\hline $\mathrm{H}$ & 10.69572800 & -1.77782400 & 6.04596600 & $\mathrm{H}$ & 8.11876900 & -5.94113000 & 6.86028700 \\
\hline $\mathrm{H}$ & 11.95835100 & -2.75690300 & 5.28696900 & $\mathrm{H}$ & 7.31855600 & -3.28171100 & 2.21984700 \\
\hline $\mathrm{H}$ & 10.26312200 & -3.04860500 & 4.88962200 & $\mathrm{C}$ & 6.05585500 & -1.24621300 & 2.56089800 \\
\hline $\mathrm{C}$ & 11.67558500 & 0.80905200 & 5.44158300 & $\mathrm{H}$ & 6.28944200 & -1.57916900 & 1.54619200 \\
\hline $\mathrm{H}$ & 10.86982300 & 0.59289700 & 6.14633400 & $\mathrm{H}$ & 5.04724500 & -1.61475000 & 2.77680900 \\
\hline $\mathrm{H}$ & 11.70020900 & 1.89088200 & 5.30624300 & $\mathrm{H}$ & 6.03703500 & -0.15859700 & 2.63792600 \\
\hline $\mathrm{H}$ & 12.62850600 & 0.50435900 & 5.89711800 & $\mathrm{H}$ & 9.23783600 & -7.61262500 & 5.38913900 \\
\hline $\mathrm{C}$ & 12.36037800 & 1.96695100 & 2.52009700 & $\mathrm{C}$ & 6.68919900 & -1.36041500 & 5.02073300 \\
\hline $\mathrm{H}$ & 13.44686900 & 1.97018400 & 2.68648500 & $\mathrm{C}$ & 5.40603900 & -1.96101100 & 5.62567700 \\
\hline $\mathrm{H}$ & 11.92336500 & 2.72732300 & 3.17064900 & $\mathrm{C}$ & 7.85369000 & -1.32612600 & 6.03193900 \\
\hline $\mathrm{H}$ & 12.18711100 & 2.26673900 & 1.48286800 & $\mathrm{C}$ & 4.78240000 & -3.14423500 & 5.20824700 \\
\hline $\mathrm{C}$ & 11.91604800 & -0.39979800 & 0.39421600 & $\mathrm{C}$ & 4.81588400 & -1.25016400 & 6.68718200 \\
\hline $\mathrm{H}$ & 12.95865200 & -0.68297500 & 0.20508100 & $\mathrm{H}$ & 8.64300500 & -0.68889800 & 5.63181300 \\
\hline $\mathrm{H}$ & 11.77767800 & 0.60960800 & -0.00415400 & $\mathrm{C}$ & 3.63377600 & -3.61878900 & 5.85179400 \\
\hline $\mathrm{H}$ & 11.27440900 & -1.08274600 & -0.17033400 & $\mathrm{H}$ & 5.18241700 & -3.70980300 & 4.37538000 \\
\hline $\mathrm{C}$ & 11.05359900 & -3.02864600 & 2.03425600 & $\mathrm{C}$ & 3.67190200 & -1.71969500 & 7.32892800 \\
\hline $\mathrm{H}$ & 10.64940600 & -3.02074600 & 1.01882600 & $\mathrm{C}$ & 3.07678300 & -2.91540400 & 6.91810400 \\
\hline $\mathrm{H}$ & 10.38738400 & -3.63192000 & 2.65178500 & $\mathrm{H}$ & 3.18107400 & -4.54731900 & 5.51312000 \\
\hline
\end{tabular}




\begin{tabular}{|c|c|c|c|c|c|c|c|}
\hline $\mathrm{H}$ & 3.24156900 & -1.14721600 & 8.14663400 & $\mathrm{C}$ & 2.70914700 & 1.30865700 & -1.00835700 \\
\hline $\mathrm{H}$ & 2.18674400 & -3.28739600 & 7.41838200 & $\mathrm{C}$ & 2.63733800 & 1.52964600 & -2.38175900 \\
\hline $\mathrm{H}$ & 6.45562400 & -0.30429800 & 4.85686500 & $\mathrm{H}$ & 2.95693000 & 0.61187500 & -4.29427300 \\
\hline $\mathrm{H}$ & 8.26576600 & -2.30407700 & 6.27890500 & $\mathrm{H}$ & 2.44076800 & 2.12190900 & -0.33793700 \\
\hline $\mathrm{H}$ & 7.50532300 & -0.86352600 & 6.95963800 & $\mathrm{C}$ & 9.96243900 & -2.15472400 & -3.85470200 \\
\hline \multirow[t]{2}{*}{$\mathrm{H}$} & 5.25869200 & -0.31024200 & 7.00722200 & $\mathrm{H}$ & 8.94867600 & -2.45902700 & -3.58024300 \\
\hline & & & & $\mathrm{C}$ & 14.73127800 & -2.96882100 & -2.32981500 \\
\hline $17-S S$ & & & & $\mathrm{H}$ & 15.27333300 & -3.74248400 & -1.76822100 \\
\hline $\mathrm{C}$ & 4.65809200 & -6.10508900 & -2.79930300 & $\mathrm{C}$ & 11.16271200 & -6.28755700 & -1.01840200 \\
\hline $\mathrm{C}$ & 6.92085800 & -6.24751400 & -3.47002300 & $\mathrm{H}$ & 10.07290700 & -6.34029700 & -0.92245200 \\
\hline $\mathrm{C}$ & 5.59006600 & -6.91750100 & -3.71060400 & $\mathrm{C}$ & 10.05937400 & -0.64456600 & -3.57622200 \\
\hline $\mathrm{H}$ & 4.31763700 & -5.21064600 & -3.33297000 & $\mathrm{H}$ & 9.28209600 & -0.11423700 & -4.13855300 \\
\hline $\mathrm{H}$ & 3.76893700 & -6.65347100 & -2.47474300 & $\mathrm{H}$ & 11.02595700 & -0.22723000 & -3.88478900 \\
\hline $\mathrm{H}$ & 5.62132500 & -7.97589000 & -3.41299600 & $\mathrm{H}$ & 9.89546800 & -0.43918700 & -2.51690200 \\
\hline $\mathrm{H}$ & 5.28560400 & -6.89447000 & -4.76339100 & $\mathrm{C}$ & 10.15298700 & -2.43462000 & -5.36050900 \\
\hline $\mathrm{C}$ & 5.55540400 & -5.65363400 & -1.58648800 & $\mathrm{H}$ & 9.43620500 & -1.85054200 & -5.95068700 \\
\hline $\mathrm{C}$ & 5.52043500 & -6.76041300 & -0.48797800 & $\mathrm{H}$ & 10.00258200 & -3.49257000 & -5.59951700 \\
\hline $\mathrm{C}$ & 4.80844800 & -4.53302800 & -0.87149500 & $\mathrm{H}$ & 11.16297500 & -2.15694900 & -5.68777200 \\
\hline $\mathrm{C}$ & 4.14234800 & -6.56853600 & 0.20126800 & $\mathrm{C}$ & 15.01415200 & -1.62268100 & -1.64029500 \\
\hline $\mathrm{H}$ & 5.65457700 & -7.76571100 & -0.90152000 & $\mathrm{H}$ & 16.08711600 & -1.39531300 & -1.65775100 \\
\hline $\mathrm{H}$ & 6.32956100 & -6.58165500 & 0.22837400 & $\mathrm{H}$ & 14.68762000 & -1.64304200 & -0.59510600 \\
\hline $\mathrm{H}$ & 4.17565100 & -6.81794300 & 1.26896100 & $\mathrm{H}$ & 14.48993500 & -0.79964300 & -2.14019400 \\
\hline $\mathrm{H}$ & 3.37479100 & -7.21548700 & -0.24517700 & $\mathrm{C}$ & 15.28494800 & -2.97567600 & -3.76828800 \\
\hline $\mathrm{C}$ & 6.89971500 & -5.48374700 & -2.29198000 & $\mathrm{H}$ & 16.36266400 & -2.77124100 & -3.76972000 \\
\hline $\mathrm{C}$ & 8.03123800 & -4.74301600 & -1.92060900 & $\mathrm{H}$ & 14.79951600 & -2.20995300 & -4.38489300 \\
\hline $\mathrm{C}$ & 8.05973900 & -6.31379900 & -4.26121800 & $\mathrm{H}$ & 15.12029700 & -3.94545100 & -4.25064700 \\
\hline $\mathrm{C}$ & 9.21226500 & -4.81709300 & -2.69377300 & $\mathrm{C}$ & 11.60681800 & -7.47257200 & -1.90147900 \\
\hline $\mathrm{C}$ & 9.18920400 & -5.60998300 & -3.85649000 & $\mathrm{H}$ & 12.69408900 & -7.46522600 & -2.04606400 \\
\hline $\mathrm{H}$ & 8.07297300 & -6.90106400 & -5.17598300 & $\mathrm{H}$ & 11.13521700 & -7.43286500 & -2.88801300 \\
\hline $\mathrm{H}$ & 10.09622300 & -5.66261600 & -4.45159900 & $\mathrm{H}$ & 11.33524800 & -8.42792700 & -1.43454100 \\
\hline $\mathrm{C}$ & 3.83958300 & -5.09968600 & -0.03465500 & $\mathrm{C}$ & 11.75626700 & -6.43529900 & 0.39487200 \\
\hline $\mathrm{C}$ & 2.76732000 & -4.32756400 & 0.40539400 & $\mathrm{H}$ & 11.42241100 & -7.37771000 & 0.84731500 \\
\hline $\mathrm{C}$ & 4.82597800 & -3.15377500 & -1.08848100 & $\mathrm{H}$ & 11.45401400 & -5.61060900 & 1.04887800 \\
\hline $\mathrm{C}$ & 2.66441600 & -3.00910500 & -0.04523400 & $\mathrm{H}$ & 12.85165400 & -6.46332100 & 0.38019100 \\
\hline $\mathrm{H}$ & 1.98761000 & -4.75302900 & 1.03318100 & $\mathrm{C}$ & 3.68740000 & -1.86697900 & -3.76250400 \\
\hline $\mathrm{C}$ & 3.68432500 & -2.37758700 & -0.78498500 & $\mathrm{H}$ & 3.94542300 & -2.78684400 & -3.23613800 \\
\hline $\mathrm{H}$ & 1.77146200 & -2.43696400 & 0.18663100 & $\mathrm{C}$ & 2.15036500 & 2.86257100 & -2.93630000 \\
\hline $\mathrm{O}$ & 7.98595000 & -4.01327000 & -0.74337100 & $\mathrm{H}$ & 1.93161700 & 3.50764700 & -2.07407100 \\
\hline $\mathrm{O}$ & 5.97292700 & -2.56507300 & -1.60438200 & $\mathrm{C}$ & 3.16098500 & -0.00959400 & 1.06749800 \\
\hline $\mathrm{P}$ & 7.29591500 & -2.50196000 & -0.57958200 & $\mathrm{H}$ & 3.55124000 & -0.99589000 & 1.33150900 \\
\hline $\mathrm{O}$ & 6.80883000 & -2.46792200 & 0.85548500 & $\mathrm{C}$ & 3.22293100 & 3.57460800 & -3.78144600 \\
\hline $\mathrm{O}$ & 8.20686200 & -1.43299100 & -1.09065400 & $\mathrm{H}$ & 2.85378600 & 4.54529100 & -4.13463800 \\
\hline $\mathrm{C}$ & 10.54963300 & -4.19107200 & -2.38741900 & $\mathrm{H}$ & 3.49344000 & 2.98184100 & -4.66384300 \\
\hline $\mathrm{C}$ & 11.50938100 & -4.94999200 & -1.67565400 & $\mathrm{H}$ & 4.13443900 & 3.74648900 & -3.19969800 \\
\hline $\mathrm{C}$ & 10.93931400 & -2.98462000 & -3.02011700 & $\mathrm{C}$ & 0.84100900 & 2.69871200 & -3.73265900 \\
\hline $\mathrm{C}$ & 12.83923900 & -4.51101900 & -1.64761900 & $\mathrm{H}$ & 0.46786500 & 3.67350900 & -4.06972600 \\
\hline $\mathrm{C}$ & 12.28149900 & -2.58875700 & -2.95298200 & $\mathrm{H}$ & 0.06280200 & 2.22655700 & -3.12286500 \\
\hline $\mathrm{C}$ & 13.25521500 & -3.34839400 & -2.29741900 & $\mathrm{H}$ & 0.99266400 & 2.07450600 & -4.62135900 \\
\hline $\mathrm{H}$ & 13.58573200 & -5.11249600 & -1.13395400 & $\mathrm{C}$ & 2.44200500 & -2.18586000 & -4.61471100 \\
\hline $\mathrm{H}$ & 12.57940300 & -1.67673900 & -3.46333300 & $\mathrm{H}$ & 2.12625500 & -1.32422700 & -5.21447500 \\
\hline $\mathrm{C}$ & 3.44492900 & -0.99185400 & -1.31934100 & $\mathrm{H}$ & 1.59555700 & -2.48203500 & -3.98462300 \\
\hline $\mathrm{C}$ & 3.39515900 & -0.77930400 & -2.72685900 & $\mathrm{H}$ & 2.65588100 & -3.00959200 & -5.30710100 \\
\hline $\mathrm{C}$ & 3.10699800 & 0.08328600 & -0.45767800 & $\mathrm{C}$ & 4.89350700 & -1.50353100 & -4.64969600 \\
\hline $\mathrm{C}$ & 3.00310000 & 0.47139400 & -3.21725500 & $\mathrm{H}$ & 5.78271600 & -1.32117000 & -4.03958900 \\
\hline
\end{tabular}




\begin{tabular}{|c|c|c|c|c|c|c|c|}
\hline$H$ & 4.69709500 & -0.60755700 & -5.25136600 & $\mathrm{H}$ & 11.74000600 & -3.32750200 & 4.66319900 \\
\hline $\mathrm{H}$ & 5.11648800 & -2.32402700 & -5.34295900 & $\mathrm{H}$ & 10.18570300 & -3.35472500 & 3.81461100 \\
\hline $\mathrm{C}$ & 1.76408800 & 0.12515400 & 1.70821100 & $\mathrm{C}$ & 11.78038700 & 0.03650800 & 5.70565900 \\
\hline $\mathrm{H}$ & 1.32502800 & 1.10848800 & 1.50255500 & $\mathrm{H}$ & 10.93718400 & -0.38609600 & 6.25621800 \\
\hline $\mathrm{H}$ & 1.82875000 & 0.01380700 & 2.79789800 & $\mathrm{H}$ & 11.78140600 & 1.10952100 & 5.90901700 \\
\hline $\mathrm{H}$ & 1.06652900 & -0.63087700 & 1.33100900 & $\mathrm{H}$ & 12.71206000 & -0.38491100 & 6.11224400 \\
\hline $\mathrm{C}$ & 4.11691400 & 1.04261000 & 1.66151900 & $\mathrm{C}$ & 12.82499300 & 1.94631400 & 3.3661780 \\
\hline $\mathrm{H}$ & 5.11863400 & 0.95345000 & 1.23029400 & $\mathrm{H}$ & 13.85154800 & 1.84555500 & 3.74605800 \\
\hline $\mathrm{H}$ & 4.20635600 & 0.92859300 & 2.74673200 & $\mathrm{H}$ & 12.26583500 & 2.54065100 & 4.09419100 \\
\hline $\mathrm{H}$ & 3.76534300 & 2.06200000 & 1.46589700 & $\mathrm{H}$ & 12.87662800 & 2.51482000 & 2.4328500 \\
\hline $\mathrm{C}$ & 11.66364700 & -0.24195400 & 4.23443500 & $\mathrm{C}$ & 12.47578600 & 0.39148500 & 0.57958400 \\
\hline $\mathrm{C}$ & 12.20163500 & 0.59744300 & 3.16223900 & $\mathrm{H}$ & 13.50400100 & 0.08425000 & 0.34973100 \\
\hline $\mathrm{C}$ & 12.06066400 & -0.09109100 & 1.93982800 & $\mathrm{H}$ & 12.43829600 & 1.48321700 & 0.50379600 \\
\hline $\mathrm{C}$ & 11.43677600 & -1.39018100 & 2.21305200 & $\mathrm{H}$ & 11.83605000 & -0.02267000 & -0.20611600 \\
\hline $\mathrm{C}$ & 11.27237600 & -1.49627400 & 3.64335400 & $\mathrm{C}$ & 11.25573500 & -2.49232700 & 1.20989000 \\
\hline $\mathrm{Ir}$ & 9.91570000 & 0.04755200 & 2.88706000 & $\mathrm{H}$ & 10.86296900 & -2.12953700 & 0.25621600 \\
\hline $\mathrm{N}$ & 8.95328200 & 1.95716700 & 3.38770900 & $\mathrm{H}$ & 10.55089000 & -3.24194300 & 1.57925100 \\
\hline $\mathrm{N}$ & 8.68446200 & 0.47858800 & 1.18998500 & $\mathrm{H}$ & 12.20685800 & -2.99995000 & 0.99998300 \\
\hline $\mathrm{H}$ & 8.85580300 & -0.09785700 & 0.35419300 & $\mathrm{H}$ & 7.24141200 & 2.52843600 & 2.22114400 \\
\hline $\mathrm{C}$ & 8.33523400 & 2.64963900 & 2.22110600 & $\mathrm{C}$ & 8.64788000 & 4.14179600 & 2.17474300 \\
\hline $\mathrm{C}$ & 8.84066000 & 1.93414800 & 0.92927600 & $\mathrm{C}$ & 9.94350500 & 4.61141300 & 2.42842600 \\
\hline S & 7.96300800 & 1.88040300 & 4.71631500 & $\mathrm{C}$ & 7.65812300 & 5.06673100 & 1.82351900 \\
\hline $\mathrm{O}$ & 8.69028500 & 1.19607100 & 5.80151800 & $\mathrm{C}$ & 10.24213700 & 5.97049200 & 2.32842400 \\
\hline $\mathrm{O}$ & 6.62831200 & 1.30813100 & 4.38422800 & $\mathrm{H}$ & 10.71101300 & 3.90352800 & 2.72385400 \\
\hline $\mathrm{C}$ & 7.68206300 & 3.60121300 & 5.32325500 & $\mathrm{C}$ & 7.95155000 & 6.42855800 & 1.72605600 \\
\hline $\mathrm{C}$ & 6.40758500 & 4.20714600 & 5.26472400 & $\mathrm{H}$ & 6.64898600 & 4.71704500 & 1.61968600 \\
\hline $\mathrm{C}$ & 8.75987700 & 4.22473500 & 5.99086700 & $\mathrm{C}$ & 9.24606500 & 6.88534500 & 1.97612500 \\
\hline $\mathrm{C}$ & 6.20531100 & 5.41768500 & 5.96630200 & $\mathrm{H}$ & 11.25292400 & 6.31747900 & 2.52972400 \\
\hline $\mathrm{C}$ & 8.53834700 & 5.44261800 & 6.66216400 & $\mathrm{H}$ & 7.16752900 & 7.13103400 & 1.45491100 \\
\hline $\mathrm{C}$ & 7.27050600 & 6.05177600 & 6.62911900 & $\mathrm{H}$ & 9.47718500 & 7.94489600 & 1.90191100 \\
\hline $\mathrm{C}$ & 5.23910900 & 3.67823100 & 4.45196000 & $\mathrm{H}$ & 9.91937200 & 2.11075000 & 0.86746200 \\
\hline $\mathrm{H}$ & 4.56960700 & 3.05263600 & 5.05594400 & $\mathrm{C}$ & 8.21601600 & 2.41489200 & -0.37312100 \\
\hline $\mathrm{H}$ & 5.55848600 & 3.06383700 & 3.61667700 & $\mathrm{C}$ & 7.12390200 & 1.77086400 & -0.96666800 \\
\hline $\mathrm{H}$ & 4.65096100 & 4.51207300 & 4.05867900 & $\mathrm{C}$ & 8.77688900 & 3.52213900 & -1.02968400 \\
\hline C & 4.81442200 & 6.02698200 & 6.02106400 & $\mathrm{C}$ & 6.59944500 & 2.22464300 & -2.17933900 \\
\hline $\mathrm{H}$ & 4.67649600 & 6.62873900 & 6.92209000 & $\mathrm{H}$ & 6.69858800 & 0.87860400 & -0.52143800 \\
\hline $\mathrm{H}$ & 4.03937700 & 5.25667200 & 6.03585200 & $\mathrm{C}$ & 8.25077500 & 3.98235100 & -2.23536700 \\
\hline $\mathrm{H}$ & 4.60493800 & 6.67804200 & 5.15959200 & $\mathrm{H}$ & 9.63188600 & 4.02856000 & -0.59005900 \\
\hline $\mathrm{C}$ & 7.06202600 & 7.40859600 & 7.27822600 & $\mathrm{C}$ & 7.15817700 & 3.33339400 & -2.81545200 \\
\hline $\mathrm{H}$ & 6.74669600 & 7.32500100 & 8.32853400 & $\mathrm{H}$ & 5.76246400 & 1.69618300 & -2.62554100 \\
\hline $\mathrm{H}$ & 6.29802600 & 7.99067800 & 6.75752300 & $\mathrm{H}$ & 8.70199300 & 4.84078800 & -2.72638700 \\
\hline $\mathrm{H}$ & 7.97665300 & 8.00578300 & 7.26291700 & $\mathrm{H}$ & 6.75542400 & 3.68061100 & -3.76346000 \\
\hline $\mathrm{C}$ & 9.67282800 & 6.08022000 & 7.44428200 & $\mathrm{~N}$ & 6.50303700 & -3.63098900 & 3.21710100 \\
\hline $\mathrm{H}$ & 9.30117700 & 6.69970200 & 8.26356200 & $\mathrm{C}$ & 6.04305400 & -2.79459300 & 4.10618900 \\
\hline $\mathrm{H}$ & 10.30597600 & 6.72001600 & 6.81211000 & $\mathrm{C}$ & 7.03951200 & -4.94716800 & 3.36799800 \\
\hline $\mathrm{H}$ & 10.32561500 & 5.32531500 & 7.88960900 & $\mathrm{C}$ & 8.04467700 & -5.31360600 & 2.46209900 \\
\hline $\mathrm{C}$ & 10.16621700 & 3.66012500 & 5.98865700 & $\mathrm{C}$ & 6.54691600 & -5.87313200 & 4.29646800 \\
\hline $\mathrm{H}$ & 10.89449400 & 4.47012500 & 5.87659200 & $\mathrm{C}$ & 8.59284000 & -6.59329700 & 2.53046400 \\
\hline $\mathrm{H}$ & 10.31547600 & 2.95747200 & 5.17340300 & $\mathrm{H}$ & 8.36726100 & -4.60879800 & 1.70241700 \\
\hline $\mathrm{H}$ & 10.38887100 & 3.13235600 & 6.92401000 & $\mathrm{C}$ & 7.10614900 & -7.15000900 & 4.35091200 \\
\hline $\mathrm{H}$ & 7.72036400 & 0.26323700 & 1.44594600 & $\mathrm{H}$ & 5.71513900 & -5.62147000 & 4.94265800 \\
\hline $\mathrm{H}$ & 8.61145300 & -0.68461800 & 3.43991800 & $\mathrm{C}$ & 8.13454700 & -7.51045300 & 3.47933300 \\
\hline $\mathrm{C}$ & 10.85835000 & -2.72464600 & 4.40250900 & $\mathrm{H}$ & 9.37535400 & -6.87236600 & 1.83350400 \\
\hline $\mathrm{H}$ & 10.34842600 & -2.46048400 & 5.33316600 & $\mathrm{H}$ & 6.72231500 & -7.86650600 & 5.07138700 \\
\hline
\end{tabular}




\begin{tabular}{|c|c|c|c|c|c|c|c|}
\hline $\mathrm{H}$ & 6.57062200 & -3.25383900 & 2.22262000 & $\mathrm{O}$ & 7.82241400 & -4.01393100 & -0.94980700 \\
\hline $\mathrm{C}$ & 5.50475800 & -1.50001100 & 3.60844500 & $\mathrm{O}$ & 5.90105300 & -2.44915700 & -1.82947700 \\
\hline $\mathrm{H}$ & 5.40995800 & -1.50075100 & 2.52308700 & $\mathrm{P}$ & 7.15796300 & -2.49484400 & -0.72302200 \\
\hline $\mathrm{H}$ & 4.53537300 & -1.30100800 & 4.07969300 & $\mathrm{O}$ & 6.58085300 & -2.51482000 & 0.67146500 \\
\hline $\mathrm{H}$ & 6.15762100 & -0.66606100 & 3.90619000 & $\mathrm{O}$ & 8.15740300 & -1.44164300 & -1.10169000 \\
\hline $\mathrm{H}$ & 8.56448600 & -8.50684000 & 3.52614900 & $\mathrm{C}$ & 10.61269200 & -4.05067400 & -2.22069500 \\
\hline $\mathrm{C}$ & 6.07960500 & -3.05826800 & 5.59696000 & $\mathrm{C}$ & 11.37755500 & -4.90893400 & -1.39260600 \\
\hline $\mathrm{C}$ & 4.75548200 & -3.61411100 & 6.14464900 & $\mathrm{C}$ & 11.18375600 & -2.84531100 & -2.69982300 \\
\hline $\mathrm{C}$ & 6.51129900 & -1.77328400 & 6.35014100 & $\mathrm{C}$ & 12.69538100 & -4.55159100 & -1.07881900 \\
\hline $\mathrm{C}$ & 3.59282200 & -3.74585800 & 5.37277000 & $\mathrm{C}$ & 12.50948700 & -2.54197300 & -2.36030400 \\
\hline $\mathrm{C}$ & 4.71116400 & -4.02385600 & 7.48734700 & $\mathrm{C}$ & 13.29278800 & -3.38839400 & -1.56964200 \\
\hline $\mathrm{H}$ & 7.44103300 & -1.35828300 & 5.95029400 & $\mathrm{H}$ & 13.29673400 & -5.22647800 & -0.47416200 \\
\hline $\mathrm{C}$ & 2.41540800 & -4.25195500 & 5.93188900 & $\mathrm{H}$ & 12.95202000 & -1.63355300 & -2.76212600 \\
\hline $\mathrm{H}$ & 3.59124400 & -3.46586700 & 4.32329200 & $\mathrm{C}$ & 3.26821500 & -1.00544700 & -1.47759700 \\
\hline $\mathrm{C}$ & 3.53848200 & -4.52943300 & 8.04511000 & $\mathrm{C}$ & 3.24015700 & -0.60766500 & -2.84480500 \\
\hline $\mathrm{C}$ & 2.38159100 & -4.64098100 & 7.26948000 & $\mathrm{C}$ & 2.83826100 & -0.08040800 & -0.48988800 \\
\hline $\mathrm{H}$ & 1.52652000 & -4.34117900 & 5.31344000 & $\mathrm{C}$ & 2.78508400 & 0.67299200 & -3.17859800 \\
\hline $\mathrm{H}$ & 3.52924400 & -4.83826900 & 9.08691100 & $\mathrm{C}$ & 2.38388400 & 1.18475800 & -0.88780500 \\
\hline $\mathrm{H}$ & 1.46634200 & -5.03309000 & 7.70389400 & $\mathrm{C}$ & 2.33940000 & 1.58712600 & -2.22118600 \\
\hline $\mathrm{H}$ & 6.85052100 & -3.81869800 & 5.77179700 & $\mathrm{H}$ & 2.76254500 & 0.95534800 & -4.22821700 \\
\hline $\mathrm{H}$ & 5.74920900 & -0.99277200 & 6.29325300 & $\mathrm{H}$ & 2.04319000 & 1.88366300 & -0.12745500 \\
\hline $\mathrm{H}$ & 6.67096000 & -2.01375400 & 7.40500800 & $\mathrm{C}$ & 10.43641100 & -1.91441000 & -3.65805100 \\
\hline \multirow[t]{2}{*}{$\mathrm{H}$} & 5.60419000 & -3.94806800 & 8.10231500 & $\mathrm{H}$ & 9.39055800 & -2.23194400 & -3.68252400 \\
\hline & & & & $\mathrm{C}$ & 14.77470100 & -3.12215500 & -1.32248200 \\
\hline TS-14-SS & & & & $\mathrm{H}$ & 15.13291500 & -3.90830500 & -0.64340100 \\
\hline $\mathrm{C}$ & 4.82155700 & -5.75289800 & -3.66802700 & $\mathrm{C}$ & 10.85461300 & -6.27774100 & -0.94883500 \\
\hline $\mathrm{C}$ & 7.15142900 & -5.80299400 & -4.09454800 & $\mathrm{H}$ & 9.76273800 & -6.25806600 & -1.01834800 \\
\hline $\mathrm{C}$ & 5.86041300 & -6.40463300 & -4.59604200 & $\mathrm{C}$ & 10.43923400 & -0.44446800 & -3.20125300 \\
\hline $\mathrm{H}$ & 4.52491500 & -4.78176300 & -4.07985300 & $\mathrm{H}$ & 9.89275500 & 0.17007000 & -3.92671800 \\
\hline $\mathrm{H}$ & 3.91252000 & -6.34755100 & -3.53755500 & $\mathrm{H}$ & 11.45579100 & -0.03684900 & -3.12838700 \\
\hline $\mathrm{H}$ & 5.86687500 & -7.49894300 & -4.48682700 & $\mathrm{H}$ & 9.93315700 & -0.34387900 & -2.23979100 \\
\hline $\mathrm{H}$ & 5.67665500 & -6.19492000 & -5.65628000 & $\mathrm{C}$ & 11.00184200 & -2.03096500 & -5.08941700 \\
\hline $\mathrm{C}$ & 5.57700700 & -5.50935900 & -2.30940400 & $\mathrm{H}$ & 10.43491000 & -1.39076100 & -5.77623900 \\
\hline $\mathrm{C}$ & 5.47016100 & -6.78996900 & -1.42480500 & $\mathrm{H}$ & 10.94901800 & -3.05788200 & -5.46577300 \\
\hline $\mathrm{C}$ & 4.74547600 & -4.53178900 & -1.48628400 & $\mathrm{H}$ & 12.05200900 & -1.71525200 & -5.12789900 \\
\hline $\mathrm{C}$ & 4.03749100 & -6.73051900 & -0.83722000 & $\mathrm{C}$ & 15.05341600 & -1.77040900 & -0.64173400 \\
\hline $\mathrm{H}$ & 5.66620100 & -7.70715800 & -1.99022000 & $\mathrm{H}$ & 16.13012600 & -1.63282000 & -0.48448200 \\
\hline $\mathrm{H}$ & 6.21122900 & -6.72766200 & -0.61960900 & $\mathrm{H}$ & 14.56265600 & -1.70917100 & 0.33646500 \\
\hline $\mathrm{H}$ & 3.98163500 & -7.16206600 & 0.16941600 & $\mathrm{H}$ & 14.69933100 & -0.93127100 & -1.25241200 \\
\hline $\mathrm{H}$ & 3.32497700 & -7.29208700 & -1.45701700 & $\mathrm{C}$ & 15.58045200 & -3.24600100 & -2.63165700 \\
\hline $\mathrm{C}$ & 6.98199300 & -5.22980400 & -2.82539600 & $\mathrm{H}$ & 16.65450400 & -3.12841100 & -2.44149300 \\
\hline $\mathrm{C}$ & 8.04737800 & -4.57149300 & -2.19910900 & $\mathrm{H}$ & 15.28088300 & -2.47653100 & -3.35310300 \\
\hline $\mathrm{C}$ & 8.38588800 & -5.77330200 & -4.72963700 & $\mathrm{H}$ & 15.42071200 & -4.22245900 & -3.10156000 \\
\hline $\mathrm{C}$ & 9.32354800 & -4.56482100 & -2.80672300 & $\mathrm{C}$ & 11.36042100 & -7.38104700 & -1.90322700 \\
\hline $\mathrm{C}$ & 9.45408100 & -5.17444500 & -4.06948200 & $\mathrm{H}$ & 12.45545700 & -7.44365200 & -1.87937300 \\
\hline $\mathrm{H}$ & 8.52200700 & -6.21448200 & -5.71387900 & $\mathrm{H}$ & 11.05528800 & -7.18682000 & -2.93595600 \\
\hline $\mathrm{H}$ & 10.43606500 & -5.17115800 & -4.53318900 & $\mathrm{H}$ & 10.96001400 & -8.35945400 & -1.60929400 \\
\hline $\mathrm{C}$ & 3.72723800 & -5.24594100 & -0.84097400 & $\mathrm{C}$ & 11.20543900 & -6.64069000 & 0.50418500 \\
\hline $\mathrm{C}$ & 2.60309800 & -4.57937000 & -0.36606900 & $\mathrm{H}$ & 10.74540900 & -7.60025700 & 0.76785400 \\
\hline $\mathrm{C}$ & 4.74099900 & -3.13401800 & -1.47662500 & $\mathrm{H}$ & 10.83551900 & -5.89178400 & 1.20952100 \\
\hline $\mathrm{C}$ & 2.50083600 & -3.20720200 & -0.59630300 & $\mathrm{H}$ & 12.28596700 & -6.75130900 & 0.65430600 \\
\hline $\mathrm{H}$ & 1.79067500 & -5.11650600 & 0.11742200 & $\mathrm{C}$ & 3.63298400 & -1.52684500 & -4.00303300 \\
\hline $\mathrm{C}$ & 3.55297900 & -2.44383300 & -1.13806900 & $\mathrm{H}$ & 3.90757300 & -2.49972000 & -3.59353200 \\
\hline $\mathrm{H}$ & 1.57469000 & -2.69748900 & -0.35458000 & $\mathrm{C}$ & 1.80862500 & 2.96151100 & -2.60762900 \\
\hline
\end{tabular}




\begin{tabular}{|c|c|c|c|c|c|c|c|}
\hline $\mathrm{H}$ & 1.56837100 & 3.48764700 & -1.67333100 & $\mathrm{H}$ & 5.19947700 & 7.33952500 & 5.06898700 \\
\hline $\mathrm{C}$ & 2.82439500 & -0.39157000 & 1.00954200 & $\mathrm{C}$ & 7.97520400 & 8.01738700 & 6.79911700 \\
\hline $\mathrm{H}$ & 3.35187900 & -1.33823100 & 1.16430300 & $\mathrm{H}$ & 7.75290600 & 8.08241300 & 7.87414400 \\
\hline $\mathrm{C}$ & 2.85941500 & 3.80511200 & -3.35391700 & $\mathrm{H}$ & 7.23767200 & 8.63441200 & 6.27998000 \\
\hline $\mathrm{H}$ & 2.47309600 & 4.81136400 & -3.55709300 & $\mathrm{H}$ & 8.94775200 & 8.48884400 & 6.64184200 \\
\hline $\mathrm{H}$ & 3.12196200 & 3.35129800 & -4.31734400 & $\mathrm{C}$ & 10.43846400 & 6.42991300 & 6.80318600 \\
\hline $\mathrm{H}$ & 3.77977700 & 3.90265200 & -2.76816400 & $\mathrm{H}$ & 10.24357000 & 7.11892500 & 7.6285470 \\
\hline $\mathrm{C}$ & 0.50642800 & 2.85569900 & -3.42530300 & $\mathrm{H}$ & 11.00242800 & 6.98733500 & 6.04074600 \\
\hline $\mathrm{H}$ & 0.10665400 & 3.85222500 & -3.64981600 & $\mathrm{H}$ & 11.10305300 & 5.65436700 & 7.19036300 \\
\hline $\mathrm{H}$ & -0.25981800 & 2.29496300 & -2.87870500 & $\mathrm{C}$ & 10.48301600 & 3.81957300 & 5.6035270 \\
\hline $\mathrm{H}$ & 0.67871000 & 2.34210700 & -4.37876200 & $\mathrm{H}$ & 11.25732200 & 4.51352900 & 5.2578480 \\
\hline $\mathrm{C}$ & 2.44957400 & -1.77376300 & -4.96052600 & $\mathrm{H}$ & 10.43073200 & 2.99190400 & 4.90236300 \\
\hline $\mathrm{H}$ & 2.13056100 & -0.85352900 & -5.46403700 & $\mathrm{H}$ & 10.80540100 & 3.41028200 & 6.5682830 \\
\hline $\mathrm{H}$ & 1.58354600 & -2.17859900 & -4.42467600 & $\mathrm{H}$ & 7.28370100 & -0.05393800 & 1.6245990 \\
\hline $\mathrm{H}$ & 2.73450500 & -2.49294200 & -5.73839300 & $\mathrm{H}$ & 8.06432800 & -1.21469400 & 3.88592800 \\
\hline $\mathrm{C}$ & 4.86440600 & -0.99333300 & -4.76028900 & $\mathrm{C}$ & 10.78805300 & -2.52990800 & 5.3099210 \\
\hline $\mathrm{H}$ & 5.71381800 & -0.87051600 & -4.08229100 & $\mathrm{H}$ & 10.31182800 & -2.04933700 & 6.1694100 \\
\hline $\mathrm{H}$ & 4.65698400 & -0.02551400 & -5.23358500 & $\mathrm{H}$ & 11.77477000 & -2.88735400 & 5.6377890 \\
\hline $\mathrm{H}$ & 5.15767000 & -1.69299500 & -5.55287100 & $\mathrm{H}$ & 10.20123600 & -3.40237700 & 5.0203920 \\
\hline $\mathrm{C}$ & 1.38751000 & -0.54518100 & 1.55348200 & $\mathrm{C}$ & 11.77325800 & 0.41061500 & 5.64581100 \\
\hline $\mathrm{H}$ & 0.82755400 & 0.39162000 & 1.44667400 & $\mathrm{H}$ & 10.91661500 & 0.46134600 & 6.3213030 \\
\hline $\mathrm{H}$ & 1.40688300 & -0.80233400 & 2.61972900 & $\mathrm{H}$ & 12.13851800 & 1.42975800 & 5.50703400 \\
\hline $\mathrm{H}$ & 0.82576800 & -1.32477000 & 1.03012300 & $\mathrm{H}$ & 12.57364600 & -0.16699400 & 6.1280380 \\
\hline $\mathrm{C}$ & 3.55316000 & 0.68973100 & 1.83209000 & $\mathrm{C}$ & 12.24266700 & 1.66100400 & 2.69786600 \\
\hline $\mathrm{H}$ & 4.58005300 & 0.83851400 & 1.48511400 & $\mathrm{H}$ & 13.33880300 & 1.58112200 & 2.71622600 \\
\hline $\mathrm{H}$ & 3.58978200 & 0.40715700 & 2.88944200 & $\mathrm{H}$ & 11.95163500 & 2.41575500 & 3.43075800 \\
\hline $\mathrm{H}$ & 3.04340300 & 1.65786600 & 1.77309500 & $\mathrm{H}$ & 11.95813200 & 2.02352000 & 1.70644500 \\
\hline $\mathrm{C}$ & 11.40633400 & -0.22039300 & 4.33294100 & $\mathrm{C}$ & 11.44343000 & -0.59599900 & $0.5555350 \mathrm{C}$ \\
\hline $\mathrm{C}$ & 11.62794600 & 0.32987000 & 3.00291500 & $\mathrm{H}$ & 12.41701000 & -0.99009000 & 0.2446500 \\
\hline $\mathrm{C}$ & 11.30546700 & -0.69465300 & 2.04331500 & $\mathrm{H}$ & 11.37938100 & 0.43829200 & 0.20589200 \\
\hline $\mathrm{C}$ & 10.87328600 & -1.87763200 & 2.75691700 & $\mathrm{H}$ & 10.68359500 & -1.18143500 & 0.0317660 \\
\hline $\mathrm{C}$ & 10.96465900 & -1.57012700 & 4.17330700 & $\mathrm{C}$ & 10.65221600 & -3.22113000 & 2.12884700 \\
\hline Ir & 9.46363200 & -0.22800000 & 3.17703300 & $\mathrm{H}$ & 10.06208100 & -3.15444000 & 1.2108510 \\
\hline $\mathrm{N}$ & 8.68980000 & 1.84328900 & 3.47085800 & $\mathrm{H}$ & 10.13828500 & -3.90491500 & 2.8055350 \\
\hline $\mathrm{N}$ & 8.27036800 & 0.12357700 & 1.43010000 & $\mathrm{H}$ & 11.61740900 & -3.66997900 & $1.8596790 \mathrm{C}$ \\
\hline $\mathrm{H}$ & 8.47181100 & -0.48143800 & 0.61485600 & $\mathrm{H}$ & 6.88911300 & 2.20364800 & 2.36169500 \\
\hline $\mathrm{C}$ & 7.97410600 & 2.38091300 & 2.28285500 & $\mathrm{C}$ & 8.18974400 & 3.87097800 & 2.02842900 \\
\hline $\mathrm{C}$ & 8.47033900 & 1.55856400 & 1.06611300 & $\mathrm{C}$ & 9.45466300 & 4.45846500 & 2.15384700 \\
\hline S & 7.93026200 & 2.16327800 & 4.91420200 & $\mathrm{C}$ & 7.12749200 & 4.66254700 & 1.57611600 \\
\hline $\mathrm{O}$ & 8.76071300 & 1.55080300 & 5.97183100 & $\mathrm{C}$ & 9.65195100 & 5.80319800 & 1.83901300 \\
\hline $\mathrm{O}$ & 6.50788400 & 1.75015700 & 4.87567500 & $\mathrm{H}$ & 10.28156500 & 3.85857800 & 2.51778800 \\
\hline $\mathrm{C}$ & 7.94135700 & 3.97421300 & 5.27199200 & $\mathrm{C}$ & 7.31942300 & 6.00900500 & 1.26229100 \\
\hline $\mathrm{C}$ & 6.73766500 & 4.71420200 & 5.28429700 & $\mathrm{H}$ & 6.14241500 & 4.21868900 & 1.45866000 \\
\hline $\mathrm{C}$ & 9.15598400 & 4.53967600 & 5.71674100 & $\mathrm{C}$ & 8.58386400 & 6.58481500 & 1.39247100 \\
\hline $\mathrm{C}$ & 6.75659700 & 6.01160300 & 5.84577000 & $\mathrm{H}$ & 10.64070400 & 6.24276300 & 1.94593200 \\
\hline $\mathrm{C}$ & 9.15207200 & 5.84132100 & 6.25173200 & $\mathrm{H}$ & 6.48056400 & 6.60574600 & 0.91364700 \\
\hline $\mathrm{C}$ & 7.95846700 & 6.58520300 & 6.29621000 & $\mathrm{H}$ & 8.73643100 & 7.63302300 & 1.14905700 \\
\hline $\mathrm{C}$ & 5.42293700 & 4.23797300 & 4.69292700 & $\mathrm{H}$ & 9.55281600 & 1.70186500 & 1.00947800 \\
\hline $\mathrm{H}$ & 4.78116700 & 3.77112600 & 5.45005500 & $\mathrm{C}$ & 7.89264100 & 1.95858800 & -0.27965000 \\
\hline $\mathrm{H}$ & 5.56069400 & 3.49870100 & 3.91091100 & $\mathrm{C}$ & 6.65016100 & 1.50981300 & -0.73768100 \\
\hline $\mathrm{H}$ & 4.88016600 & 5.08596600 & 4.26670100 & $\mathrm{C}$ & 8.64237800 & 2.81357200 & -1.10047400 \\
\hline $\mathrm{C}$ & 5.45588800 & 6.78400400 & 5.98285100 & $\mathrm{C}$ & 6.16413500 & 1.91231500 & -1.98237000 \\
\hline $\mathrm{H}$ & 5.50368200 & 7.50749400 & 6.79934500 & $\mathrm{H}$ & 6.05918800 & 0.81406600 & -0.15071000 \\
\hline $\mathrm{H}$ & 4.61642400 & 6.11887100 & 6.19975700 & $\mathrm{C}$ & 8.15787200 & 3.22447400 & -2.34096900 \\
\hline
\end{tabular}




$\begin{array}{llllllll}\mathrm{H} & 9.60980700 & 3.17151700 & -0.75693300 & \mathrm{C} & 6.98063400 & -2.32760300 & 5.67816200 \\ \mathrm{C} & 6.91407100 & 2.77289000 & -2.78518600 & \mathrm{C} & 6.02628900 & -3.41792100 & 6.20678900 \\ \mathrm{H} & 5.20592200 & 1.53866700 & -2.32477900 & \mathrm{C} & 6.74754700 & -1.03689100 & 6.48637900 \\ \mathrm{H} & 8.75463500 & 3.88891000 & -2.96035200 & \mathrm{C} & 4.89111500 & -3.89303700 & 5.53864100 \\ \mathrm{H} & 6.53475700 & 3.07993600 & -3.75631500 & \mathrm{C} & 6.29453800 & -3.93365500 & 7.48676100 \\ \mathrm{~N} & 7.03106800 & -3.20926500 & 3.32728100 & \mathrm{H} & 7.46405900 & -0.25889600 & 6.22784100 \\ \mathrm{C} & 6.91272800 & -2.11432400 & 4.14945500 & \mathrm{C} & 4.05073600 & -4.83946500 & 6.13267300 \\ \mathrm{C} & 7.57468400 & -4.50332500 & 3.45230400 & \mathrm{H} & 4.65976700 & -3.55417600 & 4.53585600 \\ \mathrm{C} & 7.48703100 & -5.29424100 & 2.28525300 & \mathrm{C} & 5.46058300 & -4.87803300 & 8.08160800 \\ \mathrm{C} & 8.14716000 & -5.07852400 & 4.60179300 & \mathrm{C} & 4.32703800 & -5.33469900 & 7.40540600 \\ \mathrm{C} & 7.93864700 & -6.60836000 & 2.27839900 & \mathrm{H} & 3.17864300 & -5.19136100 & 5.58777200 \\ \mathrm{H} & 7.06877500 & -4.85643900 & 1.38541900 & \mathrm{H} & 5.69819900 & -5.25845200 & 9.07177900 \\ \mathrm{C} & 8.60309500 & -6.39853200 & 4.57642800 & \mathrm{H} & 3.67294300 & -6.07158100 & 7.86350400 \\ \mathrm{H} & 8.22172700 & -4.53326600 & 5.52814800 & \mathrm{H} & 7.99870300 & -2.65257100 & 5.91295100 \\ \mathrm{C} & 8.50164400 & -7.17623600 & 3.42487000 & \mathrm{H} & 5.73929500 & -0.64118200 & 6.34064000 \\ \mathrm{H} & 7.85336700 & -7.19060700 & 1.36482200 & \mathrm{H} & 6.86087300 & -1.26264000 & 7.55146500 \\ \mathrm{H} & 9.03259700 & -6.81844700 & 5.48224200 & \mathrm{H} & 7.17389000 & -3.58684800 & 8.02609700 \\ \mathrm{H} & 6.79033000 & -3.00009800 & 2.34444600 & & & & \\ \mathrm{C} & 5.81694500 & -1.17360400 & 3.66122900 & & & & \\ \mathrm{H} & 5.75478000 & -1.19861000 & 2.57074500 & & & & \end{array}$




\section{References.}

1. (a) A. D. Becke, J. Chem. Phys. 1993, 98, 5648-5652. (b) C. Lee, W. Yang, R. G. Parr, Phys. Rev. B: Condens. Matter Mater. Phys. 1998, 37, 785-789. (c) P. J. Stephens, F. J. Devlin, C. F. Chabalowski, M. J. Frisch, J. Phys. Chem. 1994, 98, 11623-11627.

2. (a) W. J. Hehre, R. Ditchfield, J. A. Pople, J. Chem. Phys. 1972, 56, 2257-2261. (b) J. D. Dill, J. A. Pople, J. Chem. Phys. 1975, 62. 2921-2923. (c) M. M. Francl, W. J. Pietro, W. J. Hehre, J. S. Binkley, M. S. Gordon, D. J. DeFrees, J. A. Pople, J. Chem. Phys. 1982, 77, 3654-3665.

3. D. Andrae, U. Haeussermann, M. Dolg, H. Stoll, H. Preuss, Theor. Chim. Acta 1990, 77, 123-141.

4. Y. Zhao, D. G. Truhlar, Theor. Chem. Acc. 2008, 120, 215-241.

5. (a) R. Krishnan, J. S. Binkley, R. Seeger, J. A. Pople, J. Chem. Phys. 1980, 72, 650-654. (b) A. D. McLean, G. S. Chandler, J. Chem. Phys. 1980, 72, 5639-5648.

6. (a) P. J. Hay, W. R. Wadt, J. Chem. Phys. 1985, 82, 299-310. (b) L. E. Roy, P. J. Hay, R. L. Martin. J. Chem. Theory Comput. 2008, 4, 1029-1031. (c) A. W. Ehlers, M. Böhme, S. Dapprich, A. Gobbi, A. Höllwarth, V. Jonas, K. F. Köhler, R. Stegmann, A. Veldkamp, G. Frenking. Chem. Phys. Lett. 1993, 208, 111-114.

7. (a) V. Barone, M. Cossi, J. Phys. Chem. A 1998, 102, 1995-2001. (b) M. Cossi, N. Rega, G. Scalmani, V. Barone, J. Comp. Chem. 2003, 24, 669-681. 